\title{
Cigarette Mainstream Smoke: The Evolution of Methods and Devices for Generation, Exposure and Collection*
}

\author{
by \\ Hubert Klus ${ }^{1}$, Barbara Boenke-Nimphius ${ }^{2}$, and Lutz Müller ${ }^{3}$ \\ Oriongasse 9,3100 St. Pölten, Austria \\ Beiträge zur Tabakforschung International, Chausseestraße 51A, 10115 Berlin, Germany \\ Stralsunder Straße 1, 01109 Dresden, Germany
}

\section{SUMMARY}

The objective of this review is to support tobacco scientists when evaluating information published on smoking machines, and on cigarette mainstream smoke (in vivo and in vitro) exposure systems and collection devices.

The intriguing development of smoking machines (mainly for cigarettes) is followed for more than 170 years - from the first simple set-ups in the 1840 s to the sophisticated and fully automated analytical smoking machines available today. Systems for the large-scale production of smoke (condensate) for preparative work are equally considered. The standardization of machine smoking methods and test pieces has solved several technical problems and produced sensible rules but, at the same time, given rise to new controversies like the compatibility of artificial and human smoking, and the implementation of more intense machine smoking regimes.

Adequate space is allotted for the discussion of configurations for in vivo smoke exposure of rodent and non-rodent species and the machines generating the required smoke (condensate). Covered as well is the field of in vitro toxicity testing, including the increasingly informative new techniques of air-liquid interface exposure, which are becoming more and more refined with the use of organotypic cultures and genetic analyses.

The review is completed by the examination of the considerable variety of mainstream smoke collection devices (filters and traps) developed over time - some for very specific purposes - and refers to the perpetual problem of artifact formation by aging. [Beitr. Tabakforsch. Int. 27 (2016) 137-274]

\section{ZUSAMMENFASSUNG}

Es ist die Intention dieser Übersicht, auf dem Gebiet des Tabaks arbeitende Wissenschaftler zu unterstützen bei der Bewertung publizierter Informationen über Rauchmaschinen sowie über Systeme zur experimentellen Exposition (in vivo und in vitro) mit Zigarettenhauptstromrauch und Apparate zu dessen Sammlung.

Die sehr interessante Entwicklung von Rauchmaschinen (vornehmlich für Zigaretten) wird über einen Zeitraum von mehr als 170 Jahren nachgezeichnet - von den ersten einfachen Apparaten um 1840 bis zu den hochentwickelten und vollautomatisierten analytischen Rauchmaschinen, die heute verfügbar sind. Systeme für die Produktion von Rauch (und Kondensat) in großem Maßstab für präparative Arbeiten werden gleichfalls besprochen.

Die Standardisierung maschineller Abrauchmethoden und von Prüfstücken hat mehrere technische Probleme gelöst und zu vernünftigen Vorschriften geführt, aber zugleich neue Kontroversen hervorgerufen wie die Vereinbarkeit maschinellen Abrauchens und natürlichen Rauchens und die Anwendung intensivierter Parameter für Rauchmaschinen. 
Angemessener Raum ist zugemessen der Diskussion von Anordnungen zur in vivo Rauchbelastung von Versuchstieren (Nagern und Nichtnagern) und der Maschinen zur Produktion von erforderlichem Rauch und Kondensat. Besprochen wird auch das Arbeitsgebiet der in vitro Toxizitätsuntersuchungen, einschließlich der immer aussagekräftigeren neuen Techniken der air-liquid interface Expositionen, welche zunehmend ausgefeilter werden mit dem Einsatz organtypischer Kulturen und genetischer Analysen. Die Übersicht wird vervollständigt durch die Besprechung der ansehnlichen Vielfalt von Geräten (Filter und Fallen) zur Sammlung von Hauptstromrauch, die im Laufe der Zeit entwickelt wurden - einige für sehr spezielle Anwendungen - und spricht das permanente Problem der Artefaktbildung durch Alterung an. [Beitr. Tabakforsch. Int. 27 (2016) 137-274]

\section{RESUME}

La finalité du présent article est de prêter assistance aux scientifiques étudiant le tabac dans leur évaluation des informations publiées au sujet des machines à fumer et des systèmes d'exposition (in vivo et in vitro) et des dispositifs de collecte de la fumée principale de la cigarette.

L'évolution fascinante des machines à fumer (principalement pensés pour les cigarettes) est suivie depuis plus de 170 ans, des premiers appareils simples des années 1840 aux dispositifs analytiques, totalement automatisés, disponibles aujourd'hui. Des systèmes conçus pour la production à grande échelle de fumée (condensat), lors du travail préparatoire, ont également été considérés.

La normalisation des méthodes et des pièces d'essai pour les machines à fumer a permis de résoudre plusieurs problèmes techniques et a énoncé des règles judicieuses; cependant, dans un même temps, elle a aussi suscité de nouvelles controverses, telles que la compatibilité de la fumée humaine et de la fumée artificielle, et la mise en œuvre de paramètres de fumage plus intenses pour les ma- chines.

L'article fait une part belle à la discussion concernant les configurations pour l'exposition à la fumée in vivo d'animaux de laboratoire (rongeurs et non-rongeurs) et pour les machines produisant la fumée requise (condensat). Il couvre également le champ des essais de toxicité in vitro, y compris les nouvelles techniques d'exposition en interface air-liquide qui livrent de plus en plus d'informations et gagnent en affinage grâce à l'utilisation des cultures organotypiques et des analyses génétiques.

L'article est complété d'un examen de la vaste gamme des dispositifs de collecte de la fumée principale (filtres et pièges) mis au point au fil du temps - certains à des fins très spécifiques - et évoque le sempiternel problème de la formation d'artéfacts due au vieillissement. [Beitr. Tabakforsch. Int. 27 (2016) 137-274]

\section{ABBREVIATIONS}

$\begin{array}{ll}\text { ALI } & \text { Air-liquid interface } \\ \text { BAT } & \text { British American Tobacco } \\ \text { CORESTA Cooperation Centre for Scientific Research } \\ & \text { Relative to Tobacco } \\ \text { CRM } & \text { CORESTA recommended method } \\ \text { DIN } & \begin{array}{l}\text { Deutsches Institut für Normung } \\ \text { EC }\end{array} \\ & \begin{array}{l}\text { Concentration inducing a response halfway } \\ \text { between baseline and maximal effect }\end{array} \\ \text { ETS } & \text { Environmental tobacco smoke } \\ \text { FTC } & \text { Federal Trade Commission } \\ \text { GC/MS } & \text { Gas chromatography/mass spectrometry } \\ \text { GVP } & \text { Gas vapor phase } \\ \text { ISO } & \text { International Organization for Standardization } \\ \text { NDIR } & \text { Non-dispersive infrared analysis } \\ \text { NFDPM } & \text { Nicotine-free dry particulate matter } \\ \text { OECD } & \text { Organisation for Economic Co-operation and } \\ & \text { Development } \\ \text { QCM } & \text { Quartz crystal microbalance } \\ \text { TPM } & \text { Total particulate matter }\end{array}$




\section{CONTENTS}

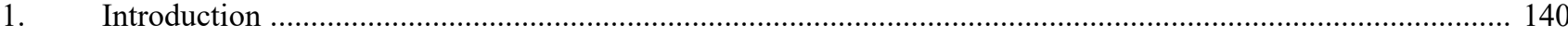

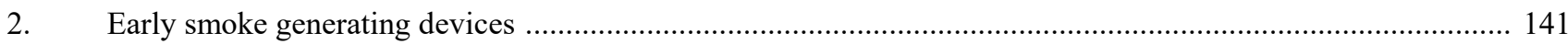

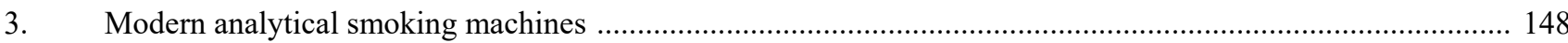

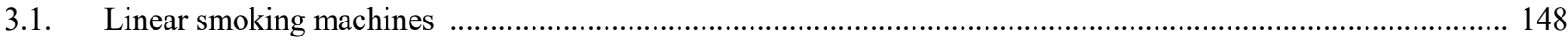

3.1.1. Development of linear smoking machines in the United States ............................................................... 148

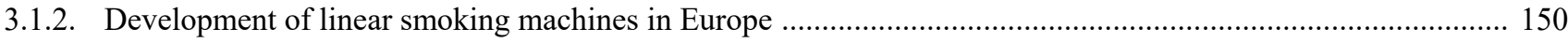

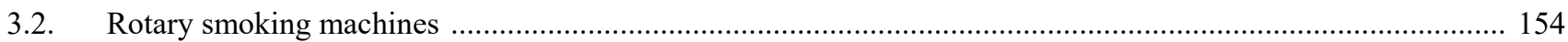

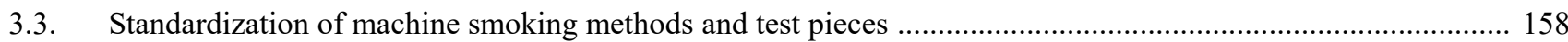

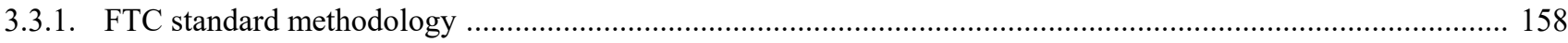

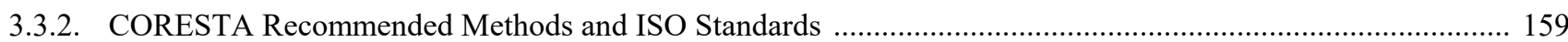

3.3.3. Kentucky reference cigarettes and CORESTA monitor test pieces ......................................................... 161

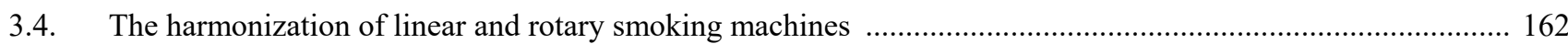

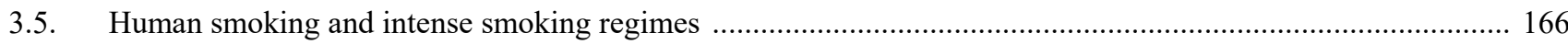

4. Smoking machines for large scale condensate production and in vivo toxicity testing ................................. 174

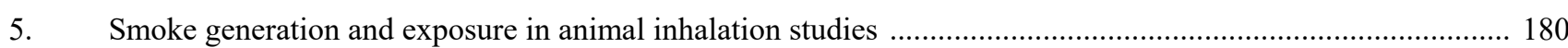

5.1. Smoke generation and exposure in inhalation studies with rodent species ................................................. 180

5.2. Smoke generation and exposure in inhalation studies with non-rodent species ........................................ 193

6. Smoking machines and exposure devices for in vitro smoke toxicity testing .............................................. 196

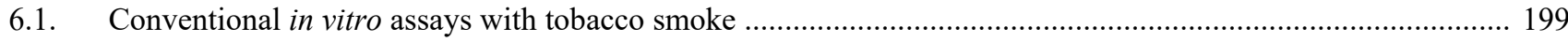

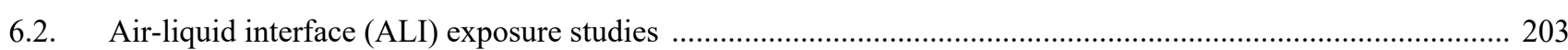

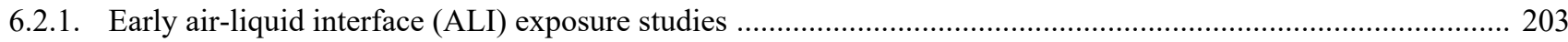

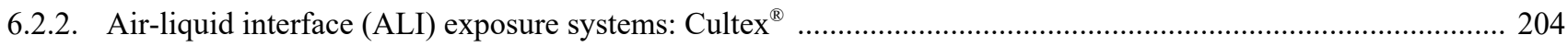

6.2.3. Air-liquid interface (ALI) exposure systems: RM20S and the BAT chamber ..............................................2 214

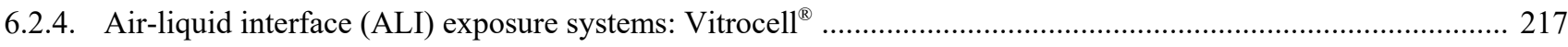

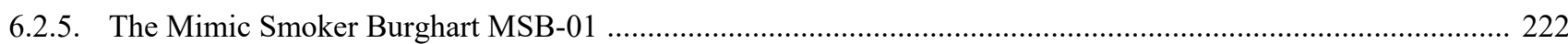

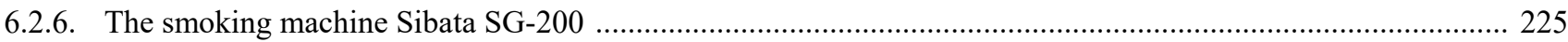

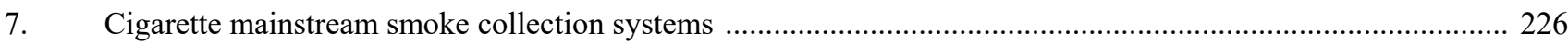

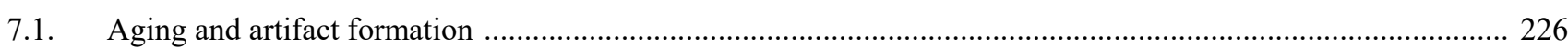

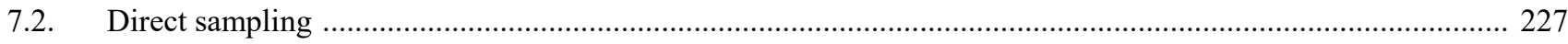

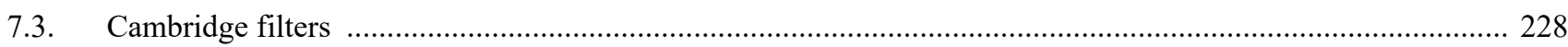

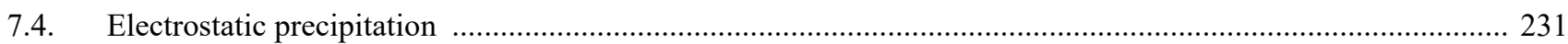

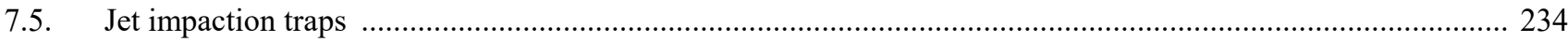

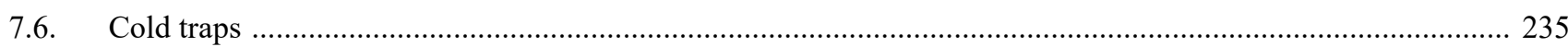

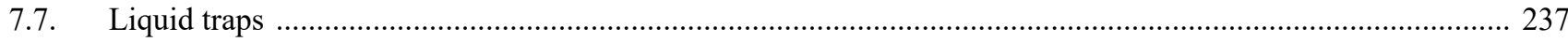

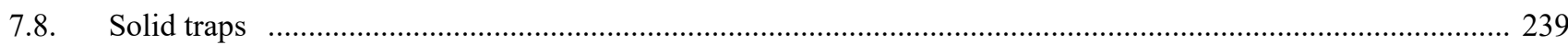

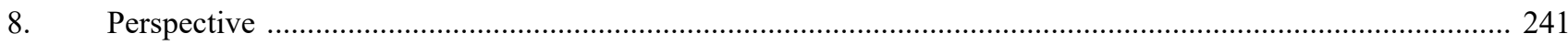

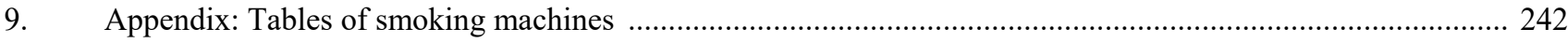

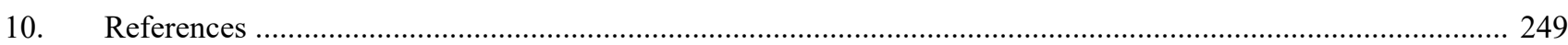




\section{INTRODUCTION}

When in the middle of the $19^{\text {th }}$ century the consumption of smoking articles, made from small tobacco pieces and a paper envelope - and called cigarettes, became more popular in Europe and the United States, it was the begin of a fascinating scientific, technological and commercial story. Laboratory research on tobacco smoke and also on cigarette smoke quickly developed in ambitious and productive ways. What would one see if the evolution of methods and devices for the generation and collection of, and exposure to, cigarette mainstream smoke was attentively followed, critically appraised and put into proper perspective?

For instance:

1843 First meaningful experimental studies on tobacco "combustion" $(1,2)$; correct proportional elemental composition of nicotine established

1908 The first cigarette mainstream smoke inhalation study with rabbits (3)

1936 Bradford's "robot for mass smoking" using standard parameters, namely a $35-\mathrm{mL}$ puff in 2 sec every minute (4)

1939 The first mouse skin painting study with genuine cigarette smoke condensate (5)

1959 The Cambridge glass fiber filter suggested for the quantitative collection of mainstream smoke particulate matter (6)

1959 The preparative 600-port "mammoth" machine for smoking up to 20,000 cigarettes per day (7)

1967 The linear 20-port syringe smoking machine (8) adopted by the U.S. tobacco industry as single design for routine analytical studies

1967 The commencement of formal cigarette testing by the U.S. Federal Trade Commission (FTC) using the "Cambridge Filter Method" (9)

1977 The first edition of ISO 3308, defining smoking parameters and specifying requirements for a routine analytical smoking machine (10)

1991 A CORESTA collaborative study (11) completed with harmonized smoking machines complying with ISO 3308:1991 (12)

1998 The first direct exposure of a mammalian lung cell line to cigarette mainstream smoke at the air-liquid interface (13).

This list, however, is just a glimpse into the multifarious developments of the devices and methods under review; and there is more to it. Several issues and ideas (seemingly recent) surfaced much earlier in time than generally known. On the other side, a number of instruments and setups were not accepted and disappeared quickly. Today, scientific concepts and technical equipment are at a remarkably high level of implementation and performance.

Special circumstances allowed this review to be prepared having regard to quite a few documents, which are not easily accessible or intelligible. Several publications are rather time-honored but nevertheless interesting and important; they are for the most part found neither on the internet nor in tobacco litigation document depositories (14). The reading room of the Staatsbibliothek in Berlin was a valuable source for unconventional documents. In addition, languages other than English played a major role in books and publications dealing with tobacco sciences and printed before the 1950s, and translations are frequently not available. Such materials add a lot of insight and color to the (historical) picture and are readily included in this review article.

The second rich source of relevant literature was the "tobacco library" now maintained by the Institute for Tobacco Research in Berlin after its transfer from the former German Association of Cigarette Manufacturers (VdC). In addition to over 6,000 books, the collection includes over 110,000 documents and published papers, a major part in pdf format. Many items relate to the historical development of tobacco science and the industry in Austria, Germany and other European countries, and would be hard to find anywhere else.

Upon examination of both old and new published material it becomes obvious that work on artificial smoking techniques was driven by rather different motivations. In the beginning, crude thermal methods of tobacco degradation were used $(1,2)$ for the generation of "combustion" products and the isolation and characterization of some smoke constituents foremost nicotine. On the other hand, a study (15) was reported in 1893 on the burning quality of cigar wrapper leaf and the visual appearance of the ash - with no interest in smoke composition at all. As analytical methodology of smoke analysis became more refined, there were first suggestions (16) that tobacco smoke for chemical analysis should be generated by a method mimicking a habitual smoker's behavior as comprehensively and closely as possible. This triggered multiple efforts to document specifics of human smoking by observation and measurement, and to deduce technical parameters recommended or mandated for (analytical) machine smoking (4, 10, 17-19). The conflict area between human and artificial smoking was defined. With the advent of cigarette mass production and vigorous product marketing, analytical smoking machines were perfected with respect to versatility, reliability and automation. Smoking-related health effects - gaining avid interest in the $1950 \mathrm{~s}$ - stimulated the development of methodology and equipment for an enormous number of biological studies with cigarette mainstream smoke in vivo (skin painting of small rodents with smoke condensate, animal smoke inhalation experiments) and in vitro (cytotoxicity and genotoxicity assays). Recently, focus on the consumption behavior and smoke uptake of the individual smoker resulted in the challenge of established standard methods by new regimes (20-22) and consequential turbulence regarding methodology and equipment - truly exciting times!

Both the capability and efficiency of devices for the production, collection and application of cigarette mainstream smoke (and its fractions) were developed and improved impressively over time. Progress in methodology and technology had major influence on the properties of smoke materials and the quality of test and study results. This review is intended to support the fitting interpretation of old and new data in their historical context.

The number of smoking machines discussed in this review article is appreciable. For the reader not to get lost, Ariadne ${ }^{1}$ - recognized as rescuer in labyrinthian situations - has provided a comprehensive table, in which the smoking machines are listed as they were developed; it is found at the end of the text.

\footnotetext{
${ }^{1}$ Ariadne, the daughter of the Crete King Minos, helped the Greek hero Theseus to escape from the labyrinth after killing the man-eating Minotauros by giving Theseus a thread to follow, which he had uncoiled when entering the monster's hideout (23)
} 
The earliest reported smoking devices used continuous suction to smoke tobacco or tobacco products. ZEISE (1) and MELSENS (2) - both in 1843 - were the first who used an aspirator to draw a continuous flow of air through a porcelain pipe with burning tobacco. Particulate matter and certain components of the gas phase were collected in a long and wide glass tube, filled with pieces of broken glass and kept cold, or in aqueous liquid traps (acidified, neutral or basic). For the production of larger amounts of "combustion" products ZEISE resorted to the dry distillation of tobacco (several pounds at a time) and subjected the products to a very involved sequence of separation steps while MELSENS succeeded in isolating nicotine from a washing bottle with diluted sulfuric acid and went on to establish its correct proportional elemental composition (though the empirical formula provided was wrong as an inaccurate atomic mass for carbon was used at that time). Starting from the correct empirical formula, $\mathrm{C}_{10} \mathrm{H}_{14} \mathrm{~N}_{2}$, PINNER (24) established in 1895 the chemical structure of nicotine unequivocally as that of 3-(1-methyl-2-pyrrolidinyl)pyridine; the $(\mathrm{S})-(-)$-enantiomer is the naturally occurring form.

Smoke generation from cigars by continuous suction was the approach taken likewise by KIßLING (25) and THOMS (26). In a study performed in 1903 in Estonia (then part of Russia) PONTAG (27) used cigarettes. These authors investigated the presence and levels of nicotine, several alkyl pyridines, carbon monoxide, hydrogen sulfide and hydrogen cyanide in smoke. $\operatorname{KI} ß \operatorname{LING}(25,28)$ referred to these substances as the highly toxic components of tobacco smoke. With special attention to nicotine he built an apparatus consisting of a holder for experimental and commercial cigars attached to a smoke absorption system of wash bottles charged with ethanol, diluted sulfuric acid and sodium hydroxide solution. This in turn was connected to a source of continuous suction, which was controlled in such a way that a cigar was smoked in about $30 \mathrm{~min}$. KIßLING'S arrangement served as a model for several studies that followed. The apparatus developed by THOMS (26) for collecting smoke constituents consisted essentially of six circularly arranged bottles with various absorption liquids, including blood (for absorption of smoke carbon monoxide), and a larger central vessel containing dry cotton

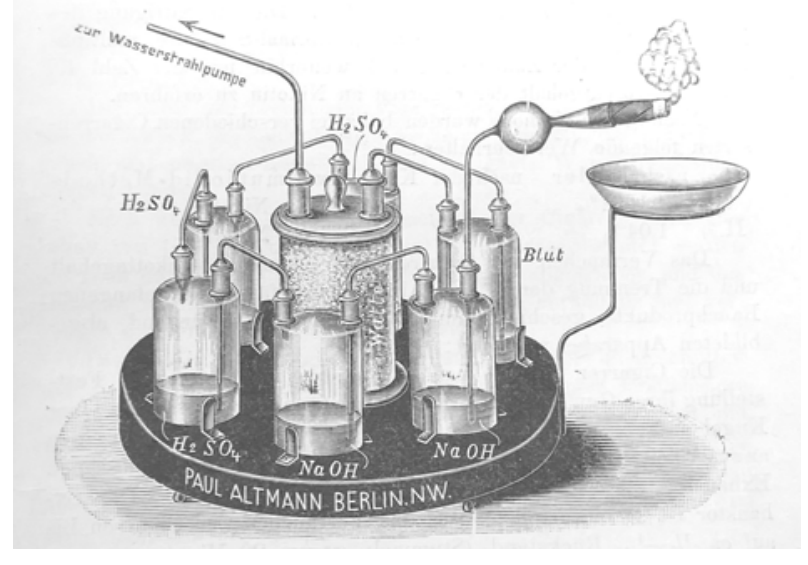

Figure 1. The smoking apparatus developed in 1900 by THOMS (26) used blood for the absorption of smoke carbon monoxide.

\section{First artificial smoking device}

In 1830, ZEISE ${ }^{1}$ was the first to use an artificial device for smoking. However, the development was quite coincidental:

"Die Benutzung des BRUNNER'SCHEN Aspirators bei verschiedenen Arbeiten führte mich auf den Gedanken, ihn als Tabaksraucher anzuwenden, um den Rauch zur genaueren Untersuchung aufzusammeln. Ich habe dieß einfach dardurch ausgeführt, daß ich den Aspirator mit einem Glasrohr verband und dieses wiederum mit einem Pfeifenkopf mit angezündetem Tabak."

["Using the BRUNNER aspirator for various tasks I had the idea of utilizing it as a tobacco smoker to collect smoke for more precise examination. I simply connected the aspirator with a glass tube and this in turn with a pipe bowl containing burning tobacco."]

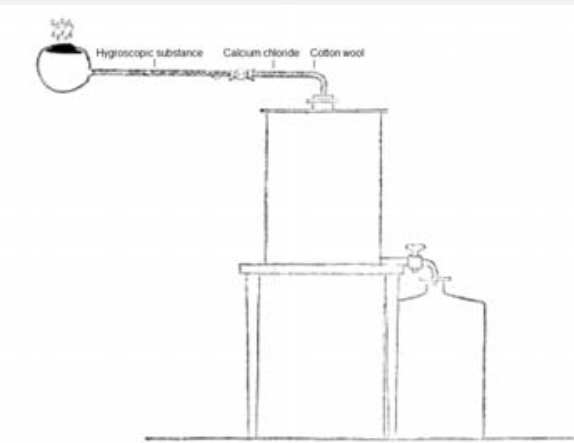

The ZEISE aspirator ${ }^{2}$

Zeise, W.C.: Untersuchung der Producte von der trockenen Destillation des Tabaks und üer die chemische Beschaffenheit des Tabakrauchs; Justus Liebigs Ann Chem 47 (1843) 212-225.

2 Adapted from: Brunner, C.: Ueber die Bestimmung des Wassergehaltes der Atmosphäre [On the determination of water content in the atmosphere]; Ann. Physik Chemie 20 (1830) 274-281.

wool, connected to an aspirator (Figure 1). Incidentally, blood was later recognized by PONTAG (27) to be a rather ineffectual absorbent for smoke carbon monoxide.

It should be pointed out, however, that - as early as 1892 researchers at the CONNECTICUT AGRICULTURAL EXPERIMENT STATION (15) noticed the inadequacy of continuous suction for smoking a cigar as it was much more liable to burn unevenly. Therefore, they described an apparatus employing intermittent suction with an aspirator, which was filled by a continuous and perfectly uniform inflow of water and emptied at regular intervals by means of a siphon (Figure 2). Remarkably, the researchers were not interested at all in the smoke produced; they aimed at examining the burning quality of wrappers, specifically the appearance of the ash formed by the controlled experimental burning of cigars.

Tobacco smoke generation by continuous suction was critically assessed at the turn of the $19^{\text {th }}$ century by HABERMANN (16). HABERMANN was professor of analytical chemistry at the K.K. Technical University of Brünn (then a city in the Kingdom of Bohemia, a part of the AustrianHungarian Monarchy; now Brno in the Czech Republic). His studies on tobacco and tobacco smoke generated from different tobacco products (cigars, cigarettes and pipes) were suggested and supported by the Austrian Tobacco 


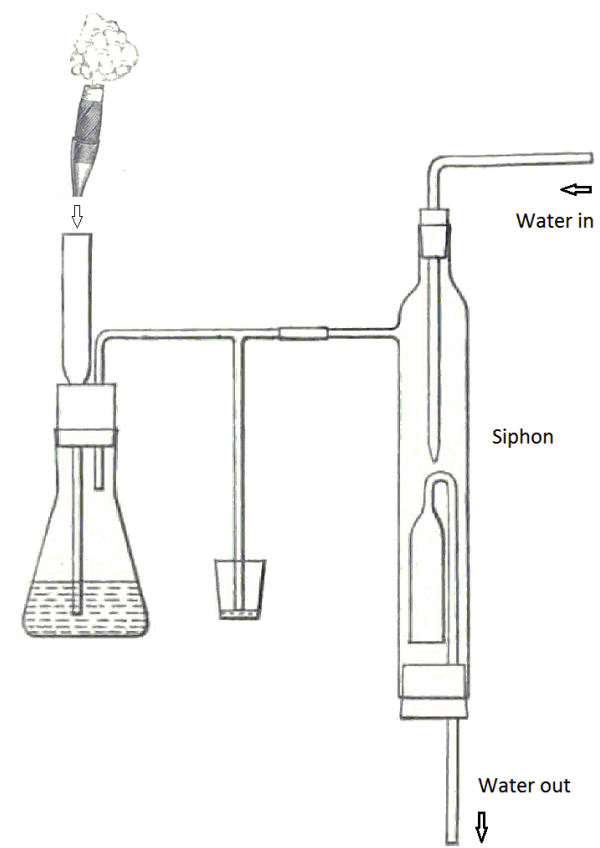

Figure 2. Intermittent puffing of cigars was accomplished in 1892 at the CONNECTICUT AGRICULTURAL EXPERIMENT STATION (15) by using a siphon.

Monopoly. He stated that the artificial smoking of tobacco products for analytical investigations should be done mimicking human smoking habits to gain an impression of a consumer's exposure to toxic smoke components. This was valid for testing all kinds of smokeable tobacco products. He wrote in 1901 (16):

"Nach meiner Auffassung wird die chemische Analyse des Tabakrauches ihren leicht erkennbaren, wichtigen Zweck erst dann erfüllen, wenn der der chemischen Analyse zu unterwerfende Tabakrauch nach einer Methode erzeugt wird, welche das beim Gewohnheitsraucher übliche Verfahren nach jeder Richtung mit thunlichster Sorgfalt nachahmt. Je vollständiger die Nachahmung des Gewohnheitsrauchers gelingt, desto grösser wird der Werth der analytischen Resultate selbst dann sein, wenn uns zur Nachweisung und quantitativen Bestimmung der einzelnen Bestandteile des Tabakrauches keine völlig einwandfreien, analytischen Methoden zur Verfügung stehen. Die analytischen Resultate werden in letzterem Falle, wie in ähnlichen Fällen, bei entsprechend gleichmässiger Handhabung des Verfahrens, zwar nicht absolut genau, aber zu einem Vergleiche untereinander und mit den Resultaten analog ausgeführter Versuche anderer Forscher sehr brauchbar sein und dadurch einen nicht geringen Werth besitzen. Dementsprechend muss das Versuchsrauchen vor Allem intermittirend gestaltet werden, da es ja einleuchtend ist, dass bei einer brennenden Cigarre die Destillation der mehr oder weniger schwer flüchtigen und die trockene Destillation der nicht flüchtigen Produkte bei einer andauernd gleichmässig vordringenden, kräftigen Gluthzone anders verlaufen, $d$. h. andere Destillationsprodukte, oder doch die Destillationsprodukte in einem anderen Mengenverhältnisse liefern wird, als wenn die Gluthzone abwechselnd für kurze Zeit, kräftig und rasch fortschreitend und sodann für relative längere Zeit in der Stärke zurückgehend und an der Cigarre langsam vorschreitend wird."

["In my opinion, the easily recognized important objective of the chemical analysis of tobacco smoke will be fulfilled only if the tobacco smoke for chemical analysis is generated by a method mimicking a habitual smoker's behavior as comprehensively and closely as possible. The more complete the imitation of the habitual smoker, the higher the value of the analytical results even if there are no really perfect analytical methods available for the detection and quantification of individual tobacco smoke components. In this case like in similar cases, with consistent methodology, the analytical results will not be absolutely correct but very useful for comparison internally and with the results of analogous studies of other researchers, and therefore have no little value. Therefore, the experimental smoking process above all has to be performed in intermittent mode because it is evident that in a burning cigar the distillation of more or less volatile products and the dry distillation of non-volatile products will proceed differently in a continuously progressing, active burning zone, that means produce different distillation products or distillation products in different quantitative ratios, than when the burning zone progresses alternatively for a short time forcibly and quickly, and then for a relatively longer period weaker and slowly advancing along the cigar."]

For his investigations, HABERMANN built a smoking device allowing the manually controlled reproducible intermittent (puff-by-puff) smoking of cigars $(16,29)$, later also of cigarettes and pipes (30). Puff volumes between 25 and $45 \mathrm{~mL}$ were possible. Puff volume, puff duration and smoldering time between the puffs varied, depending on the tobacco product and in line with observed human smoking habits (assessed, in fact, by self-analysis!). HABERMANN determined and compared the mainstream smoke levels of nicotine, nitrogen bases, hydrogen cyanide, carbon monoxide, carbon dioxide and oxygen for different kinds of cigarettes, cigars and pipe tobaccos.

In 1906, GARNER (31) of the Bureau of Plant Industry, U.S. Department of Agriculture, pointed out "that no two persons would smoke a cigar in exactly the same way, nor would the same individual smoke two cigars under exactly similar conditions. It is necessary, therefore, to use some means of smoking the cigars artificially in order to eliminate the personal equation and secure uniformity of conditions." Clearly, this statement is valid not only for cigars but for all smokeable tobacco products. For testing the burning quality of cigar tobaccos GARNER developed an apparatus for the simultaneous artificial smoking of four cigars (Figure 3). The device used in 1892 at the CONNECTICUT AgRiCULTURAL EXPERIMENT STATION (15) was modified while its principal mode of operation was maintained - suction by means of an aspirator, which was filled by a continuous inflow of water and emptied at regular intervals a by siphon. The cigars were smoked intermittently for a period of $10 \mathrm{sec}$ at intervals of $30 \mathrm{sec}$.

In 1909, GARNER (32) employed smoking by continuous or intermittent suction in his study of the effect of citric acid on the nicotine content of cigar smoke. While the addition of the acid to the tobacco used for cigar manufacturing had little effect on nicotine levels in the smoke, the fate of nicotine (destroyed or lost into the air vs. trapped in the 


\section{Tobacco smoke}

Tobacco smoke (cigarette smoke) is a complex, highly reactive and slightly charged aerosol, consisting of solid/liquid droplets (particulate phase) dispersed in the gaseous phase. It contains several thousand different compounds of various chemical classes. Due to its dynamic nature fresh tobacco smoke ages rapidly, changing its physical properties, chemical composition (artifact formation) and toxicity.

\section{Mainstream smoke}

Tobacco smoke (cigarette smoke) emerging from the mouth end of the tobacco product (cigarette) during puffing.

\section{Sidestream smoke}

Tobacco smoke emerging into the environment primarily from the glowing cone of the tobacco product (cigarette) between the puffs.

\section{Environmental tobacco smoke (ETS)}

Highly diluted, aged tobacco smoke aerosol in ambient air, consisting of about $15 \%$ exhaled mainstream smoke and $85 \%$ sidestream smoke. The toxicity of ETS is explicitly lower than that of unaged mainstream or sidestream smoke.

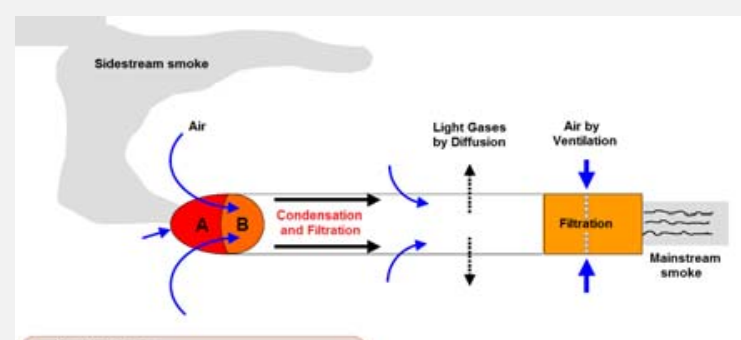

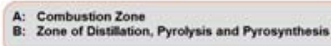

(Adapted from Thielen, A., H. Klus, and L. Müller: Tobacco Smoke: Unraveling a Controversial Subject; Exp. Toxicol. Pathol. 60 (2008) 141-156. DOI: 10.1016/j.etp.2008.0 1.014)

\section{Smolder stream}

The smoke stream flowing through a cigarette between puffs and leaving the cigarette at its mouth end.

\section{Smolder time}

The time between lighting and extinguishing a smoking article (cigarette) minus the summed duration of all puffs, equivalent to the total time between individual puffs.

\section{Diffusion stream}

The diffusion of small amounts of smoke through cigarette paper into the environment, both during puffs and between puffs.

remaining butt) was strongly influenced by whether the cigars were smoked continuously or intermittently. In the last years of World War I and the early 1920s,

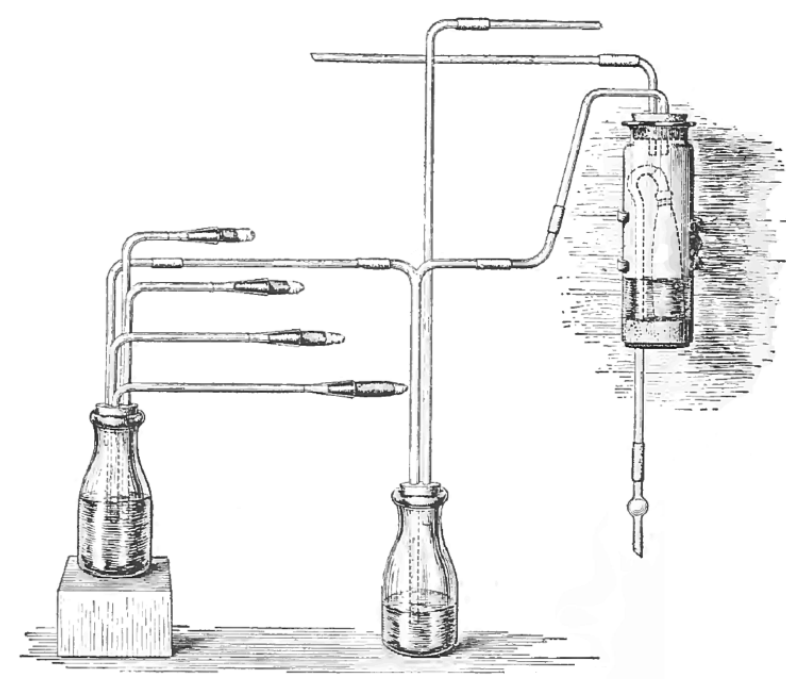

Figure 3. The burning quality of cigar tobacco was examined in 1906 by GARNER (31) using a 4-channel apparatus for intermittent smoking.

various non-tobacco materials were blended - for economic reasons - with tobacco in Germany and Austria to manufacture products intended for smoking, chewing and snuffing. The use of plant materials such as beech leaves or hops described by PREISSECKER et al. (33) - was addressed in regulatory announcements $(34,35)$. Consequently, materials similar to tobacco were included in the new German food law (36) of 1927, which was also about "Tabak, tabakhaltige und tabakähnliche Erzeugnisse, die zum Rauchen, Kauen und Schnupfen bestimmt sind" ["Tobacco, products containing tobacco and similar to tobacco, which are intended for smoking, chewing and snuffing"']. The law governed the production and distribution of food materials and tobacco products with the objective of consumer health protection. Based on the available physiological and pharmacological knowledge, health officials (37) at that time considered nicotine in tobacco and smoke to be the main cause of health risks in humans, while not neglecting other toxic smoke constituents. To our knowledge, the German food law was the first to regulate manufactured tobacco products on a scientific basis.

In response to health concerns about commercial tobacco products and at the request of the German Secretary of the Interior, PFYL and SCHMITT (38) of the German Government Health Office examined nicotine levels in the tobacco of cigarettes and cigars, some of which were claimed to be "nicotine-free", "nicotine-reduced" or "nicotine-harmless". Data did not confirm reduced nicotine levels compared to other tobacco products. As certain manufacturers proclaimed nicotine reduction in smoke, PFYL and SCHMITT also evaluated smoke nicotine levels and did not find such claims to be justified.

The apparatus used for smoke production and collection from single cigarettes and cigars consisted of a simple holder for the tobacco product (or a small tube or a pipe when tobacco was to be smoked), a row of wash bottles and a suction flask connected to a water jet pump. By manually operating a stopcock, a pattern of intermittent puffs (not defined in detail and probably quite variable) could be pro- 
duced, which was assumed to mimic human smoking. In the course of the study it was recognized that the intensity of burning (described only as "slow", "common" and "fast") exerted a major influence on the levels of nicotine found in the smoke.

As it was not possible to obtain sufficiently reproducible data for comparing smoke nicotine levels of different tobacco products this setup was subsequently modified and improved by PFYL et al. (17) (see page 146).

The fate of nicotine in the smoking process was the focus of a large study by WINTERSTEIN and ARONSON (39). Nicotine was measured in the tobacco of a wide selection of commercial cigarettes and cigars; after artificial smoking its presence in mainstream smoke, sidestream smoke and butts was assessed. A number of volunteers were asked to smoke test pieces with or without inhaling. Invoking mainstream smoke data and following the analysis of exhaled breath, the investigators arrived at numbers considered to represent the quantity of nicotine absorbed by smokers. Technically, test pieces were directly attached to a cold trap, i.e., a partially coiled glass tube, cooled in a dry ice/ether mixture. At the outlet, puffs were drawn intermittently by volunteers every $20 \mathrm{sec}$ with cigarettes and every $30 \mathrm{sec}$ with cigars. The same absorption apparatus was used to trap nicotine in exhaled breath, which was blown into the trap. In an unusual approach collected nicotine was quantified in highly diluted aqueous solutions - using a very specific biological test detecting only physiologically active substances, like nicotine, by recording the contraction of leech muscles (40).

Until the late 1920s the smoking devices in use were certainly suitable to identify many compounds in cigarette smoke and to determine the chemical composition of smoke in a basic way. Studies on the release of nicotine from tobacco during artificial and human smoking and speculations about nicotine uptake by smokers - at that time a focal point of smoking research - had led to a wealth of (in part strikingly contradictory) data. Due to the differences in equipment and smoking conditions between the various laboratories, their results could be assessed or reproduced only with restraint and considerable difficulties.

At this point, it had become obvious to leading tobacco scientists that clear reasoning behind, and the standardization of, the artificial smoking of tobacco products were urgently called for. This was also requested by both tobacco product manufacturers and official administrators including the establishment of maximum levels of nicotine in smoke.

The premise and the objectives of adequate, reproducible artificial smoking procedures were reasoned out in 1931 by WENUSCH (41). He emphasized that the production of smoke for nicotine analysis should be performed under defined and identical, or at least comparable, conditions. To make the data meaningful for physiologists and physicians when evaluating the effects of tobacco consumption in humans, the parameters of artificial smoking should correspond to human smoking so that nicotine data would be an indicator of human nicotine uptake. Specifically, artificial smoking had to use intermittent, rather than continuous, puffing. Test pieces for a particular analysis should be matched for dimensions (such as size and weight) and other physical properties, e.g., tobacco filling power and tobacco moisture. In those days, the consistency of ma- chine-made brands left much to be desired, even with regard to tobacco blend in the product. Cigarettes should be mounted in suitable holders, which would not deform the mouth end of test pieces, and smoked to the same butt length - a consideration also valid for cigars. Special attention was given to the consistency of puff volume, which would vary in the course of artificial smoking due to changing pressure drop and butt clogging. To this end, identical amounts of tobacco, assumed to produce identical volumes of smoke, were marked out, for instance, by pencil lines along the cigarette rod; when drawing a puff precisely the tobacco within two marks was burnt, requiring more or less time. The time needed for a puff became shorter with increasing puff number due to the decreasing pressure drop of the smoked cigarette.

WENUSCH had observed that smokers tended to control their behavior instinctively for intake of constant volumes of smoke; they produced a certain (bell-shaped) puff profile and achieved a negative pressure of 10-20 mm of mercury in the oral cavity. In a monograph on tobacco smoke, its analysis and human smoking behavior (published in 1939), WENUSCH (42) summarized:

"Als oberstes Gebot für alle Rauchgasanalysen mu $\beta$ daher bedingungslos gelten, daß beim Abrauchen für die einzelnen Züge solche Unterdrucke angewendet werden, die wenigstens größenordnungsmäßig jenen Unterdrucken gleichkommen, die von den Rauchern bei den Zügen in der Mundhöhle hervorgebracht werden. Die Feststellung des Unterdrucks, den die Raucher in der Mundhöhle erzeugen, ist daher die wichtigste Voraussetzung für jedes künstliche Abrauchen. ... Der erste und wichtigste Grundsatz muß sein: Nur Abrauchapparaturen verwenden, mit denen sich im Mundstückraum Unterdrucke von 10 bis $20 \mathrm{~mm} \mathrm{Hg}$ hervorbringen lassen."

["The primary rule for all smoke analyses unquestionably has to be that for all puffs drawn hypobaric pressures have to be applied of a magnitude sufficiently equivalent to those produced by smokers in the oral cavity while puffing. The ascertainment of the hypobaric pressure produced by smokers in the oral cavity is, therefore, the most important precondition for all artificial smoking. ..... The first and most important principle has to be: Use only smoking devices able to produce hypobaric pressures of 10 to $20 \mathrm{~mm}$ of mercury at the cigarette mouth end".]

Following these principles, WENUSCH (43) constructed and described a smoking apparatus, which could be operated with two different sources for suction, either a rubber balloon or a "falling mercury column". In both cases the puff was drawn directly into an intermediary receptacle (balloon or inflatable rubber pouch) and only then forced through the absorption system; this action was controlled by means of a manually operated three-way stopcock. The assembly was a remarkable departure from previously used configurations.

For producing the negative pressure of $10-20 \mathrm{~mm}$ of mercury, WENUSCH used, on the one hand, a rubber balloon with a volume comparable to the human oral cavity and elasticity suitable for simulating the pressure conditions in the human oral cavity during puffing. To take an artificial puff the balloon was compressed and then allowed to expand, this way drawing air through the test piece as long as 


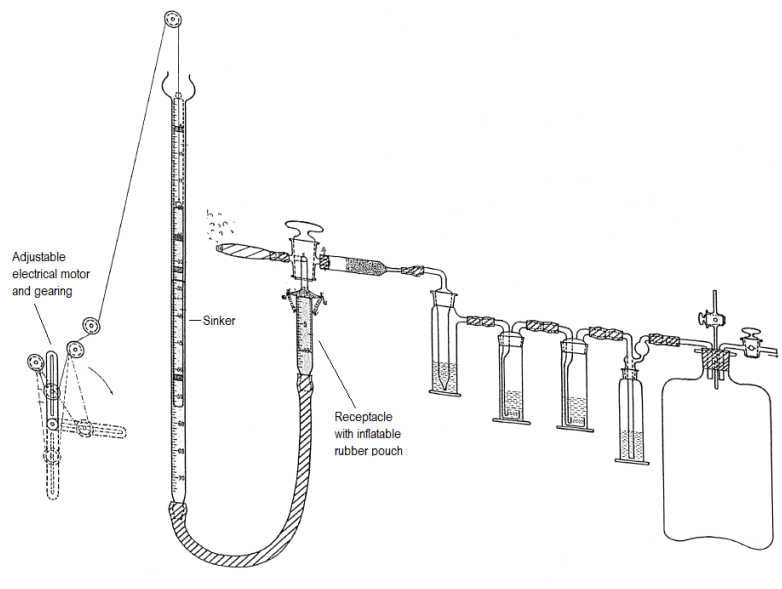

Figure 4. A column of mercury, shifting in communicating tubes, provided suction and pressure in the smoking apparatus designed by WENUSCH (43) in 1931.

the connection via the stopcock was kept open for burning identical amounts per puff of tobacco, as marked out by pencil lines. WENUSCH considered this relatively simple and inexpensive setup as suitable for all except the most sophisticated smoke analyses.

The second device for suction consisted of two communicating tubes, in which mercury - when alternately descending and ascending, following the movement of a sinker produced the negative and positive pressure for drawing a puff into an inflatable rubber pouch inside a receptacle and expelling it subsequently into the absorption system; the sequence and duration of steps was controlled by means of the three-way stopcock mentioned above. The movement of mercury was effected by an adjustable electrical motor connected to the sinker by a string (Figure 4).

Clearly, smoke collection in reservoirs as described by WENUSCH (43) is prone to artifact generation by aging. This, however, was not an issue in the 1920s and 1930s. At this time, the presence and levels of the well-known toxic components in tobacco smoke, such as nicotine, carbon monoxide, hydrogen cyanide and furfural, were the focus of research. These compounds are present in smoke in relatively large amounts and facilitate artifact formation if any - only to a negligible degree. Specific studies of artifact formation in smoke by aging were not performed until the 1960s and 1970s, for instance, by NEURATH et al. (44), KALLIANOS et al. (45) and SCHÖNHERR et al. (46) (see Chapter 7.1, p. 226).

WENUSCH considered the control of hypobaric pressure (preferably 20-30 mm mercury and definitely not below $10 \mathrm{~mm}$ ) an essential factor in artificial smoking. Effects of cigarette size on smoke nicotine yields - observed by other researchers (39) and hard to explain - had alerted him to the problem. Consequently, WENUSCH $(42,47)$ designed a novel smoking apparatus with components now arranged quite differently in comparison to the "balloon method" (43). The test cigarette was inserted into a long glass tube with two extensions branching off vertically (Figure 5), one connected to a mercury manometer and the other with an open end and equipped with the pressure regulator. Smoke was routed through a short tube with cotton wool to prevent impinger clogging, and then through a train of five wash bottles with glass filter frits, filled with highly diluted sulfuric acid (smoke nicotine determination being the main objective of the procedure). Suction was provided by an aspirator with a large glass balloon placed in between serving as a vacuum buffer. Hypobaric pressure was built up by closing the extension open end with a fingertip; its strength was checked by means of the manometer and controlled by both aspirator flow and pressure regulator adjustment. Puff duration was determined by ensuring the combustion of identical amounts of tobacco, marked out by pencil lines on the cigarette. The system allowed the effective control of hypobaric pressure but, at the same time, was prone to operating with variable air flow velocities. Pressure by administrators and manufacturers was mounting in Germany in the early 1930s to establish sensible and standardized smoking procedures for the determination of nicotine in smoke, including the potential effects of additives on nicotine release from tobacco into smoke. This was clearly expressed in 1929 in a decree of the GERMAN SECRETARY OF THE INTERIOR (48); however, technical parameters were not (yet) mandated at that time. Especially, manufacturers of tobacco products claimed to be low in nicotine asked health authorities and the GERMAN ASSOCIATION OF FOOD CHEMISTS to establish standardized analytical procedures and definitions for labeling tobacco products as "low in nicotine" or "nicotine-free" (49).

In 1933, PFYL et al. (17) took a major step forward in direction of reality based artificial smoking. In order to adjust laboratory smoking conditions towards best simulation of "natural" human smoking behavior, they performed a study to determine, by observation of smokers and physical measurements, the following key parameters:

- Puff frequency and

- Puff duration

These were recorded inconspicuously by means of a stopwatch. It turned out that both cigarette and cigar smokers on average took 2 puffs/min of $2 \mathrm{sec}$ duration

- Puff volume was measured by a small volumometric apparatus and was shown on average to be $42.5 \mathrm{~mL}$ for cigarettes and $48.6 \mathrm{~mL}$ for cigars

- Number of puffs when smoking a cigarette or cigar and

- Time needed for smoking a cigarette or cigar

- Butt length was found to be approximately $15 \mathrm{~mm}$ for plain cigarettes and $20 \mathrm{~mm}$ for cigars.

Special attention was paid to the parameter of air flow velocity through the test piece (and the apparatus), which could be calculated on the basis of puff volume and puff duration and yielded values of $20 \mathrm{~mL} / \mathrm{sec}$ for cigarettes and $25 \mathrm{~mL} / \mathrm{sec}$ for cigars. The authors raised serious doubts whether earlier smoking devices, using a falling water column or a com-

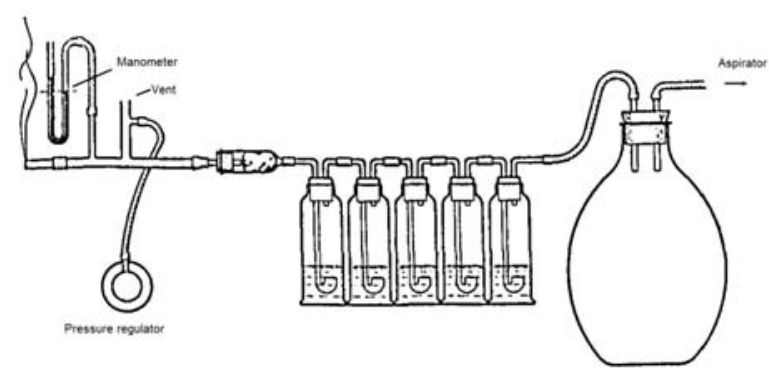

Figure 5. Effective control of hypobaric pressure was the main objective of the smoking apparatus designed in 1936 by WENUSCH (47) (picture extracted from (42)). 
pressible rubber balloon, were able to meet these conditions. To make sure that their equipment would achieve and maintain the required air flow intensity (i.e., puff volume and duration) PFYL et al. (17) combined their existing (38) absorption system with a vertically positioned pipette filled with mercury, the level of which could be raised and lowered (time-controlled) by moving a coupled-up reservoir up and down - thus producing the hypobaric pressure needed for puffing (Figure 6). Interestingly, this smoking machine was developed in cooperation with a technical enterprise, became commercially available and received in the mid-1930s recognition as part of the official "nicotine in smoke method". The definition of certain essential parameters by PFYL et al. (17) was a remarkable step towards the establishment of rules for artificial smoking, which were specified - some 40 years later - by the Scientific Research Unit of the (German) VERBAND DER CigARETTENINDUSTRIE in 1961 and 1962 $(18,50)$, in the CORESTA Standard No. 10 of $1968(51)$, in the German Standard DIN 10240 of 1969 (119), and in the International Standard ISO 3308 of 1977 (10).

Based on an entitlement in the German food law of 1936 (52), the Ordinance on Tobacco with Low or No Nicotine (53) was put into force in 1939. Tobaccos and tobacco products could be labeled "low in nicotine" if the nicotine level was not higher than $0.6 \%$ (cigarettes, cigarette tobaccos and pipe tobaccos) or $0.8 \%$ (cigar tobaccos, cigars, cigarillos and small cigars), based on dry substance, and "free of nicotine" if not higher than $0.1 \%$ (tobaccos and cigarettes) or $0.2 \%$ (cigars, cigarillos and small cigars). There were also ceilings for nicotine in smoke: "low in smoke nicotine" if nicotine content in smoke was not higher than $0.1 \%$ (cigars, cigarillos and small cigars) or $0.17 \%$ (cigarettes, cigarette tobaccos and pipe tobaccos) - and "free of smoke nicotine" if nicotine content in smoke was not higher than $0.08 \%$ (tobaccos and tobacco products). The smoke nicotine ceilings were based on the weight of tobacco smoked (water content 8 to $10 \%$ ). The Ordinance remained in effect in West Germany until 1974 when the food law was thoroughly revised $(54,55)$.

From the 1930s into the mid-1950s, the nicotine content in cigarette mainstream smoke was determined in Germany and other European countries using the apparatus (modified if necessary) and the smoking regime suggested by PFYL et al. (17) - with the exception of Austria, which continued using devices of the type developed by WeNUSCH $(43,47)$.

Over time, problems were recognized with the method of PFYL et al. (17). Two factors seemed to have a disturbing influence. The hypobaric pressure produced by the movement of mercury in the pipette was dampened by the absorption system positioned between the pipette and the test piece holder. This was shown to result in a considerable reduction of negative pressure at the mouth end of the (burning) tobacco product. In addition, changes in draw resistance unavoidably occurring during the smoking of a cigarette or cigar would alter the time required to draw the targeted volume of smoke. These conditions were not favorable to maintaining steady air flow intensity during the smoking process, which was considered to be of particular importance for obtaining correct results. The relevance and potential consequences of this situation were heavily - and emotionally discussed among scientists (56-60).

To overcome these obvious deficiencies, a manometer positioned close to the mouth end of the test piece and a flow meter with a regulating valve for maintaining an air flow

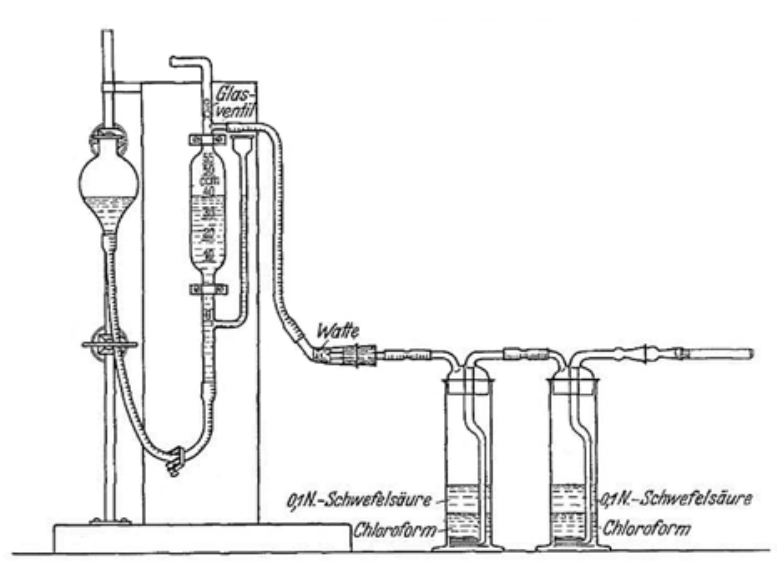

Figure 6. The smoking apparatus developed in 1933 by PFYL et al. (17) allowed the duplication and standardization of human smoking behavior and became commercially available.

during puffing of $20 \mathrm{~mL} / \mathrm{sec}$ (equivalent to a $40-\mathrm{mL}$ puff of 2 sec duration) near the pump were used as two new essential components of a smoking machine developed in 1953 by WAHL and HEIL (61). The device was described by MAREK (62) as an "extremely successful solution" to the problem of air flow control.

For their surveillance functions at the cantonal laboratory in Zurich (Switzerland) STAUB and FURRER (63) developed in 1953 a new manually controlled constant volume smoking machine, based on the system described by PFYL et al. (17). Puffing was done by a falling mercury column in combination with a water jet pump, controlled by a manually operated three-way cock. The system allowed adjustment of the hypobaric pressure between 10 and 20 mm of mercury during suction as suggested by WENUSCH(47). Cigarettes were smoked with 4 puffs of $2 \mathrm{sec} / \mathrm{min}$ and a puff volume of $40 \mathrm{~mL}$. Smoke was collected in two impinger bottles with glass frits, the first loaded with equal volumes of chloroform and $0.1 \mathrm{~N}$ sulfuric acid, and the second with $0.1 \mathrm{~N}$ sulfuric acid only. Two smoke fractions were determined quantitatively, chloroform-soluble dry "tar" and nicotine (as dipicrate). Nota bene: This was before the advent of the Cambridge filter. Moreover, the smoking system was used for the quantitative determination of the adsorption efficiency of cigarette filters for nicotine and "tar" (64).

In order to establish a reliable method of nicotine analysis in future machine smoking studies, STAUB and FURRER(65) had examined and compared in an earlier systematic investigation the two methods available at that time for the determination of nicotine (neat substance or tobacco constituent): precipitation with silicotungstic acid and gravimetry, and precipitation with picric acid followed by titration or gravimetry of the dipicrate. It was concluded that the gravimetric analysis of nicotine dipicrate was the most accurate and convenient analytical procedure.

The smoking machines developed and used in the 1950s in the research laboratories of S.E.I.T.A. (Service d'Exploitation Industrielle des Tabacs et des Allumettes, the French Tobacco Monopoly) were described by CUZIN (66) at the Second International Scientific Tobacco Congress, organized by CORESTA and held in 1958 in Brussels (Belgium). The two machines in service were both semi-automatic and made use of a vacuum tank in combination with valves controlled 
by a timer for puffing, making them constant time machines. One type drew puffs of $30-40 \mathrm{~mL}$ in $1.5 \mathrm{sec}$ twice per min simultaneously from 7 cigarettes, placed in a ring-shaped smoking head, and collected combined mainstream smoke in a scrubbing tower filled with a low boiling organic solvent, such as methanol, acetone or hexane. This trapping system had the capacity of collecting smoke from $600-1,000$ cigarettes. The other type of machine smoked simultaneously 10 cigarettes in a bar-shaped manifold; mainstream smoke condensate was collected from individual cigarettes by capillary traps as described by WENUSCH (42). The drawbacks of constant time (compared to constant volume) smoking machines regarding the variation of mainstream smoke yields were discussed by WALTZ et al. (67) and WALTZ and HÄUSERMANN (68).

In the United States, JENSEN and HALEY (69) of the Pennsylvania Agricultural Experiment Station in the mid1930s carried out an investigation of the influence of tobacco moisture and puff strength on the nicotine levels in mainstream smoke, sidestream smoke and butts. For this purpose they developed a machine for smoking cigarettes, cigars and pipes with constant puff volume, time and interval. Suction was provided by a high-vacuum pump with a vacuum reservoir and controlled by a motor-driven rotating valve. The unusual smoking parameters (one puff of $20 \mathrm{~mL}$ every $6.1 \mathrm{sec}$, puff duration $1.6 \mathrm{sec}$ ) were obviously not intended to duplicate human smoking behavior.

\section{"Stuck in tar?"}

The meaning of the term "tar" has changed as time passed:

Up to around 1960

"Tar" was a synonym for the (total) particulate matter (TPM) of smoke aerosol, independent of the collection mode (sedimentation, cotton wool, capillary coagulation, electrostatic precipitation, solvents such as chloroform)

\section{Since the early 1960 s}

"Tar" was a synonym for either

- TPM: total particulate matter (water and nicotine included) also called condensate or

- Dry condensate (total particulate matter minus water = dry "tar")

Since the mid of the 1970s

"Tar" is a synonym for NFDPM (nicotine-free dry particulate matter) 
In the early 1930s the stage was set for the evolution of efficient, highly controlled and automated smoking machines. The development was driven by two motives effective both in the U.S. and in Europe: First, consumer demand for cigarette brands, consistent in taste and "impact" (i.e., nicotine content in smoke); second, in 1950, early epidemiological studies reporting an association of cigarette smoking and lung cancer $(70,71)$. A doseresponse relationship was shown between the type and number of cigarettes smoked and the risk of lung cancer. An animal study by WYNDER et al. (72), applying cigarette mainstream smoke condensate on mouse skin ("skin painting"), revealed a dose-response relationship between the amount of condensate and the number of tumor-bearing mice. Subsequently, WYNDER and MANN (73) demonstrated that the tumor inducing effect, when applying equal amounts, was weaker with smoke condensate from cigarettes with cellulose acetate filters than from plain cigarettes. These findings supported the expectation that reducing cigarette smoke yields of "tar" by filtration might be of significant benefit to smokers. In 1964, the Report of the Advisory Committee to the U.S. Surgeon General, Lucius Terry, on "Smoking and Health" (74) - the so-called "Terry Report" - alerted the cigarette industry worldwide to the health risks of smoking. The development of "low tar" cigarettes with very efficient filters was one of the consequences.

The interrelation of filters and reduced "tar" levels (with potentially positive effects on health risks) called into play prominent manufacturers of materials for filter production (Eastman Kodak), filter rods (Cigarette Components/ Filtrona), and filter and cigarette papers (Ecusta), and involved them in the development of smoking machines for the determination of mainstream smoke condensate and constituents. This is exemplified by the invention of a "Simple Smoking Machine" (75) and the Ethel Mark VI smoking machine (76) by Cigarette Components Ltd. and the work of MUMPOWER et al. (77) at Eastman Kodak Company - all discussed below in more detail.

Consumer demand for cigarettes of good and consistent quality and the public discussion of the health effects of cigarette smoking in the U.S. and Europe called for practical and reliable smoking devices for obtaining reproducible and comparable data on cigarette smoke. A standardized smoking regime was mandatory for cigarette development and quality assurance but also for scientific research on cigarette smoke. The development of modern analytical smoking machines became an important field of activities in the U.S. and Europe.

\subsection{Linear smoking machines}

\subsubsection{Development of linear smoking machines in the United States}

The divergence of results regarding the presence and amounts of chemical constituents in tobacco smoke, which was obviously caused by differences in artificial smoking conditions, was still of major concern when, in 1936, BRADFORD et al. (4) of the American Tobacco Company

published their landmark paper on the technique of experimental smoking. They looked upon the device designed by PFYL et al. (17) as an efficient constant pressure/constant time machine, able to draw a puff of equal volume each time (a flawed assumption as has been recognized since). Only the use of mercury for generating the relatively forceful hypobaric pressure, needed in PFYL's system, seemed to be objectionable. The problem was solved by placing a flask for the gravitational deposition of particulate matter in front of low-resistance bubblers (see Figure 61 on page 238), this way allowing the use of water as hydrostatic fluid.

Three smoking machines of increasing complexity were developed. The most advanced machine included three rotating valves of metal, operated by a timer-actuated electric motor, for the alternate puffing of 1 of 4 cigarettes every $15 \mathrm{sec}$ and the stepwise distribution of air and water within the suction system, driven by a falling water column. This setup made it possible to smoke four cigarettes simultaneously (Figure 7) with a frequency of one puff per cigarette per min. Attention was given to experimental details, such as an air-tight cigarette holder made of Bakelite with a seal of concentrated dextrose syrup.

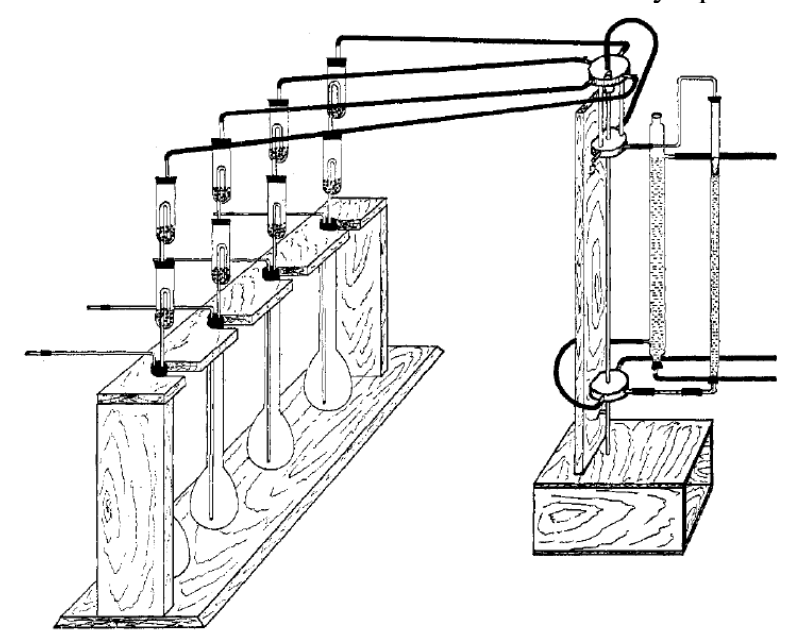

Figure 7. BRADFORD et al. (4) - in their landmark paper of 1936 - referred to their 4-channel smoking machine as a "robot for mass smoking".

For the reliable artificial smoking of cigarettes the following requirements were considered essential:

- Thorough control of smoking parameters (puff volume, duration and frequency);

- Complete description of the test cigarettes and the remaining butts (in the absence, at that time, of any standard for butt length in artificial smoking); and

- Full control of ambient conditions.

Taking guidance from the data on human smoking collected by PFYL et al. (17) the authors decided on a $35-\mathrm{mL}$ puff (chosen arbitrarily and probably due to the technical limitations of their smoking machine (78)) in $2 \mathrm{sec}$ once a min. This smoking regime was subsequently accepted widely and became an essential part of the standards mandated, for instance, by ISO (10) and FTC, as described by PILLSBURY et al. (19).

BRADFORD et al. (4) pointed at an important difference between smoking devices producing the puff by constant 


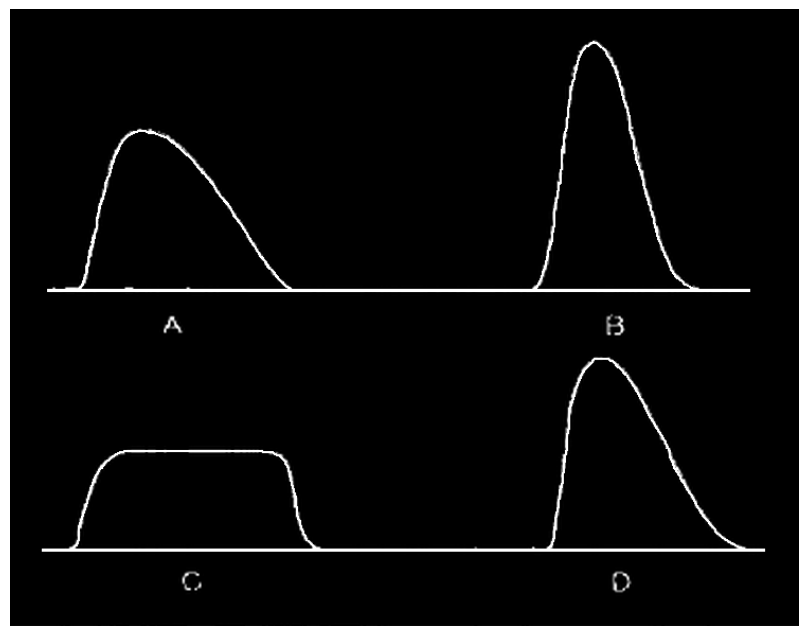

Figure 8. Kymograph tracing (4) of pressure-time relationships in cigarette puffs of identical volume, produced by human smokers ( $A$ and $B$ ) and by smoking devices operating with constant flow/constant pressure (C) and constant volume (D).

time/constant flow or by constant volume: there was a discrepancy in puff profiles. Constant flow machines produce a "square wave" profile over a preset time whereas constant volume machines produce a "bell shaped" profile (Figure 8). This is important for two reasons. As WENUSCH (41) pointed out already in 1931, humans do not draw a puff with constant hypobaric pressure. A bell shaped puff profile simulates human behavior more closely than a square wave profile. As explained by BAKER $(79,80)$ in the late 1970 s, there are distinct temperature conditions inside the cigarette combustion coal during puffing. These are caused by the amounts of oxygen available in different sections of the glowing cone - a situation somewhat different between the two puff profiles. Consequently, there may be small differences in the composition of generated smoke. These had already been observed in 1971 by SEEHOFER and WENNBERG (81).

The smoking machine of BRADFORD et al. (4) - often called the "American Tobacco Company Machine" - was the first enabling cigarette manufacturers to evaluate routinely the mainstream smoke yields of their products for quality assurance purposes, e.g., the consistency of smoke nicotine yields. After its introduction, variations of the Bradford machine were used in particular by the U.S. cigarette industry for the next 25 years (Figure 9).

The "American Tobacco Company Machine" could also be used for the evaluation of cigarette sidestream smoke. Sidestream smoke is the smoke emitted into air primarily from the glowing cigarette cone between puffs. For its collection specific devices and traps are necessary to avoid any influence on the generation and composition of mainstream smoke $(82,83)$. However, as this is beyond the scope of this review the topic is not discussed further.

By 1937, other laboratories of the tobacco industry were using constant time/constant flow smoking devices, which regulated puff duration to a pre-set number of seconds, in most cases $2 \mathrm{sec} /$ puff. However, contrary to the constant volume machines, the puff volume was dependent on the draw resistance (pressure drop) of the cigarettes being smoked as well as the pressure drop of the smoke trapping system. Consequently, puff volume did not remain constant while smoking a cigarette because pressure drop decreased as the cigarette was consumed. The differences in pressure drop were related in different ways to both types of sucking (constant flow/constant time and constant volume) and resulted in poor reproducibility. The problems related to pressure drop were eventually solved in the 1960 s by the development of constant time/constant volume machines (discussed below).

The late 1950s saw significant improvement of certain features of the "American Tobacco Company Machine" (4). KEITH and NewSOME (84) of the Liggett and Myers Tobacco Co. (Durham, NC, USA) described in 1957 the use of vacuum reservoirs (of variable strength and sufficient size) in combination with pressure drop regulators to minimize the influence of (changing) pressure drop on puff volume or duration. Advanced components of the machine were solenoid-operated valves and latex tubes for ensuring test pieces being well sealed in their holders. The system was sufficiently flexible to duplicate a wide range of human smoking characteristics and equipped to trap essentially all the normally liquid and solid components of cigarette smoke.

MUMPOWER et al. (77) of Eastman Kodak Company modified the "American Tobacco Company Machine" (4) in a similar way in their study on how filter efficiency, at constant puff volume, was influenced by puff duration. While a 35-mL puff took 4-6 sec depending on filter pressure drop and when using a falling water column, the puff duration was invariably maintained at $2 \mathrm{sec}$ by means of a surge tank in combination with an orifice, a manostat and a vacuum pump. Very informative pressure drop-time curves were obtained, and recorded on microfilm, during smoking under the two different conditions. The authors

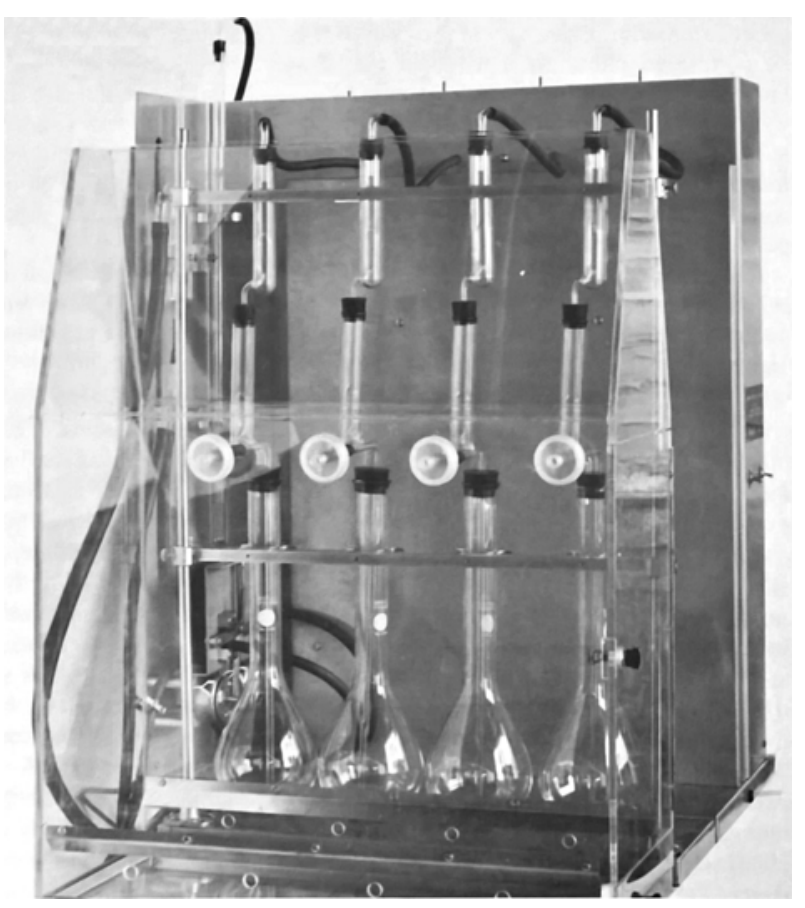

Figure 9. A commercial version of the "American Tobacco Company Machine", manufactured by Phipps \& Bird, Inc. in the 1960 s and already equipped with Cambridge filters. 


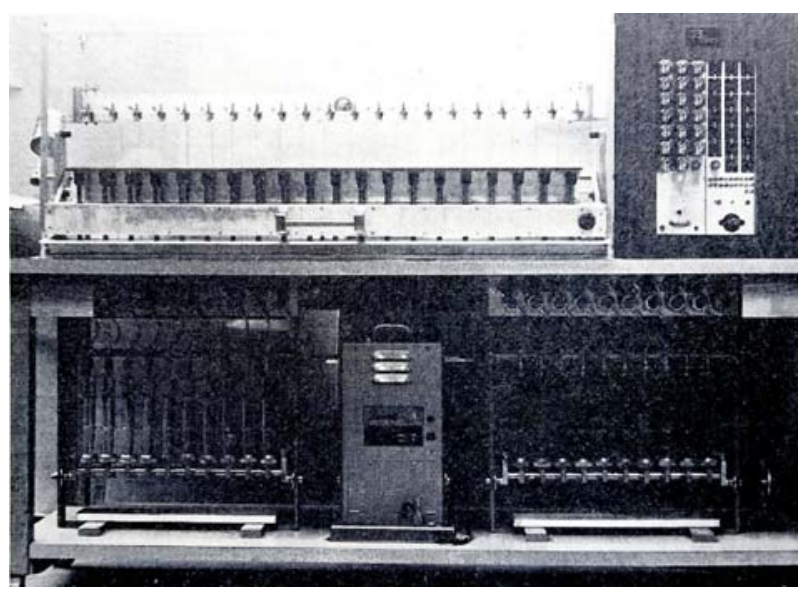

Figure 10. The 20-port linear smoking machine of WILEY and FERRI (8) became the prototype for the development of numerous commercial models.

also pointed out the importance of the dead air space behind the smoked cigarette for puff duration in constant volume machines. The extension of the pre-set 2-sec $35-\mathrm{mL}$ puff up to $4.5 \mathrm{sec}$ was observed depending on the dead air space volume.

In 1958, O'KEEFFE and LIESER (85) of Philip Morris (Richmond, VA, USA) described an 8-port constant volume smoking apparatus with individual puffing mechanisms. Due to the use of syringes for suction, puff duration was not influenced by pressure drop changes in the cigarettes and/or the trapping system during smoke collection. This type of machine brought laboratories closer to reproducible results because puff frequency was determined by a synchronous motor and puff duration controlled at the millisecond level. The smoke trap used most probably was a Cambridge filter, a disk about $2 \mathrm{~mm}$ thick and made from glass fiber. The capacity of this machine was later expanded to 20 ports. However, the modification greatly magnified the inherent problems of the synchronous lighting of cigarettes and the accurate interruption of puffing at the desired butt length, which was done manually by removing the glowing cone with pincers.

To overcome these problems the twenty-port machine was improved by WILEY and FERRI (8) of Philip Morris (Richmond, VA, USA). The new machine (Figure 10) was equipped with a simultaneously working 20-port electric lighter and with the "string cut-off technique" for butt length control. The technique used a thread placed at a mark on the cigarette indicating the desired butt length; when the glowing coal reached this point the thread burned out, and micro switch/solenoid valve deactivation effected the immediate interruption of puffing at the individual smoking port. There was also an electrical puff counting unit. Routinely, smoking parameters were a 35-mL puff of $2 \mathrm{sec}$ duration every $60 \mathrm{sec}$. Smoke condensate was retained on a Cambridge filter of $44 \mathrm{~mm}$ diameter with a capacity of collecting $300 \mathrm{mg}$ material. The Cambridge filter (CM-113, a glass fiber filter) had been recommended in 1959 by WARTMAN et al. (6) as the preferred trap for smoke condensate. The filter material showed removal efficiency, which was better than $99.9 \%$ for particles with a diameter of $0.3 \mu \mathrm{m}$ or more. No more than five cigarettes were usually smoked at each port to prevent Cambridge filter overload - plausible at a time when cigarettes had condensate levels as high as $40 \mathrm{mg}$. Properties and advantages of the Cambridge filter for condensate collection are discussed in more detail below (see Chapter 7.3, p. 228).

The 20-port syringe machine designed by WILEY and FERRI (8) was the prototype for the 20-port smoking machines manufactured and marketed by Phipps \& Bird in the U.S. and Filtrona (Cigarette Components) worldwide except in the U.S. This machine type was adopted by the U.S. tobacco industry as the one design for routine analytical purposes (86).

The smoking regime suggested by BRADFORD et al. (4) $1 \mathrm{puff} / \mathrm{min}$, puff duration of $2 \mathrm{sec}$ and puff volume of $35 \mathrm{~mL}$ - became the "standard" with the Phipps \& Bird and Filtrona machines, while there were limited possibilities for using other smoking parameters. The linear 20-port machine was suggested by the U.S. FEDERAL TRADE COMMISSION (FTC) in 1969 (19) as standard equipment for cigarette testing - a development described in Chapter 3.3.1. (p. 158).

\subsubsection{Development of linear smoking machines in Europe}

A second center for the development of modern linear cigarette smoking machines was Europe, specifically Switzerland and the United Kingdom.

The burden of numerous tobacco smoke analyses caused STAUB and FURRER from the cantonal laboratory Zurich (Switzerland) (87) to develop their manually operated apparatus (63) into a fully automated smoking machine (Figure 11) - featuring a range of improvements: Intermittent puffing was managed electronically; use of mercury was no longer required; puff duration (between 1.0 and $2.5 \mathrm{sec})$ and interval $(15,30,45$ or $60 \mathrm{sec})$ as well as hypobaric pressure and puff volume could be set with precision and were easily adjustable. As reported in collateral papers by WALTZ et al. $(67,68)$ puff volume and duration relied on the action of a piston with adjustable speed and travel in combination with a time-controlled electromagnetic valve. The control of actual puff volume was achieved by separate volumetric measurement. The new smoking machine was offered for sale by the Radag company of Kilchberg, ZH (Switzerland).

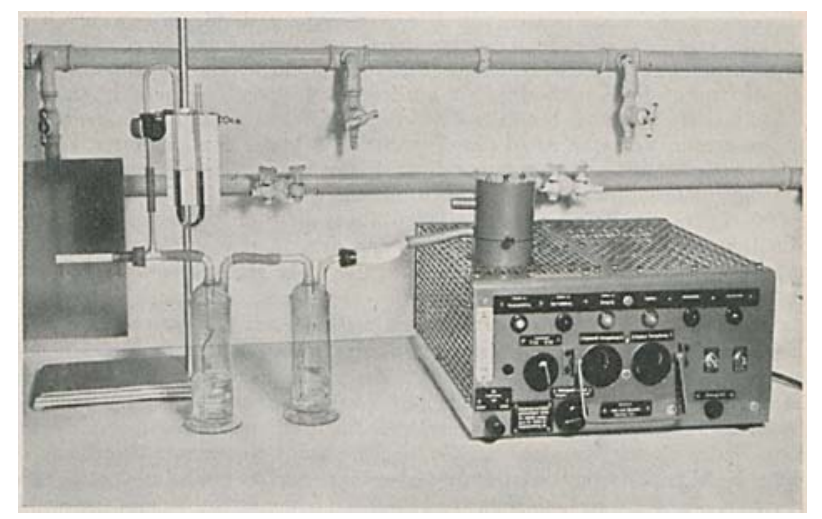

Figure 11. The electronically controlled and fully automatic smoking machine assembled in 1954 by STAUB and FURRER (87). 
With an automated smoking procedure available, which satisfied practical and also scientific requirements, STAUB and FURRER (88) put together a comprehensive and systematic discussion of the reproducibility of smoke analysis - combining theoretical considerations with experimental data. The scope of aspects included: the essential parameters of human and artificial smoking; smoking machine operation and the collection of nicotine and (chloroform-soluble) "tar"; the conditioning and handling of test pieces; the quantification of nicotine and "tar"; and the assessment of the accuracy and reproducibility data by statistical evaluation. Standard operating conditions were puffs of $35 \mathrm{~mL}$ in $1.6 \mathrm{sec}$ every $30 \mathrm{sec}$; butt length of $20 \mathrm{~mm}$; and two impingers loaded with equal volumes of chloroform and $0.1 \mathrm{~N}$ sulfuric acid for trapping "tar" and nicotine. Again, the experimental evaluation of cigarette filter efficiency in reducing cigarette mainstream smoke nicotine and "tar" was addressed in the study.

Prompted by the growing international spreading of cigarette smoking, WALTZ et al. (67) of Vereinigte Tabakfabriken in Neuchâtel (Switzerland) conducted in 1959 a thorough survey of the parameters relevant for the artificial smoking of cigarettes. The definitions as well as measurement techniques and ways of (corrective) control were outlined for puff volume, duration and interval, pressure drop and resistance to draw, volume of aspired air and flow rate, and puff profile, which is the essential resultant of all these parameters. The interplay of these factors was discussed in detail and examined experimentally. Smoke yields of "tar" and nicotine were described as depending strongly on puff volume and interval, and weakly on puff duration - all three variables basic and effectively controllable in smoking machine operation. The range of currently used equipment for smoke production and collection was reviewed and characterized. Puff profiles of the smoking machine of STAUB and FURRER (87) in combination with different smoke collection devices were compared to those produced by human smokers.

By 1960 , no country had yet established an official method for the determination of "tar" and nicotine in cigarette smoke. Each laboratory used its particular and supposedly most suitable methodology. There was, however, a growing tendency towards harmonization. In a very comprehensive publication, WALTZ and HÄUSERMANN (68) first described their own method of preparing and machine smoking test cigarettes. Basically, the commercially available smoking machine of STAUB and FURRER (87) was used in compliance with established (87) parameters: puff volume of $35 \mathrm{~mL}$ (precisely: aspired air volume); puff duration of $1.6 \mathrm{sec}$; puff interval of $30 \mathrm{sec}$. WALTZ and HÄUSERMANN (68) decided on a butt length of $20 \mathrm{~mm}$ with cigarettes $\leq 75 \mathrm{~mm}$ long, and $25 \mathrm{~mm}$ with cigarettes $>75 \mathrm{~mm}$ long. Smoke was precipitated electrostatically and weighed out after drying in situ to give "total smoke". The precipitate was further partitioned between chloroform and $0.1 \mathrm{~N}$ sulfuric acid; the organic phase after evaporation yielded chloroform soluble oven-dried "smoke tar" while nicotine was recovered from the aqueous phase and determined by photometry. Using the equipment and procedures as described an operator was able to smoke and fully analyze 20-30 cigarettes per day (higher throughput was considered desirable).
In addition, the authors provided a detailed, most informative survey of the similarities and differences of methods commonly used by other laboratories - as many as 18 sources from Europe and the United States were considered to the extent that information was available. Specifics included: sample size; test piece conditioning; basic smoking parameters (butt length as well as the volume, duration and interval of puffs); smoking machines used; smoke collection devices (electrostatic, impingers, Cambridge filters, cotton and glass wool, cold traps); definition and quantification of total smoke and smoke "tar"; determination of smoke nicotine. The authors expressed the opinion that the information available pointed to the possibility of agreeing on the fundamentals of artificial smoking, and mentioned that CORESTA had just put forward a proposal regarding standardization of the preparation and artificial smoking of cigarettes. CORESTA (Centre de Coopération pour les Recherches Scientifiques Relatives au Tabac - Cooperation Centre for Scientific Research Relative to Tobacco), an organization for international scientific collaboration in the field of tobacco, had been founded a few years earlier (1956) in Paris (89).

A versatile automatic machine for the smoking of cigarettes - and even pipes - was described in 1957 by ILES and SHARMAN (90) of the Imperial Tobacco Co. (Bristol, UK). The "Autosmoker" consisted of a smoking port ("mechanical mouth"), directly connected to a small glass tube for the electrostatic precipitation of mainstream smoke condensate (for more details on electrostatic precipitation, see Chapter 7.4, p. 231). Puffs were produced by expanding bellows in individual smoker units, of which 12 or more could be combined in a bank with a common motor and a high-voltage generator for electrostatic condensate collection. Frequency, volume and duration of puffs as well as butt length could be controlled. The authors, however, were not completely at ease with the set of smoking parameters used routinely by other researchers. It is of interest to note that the "mechanical mouth" in combination with the glass tube for the electrostatic precipitation of smoke condensate looked like the system described in 1923 by BAUMBERGER (91) for the evaluation of smoke uptake by human smokers.

The smoking machine of ILES and SHARMAN (90) with electrostatic precipitation was used by BENTLEY and BURGAN (92) for the determination of benzo $[a]$ pyrene in cigarette mainstream smoke condensate.

In a study of 1959 intended to characterize tumor-initiating $v s$. tumor-promoting substances in tobacco smoke, ROE et al. (93) produced condensate using the smoking machine of ILES and SHARMAN (90) in a version equipped with cold traps immersed in a dry ice/acetone mixture, one trap being attached to each smoking position. Commercial cigarettes were smoked with 4 puffs $/ \mathrm{min}$ and a puff volume of $15 \mathrm{~mL}$ in $2 \mathrm{sec}$. The butt length chosen was $20 \mathrm{~mm}$. A phenolic fraction of the condensate applied to the dorsal skin of mice was found to show strong tumor-promoting activity while the tumor-initiating effect of smoke condensate was judged to be weak.

In 1955, CigareTte COMPONENTS LTD. (75) designed in England a constant volume device comparable to the "American Tobacco Company Machine" (4) and offered it as a "Simple Smoking Machine". The all-glass apparatus 
was operated manually by turning a double tap. Puffs of $35 \mathrm{~mL}$ were obtained by suction produced by a falling water column and trapped by contact with solvent in a large flask. Tap control was also possible by use of a small electric motor - making equipment available which could smoke 4 cigarettes simultaneously. The apparatus was used by some European cigarette manufacturers, mainly by tobacco laboratories in the United Kingdom.

At the end of the 1950s, Cigarette Components LTD. (76) developed the constant time/constant flow machine Ethel Mark VI (Figure 12). Its primary purpose was to study the retention characteristics of various types of filters. The 4-channel machine showed flexibility in adopting different smoking regimes. A rotary vacuum pump, controlled by a valve and timing system, drew one puff per channel every $60,30,20$ or $15 \mathrm{sec}$ with puff durations of 1 , 2 or $3 \mathrm{sec}$. Deriving profit from the work of ILES and SHARMAN (90), the preferred method of condensate collection was electrostatic precipitation though the use of cold traps and Cambridge filters was also possible.

In 1962, WILLIAMSON and CLARK (94) described the 4-channel Cigarette Components smoking machine CSM 10, which was an improvement over the Ethel Mark VI. It was also a constant time smoking device. ${ }^{2}$ Puffs were drawn by an oil vacuum pump. Puff duration and interval were set with two clock type mechanical timers, and flow rate was adjusted by means of two control valves. The target puff volume was regulated automatically by a volume compensator that guaranteed constant puff volumes independent from variations in pressure drop. Puff duration could be chosen continuously over a range between 0.1 and $6 \mathrm{sec}$ for achieving puff volumes between 1.5 and $135 \mathrm{~mL}$; puffs could be taken every 1 to $60 \mathrm{sec}$. Obviously, with regard to smoking parameters this machine was extremely flexible. Smoke condensate was collected by electrostatic, cold and liquid traps or on Cambridge filters. The CSM 10 was followed by the rather similar model CSM 12 featuring electronic timers. It was further improved by the Cigarette Components affiliate, Filtrona, to become the completely solid state, 4-channel Filtrona SM 100 (95). It offered a wide range of puff frequency and duration and allowed air flow and vacuum control within narrow limits. Air flow was manually variable over the range of $12.5-20 \mathrm{~mL} / \mathrm{sec}$; distinct rates (for instance, $35 \mathrm{~mL}$ in $2 \mathrm{sec}$ ) could be maintained automatically by means of a critical flow orifice. The machine was operated with various smoke collection devices (Cambridge filters, liquid traps, etc.). Besides smoking cigarettes this extremely flexible smoking machine could also be used for smoking cigars and pipes. There was a similar 12-channel machine, called SM 101.

The Filtrona SM 100 was the last model with a vacuum pump for suction offered by Cigarette Components/Filtrona. Subsequently, the company manufactured only models operated with syringes (20-channel and 8-channel linear

Edward M. Cumming (retired Manager of the Analytical Service Laboratory of Rothmans International Services Limited, Basildon, Essex, UK) and Keith Holland (retired Director of Filtrona Instruments \& Automation Limited, Milton Keynes, Buckinghamshire, UK) kindly provided guidance for following the development of linear smoking machines manufactured by Cigarette Components and Filtrona.

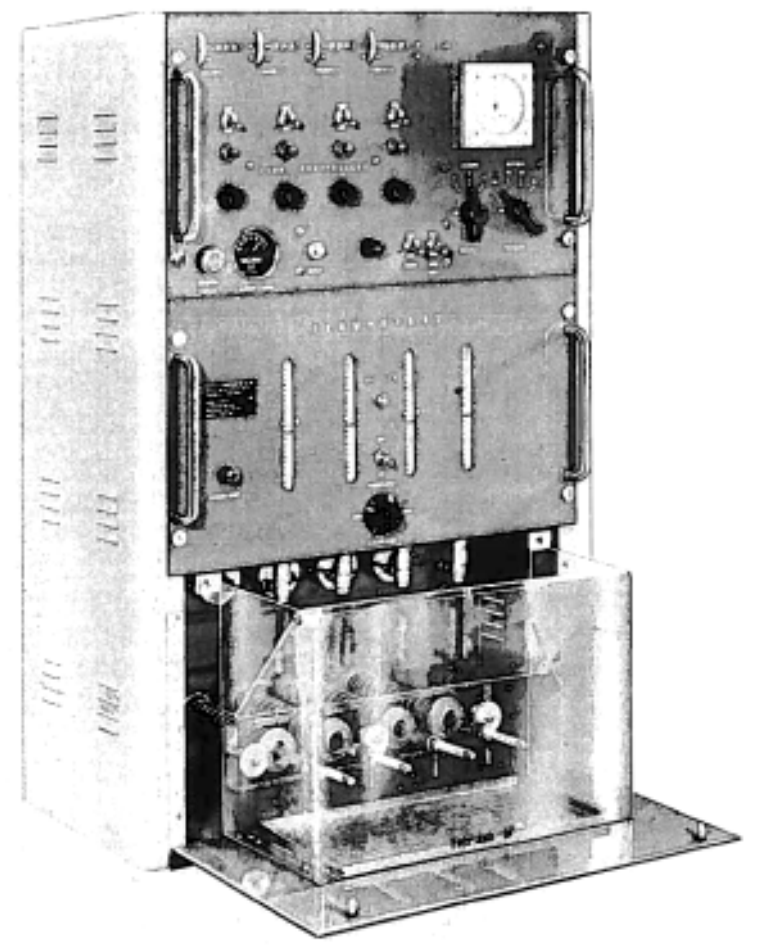

Figure 12. The Ethel Mark VI smoking machine (76) - shown under operating conditions - collected particulate matter in cold or liquid traps, on Cambridge filters or by electrostatic precipitation.

machines). Model SM 300 was a version of the automated 20-channel smoking machine of WILEY and FERRI (8), adopted for commercial production (an activity comparable to the course taken by Phipps \& Bird in the United States). It was equipped with 20 individual syringes and Cambridge filters. Compared to the Phipps \& Bird machine the SM 300 offered updated electronics and was intended for sale in the rest of the world. For the determination of total particulate matter (TPM) and smoke nicotine this configuration of smoking machine was specified in 1969 by the Federal Trade COMMISSION (19), also in 1969 by the BRITISH STANDARDS INSTITUTION (96), and in 1972 by the BRITISH TOBACCO RESEARCH COUNCIL (97) in their Research Paper 11, which - in three editions complied agreed standard methods of tobacco smoke analysis.

CUMMING (98) observed several shortcomings of the 20-channel SM 300 smoking machine in the routine analysis of certain gas phase constituents, specifically carbon dioxide, carbon monoxide and nitric oxide. Proper sampling was impeded by gas phase contact with the silicone oil used for lubricating and sealing syringe pistons, large dead volumes, and a long hold-up time before analysis. Rather than making fundamental alterations to the SM 300 CUMMING developed an eight-channel vapor phase smoking attachment. It included the eight-fold combination of an ancillary syringe with two three-way solenoids, one set for each channel placed behind the Cambridge filter, for the temporary collection of puffs, which were afterwards combined and made available for simultaneous puffby-puff measurements using special analyzers. Interest- 
ingly, an agreement was reached with Cigarette Components Ltd. regarding the manufacturing and commercial distribution of the new device.

Replacing the SM 300, a new model was offered in 1992 by Filtrona as SM 400 (99). In this machine, syringe pistons sealed with paraffin or silicone oil were replaced by oil free pistons with practically no dead volume, and labyrinth seals were substituted for artificial rubber lips in cigarette holders. For conformity with ISO $3308,3^{\text {rd }}$ edition of 1991 (12), average air flow around the glowing cone of the cigarettes during puffing and between puffs was tuned to $200 \pm 30 \mathrm{~mm} / \mathrm{sec}$. Mainstream gaseous phase was collected in small plastic bags from individual channels for the on-line quantification of carbon monoxide by non-dispersive infrared (NDIR) analysis.

Filtrona also developed an 8-channel machine, the SM 302 (100), specifically for the analysis of components in the cigarette mainstream smoke gaseous phase, featuring eight lubrication free syringes with no dead volume. Carbon monoxide could be measured puff-by-puff by passing the combined gaseous phases from all eight simultaneously smoked cigarettes though a cell for NDIR analysis; for assessing total carbon monoxide yield per cigarette the gaseous phase from each channel was combined and collected in a plastic bag, and quantified by NDIR analysis (originally off-line). However, both measurements could also be done automatically by means of the ATCOM module (101). Combined with special equipment, the machine could be used for the puff-by-puff determination of nitric oxide in mainstream smoke by chemiluminescence (102).

In the 8-channel successor model SM 342 (103), labyrinth seals were used and the dead volume behind the Cambridge filter holder was reduced. The SM 342 was in compliance with ISO 3308, $3^{\text {rd }}$ edition of 1991 (12).

In 2000, the UK-based company Filtrona Instruments and Automation was acquired by MoLINS GROUP (104) and renamed Cerulean - with headquarters retained in Milton Keynes (UK).

Today, several models of manually operated linear smoking machines are manufactured and offered by Cerulean, complying with the design and smoking requirements of the latest $\left(5^{\text {th }}\right.$ of 2012) edition of ISO 3308 (105) and allowing the use of different smoke collection devices. There is a choice of smoking channels ( 20 ports in the SM 450 series, 10 ports and 5 ports in two smaller machines), and a variety of puff volumes, puff durations, puff profiles and puff intervals is possible. A smoking machine with integrated equipment for the determination of the mainstream gaseous constituents carbon monoxide and nitric oxide is also available.

The 20-port model SM 450 (106) is capable of smoking cigarettes according to ISO (105), FTC (19), Massachusetts (20) and Canadian Intense (22) methods without loss of smoking throughput or efficiency. Its 20 channels can be controlled as banks of 5, allowing 4 different smoking regimes being conformed to simultaneously. A wide variety of cigarettes can be smoked using appropriate holders. Besides routine collection of particulate matter on Cambridge filters or of carbon monoxide in bags, a socalled "Hoffmann shelf" (106) may be integrated for the capture of mainstream smoke for the determination of

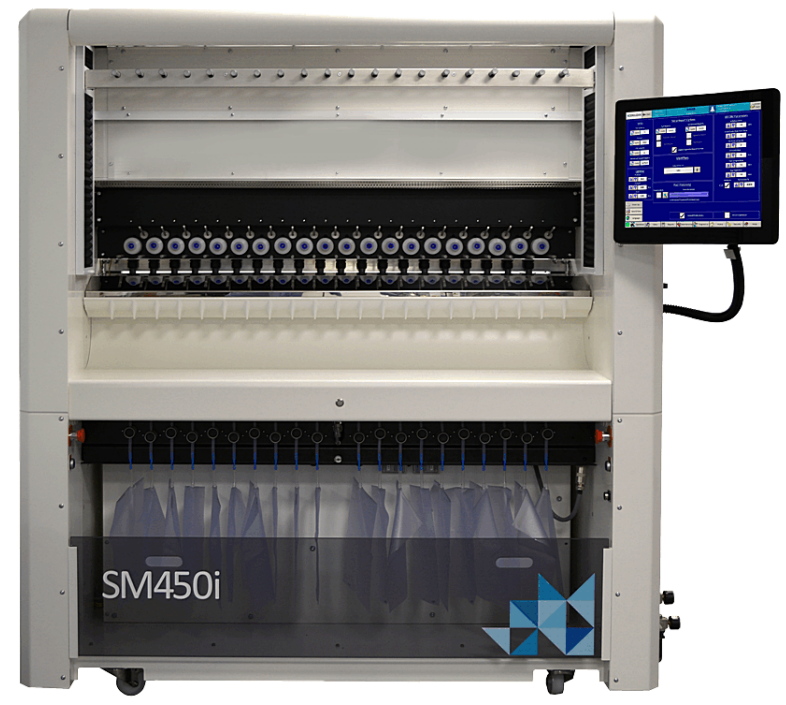

Figure 13. The semi-automated 20-port linear smoking machine SM 450i (106) with its new air management system constitutes the latest addition to Cerulean's SM 450 series.

specific "Hoffmann analytes"; the device contains sets of special carriers for impingers and holders for Cambridge filters.

The smaller models SM 405 (106) and SM 410 (106) represent smoking machines with 5 ports and 10 ports, respectively, both offering all the features of the larger SM 450 (106).

The 20-port smoking machine SM 450RH (106) is both suitable for routine cigarette smoking and flexible enough for non-routine and research work.

The upgraded 20-port SM 450N (106) features two integrated chemiluminescence analyzers, each coupled to a single (optionally independently configured) bank of 10 ports and offers a choice of analysis for $\mathrm{NO}$ or $\mathrm{NO}_{\mathrm{x}}$ $\left(=\mathrm{NO}+\mathrm{NO}_{2}\right)$ during a smoke run.

Efficient control of air flow around the glowing cone - as defined by ISO 3308 (105) - during both the puffing and smoldering phases is a requirement for achieving consistent yields of mainstream smoke nicotine-free dry particulate matter (NFDPM), nicotine, and carbon monoxide. The smoking machine SM 450 met ISO 3308 requirements for air flow (on average $200 \pm 30 \mathrm{~mm} / \mathrm{sec}$ ); however, the direction of flow is not specified in the standard. Instantaneous air flow turbulences and differences between ports may influence measured yields of "tar", nicotine and carbon monoxide; both factors should be as low as possible. The improved semi-automated 20-port linear smoking machine SM 450i (106), shown in Figure 13, addressed this problem by improvements of the ash tray (controlling the direction of air flow over the burning cigarette) and by extracting sidestream smoke not from above but from both sides of the smoking cabinet. In addition to a simpler way of setting up the air flow, the new SM 450i shows greater uniformity of flow across all the smoking ports and less individual port turbulence (107). Independently controlled puff engines in the SM 450i make up to 20 different smoking regimes possible at one time. Puff termination is optionally possible by optical means. 


\section{Free smoking}

The mouth end of the cigarette remains open between puffs; this allows a small part of the smoke, formed during smoldering, to escape through the open butt end.

Restricted smoking

The mouth end of the cigarette is sealed between the puffs.

Neither free nor restricted smoking mirror human smoking behavior, which differs not only between smokers but also per individual smoker depending on the time of day, circumstances, personal situation, etc.

Linear smoking machines for cigarette mainstream smoke analysis and research are also commercially available from BORGWALDT KC (108) - fully compliant with ISO 3308. Model LX 1 operates with a single syringe and offers high flexibility and low dead volume. The 20 individually controlled syringes of model LX20 allow up to 20 different smoking regimes to be followed simultaneously, optionally in ports grouped together.

In certain circumstances, strict compliance of a smoking machine with standard operating parameters may not be a primary objective; rather, plenty of scope for changing smoking patterns was desirable to support the development of new cigarette designs. For this purpose, LORTON and CUMMING (109) invented in 1987 a single port programmable smoking machine, which consisted of a step motor driven glass syringe connected via a three-way valve to the Cambridge filter/cigarette holder assembly. Non-standard smoking programs were controlled by a microcomputer. The machine allowed free and restricted smoking and the puff-by-puff collection of gas phase for direct analysis or trapping. Subsequently, the device was expanded to a 4-channel smoking machine with automatic electric lighting and butt termination detectors.

\subsection{Rotary smoking machines}

In the mid-1950s, striving for the automation of cigarette machine smoking resulted in the first prototypes of rotary devices. A telling example is the development of an apparatus by DECKER et al. (110) in Switzerland. Starting from a manually operated system for smoking only one cigarette at a time an automated machine was designed with 5 smoking ports (Figure 14) for the determination of nicotine and "tar".

Smoking parameters (supposedly reflecting human behavior and not yet standardized at that time) were a $40-\mathrm{mL}$ puff of $2 \mathrm{sec}$ duration every $20 \mathrm{sec}$. A camshaft and a set of valves were operated by a constant speed motor controlling puffing and aeration steps and rotating a vertical metal disk with 5 horizontally mounted cigarettes (one $5^{\text {th }}$ of the perimeter every $4 \mathrm{sec}$ ). Hypobaric pressure of $20 \mathrm{~mm}$ mercury was provided by a water aspirator and regulated in the completely closed system by means of a pinch cock and a manometer to ensure the desired volume when puffing. Smoke was trapped in four impingers filled with a mixture of diluted sulfuric acid and chloroform and cooled in ice water; nicotine was measured as dipicrate and "tar" gravimetrically.

WALTZ et al. (111) of Vereinigte Tabakfabriken AG (Neuchâtel-Serrieres, Switzerland) developed in 1961 a constant volume smoking machine with a vertically rotating smoking head, which held up to 10 cigarettes (Figure 15). A piston pump was used in combination with a magnetic valve for volume control. Smoke condensate was collected in a central electrostatic precipitator. The machine achieved constant puff volumes of $35 \pm 0.2 \mathrm{~mL}$ in $2 \mathrm{sec}$ at a cigarette pressure drop of $100 \mathrm{~mm}$ water column; puff duration increased by $0.1 \mathrm{sec}$ for every increase of $25 \mathrm{~mm}$ water column. 10 cigarettes could be smoked with a frequency of one puff per $60 \mathrm{sec}$ or 5 cigarettes with one puff per $30 \mathrm{sec}$. Practical machine performance was evaluated by WALTZ et al. (112) in connection with various collection devices (three different kinds of electrostatic precipitators and Cambridge filters), tobacco types, puff intervals of 30 and $60 \mathrm{sec}$, and methods of smoke constituent analysis. In addition, it was recognized that the increase of the burn rate (by approx. 10\%) of cigarettes smoked simultaneously in a rotary machine (compared to a single test piece) was an effect of heat radiation from smoldering cigarettes (and not of heat convection) - a phenomenon particularly effective with cigarettes mounted perpendicularly on a rotary smoking head. The new smoking machine, however, was not adopted by other European cigarette manufacturers; development activities turned towards horizontally rotating smoking machines.
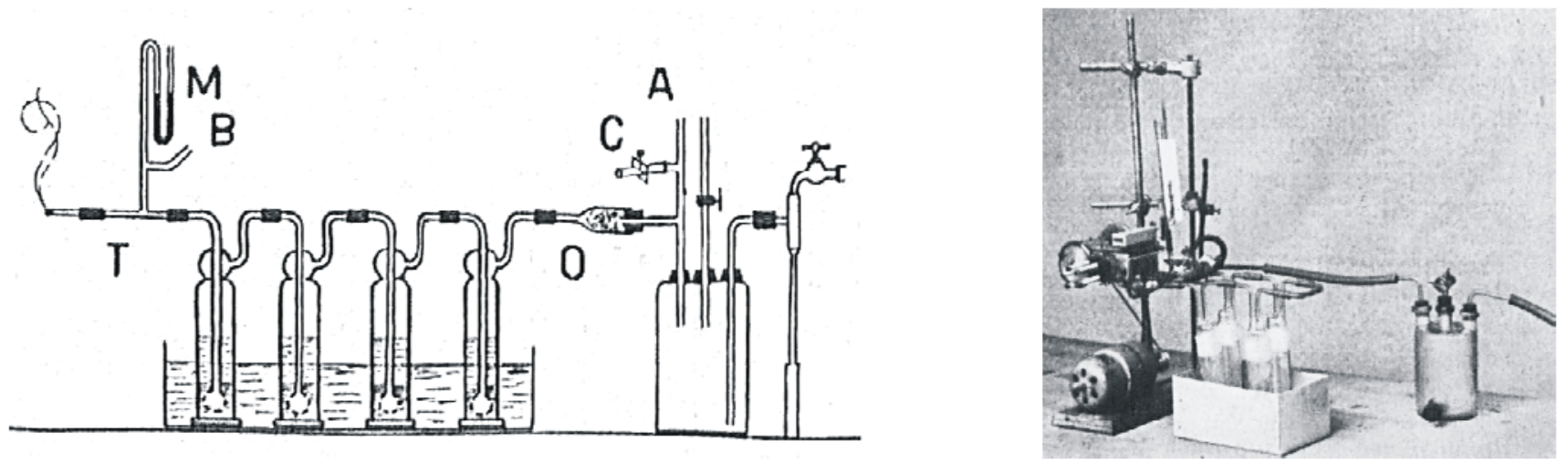

Figure 14. The conventional manually operated single cigarette smoking system (left) evolved into the automated 5-port rotating smoking machine (right) designed in 1955 by DECKER et al. (110). 


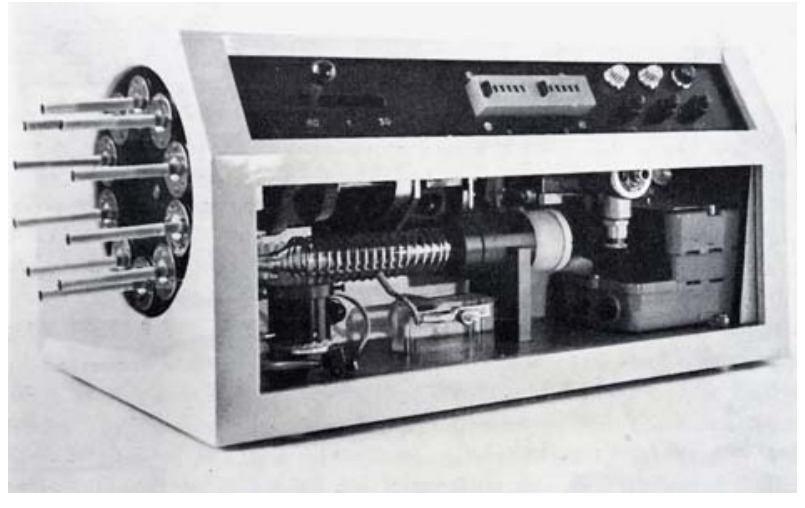

Figure 15. The 10-port rotary machine of WALTZ et al. (111) produced a constant puff volume of $35 \mathrm{~mL}$ in $2 \mathrm{sec}$ independent of variations in cigarette pressure drop.

The need for efficient quality control in cigarette manufacturing led - primarily in Germany - to the evolution of new designs of smoking machines. The developers' pragmatic challenge was high throughput and the production of useful data with reasonable accuracy rather than ultimate scientific precision. A horizontally rotating turntable served as smoking head accommodating a certain number of test pieces (initially 15 , later 30 or 20 ), which could be smoked sequentially. Mainstream smoke from all cigarettes was collected in a central trap. A variety of smoking machines of this type was developed by BAT (Hamburg, Germany) and made commercially available by the $\mathrm{H}$. Borgwaldt Company (Hamburg, Germany).

In 1959, SEEHOFER (113) described a rotary smoking machine for 15 cigarettes, later designated BAT-RM 15. The cigarettes were held in latex sleeves ("artificial lips") on the circular turntable, which rotated at $2 \mathrm{rpm}$. A puff of close to $40 \mathrm{~mL}$ was taken in approx. $1.5 \mathrm{sec}$ every $30 \mathrm{sec}$, whenever the cigarette reached a position facing the central suction port, which was connected to an oil vacuum pump. The system (like all others based on the same principle) worked with constant time and constant (hypobaric) pressure while puff volume was susceptible to (changing) cigarette pressure drop. Smoke condensate was collected centrally in a cotton wool trap according to WAHL and HEIL (61). The device was manufactured and offered for sale by Borgwaldt (as were several successor models).

The BAT-RM 15 was subsequently improved by SEEHOFER et al. $(114,115)$. In the BAT-RM 15/61, the cotton wool trap was replaced by a Cigarette Components electrostatic trap, operating at $20 \mathrm{kV}$. By including a crossed valve in the machine it was possible to obtain constant puff volumes and minimize the effect of differences in the pressure drop of the smoked cigarettes. The modified machine allowed better control of puff volumes and duration. This way, compliance was ensured with the smoking parameters becoming widely accepted in the Anglo-Saxon countries (one puff of $35 \mathrm{~mL}$ in 2 sec every $\min )$.

SEEHOFER et al. $(115,116)$ increased the capacity of the BAT-RM 15 smoking machine to 30 cigarettes. Smoking with the new "high-speed" BAT-RM 30/65 - shown in Figure 16 - was done under standard conditions (one puff of $35 \mathrm{~mL}$ in $2 \mathrm{sec}$ every min). Smoke condensate was collected in an electrostatic precipitator with high am- perage, specifically designed by H. Borgwaldt (Hamburg, Germany). Comparative batch analyses showed that the data obtained with a larger number of cigarettes, not selected for weight and draw resistance, were equivalent to those with a smaller number of selected test pieces.

Neither the BAT-RM 15 nor the BAT-RM 30/65 smoking machines were equipped with components for maintaining constant flow during puffing for eliminating the influence of cigarette pressure drop on puff volume. This problem was overcome by SEEHOFER (115) when he included flow stabilizing features in a new 20-port rotary smoking machine (the first version designated BAT-RM 20/68). Also, the distance between individual cigarettes mounted on the smoking head $(45 \mathrm{~mm})$ was sufficiently large to prevent any influence of heat radiation from neighboring cigarettes on the burn velocity during and between puffs. The importance of heat radiation had first been pointed out in 1958 by O'KEEFFE and LIESER (85).

It should not go unnoticed that SEEHOFER (115) - in his 1968 review of smoking machines for analytical determinations and for chemical and biological examinations of tobacco smoke - described a rather simple device (Figure 17) for the direct exposure of very small creatures to the smoke of one cigarette; it consisted of a motordriven syringe and the exposure tube with the cigarette holder attached.

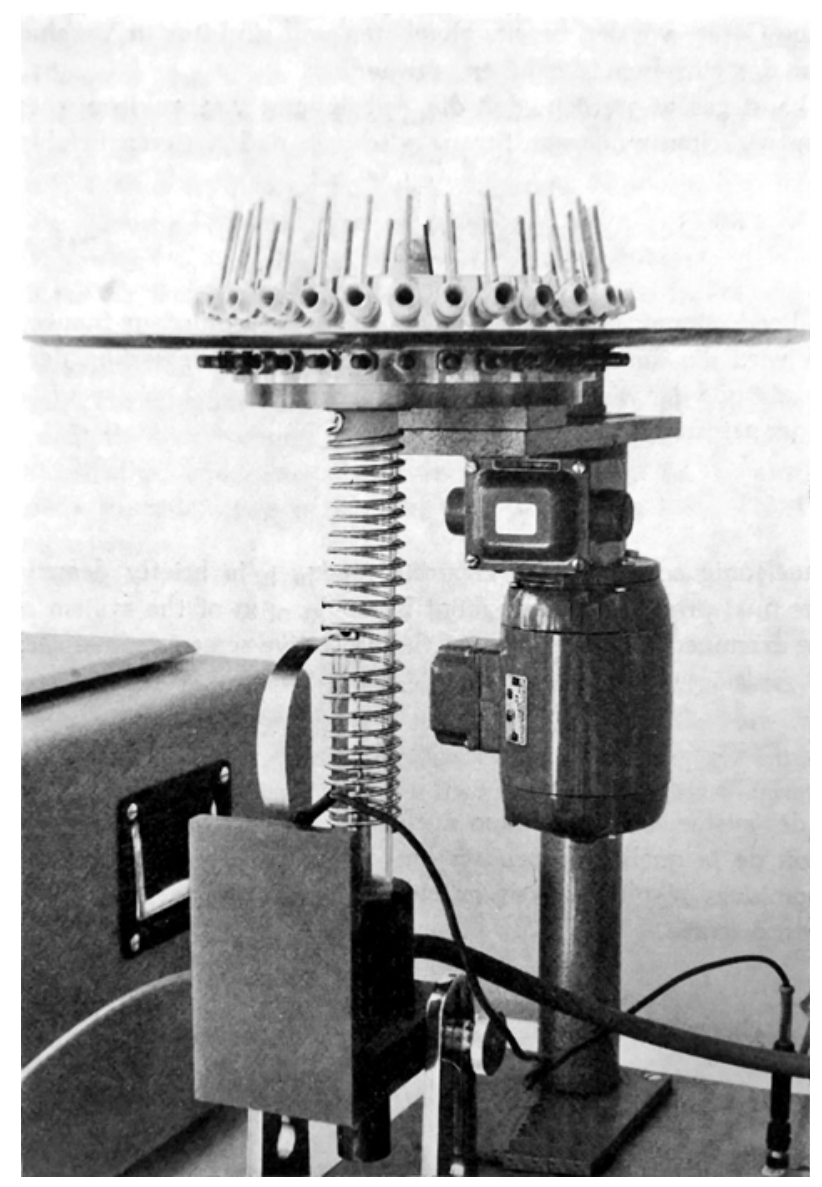

Figure 16. The horizontal 30-port smoke head (top) and the vertical electrostatic precipitator (center) are distinctive components of the BAT-RM $30 / 65$ smoking machine (116). 


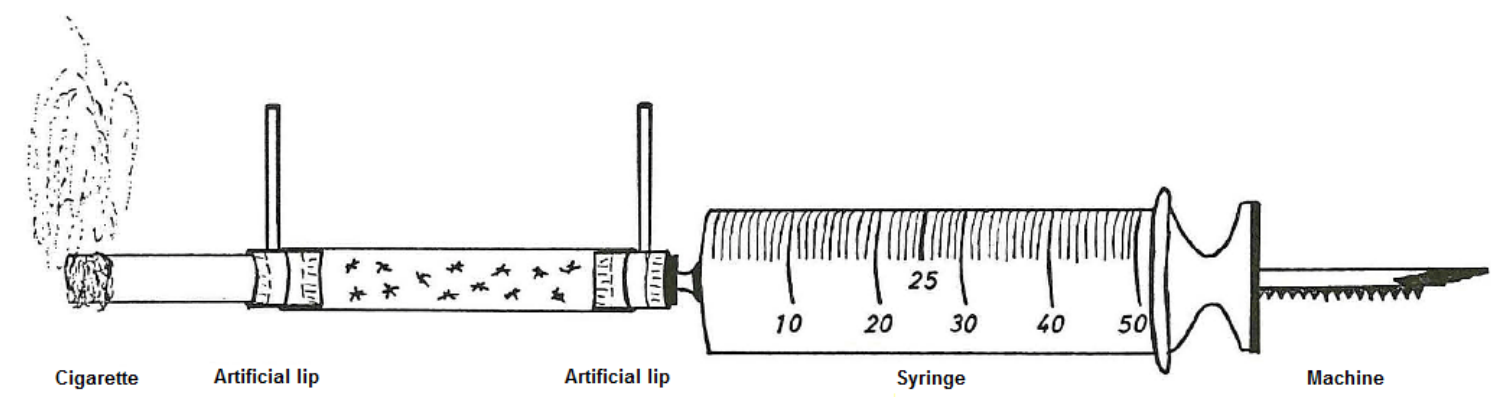

Figure 17. This simple exposure device for biological testing was disclosed by SEEHOFER (115); unfortunately, the correct Linnean name of the species under examination is not known.

In 1969, LIPP (117) compared smoke condensate and nicotine values obtained when using the rotary smoking machines BAT-RM 15 and BAT-RM 20/68, and Filtrona's linear 4-channel constant flow/constant time smoking machine CSM 10. There was good agreement of results between the BAT-RM 20/68 and CSM 10 smoking machines while the BAT-RM 15 delivered significantly lower mean smoke values for (wet and dry) condensate and nicotine. This is particularly interesting as electrostatic traps were used for smoke collection in both the BATRM 15 and BAT-RM 20/68 machines, and Cambridge filters in the CSM 10 machine.

At their plenary meeting in The Hague (The Netherlands) in September 1969, the ISO TECHNICAL COMMITTEE 126 (responsible for tobacco and tobacco products) invited CORESTA and laboratories in the United Kingdom - in Draft Resolution 15 (118) - to generate comparative data on the performance of different smoking machines. A technical committee under the chairmanship of the British Standards Institution accepted the challenge "to discuss all aspects of the various smoking machines available and to recommend, if possible, the selection of a machine, or type of machine, as a standard or reference smoking machine" (96) and asked Imperial Tobacco Group to undertake a comparative study, with the results eventually compiled in a report (96). The smoking machines examined were the Phipps \& Bird 20-channel linear machine developed by PHILIP MORRIS (8) and equivalent to the Filtrona SM 300, the 4-channel CSM 10 (94) and the slightly modified 4-channel CSM 12 machine manufactured by Cigarette Components Ltd., and the Borgwaldt BAT-RM 20/68 (115). Only the Phipps \& Bird machine operated with constant volume (suction by syringes, bell-shaped puff profile), the others with constant flow (suction by vacuum pumps, rectangular puff profile). Mainstream condensate was collected on Cambridge filters by all machines. The objective of the study (96) was to examine the different machines for their functional performance (mechanical and electrical reliability; puff volume stability; compensation; ability to maintain a symmetrical puff shape) and for their analytical performance (NFDPM, smoke nicotine, and nicotine retention on the filter; all three parameters assessed per cigarette and per smoking run). A ranking of machines was established based on the various performance criteria, which showed a mixed picture. In summary, the report concluded that the "Phipps \& Bird model unquestionably emerges as the most outstanding machine and is therefore recommended as the standard or reference machines for International Standardization" (96).

In response to two new standards for the machine smoking of cigarettes, namely the CORESTA Standard Method No. 10 of 1968 (51) and the DIN Norm 10240 of 1969 (119), and in view of activities in ISO, which in 1977 led to the International Standard ISO 3308, $1^{\text {st }}$ edition (10) these developments are discussed in detail in Chapter 3.3.2 (p. 159) - LORENZ et al. (120) reported in 1972 additional improvements of the 20-channel rotary smoking machine originally designed by BAT in Germany and developed it into the BAT-RM 20/71 mainly for routine analytical purposes - the endpoint of a consequential development of this type of smoking machine. The machine was equipped with automatic controls for puff duration, interval between puffs and puff number, automatic interruption of puffs at the preset butt length, and ejection of butts. Suction was provided by a rotating vacuum pump, producing a rectangular puff profile, or alternatively by a piston pump, producing rectangular or bell-shaped puff profiles. The machine was suitable both for restricted smoking (cigarette mouth end sealed between puffs - as required in standard procedures) and free smoking (mouth end open between puffs). Smoke condensate could be collected by electrostatic precipitation or on a central Cambridge filter (diameter $92 \mathrm{~mm}$ ) or on 20 separate Cambridge filters (diameter $44 \mathrm{~mm}$ ), each connected to a smoking port.

The smoke formed between puffs and leaving the cigarette - without suction - at the (unsealed) mouth end, the socalled "smolder stream", was examined in 1965 by SEEHOFER and SCHULZ (121). They reported that its intensity depended on physical cigarette parameters, such as pressure drop, moisture content and paper porosity/perforation, and assessed the influence of the smolder stream on mainstream smoke levels of condensate, nicotine, total phenols, total volatile aldehydes and acrolein. Effects on yields were marked but inconsistent. As they seemed uncontrollable when using smoking machines in the open smoking mode (cigarette mouth end not sealed between puffs), restricted smoking was deemed a requirement in analytical standard procedures. Consequently, restricted smoking was mandated by DIN 10240 of 1969 (119) and ISO 3308, $1^{\text {st }}$ edition of 1977 (10).

The BAT-RM 20/71 smoking machine - commercially manufactured by H. Borgwaldt (Hamburg, Germany) - also met the requirements specified for rotary machines in the revised DIN 10240, part 1 (122) of 1978 and, until 1991/ 1992, was the smoking machine accepted in Germany for 
the determination of nicotine-free dry particulate matter (NFDPM) and nicotine in cigarette smoke according to DIN 10240, part 2 (123) and part 3 (124); these data were required for imprints on cigarette packs. While the smoking parameters were those internationally accepted (one 35-mL puff of $2 \mathrm{sec}$ every min), smoke condensate was collected in a central electrostatic trap; this was different from the modalities of FTC (19) and the BRITISH STANDARD INSTITUTION (125), which required the use of Cambridge filters.

As mentioned above, the BAT-RM 20/71 analytical smoking machine (120) was able to produce two different puff profiles, rectangular and bell-shaped. It was demonstrated in 1971 by SEEHOFER and WENNBERG (81) that puff profiles affected mainstream smoke composition and yields. Dry condensate, nicotine and total steam volatile phenol yields were shown to depend on puff profile. Making use of the considerable versatility of the BATRM 20/71 smoking machine, SCHULZ and SEEHOFER (126) investigated the influence of several operating parameters on wet and dry total particulate matter (TPM), smoke nicotine and nicotine retention in the cigarette filter (means and deviations). The features compared were:

- Suction source (piston pump vs. vacuum pump)

- Puff profile (rectangular vs. bell-shaped)

- Trapping (Cambridge filter vs. electrostatic precipitation)

- Smoking mode (restricted vs. free)

- Dead volume with a central Cambridge filter (92 mm diameter) and per-port Cambridge filters (44 mm diameter)

- Number of cigarettes smoked (5, 10 or 20) affecting the collection efficiency of the central electrostatic trap.

Three commercial cigarette brands were used in the study (two German blend filter cigarettes and a Virginia plain cigarette). While the influence of certain parameters on some results was obvious, the authors did not see themselves in a position to express an unambiguous preference for one or the other. Remarkably, TPM and smoke nicotine yields were found to be significantly higher with free smoking compared to restricted smoking - confirming observations reported by AYRES and IVINSON in 1964 (127). In any case, the need was compelling for the effective control of whichever operating conditions were met.

In the manually operated rotary 20-port smoking machine RM20CS $(128,129)$, introduced by Borgwaldt in 1985, the vacuum pump was replaced by a piston pump to produce a bell-shaped puff profile and to overcome problems caused by variable cigarette pressure drop. The specifications of ISO 3308:1991 (12) necessitated the replacement of the RM20CS by version RM20CRS (129), which collected condensate on a central $92 \mathrm{~mm}$ Cambridge filter and featured an exhaust hood for controlling air flow around the cigarettes, a port in the exhaust line for measuring air velocity with an anemometer, and labyrinth seals and washers to replace the artificial lips of the cigarette ports. In 1994, Borgwaldt launched the first fully automatic rotary smoking machine, model RM200 (129). It aimed at analyzing cigarette smoke yields routinely on a large scale and with high efficiency. In compliance with ISO 3308: 1991 (12) and without operator intervention 10 smoke runs with 20 cigarettes each could be performed, automatically recording TPM and later, upon integration of a CO module, also carbon monoxide yields per cigarette and supplying the corresponding condensates on Cambridge filters for subsequent nicotine and water determination, preferentially by gas chromatography. It was also possible to examine other smoke constituents provided they were trapped on the filters; gas phase analysis - except carbon monoxide was not feasible.

The new semi-automatic smoking machine RM20 (129), introduced in 1998, was based on the concept of the RM20CS and improved for full compliance with ISO 3308:1991 (12); an embedded CO analyzer was optionally available.

The revised version RM20H (108) allowed the use of various trapping systems such as electrostatic precipitation for metal analysis following Health Canada method T-109 (130), and was particularly suitable for the determination of "Hoffmann analytes" (131).

The RM200 was improved by the integration of a CO module and an $\mathrm{NO} / \mathrm{NO}_{\mathrm{x}}$ analyzer and designated RM200A (108); the smoking machine is shown in Figure 18. An advanced version, RM200A2 (108), became available in 2015 for the routine determination of nicotine, "tar" and $\mathrm{CO}$ and optionally $\mathrm{NO}_{\mathrm{x}}$. Special features include the capability of smoking cigarettes up to $120 \mathrm{~mm}$ length and the accommodation of alternative smoke trapping systems, such as liquid and electrostatic traps.

As successor to model RM20CRS the manual rotary machine RM20D (108) was introduced in 2008. It was in compliance with ISO 3308:1991 (12) and could be combined with various trapping systems.

All BORGWALDT (108) rotary smoking machines currently available for purchase are in compliance with the design and smoking requirements of ISO 3308, $5^{\text {th }}$ edition of 2012 (105).

CERULEAN (106) as well provides semi-automatic rotary smoking machines which are in compliance with the requirements of ISO 3308 and flexible for ISO and Canadian

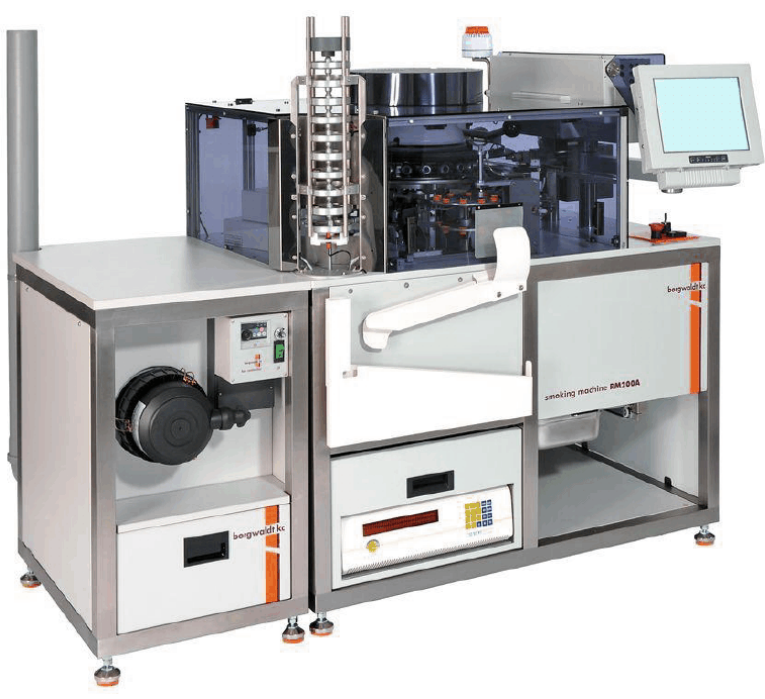

Figure 18. The automatic 20-port rotary smoking machine RM200A (108) was capable to perform 10 consecutive smoke runs without operator intervention. 


\section{Rotary smoking machines}

Ports for inserting cigarettes are arranged horizontally on the outside of a rotating ring, which makes one turn in a defined period of time. Mainstream smoke generated from all ports is collected in a central trap. A puff is drawn whenever a port holding a cigarette is linked up to the trap. Commonly, the smoking machine works with only one central suction device. However, smoke collection in individual traps is also possible.

Linear smoking machines

Separate traps for each port with a single cigarette are arranged in a horizontal row. Puffing is done simultaneously for all ports.

Intense regulatory smoking, specifically the 20-port CR20i and the 20-port CR20iE, featuring an enclosed hood to protect the operator and for more controlled air flows during smoking.

\subsection{Standardization of machine smoking methods and test pieces}

\subsubsection{FTC standard methodology}

In response to escalating marketing activities in the late 1950 s as well as statements by the U.S. Public Health Service with focus on lower "tar" and nicotine levels in cigarette smoke and potentially reduced health risks, and under its authority to regulate deceptive or unsubstantiated claims in advertising, the U.S. Federal Trade Commission (FTC) set about establishing standardized testing methodology for the determination of "tar" and nicotine in cigarette smoke. In March 1966, the COMMISSION (132) sent identical letters to all major cigarette manufacturers in regard to factual statements of "tar" and nicotine content on labels and in advertising, and required competent scientific proof by tests conducted in accordance with the "Cambridge Filter Method". This procedure had been examined by OGG (133) of the USDA Agricultural Research Service by performing a collaborative study with 12 participants, using automatic smoking machines and the smoking parameters (a 35-mL puff of $2 \mathrm{sec}$ every min), which had been chosen earlier by BRADFORD et al. (4) and were confirmed by OGG'S own real-life observations of smokers. For the collaborative study, a butt length of $30 \mathrm{~mm}$ was selected for both kinds of test cigarettes, King Size plain and King Size filtered. At the same time, the FTC authorized the establishment of a Commission laboratory to analyze cigarette smoke; it existed until 1987. Furthermore, the FTC (134) invited public comment from all interested and affected parties on modifications of the method, if appropriate, and the expression of results, requested information and suggestions on a number of quite specific technical questions, and called a public hearing on November 30, 1966.

The U.S. cigarette industry participated actively in the discourse as exemplified by two submissions: The cover letter (135) and consensus document (136) of major laboratories of the cigarette industry presenting technical data and recommendations regarding methods and operating procedures for the determination of total particulate matter (TPM), water in TPM and total alkaloids as nicotine, both dated November 28, 1966; and a memorandum (137) with supplementary observations following the November 30,1966, hearing, which endeavored in simple form to expose and to clarify the complex technological procedures involved in determining the amounts of particulate matter (PM) and nicotine in cigarette smoke, dated December 20, 1966.

Based on written presentations and oral testimony put forward at the hearing and following the satisfactory completion of trial tests by the Commission laboratory, the FTC (9) defined various technical and reporting conditions regarding the "Cambridge Filter Method" and ordered the commencement of formal testing using what has become known as the "FTC Method" (19). In a parallel news release the FTC (138) stated clearly the purpose of the agreed-upon method and the informative value it was - or was not - expected to provide: "In determining the testing method, the Commission has not attempted to gauge the test to the amount of smoke, or "tar" and nicotine, which the "average" smoker will draw from any particular cigarette. No two human smokers smoke in the same way. ..... The Cambridge Filter Method does not and cannot measure these many variations in human smoking habits. .... the uniform method determined by the Commission has as its purpose measurement of the "tar" and nicotine generated by cigarettes when smoked according to that procedure."

In the form of a scientific paper, the "FTC Method" was outlined by PILLSBURY et al. (19) - including a specific description of the smoking machine used and an evaluation of the precision of the method based on within-laboratory reproducibility. Details of method use and data reporting continued to be the topic of critical discussions between the FTC and the cigarette manufacturers. By 1971, six major and three smaller U.S. cigarette manufacturers had entered into voluntary agreements with the FTC to disclose "tar" and nicotine data as determined by the "FTC Method" in all cigarette advertising. Effective July 1980, the FTC (139) required the testing and disclosure of carbon monoxide levels in cigarette smoke and instituted the adaptation of method and equipment. To this end, the FTC laboratory had designed a new 20-port sequential smoking machine with an improved motor driven and hydraulically operated syringe device without sealing oil, and the capability of detecting carbon monoxide puff-by-puff by quantitative non-dispersive infrared spectrometry (140). The new machine was built and marketed by Phipps \& Bird, and well accepted in the United States.

In the course of time, the "FTC Method" was challenged on grounds of technical and scientific arguments (some obviously arising from commercial rivalry): In 1977 insertion depth of filter-ventilated cigarettes into the holder seal became an issue, and in the mid-1980s the problem of compensatory smoking was discussed controversially. In both cases the FTC saw no reasons necessitating changes in the method, upholding the concept that it was not intended to replicate actual human smoking.

In April 1983, the Federal Trade Commission announced its concern that the deliveries of a novel type of filter ciga- 
rette (branded "Barclay") might be underestimated when measured using current standard methodology. These cigarettes featured filters with a new channel ventilation system (drawing air directly into the smoker's mouth) in addition to conventional air holes (mixing smoke and air in the filter). It was alleged that, in practice, crushing the channels or covering them by lips could reduce the flow of air and provide the smoker with less diluted smoke; channels definitely remained open during machine smoking using standard methodology $(10,19)$. Barclay cigarettes had initially (1981) received an official rating of $1 \mathrm{mg}$ "tar" but were now, for the time being, excluded from the annual Commission reports on "tar", nicotine and carbon monoxide. Three years later, after a review of data presented by the manufacturer the Commission accepted the use of set yields per cigarette for official reporting and for advertising purposes: $3 \mathrm{mg}$ "tar" and $0.2 \mathrm{mg}$ nicotine for Barclay King size, and $5 \mathrm{mg}$ "tar" and $0.4 \mathrm{mg}$ nicotine for Barclay 100's (141). The standard method was not modified.

Interesting accounts by PEELER (142) and PILLSBURY (143) of how the "FTC Method" evolved and was actually used may be found in the National Institutes of Health (NIH) Smoking and Tobacco Control Monograph No. 7.

It was not until 2008 when the FTC (144) gave in to pressure from health advocacy groups, that it distanced itself from the "FTC Method" and rescinded the guidelines issued in 1966, which required statements concerning cigarette "tar" and nicotine yields in advertising. Scientific consensus was quoted as evidence that machine-based measurements did not provide meaningful information to consumers on the amounts of "tar" and nicotine smokers received from cigarettes. It is, however, noteworthy that a quite similar machine smoking method, namely ISO 3308 (105), is currently mandated by the U.S. Federal Drug Administration (FDA) for harmful and potentially harmful constituents (HPHC) testing and reporting (145).

\subsubsection{CORESTA Recommended Methods and ISO Standards}

In the early 1950s the European cigarette industry was confronted, rather unprepared, with smoking related health issues. There was an obvious requirement for relevant and detailed knowledge of the composition of tobacco and smoke. At that time, neither a widely accepted practical machine nor a standardized method existed in Europe for the analytical smoking of cigarettes. The challenge to develop such a method was accepted by CORESTA, an organization founded in 1956 in Paris under the leadership of tobacco state monopolies. For scientific work, CORESTA set up a number of working groups. In 1981, KUHN (146) - one of the three original members of CORESTA's Smoke Study Group, which was formed in 1959 - presented a historial review of the activities of this group. He called to mind that the need for physicochemical investigation of tobacco smoke was recognized and focused on. In response to a major issue at that time, a standard method for the determination of cigarette filter efficiency was to be established. For the artificial smoking of cigarettes the parameters widely used in the United States (4) seemed to be quite suitable. A first draft standard was tested in a collaborative study in 1961 with 10 laboratories participating; final and statistically evaluated results became available in 1962. In addition, the standardization of a method for quantifying nicotine in smoke was called for in view of the high variation of observed analytical values. However, health related aspects of any experimental results were not part of the program. From 1962 onward, the problem of filter efficiency became less important and the focus of scientific work shifted to "smoke yield of a tobacco product", namely "tar" and nicotine. Initial work was directed at developing basic working parameters for a unified smoking machine and conceiving a trap for quantitative smoke collection. Smoke trapping was addressed in a draft standard, which was tested in a collaborative study and subsequently revised in 1964. A draft standard for nicotine determination was put to the test in a joint study with 11 laboratories in 1965, and data became available the following year. All these activities resulted in four CORESTA standards, finalized and printed in 1966 (the forth being the eventual outcome of the initial work on filter efficiency):

- CORESTA Standard Method No. 1: Machine Smoking of Cigarettes and Determination of Moist and Anhydrous Smoke Condensate (147).

- CORESTA Standard Method No. 2: Determination of Total Alkaloids as Nicotine in Tobacco (148).

- CORESTA Standard Method No. 3: Determination of Total Alkaloids as Nicotine in Tobacco Smoke Condensates (149).

- CORESTA Standard Method No. 4: Determination of Nicotine Alkaloid Retention by Cigarette Filters (150). With the intention of gaining international recognition by ISO (International Organization for Standardization) the four CORESTA standards were presented - at the initiative of the German Verband der Cigarettenindustrie - to DIN (Deutsches Institut für Normung, German Institute for Standardization), revised according to DIN general editorial requirements and adopted in September 1968 (and published in 1969) as:

- CORESTA Standard Method No. 10: Machine Smoking of Cigarettes, Determination of Crude and Dry Smoke Condensate (51).

- CORESTA Standard Method No. 12: Determination of Alkaloids in Cigarette Smoke Condensates (151).

- CORESTA Standard Method No. 13: Determination of Alkaloid Retention by Cigarette Filters (152).

- CORESTA Standard Method No. 20: Determination of Alkaloids in Manufactured Tobacco (153).

CORESTA Standard Method No. 10 - in literal translation - was promptly (in April 1969) accepted as German national standard DIN 10240 (119).

The four CORESTA Standard Methods in DIN format were submitted to the new ISO Technical Committee "TC 126 - Tobacco and Tobacco Products" at their first meeting in Berlin in October 1968. Three of the four standards were approved as ISO Standards after several years:

- ISO 3400 of 1976: Tobacco and Tobacco Products Determination of Alkaloids in Cigarette Smoke Condensates - Spectrophotometric Method, $1^{\text {st }}$ edition (154); based on CORESTA Standard No. 12 ; currently $3^{\text {rd }}$ edition of 1997.

- ISO 3401 of 1977: Tobacco and Tobacco Products - 
Table 1. ISO Standards and Technical Reports.

\begin{tabular}{|c|c|c|}
\hline Standard & Title & Evolution \\
\hline ISO 8243 & Cigarettes -- Sampling & \begin{tabular}{|l|} 
ISO $8243: 2013$ \\
ISO $8243: 2006$ \\
ISO $8243: 2003$ \\
ISO $8243: 1991$ \\
ISO $8243: 1988$
\end{tabular} \\
\hline ISO 3402 & $\begin{array}{l}\text { Tobacco and tobacco products -- Atmospheres for conditioning and } \\
\text { testing }\end{array}$ & $\begin{array}{l}\text { ISO } 3402: 1999 \\
\text { ISO } 3402: 1991 \\
\text { ISO } 3402: 1978\end{array}$ \\
\hline ISO 3308 & $\begin{array}{l}\text { Routine analytical cigarette-smoking machine -- Definitions and } \\
\text { standard conditions }\end{array}$ & $\begin{array}{l}\text { ISO } 3308: 2012 \\
\text { ISO } 3308: 2000 / \text { Amd } 1: 2009 \\
\text { ISO } 3308: 1991 \\
\text { ISO } 3308: 1986 \\
\text { ISO } 3308: 1977\end{array}$ \\
\hline ISO 7210 & $\begin{array}{l}\text { Routine analytical cigarette-smoking machine -- Additional test } \\
\text { methods for machine verification }\end{array}$ & $\begin{array}{l}\text { ISO } 7210: 2013 \\
\text { ISO } 7210: 1997 \\
\text { ISO } 7210: 1983\end{array}$ \\
\hline ISO 16055 & $\begin{array}{l}\text { Tobacco and tobacco products -- Monitor test piece -- Requirements } \\
\text { and use }\end{array}$ & $\begin{array}{l}\text { ISO } 16055: 2012 \\
\text { ISO } 16055: 2003\end{array}$ \\
\hline ISO 4387 & $\begin{array}{l}\text { Cigarettes -- Determination of total and nicotine-free dry particulate } \\
\text { matter using a routine analytical smoking machine }\end{array}$ & $\begin{array}{l}\text { ISO } 4387: 2000 / A m d ~ 1: 2008 \\
\text { ISO 4387:1991 } \\
\text { ISO 4387:1987 }\end{array}$ \\
\hline ISO 8453 & $\begin{array}{l}\text { Cigarettes -- Determination of total and dry particulate matter using a } \\
\text { routine analytical cigarette-smoking machine -- Electrostatic smoke } \\
\text { trap method }\end{array}$ & $\begin{array}{l}\text { ISO } 8453: 1987 \\
\text { withdrawn }\end{array}$ \\
\hline ISO/TR 22305 & $\begin{array}{l}\text { Cigarettes -- Measurement of nicotine-free dry particulate matter, } \\
\text { nicotine, water and carbon monoxide in cigarette smoke -- Analysis } \\
\text { of data from collaborative studies reporting relationships between } \\
\text { repeatability, reproducibility and tolerances }\end{array}$ & ISO 22305:2006 \\
\hline ISO 10362-1 & $\begin{array}{l}\text { Cigarettes -- Determination of water in smoke condensates -- Part 1: } \\
\text { Gas-chromatographic method }\end{array}$ & $\begin{array}{l}\text { ISO 10362-1:1999/Amd 1:2011 } \\
\text { ISO 10362-1:1991 }\end{array}$ \\
\hline ISO 10362-2 & $\begin{array}{l}\text { Cigarettes -- Determination of water in smoke condensates -- Part 2: } \\
\text { Karl Fischer method }\end{array}$ & $\begin{array}{l}\text { ISO 10362-2:2013 } \\
\text { ISO 10362-2:1994/Amd 1:2011 }\end{array}$ \\
\hline ISO 3400 & $\begin{array}{l}\text { Cigarettes -- Determination of alkaloids in smoke condensates -- } \\
\text { Spectrometric method }\end{array}$ & $\begin{array}{l}\text { ISO 3400:1997/Amd 1:2009 } \\
\text { ISO 3400:1989 } \\
\text { ISO 3400:1976 }\end{array}$ \\
\hline ISO 10315 & $\begin{array}{l}\text { Cigarettes -- Determination of nicotine in smoke condensates -- } \\
\text { Gaschromatographic method }\end{array}$ & $\begin{array}{l}\text { ISO } 10315: 2013 \\
\text { ISO } 10315: 2000 / \text { Cor } 1: 2000 / \\
\text { Amd } 1: 2011 \\
\text { ISO } 10315: 1991\end{array}$ \\
\hline ISO 11454 & $\begin{array}{l}\text { Tobacco and tobacco products -- Determination of vapour-phase } \\
\text { nicotine in air -- Gas-chromatographic method }\end{array}$ & $\begin{array}{l}\text { ISO 11454:1997 } \\
\text { withdrawn }\end{array}$ \\
\hline ISO 8454 & $\begin{array}{l}\text { Cigarettes -- Determination of carbon monoxide in the vapour phase } \\
\text { of cigarette smoke -- NDIR method }\end{array}$ & $\begin{array}{l}\text { ISO } 8454: 2007 / A m d ~ 1: 2009 \\
\text { ISO } 8454: 1995 \\
\text { ISO } 8454: 1987\end{array}$ \\
\hline ISO19290 & $\begin{array}{l}\text { Cigarettes -- Determination of tobacco specific nitrosamines in } \\
\text { mainstream cigarette smoke -- Method using LC-MS/MS }\end{array}$ & under development \\
\hline ISO 22634 & $\begin{array}{l}\text { Cigarettes -- Determination of benzo[a]pyrene in cigarette } \\
\text { mainstream smoke -- Method using gas chromatography/mass } \\
\text { spectrometry }\end{array}$ & ISO 22634:2008 \\
\hline ISO/DIS 22634-2 & $\begin{array}{l}\text { Cigarettes -- Determination of benzo[a]pyrene in cigarette } \\
\text { mainstream smoke using GC/MS -- Part 2: Method using } \\
\text { cyclohexane as extraction solvent }\end{array}$ & under development \\
\hline ISO 13110 & $\begin{array}{l}\text { Cigarettes -- Determination of menthol in smoke condensates -- } \\
\text { Gaschromatographic method }\end{array}$ & ISO 13110:2012 \\
\hline ISO/TR 17219 & $\begin{array}{l}\text { Review of human smoking behaviour and recommendations for a } \\
\text { new ISO standard for the machine smoking of cigarettes }\end{array}$ & ISO/TR 17219:2013 \\
\hline ISO/TR 19478-1 & $\begin{array}{l}\text { ISO and Health Canada intense smoking parameters -- Part 1: } \\
\text { Results of an international machine smoking study }\end{array}$ & ISO/TR 19478-1:2014 \\
\hline ISO/TR 19478-2 & $\begin{array}{l}\text { ISO and Health Canada intense smoking parameters -- Part 2: } \\
\text { Examination of factors contributing to variability in the routine } \\
\text { measurement of TPM, water and NFDPM smoke yields of cigarettes }\end{array}$ & ISO/TR 19478-2:2015 \\
\hline
\end{tabular}

DIS: Draft Interational Standard; TR: Technical Report 
Determination of Alkaloid Retention by Filters of Cigarettes, $1^{\text {st }}$ edition (155) - based on CORESTA Standard No. 13 ; currently $2^{\text {nd }}$ edition of 1991 .

- ISO 2881 of 1977: Tobacco and Tobacco Products Determination of Alkaloids in Tobacco - Spectrophotometric Method, $1^{\text {st }}$ edition (156) - based on CORESTA Standard No. 20; currently $3^{\text {rd }}$ edition of 1992.

CORESTA Standard Method No. 10 as such (equivalent to DIN 10240 of 1969) did not progress toward international acceptance. Rather, approval was granted to:

- ISO 3308 of 1977: Routine Analytical Cigarettesmoking Machine - Definitions and Standard Conditions (10) - currently $5^{\text {th }}$ edition of 2012 (105).

ISO 3308:1977 represents a key document for cigarette analytical work; over the years it has been revised several times. The standard "defines the smoking parameters and the standard conditions to be provided for the routine analytical machine smoking of cigarettes" and "specifies requirements for a routine analytical smoking machine ... and for the auxiliary equipment". Obviously, the standard is focused on delineating technical specifications for analytical cigarette smoking rather than singling out a certain type or version of a smoking machine. This was very much in line with earlier work done by the CORESTA Smoke Study Group. Parallel to the work on standard methods, an attempt had been made in 1967 towards unifying the existing multiplicity of analytical smoking machines after two-thirds of 29 respondents to a questionnaire had expressed their dissatisfaction with the machinery available for routine analytical applications. A small scale comparison, however, led to the conclusion that "it seems impossible to standardize anyone of the smoking machines in use as a reference machine and it appears preferable to standardize only the specifications with which such a machine should have to comply" (146, page 75).

In parallel, DIN 10240 of 1969 (119) was rewritten in 1978 and split into three parts (122-124) - with part 1 conforming to ISO 3308 (10). An additional standard DIN 10240 , part 4 (157) was issued in 1985 after "channel ventilated" cigarettes (brand name "Barclay") had become commercially available. As described above, these cigarettes featured lengthwise grooves in the filter plug, which allowed air - drawn in through the (traditional) filter ventilating holes - to enter directly into the smoker's mouth without prior mixing with smoke. As this function could be modulated by smoker's lips the existing standards DIN 10240, parts 1 and $2(122,123)$ were considered inappropriate for this kind of cigarettes because of presumed underestimation of smoke yields. DIN 10240, part 4 (157) specified that channel ventilated cigarettes were to be smoked independently under two conditions, namely unaltered as well as with completely blocked ventilation channels. The arithmetic means of both determinations represented the values for "tar" and nicotine, respectively, for imprints on packages and advertising. In 1972, CORESTA (158) decided to forego the development of perfected standards in favor of producing suitable "Recommended Methods", which were subsequently to be refined by ISO Technical Committee 126.

An informative review - based on readily available published data and with focus on the United Kingdom - of the development of machine smoking parameters for mea- suring cigarette "tar" yields and their adoption by research, regulatory and quality control laboratories up to 1986 was published by JOHNSON (78).

\subsubsection{Kentucky reference cigarettes and CORESTA monitor test pieces}

The need for the comparative assessment of smoking machine performance, the validation of analytical and toxicological methods and the reliable generation of data regarding smoke yields and composition necessitated the development of standard test pieces with representative composition and known characteristics (159). Today, there are two versions of reference cigarettes which may be obtained from these sources: Kentucky reference cigarettes from the University of Kentucky, College of Agriculture, Lexington (KY); and CORESTA monitor test pieces from the Borgwaldt or Cerulean companies.

As early as 1959 - at the $13^{\text {th }}$ Tobacco Chemists' Research Conference - MORGAREIDGE (160) stated that the observed variations in analytical data on cigarette smoke could be due to differences between individual cigarettes within a sample as well as differences attributable to the analytical procedures. For assessing the "reproducibility and precision" of these procedures the establishment of a standardized reference cigarette was called for.

In 1968 the Scientific Advisory Board of the U.S. Council for Tobacco Research (CTR) initiated the development and production of reference cigarettes by the University of Kentucky, College of Agriculture within the frame of their Tobacco and Health Research Program (which afterwards became the Tobacco and Health Research Institute, subsequently renamed the Kentucky Tobacco Research and Development Center and now operating under the name of Center for Tobacco Reference Products).

The first so-called Kentucky reference cigarette became available in the same year (161). The program provides standardized cigarettes typical for the U.S. market in terms of design and tobacco composition (an "American blend" consisting of flue-cured bright Virginia, air-cured Burley and sun-cured oriental tobaccos - though without final top flavorings) and of smoke condensate and nicotine levels (plain and filter cigarettes with high, medium, low and ultra-low yields). The 1R1 reference cigarette (a plain $85 \mathrm{~mm}$ cigarette, high in smoke condensate and nicotine) was the first in a series of standardized test pieces used world-wide for research purposes.

Interestingly, initiated in 1969 , there was a collateral line of "alkaloid series cigarettes" comprising test pieces with different levels of nicotine in mainstream smoke (161, 162). The respective codes included the letter " $A$ " (rather than the " $\mathrm{R}$ " used for the reference cigarettes).

In 2014, CHAMBERS et al. (163) reported on a cooperative agreement between the Center for Tobacco Products of the U.S. Food and Drug Administration and the University of Kentucky's Tobacco Research and Development Center to continue and restructure the reference cigarette program and to establish a center of excellence on tobacco reference products. While many features of the existing scheme were maintained and affirmed (including coding the forthcoming reference batch of 50 million sticks with $1 \mathrm{R} 6 \mathrm{~F}$ ), a number of new aspects appeared on the agenda: Instrument calibra- 
Table 2. Smoke data of Kentucky reference cigarettes.

\begin{tabular}{|c|c|c|c|c|c|c|}
\hline Code $^{a}$ & $\begin{array}{c}\text { Production } \\
\text { year }\end{array}$ & $\begin{array}{l}\text { FTC tar }^{\mathrm{d}} \\
\text { (mg/cig) }\end{array}$ & $\begin{array}{c}\text { FTC nicotine }^{d} \\
(\mathrm{mg} / \mathrm{cig})\end{array}$ & $\begin{array}{l}\text { Butt length } \\
(\mathrm{mm})\end{array}$ & Reference & Characteristic \\
\hline 1R1 & 1969 & 34.3 & 2.16 & 23 & (161) & original reference cigarette \\
\hline $2 \mathrm{R} 1$ & 1974 & 36.8 & 2.45 & 23 & (161) & $2^{\text {nd }}$ manufacturing run of $1 \mathrm{R} 1$ \\
\hline 1R3 & 1974 & 22.8 & 1.46 & 23 & $(161)$ & equivalent to $\mathrm{NCl}$ standard blend \\
\hline 2R1F & 1974 & 23.4 & 1.74 & 30 & (161) & filtered version of $2 \mathrm{R} 1$ \\
\hline 1R3F & 1974 & 15.0 & 1.16 & 30 & $(161,164)$ & filtered version of $1 \mathrm{R} 3$ \\
\hline 1R4F & 1983 & 9.2 & 0.80 & 35 & $(161)$ & filtered, low smoke yields \\
\hline 1R5F & 1989 & 1.67 & 0.160 & 35 & $(161,164)$ & filtered, ultra-low smoke yields \\
\hline $2 \mathrm{R} 4 \mathrm{~F}$ & 2001 & 9.70 & 0.85 & 35 & (164) & followed 1R4F \\
\hline $3 \mathrm{R} 4 \mathrm{~F}^{\mathrm{b}}$ & 2008 & 9.4 & 0.73 & 35 & $(165)$ & followed 2R4F \\
\hline $1 \mathrm{R} \mathrm{F}^{\mathrm{b}}$ & 2016 & $8.58^{\mathrm{C}}$ & $0.721^{\mathrm{c}}$ & 35 & $(166)$ & filtered, low smoke yields, FSC paper \\
\hline
\end{tabular}

\author{
$\mathrm{F}=$ Filter cigarette \\ currently available reference cigarettes (167) \\ ISO smoking regime \\ All tar and nicotine data extracted from University of Kentucky documents
}

tion, method validation and proficiency testing. The proficiency testing program defined 19 "elements", viz. single, or combinations of, physical and chemical parameters to be determined in $1 \mathrm{R} 6 \mathrm{~F}$ reference cigarettes using both the ISO (105) and the Canadian Intense (22) smoking regimes. At present, proficiency test kits are available - or at least advertised - for the first 9 elements and designed for use with either rotary or linear smoking machines (167).

At their 1994 meeting, the CORESTA BOARD (168) approved the specifications, production and distribution of a CORESTA monitor, called test piece. It was designed for greater uniformity (made from flue-cured lamina only, no non-tobacco ingredients in the blend except $\leq 2 \%$ glycerol, with tighter weight tolerances) and specifically intended for observing long-term instrument performance in analytical routine analysis. A target yield of $18 \mathrm{mg}$ "tar" was chosen in order to obtain sufficient sensitivity when checking analytical equipment. Since 1995, seven monitors with unventilated filters (designated CM2 to CM8) were produced - the more recent versions in compliance with ISO 16055 (169), which describes the requirements and use of a cigarette monitor test piece. Also, CM8 was manufactured according to CORESTA Guide No. 8 of 2009 - CORESTA Monitor Test Piece Production and Evaluation Requirements (170). The current labeling of monitor test piece packs includes "Approved for laboratory testing" and "For non-consumer testing purposes only".

For several years now, yields per cigarette of the topical CORESTA monitor(s) have been assessed on an annual basis. Thirty-seven laboratories, using 52 smoking machines (24 linear and 28 rotary), participated in the collaborative study of the CORESTA ROUTINE ANALYTICAL CHEMISTRY SUBGROUP (171), conducted in 2013 with the recent monitor \#7 (CM7). Mainstream smoke yields according to ISO (105) amounted to $13.2 \mathrm{mg}$ NFDPM, $1.22 \mathrm{mg}$ nicotine and $12.8 \mathrm{mg} \mathrm{CO}$ - showing good consistency of smoke levels over the years (CM4 in 2004 through CM7 in 2013). In this study, smoke yields were also determined using the Canadian Intense smoking regime (22) and ran to $28.4 \mathrm{mg}$ NFDPM, $2.49 \mathrm{mg}$ nicotine and $24.7 \mathrm{mg} \mathrm{CO}$.

The latest collaborative study of the CORESTA RouTINE ANALYTICAL CHEMISTRY SUBGROUP (172) was conducted in 2014/2015 and involved 27 laboratories with 38 smoking machines (17 linear and 21 rotary). Smoking was done according to ISO (105). Two variants of monitor test pieces were examined: Once more the current CM7, existing since 2011 , to verify the stability of previously obtained smoke yields; and the new CM8 to produce data supporting its release as the next monitor. Average smoke yields of CM7 were shown to be highly consistent over the last four tests (2011 through 2014/2015). The new CM8 produced smoke yields quite in line with earlier monitor test pieces: $14.1 \mathrm{mg}$ NFDPM, $1.65 \mathrm{mg}$ nicotine and $13.4 \mathrm{mg} \mathrm{CO}$; it was concluded that CM8 was suitable for use and sale (available since August 2015).

\subsection{The harmonization of linear and rotary smoking machines}

When the mainstream smoke levels of condensate and nicotine - determined by smoking identical cigarettes with linear machines (equipped with Cambridge filters) or rotary machines (with electrostatic traps) - were compared in the time period before 1992, differences of up to $10 \%$ were observed. As smoking conditions (puffing parameters and butt length) were identical, the type of smoking machine and/or the device for condensate trapping had to be responsible for the differences, although both machine types and trapping devices were in compliance with the relevant ISO standards $(10,173,174)$ released in 1977 and 1987, respectively. The ramifications caused by these differences were discussed by BAKER (175) in his paper "The development and significance of standards for smoking-machine methodology".

The issue gained regulatory relevance when - in the mid1970s - various European countries required mainstream smoke yields of "tar" (nicotine-free dry particulate matter, NFDPM) and nicotine to be declared on cigarette packs. Analytical data had to be obtained using pertinent official methodology. This led to uncommon obligations on the 
manufacturers' part when a certain brand was produced for different countries. For example, "tar" and nicotine yields of the very same brand had to be determined for the Austrian market according to ISO 3308 (using the Filtrona 300 smoking machine and Cambridge filters for condensate collection) and for Germany according to DIN 10240 (in practice, with the Borgwaldt BAT-RM 20/71 rotary machine and electrostatic precipitation of condensate) - resulting in numbers printed on packs that were definitely not the same.

In 1988, the EUROPEAN COMMUNITY (176) announced its intention to mandate a ceiling for "tar" (NFDPM) for all cigarettes sold in member states from 1993 on. The ceiling was later extended to nicotine and carbon monoxide and, in 2001, was to be applied to all cigarette brands manufactured in the EUROPEAN COMMUNITY (177). Resulting from the lower smoke yields measured with rotary machines in combination with electrostatic trapping a given cigarette brand could be in compliance with EU regulations when tested in Germany but not so in a member state using linear machines. Therefore, the harmonization of the standards for smoking machines, at least within the European Community, became necessary.

In 1988, ISO concluded that there was a need for revising the standard smoking methods worldwide and producing a single standard method. The task was tackled in two separate steps. ${ }^{3}$ One started off in 1988 upon a request from ISO TC 126 (the ISO Technical Committee responsible for tobacco and tobacco products) to CORESTA and focused on NFDPM, nicotine and water yields obtained with different types of smoking machines; the second step was taken in 1999 at the initiative of the Scientific Commission of CORESTA and revolved around carbon monoxide measurement.

Prompting the first round of standardization activities, the CORESTA Board set up a Task Force, which was requested to survey the diversity of existing laboratory practices and to undertake the experimental work required for harmonization. The consistency of results obtained with different types of smoking machines, specified by official regulations in various countries, was one of the key points of this approach. The smoking machine manufacturers were called on to take up the following challenge as defined by the CORESTA Task Force: "Given the difference in the existing smoking machines, develop and implement additions to or changes in the design which will standardize the performance" (178). It was recognized that the direction and velocity of air flow around the cigarettes during smoking was one of the main factors. Air flow affects to some extent the temperature conditions around the cigarettes, influences the burning speed of cigarettes between puffs and is responsible for removing sidestream smoke. On the basis of exploratory studies conducted by the two leading machine manufacturers, Filtrona (linear machines) and Borgwaldt (rotary machines), the following recommendations (179) were worked out for the harmonization of smoking machines:

\footnotetext{
3 Hans-Valdemar Thomsen (retired Laboratory Manager of House of Prince A/S, Søborg, Denmark) was deeply involved in this work and kindly shared some of his views and recollections
}

- The air flow must be controlled and measurable

- The air flow must remove all sidestream smoke to prevent interference with mainstream measurements

- The air flow must be achievable within existing ambient conditions

- The air flow must be equivalent at all cigarette positions

- The air flow in a (new) duct must be similar to the present standard

- The air flow must be laminar

- The air flow must be practically unaffected by external factors

- Modifications must be practical and cost effective for existing machines.

For achieving these objectives, the rotary machines were equipped with an exhaust hood for covering the rotary smoking head and removing sidestream smoke through a central chimney. The air flow around the cigarettes was horizontal and channeled between the ashtray below the cigarettes and the horizontal part of the exhaust hood. For linear machines, Filtrona changed the smoke hood and ashtray and installed a new internal duct just above the cigarettes. To reduce the influence of ambient air turbulence the machine was equipped with a front cover to be lowered after lighting the cigarettes. Later, ambient air turbulence was further diminished when fully enclosed machine types were designed.

Due to these modifications air flow was forced to be almost laminar and mainly vertical around the cigarettes. Labyrinth seal equipped cigarette holders had to be used with both types of machines. Only Cambridge filters were allowed for trapping condensate when NFDPM and smoke nicotine had to be determined under standard conditions. The Cambridge filter holder was equipped with a slitted washer to allow the smoking of channel ventilated cigarettes. Other techniques, however, remained in use for the analysis of smoke composition under the influence of interfering factors (such as contamination, loss of analytes or artifact formation) and were in fact indispensible for the quantification of certain smoke constituents; examples are metals in samples collected by electrostatic precipitation (130), volatile organic compounds (1,3-butadiene, isoprene, acrylonitrile, benzene and toluene) in a cryogenic trap containing methanol (180), carbonyls absorbed in an acidic solution of 2,4-dinitrophenyl hydrazine (181), and volatile $N$-nitrosamines collected in a liquid trap containing ascorbic acid in an aqueous buffer ( $\mathrm{pH} 4.5$ ) solution (182). The harmonization of smoking machines resulted in the substantial reduction of differences in cigarette smoke data between linear and rotary machines. The modifications in smoking machine design as well as repeatability (r) and reproducibility $(\mathrm{R})$ data for the determination of mainstream smoke NFDPM, nicotine and water by smoking cigarettes with the modified machines were presented by CORESTA in 1991 (11). They are reflected in several revised CORESTA Recommended Methods and the corresponding international standard ISO 3308 ( $3^{\text {rd }}$ edition), published in 1991 (12). In ISO, there is the requirement that standard methods should be reviewed every five years and revised, if necessary (183). The $4^{\text {th }}$ edition of ISO 3308, published in 2000 (184), included only editorial changes. After a number of both technical and editorial 


\section{Repeatability}

- In general, repeatability is the variation in measurements taken by a single person or instrument on the same item, under the same conditions, and in a short period of time. ${ }^{1}$

- For specialists, repeatability is the precision under repeatability conditions, including: the same measurement procedure or test procedure; the same operator; the same measuring or test equipment used under the same conditions; the same location; repetition over a short period of time. ${ }^{2}$

\section{Reproducibility}

- In general, reproducibility is the ability of an entire experiment or study to be duplicated, either by the same researcher or by someone else working independently. ${ }^{1}$

- For specialists, reproducibility is the precision under reproducibility conditions, where independent test/ measurement results are obtained with the same method on identical test/measurement items in different test or measurement facilities with different operators using different equipment. ${ }^{2}$

\section{Precision}

- In general, common statistical usage defines precision as the reciprocal of the variance

- For specialists, precision is the closeness of agreement between independent test/measurement results obtained under stipulated conditions ${ }^{2}$

Wikipedia:

Repeatability; available at: https://en.wikipedia.org/wiki/ Repeatability (Version of 1 September 2016, 23:25)

Reproducibility; available at: https://en wikipedia.org/wiki/ Reproducibility (Version of 1 September 2016, 21:54) Precision (statistics); available at: https://en.wikipedia.org/ wiki/Precision_(statistics) (Version of 21 August 2016, 22:01), all accessed September 2016.

International Organization for Standardization (ISO): ISO 21748:2010 - Guidance for the use of repeatability, reproducibility and trueness estimates in measurement uncertainty estimation; available at: (https://www.iso.org/ obp/ui/\#iso:std:iso:21748:ed-1:v1:en), accessed 12 September 2016.

revisions the $5^{\text {th }}$ edition of ISO 3308 was released in 2012 (105).

The large amount of work undertaken by CORESTA between 1989 and 1992 in pursuit of the objective was described by THOMSEN (179).

The first round of harmonization had been focused on, and was restricted to, nicotine free dry particulate matter (NFDPM) and nicotine levels in cigarette mainstream smoke; the analysis and reporting of carbon monoxide numbers was optional and not included in the statistical evaluation (11). The notified introduction of ceilings for nicotine and carbon monoxide for cigarettes manufactured and/or distributed in Member States of the European Community in 2001 (177) led the CORESTA Scientific Commission in 1999 to initiate a second step in smoking machine harmonization. When the new ISO machine smoking standard 3308 (12) was published in 1991, it was known that the smoking machines were not harmonized regarding mainstream smoke $\mathrm{CO}$ generation. Therefore, a sub-committee was set up for this purpose by CORESTA under the condition that the modifications of the smoking machines, worked out in 1991, should not be touched. This second step of harmonization again demanded the close cooperation between scientists of the cigarette industry and smoking machine suppliers.

The key factor for success proved to be air flow across the cigarette in rotary smoking machines. An air flow straightener (a honeycomb arrangement) was introduced to minimize the influence of external air turbulences and to achieve laminar air flow, which unexpectedly was found to be quite turbulent. Air flow patterns were visualized in a series of tests performed by Borgwaldt using cold smoke. Quite remarkably, the two sequential harmonization steps led to very satisfying results especially when taking into account the physical differences of the two types of smoking machines. Comparable results were reached for cigarette mainstream smoke nicotine free dry particulate matter (NFDPM), nicotine and carbon monoxide satisfactory for declaration and conformity to ceilings in compliance with the Directive 2001/37/EC of the EUROPEAN COMMISSION (177). At the same time, thorough appraisal demonstrated the impossible task of the complete total harmonization of smoking machines with very different technical construction. The results for water and carbon monoxide indicated a clear, though statistically non-significant difference in yields between the two types of smoking machines. This went on record in each consecutive study evaluating CORESTA monitor test pieces. There was also a clear indication that, as smoke yields increased when using alternative intense smoking regimes, the observed yield differences in water and carbon monoxide also increased $(171,172)$. Differences in water values were already observed in the 1991 CORESTA harmonization study and regarded to be "of no practical consequence" (11).

In this context, it should be remembered that air flow around the cigarettes in rotary head machines increases during smoking as the glowing cone moves nearer to the cigarette ports - contrary to linear machine, where the flow decreases from the cigarette's lit end to the butt. Reasonable harmony has been achieved for certain parameters such as NFDPM, nicotine and carbon monoxide (at least, close enough for practical purposes) but with other analytes or under different smoking conditions harmonized machine performance is not really assured.

Repeatability (r) and reproducibility (R) are key characteristics for evaluating the performance of an analytical method. This is particularly relevant with regard to the determination of "tar", nicotine and carbon monoxide in cigarette mainstream smoke when the machinery used is significantly different (like linear and rotary smoking machines) or when operative parameters are at variance (like the ISO 3308 and Canadian Intense regimes). For assessing the acceptability of repeatability and reproducibility values, a system of annual or single collaborative studies has evolved over time pursuing specific objectives and with variable participation. The nature and technicalities of collaborative studies and their relationship with other forms of inter-laboratory testing (proficiency studies, certification studies) were instructively outlined in a presentation by CAHOURS et al. (185). 
For over 10 years now, the annual European collaborative study (EUCS) routinely involves more than 40 tobacco manufacturers, government tobacco testing laboratories and contract laboratories using the ISO 3308 smoking regime; it is organized by the Committee on Tobacco and Tobacco Smoke of the German Institute for Standardization (DIN). No data or reports are being published (186). The annual Asian collaborative study (ACS) has been conducted since 1992 with more than 60 laboratories currently participating - a sizable number from European and American countries (187); similarly, no data or reports are published.

CORESTA organizes annual collaborative studies using monitor test pieces and focused on certain (groups of) analytes. They are part of a fruitful scheme of analytical methods development and validation: A Collaborative Study, generally preceded by a Joint Experiment, produces a CORESTA Recommended Method (CRM) - first in a preliminary version and subsequently, following another trial run, in final form. Frequently, a CRM is submitted to ISO for development into an international standard in compliance with the rules of ISO.

The recent three CORESTA Collaborative Studies conducted in 2009 (188), 2010 (189) and 2011 (190) worked towards smoke yield and variability data under both the ISO (105) and Canadian Intense (22) smoking regimes. In consequence, three CRMs emerged in updated form: CRM No. 70 (180) for the determination of selected volatile organic compounds in mainstream cigarette smoke by GC/MS, CRM No. 74 (181) for the determination of selected carbonyls in mainstream cigarette smoke by HPLC, and CRM No. 75 (191) for the determination of tobacco-specific nitrosamines in mainstream cigarette smoke by LC-MS/MS.

Major dedicated collaborative studies were conducted by the CORESTA AlternATIVE SMOKING REgIMES TASK FORCE (192) in 2000-2003 with 26 laboratories to establish correlation models for smoke yields when using ISO 3308:2000 (184), the Massachusetts regime (20) and the Canadian Intense regime (22); by the ISO TECHNICAL COMMITTEE 126 WorkING GROUP 10 (193) in 2010 involving 35 laboratories comparing the ISO and Canadian Intense smoking regimes; and by the WHO TOBACCO LABORATORY NETWORK (194) with 14 government and independent laboratories to validate a standardized method for the determination of carbon monoxide, NFDPM and nicotine in mainstream cigarette smoke using an intense smoking regime corresponding to the Canadian Intense regime (22) (a puff of $55 \mathrm{~mL}$ in $2 \mathrm{sec}$, every $30 \mathrm{sec}$ with ventilation holes completely blocked).

The lighting of cigarettes was addressed in the $3^{\text {rd }}$ edition of ISO 3308 (12). The use of an electrical lighter was specified - in our opinion, a necessary and important requirement as variations in the chemical composition of lighting puffs are to be expected when making use of different lighting sources. In 2000, PARRISH and HARWARD (195) reported that levels of formaldehyde in first puffs (accounting for $30-50 \%$ of total mainstream smoke deliveries) were affected by the lighting technique whether match, butane lighter or electronic projection bulb system. However, variations could be reduced by making sure that the cigarette coal was well formed and the char line positioned evenly around the entire circumference of the cigarette. This was achieved by gentle warming of the burning end of the cigarette before lighting ("precharring"). The conditions leading to the preponderance of formaldehyde in first puffs were further explored by LI et al. (196).

LI et al. $(197,198)$ developed an analytical technique for determining polycyclic aromatic hydrocarbons (naphthalene, phenanthrene, fluoranthrene, pyrene and benzo $[a]-$ pyrene) in single puffs. Total particulate matter from 1R4F Kentucky reference cigarettes and the industry monitor IM 16 (produced and exclusively used by Philip Morris) was collected - puff by puff - by impaction onto a small disc of aluminum foil. Analysis by GC/MS showed for benzo $[a]$ pyrene that single puff relative yields were, in principle, rather uniform across the 8 puffs collected. However, this pattern was grossly disturbed by cigarette lighting devices, such as matches or conventional butane lighters, while it was essentially unaffected by the use of butane torch lighters or electric lighters. With matches and conventional butane lighters, first puff polycyclic aromatic hydrocarbon (PAH) levels were up to 20 times higher than with an electric lighter - with a slight carryover to the second puff (Figure 19); the effect of yellow flame lighters was more pronounced in case of the higher molecular weight PAHs, pyrene and benzo[a]pyrene. The increases were explained either by direct contributions from the yellow flames (indicating slow and incomplete combustion) of matches or butane lighters or by interaction of the yellow flame with tobacco or components produced by tobacco burning (Figure 20).

The effect of different cigarette lighting devices on the chemical composition of the first puff was also investigated by ADAM et al. (199), using single-photon ionisation time-of-flight mass spectrometry (SPI-TOFMS) for the on-line analysis of gaseous and semi-volatile compounds present in cigarette smoke. The implements examined were a Borgwaldt electric lighter, a commercial propane/butane gas lighter, a match, a candle and the burning end of another cigarette. The analytical method allowed the recording of 41 mass signals, which were assigned (unequivocally or alternatively) to over 75 smoke

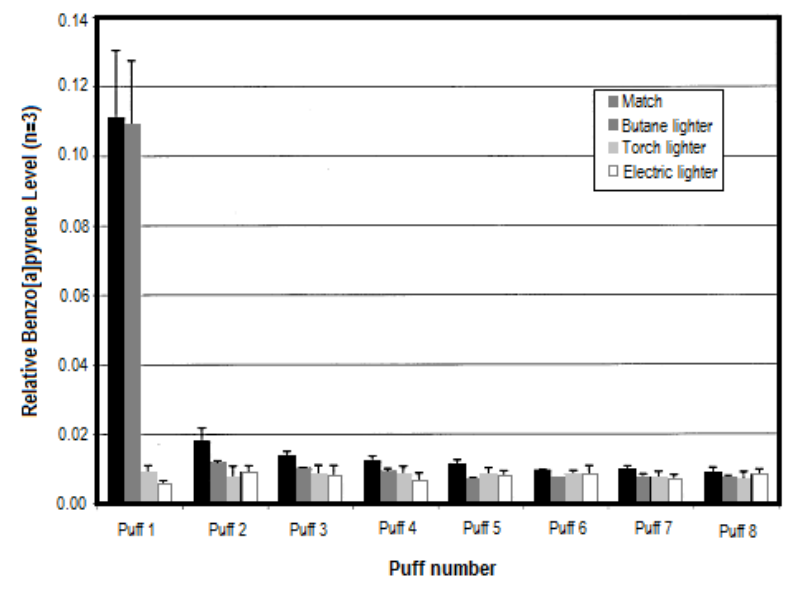

Figure 19. Matches and butane lighters produce excessive levels of benzo[a]pyrene in the first puff while torch lighters and electric lighters do not (197). 


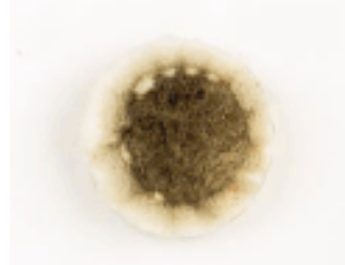

Match

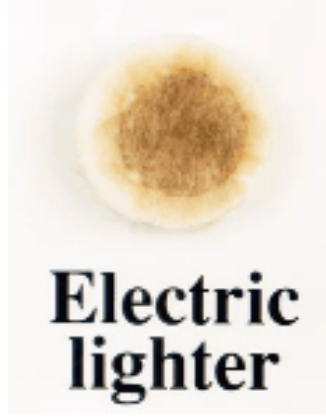

Figure 20. Images of several combined lighting puffs on Cambridge filter pads visibly depend on which lighting device was used (197).

constituents. The practical handling of the lighters proved quite non-uniform and resulted in different amounts of tobacco burnt during the first puff. After appropriate normalization it became obvious that the electric lighter performed most consistently, and the burning cigarette least effectively. With gas lighters, matches and candles the open flames influenced both lighting efficiency and first puff composition. For instance, remarkable increases of benzene and butadiene were produced by candles. The authors concluded: "The electrical lighter, which is most commonly used in routine cigarette-smoke analysis, results in a first puff with a unique chemical composition compared with the first puffs resulting from the use of the lighting devices, for example a match or a gas lighter, used by most human smokers. Use of the electric heater in laboratory experiments should therefore be reconsidered." In our opinion, however, the findings of ADAM et al. rather support the use of electric lighters as specified by ISO 3308 (12), which are distinguished by their stable performance. For the standardized characterization of cigarettes, technical elements should be as well-defined as possible and not be complicated by attempts to reproduce any conceivable human behavior. Electric lighters for machine smoking are consistent with this concept.

Subsequent work on both types of analytical cigarette smoking machines focused on the automation and computer control of the smoking process and the establishment of the concurrent determination of carbon monoxide in mainstream smoke. Dedicated types of smoking machines were constructed and marketed for the analysis of specific mainstream smoke components, such as the so-called "Hoffmann analytes" (131), e.g., the Borgwaldt RM20H (108) and the rotary Cerulean CR20iE (106).

The technical challenge of partial or full automation is much less demanding with rotary than linear smoking ma- chines. Today, highly sophisticated rotary devices are offered by both major manufacturers, BORGWALDT (108) and CERULEAN (106). These machines are primarily used for the routine analysis of cigarette smoke condensate, smoke nicotine and carbon monoxide but may be also employed for testing specific products such as roll-yourown and Bidi products, and certain small cigars. Remarkably, one of the Cerulean machines is equipped with a completely enclosed hood for better controlled air flows during smoking, and to "protect the operator" and "to reduce operator exposure" (106).

However, a problem not yet completely solved is the control and impact of air flows around cigarettes during the smoking process. Air flow velocities are indeed defined in ISO $3308(12,105,184)$ and referenced by the Health Canada Intense method (22) and the WHO TobLabNet Standard Operating Procedure (SOP) 1 (200) but details regarding directions and stability are not addressed in any of the standards. It is assumed that these factors have a certain influence on both mainstream smoke yields and the repeatability of measurements. TINDALL et al. (201) conducted a sophisticated study - smoking cigarettes on a Cerulean 20-port linear machine - to investigate the movement of air and smoke during the smoking process and the effect of certain structural features of the smoke hood on carbon monoxide yield levels and variability. It was shown that a relatively simple change in ashtray form improved the uniformity of air flow, resulting in better yield consistency across the smoking machine.

\subsection{Human smoking and intense machine smoking regimes}

The discussion of the similarities and differences of cigarette smoking by humans or machines, and of the value of comparing data thereby obtained, is one common thread that has been running through much of the development of smoking machines since the beginning of the last century. In the last decades, a good number of statements were released by scientists and regulatory agencies to assess and interpret this problem.

Already in 1967 the U.S. Federal Trade Commission (138) stated in a press release introducing the FTC standard smoking method:

"No test can precisely duplicate conditions of actual human smoking and, within fairly wide limits, no one method can be said to be either "right" or "wrong. " ... No two human smokers smoke in the same way. No individual smoker always smokes in the same fashion. The speed at which one smokes varies both among smokers, and usually also varies with the same individual under different circumstances even within the same day. Some take long puffs (or draws); some take short puffs. That variation affects the tar and nicotine quantity in the smoke generated. Even with the same type of cigarette, individual smokers take a different number of puffs per cigarette depending upon the circumstances. ... The number of puffs and puff duration (as well as butt length) will vary according to emotional state. ... Thus, the purpose of testing is not to determine the amount of tar and nicotine inhaled by any human smoker, but rather to determine the amount 
of tar and nicotine generated when a cigarette is smoked by machine in accordance with the prescribed method."

In an assessment of the relationship between smoking machine parameters and the cigarette smoke yields of "tar", nicotine and carbon monoxide, DARRALL (202) - the UK Government Chemist - referred to the smoke values tabulated in rank order and published, since 1973, by the UK Department of Health and Social Security and stated that they "have never been intended to be actual yields obtained by any one smoker, a point often misunderstood by critics, but to rank brands and allow comparison of one with another under a standard test procedure. This ranking presents the smoker with information to enable him to choose, if he so wishes, a lower yielding brand."

Statements like those made by FTC and the UK Government Chemist are supported by the findings of several studies. A convincing example is the paper of SCHERER et al. (203) titled "Relationship between machine-derived smoke yields and biomarkers in cigarette smokers in Germany" published in 2007. 274 smokers participated in the field study smoking regularly different kinds of cigarette brands. Biomarkers in smokers' 24-hour urine, which reflect the uptake of individual mainstream smoke components such as nicotine, tobacco-specific nitrosamines, acrolein, benzene and polycyclic aromatic hydrocarbons, showed strong correlation with daily cigarette consumption. Comparable results were obtained with certain hemoglobin adducts. In contrast, the elevation of biomarker levels per cigarette smoked was at best weakly correlated with the corresponding machine derived ISO smoke yields. The authors concluded that machine derived cigarette smoke yields have little or no relationship with actual human smoke component uptake, as determined by suitable biomarkers.

The revision and updating of the ISO standards did not silence the request for developing a smoking machine and regime for mimicking human smoking behavior. In the late 1990s, the COMMONWEALTH OF MASSACHUSETTS (20) and the STATE OF TEXAS (21) introduced new cigarette testing requirements. With regard to reporting requirements, enacted methodology should ensure that the nicotine yield determined "reflects, as accurately as possible, nicotine intake for an average consumer" (204).

In 1996, RICKERT (205) released a report - titled "Smoking Under Realistic Conditions: Development of Minimum and Maximum Values for Toxic Constituents in Tobacco Smoke" - on the Tobacco Characterization Program of Labstat Incorporated (Kitchener, Ontario, Canada) which had been initiated as early as 1979 . Relevant publications of RICKERT et al. from 1980 onwards, resulting from Canadian Government sponsored research, are listed in (205). Labstat's data called into question the use of machine smoke yields, determined under standard conditions, for predicting human uptake of (certain) smoke constituents.

Using existing published data of human smoking behavior RICKERT focused on the two parameters of artificial smoking, considered above all to determine smoke yields: puff volume and puff interval. He equated "minimum" smoking with ISO standard conditions (35-mL puffs every $60 \mathrm{sec}$ ) and defined "average" smoking as 44-mL puffs

\section{Biomarker}

A characteristic that is objectively measured and evaluated as an indicator of normal biological responses, pathogenic processes, or pharmacologic responses to an intervention

\section{Biomarker of exposure}

The substance, or its metabolite, or the product of an interaction between a substance (single substance or a mixture) and some target molecule or cell, that is measured in a compartment in an organism

\section{Biomarker of effect/risk}

A biomarker that indicates a risk factor for a DISEASE

Adapted from: IOM (Institute of Medicine): Scientific Standards for Studies on Modified Risk Tobacco Products; The National Academies Press, Washington, DC, USA, 2012.

every 50 or $26 \mathrm{sec}$ with ventilation holes all open, and "maximum" smoking as 56-mL puffs every $26 \mathrm{sec}$ with ventilation holes either all open or all blocked. Puff duration remained at $2 \mathrm{sec}$. All other ISO standards for smoke generation and constituent analysis were adhered to. Using the four new sets of parameters to represent different human smoking intensities (twice "average" and twice "maximum") he examined 115 Canadian brands for smoke "tar", nicotine and carbon monoxide. This set the stage for a series of new regulatory developments in Canada.

In 1998, the PROVINCE OF BRITISH COLUMBIA (206) issued the Tobacco Testing and Disclosure Regulation 282/98. It decreed - for all brands of cigarettes and cigarette tobaccos sold, offered for sale, distributed, advertised or promoted in British Columbia - the quarterly reporting of all ingredients and additives and the annual reporting of a number of smoke constituents (listed in a special schedule) of mainstream and sidestream smoke as well as smoke $\mathrm{pH}$ and filter efficiency. Constituent yields had to be determined under two smoking conditions: using the established parameters of ISO 3308:1991 (12) and a set of new parameters, which enlarged puff volume from 35 to $56 \mathrm{~mL}$, shortened puff interval from 60 to $26 \mathrm{sec}$, maintained puff duration at $2 \mathrm{sec}$ and required all ventilation holes completely closed by tape. The Regulation was amended in April 2001 (207) and specified the "modified" ISO smoking parameters in the following way: Puff volume was $55 \mathrm{~mL}$, puff interval was $30 \mathrm{sec}$, puff duration remained at $2 \mathrm{sec}$ as did vent blocking at $100 \%$. The evolution of the intense smoking regime from a " $56 / 26 / 2$ " puffing regime to a " $55 / 30 / 2$ " puffing regime was the result of scientific collaboration of Health Canada with industry scientists ${ }^{4}$ and was based on the development of actual data that verified no significant difference in smoke yields when using a more practical combination of set points than those originally specified. A key driver was the

\footnotetext{
${ }^{4}$ Michael F. Borgerding (Sr. Director, RAI Services Company, Scientific \& Regulatory Affairs, Winston-Salem, NC) kindly elucidated important steps in the development of the standard
} 
Table 3. Mandated smoking methods.

\begin{tabular}{|c|c|c|c|}
\hline Method & Origin & Smoking parameters & Document (reference) \\
\hline ISO & $\begin{array}{l}\text { International } \\
\text { Organization for } \\
\text { Standardization }\end{array}$ & $\begin{array}{l}\text { puff volume: } 35 \mathrm{~mL} \\
\text { puff duration: } 2 \mathrm{sec} \\
\text { puff interval: } 60 \mathrm{sec} \\
\text { vent blocking: none }\end{array}$ & $\begin{array}{l}\text { International Standard ISO 3308:2012 - Routine Analytical } \\
\text { Cigarette-Smoking Machine -- Definitions and Standard } \\
\text { Conditions; } 5^{\text {th }} \text { edition, ISO, Geneva, Switzerland, 2012. (105) }\end{array}$ \\
\hline \multirow[t]{3}{*}{ FTC } & \multirow[t]{3}{*}{$\begin{array}{l}\text { U.S. Federal Trade } \\
\text { Commission }\end{array}$} & \multirow{3}{*}{$\begin{array}{l}\text { puff volume: } 35 \mathrm{~mL} \\
\text { puff duration: } 2 \mathrm{sec} \\
\text { puff interval: } 60 \mathrm{sec} \\
\text { vent blocking: none }\end{array}$} & $\begin{array}{l}\text { Pillsbury, H.C., C.C. Bright, K.J. O'Connor, and F.W. Irish: Tar } \\
\text { and Nicotine in Cigarette Smoke; J. Assoc. Off. Anal. Chem. } 52 \\
(1969) 458-462 .(19)\end{array}$ \\
\hline & & & $\begin{array}{l}\text { Cigarettes - Testing for Tar and Nicotine Content; Federal } \\
\text { Register } 32 \text { (1967) 11178. (9) }\end{array}$ \\
\hline & & & $\begin{array}{l}\text { Cigarettes and Related Matters - Carbon Monoxide, "Tar" and } \\
\text { Nicotine Content of Cigarette Smoke; Description of New } \\
\text { Machine and Methods to be Used in Testing; Federal Register } \\
45 \text { (1980) 46483-46487. (139) }\end{array}$ \\
\hline \multirow[t]{2}{*}{$\begin{array}{l}\text { British } \\
\text { Columbia }\end{array}$} & \multirow[t]{2}{*}{$\begin{array}{l}\text { Province of British } \\
\text { Columbia }\end{array}$} & $\begin{array}{l}\text { puff volume: } 56 \mathrm{~mL} \\
\text { puff duration: } 2 \mathrm{sec} \\
\text { puff interval: } 26 \mathrm{sec} \\
\text { vent blocking: } 100 \%\end{array}$ & $\begin{array}{l}\text { Tobacco Testing and Disclosure Regulation; Order of the } \\
\text { Lieutenant Governor in Council, B.C. Reg. 282/98, Section } 1 \text { to } \\
6,1998 \text {, effective 1998-2000. (206) }\end{array}$ \\
\hline & & $\begin{array}{l}\text { puff volume: } 55 \mathrm{~mL} \\
\text { puff duration: } 2 \mathrm{sec} \\
\text { puff interval: } 30 \mathrm{sec} \\
\text { vent blocking: } 100 \% \\
\end{array}$ & $\begin{array}{l}\text { Tobacco Testing and Disclosure Regulation; Order of the } \\
\text { Lieutenant Governor in Council No. } 423 \text { of April 2, 2001, } \\
\text { amending B.C. Reg. 282/98, 2001, valid since 2000. (207) }\end{array}$ \\
\hline $\begin{array}{c}\text { Canadian } \\
\text { Intense }\end{array}$ & $\begin{array}{l}\text { The Government of } \\
\text { Canada }\end{array}$ & $\begin{array}{l}\text { puff volume: } 55 \mathrm{~mL} \\
\text { puff duration: } 2 \mathrm{sec} \\
\text { puff interval: } 30 \mathrm{sec} \\
\text { vent blocking: } 100 \%\end{array}$ & $\begin{array}{l}\text { Tobacco Reporting Regulations SOR/2000-273 of June } 26 \text {, } \\
2000 \text {, current to June } 17,2015 .(22)\end{array}$ \\
\hline $\begin{array}{l}\text { Massa- } \\
\text { chusetts }\end{array}$ & $\begin{array}{l}\text { The Commonwealth of } \\
\text { Massachusetts }\end{array}$ & $\begin{array}{l}\text { puff volume: } 45 \mathrm{~mL} \\
\text { puff duration: } 2 \mathrm{sec} \\
\text { puff interval: } 30 \mathrm{sec} \\
\text { vent blocking: } 50 \% \\
\end{array}$ & $\begin{array}{l}\text { Cigarette and Smokeless Tobacco Products: Reports of Added } \\
\text { Constituents and Nicotine Ratings; General Laws of } \\
\text { Massachusetts, Chapter 94, Section 307B } 105 \text { CMR 660.000, } \\
\text { 1997. (20) }\end{array}$ \\
\hline Texas & State of Texas & $\begin{array}{l}\text { puff volume: } 45 \mathrm{~mL} \\
\text { puff duration: } 2 \mathrm{sec} \\
\text { puff interval: } 30 \mathrm{sec} \\
\text { vent blocking: } 50 \%\end{array}$ & $\begin{array}{l}\text { Texas Register 22, No. 79, December 5, 1997, pp. 11939- } \\
\text { 11941. (21) }\end{array}$ \\
\hline
\end{tabular}

inability to achieve a 26 -sec puff interval on a rotary machine at that time without reducing the puff duration from 2 to $1.6 \mathrm{sec}$.

Incidentally, the amended regulation effectuated several additional changes, for instance regarding reporting schedules and the keeping of trade secrets.

In 2000, the CANADIAN FEDERAL GoVERnMENT (22) implemented nationwide cigarette yield testing regulations, which were identical to those effective in British Columbia. The intensified smoking regime with larger puffs and increased puff frequency (compared to ISO 3308 ) and with $100 \%$ vent blocking was intended "to provide data that reflects the emissions that are actually available to the consumer" (208); the method is generally referred to as "Canadian Intense". Cigarette smoking had to be done with routine analytical machines and using glass fiber filter smoke traps as specified in ISO 3308:1991 (12). "Tar" (NFDPM), nicotine and carbon monoxide were to be determined according to the Official Method T-115 (209), which in turn quoted ISO 4387:1991 (210) and other relevant ISO standards as normative references.

It was a dicey step to graft rigorously modified smoking conditions onto an established and well balanced system of machinery and procedures without prior adaptation and testing. Methodological incompatibilities were recognized within a short time and required corrective experimental and interpretational action.
In 2003, the World Health Organization (WHO) formally established the "Study Group on Tobacco Product Regulation (TobReg)" (211). This group expressed the view that "the current methods for product testing adopted by the International Organization for Standardization (ISO) and the United States Federal Trade Commission (FTC) are inadequate since they fail to provide the appropriate scientific basis for tobacco product regulation" (212). A report compiled in 2005 by the AD HOC SMOKING BEHAVIOUR REVIEW TEAM to ISO/TC 126 WG 9 (213), titled "A review of human smoking behaviour data and recommendations for a new ISO standard for the machine smoking of cigarettes", listed several knowledge gaps: "There was a general recognition within the group that no single machine regime could model human smoking behavior. All of the data reviewed indicated a) large differences between the puff parameters of individuals smoking the same type of cigarettes, b) changes in puff parameters that occurred within individuals as they smoked single cigarettes and c) smokers may vary their puff parameters when smoking the same cigarette type on different occasions." Representatives of TobReg and scientists working in the tobacco industry supported this conclusion, which, in essence, had already been reached by the U.S. FEDERAL TRADE COMMISSION in 1967 (138). The feasibility and limitations of duplicating human smoking behavior using different machine smoking 
regimes were investigated by PURKIS et al. (214). Smoking parameters were obtained by means of puff analyzers in an in-house study from 60 volunteers, who were provided with cigarettes of different design. Smokers obviously fine-tuned their smoking intensity in response to (changes in) draw resistance (the most important factor), smoke concentration and smoke temperature. A duplicator machine allowed cigarettes to be smoked in compliance with one experimental and four mandated or proposed smoking regimes as well as under the previously recorded "average" smoking parameters. It was recognized that the conditions generated in a cigarette when using intense machine smoking regimes were extreme compared to the conditions found for regimes based more realistically on human smoking. In particular, the $100 \%$ blocking of ventilation with its notable influence on draw resistance - was considered a distorting factor which did not reflect human behavior. In addition, the increase of smoke temperature seen under intense smoking conditions was not in line with the behavior of human smokers who tended to control its occurrence by reducing puff intensity.

The CORESTA Alternative SMOKING REgimes TASK FORCE (192), established by the Scientific Commission in 2000, was charged with developing "correlation models for smoke yields between ISO and representative alternative smoking regimes". Twenty-six laboratories participated and the final report was released in 2006. Smoking regimes considered were the international standard ISO 3308:2000 (184), the Massachusetts regime (20) and the Canadian Intense regime (22). The analytes in focus were NFDPM, nicotine and carbon monoxide. In a comprehensive study alternative smoking regimes were applied to approximately 90 cigarette brand styles from various regions of the world, which represented numerous designs and a variety of tobacco blends. The NFDPM, nicotine and carbon monoxide data provided the basis for correlation models between smoke yields in compliance with ISO and the alternative smoking regimes, though not with the same strength for all combinations (for instance, an adequate functional relationship was not observed for carbon monoxide yields between the ISO and Canadian Intense regimes). Correlations were further refined by examining specific cigarette traits, such as tobacco blend or regional origin. The analytical data produced in the study also provided an opportunity to compare and assess different types of smoking machine hardware and to investigate potential approaches for data normalization. A detailed discussion of machine smoking regimes other than the existing ISO regulations was published by Borgerding and KLUS in 2005 (131), DiXON and BORGERDING in 2006 (215) and PURKIS in 2013 (216).

In particular, the paper of DIXON and BORGERDING (215), presented at the $60^{\text {th }}$ Tobacco Science Research Conference in Montreal (Canada), is a significant overview concerning the application and evaluation of alternative smoking regimes introduced for regulatory purposes in the United States and Canada. The question arose whether the available smoking machines complied fully with the new requirements. These machines were designed to follow the FTC/ISO smoking regime with "accuracy and precision". The authors described the technical advances in commercially available smoking machines to fulfill alternative smoking regimes. As noted by DIXON and BORGERDING (215), "smoking machines introduced into the marketplace in recent years have been designed for complete flexibility when designating the puffing regime applied during smoking. Capabilities typically include a wide range of puff volumes, frequencies and durations. Simplicity of use is also a hallmark of recent designs. Puff parameters are set via microprocessor control from the machine's PC control panel, eliminating the need for portby-port manual adjustments or for swapping from one pneumatic panel to the next. Electronic control of puff parameters extends to the individual smoking port level for some smoking machines. With these models, it is possible to apply different smoking regimes across the smoke run.

.... Recently introduced smoking machines have been tailored to address a much wider range of measurement endpoints. Smoking machines are commercially available to test cigarette physical properties (e.g., burn rate and hot collapse), mainstream smoke (T, N,CO and Hoffmann analytes), sidestream smoke ( $T, N, C O$ and Hoffmann analytes) and biological activity (in vitro biology assays of mainstream smoke)."

DIXON and BORGERDING pointed out that "... the mechanical solution necessary to achieve the smoking parameters currently specified by the FTC and ISO smoking methods required decades of hardware development, smoking machine refinement and ultimate standardization through collaborative evaluation and testing. .... However, this logical sequence has been largely abandoned as recent alternative smoking regimes have been promulgated in some regulatory jurisdictions. New regulatory testing requirements have been introduced since the late 1990s without regard for existing smoking machine capabilities and limitations. The nature of these new machine smoking regimes suggests that the amount of effort that has been expended historically to standardize analytical smoking machines is not well understood or appreciated."

It is important to recognize that most smoking machines offered commercially in the late 1990s were extremely flexible and allowed the selection from a wide range of puff volumes, durations and frequencies as well as puff profiles. However, they were not capable of producing mainstream smoke data - using alternative intense smoking regimes - with comparable robustness regarding repeatability and reproducibility as obtained with the FTC or ISO regimes.

Besides the fact that smoking machines and regimes are hardly able to duplicate the uptake of cigarette mainstream smoke by humans, smoking regimes (20-22) more intense than ISO (105) have a strong impact on the analytical variability of smoke yields (217).

In 2005, CouNTS et al. (218) analyzed mainstream smoke yields of NFDPM and 44 individual constituents from 48 international Philip Morris brands of filtered cigarettes and the 1R4F Kentucky reference cigarette - using the three different machine-smoking regimes specified by ISO (105), the COMMONWEALTH OF MASSACHUSETTS (20) and the GOVERNMENT OF CANADA (22). Generally, smoke constituent yields were found to increase in the order "ISO $<$ Massachusetts $<$ Canadian Intense", resulting from larger puff volumes, doubling of puff frequency, reduced 
filter ventilation and larger amounts of tobacco burnt during puffing. Remarkably, the relationship "Massachusetts < Canadian Intense" was reversed for certain compounds, such as phenols. Predicting relationships were explored between "tar" and smoke constituent yields - both separately for each smoking regime and across regimes. The abundance of analytical data made it possible to enrich the publication by including a multitude of graphs and tables.

When smoking cigarettes under an intense regime - puffs of $55 \mathrm{~mL}$ in $2 \mathrm{sec}$ every $30 \mathrm{sec}$, as mandated by Health Canada (22) - in combination with $100 \%$ blocking of filter ventilation, significant increases in filter temperature and disproportionately high accumulations of tobacco moisture and mainstream smoke water in filters were observed by PURKIS et al. (217), compared to the ISO smoking regime $(105,219)$, especially for highly ventilated cigarettes. These effects may impair the mechanical stability of the tobacco column and filter of the cigarette and lead to reduced firmness, reduced absorption efficiency of cellulose acetate filters, reduced adsorption of gaseous compounds on carbon filters as well as to desorption effects from such filters. These confounding and uncontrolled factors may reduce the robustness of analytical data and explain the observed variability (reproducibility and repeatability) between laboratories using the Canadian Intense smoking regime.

Blocking filter ventilation is usually achieved by the manual application of tape from the tipping joint to the mouth end of the cigarette. Tapes are used broad enough to cover half (50\% blocking) or all (100\% blocking) of the cigarette circumference. The manual procedure may affect filter efficiency by squeezing the plug in different, uncontrolled ways. This was noted by BORGERDING and KLUS in 2005 (131). The manipulation influences the repeatability (r) and reproducibility ( $R$ ) of the analytical data for cigarette mainstream smoke. According to DIXON and BORGERDING (215) a logical alternative to manual sealing of ventilation holes in the filter tipping paper with a tape was the use of cigarette holders designed to occlude these holes. The holder was easier to design when $100 \%$ vent blocking was desired. A commercially available holder for $100 \%$ vent blocking requiring no additional taping (Figure 21) was presented at the Tobacco Science Research Conference 2013 (129).

A holder for $50 \%$ vent blocking was developed by R.J.

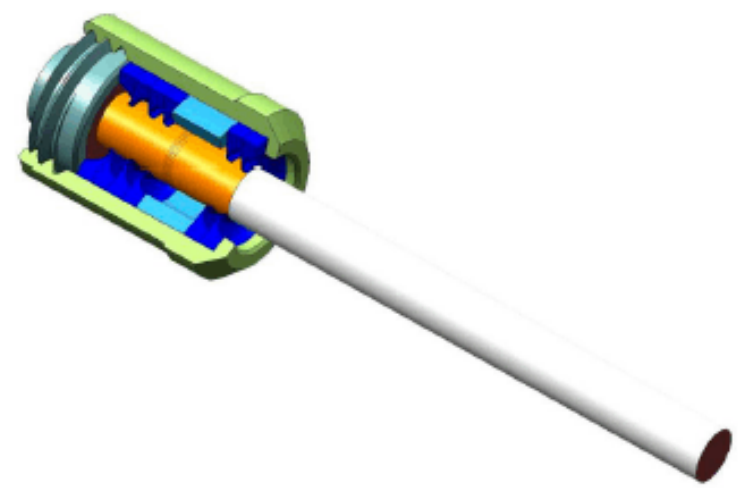

Figure 21. This cigarette holder (129) ensures $100 \%$ vent blocking without the need for additional taping of the filter.
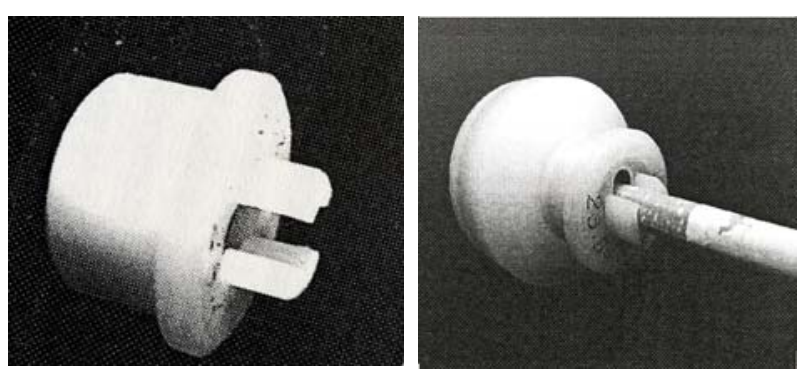

Figure 22. An experimental cigarette holder (215) occludes two opposite $25 \%$ sections of the filter circumference to achieve $\mathbf{5 0 \%}$ vent blocking.

REYNOLDS (220). The holder was based on the application of a mild "pinching force" to "seal" the filter holes. Two sections of the filter, each constituting $25 \%$ of the total circumference, were blocked by the holder on opposite sides. $25 \%$ vent blocking on two opposite sections of the tipping paper mimics the conceivable vent blocking by a smoker's fingers or lips (Figure 22).

RICKERT (221) investigated measurement variability when applying the Canadian Intense smoking regime (22). He concluded that the increase in the variability of NFDPM levels was smoking machine dependent (linear compared to rotary) and not caused by cigarette handling. The unexpected increase in variability with linear machines was probably due to the disproportionate rise in the water content of the particulate phase. Repeatability using the Canadian Intense smoking regime with a rotary smoking machine, however, remained in line with ISO 4387 (219). RICKERT pointed out that in experienced hands the taping of cigarettes was unlikely to be a significant source of smoke yield variability. In our view, the situation remained troubling.

DIXON and BORGERDING (215) discussed the effects of alternative smoking regimes on cigarette mainstream smoke composition. They considered it well established that mainstream smoke yields increased when cigarettes were smoked by more intense smoking regimes. The extent to which relative contributions from different mechanisms of smoke formation (combustion, pyrolysis, pyrosynthesis and direct transfer) influenced mainstream smoke generation was not known. DIXON and BORGERDING noted: "It is reasonable to expect that many changes will occur within the "microenvironment" of the burning cigarette when cigarettes are smoked with different puffing and ventblocking conditions. Peak temperatures during a puff and the thermal gradient along the tobacco column during smoking will each likely change to some extent with different smoking conditions. The nature of the reducing and oxidizing atmospheres in and beyond the burning zone will also be altered. Diffusion characteristics into and out of the tobacco column are unlikely to remain constant with different smoking regimes. Filter efficiencies will be affected by increased flow rates through the filter. The likelihood of achieving distinct puffing and smoldering states during the smoking process will be reduced." In 2009, MARIAN et al. (222) published a systematic literature review (including publicly available internal industry documents) on the current status of the following weighty topics: 


\section{Cigarette mainstream smoke mouth level}

The amount of mainstream smoke or a specific smoke constituent exiting from a cigarette into a smoker's mouth when taking a puff

To be differentiated from:

\section{Cigarette mainstream smoke intake}

The amount of whole mainstream smoke or a specific smoke constituent ingested into the mouth or the respiratory system of a person smoking a cigarette, prior to any deposition and adsorption

\section{Cigarette mainstream smoke uptake}

The amount of whole mainstream smoke or a specific smoke constituent, which is absorbed by the organism of a smoker

- The technique and protocols of machine smoking of tobacco products (mainly cigarettes)

- The assessment of human smoking behavior and

- The reciprocal interplay of machine performance, human smoking behavior, and risks and claims regarding smoking-related health effects.

The paper discussed a considerable range of technical, biological and legal aspects - many previously well documented and some with less attention in the past, such as the influence of mouth-holding and inhalation on the uptake of smoke constituents. Evidently, potential reduced exposure products (PREPs) were addressed as well. The review offered a wealth of useful recapitulations and interesting insights - with occasional insinuating undertones.

As discussed earlier, the design of rotary and linear smoking machines was modified in the 1990 s to produce harmonized levels of cigarette mainstream smoke condensate, nicotine and carbon monoxide under the ISO smoking regime $(105,219)$. However, as shown by RICKERT (221) and MARINER et al. (193), concordant data are not obtained when using the Health Canada Intense smoking regime (22).

MARINER et al. (193) reported the results of the ISO TC 126 Working Group 10 (WG 10) collaborative study, undertaken in 2010 in preparation for the standardization of an intense machine smoking method, to investigate intra- and inter-laboratory variability of the determination of mainstream smoke TPM, NFDPM, nicotine and carbon monoxide yields from a range of commercial cigarettes and two reference cigarettes. The cigarettes were smoked according to the ISO (105) and Canadian Intense (22) regimes. Thirty-five laboratories from 21 countries participated with 41 smoking machines (nearly as many linear as rotary machines); 6 laboratories used both types. For all parameters intra-laboratory variation tended to be lower with Canadian Intense than ISO. Inter-laboratory variation was higher in Canadian Intense data than with ISO for TPM, water and NFDPM but not for carbon monoxide. Smoking machine type was the major contributing factor; linear machines produced higher values for TPM, water and NFDPM and lower values for carbon monoxide than rotary machines. Regrettably, no further study details have been released so far.

In 2012, three CORESTA Recommended Methods (CRMs) were revised to include cigarette mainstream smoke yield and variability data generated under both the ISO (105) and Canadian Intense (22) smoking regimes. They are now available as CRM No. 58 for the determination of benzo[a]pyrene (223), CRM No. 70 for selected volatile organic compounds (180), and CRM No. 74 for selected carbonyls (181). PURKIS et al. (224) reported interesting details of the methods revision process with focus on factors influencing data variability (type of smoking machine, outliers) and presented a number of instructive graphs showing the relationship between mean yield and reproducibility for several analytes collected under ISO and Canadian Intense smoking regimes. The final collaborative studies for updating the three CRMs were conducted by 12 to 19 laboratories with the $3 \mathrm{R} 4 \mathrm{~F}$ and 1R5F Kentucky reference cigarettes, the CORESTA monitor CM6 and seven commercial cigarettes. Under Canadian Intense smoking conditions yields of certain vapor phase compounds, such as acetaldehyde, isoprene and crotonaldehyde, were consistently higher when cigarettes were smoked by linear compared to rotary machines. A smoking machine effect on benzo $[a]$ pyrene yield was not seen. Concerned about this data and the smoking machine dependent elevated water yields reported earlier by MARINER et al. (193), PURKIS (216) pointed out that significant adjustments to rotary and linear smoking machine set-ups might be necessary for bringing data in line when using the Health Canada Intense smoking regime. One of the reasons for the relatively lower key analyte yields produced (only) under the Canadian Intense regime by rotary, compared to linear, smoking machines may be the distance and path between the cigarette butt and the (more or less) remote Cambridge filter, casually called "dead volume" (Figure 23). Obviously, it is larger in rotary than linear systems. Using a single experimental arrangement that combined and mimicked the machine behavior of both types with the exception of smoke path and collections systems, TINDALL et al. $(225,226)$ observed significant condensate losses in the transfer tube, before reaching the central Cambridge filter. The higher water

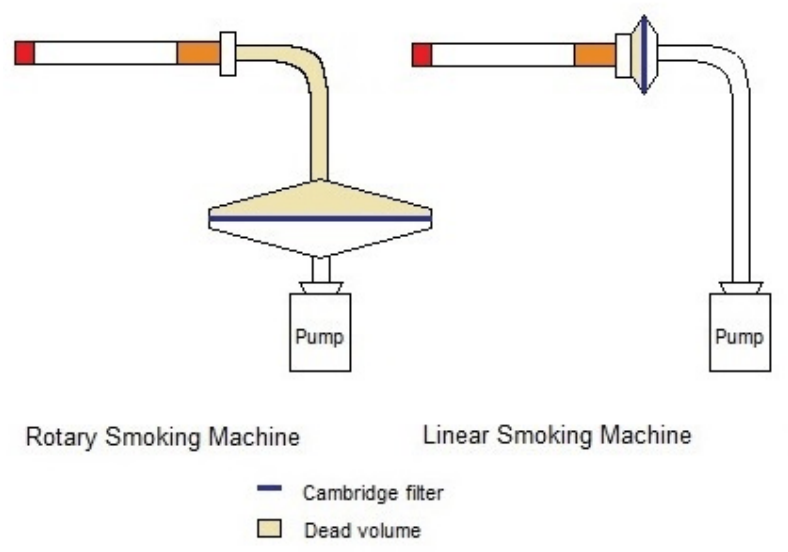

Figure 23. The basic difference in dead volume between rotary and linear smoking machines. 
content of the smoke matrix resulting from intensified smoking conditions seemed to be responsible. In addition, certain differences in condensate capturing, extracting and calculating steps between the linear and rotary systems, in all probability, had stronger bearing on yields when the Canadian Intense method was used.

Both with linear and rotary machines and under both the ISO and Canadian Intense regimes there is desorption of semi-volatile materials, including water, from the Cambridge filters during the smoking run. As found by TINDALL et al. (227) this effect was more pronounced under the Canadian Intense than the ISO regime and affected in particular the yields obtained with rotary machines. The larger volume of air passing through the central Cambridge filter was assumed to desorb more water from the pad; this effect was magnified by the higher quantities of water, which were produced under the more forceful Canadian Intense smoking conditions and initially captured on the central Cambridge filter. Consequently, the yields of total particulate matter (TPM) and water collected (and measured) on the pad were reduced.

In a consistent next step, TINDALL et al. (228) investigated the effect of the volume between smoking article and a liquid impinger system on the pressure drop of the collection system, and the resulting changes in combustion conditions. Two linear smoking machines (Cerulean SM 450 and SM 450i) with different dead volumes (19.73 and $11.26 \mathrm{~mL}$, respectively) were examined. A puff capture device was used for recording profile parameters right at the test piece. Effects were seen in the form of reduced peak flow, increased asymmetry and delayed peak maximum. This may have consequences for analytical measurements.

Obviously, the introduction of a different, more intense smoking regime - such as the one suggested by HEALTH CANADA (22) and other regulatory agencies - had serious and disturbing consequences for the continued use of both linear and rotary smoking machines under harmonized conditions in compliance with ISO $3308(105,185)$. Consequently, the problem was addressed in additional studies. Referring to the recent incorporation of the Canadian Intense smoking regime (22) into a standard operating procedure for cigarette smoking, set up by the TOBACCO LABORATORY NETWORK OF THE WORLD HEALTH ORGANIZATION (200), PURKIS (229) pointed out that the compatibility of equipment and procedures, specified and validated according to ISO, with the new intense smoking regime was questionable and certainly not demonstrated. The formation and fate of water under Canadian Intense conditions was recognized as a major disruptive factor: Considerably increased relative water content in TPM; disproportionately higher water yield in later puffs (Figure 24); the temperature of smoke from a $1 \mathrm{mg}$ "tar" cigarette elevated up to $60{ }^{\circ} \mathrm{C}$ when leaving the filter as opposed to ambient temperature under ISO conditions; precipitation of water on, and incomplete recovery from, Cambridge filter holder walls - leading to an underestimation of water and an overestimation of NFDPM. Interlaboratory reproducibility assessment with regard to NFDPM and certain other smoke constituents was consequently weakened by data scattering and complicated outlier treatment. The author asserted in " $A$ cautionary

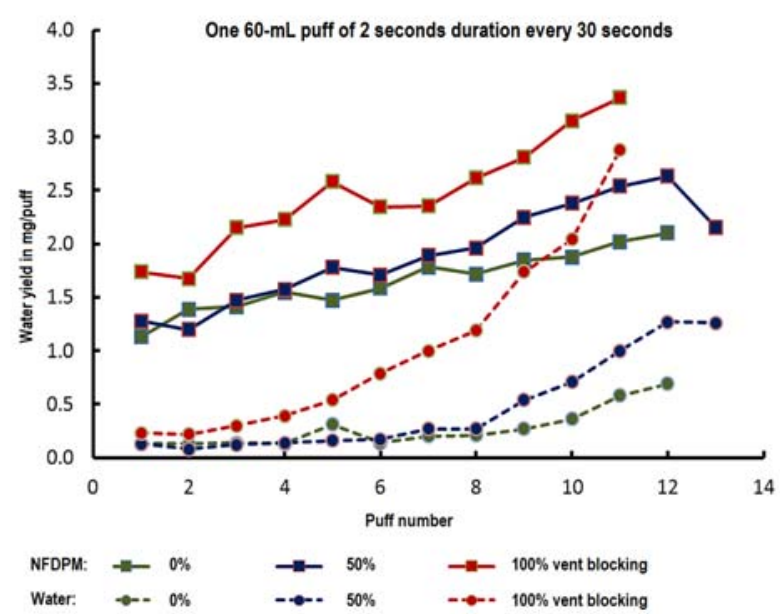

Figure 24. Water yields are increased out of proportion in later puffs under conditions of $100 \%$ vent blocking (229).

note: making reference to ISO standards is not a sufficient means of providing validation for non ISO methods" (229). A comparative trial, performed by CORESTA in 2012 and smoking CORESTA monitor test pieces (CM 7) under Canadian Intense conditions, revealed differences in TPM yields between linear (44.39 $\mathrm{mg} / \mathrm{cig})$ and rotary (38.81 mg/cig) smoking machines (171). One possible explanation for this observation was that with the rotary type not all particulate matter was trapped on the Cambridge filter but rather significant amounts of material did condense or precipitate in the smoke path before reaching the filter pad. The considerably larger dead volume between cigarette mouth end and filter in rotary compared to linear machines was a likely cause for the effect. The underlying experimental conditions had already been examined in considerable detail by TINDALL et al. (225, 226). Correspondingly, HERDT (230) reported data showing that in a rotary machine under Canadian Intense conditions (with CM 7) as much as $12.6 \%$ of TPM condensed on the cigarette holder and the sealing element and was, consequently, not trapped on the filter pad. After reduction of the dead volume in the rotary type by $76 \%$, resulting from (only schematically disclosed) design changes in the smoke path (cigarette holder and sealing element), TPM loss away from the filter was minimized and TPM yield of CM 7 increased by $3.26 \mathrm{mg} / \mathrm{cig}$ to $42.07 \mathrm{mg} / \mathrm{cig}$ narrowing the difference from the result obtained with a linear machine (44.39 mg/cig).

In summary, compared to other - more intense - smoking regimes the ISO smoking regime (105) is a validated, powerful and robust tool for research purposes as well as for quality control and product development. Cigarettes can be characterized and compared by evaluating reproducibly the effects of different tobacco components, additives and design features (filters, papers, etc.) on mainstream smoke yields. Smoking according to ISO is also a tool for checking the compliance with national and international regulations. It is, however, indubitably recognized that neither ISO nor any other regime for the machine smoking of cigarettes is able to duplicate the array of human smoking behavior to measure the uptake of smoke components for evaluating health risks related to smoking a specific cigarette brand. 
It is obvious that the standards for smoking machines and mainstream smoke trapping devices were adapted to the ISO smoking regime and vice versa. Therefore, any changes will have serious decoupling effects. The publications mentioned and discussed above indicate that this happens whenever smoking devices designed according to ISO standards are used with smoking regimes other than ISO.

The International Organization for Standardization - founded in 1947 and still the most prominent global agency developing and preserving international standards - included in the $5^{\text {th }}$ edition of ISO 3308, published in 2012 (105), a remarkably sensible and realistic statement:

"Experience and knowledge gained from the use of analytical cigarette-smoking machines has highlighted a need to specify certain requirements, which are addressed in this International Standard.

No machine smoking regime can represent all human smoking behavior:

- It is recommended that cigarettes also be tested under conditions of a different intensity of machine smoking than those specified in this International Standard;

- Machine smoking testing is useful to characterize cigarette emissions for design and regulatory purposes, but communication of machine measurements to smokers can result in misunderstandings about differences in exposure and risks across brands;

- Smoke emission data from machine measurements may be used as inputs for product hazard assessment, but they are not intended to be nor are they valid as measures of human exposure or risks. Communicating differences between products in machine measurements as differences in exposure or risk is a misuse of testing using ISO standards."
It should be pointed out that a comparable statement was made by ISO in the International Standard ISO 4387 already in 2008 (219). This statement was subsequently incorporated into national standards, e.g., in the 2011 German standard DIN/ISO 4387 (231).

In 2012, an equivalent statement was made in the preamble of the WHO TOBACCO LABORATORY NETWORK (200) Standard Operating Procedure for Intense Smoking of Cigarettes (SOP 01):

"No machine smoking regimen can represent all human smoking behaviour: machine smoking testing is useful for characterizing cigarette emissions for design and regulatory purposes, but communication of machine measurements to smokers can result in misunderstanding about differences between brands in exposure and risk. Data on smoke emissions from machine measurements may be used as inputs for product hazard assessment, but they are not intended to be nor are they valid as measures of human exposure or risks. Representing differences in machine measurements as differences in exposure or risk is a misuse of testing with WHO TobLabNet standards."

The new European Union "Tobacco Products Directive" of 2014 (232) considered imprints of "tar", nicotine and carbon monoxide smoke levels on cigarette packs as "misleading" and interdicted their use in labeling packet and outside packaging.

In our opinion, imprints on cigarette packs stating "tar", smoke nicotine and carbon monoxide yields, measured according to ISO 4387 (219), provide certain useful information to consumers. While these data are only weakly correlated with material smoke intake and uptake by humans consuming cigarettes, they are suitable as predictors of smoke taste and impact and, therefore, good indicators of the sensorial quality of a brand. 

CONDENSATE PRODUCTION AND IN VIVO TOXICITY TESTING

The analytical smoking machines described and discussed so far were (and are) employed essentially for the qualitative and quantitative determination of mainstream smoke constituents. A number of other scientific themes require smoking machines with different performance characteristics, above all, the capability of producing larger amounts of smoke condensate of constant quality. These preparative smoking machines are needed, e.g., for the capture of sufficient amounts of smoke condensate for investigating general toxic and carcinogenic effects by non-inhalational in vivo exposure methods - foremost skin painting assays.

For obtaining statistically significant results in vivo, smoke condensate has to be administered at different levels to fairly large numbers of experimental animals, predominately small rodents. Therefore, the smoke condensate produced by preparative smoking machines from bulk amounts of cigarettes should be homogeneous and its generation reproducible. The experimental procedures should be repeatable by other researchers in different locations and at different times. The generation of artifacts due to reactions between different smoke constituents and the loss of reactive components over time should be prevented by suitable collection devices. As a rule, the smoking of cigarettes should conform to standardized regimes, such as ISO (105) or FTC (19). It was, however, not before the 1950 s that tobacco derived material for in vivo toxicity testing was produced by methods with some degree of standardization.

The first attempt to induce cancer with a tobacco smoke preparation in an experimental animal was reported in 1900 by BROSCH (233), prosector at the military funeral center in Vienna, Austria (Prosector am Militär-Leichenhof in Wien). After inflicting an artificial necrosis he painted the back skin of guinea pigs with "tobacco juice" and observed epithelial proliferation but no development of cancer. Details of the generation and nature of the "tobacco juice" or the duration of application were not reported. However, based on a literature reference it can be assumed that the "tobacco juice" was produced by smoking tobacco in a porcelain pipe by means of an aspirator $(234,235)$.

In 1911, WACKER and SCHMINCKE (236) at the University of Munich (Germany) produced samples of tobacco "tar" by scouring tarry pipes with ethanol, evaporating the solvent and extracting of the residue with oil or fat, and injected them subcutaneously into rabbit ears. Atypical epithelial proliferation (generally massive) was observed. The probably first mouse skin painting study using tobacco derived material was reported in 1923 by ERICH HOFFMANN et al. (237) at the University of Bonn (Germany). White mice were treated repeatedly with "tobacco tar" which was obtained by coking tobacco (some kind of thermal decomposition; no details communicated). Interestingly, nicotine had to be removed tediously from the coking product before application as the animals did not survive exposure to the unpurified material. In addition to hair loss, the treatment resulted in signs of skin irritation like those usually preceding tumor formation. This study pointed for the first time to a possible relationship between tobacco smoke condensate and cancer.

In 1928, HELWIG (238) duplicated the work of WACKER and SCHMINCKE (236). The ethereal extract of tobacco "tar" from bowls of smoked briar pipes was painted - after mandatory nicotine removal - on mouse skin for almost one year and produced severe ulceration but no atypical growth. In addition, the "tar" extract was mixed with olive oil and injected into the skin of rabbit ears. Again, atypical proliferation was observed but no benign or malignant tumor formation. No information was provided on the doses applied.

Studies performed in the 1930 s by the Argentinean scientist ROFFO demonstrated the carcinogenicity of tobacco derived preparations after application to rabbit ears. Materials for testing were fractions of tobacco smoke obtained by various - poorly described - extraction steps $(239,240)$, tobacco "tar" fractions produced by the pyrogenic distillation of dry tobacco leaves (241), or even whole smoke formed by combustion of tobacco in a metal syringe and emitted directly onto the inner surface of the rabbit's ear (242). Generally, the toxic and carcinogenic effects on the animals were massive. At a later time, ROFFO (243) isolated a polycyclic aromatic hydrocarbon by "fractional distillation" from tobacco "tar", which showed an UV spectrum like benzo[a]pyrene, and established experimentally the high malignant potency of this compound; he considered it to be the carcinogenic agent in tobacco "tar". The obvious fact that this work had not (yet) proven the presence of benzo $[a]$ pyrene in tobacco smoke was subsequently pointed out by KOSAK (244).

In 1934, LÜ-FU-HUA (245) at the University of Freiburg/Br. (Germany) painted the ears of rabbits with tarry material produced from smoldering tobacco. No tumors were observed after 120 days.

The first mouse skin painting study with cigarette smoke condensate, produced by intermittent smoking, was reported in 1939 by the British scientist CAMPBELL (5). Puffs (14 to 15 per cigarette) were generated using an aspirator rubber bulb (Higginson syringe), and mainstream smoke was obtained from cigarettes inserted in a glass holder, which was cooled and had a small bulb attached for collecting a dark brown liquid ("condensate"). After concentration, the tarry material was used to paint mice in the usual way twice a week for 20 months. Occasional hyperplasia and cancer of the skin were observed after prolonged application. The problem of malignant primary lung tumors detected in a number of treated animals remained nebulous.

In the U.S., SUGIURA (246) isolated watery and oily distillates from tobacco heated in an iron retort, and examined them in a painting study with mice (interscapular skin) and with rabbits and rats (inside surface of the ears). Among 168 albino mice treated for 90 to 500 days only one developed a squamous carcinoma, while the positive control with coal "tar" produced progressively growing tumors at a high rate. Rat and rabbit ears showed no cancerous change at the site of application.

In 1941, FLORY (247) started off his paper on the production of tumors by tobacco "tars" with a review of clinical observations and a useful survey of experimental attempts 


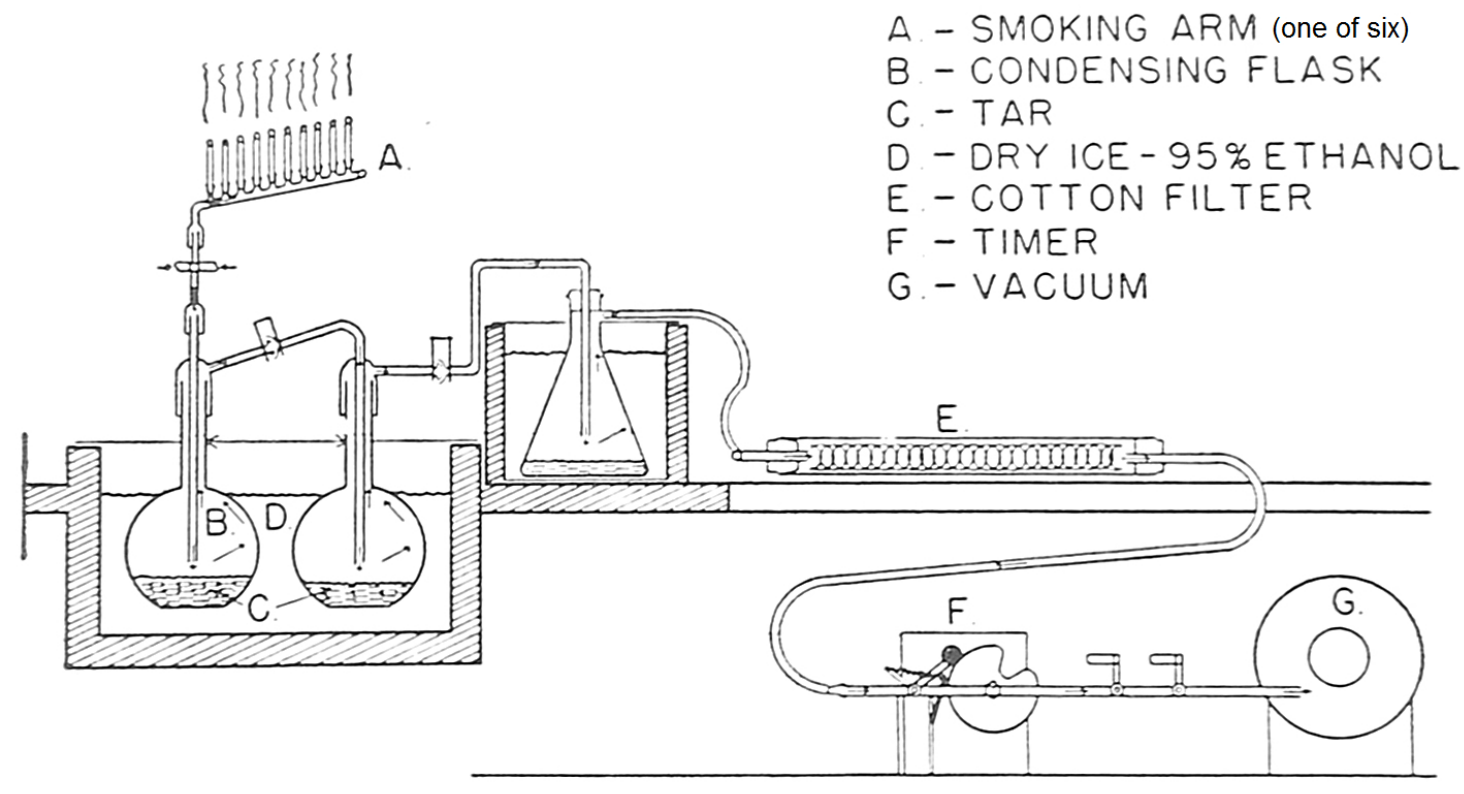

Figure 25. The smoking machine used by WYNDER et al. (72) drew a large puff of $350 \mathrm{~mL}$ at a time from the 10 cigarettes mounted in one of six manifold smoking arms.

to produce carcinomas with tobacco products in rodents and dogs. For his own study, the author obtained watery and oily fractions from cured Kentucky tobacco (whole leaf and stems) by destructive distillation in an electrically heated steel still, and produced pipe "tar" by burning tobacco in an array of small clay pipes (continuous suction provided by a water pump), collecting the smoke in water and isolating the tarry material. Certain fractions had to be denicotinized before application. Painting of five groups of mice (242 animals altogether) induced only two squamous cell carcinomas (strong response to coal "tar" control, 13 out of 15 mice developed squamous cell carcinoma) and application to the inside of rabbit ears did not give rise at all to proven carcinomas.

Until 1953 ten more detailed mouse skin painting studies in all had been performed with various kinds of tobacco "tar" as a possible carcinogen; they were compiled and assessed by WYNDER et al. (72) in preparation of their own investigations on the experimental production of carcinoma with cigarette "tar". Frequently, methodology had not been described in sufficient detail. Many of the studies were deemed to have been "carried on for too brief a period of time or with too few animals to be regarded as significant". All attempts to induce experimental cancer (involving many hundreds of mice) had produced only seven epidermoid cancers of the skin.

The need for the relatively large amounts of smoke condensate required for biological studies led to the development of manifold smoking machines - maintaining the guiding principle of simulating human smoking habits as closely and as practically as possible. One of the early examples is the apparatus used by WYNDER et al. (72), which held 60 cigarettes placed vertically and side by side in 6 arms with 10 ports each (Figure 25). Operation of the machine was based on manual cigarette loading and lighting, and butt removal. A large puff of $350 \mathrm{~mL}$ from each arm (equivalent to $35 \mathrm{~mL}$ per cigarette) was taken in
$2 \sec 3$ times per min for a butt length of $20-25 \mathrm{~mm}$. The condensate was collected in glass flasks placed in an ethanol/dry ice mixture. It was then used without fractionation for skin painting of CAF1 mice. The availability of sufficient test material prepared by standardized, reproducible procedures made it possible to treat animals with higher doses over longer time, thereby overcoming the above-mentioned deficiencies of many earlier studies. As a result, $44 \%$ of the painted animals developed histologically proven epidermal carcinomas - a level not achieved before.

In a study to assess the carcinogenicity of diligently prepared individual fractions of smoke condensate WYNDER and WRIGHT (248) used a larger manifold machine, which was capable of smoking up to 150 cigarettes at one time, taking large puffs ( $350 \mathrm{~mL}$ from 10 cigarettes) by means of a motor pump. For each cigarette, the puff volume was $35 \mathrm{~mL}$ taken in $2 \mathrm{sec}$ at a rate of 3 per min (which was later reduced to one per min). The desired butt length was $25 \mathrm{~mm}$. The machine was able to smoke at least 4,000 cigarettes per day (249).

As noted by O'KEEFFE and LIESER (85) these manifold smoking machines $(72,248)$ had their shortcomings. Like with other constant time machines with constant vacuum, changes of cigarette draw resistance during smoking led to rather variable puff volumes. With the WYNDER and WRIGHT smoking device (248) the actual puff volumes ranged from 32 to $38 \mathrm{~mL}$. In addition, puff numbers could vary as much as 2 , and butt length was $25 \pm 5 \mathrm{~mm}$.

KOSAK et al. (250) developed an apparatus that accommodated 240 horizontally positioned cigarettes in 12 manifolds, with a distance between their axes of $25 \mathrm{~mm}$. The system was set to take 2 -sec puffs, one per minute. Puffing was done by means of a rotary air pump combined with a large vacuum reservoir to allow puffs in the range of 35 to $40 \mathrm{~mL}$. Median butt length of the plain cigarettes was $25 \mathrm{~mm}$. The condensate was collected in an assembly of 
four cold traps by impact and gravity deposition. Puff profiles were controlled by kymograph recording. The condensate was fractionated and used for chemical characterization and biological testing at a later time.

In Denmark, ENGELBRETH-Holm and AHLMANN (251) constructed a somewhat unusual device for the artificial smoking of cigarettes. The upper wide opening of an upright positioned glass funnel was tightly covered by a Bakelite plate with 50 holes, in which cigarettes were mounted vertically. The lower end of the funnel reached into a glass tube, stuffed with cotton wool for absorbing smoke particles (without cooling), which in turn was connected to the vacuum source. After lighting the cigarettes with a Bunsen burner, puffs of $1 \mathrm{sec}$ duration were drawn every $30 \mathrm{sec}$ by means of a pulsator (like in a mechanical milker). Suction was regulated by a needle valve allowing the smoking of the cigarettes in 7-8 min. No data on puff volume was reported. The absorbed material was extracted from the cotton wool with acetone and used for chemical characterization and carcinogenicity testing.

In 1957, SCHUR and RICKARDS (252) of Olin Mathieson's Ecusta Paper Division described a rather sophisticated multiple smoking machine for the large scale collection of condensate, suitable for chemical and biological studies. The device could smoke 20 cigarettes simultaneously through individual "smoke channels" for producing up to $10 \mathrm{~g}$ of condensate per day from some 500 cigarettes. The constant time machine could be used either with a vacuum pump in combination with a suction regulator and a valve or with a cam actuated piston, for shaping desired puff profiles. The vacuum head rotated over a fixed horizontal circular metal selector plate with 20 cigarette ports; mainstream smoke condensate was collected separately from each smoke channel in glass coils, which were cooled by dry ice and acetone to $-80^{\circ} \mathrm{C}$ and each capable of catching the condensate of up to 30 cigarettes. Puff volume and puff duration could be varied within a limited range, corresponding to human smoking behavior. Using a kymograph, the authors had gathered data on the smoking habits of 20 male volunteers and determined an average puff volume of $37.4 \pm 10.7 \mathrm{~mL}$ and an average puff duration of $1.58 \pm 0.37 \mathrm{sec}$. Consequently, the preferred smoking regime was one 2 -sec puff/min with a volume of $35 \mathrm{~mL}$.

In their paper, the authors included an overview of the butt lengths of the plain cigarettes used by others for machine smoking (filter cigarettes were not common at that time). Butt lengths varied between $13 \mathrm{~mm}$ (47) and 20-25 mm (72). A butt length of $23 \mathrm{~mm}$ was used by BRADFORD et al. (4), HAAG and LARSON (253), WOLMAN (254) and the U.S. CONSUMERS UNION (255); it was typical for studies conducted in the U.S. In European studies shorter butt lengths were generally chosen: $13 \mathrm{~mm}$ by WENUSCH (47), $15 \mathrm{~mm}$ by PFYL et al. (17), WASER and STÄHLI (256) and PYRIKI (257), and $20 \mathrm{~mm}$ by STAUB and FURRER (63).

The machine developed by SCHUR and RICKARTS (252) was modified in 1965 by HACKNEY et al. (258) of the Liggett and Myers Tobacco Company by incorporating a fixed vacuum head. The 15 cigarettes smoked per run were mounted on a vertically rotating wheel allowing smoke condensate to be collected in a single trap. Except for the replacement (insertion and removal) of cigarettes, opera- tion of the machine was essentially automatic. Not suitable for analytical purposes, it was a device for the production of large amounts of smoke condensate, required for biological evaluations. More than 100 cigarettes per hour could be smoked, corresponding to 600-900 cigarettes in an 8-hour workday, usually representing 40-50 g of collected smoke material.

A manifold-type smoking machine for the very large scale production of mainstream smoke condensate was described in 1959 by CLARK and BOCK (7). The instrument was produced by the Process and Instruments Company and used in the late 1950 s and early 1960 s by BocK et al. $(259,260)$ for their studies of the carcinogenicity of cigarette smoke condensate in mouse skin. Two-second puffs were taken every minute for mainstream smoke generation from 600 cigarettes automatically loaded and positioned in 6 horizontal rings of 100 holders each around the cylindrical smoking head (Figure 26). 30 cigarettes were smoked simultaneously with $35-\mathrm{mL}$ puffs; consequently, the total volume of one puff was $1,050 \mathrm{~mL}$. Another model smoked 100 cigarettes simultaneously (260). For puffing, a motor vacuum pump was used. After a fixed number of puffs (usually 10) the cigarettes were automatically ejected by air pressure. Afterwards the holders were refilled with new cigarettes and the cigarettes lit by means of a bank of gas jets. Mainstream smoke condensate was collected in cold traps. Up to 20,000 cigarettes could routinely be smoked per day. Understandably, the device was nicknamed "mammoth machine", and it was suspected that condensate was needed for "painting elephants" (7).

In 1965 an improved manifold smoking machine was described by LANG and MOSHY (261) of the American Machine and Foundry Company (Springdale, CT, USA) at the $19^{\text {th }}$ Tobacco Chemists' Research Conference. Cigarettes were loaded automatically into 90 Teflon coated ports on the periphery of a horizontally rotating drum (Figure 27). Ten equally spaced stationary glass arms of a stationary manifold, which was connected to the cold trap collection system and the vacuum source (a motor driven mechanical pump), made sliding contact with the inner surface of the

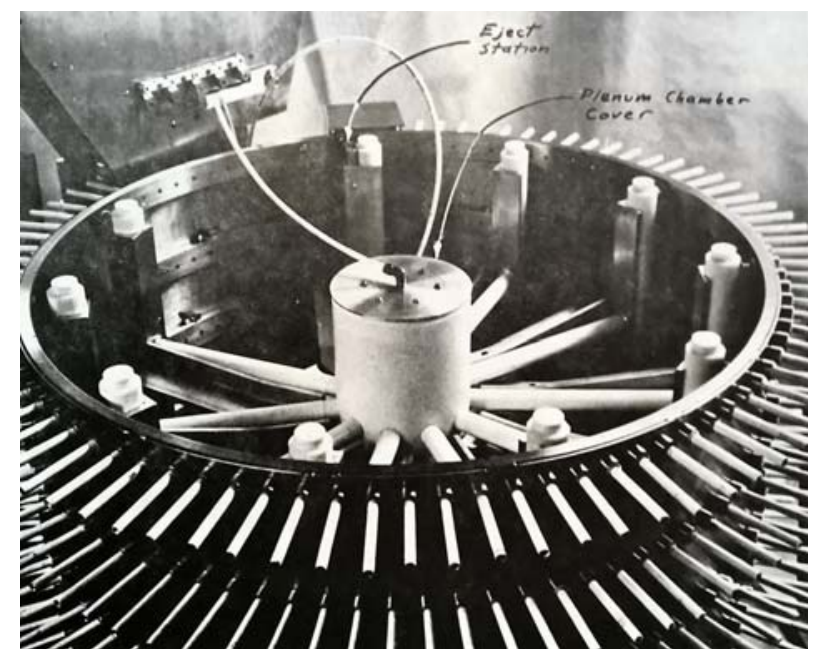

Figure 26. The features of the smoking machine of CLARK and BocK (7) were colossal: 600 cigarettes smoked concurrently; collective puffs of $1,050 \mathrm{~mL}$; enough condensate produced for "painting elephants". 


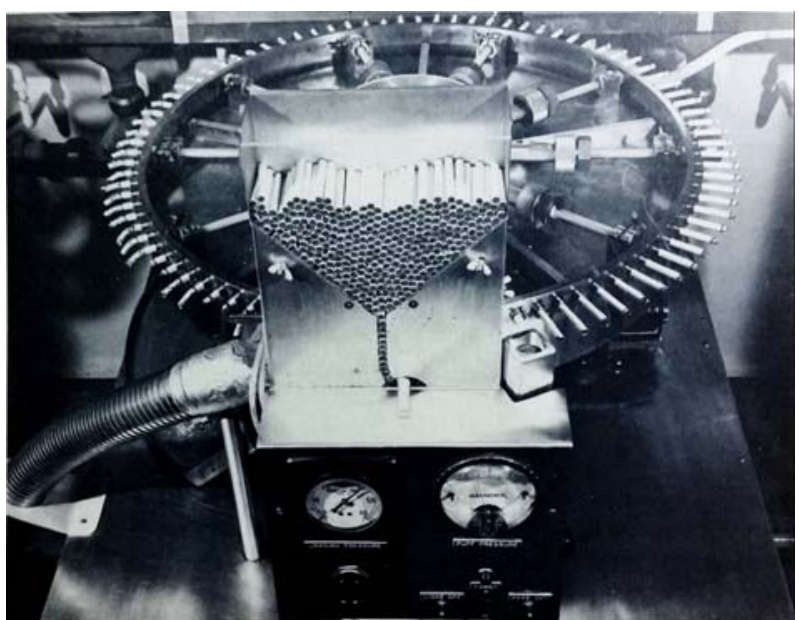

Figure 27. The smoking machine of LANG and MosHY (261) was the "trusty workhorse" for the production of smoke condensate in the U.S. program on cigarette harm reduction.

drum. Each time the stationary arms were aligned with cigarette ports, a puff was taken simultaneously from 10 cigarettes. Puff frequency and puff duration were controlled by the rotation mechanism of the drum. The machine was very flexible and could be used for free or restricted smoking. To obtain the desired puff volume of $35 \mathrm{~mL}$ in $2 \mathrm{sec}$, vacuum strength in the manifold had to be adjusted to the draw resistance of the cigarettes smoked. Butt length was defined by puff number; their relation had to be pre-determined by means of an analytical smoking machine. The apparatus was one with constant puff duration and could produce a puff profile relatively similar to that observed in smokers. Operation of the machine was fully automatic, and only feed hopper refills required manual work. With one puff taken per min, 540 cigarettes could be smoked per hour. This kind of smoking machines was used for the production of the large quantities of cigarette mainstream smoke condensate required for mouse skin painting assays in the U.S. program on cigarette harm reduction (262-266).

As mentioned before, O'KEEFFE and LIESER (85) raised objections to manifold machines employing the constant time/constant suction (by a vacuum source) mode because puff volume might vary with decreasing draw resistance as the cigarettes were consumed. In our view, there is additional variation of the puff volume for individual cigarettes when a puff is drawn at the same moment from more than one cigarette, e.g., $350 \mathrm{~mL}$ from 10 cigarettes. If the cigarettes are positioned in a row on the arms of a manifold, the cigarette with the highest, or lowest, pressure drop increases, or reduces, the actual puff volume of the other cigarettes. This objection was also raised by WALTZ et al. (111). In addition, there may be a gradient of negative pressure along the arms. Therefore, the composition of puffs drawn from the individual cigarettes may be affected, which could influence mainstream smoke toxicity. If the cigarettes are positioned (too) close, the radiation heat of the glowing cones of neighboring cigarettes may influence the burning velocity during and between puffs. Absorption of sidestream smoke during puffing cannot be excluded. As the toxicity of freshly generated sidestream smoke is higher than that of mainstream smoke (267), an influence on the toxicity of the collected smoke condensate may be expected.

WYNDER and HOFFMANN (249) stated that the critique of O'KEEFFE and LIESER (85) was probably unwarranted - a rather realistic view. The objective of the studies of KOSAK et al. (250), WYNDER et al. (72) and BOCK'S GROUP (259, 260) was to show proof of the carcinogenicity of, and the presence of carcinogens in, tobacco smoke condensate, especially cigarette smoke condensate. For this goal (a qualitative "yes or no decision"), the smoking devices used by KOSAK et al., WYNDER et al. and BOCK'S GROUP looked quite practical. However, these machines were certainly not suitable for the precise and reproducible quantitative comparison of the carcinogenicity of mainstream smoke condensates produced from different kinds of cigarettes. In Europe, systems for the large scale production of cigarette mainstream smoke condensate were primarily developed by British American Tobacco (Hamburg, Germany) in cooperation with the German Verband der Cigarettenindustrie ( $\mathrm{VdC}$ ) and the H. Borgwaldt Company (Hamburg, Germany). One approach chosen was to upgrade available analytical smoking machines and design new collection devices of sufficient capacity.

With a few mechanical adaptations, the existing analytical rotary 30-port Borgwaldt smoking machine BAT-RM 30/65 (116) was customized by ELMENHORST (268) to smoke 1,000 cigarettes a day when combined with a novel condensate collection apparatus (discussed in Chapter 7.7, p. 238), which used a solvent energized by vibration; this liquid trap had originally been described by BARKEMEYER and SEEHOFER (269).

Also for use with the modified preparative smoking machine BAT-RM 30/65, ELMENHORST (270) constructed a cold trap, which required no solvent at all and worked on the principle of capturing newly formed condensate flowing into the trap inside the already collected halffrozen material (a somehow strange procedure described in detail in Chapter 7.6, p. 237). The trap had the remarkable capacity of accommodating the precipitated smoke of 6,000 cigarettes, equivalent to $180 \mathrm{~g}$ condensate.

The preparative version of the BAT-RM 30/65 smoking machine required still a lot of manual operation. There were preparative machines with most of their functions automated, such as those described by CLARK and BOCK (7) and LANG and MoshY (261). However, these were of the manifold type and smoked several cigarettes (say, 10) in one puff with the consequence that individual cigarettes - in case of unequal draft resistance - would be smoked with different volumes per puff.

Therefore, ELMENHORST (271) held on to the principle of the rotary machine, taking each puff separately from one cigarette, and made the preparative BAT-RM 30/65 fully automatic with regard to cigarette insertion from the (new) hopper into the smoking head, lighting, smoking, puff counting, and the expulsion and extinguishing of butts. Duration of smoking was determined by the preset number of puffs (rather than butt length) after the relationship of puff number and butt length had been determined using an analytical smoking machine. It was found that - when smoking a large number of uniform cigarettes - this was a quite acceptable way of controlling the process; the amounts of condensate and smoke nicotine were rather 
consistent when comparable test pieces were smoked to either constant butt length or the (predetermined) constant puff number.

When operated in compliance with the standardized regulations of the VERBAND DER CIGARETTENINDUSTRIE (50) - a 35-mL puff of $2 \mathrm{sec}$ duration per min - and using the solvent free cold trap (270) the automatic machine could smoke over 1,000 cigarettes per day. Owing to the degree of automation, an experienced operator could supervise eight machines concurrently for an impressive throughput of 8,000-10,000 cigarettes per day.

However, achieving the desired butt length within acceptable limits by controlling the number of puffs turned out to be a problem when smoking cigarettes manufactured in small batches, which possibly differed in tobacco weight, pressure drop and, consequently, burn rate. Therefore, ELMENHORST and HENNIG (272) completed the automatic preparative smoking machine (271) by incorporating a heat sensor measuring the infrared radiation of the glowing cigarette; the automatic butt ejector was activated when the targeted butt length was reached.

Advanced equipment - an automatic preparative rotary smoking machine (271) and a solvent free cold trap (270) was used in the late 1960s by DONTENWILL et al. (273) for producing the tremendous amounts of condensate required for their skin painting studies with mice. In periods of peak research activity, 280,000-330,000 cigarettes were smoked per month, yielding $8-9 \mathrm{~kg}$ of condensate. The condensate producing equipment used proved robust, and the smoking regime followed was reproducible. DONTENWILL et al. investigated the induction of tumors in mouse skin and compared cigarettes, which had been made of different tobaccos, differently treated tobaccos and reconstituted tobaccos, as well as samples (some markedly enriched in polycyclic aromatic hydrocarbons) obtained after the fractionation of condensate.

The high level of productivity and reliability displayed by the advanced smoke condensate generating systems was, however, associated with an (often considerable) lapse of time before analytical examination or biological testing could be performed. The properties of condensate may change substantially by aging; and it may be practically impossible to assess properly the characteristics and effects of short-lived radicals and electrically charged components. All types of trapping systems (filters, cold, electrostatic or solvents) are more or less prone to undesirable reactions between constituents found in the gaseous and particulate phases (see Chapter 7.1, p. 226).

Research groups in Germany advanced the principle of condensate precipitation by coagulation. When smoke aerosol is forced at high speed through a nozzle or capillary the sharply increased collision rate and intensity of the inelastic particles make them coalesce into a precipitate. In the late 1930s, WENUSCH (42) had used this technique for condensate collection by forcing smoke through a z-shaped capillary, and CUZIN (66) of the French Tobacco State Monopoly had generated native smoke condensate for biodynamic studies in a capillary trap.

In 1963 NEURATH and KRÖGER (274) designed a novel smoking apparatus that turned to "reverse smoking", this way avoiding the need for prior collection of condensate and allowing its immediate application in a biological study. Puffing was replaced by pressing air through the lit end of a single cigarette mounted inside a specially designed, water cooled receptacle. Smoke was forced to exit from the mouth end of the cigarette through a nozzle, which brought about the instant condensation of particulates right onto the target area, for instance, on mouse skin - eliminating the risk of aging before administration. A $35-\mathrm{mL}$ flow of air through the cigarette under constant pressure occurred once per min for $2 \mathrm{sec}$. The yield of mainstream smoke condensate was $60-70 \%$ compared to electrostatic precipitation. Sidestream smoke formation and removal between puffs $(58 \mathrm{sec})$ was controlled by a mild air stream passing through the receptacle.

To our knowledge, the smoking and application device developed by NEURATH and KRÖGER was the first successful approach to utilizing absolutely fresh, un-aged cigarette mainstream smoke condensate for biological testing. In addition, mainstream smoke gaseous phase was examined. But even so, this technique was criticized by WYNDER and HOFFMANN (249) who noted first, that reverse smoking was quite different from actual human smoking; second, that the doses applied were too low for showing an effect on mouse skin; and third, that the absence of solvents weakened the effects on mouse skin. According to the U.S. FOOD PROTECTION COMMITTEE (275), "various solvents will have to be used as vehicles for applying the different kinds of substances by this route". WYNDER and HOFFMANN'S critique looks reasonable with regard to the mouse skin assay. In addition, it would be practically impossible to treat the (high) number of animals required for biological testing in view of the complicated and slow smoking procedure (274). These objections may be much less appropriate if the fresh condensate was used in vitro with cultures of microorganisms or mammalian (including human) cells.

Departing from the principle of reverse smoking, NEURATH and EHMKE (276) modified the existing specially designed, water cooled receptacle (274) and combined it with an Ethel Mark VI smoking machine (Cigarette Components, UK) for the simultaneous collection of mainstream and sidestream smoke. The system was used to follow the fate of nicotine, benzo[a]pyrene and total phenols, when smoking a plain blended cigarette, by determining their distribution into mainstream and sidestream smoke. Following additional modification, the receptacle was employed by BRUNNEMANN and HOFFMANN (277) to measure, puff by puff, the $\mathrm{pH}$ of sidestream smoke from different cigarettes and small cigars. Subsequently, HoFFMANN and his group used this device (Figure 28) for the quantification of several components in cigarette sidestream smoke, such as volatile and tobacco-specific $N$-nitrosamines $(278,279)$, nitric oxide (280), catechol (281), volatile pyridines (282) and ammonia (283).

A Pyrex glass chamber of similar design was developed in 1966 by MORRELL and VARSELL (284). Combined with a suitable lighting and puffing device it allowed the complete collection of all materials (mainstream and sidestream smoke, ash, butt) generated by cigarette smoking under standard smoking conditions. Mainstream smoke was produced by reverse puffing and sidestream smoke flushed out through a separate port. Cigarettes were lighted through the glass wall by the focused (hot) beam of a 


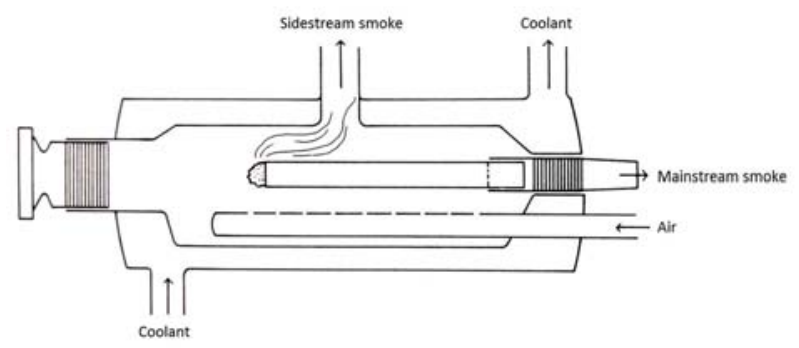

Figure 28. Both cigarette mainstream and sidestream smoke could be analyzed using the device developed by BRUNNEMANN and HOFFMANN (277) (figure adapted from (82)).

projection lamp (285) and eventually extinguished with excess nitrogen. Highly efficient devices (TPM filters, very low temperature traps and oxidation tubes) were required for complete mainstream and sidestream smoke collection. It is worth noting that the glass chamber was not wrapped with a jacket for water cooling. As pointed out by KLUS and KUHN (82) the temperature inside the glass chamber without cooling may rise during operation up to $90{ }^{\circ} \mathrm{C}$, combined with excess humidity - conditions expected to have considerable impact on the smoking process. In their study, MORRELL and VARSELL (284) analyzed the decomposition products (including $\mathrm{CO}_{2}$ ) of cigarette paper, containing ${ }^{14} \mathrm{C}$-labeled cellulose or calcium carbonate, in fractions of mainstream and sidestream smoke.

With only minor modifications of the trapping components, the system of MORRELL and VARSELL (284) was used by JENKINS et al. (286) in an examination of cigarettes containing tobacco spiked with either ${ }^{14} \mathrm{C}$-nicotine or ${ }^{14} \mathrm{C}$-dotriacontane-16,17. Combining radioactivity measured in mainstream gas phase and TPM, sidestream gas phase and TPM (the latter including the ash) and butts, the recovery of the ${ }^{14} \mathrm{C}$-label was practically $100 \%$. Following irradiation of 2R1 Kentucky reference cigarettes for neutron activation analysis, JENKINS et al. (287) determined the levels of 15 elements in the complete cigarette, the tobacco filler, the cigarette paper, and - after smoking under identical experimental conditions - in dropped ash and mainstream particulate matter.

In 1964, SEEHOFER and HANßEN (288) published the first report on a "capillary press" smoking machine. As the typical design feature, the smoking head with a single vertically positioned cigarette was linked with a piston pump, which in turn was connected to a capillary for condensate formation. The pump maintained a constant puff volume and could be adjusted to any smoking conditions; generally standard parameters were used $(35-\mathrm{mL}$ puff in $2 \mathrm{sec}$ every $\mathrm{min}$ ). Smoke was pressed through the capillary (preferred: $40 \mathrm{~mm}$ length and $0.5 \mathrm{~mm}$ inner diameter) at a speed of $100 \mathrm{~m} / \mathrm{sec}$. The accumulated condensate was allowed to drip immediately onto a surface, into a solvent or directly onto a target spot, such as mouse skin or a cell culture. Compared to electrostatic precipitation the yield with capillary precipitation was $70-80 \%$. A number of technical problems were waiting to be solved: the seals between the cigarette and the pump, and between the pump and the capillary; sedimentation inside the pump; somewhat diminished puff volumes; and differences in condensate composition (water, nicotine) compared to analytical smoking.

The smoking machine was further improved by SEEHOFER and HANßEN (289) and its capacity expanded to smoking 15 cigarettes in one run by means of a (30-port) rotary turntable; it was now called "automatic". Fifteen test pieces was the limit of the system because of the time, required per cigarette, for the $2 \mathrm{sec}$ piston induction stroke (puffing) plus the $2 \mathrm{sec}$ exhaust stroke (expulsion of the aerosol into the capillary). Up to 500 cigarettes could be smoked per day. Using the combination of turntable, piston pump and capillary, condensate collection was compared to an analytical machine (BAT-RM 30/65) with electrostatic trapping - examining a range of analytical parameters (condensate, nicotine, total phenols, water, acids and benzo $[a]$ pyrene) on a per puff and per cigarette basis. Yield with capillary precipitation was $90 \%$ compared to electrostatic precipitation.

Additional potentialities of the "capillary press" smoking machine were reported by SEEHOFER and HANßEN (290) in 1966. The system was equipped with a rotary smoking head adjustable for free and restricted smoking. Free smoking means that the mouth end of a cigarette remains open between puffs allowing a small part of the smoke formed during smoldering to escape through the open butt end. Cigarettes are smoked in a restricted way when the mouth end is sealed between puffs - the kind of smoking specified by the standards of DIN/ISO (231) and FTC (19). AYRES and IVINSON (127) had shown earlier that different yields of mainstream smoke components were produced by smoking cigarettes in a either restricted or free manner. This was confirmed by SEEHOFER and HANßEN (290) upon examination of the (partially remarkable) effects - under both smoking conditions - of tobacco moisture, butt length, puff number and pressure drop increase by a filter on condensate yield and composition (nicotine, total phenols, benzo $[a]$ pyrene and benzo $[e]$ pyrene).

A smoking machine suited best for cigarette mainstream smoke collection by impaction was developed by MATHEWSON (291). The device produced puffs of almost exactly rectangular profile and highly constant flow, and is described below (see chapter 7.5, p. 234) 


\section{SMOKE GENERATION AND EXPOSURE IN ANIMAL INHALATION STUDIES}

The mouse skin painting test is an important in vivo model to study the carcinogenic, cocarcinogenic and tumorpromoting properties of tobacco smoke condensate and its constituents and also of materials (particularly additives) used in tobacco product manufacturing. The test is mechanistically based, relatively easy to perform and produces quantitative data regarding tumor incidence, latency, multiplicity and malignancy (292). However, the relationship between mouse skin tumors and any manifestation of tobacco smoke toxicity in humans is not clear. In fact, the painting of animal skin and the human uptake of tobacco smoke differ not only at first sight.

Smoke inhalation by experimental animals obviously duplicates the human habit more closely and allows the toxicological assessment of whole smoke rather than being limited to smoke condensate. Accordingly, the reproducible availability of native whole smoke in sufficient quantity and the best possible, well controlled quality is an essential requirement. This has induced the development of ever more sophisticated and dedicated smoking machines - together with compatible exposure devices. Technically, various smoking and exposure concepts were implemented; their meaning ought to be well understood. Regarding machine smoking:

- "Normal" smoking duplicates human behavior by applying suction at the butt end of the cigarette;

- For "reverse" smoking air is forced through the cigarette by pressure applied from the lighted end (allowing smoke delivery to the animals with very short delay);

- "Free" smoking keeps both ends of the cigarette open between puffs, while with

- "Restricted" smoking air flow is cut off at the butt end between puffs.

Regarding exposure conditions:

- "Active" inhalation deals with smoke produced by the animal itself (feasible with larger species only, such as dogs and monkeys, and encumbered by poor adherence to defined smoking conditions);

- "Passive" inhalation requires mechanically generated smoke (practical with smaller rodents, such as mice, rats, hamsters and rabbits, and allowing good control of exposure intensity);

- "Intermittent" or "static" exposure describes the supply to the respiratory chamber of smoke from one or several puffs of one or a few cigarettes, which is allowed to stand for a certain time and then flushed out with fresh air;

- "Continuous" or "dynamic" exposure works with a steady stream of defined smoke delivered to the chamber.

The smoking machines and techniques as well as the equipment for exposure by inhalation developed up to the mid-1960s were chronicled and critically reviewed by WYNDER and HOFFMANN (249) in their monograph published in 1967.

When reflecting on the laboratory animals used in inhalation studies it is constructive to differentiate between the relatively small rodents (mice, rats, hamsters and rabbits) and the larger animals, such as dogs and monkeys. It is particularly important to know that the rodents are obligatory nose breathers with exceedingly well developed nasal passages. Consequently, "the animals screen out much of the smoke aerosols by their highly developed upper respiratory defense system" (249).

\subsection{Smoke generation and exposure in inhalation studies with rodent species}

It holds some nostalgic allure to describe the experimental circumstances, under which the (most probably) first animal inhalation study ever with cigarette smoke was performed. In 1908, VON ZEBROWSKI (3) at the Saint Vladimir University in Kiev (then in the Russian Empire, today the capital of Ukraine) exposed rabbits to smoke from cigarettes made from cheap Russian high-nicotine "Machorka" tobacco (about 2.5 g per cigarette). A closed glass exposure chamber - with a volume of $20 \mathrm{~L}$ and holding one or two animals - had three vertically arranged holes (about $2 \mathrm{~cm}$ diameter) in the side wall, which were used in the following way: The mouth piece of a cigarette was inserted into the bottom hole; the middle hole was fitted with a plug of cotton wool allowing air to enter depending on the plug's firmness; and the top hole was connected to an aspirator. The exposure cycle consisted of lighting the cigarette and controlling its steady burn rate during 12-15 min by both aspirator strength and cotton wool plug insertion; static exposure to the smoke in the chamber for an additional 15-30 min with bottom and top holes open; and eventual complete chamber ventilation in 5-10 min. This way, 8-10 cigarettes could be smoked each day in 6-8 hours. The process was judged to resemble human smoking behavior. Overall duration of animal exposure was between 56 and 180 days. Strong though transient effects were seen in the animals regarding physical mobility while the loss of appetite and drastic weight loss were serious and lasting consequences of smoke inhalation. Rather detailed necropsies of four longterm exposed rabbits revealed changes in cardiovascular histology; cancer was not mentioned at all.

However, by 1930 medical evidence had accumulated and was assessed by practicing clinicians, which showed a clear correlation between tobacco use and the occurrence of various forms of cancer. The comprehensive review of LICKINT (293), published in 1929, is a telling example. In particular, the increased consumption of tobacco products (specifically cigarettes) was noted to correspond to higher incidence of certain tumors. Characteristic health effects were observed for distinct forms of behavior, such as pipe smoking and lip cancer, tobacco chewing and cancer of the tongue, and inhalation of cigarette smoke and lung cancer. There was a certain amount of speculation regarding the causative factors for carcinogenesis. Combustion products of tobacco were clearly pointed to but the impact of mechanical or thermic damage (or even microbial involvement) was also taken into consideration. Interestingly, in the course of time nicotine was less and less in the firing line.

It was disturbing to scientists that various forms of tobacco juice or "tar" applied to laboratory animals had indeed produced atypical epithelial proliferation but no genuine 


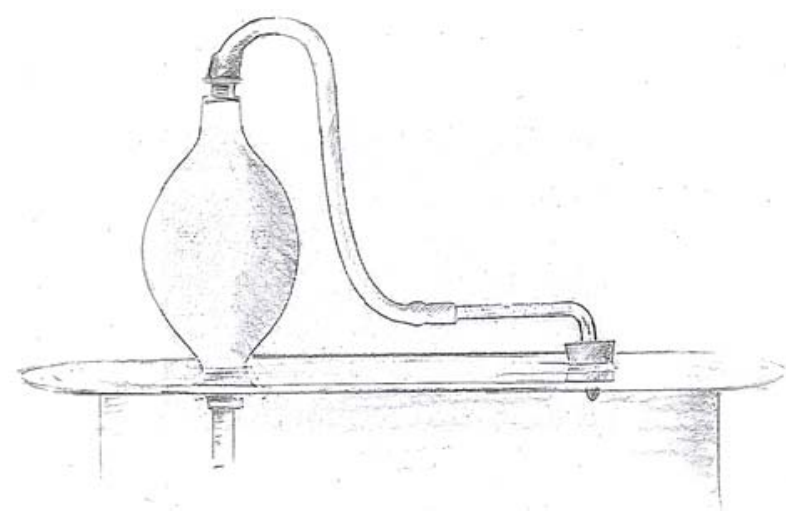

Figure 29. An ancient device from the late 1920s for producing smoke for animal exposure in inhalation studies (294).

carcinoma - in obvious contrast to clinical observations in human smokers. Therefore, inhalation came to the fore as a way of exposing experimental animals to tobacco smoke. In early studies performed between 1928 and 1933 and reported in two parts, MERTENS $(294,295)$ let mice "smoke" by exposing them to whole cigarette smoke for an extended period of time. Up to eight animals were kept in a 10-L glass container, whose flat top opening was closed with a metal plate featuring two holes. A cigarette was fitted into the opening of a modified aspirator rubber bulb and inserted (after being lit) into the container through one of the holes. On its other end the bulb was tightly connected to the second hole (Figure 29). By rhythmic pumping puffs were generated filling the container with smoke dense enough to make the animals hardly visible. Logically, all sidestream smoke remained in the container as well. Daily exposure varied from 2-3 hours but quite often up to 5-7 hours, and lasted for a maximum of 275 days. In the second part of the study, inhalation was combined in some animals with skin painting though without showing any additional effects.

Generally, smoke exposure under the conditions described had devastating consequences to the animals' health, such as convulsions and lethal intoxication. Necropsy revealed widespread suppuration, severe mucosal disintegration and a multiplicity of organ damage. In the respiratory tract extensive epithelial metaplastic changes were observed but - in both parts of the study with 254 animals combined only one lung carcinoma of doubtable origin.

The experimental approach to inhalation chosen by MERTENS was subsequently not adopted by others - a development to be strongly acclaimed from the humane point of view.

A more adequate mouse inhalation study was carried out in 1936 by CAMPBELL (296). The incidence of primary lung tumors was assessed with the suspected carcinogens: "tar" dust, exhaust fumes from petrol operated vehicles and tobacco smoke. Virginia type cigarettes were puffed one at a time (14 to 15 pumps required) using an aspirator rubber bulb (Higgins syringe), which - on one end - was attached to the glass cigarette holder and - on the outlet to the lid of the respiratory chamber; the system included two small receptacles for trapping any condensing fluid and tarry matter. Mice in the chamber were exposed to diluted smoke (1:25 and 1:50) from 12 cigarettes per day for $7 \mathrm{~h}$ on 5 days each week for most of their lifetime (about 2 years). Nicotine, carbon monoxide and other gases (carbon dioxide, hydrogen, oxygen, methane, ethane and unsaturated hydrocarbons) were determined in the smoke before dilution in the respiratory chamber; carbon monoxide was also measured in the chamber. Intensity of smoke exposure was deemed to resemble that of a human smoker. There was no effect of any importance upon general health or specifics, such as death rate, body weight, rate of growth and fertility. The incidence of primary lung tumors in the smoke exposed group was "on the high side"; as it is known that lung tumors are numerous only at the end of the life span and these mice survived longer than the other groups this increase was considered "more apparent than real".

At the $35^{\text {th }}$ Annual Meeting of the American Association of Cancer Research, LORENZ et al. (297) reported in 1942 a study with mice, exposed in a chamber to tobacco smoke (1 mg particulate matter per L of air) for several hours daily and up to 250 days (maximum cumulated exposure time was 693 hours). The average number of spontaneous lung tumors was not increased in the treated animals. No information was provided on the tobacco product or the "automatic tobacco smoking machine" used for smoke generation.

The study of LORENZ et al. (297) was criticized by ESSENBERG (298) on the grounds that exposure time was too short and smoke concentration in the breathing air too low. To overcome these deficiencies (and the shortcomings of other studies), ESSENBERG designed a new smoking machine, which consisted of a vertically positioned rotary cigarette carriage holding 12 cigarettes, a programmed electrical lighter for smoking 1 cigarette every hour for 12 hours per day, and a vacuum pump creating suction just sufficient to burn the cigarette and pull mainstream smoke into the exposure unit (Figure 30). This chamber (as well as the control unit with fresh air) had a volume of $56 \mathrm{~L}$ housing the test animals (strain A albino mice with an appreciable rate of spontaneous tumor development) during the whole experiment. Smoking of the (plain) cigarettes was done with one continuous puff without leaving

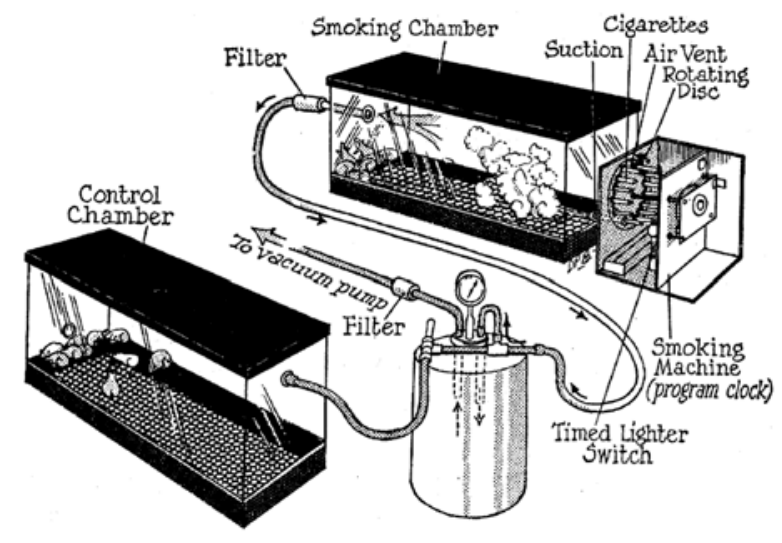

Figure 30. The exposure system designed by ESSENBERG (298) combined a rotary smoking apparatus with a large exposure chamber and included a separate control chamber. 


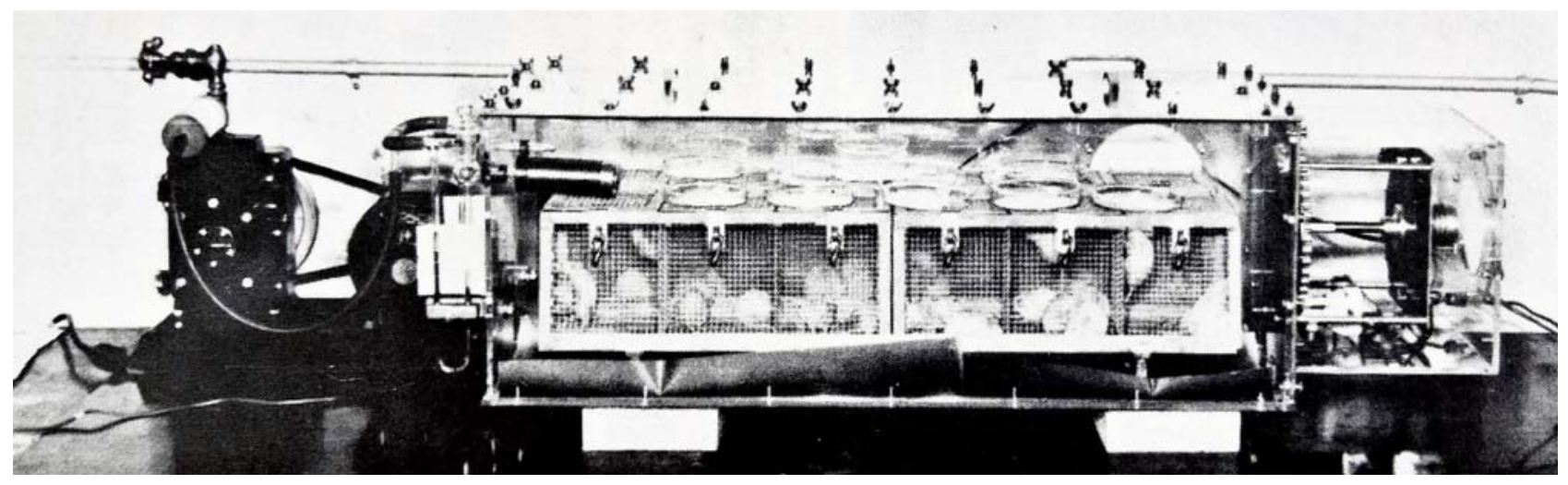

Figure 31. In the configuration developed by LEUCHTENBERGER et al. (299) the animal exposure chamber (center front) and the control chamber (center rear, concealed) were positioned between the vacuum pump (left) and the cigarette house (right) (extracted from (302)).

a butt, which required about 3 to 4 min. The chamber was filled up with smoke soon after the cigarette was lit, remained so for 6 to $7 \mathrm{~min}$, and was flushed clear again within about 2 min. After an exposure time of 12 months ESSENBERG observed lung tumors in 21 of the 23 exposed mice $(91.3 \%)$ and in 19 of the 32 controls $(59.4 \%)$, and concluded "that the preponderance of tumors in the experimental mice was induced by cigarette smoke".

The system developed by ESSENBERG (298) - smoking machine with uninterrupted puffing and dual exposure chambers - was used with some modifications (Figure 31) by LEUCHTENBERGER et al. (299-301) and LEUCHTENBERGER and LEUCHTENBERGER (302) at the Swiss Institute for Experimental Cancer Research in Lausanne in their studies of the cytological, cytochemical and histopathological changes, caused by inhaled cigarette smoke in mice. Bronchogenic carcinomas were not observed even after chronic exposure.

To study the effects of cigarette smoke on the respiratory system of rabbits, HolLAND et al. (303) housed and treated animals individually in compartments, through which continuous suction was maintained. For supplying smoke, a single cigarette was inserted into a sealable port; after lighting an electrically controlled selenoid valve allowed smoke (30-60 mL) to enter the compartment during $2 \mathrm{sec}$ every min. Interestingly, the study design allowed the exposed rabbits to live out their normal life span (4 to 5 years).

Thirty rabbits exposed daily to the smoke of 20 cigarettes and 31 control litter mates of the same sex and age were examined histologically (304). Smoke exposed animals showed more definite pathological changes in the respiratory epithelium of the nose and tracheobronchial mucosa, and emphysema developed earlier, more diffusely and more frequently. Only two breast cancers were found one each in the exposed and the control group - and considered to be spontaneous in origin. Longevity of the control rabbits was significantly longer than was observed in the exposed animals.

A smoking machine for taking a combined puff of $420 \mathrm{~mL}$ from 12 horizontally positioned cigarettes by means of a moving diaphragm was described in 1960 by HAAG et al. (305). Puffs of $35 \mathrm{~mL}$ were drawn in 2 sec once a min and discharged through a smoke distributor into a $23-\mathrm{L}$ expo- sure device with two cages, each holding 8 rats; this resulted in a dilution of about 1:40. After each puff (10 in total per cigarette) a blower cleared the chamber, leading to an exposure time of $33 \mathrm{sec}$ of each min. Fourteen batches of cigarettes were smoked in a day with $30 \mathrm{~min}$ intervals. Animals were exposed long term, either for two years or their life span. Endpoints of the study included various behavioral and physiological parameters, observed while the rats were alive and at autopsy; group differences were noted with regard to weight gain only.

To allow albino mice to be exposed to tobacco smoke up to the limits of tolerability, Отто (306) used a simple approach, dispensing with a separate smoking machine. Pressure was reduced to $600-650 \mathrm{~mm}$ of mercury in a 400-L chamber accommodating up to 60 mice and then equalized by the intermittent opening of an attached manifold with burning cigarettes in a way supposedly reflecting human smoking behavior (no details available). Animals were exposed each day for $90 \mathrm{~min}$ to the smoke of up to 12 cigarettes, collected in the chamber, and were treated as long as they lived. The rather involved study (including repetitions) took almost 7 years to complete, included 11 groups (general maximum: 30 mice) with altogether nearly 340 animals and consumed a total of 27,000 cigarettes. Summarizing a large amount of data, several cases of bronchial epithelial proliferation, single squamous cell carcinomas and an increased rate of lung adenomas were observed.

A rather similar system was used by WYNDER et al. (307) in their studies with male C57BL6 mice, exposed to the smoke of cigarettes without and with filters (cellulose acetate with and without charcoal), to assess pathologic changes in the respiratory tract. A $17-\mathrm{L}$ vacuum tight container - used as exposure chamber for 10 mice and evacuated to a pressure of $600-650 \mathrm{~mm}$ of mercury - served as vacuum source. Puffing was controlled manually by opening a two-way stopcock connected by a glass orifice to the holder of a burning cigarette. The puffs of 2-sec duration every min were claimed to have a volume of some $40 \mathrm{~mL}$. However, when compared to an analytical smoking machine (Cigarette Components CSM 10), the system needed only 7-8 puffs to smoke a cigarette while the yield (measured by Cambridge filter trapping) was about $50 \%$ higher. Obviously, smoking intensity was considerably 
higher using this system. No information on its robustness and reproducibility was provided. Mice, kept in the chamber, were exposed five times weekly for $15 \mathrm{~min}$ to the smoke of a test cigarette (eight different variants were examined); this was the highest load tolerated by the animals. A broad spectrum of exposures was performed, numbering from only 10 to as many as 315 - corresponding to study periods between 2 and 63 weeks. For comparison, mice were also exposed to a synthetic mixture of volatile acids, volatile aldehydes and phenol as well as to nitrogen dioxide; these materials were injected directly into the inhalation chamber. In all study groups of mice, increased incidence of (reversible) reactive hyperplasia and metaplasia was observed, but no progression to squamous cell cancer.

Following the concept of ESSENBERG (298) and LEUCHTENBERGER et al. (299) - evacuating the smoking chamber containing the test animals (by now, at constant intervals) for building up the low pressure required for cigarette puffing - DONTENWILL and MOHR (308) exposed a group of 10 golden hamsters over one year to the mainstream smoke of 10 cigarettes per day. Smoking was done automatically at a stretch, allowing animals to breathe fresh air for $3 \mathrm{~min}$ between cigarettes, and in compliance with standard conditions (1 puff per min, puff duration $2 \mathrm{sec}$, puff volume $35 \mathrm{~mL}$, butt length $23 \mathrm{~mm}$ for plain cigarettes). The system, however, was marred by serious deficiencies: high dilution of the smoke and intense precipitation of condensate on the surfaces of the relatively large smoking chamber (volume was $20 \mathrm{~L}$ ); in addition, as noted by WYNDER and HOFFMANN (249), hamsters are known to sit together in the exposure chamber and hide their snouts in each other's fur. Consequently, exposure of the animals was probably much reduced and resulted in benign metaplastic changes only but no tumors.

DONTENWILL (309) improved experimental conditions by designing a single chamber of considerably reduced volume (280-370 $\mathrm{mL}$ ) solely for smoke collection and equipping it with eight radially arranged tubes holding the animals individually with only their snouts protruding into the smoke collection chamber (Figure 32). The setup exposed animals to fresh smoke diluted with the given volume of collection chamber air and was referred to as a

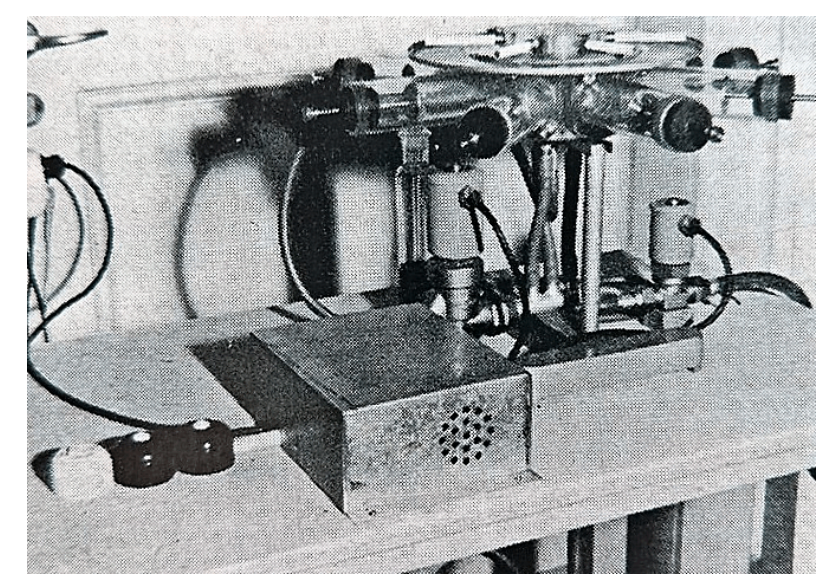

Figure 32. The "closed" smoking and exposure system type "Dontenwill" (309) held animals in circular arrangement around the central smoke chamber. "closed" system. It was estimated that this way the golden hamsters were exposed to smoke, over 100 times more concentrated than in the earlier study (308). Cigarettes were smoked under standard conditions, and animals were forced to breathe each puff for $10 \mathrm{sec}$, followed by fresh air for $50 \mathrm{sec}$ - a rhythm avoiding carbon monoxide poisoning. Exposure of eight animals to $8-16$ cigarettes per day for 9 months produced only very small papillomas of the trachea and metaplastic changes in the bronchial mucosa.

The new system represents significant experimental progress by changing from whole body to head-only exposure. The use of chambers for keeping and exposing (generally several) unrestrained animals is inevitably associated with high dilution and aging of smoke and the deposition of particulates on surfaces and furs (with the risk of uncontrolled uptake by licking). Head-only exposure in combination with small smoke collection and dilution receptacles reduces the distance between cigarette mouth end and the animal's nose and allows the controlled use of higher smoke concentrations.

Using the same equipment (309) DONTENWILL and WIEBECKE (310) increased the number of golden hamsters in a further study and expanded conditions to five levels of exposure. In every single smoking run under standard conditions, 10 puffs were drawn from 2 cigarettes simultaneously, and the 8 animals were exposed to the smoke of each puff for $10 \mathrm{sec}$ followed by $50 \mathrm{sec}$ with fresh air. Runs were repeated 4 times, 8 times or 16 times daily. The main findings were changes in the epithelium of the trachea, which were severe only after a period of more than 240 and up to 540 days; no tumors were detected.

A different instrumental approach was put forward by DONTENWILL et al. (311) when he described the modification of a smoke generation and exposure system used earlier by HARRIS and NEGRONI $(312,313)$. The adapted Imperial Tobacco Company (ITC) smoking machine, reported on by WYNDER and HOFFMANN (249), had been adapted to produce smoke:air mixtures of variable proportions for the continuous exposure of mice, which were constrained individually in the metal boxes of a new type of manifold exposure facility. While the smoke generation and dilution device was used without change, DONTENWILL et al. modified the manifold for housing hamsters under more tightly controlled breathing conditions. The availability of a smoke:air admixture with adjustable concentration was characteristic of an "open" smoking system and had significant advantages for controlling carbon monoxide and nicotine intoxication during animal exposure. The paper (311) includes a comparison of the new approach with a previous method (309) and discusses several aspects relevant for inhalation studies, such as the build-up during, and the decline after, smoke exposure of carboxyhemoglobin and nicotine (in the lung) levels, the quantification of inhaled smoke, the influence of nasal filtration and the choice of the experimental species.

However, evaluative comparison of the available smoking/ inhalation systems revealed several shortcomings. The "closed" DONTENWILL device (309) showed technical vulnerability, carried the risk of animal intoxication in case of control failure and allowed undesirable smoke aging and 


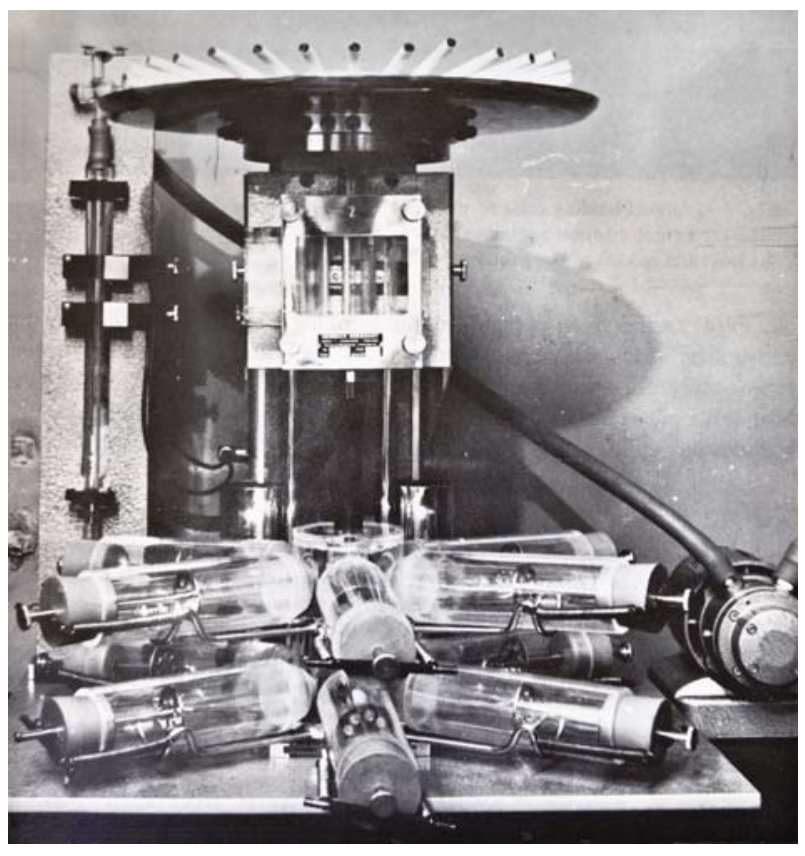

Figure 33. In the "open" smoking and exposure system type "Hamburg" (314) admixture of fresh air minimized CO intoxication of animals.

deposition; the "open" modified (311) Harris-Negroni system was impaired by qualitatively and quantitatively inconsistent long-term smoke production, long smoke retention periods with the likelihood of aging and the probability of smoke deposition. This led DONTENWILL et al. (314) to design a new "open" smoke generation and exposure apparatus, the first in a system subsequently called "Hamburg". It consisted of the 30 port smoking machine BAT-RM 30/65 developed by SEEHOFER and MILLER (116), operating under standard conditions (one $35-\mathrm{mL}$ puff of $2 \mathrm{sec}$ duration per min) with constant puff volume and frequency; a dual-chamber unit for smoke collection and mixing with air (1:4 to $1: 8$ dilution was possible for the adequate control of $\mathrm{CO}$ levels); and a lowvolume exposure chamber positioned directly underneath with six tightly attached tubes, which were replaceable for holding different species of laboratory animals (Figure 33). The instrument was engineered in cooperation with the Burkhardt Company (Hamburg, Germany), and subsequently manufactured - for use with ten animals - by the H. Borgwaldt Company (Hamburg, Germany) and marketed as Inhalation Apparatus Hamburg II (315). The novel device had the following advantages: The breathing air offered to the animals contained high concentrations of smoke while the risk of carbon monoxide intoxication was manageable. The smoke:air ratio was stable and reproducible. Due to the short distance between smoke source and the animal heads the time available for aging of the smoke was very short, and artifact formation quite unlikely.

A study of RÜCKER and DONTENWILL (316) compared the two actual systems - vacuum-controlled single smoke chamber with animals in separate tubes (309) and the BAT-RM 30/65 machine with the dual-chamber smoke collection and mixing unit, i.e., type "Hamburg" (314) with regard to breathing behavior of rats and hamsters, specifically respiratory volume and frequency. It turned out that carbon monoxide burden had major effects; smoke inhalation was considerably reduced if carbon monoxide intoxication exceeded a certain level. Smoke supply at fixed intervals (309) involved a higher risk of reflex apnea. The study demonstrated that relatively small carbon monoxide intake, allowing sufficient respiratory frequency and adequate 1-min respiratory volume, was a condition for effectual smoke exposure in inhalation studies while avoiding undue danger to the experimental animals.

The toxicity of cigarette smoke was assessed by RECKZEH et al. (317) in an inhalation study using the open smoking system "Hamburg I" (314). Syrian golden hamsters, Wistar $\mathrm{AF} / \mathrm{Han}$ rats and two strains of mice $(\mathrm{BALB} / \mathrm{c}$ and ICI) were exposed for distinct periods of time to smoke generated from experimental cigarettes and commercial cigarillos with different smoke levels of carbon monoxide and nicotine. Endpoint for acute toxicity was survival time until spontaneous respiratory arrest; chronic toxicity was measured by recording food consumption, body weight development and life span. Acute and chronic toxicity data allowed the development of recommendations regarding the selection of tests pieces and conditions of exposure for conducting meaningful inhalation studies.

In a paper presented in 1969, DONTENWILL (318) reviewed the different smoke generation/inhalation systems he had developed since the early 1960s and summarized in considerable detail the physiological, histological and toxicological effects he had observed in whole smoke inhalation studies with hamsters, mice and rats.

Using cigarettes, which were labeled by dispersing a solution of ${ }^{14} \mathrm{C}$-dotriacontane-16,17 in cyclohexane uniformly

Table 4. The types of smoke inhalation systems used by DONTENWILL.

\begin{tabular}{l|l|c|c|c|c}
\hline \multirow{2}{*}{ Year } & \multirow{2}{*}{ Type } & \multicolumn{4}{|c}{ References for } \\
\cline { 3 - 6 } & & Description of system & Inhalation physiology & Acute / chronic toxicity & Histopathology \\
\hline 1962 & $\begin{array}{l}\text { "Essenberg” } \\
\Rightarrow \text { large vacuum chamber }\end{array}$ & $(308)$ & - & - & $(308)$ \\
\hline 1964 & $\begin{array}{l}\text { "Dontenwill” } \\
\Rightarrow \text { closed smoking system }\end{array}$ & $(309,311)$ & $(311,316)$ & - & $(309,310)$ \\
\hline 1966 & $\begin{array}{l}\text { "Harris" } \\
\Rightarrow \text { open smoking system }\end{array}$ & $(311)$ & $(311)$ & - & - \\
\hline 1967 & $\begin{array}{l}\text { "Hamburg” } \\
\Rightarrow \text { open smoking system }\end{array}$ & $(314,315)$ & $\begin{array}{l}(316,318) \\
(320,325)\end{array}$ & $(317)$ & $(318,326)$ \\
\hline
\end{tabular}

a The particular versions "Hamburg", "Hamburg I" and "Hamburg II" all made use of the same principles of smoke generation, dilution and exposure. 
along the axis of an intact test piece (319), REZNIK et al. (320) examined the distribution pattern of radioactivity in TPM (mainstream and sidestream smoke, precipitate on route, butts and ash). The methodology used was described by REZNIK and MOHR (321). The presence of dotriacontane $\left(\mathrm{C}_{32} \mathrm{H}_{66}\right)$ in tobacco and tobacco smoke had been demonstrated by CARRUTHERS and JOHNSTONE (322) and by CARUGNO (323). Most importantly, the alkane had been shown to be transferred into cigarette smoke with minimal pyrolysis and be found exclusively in the particulate phase (324). Two different smoking/inhalation systems were compared. One assembly consisted of a BATRM 20/68 smoking machine (115), which was modified to smoke 4 cigarettes only and combined with a glass chamber of 1,000 mL volume, in which a Syrian golden hamster was exposed to the smoke individually and wholebody. The other system, described by DONTENWILL (314), made use of the BAT-RM 30/65 machine (type Hamburg II) and an arrangement of smoke receptacles and tubes that allowed the simultaneous exposure of ten hamsters noseonly. The authors concluded, by inference, that the total uptake of radioactively labeled ${ }^{14} \mathrm{C}$-dotriacontane- 16,17 by the animals was clearly higher with the BAT-RM 20/68 smoking machine and individual exposure than with the BAT-RM 30/65 smoking machine and simultaneous exposure of several animals. The series of studies was completed by mapping the distribution of smoke particles inside the hamster respiratory tract (325).

In a very large chronic inhalation study, using the apparatus Hamburg II $(314,315)$, DONTENWILL et al. (326) exposed 4,400 male and female Syrian Golden hamsters in all $(3,610$ for their entire lifetime) to mainstream smoke from 8 cigarette prototypes and to combinations of smoke and carcinogens. The cigarettes contained the same German standard blend (with one exception consisting largely of air-cured dark tobaccos); additionally, reconstituted tobacco sheet (made from the German standard blend), nitrate or the combination of both were included in three prototypes. Three prototypes had a filter: crimped and unsized paper, cellulose acetate or charcoal. Upon evaluation of the changes induced by smoke exposure striking differences were found between experimental groups. Histological alterations were most pronounced in the larynx and depended on duration of treatment and dosage.

The original smoking and exposure system type Hamburg (with a capacity of ten rodents arranged in two tiers) was considerably enlarged by BATTELLE PACIFIC NORTHWEST (327) to 6 tiers, increasing the number of animals per machine to 30 (Figure 34). This configuration was used in a chronic inhalation study with hamsters examining the effects of asbestos, cigarette smoke and a combination of both; carcinogenic effects were observed with none of the materials tested.

Starting in 1966, researchers at the Tobacco Research Council Laboratories in Harrogate (UK) developed an "animal smoking apparatus", which is occasionally referred to as "Harrogate smoker" and was essentially built from "perspex" (the transparent thermoplastic poly(methyl methacrylate)) and brass (Figure 35). Details of construction and operation were described by DAVIS et al. (324, 328). The device consisted of a central cylindrical smoke

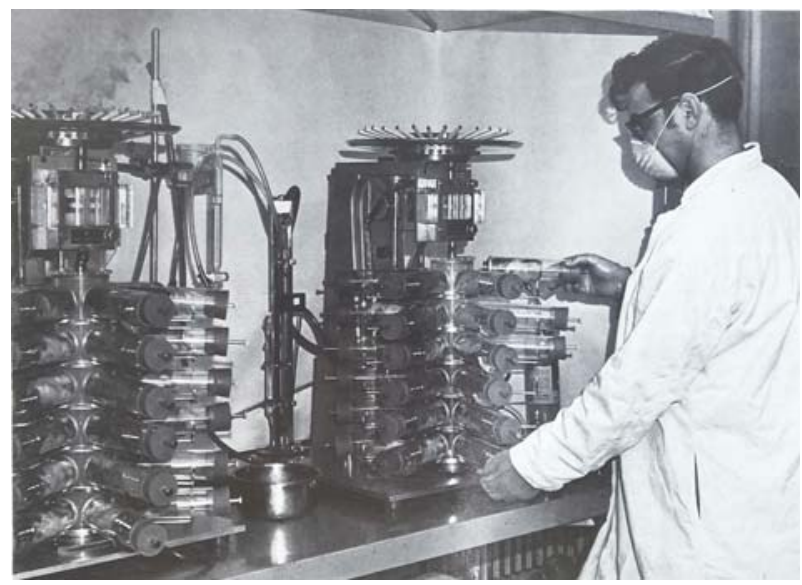

Figure 34. The smoking and exposure system type "Hamburg" was expanded by BATELLE NORTHWEST (327) to accommodate 30 rodents in 6 tiers $(2$ machines shown in operation).

chamber of $100 \mathrm{~mL}$ capacity for smoke collection, dilution and holding - fitted with several controllable inlet and exit valves. On one side, the chamber was capped by a latex diaphragm, which could be drawn into a $25 \mathrm{~mL}$ dome shaped cavity at distinct intervals by time-controlled administration of hypobaric pressure. On the opposite side, the head of an experimental animal in a nose cone reached into the smoke chamber for typical "nose only" exposure while its body was held in a cylindrical tube of selectable diameter. Smoke puffs $(1 / \mathrm{min})$ of $25 \mathrm{~mL}$ were produced in $2 \mathrm{sec}$ by connecting the burning cigarette to the smoke chamber and retracting the diaphragm into the dome. Immediately, the smoke was mixed with the air $(100 \mathrm{~mL})$ present in the smoke chamber and then held in it for $15 \mathrm{sec}$; subsequently, the diaphragm was slacked and the chamber flushed with fresh air, completing the 1-min operating cycle. Manual action was required for cigarette loading, lighting and extinguishing as well as butt extraction. The system worked on the principle that in each unit the animal received smoke from its own individual cigarette for intermittent exposure. For major long-term studies, batteries with up to 24 units were assembled.

Cigarettes spiked with ${ }^{14} \mathrm{C}$-dotriacontane-16,17 (319) were used to determine the amount total particulate matter (TPM) inhaled and retained by rats exposed to mainstream smoke generated by the "Harrogate Smoker" (324). After 20 animals had each been exposed to mainstream smoke of one spiked cigarette the mean amounts of ${ }^{14} \mathrm{C}$-dotriacontane-16,17 deposited in the head, and in the larynx, trachea and lungs (as a whole) of each animal were found to be $2.2 \pm 1,4 \%$ and $7.0 \pm 3.1 \%$, respectively, of the total ${ }^{14} \mathrm{C}$-dotriacontane-16,17 content of mainstream TPM.

Cigarette mainstream smoke produced by the "Harrogate smoker" was used in the early 1970s in behavioral studies with rats. The animals were exposed to smoke either unfiltered or after passage through a Cambridge filter, a layer of activated carbon or a combination of both. Animal performance was assessed by BÄTTIG and HRUBES (329) by measuring its swimming endurance (with extra weight attached to their tails), and by DRISCOLL and BÄTTIG (330) by recording its avoidance behavior (escape in conditioned response to a light/sound signal). In both studies, there 

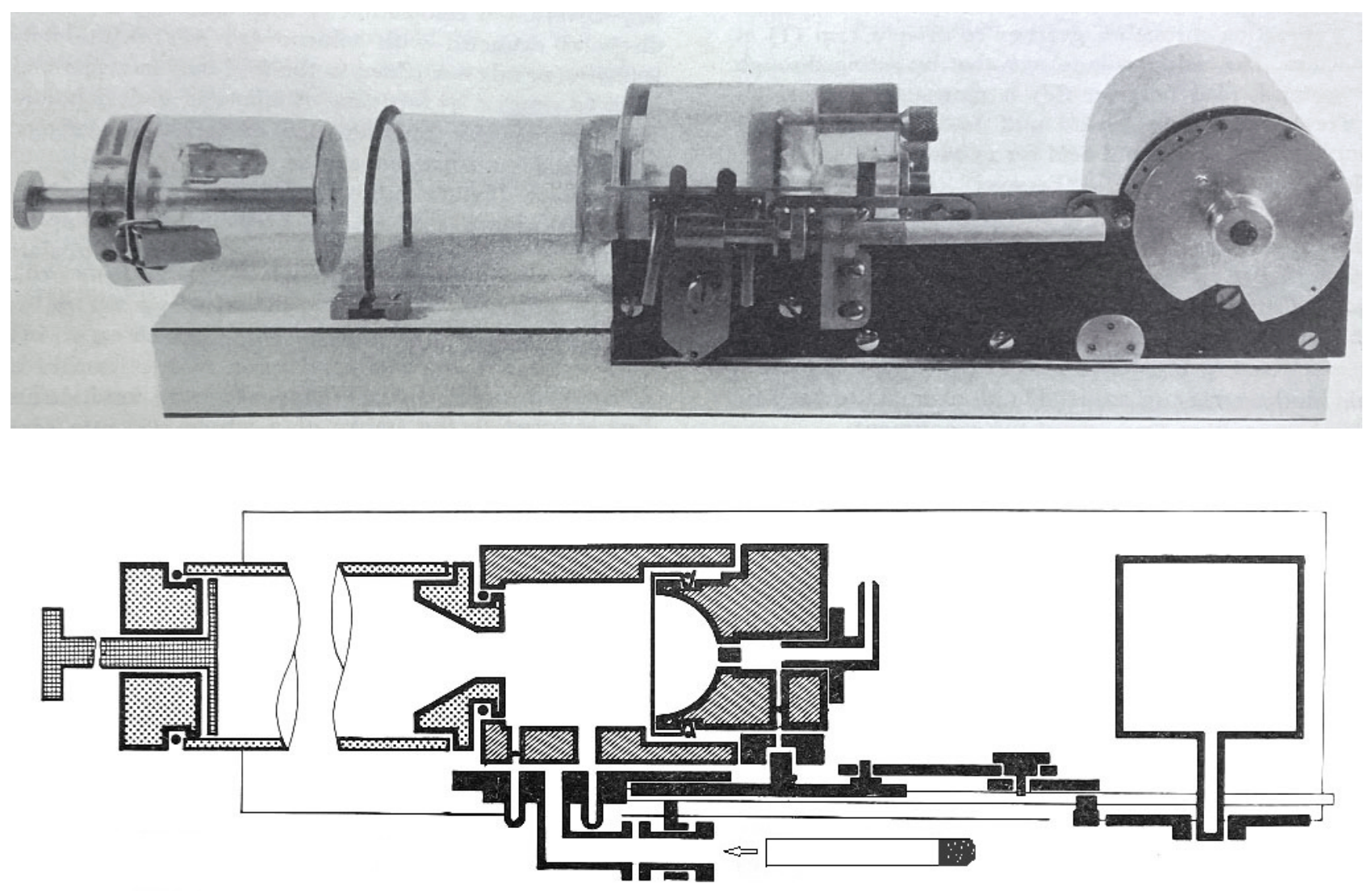

Figure 35. The "Harrogate smoker" (324) produced puffs by drawing a latex diaphragm into a $25 \mathrm{~mL}$ dome-shaped cavity at distinct intervals.

were occasional (and sometimes contradictory) tendencies of performance deterioration following exposure to the different kinds of smoke. The indistinct test outcome was obviously influenced by a multitude of - partially uncontrollable - factors, such as smoking machine technicalities, animal pre-training time and adaptation to smoke exposure, stress on animals as revealed by "illness" and weight loss, peculiar breathing patterns under different smoke filtration conditions, and the potentially antagonistic (performance enhancing or inhibiting) effects of specific smoke constituents like nicotine, carbon monoxide and hydrogen cyanide.

In the United States, starting from earlier patents assigned to WALTON (331) and MORRISSEY (332) the Walton Horizontal Smoking Machine (WHSM) was developed (and manufactured) by the Process and Instruments Corporation, Brooklyn (New York). The instrument described and characterized by STOKELY et al. (333) operated on the principle of reverse puffing: Smoke (from up to 3 cigarettes simultaneously) was fed through the holder directly into an animal exposure chamber and mixed rapidly by means of a fan. Puffs were produced generally under standard conditions (one 35-mL puff of $2 \mathrm{sec}$ duration per $\mathrm{min}$ ) - by placing a reciprocating puffing dome over each smoldering cigarette and forcing through a pre-calibrated amount of air, which was logically smaller than the chosen puff volume (usually $35 \mathrm{~mL}$ ) and not affected by draft resistance changes. Smoking was unrestricted. Smoke transit time into the chamber was less than $0.1 \mathrm{sec}$. Typically, the diluted smoke remained in the chamber for $30 \mathrm{sec}$ (maximum $45 \mathrm{sec}$ ) and was then flushed out by clean air, which remained until the 1-min cycle was completed. With a $35-\mathrm{mL}$ puff from one cigarette and using a $384 \mathrm{~mL}$ chamber smoke concentration was about $9 \%(v / v)$. Up to 10 rats or hamsters and up to 20 mice were placed horizontally in conical restraints, positioned in a vertically mounted wheel, which was enclosed in the cylindrical disc-shaped exposure chamber. All operations were automatic with the exception of cigarette lighting.

The Walton Horizontal Smoking Machine used "standing" rather than flowing smoke for exposure. The diluted smoke was examined regarding its composition and fate during the exposure period (generally $30 \mathrm{sec}$ ). STOKELY et al. (333) demonstrated that the yields of total particulate matter, water and "tar" were in close agreement for cigarettes smoked on the WHSM and on a standard analytical smoking device. This was also the case with smoke sampled from the exposure chamber. GUERIN et al. (334) characterized the organic composition of the particulate matter in the exposure chamber by profiling chromatographic peak distribution and sizes, and assessed potential changes at different time points in the presence and absence of animals in the chamber. The profiles of smoke generated under analytical smoking conditions were very similar to smoke sampled from the exposure chamber without animals $5 \mathrm{sec}$ or $25 \mathrm{sec}$ after puffing. With animals undergoing exposure, however, the pattern after $25 \mathrm{sec}$ was the same but peak sizes were clearly reduced, indicating a decrease in concentration. Identical observa- 
tions were made when the levels of vapor phase organic components were examined by chromatographic profiling. Not surprisingly, there was a marked increase of carbon dioxide in samples taken from the chamber $25 \mathrm{sec}$ after puffing with animals present.

The physical characteristics of cigarette mainstream smoke aerosol generated by the Walton smoking machine (333) from plain 2R1 Kentucky reference cigarettes were determined in 1990 by CHEN et al. (335). Puffing regime was a $35-\mathrm{mL}$ puff in $2 \mathrm{sec}$ once per min. Puff volume and amount of TPM were consistent except TPM in the first puff, when both puffing and lighting were performed simultaneously; per puff, average volume was $35.6 \mathrm{~mL}$ and average amount of TPM was $3.37 \mathrm{mg}$. Particle size distribution was strongly dependent on aging time. Mass median aerodynamic diameter at a dilution of $1: 21.7$ was calculated to be $0.45 \mu \mathrm{m}$. It seemed, however, that there was a dilution threshold impacting on rapid vaporization and final particle size, beyond which further dilution had little effect. Particle density, number concentration and coagulation coefficient of the cigarette smoke aerosol were estimated.

A smoke inhalation/exposure device for rodents was designed and developed in-house at the Oak Ridge National Laboratory (ORNL - Oak Ridge, TN, USA) and described in 1978 by MADDOX et al. (336). Operating principle was intermittent exposure (35-mL puffs of $2 \mathrm{sec}$ duration generated by a piston pump). Smoking was accomplished by inserting a cigarette into the holder section of a three-way smoke admittance valve, which was integrated in the wall of the sealable cylindrical exposure chamber. Upon evacuation of the chamber and under appropriate control by a set of valves, meters and timers puffs were taken, complying with standard conditions $(35 \mathrm{~mL}$ in $2 \mathrm{sec}$ once every $\mathrm{min}$ ), and immediately mixed in the $350 \mathrm{~mL}$ chamber with fresh air. Animals were exposed to the diluted smoke for $30 \mathrm{sec}$; smoke concentrations could be adjusted to 5-20\% $(v / v)$ though $10 \%$ was typical for chronic studies. Next, the chamber was flushed with fresh air and animals were exposed to it for the remainder of the 1-min cycle. It was possible to set up two smoking/exposure units simultaneously (dual channel mode) with one pump when puffs were taken in turn. The animals were kept in specially designed containers for nose-only inhalation, which were attached to the five exposure ports on each flat side plate of the cylinder. Up to 20 hamsters or rats or up to 40 mice could be treated in one smoking run when the dual channel system was used.

Yields produced by the smoke generator were found to be very comparable to those with a standard analytical machine except for nicotine, which was slightly higher due to free smoking. TPM deposition in the lung of hamsters was assessed by puffing cigarettes spiked with ${ }^{14} \mathrm{C}$-dotriacontane-16,17 and subsequently subjecting digested lung tissue to ${ }^{14} \mathrm{C}$ liquid scintillation spectrometry as described by DAVIS et al. (324).

Concerted efforts of the Oak Ridge National Laboratory (TN, USA) and the Process and Instruments Corporation of Brooklyn (NY, USA) led to the development of a largescale automatic smoking machine for the long-term "noseonly" exposure of animals to whole cigarette mainstream smoke, which was designated "SEM II" and described by
MONEYHUN et al. (337). The system used a rotary smoking head, holding 30 cigarettes, with automatic loading, lighting, puffing, smoke distribution and butt ejection/ extinguishment. The entire mechanism was enclosed under a sealed dome, which could be pressurized - creating the conditions for reverse puffing and free smoking. No vacuum generating device was in the smoke line. The machine could be run in single cycle or recycle mode and supplied a continuous stream of smoke, delivered to the animals in less than $2 \mathrm{sec}$. This way, the risk of smoke aging and depletion of aerosol by breathing was minimized. By adding air at the butt end of the cigarettes, smoke concentrations as low as $5 \%$ could be produced. Operating a single machine, up to 120 mice - as many as 480 in a later version (338) - could simultaneously be exposed for the major part of their lifetime nose-only via nasal orifice - with their bodies sealed from the smoke path.

While conformance to standard smoking conditions was certainly aimed at, operating this heavy-duty machine required some compromise: puff volume was influenced by changing pressure drop as cigarettes burned down; the preset puff number resulted in non-uniform butt lengths and - in addition - had to be reduced to one less to guarantee reliable butt extraction; puff duration, however, was under full control. Heat development inside the dome was another problem to be mastered.

The large-scale machine SEM II (337) and the Walton Horizontal Smoking Machine (333) for smaller numbers of animals were examined and compared by HENRY et al. (339) with regard to the deposition and internal distribution of TPM in mice. After exposure to smoke from cigarettes spiked with ${ }^{14} \mathrm{C}$-dotriacontane-16,17 the animals were sacrificed immediately and tissues of interest removed, solubilized and counted. The effects of smoke concentration and exposure time on TPM deposition and tissue distribution were found to be remarkably similar with both machines. Under typical conditions, deposition of TPM in the lungs amounted to 134 and $123 \mu \mathrm{g}$, and percentages of total body distribution were - in the lungs $70 \%$ and $80 \%$ and - in the entire respiratory tract $-88 \%$ and $86 \%$, with the SEM II and the Walton machines, respectively.

Using the SEM II (337) smoking/inhalation system, HENRY et al. (338) conducted another - principally similar but more expanded - study on the deposition of TPM from ${ }^{14} \mathrm{C}$-dotriacontane-16,17 labeled cigarettes. Complying with standard smoking conditions, 30-200 $\mu \mathrm{g}$ TPM were deposited in the lungs of mice after $50-540 \mathrm{sec}$ (cumulated) of intermittent smoke exposure. $80-90 \%$ of TPM deposits were found in the respiratory tract. This outcome remained the same over a 6-month study period.

In an exceptionally instructive and generously illustrated paper, HENRY et al. (340) discussed the facilities and equipment developed for standardized large-scale noseonly inhalation studies with mice. The SEM II smoking machine was used for smoke generation and combined with a sophisticated animal containment system. Procedures and the experimental potential of the system were commented on in considerable detail.

SEM II smoking machines were used by HENRY and KOURI (341) in a large scale chronic inhalation study to 
examine the effects of long term exposure to the plain Kentucky 2R1 reference cigarette smoke on mice. Two machines generated smoke for treating a starting cohort of over 2,000 mice for 110 weeks while one machine was needed for the over 1,000 sham controls. Only few alveolar adenocarcinomas were observed, and the difference between smoke exposed and sham treated animals was not significant. The loss rate of animals during the study due to "exposure-equipment-related problems" was quite remarkable.

In 1974, a Tobacco Smoke Inhalation Workshop on Experimental Methods in Smoking and Health Research was held in Bethesda (MD, USA), with 52 experts attending (342). In response to a request issued prior to the meeting participants had forwarded information concerning the types of inhalation instrumentation used in their laboratories. GUERIN et al. (343) reviewed the information, which showed that "a large variety of devices ranging from the ultrasimplified to the ultrasophisticated" was in use. In view of the complex situation, GUERIN et al. compiled the requirements for machines used for tobacco smoke inhalation bioassays, which had been suggested over the years by scientists, and contributed their own concise list of essentials. The relevant criteria may be summarized as follows:

- Cigarettes should be smoked according to FTC standard smoking parameters (one puff of $2 \mathrm{sec}$ duration with a volume of $35 \mathrm{~mL}$ once a $\mathrm{min}$ ).

- Free rather than restricted smoking should be used.

- Cigarette smoke offered to animals should be physicochemically comparable to smoke available to smokers, i.e., the smoking/exposure system should produce smoke for inhalation that does not differ considerably from what man inhales.

- Smoke for inhalation must not be contaminated by sidestream smoke.

- The smoke should be delivered to the test animals with minimum delay in order to prevent chemical or physical changes due to aging.

- A reliable means for providing a known amount of air dilution should be provided.

- Convenient methods for monitoring machine performance with respect to smoking parameters and for collecting smoke samples for analysis should be incorporated into the design.

- The design should warrant the capability of exposing large numbers of animals simultaneously.

Heeding the criteria compiled by GUERIN et al. (343) with their focus on standard smoking conditions, prevention of physical and chemical changes of smoke due to aging, reliable smoke dilution, convenient machine performance monitoring and sampling procedures, and the sufficient number of animals - SCHULTZ and WAGNER (344) of the Lorillard Research Center (Greensboro, NC, USA) developed a new smoking machine for use in animal inhalation studies (components for animal exposure were not specifically addressed). Reverse puffing was chosen for the generation of smoke because it could be routed directly to the animals with a minimum of manipulation and lost time. Cigarettes were placed in two vertically positioned carousels, holding 15 cigarettes each, with puffing executed in turns by means of a small puff chamber moving over the cigarette and air pressure applied after sealing. The machine was capable of automatic cigarette loading, lighting and butt ejection after a preset number of puffs. Smoke could be routed to any or all of four animal exposure chambers. Digital logic circuits operated the switches and air valves controlling the various smoking and smoke dilution steps.

While puff duration ( $2 \mathrm{sec})$ and frequency $(1 / \mathrm{min})$ could reliably be preset, the puff volume needed to be calibrated - by a rather demanding procedure - to an average of $35 \mathrm{~mL}$ over the whole smoking cycle, based on output smoke rather than input air. It should be noted that the pressure drop of cigarettes changed during smoking, in consequence of which a constant target volume of $35 \mathrm{~mL}$ within $2 \mathrm{sec}$ for each puff was - as a matter of principle not possible. In any case, it was necessary to use cigarettes of relatively uniform pressure drop in order to maintain the calibrated puff volume. Pre-selection of the test cigarettes by weight and pressure drop was recommended. By proper dilution smoke concentrations could be made available in the range of 5-20\%.

SCHULTZ and WAGNER (344) made substantial efforts to compare the new machine to various established analytical smoking machines with regard to the chemical equivalence of both the particulate and gaseous phases of smoke produced from reference cigarettes. Within the expected analytical variations, no differences were observed.

In 1976, BEVEN (345) of the British American Tobacco Co. in Southampton (UK) defined a number of basic requirements for a smoking/exposure system to be used in inhalation toxicity studies with small animals (mice, rats, guinea pigs and hamsters): Smoking of cigarettes under standard conditions of puff volume, frequency, duration and profile; "free smoking" with the butt end open between the puffs; rapid dilution of the smoke aerosol to limit particle growth; low velocity passage of the aerosol through wide-bore, unrestricted tubing; continuous or intermittent exposure head-only or snout-only. For smoke generation, BEVEN chose the apparatus manufactured by R.G. Mason (Clevedon, Avon, UK) and used by BAT in the United Kingdom. Its 24-port rotating smoking head was positioned vertically (increasing the probability of sidestream smoke contaminating the mainstream smoke). The machine was connected with a smoke collection chamber $(74 \mathrm{~mL})$, which incorporated the dilution device, and the $750 \mathrm{~mL}$ exposure chamber around which animal restraining tubes were arranged (up to 20 in future studies). The system included three sampling points along the smoke path for TPM collection on Cambridge filters. For the different cigarette types smoked, chamber concentrations of TPM were very consistent over 6 weeks, indicating reproducible machine performance and exposure conditions. TPM losses did not exceed $10-15 \%$ across the complete exposure system.

Using the new smoking/inhalation system (345) dosimetry studies were done by BINNS et al. $(346,347)$ on mice, rats, guinea pigs and hamsters. To examine the "dosing" of animals treated with smoke aerosol, TPM deposition was assessed in the nasal region and the lower respiratory tract. For biochemical research in rodents, the BAT smoking/ inhalation system (345) was used by BILLIMORIA and ECOBICHON (348-350) of McGill University (Montreal, 


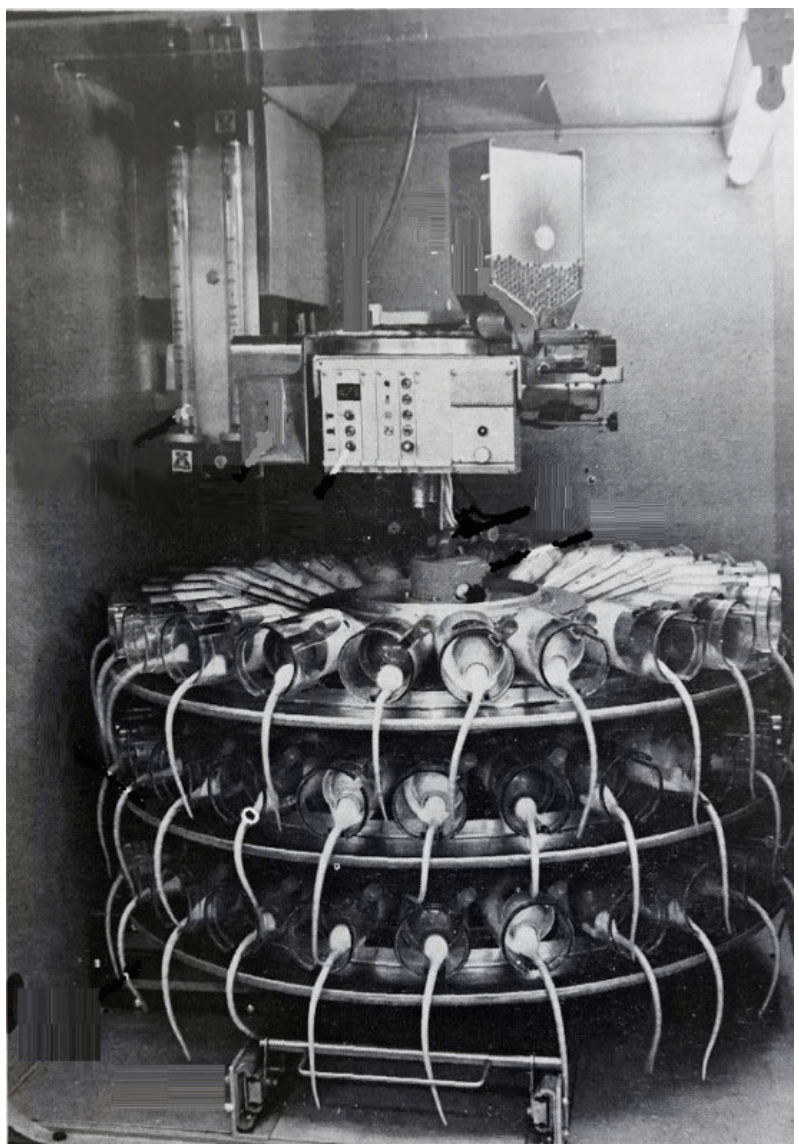

Figure 36. Up to 72 animals are simultaneously exposed to freshly diluted smoke in the smoking/inhalation machine Mark III (A) developed by BAUMGARTNER and Coggins (352).

Canada). They studied the induction of aryl hydrocarbon hydroxylase by mainstream smoke in liver, lung and kidney $(348,349)$ as well as the effects of mainstream and sidestream smoke on the water soluble cytoplasmic antioxidants, ascorbic acid and reduced glutathione (350). In the 1970s the UK-based tobacco company Gallaher examined the potential health benefits of Cytrel $^{\circledR}$ (a cellulose-based product) developed by CELANESE (351) as a tobacco substitute; the material showed considerable promise due to its low density and the virtual absence of nitrogen compounds. Gallaher considered it essential that the biological examination of (experimental) test pieces would not only rely on the well established approach of mouse skin painting but also include meaningful animal inhalation studies. To this end, Gallaher requested the Battelle Centre for Toxicology and Biosciences in Geneva (Switzerland) to design and build appropriate equipment to evaluate the carcinogenic potential of mainstream smoke generated from cigarettes containing Cytrel $^{\circledR}$ in the tobacco blend.

In 1980, the new smoking/inhalation system was described by BAUMGARTNER and COGGINS (352). The apparatus allowed the simultaneous exposure of a large number (up to 72) of small animals to continuously flowing, freshly diluted cigarette mainstream smoke. A horizontal rotary smoking head was loaded with 30 cigarettes, held in rubber labyrinth seals (Figure 36). Cigarette insertion and lighting with a gas burner as well as the ejection of butts at preset lengths were carried out automatically. Smoking was done under standard conditions $(35-\mathrm{mL}$ puff in $2 \mathrm{sec}$ once every $\mathrm{min}$ ), using a lubrication free 4-piston barrel pump. Between puffs cigarettes were allowed to smolder freely. Puffs were immediately mixed with a continuous air flow at the desired rate (for concentrations of $0.5-20 \%$ $(v / v)$; optimal working range 5-20\%) and distributed to the exposure system underneath. Animals were kept in three superposed trays, each containing 24 specially developed holders arranged in horizontal, radial position. The holders were designed in such a way that only nose and mouth of the animals were exposed to smoke but not the body itself. The continuous supply of fresh, diluted smoke through an inlet tube and its extraction through an exit tube - kind of a "flow-past" design - kept the animals from breathing stale (expired or aged) smoke. When reaching the animal's nose, the age of the diluted smoke was estimated to be about $1.5 \mathrm{sec}$. This proved a very short time lapse in handling and transferring smoke to the dilution and exposure systems. By means of a valve arrangement, smoke in the exposure system could be flushed out with $100 \%$ fresh air.

BAUMGARTNER and COGGINS considered their smoking/ inhalation machine to meet the requirements compiled at the 1974 Tobacco Smoke Inhalation Workshop (342). The machine was manufactured and marketed by Aeromechanique AMESA, Châtelaine, Geneva (Switzerland) and called - in later publications - Battelle Mk III $(353,354)$ or AMESA Mark III (A) $(355,356) .^{5}$

The elaborate chemical evaluation of the smoke in undiluted and diluted form, generated in this system (352), was reported by BRIGGS et al. (357). Different model cigarettes were used including test pieces containing the tobacco substitutes Cytrel $^{\circledR}$ or NSM (358); the latter was the subject of a major 1970s joint research project between Imperial Chemical Industries and Imperial Tobacco. Blends consisted of $100 \%$ tobacco, $\mathrm{Cytrel}^{\circledR}$ or NSM as well as 50:50 mixtures of tobacco with either tobacco substitute. Yields of major smoke constituents (NFDPM, nicotine, carbon monoxide and a few other analytes) were determined. BRIGGS et al. selected three sampling points of the system (at the smoke pump, at the dilution chamber and variably at the animal ports) for smoke analysis and compared the data to those obtained with two standard analytical smoking machines. Smoke yields (NFDPM and nicotine) immediately behind the smoke pump were lower (roughly 80-90\%), largely due to differences in puff number and deposition inside the pump (not possible in an analytical machine with pump positioned behind the Cambridge filter). Yields in diluted smoke were found to be further (slightly) reduced.

The new system (352) was the equipment used in a 12 week inhalation study conducted by CoGGINS et al. (354) to compare the pathological effects induced in the respiratory tract of rats by whole cigarette smoke and its particulate and vapor phases. Both types of tested cigarettes contained a flue-cured tobacco blend typical of the

5 Prof. Rudolph Jaeger (President and Chief Scientist at CH Technologies (USA) Inc.) and Henri Baumgartner kindly provided a lot of valuable information regarding the AMESA smoking machines. 
UK market. From the one cigarette with a dual filter of creped cellulose and cellulose acetate whole smoke was produced as well as vapor phase after passage through an inserted Cambridge filter. The other cigarette also had a dual filter comprising carbon granules bonded on polyethylene and cellulose acetate; its smoke was considered to represent "particulate matter" following the absorption of most volatile and some semi-volatile smoke constituents by the carbon - a practical approximation to filter-collected TPM. The most evident effect of exposure was marked squamous metaplasia of the laryngeal epithelium at the base of the epiglottis while no neoplastic changes were observed. Overall, it was concluded that most of the changes produced could be attributed to exposure to "particulate matter" - with some evidence of synergistic effects of the particulate and vapor phases.

The BAUMGARTNER-COGGINS smoking machine (352) was subsequently used in two biological studies assessing the toxicological potential of cigarettes containing different amounts of the cellulose-based tobacco substitute Cytrel $^{\circledR}$. In a full tumorigenicity experiment with mice CogGins et al. (359) used cigarette smoke condensate, trapped in acetone, for skin painting for a period of 104 weeks. A chronic 18-month inhalation study was performed by CoGGINS et al. (353) with rats. In both studies, the reduction of toxicological effects was approximately proportional to the levels of included tobacco substitute.

To improve the endurance and minimize the maintenance of the AMESA Mark III smoking machine (352) CHEN et al. (355) modified certain components (lighting unit, dumping mechanism and smoke exhaust system) and turned to using a peristaltic pump for intensified puffing. In an ambitious study cigarette smoke exposure atmospheres were compared in different puffing and exposure modes. Three smoking regimes were considered: the standard 35-mL puff in $2 \mathrm{sec}$ once per min; a puff of double the standard volume (i.e., $70 \mathrm{~mL}$ ) once per min; and a double puff twice per min. The three inhalation exposure modes examined were: intermittent nose only simulating traditional periodic exposure; continuous nose only with constant smoke concentration maintained for several hours; and continuous whole-body. Data were collected for mass median aerodynamic diameter and particle size distribution, gas phase concentrations and particle chemical composition. They suggested that there was no substantial difference in physical characterization and chemical composition of the smoke atmosphere among the three puffing regimes and the three exposure modes.

In 1990, AYRES et al. (360) summarized several modifications of the first prototype of the smoking/inhalation machine of BAUMGARTNER and COGgINS (352). These included a simplified peristaltic pump for drawing puffs from the cigarettes, replacement of the gas burner by an electric filament for cigarette lighting, computer use for the control and display of exposure conditions, improved cigarette holders, and - under special circumstances - the addition of a second puffing port to allow pre-ignition of cigarettes. The last customization in particular should be seen in context with the research activities of R.J. REYNOLDS TOBACCO CO. (361) on a novel cigarette type, which heats rather than burns tobacco and, consequently, does not burn down to a butt. These cigarettes contain a piece of carbon as heat source, requiring warming of the cigarette tip at the additional port before lighting.

Using the (original) AMESA smoking/exposure machine Mark III A (352), CHEN et al. (356) observed a number of technical problems, particularly the deposition of smoke particles inside the barrel pump, the cigarette ejection sensor and assembly module, and the control box; this required intensive daily maintenance and caused frequent pump failures. In addition, the invariable standard smoking conditions (35-mL puff in $2 \mathrm{sec}$ once every $\mathrm{min}$ ) were felt to be restrictive. Therefore, a new smoke generator was designed by AMESA Technologies (designated AMESA Type 1300 generator). Essentially, the barrel pump was replaced by a peristaltic smoke pump, and the turntable was fitted with three additional smoking ports. Peristaltic pumping produced a more uniform puff profile and reduced particle loss slightly, increasing mass concentration output by $10-20 \%$. In addition, puff volume could be set at $35 \mathrm{~mL}$ or $70 \mathrm{~mL}$. When using two or three smoking ports (each connected to a separate pump) puff frequency could be stepped up to 2 or 3 puffs per min. This way, it became possible either to supply more than one exposure chamber with smoke from one generator or to feed larger amounts of smoke into a single chamber.

CHEN et al. (356) also compared the two AMESA machines with regard to mainstream smoke composition and particle size distribution. The Mark III A was used under standard smoking conditions (one 35-mL puff of $2 \mathrm{sec}$ duration every min), while the Type 1300 was operated under standard conditions, with one $70-\mathrm{mL}$ puff in $2 \mathrm{sec}$ per min or with two $70-\mathrm{mL}$ puffs in $2 \mathrm{sec}$ per min. Upon examination of a range of analytes in both mainstream smoke particulate and gas phase no significant qualitative differences were observed between the four modes of smoke generation, indicating that pump type and puff volume and frequency had no effects on smoke composition.

Particle size distribution was measured using a cascade impactor and a parallel-flow battery. The mass median diameter of smoke particles generated by $35-\mathrm{mL}$ puffs was $0.45 \mu \mathrm{m}$, which was slightly larger than the $0.37 \mu \mathrm{m}$ diameter obtained with $70-\mathrm{mL}$ puffs. This could be explained by the higher flow rate and shorter residence time in the system of particles from the $70-\mathrm{mL}$ puffs, providing less opportunity for particle growth by coagulation. Based on earlier experience, the authors expected no differences in total deposition efficiencies in the respiratory tract of test animals and pertinent size related health effects.

After the AMESA companies had ceased operations in 1993 their technology was transferred to CH TECHNOLOGIES (362) in Westwood (NJ, USA). This company redesigned the original Mark III A (352) and AMESA 1300 (356) machines and made a series of automated rotating 30-port smoking machines available to the research community for analytical and animal inhalation studies. These machines were used by a number of medical, engineering and industrial laboratories, mainly in North America and Japan. (Figure 37)

As a component of the clean room configuration of a 


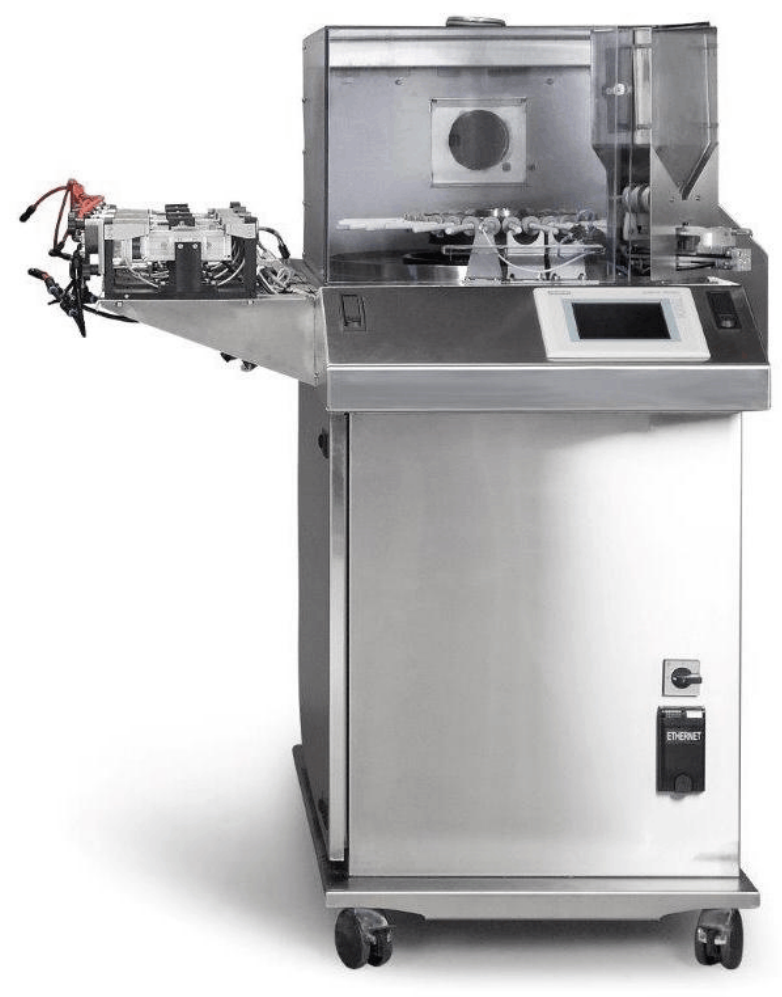

Figure 37. Highly versatile cigarette and e-cigarette smoking machine, developed by CH TECHNOLOGIES to support a wide range of in vivo and in vitro exposure studies (362).

smoke inhalation laboratory for small animals, REININGHAUS and HACKENBERG (363) described a smoking machine for continuous smoke production performing on the principle of reverse smoking (i.e., positive pressure smoking). Its features included automatic cigarette loading and lighting as well as butt ejection. Thirty cigarettes were inserted in a rotary smoking head, located in a closed and permanently hyperbaric chamber (excess pressure typically $100 \mathrm{~mm}$ water column). The chamber was purged by a gentle flow of air to remove sidestream smoke and excess heat - exhausted through a flap valve, which in addition regulated chamber pressure to ensure the desired puff volume. When during smoking head rotation at $1 \mathrm{rpm}$ a cigarette became aligned with an opening in the central tube, the butt end was exposed to ambient pressure for $2 \mathrm{sec}$ producing a puff of $35 \mathrm{~mL}$ smoke not affected by any pumping process - all in accordance with ISO 3308 (10). However, as pointed out by VANSCHEEUWIJCK et al. (364), there were minor deviations for technical reasons when using reverse smoking; these related to puff profile (rectangular instead of bell-shaped), free (open-end) smoking and air velocity around cigarettes. The continuous output of smoke was immediately diluted, as desired, with conditioned air flowing through the central tube and carried to the inhalation chamber. Its central part was a narrow chute-like chamber perfused from top to bottom with diluted smoke. Racks could be attached, which held up to 120 rodents in individual polycarbonate tubes, leaving only the heads of the animals exposed to diluted smoke. Due to the very short smoke path the age of diluted smoke when reaching the animals was only about $15 \mathrm{sec}$.
Using the 30-port reverse smoking machine described by REININGHAUS and HACKENBERG (363) cigarette sidestream smoke was collected by HAUSSMANN et al. (365) with the help of a circular hood made of glass and stainless steel placed on top of the apparatus; mainstream smoke was exhausted. A subchronic inhalation study with rats examined both fresh side stream smoke (three concentrations) and room-aged sidestream smoke (generated during 1.5 hours in a mock-up living room). Animals were exposed head-only in chambers of octagonic cross section consisting of glass, stainless steel and brass, which were equipped with made-to-size glass tubes that were conical at the front end to fix the rat's head protruding into the stream of smoke.

The mainstream smoke production system of REININGHAUS and HACKENBERG (363) was modified - and designated SM 85 (366) - by VANSCHEEUWIJCK et al. (364) for use in a subchronic inhalation study with male and female Sprague-Dawley rats to assess the potential toxic effects of (as many as 333) tobacco additives utilized in cigarette manufacturing. Cigarettes were lit at the puffing port with a halogen spot lamp and smoked by reverse puffing to a butt length of approximately $35 \mathrm{~mm}$ rather than to a preset number of puffs. The mainstream smoke generated continuously from test cigarettes was mixed with filtered conditioned air at a dilution rate of 1:150 to 1:170, depending on TPM content. At this point, $30-40 \%$ of particle mass were typically lost due to the redistribution of initially condensed volatiles into the gas/vapor phase of the diluted smoke. Animals were exposed nose-only in the exposure chambers described by HAUSSMANN et al. (365).

Smoking machines of the SM85 type were used by CARMINES et al. (367) and GAWORSKI et al. (368) for the production of mainstream smoke from 1R4F Kentucky reference cigarettes in inhalation studies, which examined effects on prenatal and postnatal development in rats.

Departing from the technique of reverse smoking the SM85 smoking machine was equipped with one or four piston pumps and an active sidestream exhaust, designated as SM85i and used in numerous inhalation studies.

Equipment of the SM85/SM85i type evolved into the "Condor" smoking machines, manufactured by KC Automation, which was to be completely merged with Borgwaldt in 2005. APPLETON et al. (369) reported on the validation of a new system for the generation of mainstream and sidestream smoke for rodent inhalation studies. The 30-port rotary machine featured automated cigarette

Reverse smoking

Instead of drawing puffs from the mouth end of a cigarette a defined volume of air is pressed within a fixed time through the lit end passing together with the generated smoke through the cigarette. Reverse smoking is used for smoking radioactively labeled cigarettes for collecting total mainstream and sidestream smoke, ash and butt. It is also used in some devices for animal inhalation studies. In this case benefit of reverse smoking is that no traps for smoke collection are needed, dilution with fresh air down to the desired concentration is immediately easily possible and time between smoke generation and animal exposure will be extremely short. 
loading, lighting, smoking, and butt ejection. An infrared puff-termination device allowed cigarettes to be smoked to a standard butt length rather than a fixed number of puffs. Machine performance was examined by smoking 1R4F Kentucky reference cigarettes under "ISO-like" conditions, and collecting mainstream particulates on a Cambridge filter and gas phase in a sampling bag. The values determined for TPM, nicotine and carbon monoxide were only slightly higher than those obtained with an "ISO harmonized" 20-port analytical smoking machine.

The functionality of the Condor smoking machine was evaluated by APPLETON et al. (370) following an inhalation study protocol, which distinctively interrupted the daily exposure time of 2 hours by a half hour rest period with fresh air to avoid carbon monoxide intoxication. Fischer 344 rats were exposed 5 days per week for a total of 6 weeks to TPM concentrations of 100, 300 and $600 \mathrm{mg} / \mathrm{m}^{3}$. The test atmosphere was produced from 1R4F Kentucky reference cigarettes and from a new additivefree light King size reference cigarette, which was developed by BROWN \& WILLIAMSON (371) specifically for biological studies. This cigarette contained a standard experimental blend of flue-cured Virginia, Burley, Oriental and reconstituted tobaccos; neither expanded tobacco nor stems were included. Per cigarette, ISO smoke yields were $7.55 \mathrm{mg}$ "tar" (NFDPM), $0.74 \mathrm{mg}$ nicotine and $10.2 \mathrm{mg}$ carbon monoxide. In the inhalation study, mean carboxyhemoglobin $(\mathrm{COHb})$ concentrations (with $600 \mathrm{mg}$ TPM per $\mathrm{m}^{3}$ ) were $42 \%$ and $49 \%$ in male and female rats, respectively. No mortalities were recorded. Dose dependent changes in body weight and histopathology were observed typical of those reported in the literature.

Mainstream smoke-induced biomarker response in two mouse strains was the endpoint in a subacute inhalation study performed by ОВОт et al. (372). Using Condor smoking machines, animals were exposed to smoke of 2R4F Kentucky reference cigarettes at TPM levels of 75, 250 and $600 \mathrm{mg} / \mathrm{m}^{3}$ for 2 hours/day on 7 consecutive days. Biomarkers of exposure in blood $(\mathrm{COHb}$, nicotine and cotinine) were recorded as were respiratory function and histopathology. To assess biomarkers of effect, which may be predictive for pulmonary emphysema, concentrations of several characteristic components were measured in bronchoalveolar lavage fluid: oxidized/reduced glutathione symptomatic of oxidative stress, lactate dehydrogenase and total protein showing potential lung injury, and - most importantly - a full range of cytokines as indicators of inflammatory or immunological events. It was concluded that the multiple effects that were recorded needed to be followed up on in chronic exposure studies.

In 1998-2007 CogGins brought out three papers (373-375) and a Letter to the Editor (376) to review representative chronic inhalation studies with mainstream cigarette smoke performed with five main species of laboratory animals; the endpoint of concern was neoplastic disease. Studies had to be published in the peer-reviewed literature and meet these requirements: overall duration consistent with the duration of carcinogenesis and/or detailed histopathological description. With regard to rodent studies done up to the year 2000 the author concluded that "significant increases in the numbers of malignant tumors of the respiratory tract were not seen in rats, mice, hamsters ..... exposed for long periods of time to very high concentrations of cigarette smoke" (375).

However, two studies - conducted around the turn of the century at the Lovelace Respiratory Research Institute in Albuquerque (NM, USA) and reviewed by HAHN et al. (377) - demonstrated increased incidence of lung and nasal tumors in both rats and mice after prolonged exposure to high concentrations of mainstream cigarette smoke. MAUDERLY et al. (378) exposed male and female F344 rats whole body for 6 hours/day and 5 days/week over near life span (up to 30 months) to 100 or $250 \mathrm{mg} \mathrm{TPM} / \mathrm{m}^{3}$ from the plain 1R3 Kentucky reference cigarettes. For female rats the higher concentration was obviously rather close to the maximum tolerated dose (MTD). Significantly increased incidences of non-neoplastic and neoplastic proliferative lung lesions were seen in females, while non-significant increases were observed in males. In another study, performed by HUTT et al. (379), female B6C3F1 mice were similarly exposed whole body for 6 hours/day and 5 days/week over near life span (918-930 days) to $250 \mathrm{mg}$ $\mathrm{TPM} / \mathrm{m}^{3}$ from the plain 2R1 Kentucky reference cigarettes. Marked increases were induced in the incidence of focal alveolar hyperplasias, pulmonary adenomas, papillomas and adenocarcinomas. In both studies, cigarette mainstream smoke for exposure was generated by a modified AMESA Type 1300 smoke generator as described by CHEN et al. (356).

Slightly widening the scope of this review article, reference is made to the (relatively inexpensive) system developed by TEAGUE et al. (380) for the small-scale production of aged and diluted cigarette sidestream smoke as a substitute for environmental tobacco smoke (ETS) in animal and in vitro exposure studies. The smoke generation part consisted of the following components (Figure 38): The automated cigarette handling and smoking device for loading, lighting and smoking one cigarette under FTC conditions, which used adjustable flow and was controlled by a mass flow meter and selenoids; the dilution chimney, which completely enclosed the burning end of the cigarette, collected smoke from the smoldering cigarette by means of a constant and moderate passing air stream and routed it together with additional diluting air - last - to the conditioning chamber for mixing with a fan and storage. Flow through the dilution chimney and the conditioning chamber was maintained by an exhaust pump; variations of flow rates produced the desired concentrations. For exposure, the smoke producing system could be combined with two chambers holding animals $\left(0.44 \mathrm{~m}^{3}\right.$ capacity each $)$ or a temperature-controlled incubator for cell cultures $(150 \mathrm{~L}$ capacity). The chambers could be connected separately or in parallel, and used with additional chamber-specific smoke dilution with air if required.

Using 1R4F Kentucky reference cigarettes a number of analytes were measured in the aged and diluted sidestream cigarette smoke to assess the performance of the system. Total particulate matter (TPM) and carbon monoxide were continuously monitored in the conditioning and exposure chambers as were temperature and relative humidity in the exposure chamber. Nicotine and several vapor phase constituents were sampled in the exposure chamber and quantified. In the exposure chamber, TPM levels of 


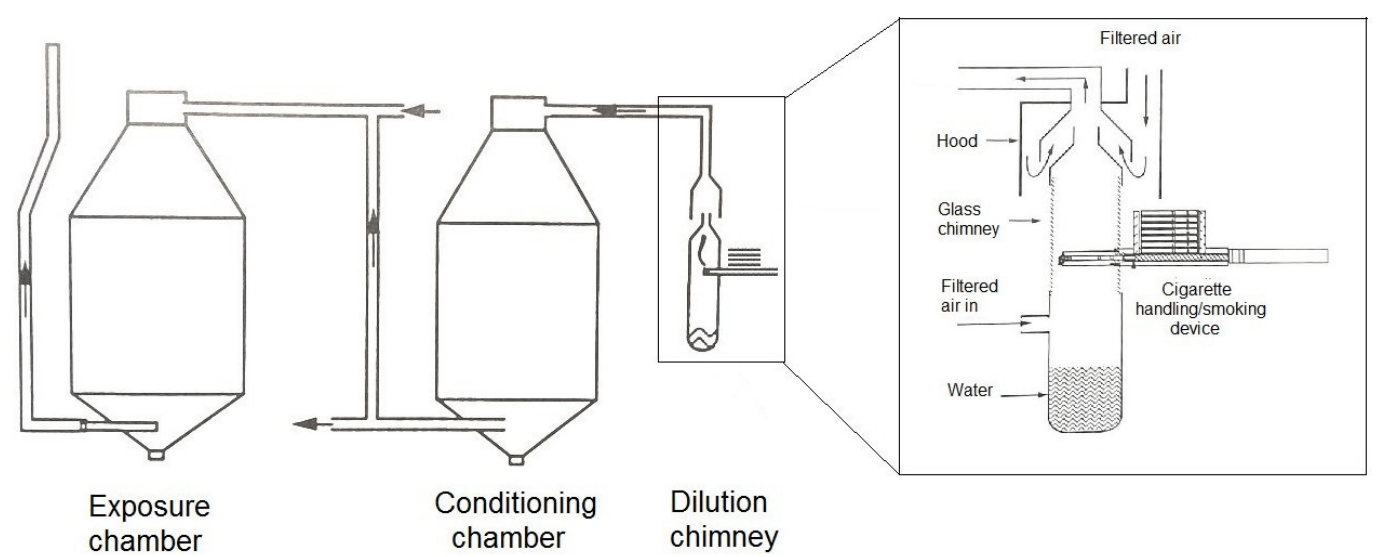

Figure 38. The dilution chimney with two controllable air inlets was the particular component of the sidestream smoke generation and exposure system developed by TEAGUE et al. (380).

$100-3,000 \mathrm{mg} / \mathrm{m}^{3}$ were maintained consistently over the exposure period of 6 hours. There was a clear linear correlation between TPM concentration and air flow volume through the conditioning chamber. When the levels of TPM, carbon monoxide, nicotine and sixteen selected vapor phase constituents, measured in the exposure chambers during a single exposure period, were compared to published ETS data it was concluded that the aged and diluted sidestream smoke, as produced by the system, was "environmentally relevant".

Consequently, this form of smoke was used as a surrogate for ETS in studies (discussed in chapter 6.2.1 and 6.2.2, starting page 203), which assessed cytotoxic and genotoxic potential under conditions of air-liquid interface (ALI) exposure (381-383). The interpretation of such study data, however, requires good awareness of the toxicological discrepancies between sidestream smoke and ETS, which are only gradually evened out by aging and dilution and affected by exhaled mainstream smoke as a (minor) component of ETS.

\subsection{Smoke generation and exposure in inhalation studies with non-rodent species}

Mice, rats, hamsters and rabbits are all obligatory nose breathers; inhaled material travels through the upper nasal passages before reaching the bronchial tree and the lung alveoli. This will inevitably result in trapping of smoke constituents and quite likely induce aging. In "passive" inhalation studies with rodents, only a portion of smoke gets as far as the bronchial system, including the lungs. In experimental practice, a change of rodent breathing patterns is nearly out of reach; a dubious trial to close the nostrils of hamsters surgically did not meet with success (309).

When smoking, humans practice "active" or "direct" inhalation - bypassing the nasal passages. This can be duplicated in the laboratory with larger non-rodent animals. Depending on the experimental setting the application of smoke is by forced insufflation, learned voluntary inhalation (particularly with monkeys) or tracheotomy.

The apparatus for direct inhalation in beagle dogs, described by BAIR et al. (384) of Battelle Northwest, Richland (WA, USA), consisted of a mask connected to a ciga- rette holder, several valves and the control system for regulating puff frequency and volume. The mask was molded from a polyethylene bottle made pliable by heat and pressed over the plaster model of a dog's muzzle; after cooling it retained its desired shape. For exposure, the dog inhaled directly a smoke bolus of controlled volume followed by fresh air. After habituation dogs tolerated as many as 20 cigarettes in an 8 hour period. This equipment was employed by CROss et al. (385) in a study, which examined the effect of chronic cigarette smoke exposure on pulmonary lesions in beagle dogs. An ancillary observation revealed that about $30 \%$ of inhaled TPM from cigarettes spiked with ${ }^{14} \mathrm{C}$-dotriacontane-16,17 was deposited in the lungs of two (sacrificed) dogs. The focus of this investigation, however, was on radon, radon daughters and uranium ore dust.

PARK et al. (386) used a slightly modified form of the face mask developed by BAIR et al. (384) to assess the effects of diluted smoke, actively inhaled by beagle dogs, on pulmonary defense and function and on lung structure. Using a simple smoke generator permitting both forced and spontaneous smoke intake CAHAN and KIRMAN (387) exposed beagle dogs, which had undergone tracheotomy for tube insertion. Following adaptation the dogs tolerated a maximum of 12 unfiltered cigarettes per day. After 60 weeks (five of ten dogs lived that long), animals were examined for pulmonary fibrosis and emphysema. When the same group of researchers $(388,389)$ continued their work, it was observed that "after a few weeks, the dogs became habituated to cigarette smoking and seemed to enjoy it as indicated by tail wagging and jumping into the "smoking box" voluntarily" (388). Consequently, the use of a pump was no longer required as the dogs smoked filter and non-filter cigarettes willingly. Almost 100 dogs (four 4 groups and 1 control) were treated for up to 27 months. One investigation (388) examined the animals for pulmonary emphysema and fibrosis and found histopathological changes in all smoking dogs, most pronounced in the lungs of dogs smoking non-filter cigarettes. The other investigation (389) focused on pulmonary neoplasms and demonstrated non-invasive bronchiolo-alveolar tumors in all groups (few in non-smoking dogs, more with filter cigarettes and significantly more with non-filter cigarettes); invasive bronchiolo-alveolar tumors were found only in 
dogs heavily exposed to the smoke of non-filter cigarettes. For use with non-rodent laboratory animals, specifically chickens and dogs, BATTISTA et al. (390) developed a specialized smoke generation and exposure system. The smoking device was designed by the Life Sciences Division of Arthur D. Little, Inc. (Cambridge, MS) and named the ADL machine. It consisted of a cigarette holder, several solenoid valves, pressure regulators and two syringes in tandem, operated by air pressure and spring tension under cam timer control. Smoke from one puff $(35 \mathrm{~mL}$ in $2 \mathrm{sec})$ was collected in a syringe and then expelled undiluted into smoke-holding tubes. These were part of the dedicated inhalation units. With chickens the holding tube was inserted into the bird's mouth and secured air-tight by means of a latex face mask around the beak. Dogs had a tube implanted by tracheotomy, which was connected to the smoke-holding tube. Animals took breaths spontaneously and were allowed to inhale fresh air between puffs for preventing sustained anoxia. For chemical analysis, mainstream smoke from the ADL machine was sampled at three points of the smoke path and compared to smoke produced by a standard analytical machine, using gas chromatographic profiling of TPM and gas phase, and by the quantification of selected analytes, in particular TPM, water, nicotine, acetaldehyde, acrolein, phenols, carbon monoxide and carbon dioxide. While the smoke collected in the syringe right behind the cigarette port was rather similar to "analytical" smoke, a considerable loss of TPM (also reflected in nicotine and water levels) was obvious in the syringe exhaust and - even more so - in the smoke-holding tube exhaust. Gaseous constituents were much less affected when moving through the system.

The ADL smoking machine was used later on in several large chronic smoke exposure studies with permanently tracheotomized beagle dogs. An inhalation bioassay performed by HAZLETON LABORATORIES AMERICA (391) investigated the effect of cigarette smoke containing high or low levels of nicotine and, in addition, enriched with carbon monoxide or not on the development of atherosclerosis in beagles fed an atherogenic diet. In a joint effort, BATTELLE NORTHWEST LABORATORIES AND BORRISTON LABORATORIES (392) exposed beagles to the smoke of experimental cigarettes with low, medium or high nicotine content and assessed pulmonary physiology, clinical chemistry data and tissue changes by gross inspection and microscopy. BRAZELL et al. (393) at the Oak Ridge National Laboratory (TN,USA) examined the influence of cigarettes with three different smoke nicotine yields on the plasma levels of nicotine and cotinine in Beagle dogs exposed to mainstream smoke.

As pointed out by CoGGINS (374), introducing smoke directly into the trachea by means of tracheotomy and, this way, bypassing the nasal passages and the larynx is an extremely invasive and non-physiologic experimental approach. Most studies of this kind were marred by serious infectious and pulmonary complications in the animals and high death rates.

The fascinating fact that monkeys "smoke tobacco with pleasure" was personally observed and communicated by CHARLES DARWIN (394) in his famous work "The Descent of Man and Selection in Relation to Sex" when he con- sidered "the mental powers of man, in comparison with those of the lower animals". In the 1960s, JARVIK (395) succeeded in inducing a small group of monkeys (species not identified) to puff smoke from cigarettes or vapors from thermally volatilized tobacco. Once acquired, the smoking behavior was maintained with no other incentive and on a free choice basis while it was not clear whether the animals had at all inhaled into the lower respiratory tract.

A complex study to initiate, maintain and influence cigarette smoking behavior in rhesus monkeys was conducted in 1981 by ANDO and YANAGITA (396). Elaborate training was required for getting the animals accustomed to sucking on a pipe - first with water, then with a sweetened liquid reinforcer, later with additionally offered smoke, and eventually with the reinforcer withdrawn. Most animals continued "voluntary" smoking under these conditions two monkeys for over 2 years. For smoke delivery, the pipe was connected to a smoking machine with a turntable holding 18 cigarettes and a device, which automatically lighted the cigarette when first puffed. Interestingly, the monkeys were allowed to observe directly through a transparent panel the functioning of the smoking machine and several colored indicator lights. The study included monitoring of smoking behavior related to switching cigarette brands with high, low or no nicotine yields, respectively.

MCGILL et al. (397) demonstrated the feasibility of a baboon model for smoking studies. A stepwise training process over several months was necessary, using water rewards for teaching the animals - first - to suck rather than lick water from a pipe and, subsequently, draw sufficiently large puffs through a holder from unlit or burning cigarettes. Smoking behavior could be controlled by regulating water rewards, and consumption was as high as 48 cigarettes in a day. Inhalation intensity was determined by measuring carbon monoxide in venous blood; the average $\mathrm{COHb}$ level $(5.9 \%)$ was comparable to that in human smokers. It is worth noting that the model operated without surgical manipulation or obvious stress.

This animal model (397) was used by RoGERs et al. (398) with 36 baboons, which were fed an atherogenic diet and divided in a smoking (on average, 43 unfiltered cigarettes a day) and a sham group. While after 14-19 months typical smoking related effects were observed (elevated levels of blood carbon monoxide and thiocyanate and of urinary cotinine as well as increased lymphocyte count), there were no differences between the two groups regarding serum cholesterol (total cholesterol; very low density, low density and high density lipoproteins) and triglycerides. In two separate studies of similar design with 55 baboons in all, ROGERS et al. (399) found little effect of long-term (2-3 years) cigarette smoke inhalation on nutritionally induced atherosclerosis.

The deposition of smoke particulate matter in the lungs of baboons was determined quantitatively by ROGERs et al. (400) using cigarettes spiked with ${ }^{14} \mathrm{C}$-dotriacontane-16,17 (319). In order to avoid the need for killing the animals the investigators resorted to bronchoalveolar lavage for recovering inhaled particles. For assessing the efficiency of recovery, animals were artificially loaded - by forced inspiration - with known amounts of labeled TPM and then 
subjected to lavage. When smoking actively in their normal fashion the baboons were found to retain an average of $9 \%$ of total particulate matter. However, retention levels estimated for individual animals varied quite considerably due to large differences in their smoking behavior (number, volume, duration and pressure of puffs) - an observation comparable to what is generally seen in humans.

In 1982, RAYMOND et al. (401) reported a study with adult male stump-tailed macaques, kept on a standard laboratory diet, on the effects of cigarette smoke on total and lipoprotein plasma cholesterol. Low dose animals received the smoke of 3-5 cigarettes/day (equivalent to $1 \mathrm{pack} /$ day for a human smoker) seven days each week for 3-5 years; levels for high dose animals were 6-13 cigarettes/day (equivalent to 3 packs/day). The macaques were fitted with an aluminum inhalation mask connected to a multiport reverse puffing smoking machine and control unit, which provided every $40 \mathrm{sec}$ smoke (1:10 diluted) for $3 \mathrm{sec}$. The mask was equipped with restraining features: a gag bit holding the mouth open and a balloon occluding the nostrils when inflated. Obviously, this procedure represents compulsory smoke dispensation rather than cigarette puffing by the experimental animals.

The same exposure system was used by SOPORI et al. (402) in an inhalation study with stump-tailed macaques examining the chronic effect of cigarette smoke on several parameters of their immune response.

As discussed earlier (see page 192) CoGGINS reviewed in 1998-2007 representative chronic inhalation studies with mainstream cigarette smoke performed with five main species of laboratory animals; the endpoint under con- sideration was neoplastic disease. The two papers dealing with dogs and monkeys were published in 2001 (374) and 2007 (375). The author concluded that "significant increases in the numbers of malignant tumors of the respiratory tract were not seen in ..... dogs or nonhuman primates exposed for long periods of time to very high concentrations of cigarette smoke" (375).

\section{Analytical smoking machines}

Smoking machines designed for analytical purposes and for preparing small amounts of condensate for in vitro testing, such as Ames test, NRU assay, etc.

\section{Preparative smoking machines}

Smoking machines designed for smoking large numbers of cigarettes for the preparation of substantial amounts of mainstream smoke condensate

Smoking machines for animal inhalation studies

Smoking machines designed for nose-only or whole-body exposure of animals to cigarette mainstream smoke

Smoking machines for whole smoke in vitro studies

Smoking machines designed for the exposure to whole mainstream smoke of bacterial, cell and tissue cultures, especially in air-liquid interface (ALI) systems 

TESTING

In addition to in vivo exposure studies (skin painting, inhalation), in vitro assays have been in use for many years for the assessment of cigarette smoke toxicity. Recently, methodology has advanced considerably in sophistication and importance due to technical progress and the increasing financial and regulatory burdens encountered when performing animal studies. In fact, experiments with animals for the development of tobacco products have been outlawed since 1998 in Germany (403), and other countries did follow: Great Britain, Belgium, Estonia, Slovakia (404). Ethical concerns raised over the use of (vertebrate) animals in toxicity studies constitute a factor of growing influence. The goal of animal welfare advocates is achieving the "Three Rs": the reduction of the number of animals used, the refinement of methods to alleviate or minimize potential pain, suffering or distress for the animals, and the replacement of animal studies by in vitro methods. The discussion gained momentum after the introduction of the EC Regulation concerning the Registration, Evaluation, Authorisation and Restriction of Chemicals (REACH) in 2006 (405). While the program was expected to require the expenditure of millions of animals for toxicity testing, it called at the same time for "the use of alternative test methods, suitable for the assessment of health and environmental hazards of chemicals, wherever possible" and termed animal studies the "last resort".

Against this background the ethical debate and scientific discussions gained momentum and gave reason for meetings such as the workshop organized in 2007 by FRAME (Fund for the Replacement of Animals in Medical Experiments (406), a UK based charity founded in 1969 and dedicated to the promotion of the "Three Rs"). The workshop report by BÉRUBÉ et al. (407) is an extensive, detailed and instructively illustrated account presenting inter alia overviews of the respiratory system, lung related diseases and equivalent in vitro models; approaches to inhalation toxicity testing with cellular and tissue cultures; and air-liquid interface (ALI) techniques of aerosol exposure in vitro (including the Cultex ${ }^{\circledR}$ system and the BAT

Table 5. Mammalian cell lines and organotypic cultures used for in vitro toxicity testing.

\begin{tabular}{|c|c|c|c|c|c|}
\hline Cell line / culture & Species & Tissue & Morphology & Origin & Pertinent studies \\
\hline CHO-WBL & $\begin{array}{l}\text { Chinese } \\
\text { hamster }\end{array}$ & ovary & epithelial & parental subclone & $(267,409)$ \\
\hline $\mathrm{CHO}-\mathrm{K} 1$ & $\begin{array}{l}\text { Chinese } \\
\text { hamster }\end{array}$ & ovary & epithelial & parental subclone & $(421,452)$ \\
\hline A549 & human & lung alveolar & epithelial & adenocarcinoma & $\begin{array}{c}(421,515,445,527 \\
446,528,459,462 \\
502,460,506)\end{array}$ \\
\hline BEAS-2B & human & lung bronchial & epithelial & virally transformed and cloned & $\begin{array}{l}(381,519,502 \\
486,477,506)\end{array}$ \\
\hline $3 \mathrm{~T} 3$ & mouse & embryo & fibroblast & immortalized & $\begin{array}{r}(410,411,431 \\
416,490,491)\end{array}$ \\
\hline Calu-3 & human & lung bronchial & epithelial & adenocarcinoma & $(460)$ \\
\hline HEP-G2 & human & liver & epithelial & hepatocarcinoma & $(515,517,525)$ \\
\hline L5178Y & mouse & whole embryo & lymphoblast & lymphoma & $(431,416)$ \\
\hline V79 & $\begin{array}{c}\text { Chinese } \\
\text { hamster }\end{array}$ & lung & fibroblast & $\begin{array}{l}\text { cloned after spontaneous } \\
\text { transformation }\end{array}$ & $\begin{array}{l}(13,416 \\
525,431)\end{array}$ \\
\hline $\mathrm{NCl}-\mathrm{H} 292$ & human & lung & epithelial & mucoepidermoid carcinoma & $(472,479,474)$ \\
\hline HFBE21 & human & lung bronchial & epithelial & fetal & $\begin{array}{r}(440,382,441 \\
444,445,383) \\
\end{array}$ \\
\hline CCD-11Lu & human & lung & fibroblast & normal tissue & $(421)$ \\
\hline Lk004 & human & lung & fibroblast & normal tissue & $(441)$ \\
\hline 16HBE140- & human & lung bronchial & $\begin{array}{r}\text { epithelial, cilia forming } \\
\text { under ALI conditions }\end{array}$ & immortalized & $(458,460)$ \\
\hline MucilAir $^{\mathrm{TM}}$ & human & nasal & epithelial, ciliated & primary cells, reconstituted & $(509)$ \\
\hline MucilAir ${ }^{\mathrm{TM}}$-HF & human & nasal & epithelial & $\begin{array}{l}\text { primary cells, reconstituted } \\
\text { with fibroblasts }\end{array}$ & (513) \\
\hline MucilAir $^{\mathrm{TM}}{ }_{-} \mathrm{HF}$ & human & bronchial & epithelial & $\begin{array}{l}\text { primary cells, reconstituted } \\
\text { with fibroblasts }\end{array}$ & (513) \\
\hline EpiAirway ${ }^{\mathrm{TM}} \mathrm{AIR}-100$ & human & bronchial & epithelial & normal cells, differentiated & $(510)$ \\
\hline EpiOral $^{\mathrm{TM}}$ & human & oral & $\begin{array}{l}\text { non-cornified buccal } \\
\text { phenotype }\end{array}$ & $\begin{array}{l}\text { keratinocytes, co-cultured with } \\
\text { fibroblasts }\end{array}$ & $(510)$ \\
\hline EpiGingival $^{\mathrm{TM}}$ & human & oral & $\begin{array}{l}\text { cornified gingival } \\
\text { phenotype }\end{array}$ & $\begin{array}{l}\text { keratinocytes, co-cultured with } \\
\text { fibroblasts }\end{array}$ & $(514)$ \\
\hline
\end{tabular}




\section{Cytotoxicity Test Systems}

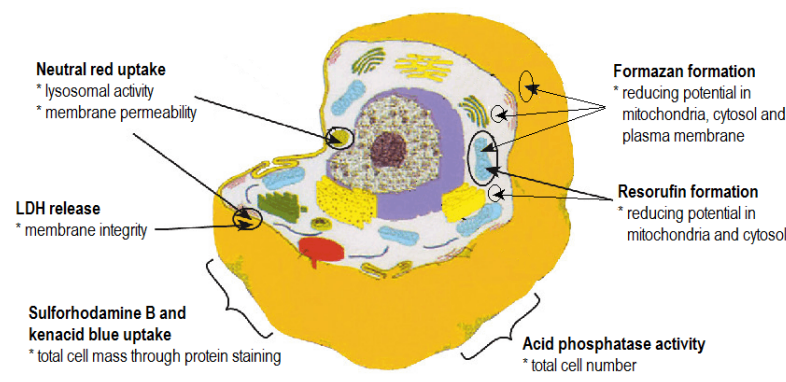

Figure 39. Points of action in cytotoxicity testing (figure modified from (641)).

chamber for whole smoke exposure, both discussed below).

Compared to animal studies, in vitro tests offer remarkable advantages; generally, less cost is incurred, less time required and less space needed. Regarding experimental conditions, in vitro tests allow higher flexibility, tighter control and greater opportunity for intervention. The quantification of endpoints at large is simplified. Substances, preparations and products can be examined for cytotoxicity (cell viability and growth rates) as well as genotoxicity with DNA as the target (mutagenic and clastogenic events, which include point mutations, frame shifts, micronucleus formation, chromosome aberration, sister chromatid exchange, unscheduled DNA synthesis and - quite importantly - DNA adduct formation). Methods of particular importance are discussed more thoroughly below.

A very broad range of bacterial and mammalian cells is available for use. For mechanistic (particularly genotoxicity) studies strains of Salmonella typhimurium (some selected for targeted specificity and highest sensitivity) and Escherichia coli are widely used. The well accepted mammalian cell lines include mouse lymphocytes, mouse fibroblasts (3T3), hamster lung cells (V79), Chinese hamster ovary cells and Chinese hamster lung cells. For disease related research human cells are ideal and accessible from a miscellany of target organs, such as lung, liver, nose and oral cavity. Many cell types (e.g., A549, BEAS-2B, Calu-3 and 16HBE14o-) are commercially available, some as ready-to-use systems. Cloned cell strains and continuous cell lines allow the study of relatively homogenous cell populations. Recently, threedimensional primary organotypic cultures of human bronchial epithelial cells have become reliably procurable and are supplied in inserts ready for air-liquid interface (ALI) exposure (see Chapter 6.2, p. 203).

Certain limitations, however, need to be kept in mind, especially when working with mammalian cells, which are sustained outside their normal physiological environment: The cells are lacking most of the protective and detoxifying elements present in vivo, and the systems are often designed to be extremely sensitive. Bacterial cells, on the other hand, are unable to metabolize (activate) chemicals via the cytochrome $\mathrm{P} 450$ route; therefore, an exogenous mammalian metabolic activation system, such as the rat liver microsomal preparation S9-mix, must be included for exploiting the assay full scope.

Generally applicable guidelines for in vitro toxicity testing have been developed by several international bodies, most prominently by the Organisation for Economic Co-operation and Development (OECD). Methods considered by OECD to date (408) include \# 471: Bacterial Reverse Mutation Test (Ames-Test); \# 473: In vitro Mammalian Chromosome Aberration Test; \# 476: In vitro Mammalian Cell Gene Mutation Tests Using the Hprt and xprt Genes; \# 479: In vitro Sister Chromatid Exchange Assay in Mammalian Cells (deleted in April 2014); \# 487: In vitro Mammalian Cell Micronucleus Test; and \# 490: In vitro Mammalian Cell Gene Mutation Tests Using the Thymidine Kinase Gene.

Cytotoxicity is revealed by the injury inflicted on the structural and/or functional integrity of cells. In a living cell a great many functions and processes are in operation at any time and may be focused on for assessing cellular viability. Cytotoxic effects can be quite variable, and so are the methods for assessing the cytotoxic potential of individual substances and more complex materials. Cellular features that may be affected include the uptake, conversion or exclusion of dyes, the activity of mitochondrial and cell membrane bound enzymes and of intracellular redox systems, the binding of dyes to intracellular proteins, and - last but not least - direct damage to the cell plasma membrane.

Methods frequently or occasionally employed are (Figure 39):

- The very widely used neutral red uptake (NRU) assay (discussed in detail below) determines membrane permeability and lysosomal activity;

- The conversion of certain tetrazolium dyes by $\mathrm{NAD}(\mathrm{P}) \mathrm{H}$-dependent cellular reductases to the corresponding formazans is a measure for cell number and viability. Tetrazolium substrates in use are MTT $=3$ (4,5-dimethylthiazol-2-yl)-2,5-diphenyltetrazolium bromide, XTT = 2,3-bis-(2-methoxy-4-nitro-5-sulfophenyl)-2H-tetrazolium-5-carboxanilide, and WST-1 = 2-(4-iodophenyl)-3-(4-nitrophenyl)-5-(2,4-disulfophenyl)-2H-tetrazolium, monosodium salt. XTT and WST-1 offer the technical advantage of giving rise to water soluble formazans, while the product from MTT is not soluble in aqueous medium and needs to be solubilized before absorbance reading (Figure 40);

- The resazurin assay is based on the NAD $(\mathrm{P}) \mathrm{H}-d e p e n-$ dent irreversible intracellular conversion of the minimally toxic redox dye, resazurin, into the highly fluorescent resorufin (Figure 40);

- The activity of lactate dehydrogenase (LDH), released from cytoplasm into extracellular space, indicates the degree of direct damage to cell plasma membrane integrity;

- The binding of the vital dyes, sulforhodamine B and kenacid blue, by cellular proteins is a measure for total cell mass;

- The activity of membrane bound acid phosphatase relates to cell number, which may be affected by direct cell damage.

The exact assessment of cytotoxicity is fundamental for determining appropriate doses for other in vitro assays, specifically genotoxicity tests. 

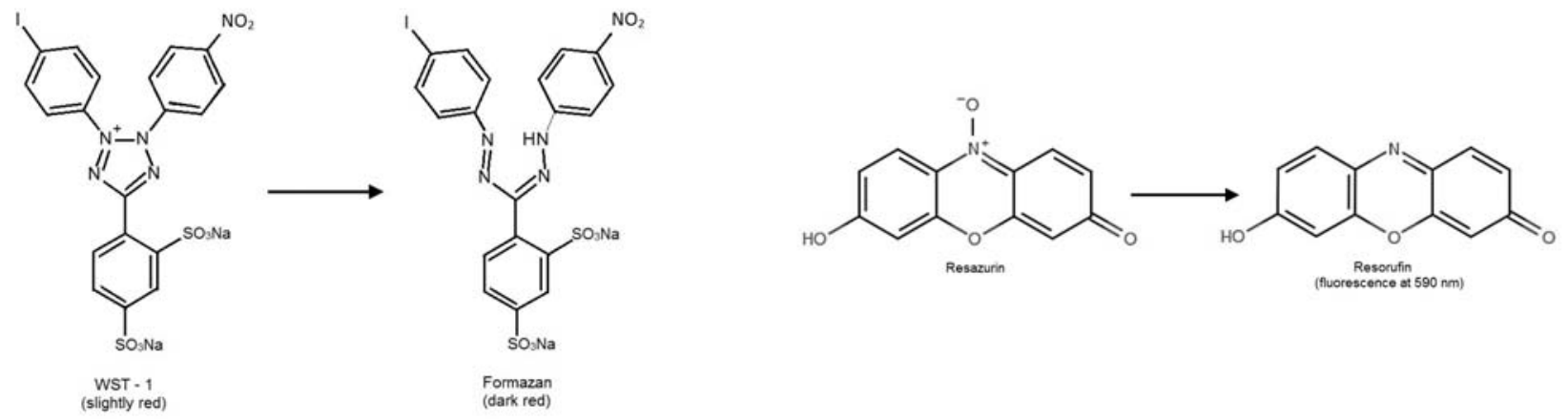

Figure 40. Two colorimetric reactions for the assessment of cellular viability: the mitrochondrial cleavage of tetrazolium salts to formazans (left) and the irreversible intracellular reduction of resazurin to resorufin (right).

The eight in vitro cytotoxicity assessment methods listed above were compared by PUTNAM et al. (409) using Chinese hamster ovary cells (CHO; WBL strain), which were exposed for time periods between 1 and 24 hours to increasing amounts of cigarette smoke condensate, produced from 1R4F Kentucky reference cigarettes and dissolved in dimethyl sulfoxide. The assays used were neutral red uptake by active endocytosis, mitochondrial reduction of MTT and XTT, cleavage of $p$-nitrophenyl phosphate by membrane-bound acid phosphatase, irreversible bioreduction of resazurin, protein binding of kenacid blue and sulforhodamine $\mathrm{B}$, and the release of cytoplasmic lactate dehydrogenase $(\mathrm{LDH})$ into the medium. At 1-hour exposure, LDH release was by far the most sensitive endpoint while after 24 hours of exposure, the neutral red uptake (NRU) and kenacid blue staining assays - both measuring total cell number - showed the highest signals. The acid phosphatase and resazurin assays failed to detect cytotoxicity under any of the conditions tested. The paper (409) provides experimental details and instructive mechanistic information on each of the methods used.

The neutral red uptake (NRU) test is the most frequently used in vitro cytotoxicity assay due to its mechanistic and experimental simplicity, high level of sensitivity and reproducibility, and very favorable speed and economy. Neutral red is a phenazine dye, which is not charged at neutral $\mathrm{pH}$ and readily diffuses through plasma and lysosomal membranes. However, in the acidic environment of lysosomes $(\mathrm{pH}<5)$ the dye gets protonated, changes its color to deep red, becomes unable to pass the lysosomal membrane and is, consequently, trapped inside the organelles. Damaged or dead cells with impaired lysosomal integrity have reduced or no potential to absorb and retain neutral red. The method was developed in 1984 by BORENFREUND and PUERNER (410) at the Rockefeller University (New York, NY, USA) - with the expressed intention (411) "to serve as a potential replacement for the Draize rabbit eye irritancy test $t$. The procedure calls for trypsinized cell cultures (e.g., 3T3 mouse fibroblasts) to be treated with the test agent in concentrations over 3-4 orders of magnitude for up to 24 hours. Neutral red solution is then added for a 3-hour incubation period and subsequently removed completely. Neutral red incorporated into lysosomes is finally released by dissolution in acetic acid/ethanol, quantified photometrically at $540 \mathrm{~nm}$ and compared to control (results usually stated in \% viability remaining).

The neutral red uptake (NRU) test was examined in a very practical way by REPETTO et al. (412), including a wealth of detailed advice for its proper execution. As a matter of fact, cytotoxicity studies are the prerequisite for properly executed additional in vitro toxicity testing, specifically genotoxicity assays.

The most popular of all in vitro genotoxicity tests is the Salmonella/microsome mutagenicity assay, developed in the early 1970s in the laboratory of AMES $(413,414)$ hence generally called the "Ames test"; a comprehensive review providing historical aspects of method development as well as practical advice for performing the test was published by MORTELMANS and ZEIGER (415). The assay uses a number of Salmonella strains each carrying different preexisting mutations in various genes in the histidine operon (the functioning unit of promoter, operator and structural genes), making them dependent on the external supply of histidine in the growth medium. These mutations are the targets for action by potential mutagens, resulting in reversion to histidine independence in case the gene defect is corrected by mutation. If so, the bacterium would be able to grow in a histidine-free environment. The mutations in the various Salmonella strains are engineered in such a way that they are susceptible to mutagens with different modes of action (base pair substitution or frameshifts, i.e., the addition or deletion of base pairs). However, it was quickly recognized that most mutagens required metabolic activation by the cytochrome $\mathrm{P} 450$ based oxidation system (absent in bacteria), preferentially in form of a rat liver microsomal preparation (S9-mix).

The original exposure technique was a spot test: The test material was applied to the center of an agar plate seeded with the Salmonella test strain; following radial diffusion of the test material into the medium revertant colonies were produced along the concentration gradient. Subsequently, AMES developed the plate incorporation assay - improving the sensitivity and quantification of the test: Test material, test strain and (optionally) the microsomal preparation were premixed with overlay agar and poured on minimal agar plates; as an alternative, components could be pre-incubated for $20 \mathrm{~min}$ or more prior to the addition of overlay agar. After an incubation period of 2-3 days the revertant colonies formed could be counted. This technique was in the course of time modified and adapted to a variety of requirements, such as suitability for 
volatile test materials, small samples and special atmospheres. The bacterial reverse mutation test is discussed and described in the OECD in vitro Test Guideline \# 471 (408).

The mouse lymphoma assay is able to detect gene and chromosome mutations at the thymidine kinase locus of L5178Y lymphoblasts by measuring their resistance to the lethal nucleoside analogue, trifluorothymidine. Following exposure only cells with the locus damaged survive in semi-solid agar or microtiter plates (containing the analogue) and can be counted, while unaffected cells die from unimpaired thymidine kinase activity and resultant analogue incorporation into DNA. The test is described and assessed in OECD in vitro Test Guideline \# 490.

Structural and numerical chromosome anomalies in mammalian cells are discovered in the reliable and rather easily performed micronucleus assay (OECD in vitro Test Guideline \# 487) and the more complicated chromosome aberration test (OECD In Vitro Test Guideline \# 473). In the micronucleus assay exposed cells are required to go through at least one cell division (limitation to one division is possible by preventing cytokinesis with cytochalasin B) and then harvested, stained and scored for small erratic nuclei in cytoplasma, which may have formed during the anaphase of mitosis or meiosis. Chromosome or chromatid damage from clastogenic events is assessed in the chromosome aberration test. Cells are exposed and treated at appropriate intervals with colchicine for arresting metaphase. After harvesting and staining, cells are examined microscopically for the presence of chromosome-type and chromatid-type aberrations.

Sister chromatid exchange (SCE) constitutes the reciprocal exchange of whole DNA duplexes between two sister chromatids of a duplicating chromosome. Occurring normally at a very low rate it may be intensified considerably in pathologies or by mutagens interacting with DNA. For testing (OECD In Vitro Test Guideline \# 479 - deleted in April 2014), human peripheral blood lymphocytes are stimulated to divide by an antigen, like phytohemagglutinin, exposed and allowed to complete two replications in the presence of bromodeoxyuridine (BrdU) before being blocked in metaphase with colchicine, harvested and stained. SECs are detected on the basis of differential labeling by BrdU incorporation.

\subsection{Conventional in vitro assays with tobacco smoke}

Test materials for in vitro assays are procured in different forms. Traditionally, cigarette smoke condensate was preferred because it was the most conspicuous product of smoking, had shown remarkable biological activity in in vivo assays and could be collected rather easily as total particulate matter (TPM) on Cambridge filters. Comparatively, gas phase constituents received much less attention. The relationship of the amount of inhaled smoke particulate matter and the incidence of lung cancer (71) was, from the 1950s onward, the most topical issue concerning tobacco use. For smoke condensate collection, cold traps, impaction traps and electrostatic devices were also in use; in these traps as well, certain semi-volatiles (but generally no volatiles) were at least partly co-precipitated. For testing, smoke condensate was usually taken up in a solvent, such as dimethyl sulfoxide (DMSO), and added to the cell culture medium. A number of specific constituents of the gaseous phase of smoke - after passing through a Cambridge filter pad or an electrostatic precipitator - had to be collected in liquid traps filled with water adjusted to acidic or basic $\mathrm{pH}$ or with phosphate buffered saline. In the process, depending on their physico-chemical properties (in particular solubility), gas phase constituents were retained to rather different degrees - falsifying the authentic composition of the smoke fraction. Whole smoke when tested was generally bubbled directly through media. Using these approaches, however, smoke and its fractions were prone to artifact formation, aging and interaction with foreign matter in the medium.

In view of the importance of cigarette smoke condensate as a substrate for in vitro testing CROOKS et al. (416) examined the effects of long term storage. Total particulate matter from 3R4F Kentucky reference cigarettes and M4A cigarettes (a long-standing BAT internal control) was collected on Cambridge filters, extracted in dimethyl sulfoxide and then stored at $-80{ }^{\circ} \mathrm{C}$ for 2 years. At seven time points during this period the material was examined for cytotoxicity (neutral red uptake assay) and genotoxicity (Ames test, micronucleus assay and mouse lymphoma assay). Only occasionally significant differences were observed between fresh and stored condensate but these were minor and did not show any consistent trend.

RICKERT et al. (417) drew attention to the effects smoking conditions might have on the in vitro biological activity of TPM and gas vapor phase in the Ames test (for genotoxicity) and the neutral red uptake assay (for cytotoxicity). Smoke fractions were compared from three different Kentucky reference cigarettes and the CIM-7 Canadian industry monitor test piece (made from 100\% flue-cured tobaccos by the Canadian Tobacco Manufacturers Association) - all smoked according to the ISO (105) or the Canadian Intense (22) regimes. It was confirmed that - if related to mg TPM or equivalent amounts of gas vapor phase - Canadian Intense smoking produced material with reduced activity in genotoxicity (lower number of revertants) and cytotoxicity (increased $\mathrm{EC}_{50}$ values, in $\mu \mathrm{g} \mathrm{TPM} / \mathrm{mL}$ ). It was reasoned that, in addition to increased water content in TPM generated by Canadian Intense smoking (22) - as discussed in Chapter 3.5 (p. 166) changes in the relative amounts of pyrolysis products and differences in particle-gas partitioning might be responsible for the effects.

There may be an additional explanation for the reduction of the toxicity of smoke generated under the Canadian Intense regime:

Due to ventilation blocking, mandated by the Canadian Intense regime, the full volume of a puff flows through the glowing cone. Therefore, more oxygen is available compared to ISO 3308 (105) smoking. Consequently, temperatures in the cone are elevated and combustion is more complete, which leads to increased amounts of carbon dioxide and water in mainstream smoke - as observed by COUNTS et al. (218). MUMPOWER and TOUEY (418) reported that higher amounts of water in smoke and, in consequence, cellulose acetate filters resulted in increased efficiency of removing phenol. Similarly, CounTs et al. (218) speculated that mainstream water elevated at Canadian Intense 


\section{$E C_{50}$ - median effective concentration}

Statistically derived concentration of a substance in an environmental medium expected to produce a certain effect in $50 \%$ of test organisms in a given population under a defined set of conditions.

\section{$E D_{50}$ - median effective dose}

Statistically derived dose of a chemical or physical agent (radiation) expected to produce a certain effect in $50 \%$ of test organisms in a given population or to produce a half-maximal effect in a biological system under a defined set of conditions.

\section{$I C_{50}$ - inhibitory concentration}

Concentration of a substance that causes a defined inhibition of a given system: $\mathrm{IC}_{50}$ is the median concentration that causes $50 \%$ inhibition.

\section{$I D_{50}$ - inhibitory dose}

Dose of a substance that causes a defined inhibition of a given system: $I_{50}$ is the median dose that causes $50 \%$ inhibition.

Reference: International Union of Pure and Applied Chemistry (IUPAC): IUPAC Compendium of Chemical Terminology - The Gold Book. Available at: http://goldbook.iupac.org/index.html (accessed September 2016).

conditions intensified filter retention of phenol and quinoline. This may well have contributed to the reduction of TPM genotoxicity and cytotoxicity observed by RICKERT et al. (417).

An early report of cytotoxicity testing of cigarette smoke using a dye was published in 1981 by HoPKIN et al. (419). Cigarette smoke condensate obtained from three popular British commercial brands (with low, middle or high levels of "tar" $)^{6}$, polymorphonuclear leukocytes from human donors and the non-toxic synthetic mixture of black dyes, nigrosin, were components of the study. Nigrosin when used for staining is excluded from living cells; their shapes and sizes are seen as color-free structures against a dark background (420). In a group of 40 healthy subjects (males and females, smokers and non-smokers), viability response of polymorphs (generally dose dependent) was of very different intensity and not related to age, sex or smoking habit. Interestingly, these observations were reproducible when individuals were re-examined after one month. When two groups of heavy smokers with 10 participants each either with clinical, physiological and radiological evidence of severe, irreversible pulmonary obstruction and probable emphysema or with no signs of respiratory disability - were examined, cellular responses were (again) dose related but revealed considerable differences between

\footnotetext{
6 "Tar" classification for cigarettes as defined in the U.K. between 1973 and 1984: Low "tar" level: 0-10 mg/cig.; Middle "tar" level: 17-22 mg/cig.; High “tar” level: more than $28 \mathrm{mg} / \mathrm{cig}$ (202)
}

the individuals within each group. There was some overlap between the two groups at each level of testing; as a whole, the polymorphs of the group without manifest pulmonary problems showed higher resistance to the cytotoxic effects of smoke condensate.

Following its introduction in 1984 (410), the neutral red uptake (NRU) assay has gained remarkable acceptance as the preferred cytotoxicity test using the principle of staining cultured cells. The procedure was adapted in 1995 by BOMBICK and DOOLITTLE (421) to examine the potency of certain chemical classes of compounds found in tobacco smoke (low molecular weight aldehydes and pyridines) and the responses among several diverse cell types (Chinese hamster ovary-K1 cells, human lung fibroblasts CCD-11Lu, A549 human lung carcinoma cells, WB rat liver epithelial cells and mouse keratinocytes). In a followup study BOMBICK et al. (267) exposed Chinese hamster ovary cells or WB rat liver epithelial cells directly to whole smoke or the vapor phase of mainstream or sidestream smoke from three Kentucky reference cigarettes (high "tar" non-filter 2R1, low "tar" filtered 1R4F and ultra low "tar" filtered 1R5F) - using a special "cellular smoke exposure technique" (discussed on page 203). Data obtained by means of the neutral red uptake (NRU) test allowed to reach the following conclusions, all based on $\mathrm{EC}_{50}$ values and expressed on a per cigarette basis (number of cigarettes $/ \mathrm{m}^{3}$ of air): Cytotoxic potency of mainstream smoke of the three test cigarettes followed clearly the ranking of "tar" yields while sidestream cytotoxicity did not differ between the three test pieces; sidestream smoke samples (whole smoke as well as vapor phase) were always considerably more cytotoxic than those of mainstream smoke; and regarding both mainstream and sidestream smoke the vapor phases were in all cases the overwhelming contributors to cytotoxic effects.

Data from Ames tests with cigarette smoke condensate were published as early as 1974 and 1975 . At that time, the carcinogenic potential of cigarette smoke had already been demonstrated in mouse skin painting studies and by the strength of statistical evidence based on lung cancer incidence in human cigarette smokers. Scientists were now concerned with discovering mutagens (i.e., likely carcinogens) in condensate (fractions) by means of a quick and easy in vitro mutagenicity test. KIER et al. (422) in Bruce Ames' laboratory used Salmonella typhimurium strains TA 1536, TA 1537 and TA 1538 (sensitive for frameshift mutagens), and TA 1535 (sensitive for base-pair substitution mutagens). For the metabolic activation of potential mutagens, microsomal S9-mix preparations were used not only from rat liver but also (ingeniously) from rat lungs. Smoke condensate from commercial non-filter cigarettes (either unmodified or after attachment to an experimental filter holder with $300 \mathrm{mg}$ activated charcoal) showed mutagenic activity only with TA 1538 and following microsomal activation. The outcome was different with condensate from cigarettes after addition of $10 \%$ magnesium nitrate to the tobacco. WYNDER and HOFFMANN (423) had previously reported that mainstream smoke condensate from cigarettes made from tobaccos rich in nitrate showed lower tumor bearing rates on mouse skin than cigarettes made from low nitrate tobaccos. This, however, was not corroborated by KIER et al. (422), who 


\section{In vitro cell viability and genotoxicity assays}

Neutral red uptake (NRU) test:

A quantitative spectrophotometric procedure, measuring the uptake of neutral red, a phenazine dye, by the lysosomes of viable cells

Formazan formation (assays using the tetrazolium substrates MTT, XTT or WST-1):

Spectrophotometric measurement of cell viability by assessing the activity of cellular dehydrogenases, which cleave tetrazolium salts to form deep-colored formazans (purple, orange or dark red)

Lactate dehydrogenase (LDH) assay:

Assessment of cell membrane integrity by measuring the release of cytoplasmic LDH into the medium

\section{Resazurin assay:}

Measurement of the reducing potential of viable cells to convert colorless resazurin to fluorescent resorufin

Acid phosphatase assay:

A quantitative indicator of viable cells by determining the activity of the cell membrane associated acid phosphatase

Sulforhodamine B assay:

Determination of total cell mass by means of protein staining with sulforhodamine $B$

Kenacid blue assay:

Determination of total cell mass by means of protein staining with kenacid blue

\section{Bacterial reverse mutation test (Ames test):}

The principle of this test is that it detects mutations, which revert mutations present in the test strains and restore the functional capability of the bacteria to synthesize an essential amino acid. The revertant bacteria are detected by their ability to grow in the absence of the amino acid required by the parent test strain (OECD Guideline \# 471 (408)). For the popular Ames test, histidine-dependent Salmonella tester strains are used

In vitro mammalian cell gene mutation tests:

The in vitro mammalian cell gene mutation tests can be used to detect gene mutations induced by chemical substances. The cell lines used in these tests measure forward mutations in reporter genes, specifically endogenous hypoxanthine-guanine phosphoribosyl transferase genes (OECD Guideline \# 476 (408)) or endogenous thymidine kinase genes (OECD Guideline \# 490 (408))

In vitro mammalian cell micronucleus test:

The in vitro micronucleus test is a genotoxicity test for the detection of micronuclei in the cytoplasm of interphase cells. Micronuclei represent damage that has been transmitted to daughter cells (OECD Guideline \# 487 (408))

In vitro mammalian chromosomal aberration test:

The purpose of the in vitro chromosomal aberration test is to identify substances that cause structural chromosomal aberrations in cultured mammalian cells. Structural aberrations may be of two types, chromosome or chromatid (OECD Guideline \# 473 (408))

observed two effects when tobacco was supplemented with magnesium nitrate: frameshift mutations in strain TA 1538 even without microsomal activation, and base-pair substitution mutations in TA 1535 . It was reasoned that smoke condensate from cigarettes enriched with nitrate contained either much higher levels of mutagens or new types of mutagens.
In addition, KIER et al. (422) examined fractions of condensate from the 1A1 Kentucky reference cigarette (a nonfilter low nicotine prototype made in 1969).

HUTTON and HACKNEY (424) studied condensate fractions obtained from the 1R1 Kentucky reference cigarette (nonfilter, the original reference produced in 1969), using microsomal homogenates isolated from rat and human livers, and rat lungs. Both research groups were strongly 
interested in the various chemical classes of mutagenic smoke constituents acting by different molecular mechanisms, and in the prospects of reducing the health risks of smoking by product modification (blend composition, filters) - a challenge that has not disappeared since.

Sister chromatid exchange (SCE) induction was first used in the late 1970s as an endpoint in in vitro toxicity studies with cigarette smoke condensates. Three European commercial brands were examined by DE RAAT (425) using Chinese hamster ovary cells. HOPKIN and EvANS $(426,427)$ exposed cultured human lymphocytes donated by healthy non-smokers, healthy smokers, smokers with histologically proven but untreated lung cancer and smokers with health disorders other than any form of cancer, and compared SCE responses produced by increasing doses of smoke condensate from a British commercial cigarette; the data obtained in their study was not readily amenable to straightforward interpretation. In all studies, smoke condensates were produced by machine smoking under ISO/FTC standard conditions, and activation with microsomal preparations was not performed.

Scores of studies using in vitro methods were conducted over the years to assess the toxicological properties of tobacco and cigarette constituents, and cigarette smoke (and its fractions). Recently, conscious steps were taken by the scientific community to develop systematic and specifically designed experimental approaches.

The development of a sound rationale and meaningful methods for the in vitro toxicological assessment of tobacco smoke (fractions) was stimulated by increased concern over the biological effects of certain smoke constituents and the need, recognized by cigarette manufacturers, to design products with potentially reduced risk for human health. In 2003, ANDREOLI et al. (428) reviewed the in vitro methods in use for the toxicological evaluation of tobacco smoke and proposed a tiered approach following the guidelines of regulatory agencies, specifically the Organisation for Economic Co-operation and Development (OECD) and the International Council for Harmonization (ICH), which is active in Europe, Japan and the United States and works towards achieving greater harmonization in the interpretation and application of technical guidelines and requirements for pharmaceutical product registration (429). The essential components of the test battery, proposed by the authors (428), included the neutral red uptake (NRU) assay for examining cytotoxicity, the Ames assay for assessing mutagenicity, and the micronucleus assay for identifying chromosome damage not detected by the Ames assay.

A systematic review of the literature published (essentially) after 1980 to evaluate in vitro assays for assessing the toxicity of cigarette smoke and smokeless tobacco was released in 2009 by JOHNSON et al. (430). Emphasis was put on methods relevant for cancer pathways. Major parts of the review addressed topics such as the generation and collection of tobacco smoke for testing, the preparation of smokeless tobacco extracts for testing, assays for a variety of cytotoxicity endpoints, a wide range of genotoxicity tests, the choice of cell types and culture conditions, the extrapolation of in vitro toxicity studies to experimental animals and humans, possible criteria for assay validation, and - finally - the development of test batteries.
The rationale and strategy for conducting in vitro toxicity testing of tobacco smoke was dealt with comprehensively in 2002-2004 by a CORESTA TASK FORCE (431). Against the background of increasing regulatory demands "key procedures based upon internationally recognized guidelines, adapted to accommodate the nature and unique properties of tobacco smoke" were identified. The report included the discussion of specific assays with regard to their utility for testing tobacco smoke (components), methods for the preparation of test materials, standard procedures, and the evaluation and interpretation of results. In conclusion, the TASK FORCE recommended a test battery composed of the following assays: A cytotoxicity assay conducted with an appropriate mammalian cell line (the neutral red uptake test); a bacterial mutagenicity assay (the Ames Salmonella mutagenicity assay); and a mammalian cell assay for cytogenetics/mutation (the micronucleus assay, the chromosome aberration assay or the L5178Y mouse lymphoma assay).

The (scientific and regulatory) basis, procedures and value of in vitro toxicity testing were dealt with in great detail in a recent document issued by a standardization committee of the German DIN (Deutsches Institut für Normung) and titled "Toxicological Assessment of Additives for Tobacco Products - A Guidance" (432). A review of the regulatory status worldwide and the approval requirements concerning additives for tobacco products - defined in the European Community Tobacco Products Directive 2001/37/EC (177) as "any substance or any constituent except for tobacco leaf and other natural or unprocessed tobacco plant parts used in the manufacture or preparation of a tobacco product and still present in the finished product, even if in altered form, including paper, filter, inks and adhesives" - had revealed that there were no assessment strategies, mandatory procedures or national or international norms for their toxicological evaluation quite different from the situation with chemicals and food additives. In line with the report's focus on tobacco additives, specific problems were addressed, such as the nature of the test materials (neat $v s$. pyrolyzed substances), the appropriate forms of test matrix, and ways of smoke generation. Several other subjects of discussion, however, were of general significance: the experimental use of the particulate and gaseous phases or of whole smoke; the scientific weight of in vitro vs. in vivo assays; the detailed review of available in vitro methods; the distinct aim to arrive at sensible recommendations, practical methods and agreements with regulatory authorities - all with the goal of assessing the health risks of tobacco products to avoid any potential increase from the use of additives (Figure 41). It should be noted that the definition of "additive" in the recently revised tobacco products Directive 2014/40/EU (433) as " $a$ substance, other than tobacco, that is added to a tobacco product, a unit packet or to any outside packaging" is more expansive than what was stated in the original Directive 2001/37/EC (177) and dealt with in the DIN document.

Traditional ways of in vitro smoke toxicity testing employ as substrates either condensate or gas phase but not whole smoke. The procedures used to obtain smoke fractions carry the risk of random capture and uncontrolled interaction and aging of constituents due to lapse of time. 


\section{Unburned Additive}

- Preparation of a toxicological dossier with available chemical and toxicological data

- Missing data to be completed by toxicological tests of the additive analogous to the assessment of chemicals
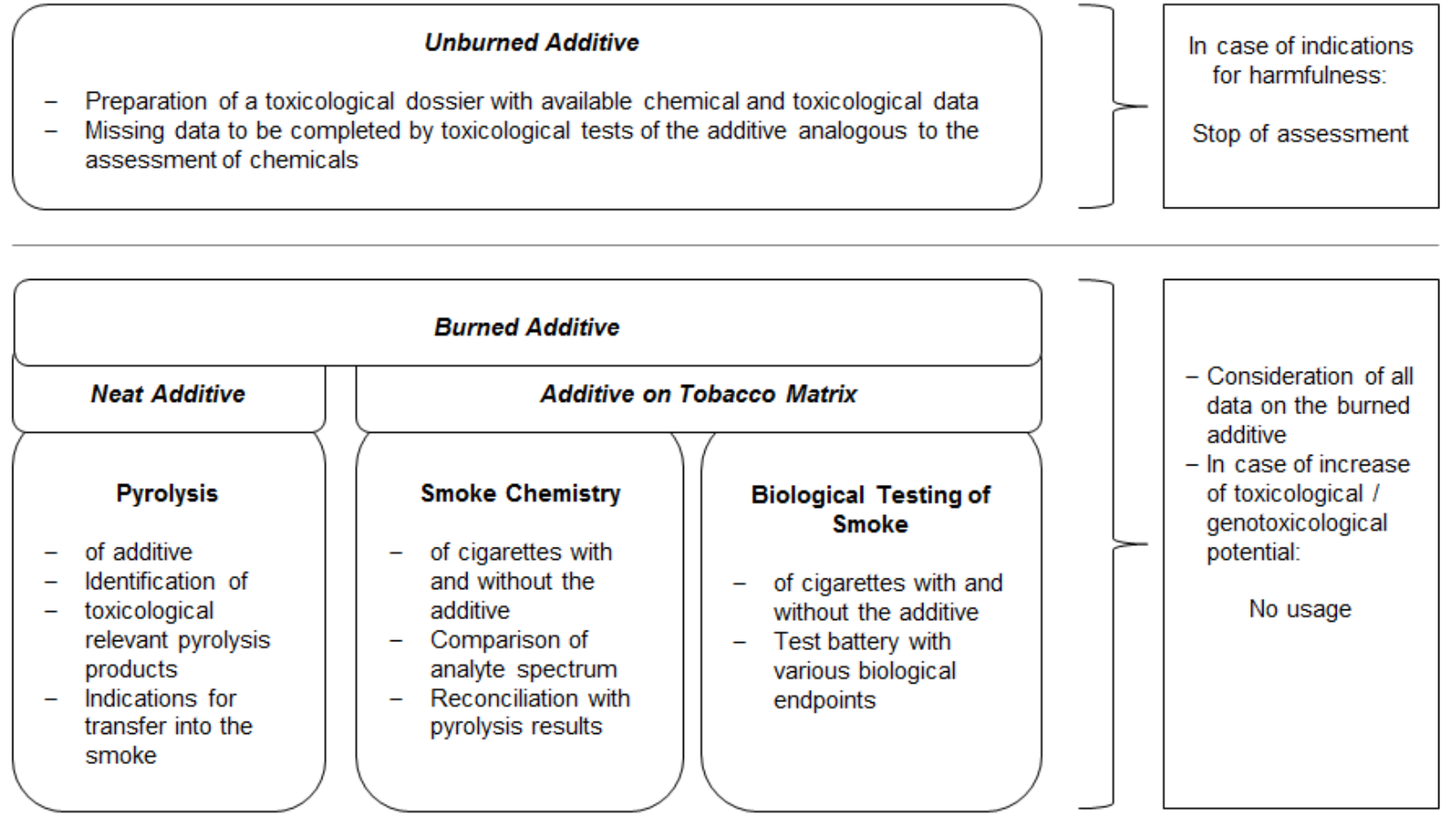

Figure 41. The DIN program (432) for the toxicological assessment of additives used in tobacco product manufacturing.

Potential interactions between particulate and vapor phase components remain unobserved. The practice of testing native whole smoke, likely to minimize or avoid such problems, was implemented by BOMBICK et al. (267) by using a "cellular smoke exposure technique" (CSET). Whole smoke was drawn at an adjusted flow rate through temperature-controlled flasks containing cells in submerged culture; the flasks were mounted and moved on a rocking platform in such a way that medium was temporarily drained off the cells for periodical intermittent direct exposure to aerosol with only a thin medium overlay (seven cycles per minute). In a similar way, cells were exposed to the gas phase of smoke after passage through a Cambridge filter.

Obviously, the "cellular smoke exposure technique" was a pragmatic step in the direction of outright exposure at the air-liquid interface (ALI), which was expected to allow the much more realistic toxicity assessment of cigarette smoke and other aerosols (Figure 42).

\subsection{Air-liquid interface (ALI) exposure studies}

\subsubsection{Early air-liquid interface (ALI) exposure studies}

Submerged cell cultures are an implausible model when used for in vitro toxicity testing of airborne inhalable materials; neither the natural anatomical orientation (polarity of differentiation) of the cells nor their physiological environment (gas phase, alveolar fluids and bronchial secretions) are properly reflected. In addition, medium covering the cells may impede the access of test materials to cell surfaces, bringing into play inherent solubility and diffusion properties with their variable effects on the different smoke constituents.

Taking a novel approach, VoISIN et al. $(434,435)$ succeeded in maintaining cells alive on a porous membrane, which was positioned on the surface of nutrient fluid inside a supporting plastic ring - all contained in a Petri dish. Alveolar macrophages were collected from guinea pigs by bronchoalveolar lavage, processed by centrifugation and re-suspension, and placed onto the center of the membrane. In order to reconstitute the physiological alveolar or bronchial micro-environment, a suspension of biological material, obtained in purified form from lavage supernate, was added to the cells. This way, cells could survive at the ALI with their apical side exposed to the atmosphere and their basal side brought in close contact - by capillary diffusion through the membrane - with the nutrient medium.

To demonstrate the good quality of the cells they were inspected by light and electron microscopy and examined for phagocytic and bactericidal activity. To assess viability, intracellular adenosine triphosphate (ATP) content was measured.

As a first application, VoISIN et al. (436) exposed guinea pig and human alveolar macrophages, cultivated at the ALI, at their apical side for $30 \mathrm{~min}$ to a continuous flow of nitrogen dioxide $\left(\mathrm{NO}_{2}\right)$ in air at various (relatively low) concentrations and observed dose-dependent morphological changes, loss of bactericidal activity and reduction of intracellular ATP content. 


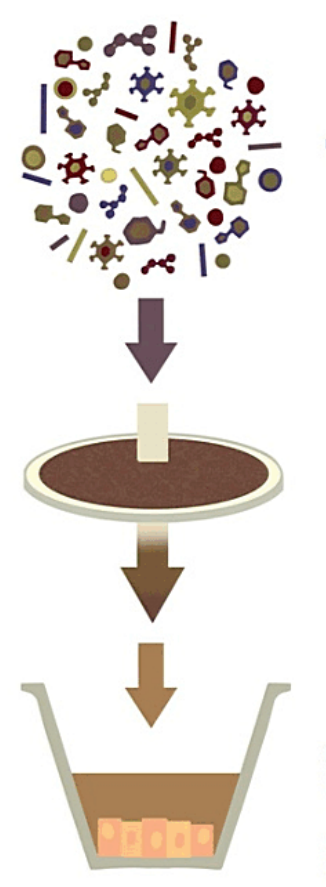

A

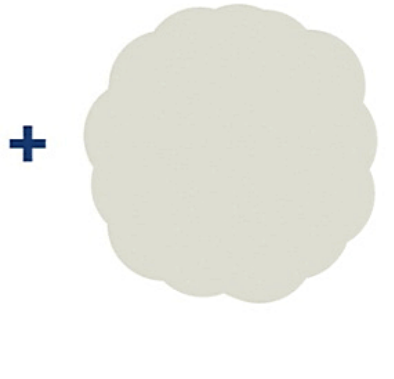

Cambridge filter pad trapped particulate

Eluted with solvent

Particulate matter diluted in medium

Cells (submerged)
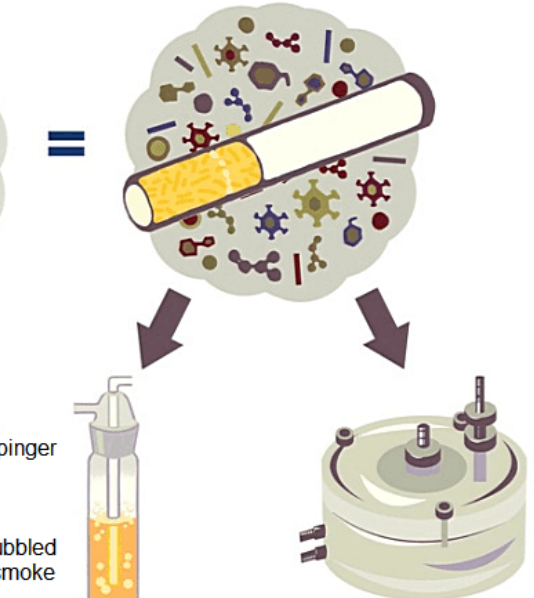

Exposure

chamber

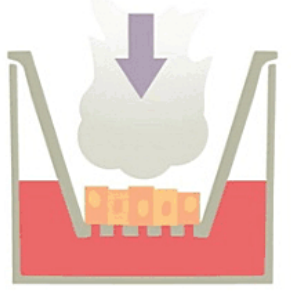

Whole smoke

Cells with ALI

Porous membrane

Media

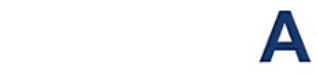

Figure 42. Submerged cells are used for toxicity testing of smoke particulate matter (A) and extracts (B) while exposure at the air-liquid interface is the preferred method for the examination of native whole smoke (C) (642).

Employing the ALI technique, a number of cultivation and exposure chambers were developed in the course of time. Significant progress in design was made when TARKINGTON et al. (437) developed - for a study of ozone effects on human respiratory epithelial cells - an exposure vessel containing five culture vials (collagen-precoated Milli$\mathrm{cell}^{\mathbb{B}}$-CM culture plate inserts) for direct luminal exposure; rat tracheal explant cultures were as well examined. At the top of the vessel the gaseous test material entered from above "through a jet oriented so that the atmosphere is injected tangentially to the wall and swirls across the tops of the culture vials", this way promoting the "mixing and even exposure among each of the five vials". The new technique exposed cells or tissue to a dynamic direct stream of gas rather than to static gas just filling, or flowing undirected through, a container.

The first study exposing cells at the air-liquid interface to tobacco smoke was conducted in 1995 by SuN et al. (381). Specifically, aged and diluted sidestream smoke was used as a surrogate for environmental tobacco smoke (ETS) owing to concerns soaring in the 1990s over ETS as a potential health risk. Sidestream smoke was generated from 1R4F Kentucky reference cigarettes under FTC conditions (19) by means of the smoke collection and exposure system developed by TEAGUE et al. (380) using an ADL II smoking machine (390) for puffing (presumably) two cigarettes by turns. Human bronchial epithelial cells (line BEAS-2B, immortalized by viral transformation) were grown on Millicell ${ }^{\circledR}$ microporous inserts $(0.4 \mu \mathrm{m}$ pore size $)$ and ex- posed in the incubator at the apical side for up to 6 hours to a concentration of $1 \mathrm{mg}$ TPM per $\mathrm{m}^{3}$. When cell viability was examined by several established methods (cell count; ${ }^{3} \mathrm{H}$-thymidine incorporation; MTT assay; resazurin assay) clear dependency of effects on exposure time was observed. In addition, cellular survival rates were shown to be dose dependent in the range of 1-9 mg TPM per $\mathrm{m}^{3}$.

At that time, the stage was clearly set for the challenging scientific and technical work to develop the concept of airliquid interface (ALI) exposure (Figure 43) into the variety of innovative methods available today for efficient in vitro toxicity testing.

\subsubsection{Air-liquid interface (ALI) exposure systems: Cultex $^{\mathbb{B}}$}

Efficient control of nutrient supply and temperature regulation were the key features of the cultivation and exposure chamber designed in 1999 by AUFDERHEIDE and MOHR (438) - referred to as Cultex ${ }^{\circledR}$ (which was to be developed into a sophisticated system of devices in the years to come). The original design and later improvements were developed at the Institute of Experimental Pathology of the Medical School, Hannover (Germany); a first patent was granted in 1999 to MOHR (439) for a culture apparatus and a method for cultivating cells or tissue components. Transwell $^{\circledR}$ inserts with porous polyethylene terephthalate (PET) membranes were turned to as ready-to-use permeable support for the cultivation of cells both under submerged 


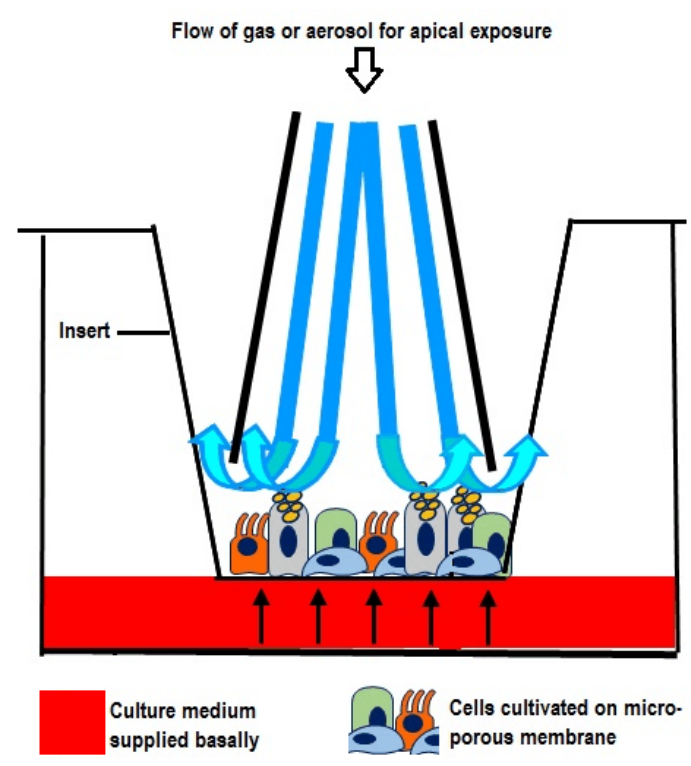

Figure 43. Concept of cell exposure at the air-liquid interface (ALI) to a direct vertical flow of gas or aerosol (Figures 43-45 used by courtesy of Cultex ${ }^{\circledR}$ Laboratories $\mathrm{GmbH}$, Hannover, Germany).

conditions and at the ALI. Generally, Transwell ${ }^{\circledR}$ inserts are available in a range of dimensions, pore sizes and different membranes. Three inserts - with a growth area of $1 \mathrm{~cm}^{2}$ each - were placed inside an elongated glass cultivation module (allowing an assay to be performed "automatically" in triplicates), and four modules were arranged side by side in the chamber (Figure 44). Each module was fitted with inlet and drainage nozzles for the supply of nutrient, the level of which in the inserts was controlled by an infrared sensor, as well as circulating water for temperature control.

In a first study (440), HFBE21 (human bronchial epithelial) cells were cultivated for 4 days, trypsinized and seeded onto porous Transwell ${ }^{\circledR}$ membranes. Cultivation could then be continued either submerged in a conventional incubator or in the Cultex ${ }^{\circledR}$ chamber under conditions of intermittent supply of nutrient: cells were covered by medium for a short period of time (minutes), which was then drained off, leaving the cells at the ALI for a considerably longer time (half an hour) - the cycle repeated for two days. Viability of the cells was examined by electronic cell counting and the WST assay. Both endpoints showed that cellular activity was much better preserved in cultures with intermittent nutrient supply, indicating that nutrient manipulation and the periodic occurrence of ALI phases did not impair cell number and vitality.

Titanium dioxide $\left(\mathrm{TiO}_{2}\right)$ particles and diesel soot were used in exposure experiments with cells grown under submerged conditions or with intermittent nutrient supply. The test materials were applied to the cells in the Transwell ${ }^{\mathbb{R}}$ inserts conventionally, i.e., suspended in basic medium at different concentrations. It is worth noting that in the case of intermittent nutrient supply cells were directly exposed to all particles during the pumping-off periods while with the constantly submerged cells particles remained in suspension during the whole exposure time of 24 hours. Cytotoxicity was assessed by cell count and the WST assay. Effects were variable, showing no cytotoxicity with $\mathrm{TiO}_{2}$ and a dose dependent reduction of metabolic activity by diesel soot.

As the next step, the Cultex ${ }^{\circledR}$ cultivation module with the vessels for three Transwell ${ }^{\circledR}$ inserts, medium supply and the possibility to regulate the temperature in the system was completed by a specially designed all-glass exposure top for introducing a gas or an aerosol, achieving homogeneous distribution above the cell cultures by establishing a continuous gas/aerosol flow. Cigarette smoke was examined by AUFDERHEIDE et al. (382) under conditions of ALI exposure. Interestingly, machine-generated sidestream smoke of 1R4F Kentucky reference cigarettes was used as experimental substitute for environmental tobacco smoke (ETS) - calling to mind the earlier study of SUN et al. (381). The overall instrumental set-up consisted of the smoking machine with a chimney for sidestream smoke collection, described by TEAGUE et al. (380) and discussed earlier (see page 192); a chamber for smoke aging and dilution; a multi-stage aerosol mixing system; and the Cultex ${ }^{\circledR}$ cultivation and exposure device. For aging and dilution, sidestream smoke and air were drawn into the $78 \mathrm{~L}$ chamber by a vacuum pump - with the objective of supplying diluted smoke of constant concentration over long periods of time; particulate and gas phase were monitored online by light scattering and $\mathrm{CO}$ measurements. The aerosol was then fed into a low flow linear mixing system (manufactured by the Fraunhofer Institute for Toxicology and Aerosol Research, now Fraunhofer Institute for Toxicology and Experimental Medicine) for dilution with mildly compressed air in four decimal steps; dilution rates of $1: 10$ to $1: 10,000$ could be achieved depending on which of four outlets the aerosol was drawn from. Again, light scattering and $\mathrm{CO}$ measurements were used for monitoring at the different stages. Following the production of aerosol continuously for three hours (collected from the large chamber and at different stages of dilution) it was shown that the technical efficiency and the time constancy of the system were impressive.

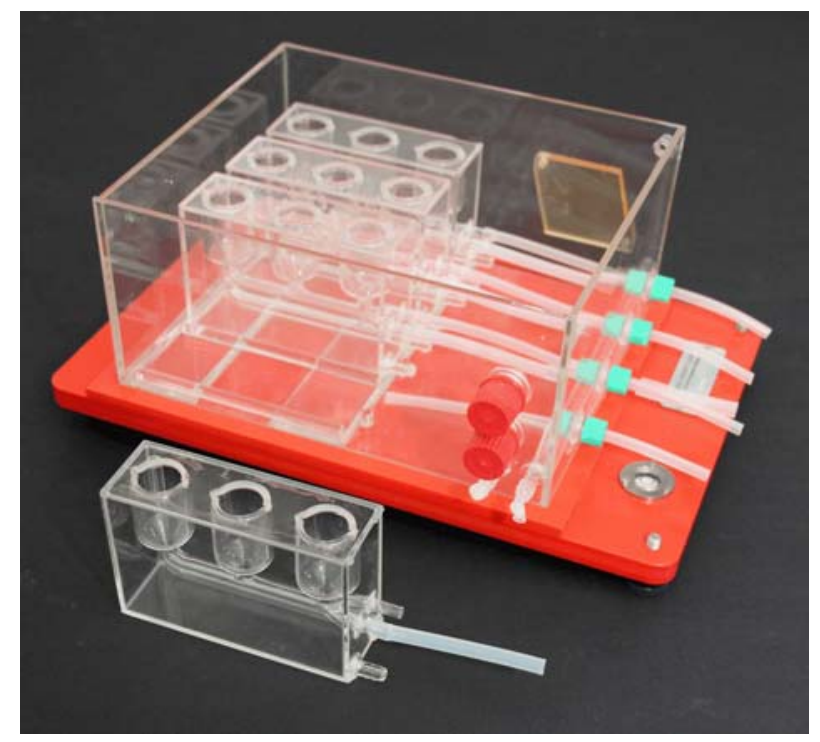

Figure 44. In the earliest Cultex ${ }^{\circledR}$ system (438) cells in PET membrane inserts were exposed to test particles suspended in the culture medium. 
Human bronchial epithelial cells (line HFBE21, sourced from the Medical School Hannover, Institute for Experimental Pathology) were seeded onto PET membranes of Transwell ${ }^{\circledR}$ inserts, grown for $54 \mathrm{~h}$ up to confluence, washed with phosphate buffered saline and finally placed in the Cultex ${ }^{\circledR}$ exposure unit (438). The level of nutrient medium was adjusted to moisten the membranes from below, this way creating ALI conditions. Test materials (clean air; appropriately diluted sidestream smoke - whole as well as filtered for particle elimination) were drawn into the exposure modules by negative pressure and fed through glass and teflon tubes directly into the individual inserts by means of the specifically designed exposure top, which allowed homogeneous aerosol distribution above the cell cultures. In this context, specific problems addressed in the study were the best aerosol flow rate (very important and eventually found to be $8.3 \mathrm{~mL} / \mathrm{min}$ per Transwell ${ }^{\circledR}$ insert) and the right exposure time $(60 \mathrm{~min})$.

Effects of aerosol exposure on cell viability were assessed by electronic cell counting and the WST assay, backed up by the determination of the intracellular content of total glutathione (reduced glutathione being a vital antioxidant in the maintenance of the intracellular redox balance). Dose dependent effects of smoke were observed for all three parameters (382).

In a follow-up study, WoLz et al. (383) duplicated accurately earlier work of AUFDERHEIDE et al. (382) except for the biological endpoint, which was now the genotoxic potency of aged and diluted sidestream smoke - as experimental substitute for environmental tobacco smoke (ETS) in two concentrations; synthetic air and nitrogen dioxide served as controls. DNA damage was determined by means of the very sensitive comet assay. The procedure called for exposed and dispersed cells to be embedded on a slide in agarose, lysed with detergent and salt to thoroughly disrupt cellular and molecular structures, and subjected to gel electrophoresis. This induces DNA fragments in single cells to migrate through the gel towards the anode (the smaller, the farther) forming a pattern, which often resembles a comet; consequently, data obtained by staining with ethidium bromide and fluorescence microscopy were expressed as "tail moment values", calculated as the product of the amount of DNA in the tail and the mean distance of migration in the tail and indicating the extent of DNA damage. At a smoke concentration with no observed loss of viability (assessed by electronic cell counting and the WST assay) - and after smoke exposure for 1 hour pronounced induction of DNA strand breaks was observed.

The efficiency and practicability of the Cultex ${ }^{\circledR}$ exposure unit with the specifically designed exposure top (382) was further examined by RITTER et al. (441) using human lung fibroblasts (Lk004) and human bronchial epithelial cells (HFBE21), exposed to air, ozone $\left(\mathrm{O}_{3}\right)$ or nitrogen dioxide $\left(\mathrm{NO}_{2}\right)$. Relevant biological effects were observed with ozone and nitrogen dioxide in the course of $120 \mathrm{~min}$ exposure time. Of particular interest was an experiment to determine - in the absence of cells - the reproducibility and intensity of test gas contact with the Transwell ${ }^{\circledR}$ membranes. The membranes were loaded with a reagent (sulfanylic acid and naphthylethylenediamine dihydrochloride), which - on contact with $\mathrm{NO}_{2}$ - forms a red azo dye, suitable for photometry. To assess reproducibility, an exposure time of $5 \mathrm{~min}$ was chosen for 10 separate tests, and remarkably small differences in delivery $(<5 \%)$ were consistently seen between the three inserts in a glass module. For quantification membranes were exposed for $30 \mathrm{~min}$ to $\mathrm{NO}_{2}$ (150-1,200 ppb); recovery rates (indicating gas-membrane contacts) were $20 \%$ for the lowest and $10 \%$ for the highest $\mathrm{NO}_{2}$ concentration used.

A well organized and instructively illustrated review of the work done by then on the development and applications of the Cultex ${ }^{\circledR}$ system was published in 2002 by AUFDERHEIDE et al. (442). It was amplified by another review in 2003 (443).

By then, a number of typical environmental pollutants (dust aerosol, ozone, nitrogen oxide, diesel exhaust and machine-generated diluted cigarette sidestream smoke) had been examined using ALI technology and Cultex ${ }^{\circledR}$ exposure modules. After discussions with, and supported by, scientists from the cigarette industry the scope of investigations was now expanded to include cigarette mainstream smoke.

First results of assessing the behavior and (toxicological) effects of native, physically unmodified cigarette mainstream smoke under ALI conditions, employing the Cultex $^{\circledR}$ system, were reported in 2003 by RITTER et al. (444) and AUFDERHEIDE et al. (445); "unmodified" relating

Table 6. Development of Cultex ${ }^{\circledast}$ exposure systems.

\begin{tabular}{|c|c|c|c|c|}
\hline Year & Type & Exposure top & Culture base & References \\
\hline 1999 & Cultex ${ }^{\circledR}$ & $\begin{array}{l}\text { not yet available (test material } \\
\text { supplied in the medium) }\end{array}$ & $\begin{array}{l}\text { glass chamber holding } 4 \text { glass } \\
\text { cultivation modules with } 3 \text { inserts } \\
\text { each }\end{array}$ & $(439,438,440)$ \\
\hline 2001 & Cultex $^{\circledast}$ & $\begin{array}{l}\text { all-glass exposure top; test material } \\
\text { supplied through teflon tubes }\end{array}$ & \multirow{3}{*}{$\begin{array}{l}\text { all-glass stand-alone cultivation } \\
\text { module with } 3 \text { inserts, medium supply } \\
\text { and temperature control }\end{array}$} & $\begin{array}{l}(382,441,383 \\
442,443,527)\end{array}$ \\
\hline 2003 & Cultex $^{\circledR}$ & $\begin{array}{l}\text { all-glass exposure top; test material } \\
\text { supplied through trumpet- shaped } \\
\text { inlets }\end{array}$ & & $(444,445,446)$ \\
\hline 2005 & Cultex $^{\circledast}$ & \multirow{2}{*}{$\begin{array}{l}\text { steel exposure top; test material } \\
\text { supplied through trumpet-shaped } \\
\text { inlets }\end{array}$} & & $(448,451,452)$ \\
\hline 2004 & Cultex-B $^{\circledast}$ & & $\begin{array}{l}\begin{array}{l}\text { cultivation module accepting } 3 \text { petri } \\
\text { dishes }\end{array} \\
\end{array}$ & $\begin{array}{l}(453,454,455 \\
449)\end{array}$ \\
\hline 2011 & Cultex $\mathrm{RFS}^{\circledR}$ & \multicolumn{2}{|c|}{$\begin{array}{l}\text { novel modular system for radial flow distribution of test material and } \\
\text { exposure in } 3 \text { inserts or } 6 \text { inserts (compact size) }\end{array}$} & $\begin{array}{c}(458,459,462 \\
460,465,467,468)\end{array}$ \\
\hline
\end{tabular}


to the fact that the smoke was neither heated or humidified nor enriched with carbon dioxide - manipulations with strong potential influence on particle quality and number. The studies used the 1R4F Kentucky reference cigarette and two experimental cigarettes with different ISO "tar" levels $(9.0 \mathrm{mg}$ and $1.4 \mathrm{mg})$. The instrumental set-up included the very versatile Borgwaldt single port, pistondriven smoking machine RM 1/G (315) delivering distinct puffs according to ISO/FTC parameters; a dilution tool absorbing the puffs into a stream of synthetic air and thereby creating (reproducibly) a continuous flow of test atmosphere of desired concentration; the Cultex ${ }^{\circledR}$ module with the Transwell ${ }^{\circledR}$ inserts for ALI exposure in combination with the specifically designed exposure top and a vacuum pump; and photometric online monitoring for particle analysis by light scattering and for $\mathrm{CO}$ and $\mathrm{CO}_{2}$ measurements by non-dispersive infrared (NDIR) analysis. It was shown that the smoke bolus, embedded in the constant stream of air, reached the exposure chamber after approx. $6 \mathrm{sec}$ - roughly comparable to the transit time observed in human smokers. Resulting from the continuous supply of fresh air, the smoke was cleared completely from the exposure unit before the next puff arrived. Particle deposition on membranes (without cells) was quantified by fluorescence after extraction with methanol in experimental runs, smoking one 1R4F cigarette with seven puffs and moderate smoke dilution, and found to amount to roughly $3.56 \mu \mathrm{g}$ particle mass per membrane. Starting from this number, RITTER et al. (444) gave themselves over to interesting (and somewhat courageous) guesswork correlating the quantities of particulate matter deposited onto Transwell ${ }^{\circledR}$ membranes and in a smoker's lungs.

Target cultures used in the studies were HFBE (human fetal bronchial epithelial) cells and A549 (adenocarcinomic human alveolar basal epithelial) cells. Cell viability was determined by electronic cell counting and by means of the WST assay; intracellular status was characterized by the adenosine triphosphate/adenosine diphosphate (ATP/ADP) ratio, glutathione level, and the ratio of oxidized and reduced glutathione (GSSG/GSH). No statistically significant changes of these parameters were observed with exposure to air for $60 \mathrm{~min}$, demonstrating the stability of the system. While exposure to mainstream smoke (from at most 4 cigarettes within $28 \mathrm{~min}$ ) showed limited effects with the low "tar" experimental cigarette, the viability of HFBE cells was dose dependently reduced by more intense exposure to smoke from the cigarettes with higher "tar" yield. Interesting effects were seen when HFBE cells were examined for intracellular parameters after short term exposure to only 6 puffs from the higher "tar" yield cigarette, followed by a 24-hour recovery period: ATP/ADP ratio was reduced by about $50 \%$, compared to control, immediately after exposure and increased to about $75 \%$ after 24 hours. In contrast, glutathione was down to $10 \%$ after exposure but had shot up to about $175 \%$ after recovery - an "overshoot" reaction typical for intensified cellular defense response.

The authors $(444,445)$ underlined that the in vitro exposure system using ALI technology minimized smoke transformation due to physical and chemical changes during generation and exposure. Intermittent smoke exposure with intermediate availability of fresh air was coming quite close to the physiological situation.

Using the same equipment - as in the two studies (444, 445) just discussed - for smoke generation and dilution, online monitoring and the exposure of cells at the ALI, RITTER et al. (446) took a functional approach to the comparative in vitro testing of cigarette mainstream smoke toxicity. The test battery included the 1R4F Kentucky reference cigarette ( $9.2 \mathrm{mg}$ "tar") and two commercial light filter brands from Italy and Greece with identical (imprinted) "tar" yields of $7.0 \mathrm{mg}$. However, when smoke was characterized it was quickly recognized that the two commercial brands, when smoked under standard conditions, differed in puff number and, when smoke was diluted 1:4.33 during cell exposure, in the concentrations of particles, carbon monoxide and carbon dioxide observations attributable to different tipping length and tobacco burn quality of the two cigarette brands. Both native whole smoke and gas phases were examined over a range of concentrations obtained by appropriate dilution. The human alveolar type II-like lung cell line A549 was used; and intracellular (reduced) glutathione content - as a meaningful endpoint of toxicological impact - was measured immediately after the exposure period of $32 \mathrm{~min}$. Impressive dose dependent reductions of glutathione were observed with all three test pieces for both whole smoke and gas phase (across all tests significantly less effective than whole smoke) and documented not only by unequivocal dose-response curves but also by the calculation of various $\mathrm{ED}_{50}$ values as a result of regression analysis. For this, the relative decrease compared to control was related to the number of cigarettes (the most realistic option) or puffs with due consideration of dilution, and to the concentration of particulate matter or carbon monoxide (itself inert but in the authors' view representing the cytotoxic gas phase). While, as expected, the 1R4F Kentucky reference cigarette produced the strongest reductions of intracellular glutathione, the two commercial brands (different tobacco blends but identical "tar" yields) showed strikingly different effects - the Greek brand considerably weaker than the Italian brand. The authors considered the methodology employed sufficiently sensitive and reproducible for detecting differences in the toxicological effects of smoke from different types of cigarettes.

In 2007, the CORESTA IN VITRO TOXICOLOGY TASK FORCE (447) released a summary report on an international collaborative study of in vitro exposure of cells to smoke at the air-liquid interface. Seven laboratories participated with the equipment available to them at the time: three laboratories used authentic continuous ALI exposure systems (twice Cultex ${ }^{\circledR}$ and in one case the BAT chamber described on page 214); two laboratories intermittent ALI exposure in conventional culture flasks on a rocker platform - in principle the "cellular smoke exposure technique" described by BOMBICK et al. (267); one laboratory standard 96-well cell culture plates with overlaying medium; and one laboratory smoke solutions under submerged conditions (TPM in dimethyl sulfoxide (DMSO) and gas vapor phase in buffered saline - also recombined to represent whole smoke). Test pieces were the $2 \mathrm{R} 4 \mathrm{~F}$ Kentucky reference cigarette and three trial cigarettes (made from single grade Burley, single grade flue-cured or a 50:50 blend of both). Experimental conditions were rather 

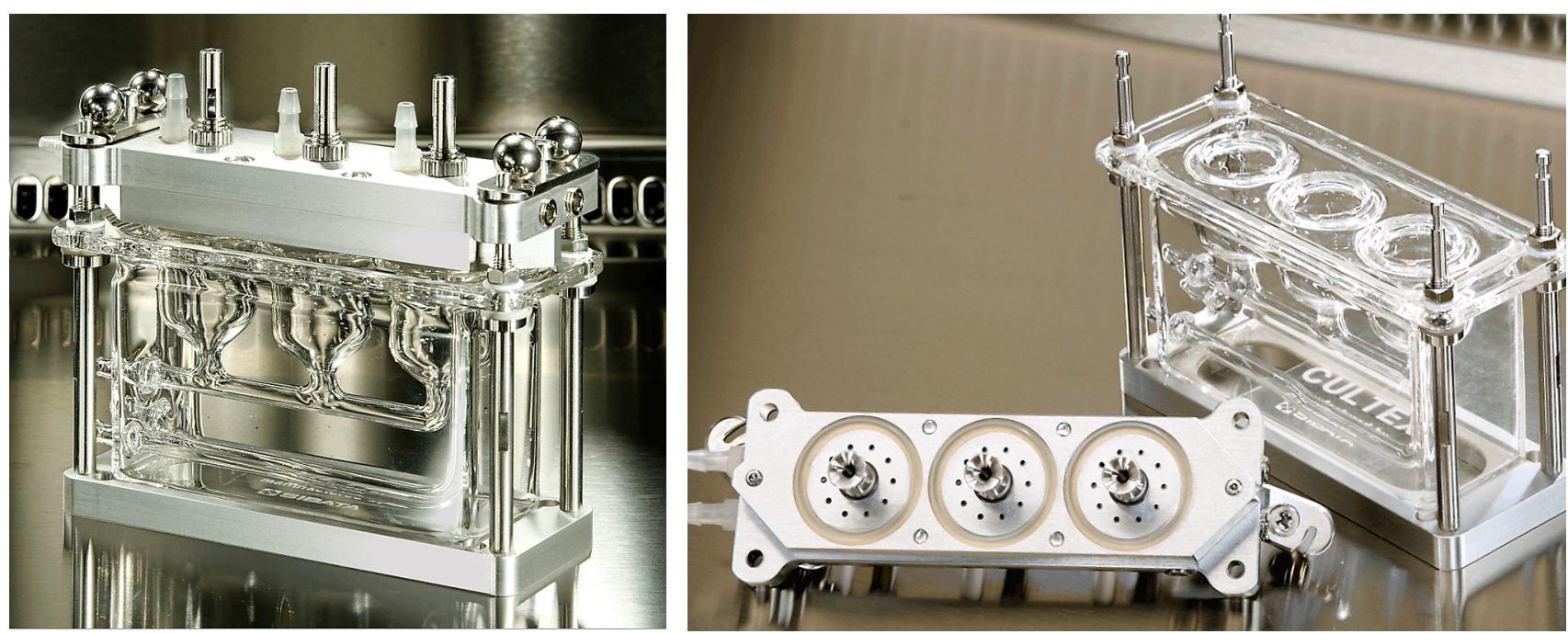

Figure 45. An advanced Cultex ${ }^{\circledR}$ system (448) featured an all-glass culture base combined with a stainless steel exposure top (left); viewed from below (right) the exposure top shows the central "trumpet-shaped" gas inlets and the circularly arranged gas outlets.

dissimilar regarding smoke generation, cell lines, and exposure and recovery periods. The biological endpoint of testing was cytotoxicity, assessed by the neutral red uptake assay as specified in a standard procedure. Participating laboratories were asked to rank the test pieces based on 50\% reduction of cell viability and to determine the contribution of particulate and vapor phases to the cytotoxicity of whole smoke. The outcome of the study showed a certain degree of conformity in ranking and good consistency at least among laboratories using ALI technology regarding the cytotoxic potency of the gas vapor phase (70-80\% of whole smoke). In a comprehensive and detailed paper, AUFDERHEIDE (448) summarized the historical development of in vitro toxicity testing, critically appraised the characteristics and value of different in vitro methods with special emphasis on air-liquid interface (ALI) exposure techniques, and reviewed the most recent developments of the Cultex ${ }^{\circledR}$ system. Specifically, its exposure top was now largely made from stainless steel with improved design of the ducts for gases and aerosols: a "trumpet-shaped" central inlet and several surrounding circularly arranged outlets (Figure 45). To continue an exacting and productive in vitro research program on the biological properties of cigarette mainstream smoke a suitable and dedicated smoke production system was required. Accordingly, the research group of MOHR and AUFDERHEIDE (at the Fraunhofer Institute of Toxicology and Aerosol Research, Hannover, Germany; afterwards Cultex ${ }^{\circledR}$ Laboratories $\mathrm{GmbH}$ ) set out to develop - with advice from experts in the tobacco industry - a smoking robot specifically designed for cell culture work, which was manufactured by Vitrocell ${ }^{\circledR}$ Systems (Waldkirch, Germany) and called "smoking robot" VC $10^{\circledR}$.

The smoking robot VC $10^{\circledR}$ (Figure 46) is a single syringe machine with a horizontally positioned rotary head, which can be automatically loaded with up to 10 cigarettes. It is placed under a hood with an integrated fan connected to a chimney for extracting sidestream smoke. Air flow at the cigarette holders is adjusted by fan speed and may be controlled by a hand-held anemometer. The smoking robot can operate in single or serial smoking mode for a defined number of cigarettes. Lighting of the cigarettes is effectuated by a contact-free electric lighter. All smoking parameters, such as puff duration, puff volume, puff frequency, puff profile and exhaust duration, can be controlled individually for compliance with ISO 3308 (105), Canadian Intense (22) or other regimes - with all data processed electronically. The butt extractor operates, after a pump stop, to a fixed puff number or a signal from the IR sensor, which stops puffing at the desired butt length. The smoke may exit through one or several (up to 5) ports for transfer to a continuous flow dilution system. Air is introduced through two facing inlets into a hollow bar to ensure turbulent mixing with the aerosol, which is then drawn towards the exposure module(s) by negative pressure. Final concentrations can be adjusted by setting diluting air flow at different rates. The new smoke generating device was clearly documented (description and figures) in a review by AUFDERHEIDE (449).

Following the completion of its development the smoking robot $\mathrm{VC} 10^{\circledR}(450)$ became and still is commercially available from Vitrocell ${ }^{\circledR}$ Systems GmbH (Waldkirch, Germany).

OKUWA et al. (451) investigated the effect of mainstream whole smoke and gas vapor phase, produced (most likely) with a VC $10^{\circledR}$ smoking robot from $2 \mathrm{R} 4 \mathrm{~F}$ Kentucky reference cigarettes according to ISO 3308 (105) or Canadian Intense (22), on the induction of micronuclei in Chinese hamster lung cells. The cells in microporous membrane inserts were exposed in Cultex ${ }^{\circledR}$ modules (448) at the ALI. The formation of micronuclei was related to the amount of smoke entering the exposure module (either calculated or monitored by means of a light scattering photometer) and found to be dose dependent - though not linear - under both smoking regimes. When response, however, was evaluated based on TPM equivalents, both whole smoke and gas vapor phase produced under ISO conditions seemed to be more effective. No different conclusion was reached with dry particulate matter (DPM = water-free TPM) chosen as reference in an attempt to eliminate the potential influence of increased water formation under Canadian Intense conditions. These findings are corroboratory evidence in support of the observations made by RICKERT et al. (417). 


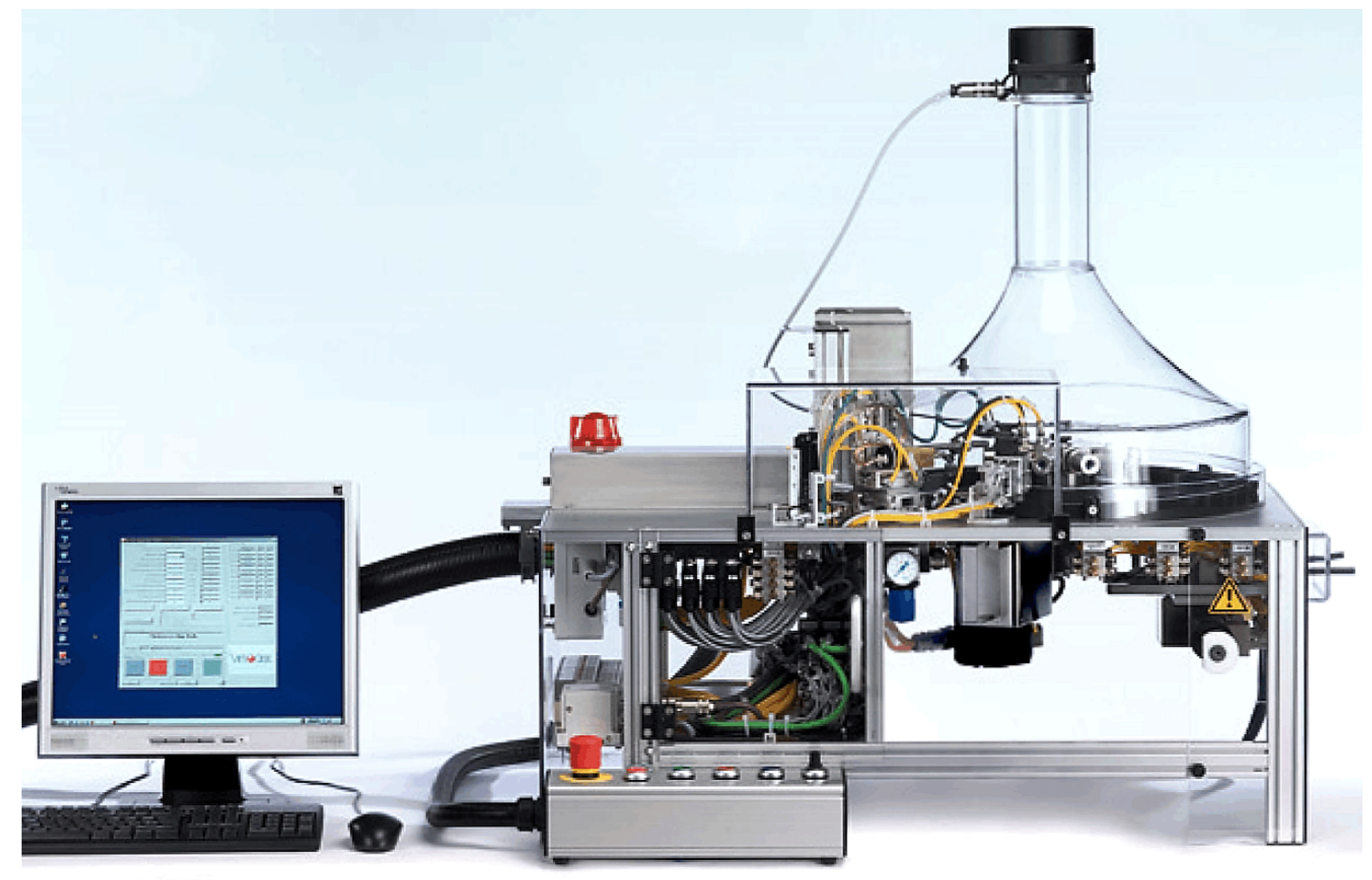

Figure 46. The Vitrocell ${ }^{\circledR}$ VC $10^{\circledast}$ smoking robot was specially designed for in vitro research and distinguishes itself by a short distance between smoke generation and exposure (450).

The cytotoxic potential of the water-insoluble gaseous fraction of cigarette mainstream smoke was investigated by NARA et al. (452). This particular test material - not examined before - was produced with a VC $10^{\circledR}$ smoking robot and by passing the smoke of modified $2 \mathrm{R} 4 \mathrm{~F}$ Kentucky reference cigarettes first through a glass fiber filter and then through 6 impingers in series, loaded with ice-cold phosphate buffered saline. Test cigarette modification consisted in replacing the original filter with a non-ventilated cellulose acetate filter with high efficiency and created a test piece with a "tar" yield of $10.6 \mathrm{mg}$. It was shown that the typical water-soluble constituents of the gas vapor phase were retained quantitatively in the (first two) impingers by analyzing eight carbonyls as dinitrophenylhydrazones. Chinese hamster ovary cells (CHO-K1), seeded on the microporous membranes of cell culture inserts, were exposed in Cultex ${ }^{\circledR}$ modules (448) at the ALI to appropriate dilutions of the gaseous phase flowing from the impingers and then tested in the neutral red uptake assay. Significant dose-dependent cytotoxic effects were observed. However, data revealed quite clearly that the principal cytotoxic potential of the mainstream smoke whole (unfractionated) gas phase resided in its water-soluble constituents.

The further development of the Cultex ${ }^{\circledR}$ system took a new turn when AUFDERHEIDE and MOHR (453) reported its technical adaption to performing the in vitro bacterial reverse mutation test (the classical Ames test). After the advantages of rapid and direct exposure of cells (at the ALI) to unchanged cigarette smoke had been clearly established, the continued use of smoke (fractions), taken up and kept in dimethyl sulfoxide (DMSO) or buffered saline, in the Ames assay left uneasy feelings. The use of spread cultures - bacteria plated on agar - for the static exposure to gases in closed vessels (for up to $24 \mathrm{~h}$ ) or for exposure by continuous flow (for 30-60 min) produced results that were strongly dependent on experimental conditions. For the delivery of physically and chemically unchanged native cigarette mainstream smoke directly onto the surface of suitable Salmonella cultures, grown conventionally in small Petri dishes without overlay agar according to the spread culture method, the top part of the exposure module was redesigned and (subsequently) called Cultex ${ }^{\circledR}$ B. It enclosed three individual gas inlets, which were arranged side by side, separately supplied with smoke and surrounded by a circular arrangement of openings for smoke removal through separate outlets. Each vertically oriented inlet had the form of a small pipe with a hyperboloid opening at the bottom end - shaped like a trumpet for the specific purpose of distributing air or aerosol uniformly to the surface of the bacterial cultures. This design was based on fundamental considerations of fluid mechanics and confirmed by numerical computer simulation. For exposure, the base of the module, designed to be manually movable and to hold three Petri dishes, was positioned closely under the three inlets, lifted and tightly connected to the exposure top.

For demonstrating the feasibility of the new instrumentation, bacteria of strain Salmonella TA 98 were exposed intermittently to mainstream smoke produced under ISO 
standard conditions (184) from 1R4F Kentucky reference cigarettes (smoking robot VC $10^{\circledR}$ ). Mutagenic effects (i.e., the induction of revertants) were clearly dose dependent; they increased with the number of cigarettes smoked (up to a certain point when cytotoxicity became a harmful factor) and with reduced dilution.

The experimental conditions of using the Cultex ${ }^{\circledR} \mathrm{B}$ exposure module and possible scientific findings were further explored by AUFDERHEIDE and GRESSMANN (454). To this, 2R4F Kentucky reference cigarettes were smoked under ISO standard conditions on the VC $10^{\circledR}$ robot; the gas vapor phase (GVP) was obtained after mainstream smoke passage through Cambridge filters. Dilution of smoke was achieved by mixing it, at different ratios, with a continuous stream of air. Flow rates through the exposure module, driven by negative pressure and a very influential parameter, could be set over a wide range. The Salmonella typhimurium strains employed were TA 98 (frameshift mutations) and TA 100 (base pair substitution) - in the presence or absence of S9 activation mix.

For estimating the efficiency of whole smoke particle deposition Petri dishes were loaded with Cambridge filters on a metal disc instead of bacteria on agar, and precipitated material was quantified after filter extraction by fluorescence. The most striking observation was the powerful influence of the flow rate through the module on relative deposition, which was about 10 times higher at $8 \mathrm{~mL} / \mathrm{min}$ compared to $100 \mathrm{~mL} / \mathrm{min}$.

Whole smoke, which was examined with strains TA 98 and TA 100 and always required S9 activation, showed higher induction of revertants with similar smoke supply in spread culture assays with direct exposure (at $8 \mathrm{~mL} / \mathrm{min}$ flow rate) compared to conventional plate incorporation tests.

Nitrogen dioxide $\left(\mathrm{NO}_{2}\right)$ was used as positive gas control and found with preincubation and direct exposure to produce maximum revertant rates at the highest flow rate in the exposure module tested $(100 \mathrm{~mL} / \mathrm{min})$ - a definitely different observation compared to what was seen with the aerosol (whole smoke). This was confirmed in tests with GVP, which (after preincubation) was mutagenic only in TA 100 and in the absence of S9-mix, and required the top flow rate for strongest effects.

To assess the contribution of the gas vapor phase to total mutagenicity of whole smoke, both materials were tested (using TA 100) under optimum conditions (whole smoke: $\mathrm{S} 9$ activation and $8.0 \mathrm{~mL} / \mathrm{min}$ flow rate; gas vapor phase: preincubation, no $\mathrm{S} 9$ activation and $100 \mathrm{~mL} / \mathrm{min}$ flow rate). Revertant numbers were compared on the basis of "tar" and the equivalent quantum of gas vapor phase. It turned out that the contribution of GVP was minimal.

While instrumentation for smoke generation and exposure was identical to that used in the work (454) discussed above, the range of bacterial strains (several Salmonella typhimurium and one E. coli) and test cigarettes was considerably widened in a new study by AUFDERHEIDE and GRESSMANN (455), published in 2008. Besides the 2R4F Kentucky reference cigarette ("tar" yield of $9 \mathrm{mg}$ ), 4 commercial brands with "tar" levels of 1, 4, 6 and $10 \mathrm{mg}$ (no further details available) were used for the generation of whole smoke and gas vapor phase (GVP). Nitrogen dioxide $\left(\mathrm{NO}_{2}\right)$ served as positive control. Test strains included Salmonella TA 98, TA 100, TA 102, TA 1535 , TA 1537 and TA 1538 as well as six YG descendants of TA 98 and TA 100; in the YG strains the introduction of further plasmids results in increased sensitivity to the mutagenic activity of nitroarenes and aromatic amines. The E. coli strain used was WP2uvrApKM101, exhibiting tryptophan dependency. Based on earlier experience, flow rate through the exposure module was $8.0 \mathrm{~mL} / \mathrm{min}$ with whole smoke and $100 \mathrm{~mL} / \mathrm{min}$ with $\mathrm{GVP}$ and $\mathrm{NO}_{2}$; $\mathrm{S} 9$ metabolic activation mix was added with whole smoke and absent with GVP and $\mathrm{NO}_{2}$; preincubation for 5 hours was required with GVP and $\mathrm{NO}_{2}$. All strains used were tabulated according to their mutations (frameshift or missense) and their gene and plasmid configurations. First, mutagenic potency was measured and documented for the 2R4F Kentucky reference cigarette (whole smoke and GVP) in combination with all test strains. Sensitive strains were identified (remarkably, not the same for whole smoke and GVP), and results were interpreted considering certain strain characteristics (presence of plasmids such as pKM101; mutations in the his gene).

In an attempt to rank the mutagenicity of cigarettes with different "tar" yields the 2R4F Kentucky reference cigarette and the four commercial brands were examined using the most suitable strains: TA 98 with whole smoke and TA 100 with GVP. Dose dependent mutagenic potency was convincingly demonstrated for whole smoke of all test pieces, though not completely in line with "tar" yields. In principle, the mutagenicity of GVP also increased with "tar" content but the effect of the $2 \mathrm{mg}$ "tar" brand was rather weak and of the $1 \mathrm{mg}$ "tar" brand not detectable.

In a comprehensive review, published in 2008, AUFDERHEIDE (449) summarized the concepts and evolution of the air-liquid interface (ALI) exposure technology from its beginning in the 1970s to the present state of development, which was represented by the Cultex ${ }^{\mathbb{B}} \mathrm{B}$ system. The paper included a large number of very instructive figures (graphs and photos) and recapitulated the considerable number of studies done so far on gases and complex mixtures, such as cigarette smoke and Diesel exhaust. Particular attention was paid to technical equipment and the evaluation of toxicological endpoints (cytotoxicity and genotoxicity). In an exacting consensus document from a 2009 workshop on exposure studies for in vitro toxicity testing of engineered nanoparticles - found in a rapidly increasing number of industrial and consumer products - PAUR et al. (456) presented an overview of the main issues regarding nanoparticle exposure, lung physiology, biological mechanisms of (adverse) reactions, in vitro cellular exposure systems, realistic doses and risk assessment. For the evaluation of lung toxicity, clear preference was expressed for air-liquid interface (ALI) rather than submerged exposure systems. Current methods for in vitro toxicity testing of airborne chemicals and more advanced exposure techniques - with special emphasis on cells cultured on permeable microporous membranes in Transwell ${ }^{\mathbb{B}}$ inserts - were reviewed in 2011 by BAKAND and HAYES (457).

A redesigned apparatus, the Cultex ${ }^{\circledR}$ RFS (radial flow system), for exposure to airborne particulate materials at the air-liquid or air-agar interface was introduced in 2011 by AUFDERHEIDE et al. (458). The new device was depicted in the publication but not described in technical 
detail. It was reported to work on the principle of "radial distribution of the test atmosphere from one sampling point to the cells, resulting in a homogeneous deposition of particulate matter on the cells which can be calculated according to a simplified model ...... . The physical forces driving the deposition of airborne material are: (1) sedimentation, (2) diffusion, (3) electrical forces and (4) inertial impaction when using a very small flow through the module .....". The outcome of a number of assays was reported, which used proven ways of cigarette whole smoke and gas vapor phase generation in combination with the Cultex $^{\circledR}$ RFS system. Test pieces, smoked on a VC $10^{\circledR}$ smoking robot under standard conditions (184), included the Kentucky reference cigarettes 3R4F (9.4 mg "tar") and 1R5F (1.67 mg "tar"), the CORESTA monitor CM 5 (15 mg "tar") and an unidentified commercial brand (7 mg "tar"). Using very similar methodology, findings of earlier studies $(454,455)$ were fully confirmed regarding the mutagenicity of whole smoke and gas vapor phase. When human immortalized bronchial epithelial cells (line 16HBE14o-) were exposed to whole smoke from the two Kentucky reference cigarettes, very impressive doseresponse curves were obtained (dose $=$ number of cigarettes smoked; response $=\%$ cell viability compared to control, measured with the WST assay); they reflected clearly the different "tar" yields of the test pieces.

The electronic supplementary material of the publication (458) is worthwhile looking at; it contains instructive theoretical considerations regarding the physical mechanisms effecting the deposition of airborne particles.

In 2013, AUFDERHEIDE et al. (459) supplemented the pictorial representation of the Cultex ${ }^{\circledR}$ RFS device by providing more detailed information on its construction and function (Figure 47). It was built from five different parts (an adaptor and four modules): (I) the inlet adapter with a specifically designed jet nozzle, which constituted the connection with the aerosol source (smoking machine or dust generator); (II) the aerosol guiding module for conducting and distributing the aerosol to the cell cultures for direct deposition and eventual emission; (III) the sampling module with the three exposure chambers, which held cell culture inserts or small Petri dishes and were each separately supplied with nutrient medium; (IV) the socket module supporting the sampling module and providing space for additional functions; and (V) the locking module as a platform for sliding the combined sampling and socket modules manually along rails into the right positions for loading and exposure. Optionally, the guiding and sampling modules were operated under temperature control. The whole system was made complete by incorporating a computerized dust generator, which produced - to particle size - dry aerosols by scraping off material from highly compressed powder cakes prepared by means of an electronically controlled pneumo-hydraulic press. The dust generator was combined with an elutriator - an apparatus capable of removing oversized particles by sedimentation in a vertical upward flow of air. Another option was the inclusion of an electrical device for increasing particle deposition efficiency.

The main improvement in the Cultex ${ }^{\circledR}$ RFS system rested on the technical design of the guiding module. Computational fluid dynamics analysis for the simulation and

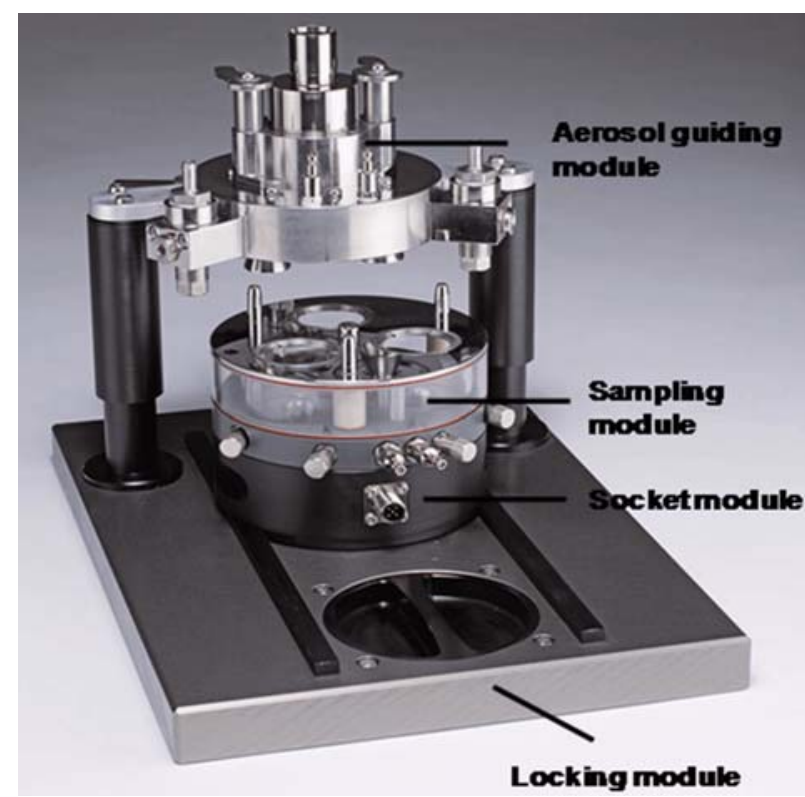

Figure 47. Four separate modules with specific functions are combined to form the Cultex ${ }^{\circledR}$ RFS aerosol exposure system (458).

optimization of particle flow within the system (elutriator, tubing and exposure modules) was heavily relied on for refining the geometry of the aerosol inlet and achieving stable, reproducible and homogeneous deposition of particles.

The utility of the Cultex ${ }^{\circledR}$ RFS system was demonstrated (459) by exposure tests with a number of dry aerosols (dusts) prepared from lactose monohydrate, copper(II) oxide micro particles (primary size: $5 \mu \mathrm{m}$ ), copper(II) oxide nano particles (primary size: $40-80 \mathrm{~nm}$ ) and copper(II) sulfate. Particle number and mass distribution were recorded with an aerodynamic particle sizer. Particle mass was determined gravimetrically with a precision balance after collecting samples from the aerosol stream on glass fiber pads at three points: directly at the elutriator outlet; at the outlet of the guiding module immediately before entry into the exposure chamber; and at the insert membrane surface. The number of deposited particles could then be calculated drawing on data obtained with the aerodynamic particle analyzer. Deposition rates of $10-35 \%$ were generally achieved with the Cultex ${ }^{\circledR}$ RFS system.

To compare the biological activity of the four powder aerosol preparations, A549 cells were exposed, with comparable particle mass deposition, for 15,30 and $60 \mathrm{~min}$ at the ALI, and cell viability was analyzed using the WST test. While lactose monohydrate was only slightly cell damaging, all copper compounds showed significant dosedependent cytotoxic effects; the water soluble copper(II) sulfate was the most potent agent.

Without further changes of the Cultex ${ }^{\circledR}$ RFS system, RACH et al. (460) reported the outcome of dose response studies of mainstream cigarette whole smoke and dust aerosols in different lung epithelial cell lines. The 3R4F Kentucky reference cigarette was smoked on a VC $10^{\mathbb{B}}$ smoking robot under ISO standard conditions (105). Besides using lactose monohydrate as negative control, aerosols were prepared from pressed powder cakes of copper(II) sulfate, titanium 
dioxide $\left(\mathrm{TiO}_{2}\right.$; primary particle size: $\left.25 \mathrm{~nm}\right)$ and cerium dioxide $\left(\mathrm{CeO}_{2}\right.$; primary particle size: $\left.50-80 \mathrm{~nm}\right)$. The cells tested were the immortalized human bronchial epithelial cell line 16HBE14o-, the adenocarcinoma derived human submucosal gland cell line Calu-3, and the human lung adenocarcinoma cell line A549. They were all first cultivated on microporous polycarbonate insert membranes under submerged conditions, and then transferred to the Cultex ${ }^{\circledR}$ RFS device and directly exposed at the ALI.

Following exposure to diluted smoke from up to eight 3R4F Kentucky reference cigarettes a dose dependent decrease of cell viability was observed with 16HBE140cells. The picture was remarkably different with Calu-3 cells: Viability increased dose dependently after exposure to one and two cigarettes and then decreased dose dependently, barely dropping below $100 \%$ of control when as many as 8 cigarettes were smoked. It was speculated that Calu-3 cells might be protected by the formation of functional tight junctions, when cultivated under ALI conditions - a protective mechanism not available to 16HBE14o- cells. Cell viability was determined by means of the resazurin assay. Concurrent multiple controls (clean air exposure or incubation only) confirmed that cells were remarkably stable under the conditions of Cultex ${ }^{\circledR}$ RFS use.

The effect of mainstream whole smoke on the content of cytoskeletal $\beta$-tubulin (a globular protein for the assembly of microtubules) was examined in 16HBE14o- cells. Using western blot analysis, $\beta$-tubulin was shown to be markedly reduced after exposure to diluted smoke from four and eight 3R4F Kentucky reference cigarettes.

Cell viability after exposure to dust aerosols was studied with A549 cells, using the WST-1 assay. In all tests, deposition intensity was adjusted to $25 \mu \mathrm{g} / \mathrm{cm}^{2}$ per $15 \mathrm{~min}$ (corresponding to $184 \mathrm{pg}$ per cell) - measured gravimetrically. Deposit mass was controlled by treatment time (15, 30 or $60 \mathrm{~min}$ ). Dose dependent and substance specific effects were observed in all exposure tests. As seen in an earlier study (459), cell viability was only slightly affected by lactose monohydrate but decreased in a pronounced way with water soluble copper(II) sulfate. Aerosols of $\mathrm{TiO}_{2}$ and $\mathrm{CeO}_{2}$ produced moderate cell damage, $\mathrm{TiO}_{2}$ being the more powerful compound. As both compounds are chemically non-toxic the effects were attributed to particle size in the nanometer range.

Using solanesol and acetaldehyde as markers for the particulate and gas/vapor phases, IsHIKAWA et al. (461) compared cigarette smoke deposition in the Cultex ${ }^{\circledR}$ RFS system and retention in the human respiratory tract (mouth, bronchi and alveoli assessed separately). For chemical analysis 3R4F Kentucky reference cigarettes were smoked on a VC $10^{\circledR}$ robot in compliance with ISO (105) and Health Canada Intense (22) regimes, and yields of solanesol (collected on Cambridge filters) and acetaldehyde (absorbed in gas tubes containing silicagel impregnated with 2,4-dinitrophenylhydrazine for immediate derivatization) were both determined by HPLC. For producing deposition data each chamber of the Cultex ${ }^{\circledR}$ RFS sampling module was equipped with two nested inserts with impermeable bottom (no membranes and no cells), which were filled with trapping solutions, namely methanol for solanesol and water for acetaldehyde, providing surface areas of $0.9 \mathrm{~cm}^{2}$. Quantification was done again by means of HPLC (after dinitrophenylhydrazone formation in case of acetaldehyde); data was evaluated on a per cigarette basis. Mean deposition of both markers was in the low $\mu \mathrm{g} /$ cigarette range with modest smoke dilution (approximately 1:15) and proportional to ISO and Canadian Intense yields, respectively. The highest deposition rates for both markers observed under all experimental conditions (on the rather small surface of $0.9 \mathrm{~cm}^{2}$ ) hardly exceeded $0.2 \%$ of marker amounts in smoke.

The human retention study was conducted with a $1 \mathrm{mg}$ "tar" Japanese commercial cigarette. Retention (uptake) of the two markers was estimated as the difference between intake as smoke and elimination in exhalate. By means of artificial smoking under a rather wide range of conditions (puff volume and interval), suitable correlations were established between solanesol retained in the filter and the amounts of solanesol and acetaldehyde in smoke available for inhalation, which allowed the determination of intake from each cigarette by filter analysis. Exhaled smoke was collected on Cambridge filters and in sampling bags for analysis. Study participants followed three different inhalation patterns: 1) "mouth hold" = taking a puff and holding the smoke in the mouth for 2 sec before exhaling; 2) "restricted inhalation" = after holding the smoke in the mouth, inhaling $150 \mathrm{~mL}$ of air from a bag (filling up also the bronchial region); 3) = "normal inhalation" = after holding the smoke in the mouth, inhaling normally with smoke permeating the whole respiratory tract. This way and after appropriate calculation, retention could be estimated differentially for the mouth, the bronchial region and the alveoli. Solanesol was taken up in comparable quantities in each of the three respiratory regions (in total, more than $60 \%$ of available solanesol) while acetaldehyde was predominantly retained in the mouth and the bronchi and very little in the alveoli (in total, nearly $90 \%$ of available acetaldehyde) - very interesting information but, unfortunately, no specific data like variability between the seventeen study participants was released. Nota bene, these percentages were brought about by the very large surface area of the respiratory tract - forming the basis for comparative reflections regarding deposition intensity in vitro and in vivo.

No doubt, the reduction and eventual replacement of animal use in certain toxicological tests is the forceful driving (scientific and ethical) motive for the development of the ALI technology. Towards this goal, documented validation and regulatory acceptance are indispensable requirements. STEINRITZ et al. (462) started out to assess the intra- and inter-laboratory reproducibility of the Cultex $^{\circledR}$ RFS system for formal acceptance as an alternative to animal testing. The process followed the modular approach for test validation (463) as defined by the European Centre for the Validation of Alternative Methods ECVAM (464). Three independent laboratories participated in the study, which examined a number of inorganic fine dusts and nanoparticles for cytotoxic effects on human lung epithelial cells (A549). The technical equipment consisted of a hydraulic press for producing powder cakes of the test substances and a scraping dust generator combined with an elutriator for trapping oversized particles (both devices in-house developments by Cultex ${ }^{\circledR}$ Laboratories $\mathrm{GmbH}$, supported by the German Federal Department of 
Education and Research), two Cultex ${ }^{\circledR}$ RFS modules (for aerosol testing and in parallel for clean air control) and a vacuum unit. The following test materials were used: Lactose monohydrate (non-toxic reference), barium sulfate (non-soluble reference), copper(II) sulfate (exhibiting very high cytotoxicity), carbon black, titanium dioxide, cerium(IV) oxide, copper(II) oxide nanoparticles, copper(II) oxide microparticles, and magnesium oxide. Powder cakes were prepared for the production of particulate aerosols by scraping, which were then routed through the elutriator to the exposure module. The viability of A549 cells was determined by the WST-1 assay after exposure periods of 15,30 or $60 \mathrm{~min}$ and 24 hours post-exposure recovery. In a first assessment round, $60.9 \%$ of all (twenty-three) comparisons showed non-significant differences (indicating good inter-laboratory reproducibility). Shaky (and so far unexplainable) clean air controls in two participating laboratories (reduced clean air viability compared to incubator controls) seemed to have contributed to the remaining discrepancies. When tighter quality criteria were retrospectively - applied and a number of comparisons excluded from the evaluation the rate of good inter-laboratory reproducibility increased to $76.5 \%$.

Nearly all ALI exposure studies done so far had examined effects of acute toxicity. In contrast, conditions of repeated exposure used by AUFDERHEIDE et al. (465) allowed the assessment of sub-chronic effects of cigarette mainstream smoke exposure in non-toxic concentrations on ciliated cells. Freshly isolated primary normal human bronchiolar epithelial (NHBE) cells with in vivo like morphology were grown on coated insert membranes to $100 \%$ confluency, allowed to differentiate for 10 days and then subjected to eight daily exposures in the Cultex ${ }^{\circledR}$ RFS system within 10 days (with two recovery days after the sixth exposure). Mainstream smoke was generated by a VC $10^{\circledR}$ smoking robot under ISO conditions (105) from Kentucky 3R4F reference cigarettes in two versions - one with original cellulose acetate filters and the other fitted instead with charcoal filters. Each exposure day, mainstream smoke was produced from four cigarettes, amply diluted with synthetic air ( $1 \mathrm{~L}$ air per puff in $1 \mathrm{~min}$ ) and drawn into the module at a rate of $5 \mathrm{~mL} / \mathrm{min} / \mathrm{insert}$; synthetic air served as control in a second module. Integrity of cilia was the study endpoint, expressed as number of ciliated cells and altered length of cilia; beat frequency was not included. Smoke from cigarettes with cellulose acetate filters showed pronounced effects: After 4 exposure days, both the number of ciliated cells and the length of cilia were significantly reduced; after 6 exposure days, all cilia had disappeared. Smoke, however, that had passed through a charcoal filter had only a marginal and transient effects on these variables. In an earlier study examining the impact of cigarette smoke gas phase (whole as well as specific components) on ciliated mussel tissue WALKER and KIEFER (466) had identified hydrogen cyanide as a major contributor to the ciliastatic activity of smoke. The experimental set-up (465) was considered a stable and relevant model for in vitro studies with the potential of reducing or replacing animal testing.

Recently, the design of the Cultex ${ }^{\circledR}$ RFS exposure module was changed to a compact size (Cultex ${ }^{\circledR}$ Compact), able to house six inserts, and put to the test in an evaluation of the

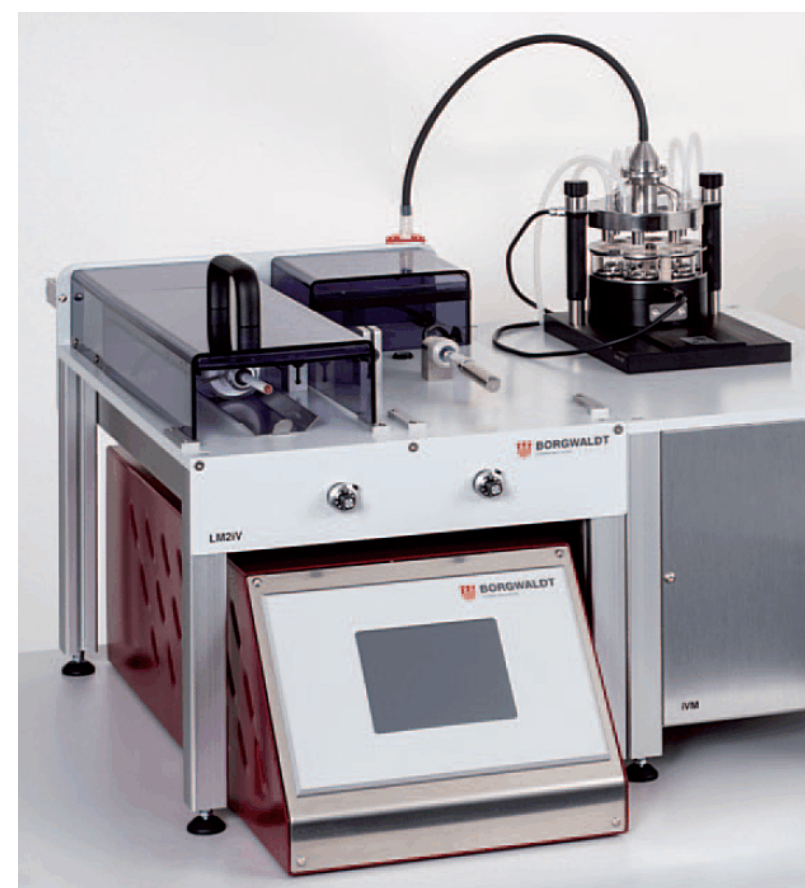

Figure 48. The compact in vitro testing system (108) consisting of the Borgwaldt pump PM 1 with control unit (lower left) and the cigarette module (upper left) combined with the Cultex ${ }^{\circledR}$ Compact exposure module (upper right).

cytotoxicity of e-cigarette vapor and cigarette mainstream smoke $(467,468)$. This was done in connection with the development of a new Modular Smoking Machine Series (108) by Borgwaldt KC of Hamburg (Germany). This system includes as center piece a precise but robust piston pump PM1 with an integrated control unit, which can be hooked up to different modules capable of smoking cigarettes and cigars under a range of conditions or generating aerosol from all kinds of e-cigarettes as well as detecting and trapping smoke and aerosol constituents in various systems. The compact Cultex ${ }^{\circledR}$ RFS exposure module may be connected to all smoke or vapor producing modules of the series for direct toxicological assessments (Figure 48). In a cytotoxicity dose-response study (468) using the resazurin assay, e-cigarette vapor was generated using a VC $10^{\circledR}$-type smoking robot for suction, and smoke from Kentucky 3R4F reference cigarettes with the new Borgwaldt PM1 piston pump combined with the Borgwaldt LM2 linear cigarette module. The viability of normal human bronchial epithelial (NHBE) cells, exposed in the compact RFS module, was reduced 6-10 times more by cigarette mainstream smoke compared to e-cigarette vapor. The differentiation phase of normal human bronchial epithelial (NHBE) cells may last as long as 28 days, and the manual handling of cells growing on Transwell ${ }^{\circledR}$ inserts is both time-consuming and prone to technical mismanagement. To produce fully differentiated $3 \mathrm{D}$ constructs for ALI exposure studies under tight and reproducible conditions, a dynamic and stable computer-controlled system was developed by AUFDERHEIDE et al. (469) and called Cultex ${ }^{\circledR}$ LTC-C. Its incubator module is capable of holding 24 inserts; it is combined with the liquid handling module with two peristaltic pumps for medium supply and discharge, a heat exchanger, and with the electronic control 

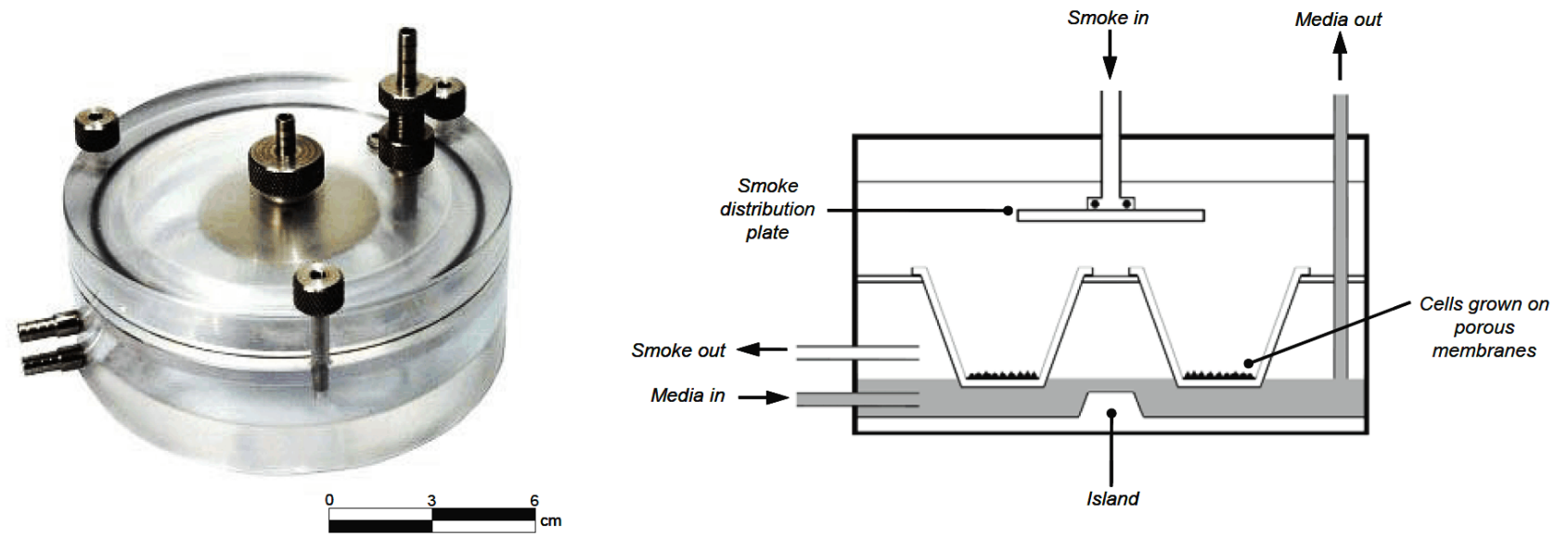

Figure 49. The "BAT exposure chamber" for the toxicological testing of tobacco smoke at the air-liquid interface (extracted from (474)).

unit. Fresh NHBE cells were cultivated for up to 38 days and exhibited in vivo-like pseudo-stratified cell organization as well as differentiation characteristics like mucusproducing and cilia-forming cells.

\subsubsection{Air-liquid interface (ALI) exposure systems: RM20S and the BAT chamber}

A paper published in 1998 by MASSEY et al. (13) is an indicator for the early cooperation between the BAT R\&D Centre in Southampton (UK) and the group at the Fraunhofer Institute of Toxicology and Aerosol Research in Hannover (Germany), which was to develop into Cultex ${ }^{\circledR}$ Laboratories. The goal was to produce preliminary data demonstrating the feasibility of a system for exposing cells to fresh cigarette smoke directly without an intervening layer of liquid (solvent or medium) between cells and smoke, i.e., at the air-liquid interface (ALI). Chinese hamster lung cells V79 were used for examining cell proliferation and micronucleus formation (without S9 activation). The instrumental set-up consisted of a smoke generator, taking puffs (presumably from a cigarette with $16.4 \mathrm{mg}$ TPM yield) under ISO standard conditions by means of a step motor driven glass syringe, and a commercial incubator equipped to dilute whole smoke with conditioned air to desired ratios (overall 1:8 to 1:200) and to accommodate the new exposure chamber, which was made from steel and teflon and had room for six Transwell ${ }^{\mathbb{R}}$ inserts with porous membranes on a support frame. Medium was supplied to the cells from the bottom. The internal smoke distribution panel at the top of the chamber delivered smoke through six cylindrical holes, located above the Transwell ${ }^{\circledR}$ inserts $(24 \mathrm{~mm}$ diameter and $3.0 \mu \mathrm{m}$ pore size) and fitted with fine-meshed screens for uniform and radial flow over the cells. Cells seeded onto the membranes could be exposed to air for 3 hours without losing viability. Smoke with TPM concentrations of up to $840 \mathrm{mg} / \mathrm{m}^{3}$ (monitored by a gravimetrically calibrated inline aerosol photometer) was routed puff by puff into the exposure chamber, left there for static exposure (10-40 sec) and then cleared out with conditioned air for the remainder of the 60-sec puff interval; standard total exposure time was 3 hours. Generally, the inhibition of cell proliferation increased with higher TPM concentrations and longer exposure time per puff. Regarding the induction of micronuclei, results were inconsistent. When particulate matter was removed from whole smoke using Cambridge filters and only the gas vapor phase examined, roughly comparable effects were observed with respect to the inhibition of cell proliferation and the induction of micronuclei. On the technical side, it was noted that a considerable portion of particulate matter was obviously lost by deposition, especially in the tubing of the smoke distribution lines; the overall loss was estimated to be as high as $36 \%$.

A study similar to the one just discussed (13) was briefly reported in 2002 in form of an abstract by PHILLIPS et al. (470).

A patent was applied for in the UK in 2002 - with an international patent (471) published in 2003 - by British American Tobacco (inventors: MASSEY, WILLIAMSON and PHILLIPS) for "an exposure device", which obviously followed the principles underlying the exposure chamber described by MASSEY et al. (13) though with a quite differently designed top portion featuring a horizontal discshaped metal plate for homogeneous smoke dispersion and three holes in the base for housing inserts. This chamber (Figure 49) - called "BAT exposure chamber" in several publications - was used in a number of BAT research projects (472-477), discussed below, in combination with the Borgwaldt smoking machine RM20S.

The Borgwaldt RM20S is a cigarette smoking machine (first commercialized in 2002) for the generation, dilution and transfer of mainstream smoke for direct in vitro exposure of cells and tissues (108). It was designed in cooperation with British American Tobacco, Research and Development (Southampton, UK) and is based on the rotary head principle with cigarettes smoked in horizontal position (Figure 50). Puffs are taken under operator-selected conditions simultaneously from (originally) four different cigarettes by four independent syringes - with the option of diluting the smoke sequentially over a remarkable range (1:2-1:750,000; practical dilution for biological exposures: 1:20-1:250). Machine operation is completely automatic (cigarette loading, lighting, butt control, butt extraction). The RM20S is able to smoke up to 20 cigarettes successively and provide exposure systems with diluted 


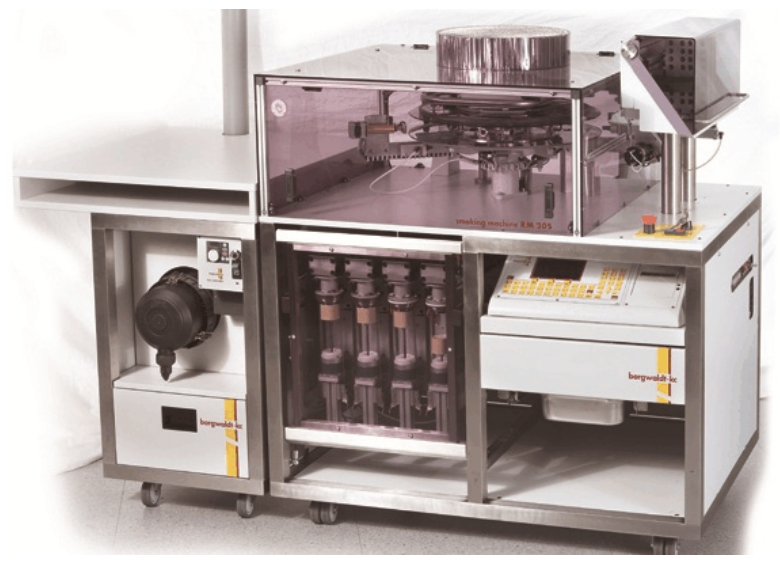

Figure 50. The rotary Borgwaldt RM20S (108) generates and dilutes smoke from four different cigarettes simultaneously for in vitro cell culture testing.

smoke for several hours. The machine is enclosed in a chamber and equipped with an exhaust for maintaining correct air flow around the glowing cones according to ISO 3308 (184); air flow is monitored by an anemometer. KAUR et al. (478) analyzed the technical performance of the RM20S machine regarding the supply of accurate smoke doses for exposure and dosimetry. Exposure chambers were not used in the study, which was rather focused on physical parameters such as machine accuracy and precision (repeatability and reproducibility). For a subset of measurements, RM20S machines were operated in different locations (Canada and the UK - here also equipped with $2 \times 4$ syringes), this way producing data for reproducibility assessment. In a pragmatic approach, dilution efficiency was determined with two reference gases, methane (10\% in argon or nitrogen; three dilution levels) and carbon monoxide (10\% in air; 4 dilution levels). For judging the quality of smoking and smoke dilution (three levels), solanesol - a marker for TPM produced from 3R4F Kentucky reference cigarettes - was measured at two points: in filter tips (for judging actual smoke production) and after dilution collected on Cambridge filters. Summarizing a wealth of data and after appropriate statistical evaluation the authors concluded that "the Borgwaldt RM20S smoking machine is a reliable tool to generate and deliver repeatable and reproducible doses of whole smoke to in vitro cultures".

The experimental potential of the combination RM20S smoking machine (108) and BAT exposure chamber (471) was explored by PHILLIPS et al. (472). Smoking two filter cigarettes with identical flue-cured tobacco blend (one unventilated with $17.1 \mathrm{mg}$ TPM, the other ventilated with $5.9 \mathrm{mg}$ TPM) under ISO standard conditions (105) the efficiency of the syringe dilution system was assessed by measuring particle concentrations in the exposure chamber by means of a light scattering particle counter, and particle deposition on blank Transwell ${ }^{\circledR}$ inserts (without cells) by extraction of the exposed membranes and fluorescence reading of the extract. Credible reciprocal relationships were observed between smoke dilution rates, and particle chamber concentrations and particle deposition quantities, respectively. The different yields of the two test cigarettes were clearly reflected in the data.
Confluent monolayers of NCI-H292 human pulmonary epithelial carcinoma cells on Transwell ${ }^{\circledR}$ membranes were exposed for $30 \mathrm{~min}$ under ALI conditions to different smoke concentrations, followed by a 20 hour recovery period in submerged culture. Cytotoxicity was evaluated using the neutral red uptake assay. Cell viability was dose dependently reduced, allowing the calculation of $\mathrm{ID}_{50}$ values (expressed as dilution factors of whole mainstream smoke producing 50\% inhibition of cell viability), which differed considerably for the two cigarettes in the test. Mainstream smoke cytotoxicity was much lower with the ventilated, low delivery cigarette than with the unventilated product. To assess cell functional ability after low dose ALI exposure the expression of the gene coding for secreted mucin, MUC5AC, was determined and messenger ribonucleic acid (mRNA) levels were found to be upregulated dose dependently. Further, the secretion of the pro-inflammatory cytokines, IL-6 and IL-8, was induced (quantified in the 20-hour post-exposure recovery medium by means of the enzyme-linked immunosorbent assay); effects were significant but not dose dependent.

The RM20S smoking machine (108) and the BAT exposure chamber (471) with Transwell ${ }^{\circledR}$ culture inserts were key components in the first study - performed in 2007 by MAUNDERS et al. (473) - to characterize the whole genome response of primary human bronchial mucociliary epithelial cells, following exposure to mainstream cigarette smoke at the air-liquid interface (ALI). Cells were obtained from three non-smoking donors and treated for 1 hour with smoke from 2R4F Kentucky reference cigarettes, generated under ISO standard conditions (105) and diluted 1:50 before use. Study focus was on the transcriptome, which constitutes the set of all messenger ribonucleic acid (mRNA) molecules in a cell (population) and is specified by amount or concentration. The transcriptome is readily influenced by agents, environmental factors or disease conditions - as a result of changes in gene expression, i.e., the upregulation or downregulation of individual genes. Established methodology was followed for analysis ("genetic profiling"): Isolation of total cellular RNA, formation of double stranded complementary DNA (cDNA) with reverse transcriptase, amplification of cDNA by means of the polymerase chain reaction (PCR), identification and quantification of differentially expressed genes (smoke compared to air control). For these biochemical steps and for the evaluation and interpretation of data, sophisticated tools (reagent kits, whole genome high-density microarray chips, data base information and data analysis programs) are commercially available and were used in the study for examining cells at 1,6 , and 24 hours post-exposure. Genes regulated by smoke were assigned to functional categories and mapped to signaling pathways. Effects were observed on many cellular processes; these included xenobiotic metabolism, oxidant/ antioxidant balance, DNA damage and repair, and the transforming growth factor- $\beta$ pathway.

The assessment of oxidative DNA damage in cultured NCI-H292 human pulmonary carcinoma cells resulting from exposure to mainstream cigarette smoke at the ALI was the objective of a study performed by THORNE et al. (479). Smoke from 2R4F Kentucky reference cigarettes was produced with a 4-port RM20S machine (108) and 
used in a range of dilutions for treating cells on Transwell ${ }^{\circledR}$ membranes for $30 \mathrm{~min}$ in BAT exposure chambers (471). DNA damage included strand breaks, the formation of alkali-labile sites, alkylations and oxidative DNA lesions, and was measured by means of the comet assay (see page 206). The test was refined by including base excision repair enzymes for the detection of oxidative purine and pyrimidine modifications. Damaging effects on cell DNA were considerable. Interestingly, strand breaks - but not oxidative lesions - were repaired during a 20-hour recovery period.

To increase its capacity the RM20S smoking machine was fitted with a second 4-syringe system. This way, the number of available whole smoke doses per run could be increased and the number of experimental runs reduced, which were required, for instance, for recording an entire dose-response curve. The performance of the new instrument with eight independently operating syringes had already been examined - in the absence of exposure devices - by KAUR et al. (478) and was now assessed in combination with the BAT exposure chamber $(471,473)$ by ADAMSON et al. (474). First, following the earlier approach (478), syringes were examined - individually (Figure 51) and collectively - for precision and accuracy when performing dilutions at two levels; methane $(10 \%$ in nitrogen) was used as gas standard. Total gauge repeatability and reproducibility ( $R \&$ r) estimates, which reflected the consistency and stability of syringe performance across the entire system, were within acceptable limits (all values $<10 \%$ ).

The flow of smoke (produced from 3R4F reference cigarettes) along the various steps of generation, dilution and exposure was tracked in three different ways: Combining a single RM20S syringe with the same exposure chamber,

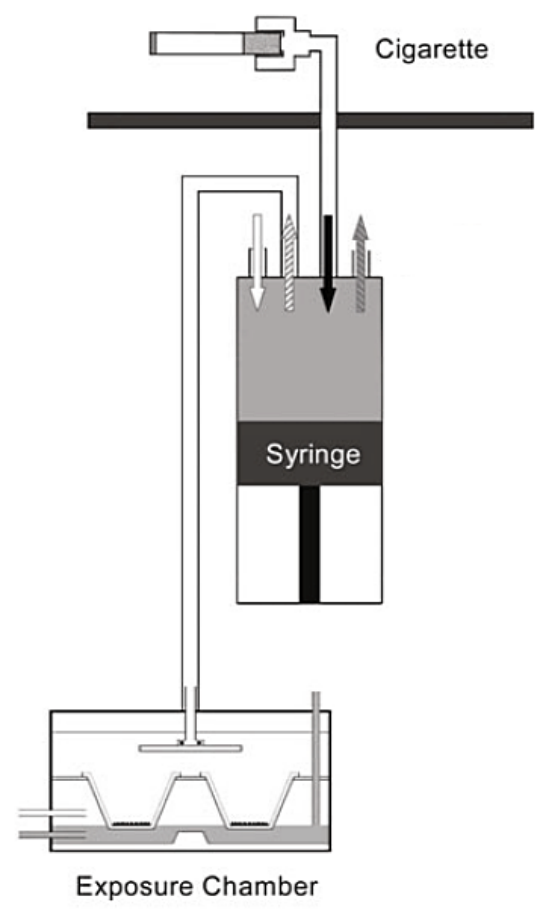

Figure 51. A single syringe of the RM20S smoking machine combined with the BAT exposure chamber - depicted in cross-section (474). diameter and concentrations of particulate matter were measured in-line and real-time by means of an electrical differential mobility spectrometer, as used by MCAUGHEY et al. (480), in samples collected at three points of the smoke path: at the exhaust port at the top of the syringe (the earliest accessible point for sampling); at the entry into the exposure chamber; and at the outlet of the chamber. After intricate recalculation of the photometric data it was possible to compare the TPM levels at the different sampling points to what was measured with ISO methodology at a simulated smoke generation point - with the following revealing results (rounded): $14 \%$ of particle mass was lost between puff generation point and the top of the syringe (i.e., in the smoking machine and clearing tubing); $33 \%$ were deposited inside the syringe and the down-stream tubing (meaning that only $53 \%$ of particle mass entered the exposure chamber); and $37 \%$ left through the chamber outlet. Accordingly, as much as $16 \%$ of particle mass ended up in the exposure chamber.

Also, particle deposition onto the membranes in inserts without cells was measured spectrofluorometrically and found to be linearly correlated to smoke concentration over 2 orders of magnitude for smoke dilution.

Finally, a biological endpoint was used to check the uniformity of smoke deposition onto the cells of six Transwell ${ }^{\circledR}$ inserts inside a single exposure chamber. The viability of human pulmonary carcinoma cells NCI-H292 after 30 min of intermittent static puff-by-puff exposure at the ALI to whole smoke (diluted to $\mathrm{EC}_{50}$ strength) was determined by the neutral red assay. Within a run, there were no significant differences between the inserts in a chamber; between-run variability, however, seemed to be larger. Summing up the authors described the combination of the RM20S smoking machine and the BAT exposure chamber as rendering possible "a reliable, repeatable, robust and potentially a more physiologically relevant method of generating and exposing in vitro cellular and tissue cultures to whole cigarette smoke at the ALI" (474). Dependable dosimetric data are essential for the interpretation of in vitro toxicity findings and their extrapolation to human exposure scenarios. Linking up the RM20S smoking machine (108) and BAT exposure chamber (471), ADAMSON et al. (475) examined the applicability and reliability of a quartz crystal microbalance (QCM) system for the real-time assessment of cigarette smoke particle deposition. Changes in oscillation frequency of a quartz crystal, held between two electrodes, were recorded under the influence of increased particle deposition - a gravimetric approach first explored and developed in 1959 by SAUERBREY (481). In the exposure chamber (without cells) the QCM replaced one of the three inserts. Two test pieces (the 3R4F Kentucky reference cigarette with $9.4 \mathrm{mg}$ "tar" and a highly ventilated commercial $1 \mathrm{mg}$ "tar" cigarette) were smoked consecutively for $30 \mathrm{~min}$ (five cigarettes with six puffs each); the smoke was then diluted to different degrees (1:5-1:400). Filling time of the chamber was $8 \mathrm{sec}$, followed by a $52 \mathrm{sec}$ still period before the next puff was introduced. The QCM showed high sensitivity with a resolution of $10 \mathrm{ng} / \mathrm{cm}^{2} / \mathrm{sec}$; remarkably, with readings taken every $2 \mathrm{sec}$, mass gain was not only recorded on a puff-by-puff basis but fine structures of mass deposition (particle sedimentation $v s$. escape of semi-volatiles) could 
be charted even within the 60 -sec puff periods. With the two test cigarettes and over the full range of dilutions, particle deposition amounted to $0.13-25.75 \mu \mathrm{g} / \mathrm{cm}^{2}$ in 30 min. When QCM deposition measurements were compared to (traditional) TPM determinations by spectrophotometry, a statistically significant correlation was observed $\left(\mathrm{R}^{2}=97.4 \%\right)$.

In a very similar follow-up study, ADAMSON et al. (476) equipped the BAT exposure chamber (471) after slight modification with identical quartz crystal microbalances (QCMs) in each of the three wells, calling the new tool triplicate QCM chamber. It provided immediate triplicate data for individual runs and allowed to examine the uniformity of particle deposition within the three positions. Diluting the smoke of 3R4F Kentucky reference cigarettes 1:5-1:400, particulate matter deposited during 30 min runs amounted to $0.21-28.00 \mu \mathrm{g} / \mathrm{cm}^{2}$. No significant differences were detected between the three positions in the chamber at any of the dilutions.

A review of the currently available smoking machines and exposure modules for in vitro toxicity testing of cigarette whole smoke, which distinguishes itself by a multitude of technical details of the various systems, was published in 2013 by THORNE and ADAMSON (482). Both the main commercially available components (the Borgwaldt RM20S, Burghart MSB-01 (see page 222) and Vitrocell ${ }^{\mathbb{R}}$ VC $10^{\circledR}$ machines for smoke generation, dilution and delivery as well as the Curbridge Engineering (BAT), Cultex $^{\circledR}$ and Vitrocell ${ }^{\circledR}$ exposure modules) and custommade ("bespoke") systems $(483,484)$ were described and evaluated. In addition, physical, chemical and gravimetric methods for the measurement of smoke dose and exposure and the assessment of particle deposition were critically reviewed.

As a novel endpoint for in vitro genotoxicity testing of tobacco smoke, GARCIA-CANTON et al. (485) proposed to measure $\mathrm{H} 2 \mathrm{AX}$, a phosphorylation product of the histone $\mathrm{H} 2 \mathrm{AX}$, which is involved in the packaging of DNA into nucleosomes. The formation of $\mathrm{H} 2 \mathrm{AX}$ is a rapid and proportional damage response to DNA double strand breaks. Following an instructive survey of currently available regulatory and non-regulatory in vitro genotoxicity assays, the authors reviewed the use of $\mathrm{H} 2 \mathrm{AX}$ - since its discovery in 1998 - in pre-clinical drug assessment, as a biomarker of DNA damage and for in vitro mechanistic studies, discussed the methods available for H2AX determination and considered its use for examining the genotoxic potential of tobacco smoke.

It was only logically consistent that GARCIA-CANTON et al. (486) set out to develop a practical method for the determination of $\mathrm{H} 2 \mathrm{AX}$ and to assess the sensitivity, specificity and accuracy of the assay. With exposure at the ALI in mind, fluorescence microscopy based automated cellular image acquisition (of H2AX labeled by immunostaining) was decided on in combination with High Content Screening (HCS) software for quantitative analysis (HCS being defined as the high throughput phenotypic screening of cells for the - increased or decreased - formation of products, such as proteins, or for changes in morphology). This approach was preferred over flow cytometry scoring, which above all requires cells in suspension. Human bronchial epithelial cells BEAS-2B (non-cancerous and immortalized) were exposed in 96-well microplates for 3 or 24 hours to a battery of 22 compounds, which are deemed either genotoxic (12 compounds, of which 10 were classified by the International Agency for Research on Cancer, IARC (487) as carcinogenic (group 1), probably carcinogenic (group 2A) or possibly carcinogenic (group 2B) to humans) or non-genotoxic (10 compounds; including two aneugens, which interfere with chromosome formation rather than interacting directly with DNA). In comparison to established in vitro genotoxicity tests, the $\mathrm{H} 2 \mathrm{AX}$ assay performed quite impressively: In terms of sensitivity (clear identification of genotoxic compounds) the score was 11 out of 12 (Ames test: 9/12; in vitro mammalian test: 12/12); regarding specificity (clear identification of non-genotoxic compounds) the score was 8 out of 10 (Ames test: 8/10; in vitro mammalian test: $5 / 10$ ). In summary, the accuracy of the H2AX assay was $86 \%$ (both Ames test and in vitro mammalian test: $77 \%$ ). Using the in vitro $\mathrm{H} 2 \mathrm{AX}$ assay by High Content Screening (486) GARCIA-CANTON et al. (477) evaluated the genotoxicity of whole cigarette mainstream smoke of two test pieces. The 3R4F Kentucky reference cigarette $(9.4 \mathrm{mg}$ "tar") and the BAT in-house experimental cigarette M4A (10 mg "tar") were smoked continuously under ISO conditions on a Borgwaldt RM20S 8-syringe smoking machine. BEAS-2B cells - used before (486) - were seeded into Transwell ${ }^{\circledR}$ inserts and exposed in the BAT chamber (471) for 3 hours to a range of smoke dilutions at the ALI. Smoke from both test pieces produced a significant positive genotoxic response (i.e., increase in H2AX frequency) at all dilutions tested $(1: 500-1: 16,000)$. However, dose-response relationships were not visible, presumably due to unreliable performance of the syringe smoke dilution system.

\subsubsection{Air-liquid interface (ALI) exposure systems: Vitrocell $^{\circledR}$}

The exposure modules - invented and patented by MOHR and Aufderheide (Cultex ${ }^{\circledR}$ Laboratories GmbH, Hannover, Germany) - were until 2006 marketed under the name Vitrocell ${ }^{\circledR}$. After that the entities Cultex ${ }^{\circledR}$ Laboratories $\mathrm{GmbH}$ and Vitrocell ${ }^{\mathbb{R}}$ Systems went their own ways regarding new developments and commercial distribution while Vitrocell ${ }^{\circledR}$ continued to sell the Cultex ${ }^{\circledR}$ glass modules under its own name. This explains both traditional similarities and the obvious and significant differences in more recent technology and devices.

The paper of LI et al. (488), published in 2013, followed the established approach for assessing the value of in vitro smoke exposure equipment but stands out for including illustrative depictions of the Vitrocell ${ }^{\circledR}$ system used in the study. Chinese hamster ovary $(\mathrm{CHO})$ cells seeded onto microporous insert membranes were exposed at the ALI to whole smoke produced under ISO conditions from 3R4F Kentucky reference cigarettes (and two undefined experimental test pieces). The Vitrocell ${ }^{\circledR}$ system was composed of the smoking robot VC $10^{\circledR}(450)$, four smoke dilution devices featuring mass-flow and valve controls, and five exposure modules (one of them for air control), "which are similar to the Cultex ${ }^{\circledR}$ exposure modules" - presumably the ones described in 2005 by AUFDERHEIDE (448). Cell 


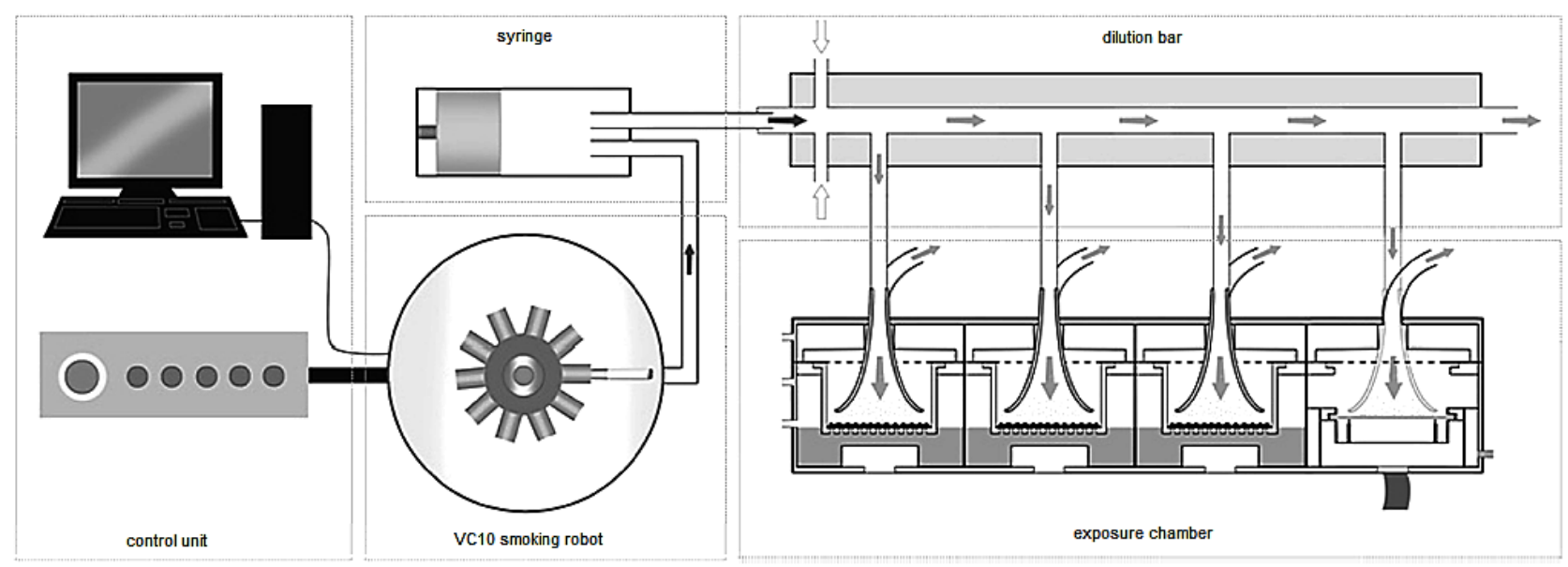

Figure 52. The performance of a Vitrocell ${ }^{\circledR}$ system (VC 10 smoking robot + dilution bar + 4-well exposure chamber) was assessed by THORNE et al. (490).

viability was estimated by means of the neutral red uptake assay for measuring the cytotoxic effects of whole smoke in quantities determined by both dilution (up to 1:6) and the number of cigarettes smoked in a test. Exposure to air for $60 \mathrm{~min}$ at a constant flow rate of not more than $5 \mathrm{~mL} / \mathrm{min}$ through the exposure module (higher flows rates were injurious) did not reduce cell viability after a post-exposure period of 24 hours, intended to allow pathological changes to manifest. With increasing amounts of whole smoke a clear dose-response relationship was observed. FIELDS et al. (489) validated the VC $10^{\circledR}$ cigarette smoking robot producing mainstream smoke for several in vitro genetic toxicity assays: neutral red uptake, Ames assay and sister chromatid exchange. Based on "critical function and assay assessments via the installation, operational, performance and validation protocols", the validity of the use of the VC $10^{\circledR}$ system was confirmed. Unfortunately, a report of this interesting study is so far available in abstract form only; a full publication is said to be in preparation. A straight-forward performance assessment of the Vitrocell ${ }^{\circledR}$ smoke generation and exposure system was conducted by THORNE et al. (490) using two physical and two biological methods for analysis. A VC $10^{\circledR}$ smoking robot was combined with a Vitrocell ${ }^{\circledR}$ 6/4 CF stainless steel or a Vitrocell ${ }^{\circledR}$ Ames exposure module (Figure 52). Smoke was produced from 3R4F Kentucky reference cigarettes under ISO conditions. Different dilutions were made (in an elongated bar) by the continuous and turbulent admixing of air with distinct flow rates $(0.5-4.0 \mathrm{~L} / \mathrm{min})$, and then drawn through the 4-well exposure chamber by a vacuum at a rate of $5 \mathrm{~mL} / \mathrm{min} /$ well. The four parameters measured in the study were: Particle deposition on quartz crystal microbalances (QCMs) placed in the module wells, carbon monoxide as a marker for smoke gas phase, cytotoxicity using the neutral red uptake assay, and genotoxicity by means of the Ames test. For evaluation, data were correlated with smoke dilutions, expressed as dilution air flow rates, or deposited mass, measured in situ while conducting the biological tests. QCM measurements are essentially useful for following the deposition process while exposure parameters for achieving proper doses need to be determined in advance.
For estimating particle deposition, the 6/4 CF stainless steel module was equipped with four QCMs. With $24 \mathrm{~min}$ exposure time (3 cigarettes smoked), deposition rates of $0.6-5.9 \mu \mathrm{g} / \mathrm{cm}^{2}$ were observed depending on smoke dilution. No statistically significant differences were detected across the QCMs in the four wells. Carbon monoxide was quantified either directly with a gas analyzer attached to the dilution bar or post-exposure after collection in a bag (a method producing more consistent values due to samples of greater homogeneity). Smoke dilutions (reciprocal air flows) correlated convincingly with particulate deposition $\left(\mathrm{R}^{2}=0.975\right)$ and vapor phase dilution ( $\mathrm{CO}$ analyzed after bag sampling; $\mathrm{R}^{2}=0.987$ ).

Cytotoxicity was estimated in mouse fibroblasts (Balb/c 3T3 cells) on microporous Transwell ${ }^{\circledR}$ inserts in three wells using the neutral red uptake assay (exposure time: $184 \mathrm{~min}$ corresponding to 23 smoked cigarettes) - with concurrent deposition control in situ by a QCM in the forth well. For genotoxicity assessment, the Vitrocell ${ }^{\circledR}$ Ames module was employed, also with a QCM in the forth well. Bacteria of Salmonella typhimurium strain YG 1042 on $35 \mathrm{~mm}$ agar plates were exposed with S9 activation for 24 min (spread plate method). Both biological tests showed consistent responses and positive, though somewhat moderate, correlations with deposited mass.

With the experimental arrangement (VC $10^{\circledR}$ and Vitrocell $^{\circledR}$ 6/4 CF exposure module, equipped with one QCM; 3R4F Kentucky reference cigarettes smoked under ISO conditions; dilution flow rates of $1-10 \mathrm{~L} / \mathrm{min}$ and chamber flow rate of $5 \mathrm{~mL} / \mathrm{min} /$ well $)$ used in the earlier study (490), THORNE et al. (491) examined the neutral red uptake test with ALI exposure of Balb/c 3T3 mouse fibroblast cells. Both whole smoke and gas vapor phase (obtained by passing smoke through a Cambridge filter placed between the VC $10^{\circledR}$ smoking head and the syringe piston) were analyzed. When cell survival rates were plotted as a function of aerosol or gas concentration (expressed as $\log _{10}$ dilution air flow) significant dose-for-dose differences between the (higher) cytotoxicity of whole smoke and the (lower) cytotoxicity of the gas vapor fraction were observed. However, the use of dilution air flow rates - a simple and easily controllable parameter - in addi- 
tional ambitious calculations looks like a daring exercise as long as their relationship with exposure intensity and particle deposition (measured by QCMs with whole smoke) is yet insufficiently understood.

Following up on their earlier studies $(475,476)$, in which the RM20S smoking machine (108) was combined with the BAT exposure chamber (471), ADAMSON et al. (492) documented the deposition of smoke particles in real time by means of a quartz crystal microbalance (QCM) system (technical details explained above), now integrating the VC $10^{\circledR}$ smoking robot (450) and the dilution system with the Vitrocell ${ }^{\circledR}$ mammalian $6 / 4$ stainless exposure module. 3R4F Kentucky reference cigarettes were smoked under ISO conditions in single mode (one cigarette only smoked at a time, and three cigarettes smoked consecutively with eight puffs each for a total exposure time of $24 \mathrm{~min}$ ). Cell culture inserts were replaced in all four wells of the module by identical QCM units, which output particle deposition as mass per surface area - a straightforward and convenient parameter. Variables investigated included air flow to the dilution bar $(0.5-4.0 \mathrm{~L} / \mathrm{min})$ and the flow through the exposure chamber $(5-100 \mathrm{~mL} / \mathrm{min} /$ well $)$, which both exerted a strong influence not only on the absolute amounts of particles deposited but also on the pattern (gradient) of deposition across the four wells in the module. Deposition was highest with the lowest air flow into the dilution bar (corresponding to minimal dilution) and with the lowest air flow through the chamber; at $5 \mathrm{~mL} / \mathrm{min} /$ well it ranged from $0.72-8.65 \mu \mathrm{g} / \mathrm{cm}^{2}$, depending on smoke dilution. Uniformity across wells was best at the highest chamber flow rate $(100 \mathrm{~mL} / \mathrm{min} /$ well not favorable for biological testing) and significantly disturbed at the lowest $(5 \mathrm{~mL} / \mathrm{min} /$ well $)$, which is the one preferred for cell exposure. The employment of QCMs allows the precise quantification of particle deposition at each well and under any condition of air flow to the dilution system and smoke flow through the module. This way, different smoke generation and dilution systems can be characterized and compared directly regarding their performance and potential to supply suitable atmospheres for exposure studies.

After three earlier studies $(475,476,492)$ had convincingly demonstrated the value of quartz crystal microbalances (QCMs) for the quantitative assessment of cigarette smoke particle deposition, ADAMSON et al. (493) used QCM technology for a comparative evaluation of Vitrocell $^{\mathbb{B}}$ smoke generation and exposure systems. The direct gravimetric measurement of precipitated mass per surface area in real time and in situ offered considerable advantages over traditional methods, such as measurement of fluorescence or differential mobility spectrometry backed by specific air flow rates. Six individual VC $10^{\mathbb{R}}$ smoking robots - operated in four independent laboratories in three countries (two laboratories in the UK, one in Germany and one in China) - were combined with mammalian stainless steel exposure modules, either the $6 / 3 \mathrm{CF}$ model with three wells or the $6 / 4 \mathrm{CF}$ model with four wells. Smoking robots and exposure modules were connected via (single or multiple parallel) dilution bars with three or four smoke outlets, respectively. In the exposure modules, all wells were equipped with QCMs rather than the customary microporous membrane inserts. 3R4F Kentucky reference ciga- rettes were smoked under ISO conditions in single mode ( 3 cigarettes in sequence with 8 puffs each), and smoke was diluted with five different air flows $(0.25-4.0 \mathrm{~L} / \mathrm{min})$. Exposure time was $24 \mathrm{~min}$ at a flow rate of $5 \mathrm{~mL} / \mathrm{min} /$ well.

Both for individual and the ensemble of machines (i.e., within-machine and between-machine), deposition data were evaluated with regard to dilution efficiency and precipitation gradients across the modules (three or four positions). Data were compared for both specific and for all dilutions used; dose-response measurements obtained from all machines across the four different locations produced a gauge repeatability and reproducibility ( $\mathrm{R} \& \mathrm{r}$ ) estimate for the entire system of $7.7 \%$, demonstrating good agreement between machines. At the same time, the study revealed that system performance was liable to be hampered by certain adverse technical conditions, such as low flow rate of diluting air $(0.25 \mathrm{~L} / \mathrm{min})$ resulting in turbulence and transit time in the dilution bar insufficient for aerosol homogeneity; poorly controlled ambient environment during machine operation; and imperfect compliance with machine service requirements - valuable insight for laboratory staff.

The scaled down spread-plate version of the Ames test, which had been explored by AUFDERHEIDE and GRESSMANN (455) using Cultex ${ }^{\circledR}$-B exposure modules, was further investigated by KILFORD et al. (494). They combined the Vitrocell VC $10^{\circledR}$ smoking robot with Vitrocell ${ }^{\circledR}$ Ames 4 stainless steel modules and produced whole smoke under ISO conditions from 3R4F Kentucky reference cigarettes in 4 concentrations by stepwise dilution. Four Salmonella typhimurium strains (TA 98, TA 100, YG 1024 and YG 1042) and one E. coli strain (WP2 uvrA pKM101) - altogether susceptible to a range of mutagens of different chemical classes - were exposed (with and without S9 activation) to either three or eight cigarettes smoked over 24 or 64 min, respectively. Module flow rate was $5 \mathrm{~mL} / \mathrm{min} /$ well. Three wells in the module were used for replicate assays while the forth well was equipped with a quartz crystal microbalance (QCM) for the quantification of particulate depositions. Concentration related increases of revertants were observed with S9 activation with smoke from three cigarettes in TA 98 and TA 100 (moderate effects only) and in YG 1024 and YG 1042 (pronounced). Cytotoxicity was noticed occasionally at the highest smoke concentration. There was no clear increase of revertants in the E. coli strain. When strains TA 100 and WP2 uvrA pKM101 were exposed to the smoke from eight cigarettes response was not significantly increased. There was also no effect in all strains in the absence of S9 activation. Comparing the performance of two different VC $10^{\circledR}$ smoking robots the response in the YG 1042 strain was remarkably consistent.

The experimental approach of KILFORD et al. (494) was duplicated in terms of equipment and procedures in a study by THORNE et al. (495) with the objective of expanding the number of strains suitable for whole smoke genotoxicity testing with the $35 \mathrm{~mm}$ spread-plate Ames air-agar interface technology. Initially, Salmonella typhimurium strains TA 97, 102, 104, 1535 and 1537 - not investigated so far - were taken into consideration but TA 1537 was excluded from further examination due to its marginal quantitative responsiveness in the $35 \mathrm{~mm}$ system. In strains 
TA 97, 102 and 1535 no increase in revertants was observed after exposure to any dose of whole smoke without and with S9 activation; only TA 104 showed effects of some statistical significance with maximal deposited mass, measured with a quartz crystal microbalance (QCM) and amounting to $3.48 \mu \mathrm{g} / \mathrm{cm}^{2}$ from 3 cigarettes smoked in $24 \mathrm{~min}$ and $9.36 \mu \mathrm{g} / \mathrm{cm}^{2}$ from 8 cigarettes in $64 \mathrm{~min}$, respectively. With all strains, appropriate positive controls were conclusive. The paper includes an informative compilation of the characteristics and responsiveness to cigarette smoke (particulate phase and aerosol) of 16 Salmonella typhimurium strains and one E. coli strain. Combining the results of the two studies $(494,495)$ the authors proposed a battery of strains for testing the genotoxicity of TPM generated from (conventional or modified) tobacco containing smoking products using either traditional or air-agar interface Ames methodology. Instrumentation for assessing the contribution of single gaseous components in cigarette mainstream smoke to cytotoxic and genotoxic effects was developed by BREHENY et al. (496). Ethylene oxide - a (minor) constituent of fresh tobacco smoke with known direct in vitro and in vivo genotoxicity and a carcinogen in rodents - was chosen as test gas. Consequently, whole cigarette smoke from 3R4F Kentucky reference cigarettes, ethylene oxide and whole smoke spiked with different amounts of ethylene oxide were used in Ames assays (spread-culture method) with the Salmonella typhimurium strain YG 1042, both with and without S9 activation. The Vitrocell ${ }^{\circledR}$ smoking robot VC $10^{\circledR}$ with up to four dilution bars was combined with a Vitrocell $^{\circledR}$ valve spiking setup and a Vitrocell ${ }^{\circledR}$ Ames 4 stainless steel exposure module, holding $35 \mathrm{~mm}$ agar plates. Bacteria were exposed for $24 \mathrm{~min}$ at a module flow rate of $5 \mathrm{~mL} / \mathrm{min} /$ well and incubated for three days before scoring. Whole smoke (generated under ISO conditions) was tested at different concentrations; final testing concentrations of neat ethylene oxide, achieved by variation of dilution air flow rates, were in the (evaluable) range of $1,000-4,500 \mathrm{ppm}$ (eminently higher than what is found in cigarette smoke). As expected, distinct dose dependent increases in the number of revertants were observed with neat ethylene oxide and with whole smoke (only with S9 activation). For the smoke spiking studies ethylene oxide was first introduced at varying flow rates into whole smoke and then mixed in the dilution bar with air of different flow rates to the desired concentrations (ethylene oxide up to 9,500 ppm). Surprisingly, the addition of ethylene oxide did not increase the genotoxic potency (with S9 activation) of whole smoke significantly at any of the (really high) concentrations tested. To explore the possibility of whole smoke interfering with the genotoxic activity of ethylene oxide, the two materials were combined in appropriate concentrations and tested without S9 activation (this way suppressing intrinsic smoke toxicity). Dose dependent - though weaker - genotoxic effects were detected; an observation not conducive to understanding the lack of genotoxic synergy between smoke and ethylene oxide in combination.

To shed light on these observations, it should be remembered that ethylene oxide is an extremely reactive substance. BINDER and LINDNER (497) confirmed reliably the presence of (estimated) $5 \mu \mathrm{g}$ ethylene oxide in the unaged mainstream gas phase of one cigarette. However, the epoxide reacts rapidly with various tobacco/smoke components, such as nicotine, forming a tertiary-quaternary amino base (498), or methylamine forming $N$-methylamino ethanol (499). In the past, treatment of tobacco with ethylene oxide was used for nicotine reduction $(500,501)$. In our opinion, the reactivity of ethylene oxide may explain the reported lack of genotoxic synergy between smoke and ethylene oxide.

For assessing the genotoxic potential of tobacco aerosols in human lung cell lines WEBER et al. (502) exposed human adenocarcinoma cells (A549) and bronchial epithelial cells (BEAS-2B) and determined DNA damage by means of the very sensitive comet assay (see page 206). Data obtained by staining and fluorescence microscopy were expressed as "\% tail intensity" (of whole comet intensity) - indicating the extent of DNA damage. Whole smoke was produced from 3R4F Kentucky reference cigarettes by a VC $10^{\mathbb{R}}$ smoking robot (450) under ISO 3308 (184) conditions and immediately diluted puff-wise in a Vitrocell ${ }^{\circledR} 6$-fold dilution system (503) with 6 separate elements, where the smoke was mixed dynamically with humidified synthetic air injected at five different flow rates $(0.2-2.0 \mathrm{~L} / \mathrm{min})$, thereby creating defined smoke dilutions; the sixth element was reserved for synthetic air control. As two sequential smoking runs with 5 cigarettes and 7 puffs each were carried out, total exposure time was $14 \mathrm{~min}$.

Exposure of cells on membranes was performed in a Vitrocell $^{\circledR} 24$ cultivation and exposure module (504), featuring an aerosol exposure top with 24 specially shaped inlets and a 24-well cultivation base ( 6 rows with 4 inserts each). The system allowed each row to be supplied separately with test aerosol $(2 \mathrm{~mL} / \mathrm{min}$ freshly diluted whole smoke at each well) and culture medium. This way, five smoke concentrations were examined in the same experiment. Comet assay response in both A549 and BEAS-2B cells was significant and dose-dependent. Cell viability, determined by an automated cell counter, was above $75 \%$ in most cell culture samples - making it unlikely that cytotoxic effects contributed considerably to DNA damage. Cursory examination indicated acceptable repeatability and reproducibility.

The most ambitious Vitrocell ${ }^{\circledR}$ cultivation and exposure system is the 24/48 module (505) with a capacity of 48 wells arranged in 8 rows, which allows 6-replicate exposures with - for instance - 7 different doses and one control (Figure 53). Technically, it represents the straight doubling of the 24-well system (504) and is housed in a climatic chamber. MAJEED et al. (506) examined the smoke dilution and exposure capabilities of the Vitrocell ${ }^{\circledR} 24 / 48$ in combination with the fully automated 30-port carousel SM2000 smoking machine (507). This machine was manufactured by Burghart Messtechnik GmbH (Wedel, Germany; the company ceased operations in 2015) according to Philip Morris specifications and became commercially available. It consists of the smoke generator S-GEN-B 30 and the programmable dual syringe pump SP-Duo. Operating parameters can be adjusted for compliance with ISO or more intense smoking regimes. In the study, two 3R4F Kentucky reference cigarettes were smoked in turn under Canadian Intense conditions (22) for delivering four puffs per minute to the dilution system. This cycle was completed three or 
five times, depending on how many cigarettes were scheduled to be smoked ( 6 cigarettes in $18 \mathrm{~min}$ or 10 cigarettes in $28 \mathrm{~min}$ ). Dilution air flows of $0.1-3.0 \mathrm{~L} / \mathrm{min}$ were used for producing smoke concentrations of $69 \%, 54 \%$, $32 \%, 19 \%, 13 \%, 10 \%$, and $7 \%$. Flow rate of diluted smoke for exposure was $2 \mathrm{~mL} / \mathrm{min}$. Various interesting approaches were taken to assess the performance of the Vitrocell $^{\circledR} 24 / 48$ system:

Insert-to-insert variability was examined with the watersoluble tetrazolium salt WST-1, which is widely used in cell vitality assays and was found to react also with the reducing constituents of tobacco smoke to form the corresponding formazan dye. All inserts in the base module were filled with reagent and optical density was measured at $430 \mathrm{~nm}$ after exposure to $32 \%$ smoke from 6 cigarettes (66 puffs in $18 \mathrm{~min}$ ). Overall, the distribution appeared uniform, and no regional pattern or gradient was observed. When different concentrations of smoke were examined a linear correlation between optical density and dilution was shown. In the very same experiment, quartz crystal microbalances (QCMs) were installed into each of the seven pipe connectors between the smoke dilution elements and the base module - and not placed in any of the wells as done by ADAMSON et al. $(475,476,492,493)$ in a number of earlier studies. Particle deposition was monitored online and the final mass determined at the end of the smoking run. Total deposition was found to be uniform at specific points over the seven supply lines. However, contrary to the optical density measurements, deposited mass was linearly correlated to smoke concentration only at smoke concentrations $\leq 20 \%$ (the range relevant for cell exposure). It should be noted that the WST-1 test indicates effects of whole smoke (including gaseous constituents) while quartz crystal microbalances (QCMs) respond to particulate matter deposition, which may be affected specifically and differently in a concentration dependent way by physical factors, such as droplet diameter, impaction or electrical charging. Computational fluid dynamics (CFD) modeling may be called for to clarify this kind of questions.

Turning to individual smoke constituents, nicotine was measured in differently concentrated smoke (7-69\%) by connecting columns with acid-impregnated diatomaceous earth directly to the outlets of the dilution elements; after elution nicotine was quantified by gas chromatography and flame ionization detection (FID). Eight carbonyls (six aldehydes and two ketones) were trapped in the 24/48 base module by filling the wells with phosphate-buffered saline (with no reagent). Following derivatization, 2,4-dinitrophenylhydrazones were analyzed by LC/MS. With all analytes tested (nicotine as well as the carbonyls) the amounts detected were linearly and convincingly correlated with smoke concentrations.

Finally, human adenocarcinoma A549 cells and bronchial epithelial BEAS-2B cells, prepared as monolayers in polyethylene terephthalate transparent membrane inserts, were exposed in the base module over $18 \mathrm{~min}$ to smoke from 6 cigarettes at concentrations of $10 \%$ and $15 \%$. Following post-exposure periods of 4, 24 and 48 hours, viability was assessed by the resazurin assay. The effect of $15 \%$ smoke was consistently stronger than with $10 \%$ smoke, and BEAS-2B cells appeared to be more sensitive

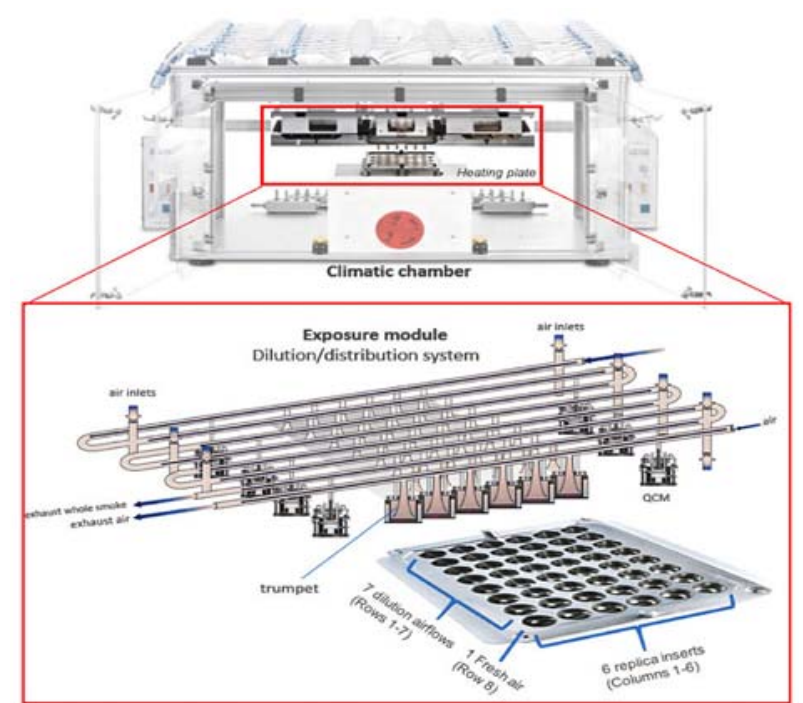

Figure 53. The sophisticated Vitrocell ${ }^{\circledR}$ dilution/distribution system supplying a 48-well module with aerosol for air-liquid interface exposure was evaluated by MAJEED et al. (506).

than A549 cells.

Monolayers of bronchial cells on microporous membranes are certainly a test model physiologically more relevant than cells in suspension. A step forward, however, is the use of three-dimensional primary organotypic cultures of human bronchial epithelial cells, which are commercially available from different sources and supplied in cell culture inserts ready for air-liquid interface (ALI) exposure. Generally, the cells come from a single (nonsmoking) donor and are genetically homogeneous. They form a multilayered pseudo-stratified tissue composed of the cells, which form the lung bronchial epithelium in vivo (508): ciliated cells, non-ciliated cells, mucin producing goblet cells on the apical side and basal cells - with functional tight junctions. The cells are morphologically and functionally differentiated and have xenobiotic metabolizing activity. It has even been said that they regenerate after mechanical or chemical injury.

The xenobiotics metabolism capability of a representative three-dimensional primary organotypic tissue culture system was recently assessed in a short term assay by BAXTER and MINET (509), using ciliated and mucus producing human epithelial cells of nasal origin (from six different donors), cultured in Transwell ${ }^{\circledR}$ inserts under airliquid interface conditions. Morphological stability (claimed to be retained for up to a year) was confirmed over the study time of 37 days by proving unvarying percentages of ciliated surface area and cilia beat frequencies. Using reporter probes and quantitative reverse transcription-polymerase chain reaction (RT-PCR) methodology, expression profiles were established for over 40 genes relevant for oxidative and conjugative metabolism. In parallel, the activity of several respiratory metabolic enzymes, which are involved in the bioactivation of certain tobacco toxicants, was measured.

Organotypic human bronchial epithelial tissue culture was used by MATHIS et al. (510) to examine biological effects of cigarette whole mainstream smoke. Single 3R4F Kentucky reference cigarettes were smoked (seven puffs per cigarette) on a VC $10^{\circledR}$ smoking robot (450) under ISO 
(184) conditions, and tissues exposed for 7, 14, 21 and $28 \mathrm{~min}$ at the air-liquid interface to either $15 \%$ smoke or synthetic air in a Vitrocell ${ }^{\circledR} 24 / 24$ system (504). Post-exposure time in fresh culture medium was $0.5,2,4,24$ and 48 hours before further analysis. To control the responsiveness of the tissue system to whole smoke exposure, the secretion of MMP-1, an interstitial collagenase, into the medium was measured by immunoassay. A very strong dose-dependent effect was observed.

The main objective of the study, however, was to examine whether major gene related effects known to occur in smokers could be replicated in organotypic epithelial tissue by ALI smoke exposure, after PEZzUlo et al. (511) had shown that the transcriptional profile of differentiated primary cultures grown at the ALI resembled very closely that of in vivo airway epithelium. Two kinds of genetic response to a single whole smoke exposure were investigated (510) in organotypic endothelial tissue using microarray technology. First, in vivo gene signatures (expression profiles) in bronchial epithelial cells obtained from smokers were extracted from four publicly available databases. Using gene set enrichment analysis (GSEA), which documents and interprets genome-wide expression data (512), the four smoker gene signatures were shown to be significantly enriched in the expression profile of organotypic tissue samples after in vitro ALI exposure to cigarette smoke - both in terms of up- and down-regulated genes. Second, the effects of smoke exposure were examined at the level of transcriptomics. Expression profiles of (noncoding/regulating) micro-ribonucleic acid (microRNA) indicated (based on limited data) that the response of several microRNA species was equally strong in vivo (smoker epithelium) and in vitro (organotypic epithelial tissue). It was the authors' conclusion "that differentiated NHBE [normal human bronchial epithelial] cells cultured at the air-liquid interface and exposed to a single exposure of CS [cigarette smoke] recapitulate many of the biological perturbations observed in the airway epithelium of smokers".

Vitrocell ${ }^{\circledR}$ exposure modules (type not specified) were used in the following two additional studies when exposure to whole cigarette smoke at the air-liquid interface was essential for determining tissue response to xenobiotic challenge.

Transcriptomic data was combined by ISKANDAR et al. (513) with a network model, designed to capture biological mechanisms of xenobiotic metabolism using causal relationships, in order to assess quantitatively the biological risk of the xenobiotic agent, cigarette smoke. Three-dimensional organotypic tissue cultures of human bronchial and nasal epithelium were exposed at the air-liquid interface to smoke from 3R4F Kentucky reference cigarettes. Smoke from 4 test pieces per exposure was produced under Canadian Intense conditions (22) by a SM 2000 machine (507), diluted to $16 \%$ and sequentially applied with a 1 hour rest time between cigarettes; post-exposure time was 4,24 or 48 hours. In a quantitative systems-level approach, the xenobiotic metabolism network model allowed the robust comparison of transcriptional data upon smoke exposure, obtained from different sources (in vivo data from public databases; in vitro data from ALI exposures): 1) nasal versus bronchial tissues in vivo, 2) nasal versus bronchial tissues in vitro, and 3) nasal and bronchial tissues in vivo versus in vitro. Xenobiotic responses in the bronchial and nasal epithelial cells of smokers were similar to those in the respective organotypic tissues exposed to cigarette mainstream smoke. The results suggested that nasal tissue, which can be sampled rather easily, was a reliable surrogate to measure in vitro xenobiotic responses in bronchial tissue and could be useful to examine smoking effects occurring in vivo.

SCHLAGE et al. (514) used oral mucosa in the form of organotypic buccal and gingival epithelial tissues, which were cultured on Transwell ${ }^{\mathbb{B}}$ permeable porous membranes and showed impressive in vivo-like morphology (thickness of 20-30 layers; inclusion of fibroblasts and inflammatory cells). In practice, it is much less invasive to obtain samples of oral than of bronchial mucosa. Whole smoke was produced from 3R4F Kentucky reference cigarettes by a SM 2000 machine (507) under Canadian Intense conditions (22) and diluted to concentrations of approximately $20 \%$ and $40 \%$. Smoke from 4 cigarettes per exposure was dispensed (6-7 min per cigarette) with a 1-hour rest time between cigarettes, simulating human smoking behavior. Post-exposure periods of 4,24 or $48 \mathrm{~h}$ were observed. Cigarette smoke showed weak cytotoxicity $(<20 \%$, measured as lactate dehydrogenase (LDH) release), increased the secretion of pre-inflammatory mediators, and induced several cytochrome P450 enzymes. Microarray technology, gene set analysis, functional annotation and causal biological network modeling were used to diagnose the effects of cigarette smoke on xenobiotic metabolism pathways and inflammatory processes. When in vitro gene expression profiles of buccal organotypic epithelial tissue were compared to analogous in vivo data (obtained from publicly available datasets derived from smoker biopsies) a considerable degree of congruity was observed, demonstrating the applicability of organotypic tissues for in vitro testing.

\subsubsection{The Mimic Smoker Burghart MSB-01}

In the late 1990s, the Heinrich Burghart GmbH (Wedel, Germany) developed - at the request of, and in cooperation with, the German cigarette manufacturer Reemtsma - a mimic smoker for exposing cells directly to "native" cigarette mainstream smoke; the device was introduced by RÖPER and WIECZORECK (515) at the 2002 CORESTA Congress in New Orleans (LA, USA). It consisted of a single port piston smoking machine linked to a glass covered exposure chamber (about $1.7 \mathrm{~L}$ volume) containing a multiwell plate (microtiter plate). Three cigarettes were smoked consecutively (with 8 puffs each) using ISO 3308 (184) parameters, producing a total of 24 puffs for immediate transfer into the chamber. During puff intervals the chamber was flushed repeatedly with small boluses of ambient air. A motor controlled stainless steel sliding lid above the multiwell plate was used to cover or display wells, thus rendering the apparatus capable to expose distinct rows to different numbers of smoke puffs. Electronic valve and piston speed control allowed various between-puff dilution and air flushing regimes. The smoke exposure system was intended to mimic human smoking and breathing behavior. Monolayers of HEP-G2 cells 


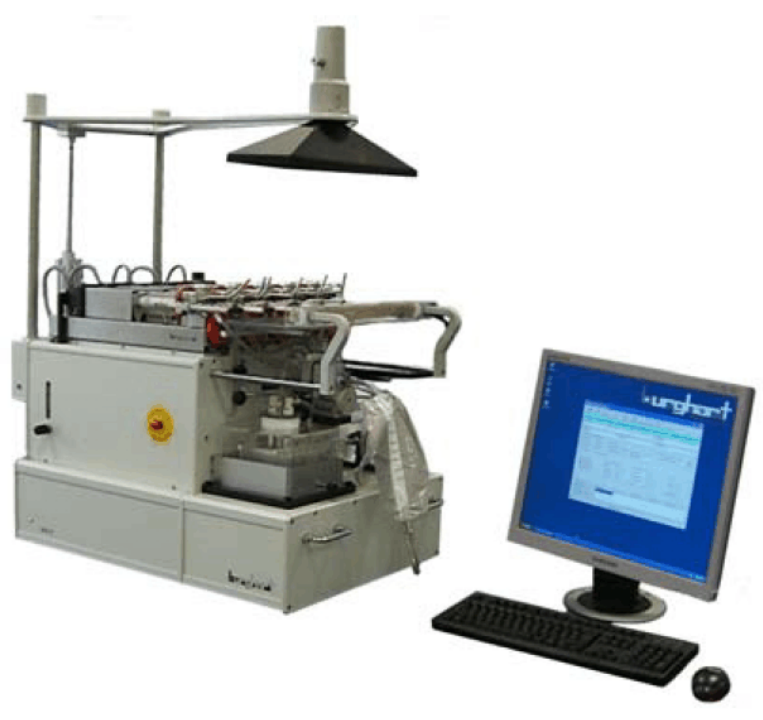

Figure 54. The five independent piston pumps of the Mimic Smoker MSB-01 (518) produce smoke for in vitro exposure of cells in a 96-microwell plate.

(human liver hepatoma, ATCC-8065) cultured in serumfree medium were exposed to whole smoke aerosol. Cytotoxic effects were measured by means of the neutral red uptake (NRU) and the (tetrazolium-based) MTS (516) viability assays. Dose response curves showed pronounced differences between several experimental cigarettes with different kinds of filters and variable degrees of filter ventilation.

A modified version of the smoking/exposure system, called $\mathrm{Bt} 020$, and its validation were described in 2005 by WIECZOREK et al. (517). The single port smoking machine was directly connected with a new exposure chamber accommodating a cell culture plate with 96 round-bottom wells and fitted with a sliding lid smoke distribution mechanism. The whole system was validated by measuring smoke particle deposition (as optical density at $370 \mathrm{~nm}$ ) under two conditions: Across the whole 96-well plate with a given dilution (range examined was 1:8-1:25), standard deviation of treated wells was less than $10 \%$, and the optical density values at different (numerically transformed) dilutions formed a linear function. Using the sliding lid optical density, determined with three different dilutions, was again linearly related to the number of puffs rows were exposed to. When cytotoxicity was assessed in monolayers of human HEP-G2 hepatoma cells using the neutral red uptake assay, exposure to mainstream whole smoke and gas phase from three cellulose acetate filtered cigarettes (with contrasting levels of tip ventilation) and a plain cigarette produced impressively divergent and very plausible $\mathrm{EC}_{50}$ values. Comparing pertinent data it was shown that the contribution of the gas phase to cytotoxicity compared to whole smoke was $2.0-24.8 \%$, clearly depending on the design of the 4 test pieces.

The mimic smoker was subsequently scaled up into a multi-port system, called Mimic Smoker Burghart MSB-01. The apparatus (Figure 54) became commercially available in 2007 (518) but is no longer on the market after the Heinrich Burghart GmbH closed operations in 2015. The device was capable of smoking up to 5 cigarettes simul- taneously, each port with an independent piston pump, which could in addition be used to dilute the cigarette smoke up to 10-fold with filtered ambient air. The smoke was then combined into a common mixing bag. This way, mainstream smoke variations resulting from unavoidable cigarette differences were minimized. The smoke was then moved forward by an additional pump, which allowed even higher dilution (up to a maximum of $1: 150$ ). The MSB-01 exposure system consisted of an integrated 96microwell plate (rather than a receptacle holding cell culture inserts) combined with a smoke distribution manifold (some $7 \mathrm{~mm}$ above the plate) featuring 96 positions, each with one delivery and two exhaust ducts. Smoke ( $35 \mathrm{~mL}$ per puff for the entire plate) could be delivered either to all wells simultaneously or - guided by a retractable stainless steel cover between the manifold and the culture plate - stepwise to specific well sections, such as a row. After treatment smoke was flushed out with air, aided by a vacuum. The time needed from puff generation to delivery for exposure was approximately 6 sec. Smoking and exposure conditions (puff volume and frequency, smoke dilution, exposure time, aeration of exposure chamber, etc.) were controlled and recorded electronically. The Mimic Smoker MSB-01 was characterized by SCIAN et al. (519) in a study, which analyzed the consistency over time of particle deposition and smoke cytotoxicity. In addition, losses of particulate matter during smoke flow and dissimilarities in particle size distribution in differently diluted smoke were determined. Four 2R4F Kentucky reference cigarettes were smoked simultaneously under ISO conditions, and the smoke was examined undiluted and diluted $1: 1$ or $1: 3(v / v ;$ smoke:air). Microwells were exposed either across the entire plate or by row with the retractable cover sliding forward after each puff, this way creating a concentration gradient (Figure 55).

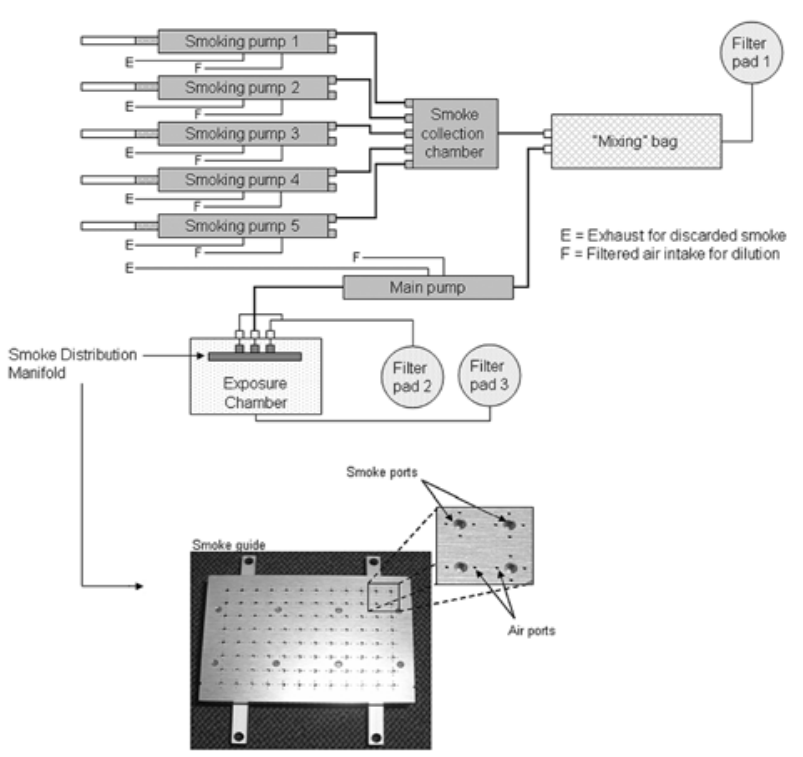

Figure 55. In the MSB-01 system, single smoke puffs flow into the collection chamber and the mixing bag, and are then delivered by the main pump to the distribution manifold in the exposure camber; manifold design features are shown on an enlarged scale (519). 
Plate deposition of particulate matter was measured in each well, after dissolution in dimethyl sulfoxide, photometrically at $370 \mathrm{~nm}$ - with condensate collected on Cambridge filters for the production of a linear standard curve. Whole plate exposure showed no significant differences between rows while linear puff dependency was observed in gradient experiments with all three dilutions tested. In these experiments, within-day and between-day variation was estimated at less than $11 \%$ and $13 \%$, respectively. Viability was assessed in nonmalignant human bronchial epithelial BEAS-2B cells by means of the neutral red uptake assay. Cells were exposed to diluted smoke $(1: 1)$ using a concentration gradient of $0-8$ puffs, leading to significant dose dependent reductions of viability. After the calculation of $\mathrm{EC}_{50}$ values (expressed as the number of puffs at the tested dilution necessary to induce a $50 \%$ decrease in viability) based on a sigmoid function, withinday variation was calculated at $13.1 \%$ and between-day variation at $20.3 \%$.

For the assessment of any losses of particulate matter during transfer, smoke samples (undiluted or 1:3 diluted) were collected on Cambridge filters at five points along the smoke path between the smoking port and the smoke manifold and quantified after extraction with methanol by photometry at $350 \mathrm{~nm}$. The data showed that - regardless of dilution - as much as $45-50 \%$ of the material, detected at the smoking port, was lost in transit prior to delivery at the multiwell plate, particularly within the connecting tubing and pump valves. This may have been caused not only by diffusion, impaction and sedimentation but also by direct electrostatic or chemical interactions between smoke components and the tubing. Particle size distribution (mass median aerodynamic diameter and geometric standard deviation) was assessed with samples taken at four points along the smoke path using a cascade impactor with subsequent photometric quantification of the fractions dissolved in methanol. It was shown that smoke particle size distribution did not change along the smoke path (regardless of dilution). However, mass median aerodynamic diameter was noted to be significantly reduced in diluted (1:3) compared to undiluted smoke; the higher proportion of air was speculated to decrease particle coagulation and promote the release of semi-volatiles from particles - with potentially disturbing effects on smoke composition.

Differences in chemical composition depending on particle size had been observed in earlier studies by BERNER and MAREK (520), JONES et al. (521), MORIE and BAGGET (522), and JENKINS et al. (523).

In view of the heavy depletion of particulate mass in the MSB-01 system it is not surprising that the authors recognized the need for regular cleaning of the equipment (after smoking approximately 100 cigarettes) and the periodic replacement of expendable parts.

The disturbing loss of about half of the particulate smoke material during transport prompted SCIAN et al. (524) to conduct a thorough - qualitative and quantitative - analysis of Cambridge filter precipitates, collected at the start and the end of the smoke path in the MSB-01 system, i.e., right at the smoking port and after the smoke manifold. In addition, after dismantling and cutting into small pieces, the plastic tubing and the "mixing" bag of the MSB-01 were examined for extractable deposits (after smoking approximately 84 cigarettes). Smoke was produced from 2R4F Kentucky reference cigarettes (five cigarettes from different packs smoked in succession) under ISO conditions using the mimic smoker with appropriate settings, and examined - in terms of mass and composition - either undiluted or after 1:1 dilution with filtered air. Particulate matter was collected on Cambridge filters and quantified either gravimetrically or by spectrophotometry following extraction from the filter pads with methanol or by LC/MS using solanesol as a marker; TPM chemical component analysis was done by GC/MS. Data obtained with material trapped at the smoking port served as $100 \%$ reference. Particle mass was confirmed to be reduced by $50 \pm 10 \%$ when sampled after the smoke manifold - based on remarkably consistent data acquired with the three different methods of determination.

A total of 110 individual chemical constituents were measured in smoke. After passage from the smoking port to the smoke manifold overall reductions to $35 \pm 13 \%$ (undiluted smoke) and $23 \pm 8 \%$ (diluted smoke) were observed. Individual compounds were lost in transport to a very variable extent, which did not depend on chemical class. Many constituents showed deliveries of $40-60 \%$, consistent with the known losses in particulate mass and likely to result from deposition. Many others were reduced to $10-40 \%$, and a large share to below $10 \%$. Evaporation was assumed to play an enhancing role in the case of volatile and semivolatile compounds. As gas phase constituents were not analyzed in the study this notion was not further explored. A number of compounds, mainly nitrogen containing and aromatics, were completely lost and no longer detected after the smoke manifold, for example pyridine and some alkyl pyridines, several pyrazines and a range of alkyl derivatives of benzene, indene and naphthalene.

In addition, when particulate matter mass and smoke constituent levels were compared in samples of undiluted and diluted smoke, taken at the smoking port, it became quite apparent that mixing in the smoking pump was not functioning perfectly.

The studies by SCIAN et al. $(519,524)$ demonstrated clearly the dramatic - quantitative and qualitative - changes smoke aerosol was subjected to in the MSB-01 system after generation and before contact with cells in the exposure chamber. These findings emphasize the importance of characterizing not only the quantitative availability of smoke for in vitro whole smoke exposure but also the composition of the delivered aerosol when assessing the biological effects of smoke. In case of the MSB-01 this was done in an approach more complete than with other devices used for in vitro exposure to native whole cigarette smoke, where comparable examinations may not have been conducted with sufficient attention.

Recently, WIECZOREK (525, page 19) described a newly developed smoke exposure in vitro system (SEIVS) in use at the Imperial Tobacco BioLab. Smoke was generated from up to five products using separate smoking pumps combined with additional mixing and diluting pumps. Two parallel ALI exposure chambers with multiwell plates (24 or 96 size) permitted the simultaneous analysis of various products, and the comparative testing of whole smoke and its gas phase; different mammalian cell lines (HEP-G2 and 
V79) and Salmonella typhimurium cells on agar plates were used as well as distinct assays (such as neutral red uptake, micronucleus formation and Ames mutagenicity).

\subsubsection{The smoking machine Sibata SG-200}

The SG-200 smoking machine was developed at the turn of the century by Sibata Scientific Ltd of Sōka City, Saitama, Japan (526) for the purpose of generating cigarette mainstream smoke for inhalation studies with small animals and for the establishment of an in vitro model for chronic obstructive pulmonary disease (COPD). It was not intended to be used in smoke analysis. The rotating smoking head was positioned vertically in a relatively small plexiglas box and able to hold up to 40 cigarettes, which were loaded and lighted automatically. Puffs of $35 \mathrm{~mL}$ in $2 \mathrm{sec}$ (fixed setting) were taken by means of a step motor driven syringe at an adjustable frequency of 1-12 puffs per min. The smoke could be diluted up to $1: 20$.

It is obvious that the Sibata SG-200 smoking machine did not meet essential criteria of ISO 3308 (184) or the requirements defined by BEVEN (345) for smoking devices used in animal inhalation studies. The air flow around cigarettes could not be measured and controlled. The vertical position of the cigarettes boosted temperature dependent air convection in the smoking chamber, affecting the burn and smolder velocities of individual cigarettes in an erratic way. Due to the small size of the smoking box sidestream smoke, projected to be removed by means of a fan, did inevitably mix with mainstream smoke - distorting its composition and toxicity.

Cultex ${ }^{\circledR}$ technology in combination with the Sibata SG-200 smoking machine was used by FUKANO et al. (527) in a study of the cytotoxic effects of cigarette mainstream smoke. 2R4F Kentucky reference cigarettes were smoked under standard conditions. The experimental set-up included 5 Cultex ${ }^{\circledR}$ exposure modules (382), operated concurrently and including the option of individual conditions of smoke dilution. Each module was closely linked to an aerosol monitoring device fed with branched-off smoke. Human lung epithelial type II cells (A549) were seeded onto microporous membranes of culture inserts and exposed to whole smoke or gas vapor phase (after smoke passage through a Cambridge filter). The usual exposure conditions were modified in two ways: A portion of fresh smoke was delivered to the exposure module with cells still being covered by medium, which was subsequently drained off slowly forming the ALI; cells were then exposed for $24 \mathrm{sec}$ and again flooded with medium, and residual smoke was removed from the module - this cycle of $1 \mathrm{~min}$ duration repeated at will. Furthermore, as the intact microporous membranes did not allow the speedy displacement of medium, five holes were pierced through each membrane (somewhat of a devious technique). Under these conditions, cell viability was not reduced by exposure to air after 5 hours, equivalent to 300 simulated puffs. Cytotoxicity was assessed after an 18-hour recovery period under submerged conditions by two methods: the tetrazolium (WST-1) assay and the neutral red uptake test. Dose-dependent effects were observed over a large range of particulate matter in whole smoke, while gas vapor phase (produced by inserting a Cambridge filter in front of each exposure module) was - at equivalent doses - clearly less effective. When the original $2 \mathrm{R} 4 \mathrm{~F}$ cellulose acetate filter was replaced by either a different cellulose acetate filter or a cellulose acetate/charcoal filter (both modifications identical in pressure drop to ensure comparable mainstream smoke total particulate matter yields), the cytotoxic potency of the gas vapor phases of the two test cigarettes was markedly different (and more reduced by the charcoal filter).

Using an identical combination of instrumentation and manipulation (including 2R4F Kentucky reference cigarettes, human lung epithelial A549 cells and the piercing of holes through the insert membranes to enhance particle sedimentation) FUKANO et al. (528) explored a new biological endpoint of ALI exposure to whole smoke and its gas vapor phase: the induction of messenger ribonucleic acid (mRNA) coding for an isoform of heme oxygenase (HO-1). This enzyme degrades heme to biliverdin and is assumed to play a role in controlling oxidative stress in the lungs. Increased mRNA expression was measured by means of the reverse transcription - real-time quantitative polymerase chain reaction (RT-qPCR), which consists of the transcription of mRNA into complementary deoxyribonucleic acid (cDNA) and coupled amplification and detection/quantification of formed cDNA. Dose dependent effects were observed over a range of whole smoke concentrations; this outcome was maintained - though with reduced intensity - when equivalent amounts of gas vapor phase were applied. Replacement of the reference cigarette's original filter by either a simple cellulose acetate filter or a filter containing charcoal demonstrated clearly reduced gas vapor phase activity with charcoal in the filter. The cigarette smoke generator Sibata SG-200 was also used by Japanese research groups (529-533) for the production of mainstream smoke for a number of mouse inhalation studies - in one investigation (533) deviating considerably from standard smoking conditions. 

COLLECTION SYSTEMS

There are many methods of analyzing or testing whole smoke or its components in native form and real time without prior trapping or collection. Examples are the physical characterization of particulate matter and the quantification of gas phase constituents online, toxicity testing of aerosol using air-liquid interface methodology and inhalation studies with experimental animals. For numerous other preparative and analytical purposes, however, the collection of smoke condensate and volatile compounds constitutes an indispensable requirement. Consequently, a broad range of techniques and a large variety of devices were developed and are available.

The role of trapping systems in the standardized preparation and fractionation of cigarette smoke condensates was discussed in 1972 by BENNER and KeENE (534). These materials were produced as a service to tobacco and health investigators elsewhere at the Tobacco and Health Research Institute of the University of Kentucky. For routine large scale condensate preparation the solvent free cold trap of ELMENHORST (270) was used. Four systems were available for small scale condensate production: Impaction traps, Cambridge filter pads, small cold traps with helices, and - most expeditiously - direct trapping into an appropriate solvent, energized by vibration, as described by ELMENHORST (268).

At the 1982 Tobacco Chemists' Research Conference DUBE and GREEN (535) presented a review of the methods and devices used up to the early 1980s for collecting cigarette smoke for analytical purposes. They pointed out:

"Methods used to generate and trap the smoke aerosol exert profound effects on the composition of the condensate. Standardization of smoking parameters has minimized the variability resulting from artificial smoking; however, the traps used to collect cigarette smoke remain a major contributor to non-reproducibility. ..... Artifact formation is a major drawback associated with many of the trapping procedures."

The authors compiled a wish list for the idealized smoke collection device:

- Efficient

- Simple construction

- Easily cleaned

- Reproducible

- Reliable

- Low pressure drop

- Minimum volume

- Eliminating possibilities of artifact formation

- Quantitative.

While none of the currently available collection systems fulfills all requirements, there are several devices that have proven very useful for the purpose intended. In all situations and circumstances, however, particular attention has to be turned to the stability of the collected materials and the physical and chemical changes that may take place in the process.

\subsection{Aging and artifact formation}

The many reactive species in fresh tobacco smoke may actually bring about the formation of several of its identified components or their depletion during sampling and by aging. This is likely to influence the results obtained in analytical determinations and toxicological testing of cigarette mainstream smoke. This phenomenon was first identified in 1962 by Rochus (536) in his fundamental reflections on the chemical composition of tobacco smoke and the reactivity of its components.

The first (biological) investigation showing that such effects may really occur was published in 1967 by DAY (537), a scientist working at the British Tobacco Research Council Laboratories in Harrogate (UK). He compared fresh mainstream smoke condensate (not older than $24 \mathrm{~h}$ ) to aged condensate (evaporated to constant weight for removing solvent, water and volatiles, and stored at $-29^{\circ} \mathrm{C}$ for least 1 month) in a mouse skin painting carcinogenicity study. At equivalent doses, fresh condensate was somewhat more active but it remained unclear whether the reduced potency of the stored material was due to processing (loss of semi-volatiles) or aging.

In the 1970s, scientists at the Research Institute of the German Cigarette Industry (Hamburg, Germany) addressed systematically the problem of artifact generation in cigarette smoke condensates.

SCHÜLLER et al. (538) investigated the qualitative composition of "gas phase condensate", which was collected in a cold trap at $-80{ }^{\circ} \mathrm{C}$ (539) and believed to contain the vapor phase components of cigarette mainstream smoke with the exception of highly volatile gases, such as nitrogen, oxygen, carbon dioxide, carbon monoxide and methane. When the oily phase of the condensate, which formed at room temperature, was analyzed by gas chromatography norbornene derivatives were identified only in aged samples and absent when the material was fresh and still below ambient temperature. The norbornene compounds were obviously produced by Diels-Alder reactions (540) of cyclopentadiene and methylcyclopentadiene with crotonaldehyde or acrolein (541).

In a more methodical approach, SCHÖNHERR et al. (46) used cold traps at $-80{ }^{\circ} \mathrm{C}$ to generate whole smoke condensate, subsequently dissolved in acetone, and "gas phase condensate" (539), undissolved or in acetone solution. Samples were stored for up to three weeks at temperatures between $-19{ }^{\circ} \mathrm{C}$ and $+20{ }^{\circ} \mathrm{C}$, followed by the determination of "dry condensate", i.e., the residue left after stepwise intense evaporation. The amounts of dry condensate increased notably during storage with particulate components making the major contribution compared to volatile constituents. Gel chromatography of dry condensates revealed polymerization being a factor in the changes of condensate composition due to aging.

In view of the fact that acetone had become a preferred solvent for taking up cigarette smoke condensate it is noteworthy that EINOLF et al. (542) identified three methylated piperidones, which were formed as artifacts by reactions between the solvent, acetone, and two smoke constituents, acetaldehyde and ammonia. 
NALL (543) reported that up to $64 \%$ of total hydrogen cyanide of cigarette mainstream smoke was trapped in complex form, presumably cyanohydrins, depending on the type of smoke collection system. The collection of smoke in a liquid trap with methanol/water at $-80{ }^{\circ} \mathrm{C}$ favored the generation of cyanohydrines from aldehydes and hydrogen cyanide. Collection of smoke in a water-free cold trap did not produce these compounds as reported by DUBE and GREEN (535).

With regard to aging, the most problematic smoke components are nitrogen oxides. NORMAN and KEITH (544) established that nitric oxide (NO) was the primary nitrogen oxide in fresh cigarette mainstream smoke, and nitrogen dioxide $\left(\mathrm{NO}_{2}\right)$ was present in trace amounts at most. This was confirmed by BARKEMEYER and SEEHOFER (545). They reported that only a trace of nitrogen dioxide was present in fresh mainstream smoke of a U.S. blend cigarette $(5 \mathrm{sec}$ old $)$ but a considerable amount was generated within 1 min from nitric oxide by aging. The reaction kinetics of the oxidation of nitric oxide to nitrogen dioxide was analyzed by GREIG and HALL (546) in experimental mixtures of nitric oxide and oxygen in nitrogen, and by SLOAN and KIEFER (547) in cigarette smoke. COOPER and HEGE (548) followed the kinetics of the conversion of nitric oxide to nitrogen dioxide in aging smoke from nitrate-enriched Burley cigarettes by Fourier transform infrared spectroscopy - with the complete spectrum recorded every $7 \mathrm{sec}$. Nitrogen dioxide formation occurred markedly only in concentrated smoke and was negligible in normally diluted smoke from commercial cigarettes while nitric oxide remained the dominant species.

The purposeful oxidation of nitric oxide in mainstream smoke vapor phase is the basis for its determination using a method developed by BAT in the UK (549). Admixture of ozone to the gas vapor phase converts nitric oxide to an excited form of nitrogen dioxide, which relaxes to the ground state by emitting light and could be quantified with a chemiluminescence detector. Considering the high reactivity of nitric oxide immediate puff-by-puff analysis was called for.

Nitric oxide and nitrogen dioxide may give rise to a number of artifactual substances in cigarette smoke. Methyl nitrite, reported in 1959 as a component of mainstream smoke vapor phase by PHILIPPE and HACKNEY (550), was shown by VILCINS and LEPHARDT (551) not to be present in fresh smoke ( $2-5 \mathrm{sec}$ old) but to be formed over time by the reaction of methanol and nitrogen oxides $\left(\mathrm{NO}\right.$ and $\mathrm{NO}_{2}$ ) at relatively high concentrations. The reaction was not expected to take place under conditions of actual smoking due to the high dilution of the smoke.

KALLIANOS et al. (45) established that 4-nitrocatechol was generated - to a limited extent - by the reaction of catechol with nitrogen oxides when tobacco smoke was collected in cold traps.

In 1974, KLUS and KUHN (552) detected more than 20 different nitrophenols in cigarette mainstream smoke condensate. The authors discussed the possibility that these nitrophenols were artifacts formed in the cold traps used. For the collection of mainstream smoke NEURATH et al. (553) used an assembly of five traps in a row (cotton wool, water and three wash-bottles with pentane at $-80{ }^{\circ} \mathrm{C}$ ). Using a new sensitive analytical method - reduction of the
$N$-nitrosamines with lithium aluminium hydride to the corresponding asymmetric hydrazines and formation of 5 nitro-2-hydroxy-benzal derivatives with 5-nitro-2-hydroxy benzaldehyde (554) - and examining smoke from cigarettes with normal and relatively high (natural) content in nitrate and volatile bases as well as from cigarettes spiked with considerable amounts of nitrate and di-n-butylamine, volatile $N$-nitrosamines were determined in the different traps. The presence of three $N$-nitroso compounds was confirmed in the pentane traps; this was considered artifactual and promoted by cold temperatures and an anhydrous environment. Under special experimental circumstances, volatile $N$-nitrosamines were also found in the traps with cotton wool and with water. Holding the aerosol in a tube for $40 \mathrm{sec}$ before entering the cotton wool trap produced particularly high levels, indicating a timedependent gas phase reaction (44). In model experiments both nitric oxide (NO) and nitrogen dioxide $\left(\mathrm{NO}_{2}\right)$ were shown to be required for nitrosation - the (relatively slow) conversion of $\mathrm{NO}$ to $\mathrm{NO}_{2}$ being the rate limiting step, aided by elevated oxygen concentrations $(44,555)$.

In 1972, MIRVISH et al. (556) showed that the formation of $N$-nitroso compounds could be blocked at low $\mathrm{pH}$ by ascorbic acid, competing effectively for the nitrosating agent. This led HOFFMANN et al. (557) to recommend the collection of smoke in liquid traps filled with an ascorbic acid containing aqueous buffer solution at $\mathrm{pH} 4.5$ in order to prevent the artifactual formation of volatile $\mathrm{N}$-nitrosamines. This trapping method was adopted and used widely by other researchers.

\subsection{Direct sampling}

The temporary trapping of mainstream smoke gas phase without the involvement of lowered temperatures or solvents - and the subsequent availability of measured volumes for analysis is termed "direct sampling" and uses either syringes, sample loops or other intermediate containers. The technique is not suitable for particulate phase components and does not include in-line and flow-through methodology.

At an early stage of development, GROB (558) described the advantages of the instant analysis of fresh gas phase:

- No losses from incomplete condensation and revaporization,

- Avoidance of secondary reactions in concentrated samples,

- Gain of time.

While potential shortcomings were not concealed:

- Very low concentration of most components in the genuine gas phase,

- Immediate analysis of individual puffs only,

- Chemical or physical changes in material collected from several puffs or cigarettes,

- Interaction of gas phase constituents with the surfaces of different materials.

When collecting gas phase samples, additional aspects should be considered (558). Concentrations of some gas phase constituents are known to increase from the beginning to the end of a puff to more than twice their level, requiring special attention to which fraction is being withdrawn for analysis. In addition, gas phase composition 
cannot be expected to reflect precisely what is being "produced" in a particular puff; there is always trapping of components in the tobacco rod (butt) and revolatilization of compounds retained during earlier puffs - processes that have a "buffering" effect over several puffs and are even more effective in the presence of a filter.

The inherent physical and chemical lability of fresh gas phase was examined experimentally. When syringes made from different materials were used as intermediate containers (glass, steel, silicon rubber and surfaces already coated with a layer of gas condensate) adsorption effects of different intensity were observed depending on the volatility and chemical nature of the gas phase constituents. However, inside surface area was recognized to have higher influence than syringe material. Consequently, a $500 \mathrm{~mL}$ steel syringe with a teflon piston and $35 \mathrm{~mL}$ marks was considered the preferred tool for gas phase collection and mixing.

MORIE and SLOAN (559) collected the gas phase of smoke from plain and filter cigarettes in a $500 \mathrm{~mL}$ syringe (puffwise or whole cigarette) and used a gas chromatograph equipped with both thermal conductivity and flame ionization detectors for the determination of nitrogen, oxygen, carbon monoxide, carbon dioxide and over 20 organic compounds, applying a cryogenic temperature program.

Sample loops are devices which can hold and process accurate volumes of gas phase for subsequent analysis by gas chromatography or other methods. In their studies of minor gaseous cigarette smoke components - as many as eighty organic and few inorganic compounds - NEWSOME et al. (560) and NORMAN et al. (561) used solvent-based trapping systems (for "absorbable" gases), reservoirs (especially useful for oxides of nitrogen) and sample loops (providing material for immediate analysis by gas chromatography).

Automated Fourier transform infrared analysis (FT-IR) was used by KOLLER et al. (562) in 1990 for the simultaneous quantitative determination puff-by-puff of four mainstream smoke gas phase constituents: acetaldehyde, hydrogen cyanide, nitric oxide and carbon monoxide. Using an (unspecified) five port smoking machine, smoke from single puffs was passed through Cambridge filters and combined $(175 \mathrm{~mL})$ for collection in a large sample loop. Following dilution with nitrogen, an aliquot of $50 \mathrm{~mL}$ was allowed to flow through a transmission gas cell, where peak areas of selected absorption lines, specific for each analyte, were recorded in $30 \mathrm{sec}$ - in time before the arrival of the next puff. The method replaced separate procedures for each of the four gases.

In 2001, THOMAS and KOLLER (563) developed a fully automated multiplex (timed sequential injections onto a single column) GC/MS method for the gas phase analysis of individual puffs of a single cigarette, which was more comprehensive and for many smoke constituents more sensitive than Fourier transform infrared analysis (FT-IR). The $35 \mathrm{~mL}$ volume of a puff was trapped temporarily in a sampling loop for pressurization with helium followed by the sweeping of an aliquot onto the column. Twenty-five gas phase constituents of special interest were assessed covering a broad spectrum of compound classes.

For the measurement of ethylene and isoprene VILCINS (564) smoked cigarettes on an (unspecified) five port syringe type machine and transferred the gas phase puffby-puff by means of a solenoid valve system to a onemeter gas cell where it was held for the duration of the infrared analysis $(75 \mathrm{sec})$.

Beside of syringes, cells and loops, collection bags made of various plastic materials serve as intermediate containers for the direct sampling of gas phase components. Collins and Williams (565) developed an automated procedure for measuring carbon monoxide by non-dispersive infrared (NDIR) spectroscopy in the gas phase of mainstream smoke. Cigarettes were smoked on a 20-port syringe type machine (8) and the $700 \mathrm{~mL}$ combined volume of gas phase per puff was collected in a gas-tight polyethylene bag with a volume of $946 \mathrm{~mL}$, which was enclosed and manipulated inside a 2-liter aspirator. After thorough mixing - an essential step - the infrared absorption of a small sample (a few $\mathrm{mL}$ only) was recorded in the measuring cell.

The same kind of polyethylene bag was used by WILLIAMS (566) for collecting the combined puff volume of $280 \mathrm{~mL}$ gas phase from an eight port smoking machine for the puffby-puff determination of nitric oxide by NDIR spectroscopy - quite possible in combination with carbon monoxide and carbon dioxide. The system allowed the measurement of nitric oxide within $15 \mathrm{sec}$ of the puff but deliveries were lowered by oxidation and possible reactions with other smoke constituents, such as amines and alcohols.

The utilization of a 500-mL Teflon bag for collecting the gas phase from a single port smoking machine was described by SLOAN et al. (567). Twenty-two organic compounds (previously identified by mass spectrometry) were analyzed in a 2-mL sample by gas chromatography and flame ionization detection. Stored data were evaluated by a dedicated computer program.

Gas phase collection in a bag is an essential step in several standardized procedures, such as the automated measurement of total carbon monoxide per cigarette by means of the ATCOM module attached to the 8-channel Filtrona SM 302 smoking machine (101), the CORESTA Recommended Method No. 5 (568) for the determination of carbon monoxide in mainstream smoke of cigarettes by non-dispersive infrared analysis, and the HEALTH CANADA method T-115 (209) for the determination of "tar", nicotine and carbon monoxide in mainstream tobacco smoke.

\subsection{Cambridge filters}

In 1959, WARTMAN et al. (6) of the American Tobacco Company (Richmond, VA, USA) suggested the use of a glass fiber filter, specifically the Cambridge filter medium CM-113 (Cambridge Filter Corp., Syracuse, NY, USA), for collecting total particulate matter in concentrated cigarette smoke aerosols. The filter disk $(1.5 \mathrm{~mm}$ thick; diameter: $44 \mathrm{~mm}$; effective diameter: $38 \mathrm{~mm}$ ), mounted in a suitable holder, was expected to retain at least $250 \mathrm{mg}$ of smoke particulates (from at least five cigarettes of any type). Collected material may then be extracted from the filter for the determination of nicotine and other smoke constituents. In 1968, the utility of the Cambridge filter was assessed jointly by major laboratories of the U.S. cigarette industry (569) in a review of methods and procedures for the determination of total particulate matter (TPM), 
water in TPM, and total alkaloids as nicotine. The use of glass fiber filters for the determination of "tar" and nicotine in cigarette mainstream smoke was specified in the FTC "Cambridge Filter Method" (19) as well as ISO 4387:1987 (173).

The Cambridge filter used today is a glass fiber filter, 1-2 $\mathrm{mm}$ thick, stabilized by an organic binder, such as polyvinyl alcohol or polyacrylate, not exceeding $5 \%$ of total filter mass. The filter pad has a removal efficiency of at least $99.9 \%$ when measured with an aerosol of dioctyl phthalate particles of at least $0.3 \mu \mathrm{m}$ diameter at a linear air velocity of $140 \mathrm{~mm} / \mathrm{sec}$. The pressure drop of the filter assembly shall not exceed $900 \mathrm{~Pa}$ at this air velocity; its increase after smoking must not exceed $250 \mathrm{~Pa}$, as defined by ISO 3308 (105).

The pressure drop of a Cambridge filter is extremely low and practically negligible, and does not influence the chosen parameters of cigarette machine smoking. The glass fiber material is not hygroscopic. An additional advantage is the collection of smoke particulates at ambient temperature and without need for additional means, such as solvents or an electrical field. After smoking, total particulate matter is determined gravimetrically. For the analysis of TPM constituents the filter pad is extracted with a suitable solvent, such as methanol or isopropanol. Levels are then quantified using ISO methodology - water $(570,571)$ and nicotine $(572)$ - or CORESTA Recommended Methods - benzo[a]pyrene (223) and tobaccospecific nitrosamines $(191,573)$. Equally, more intense smoking regimes, as mandated by HEALTH CANADA (22), MASSACHUSETTS (20) and TEXAS (21), specify Cambridge filters as collection tools for the quantitative determination of cigarette mainstream "tar" and nicotine (209) and other particulate phase constituents, such as benzo $[a]$ pyrene (574).

The gravimetric determination of TPM collected on Cambridge filters is an unsatisfactory procedure with "ultra low tar" cigarettes (according to ISO: less than $1 \mathrm{mg}$ "tar"/cigarette). Even under the most controlled conditions, nominally negative TPM levels may be obtained due to weighing errors or the loss of pad moisture during smoking. Spectrophotometric methods are suitable to overcome this problem; examples for their general use are the following:

In a study focused on cigarette filter efficiency, MCCONNELL et al. (575) developed a spectrofluorometric method for the quantification of cigarette mainstream smoke particulate matter. Smoke was allowed to sediment inside a flask; after being dissolved in methanol the sample was excited at $365 \mathrm{~nm}$ and integrated fluorescence measured in the range of 400-600 nm. Fluorescence intensity was shown to be linearly proportional to the weight of smoke solids in solution. BURTON et al. (576) used excitation at $475 \mathrm{~nm}$ and fluorescence reading at $545 \mathrm{~nm}$ for the determination of "tar" in cigarette smoke at low levels (as used in animal inhalation studies). Cigarette filter efficiency was measured by SLOAN and CURRAN (577) by relating the absorbance at $310 \mathrm{~nm}$ of TPM in methanol extracts of cigarette filters and Cambridge filters. In 1980, THOMAS (578) developed an automated method (using an AutoAnalyzer) for the quantification of FTC "tar" in low "tar" yield brands. Isopropanol extracts of TPM, collected on
Cambridge filters, were excited at $475 \mathrm{~nm}$ and measured at $520 \mathrm{~nm}$. FTC "tar" showed a linear correlation with fluorescence and could be determined in a wide range of cigarettes (from low yield up to $50 \mathrm{mg} /$ cigarette, depending on the specifics of sample preparation).

The quantitative determination of smoke particulate matter by spectrophotometry using a range of wavelengths between $310 \mathrm{~nm}$ and $384 \mathrm{~nm}$ was reported in 1990 by BORGERDING et al. (579). The technique was essential for characterizing cigarettes that heat instead of burn tobacco (361) as they are not amenable to "tar" analysis by the FTC methodology.

Cambridge filters are important devices for the isolation of smoke volatiles and their separation from particulate matter. This was first done in 1958 by IRBY and HARLOW (580) when they examined the composition of the mainstream smoke gas phase of three cigarette prototypes. Compounds passing the Cambridge filter were collected in a trap immersed in liquid nitrogen, allowed to evaporate stepwise with rising of temperature and then separated by gas chromatography. Eleven low molecular weight volatiles were identified and quantified.

The use of Cambridge filters is an element of several standardized methods for the determination of gases in cigarette mainstream smoke, such as carbon monoxide (209, 581) and nitric oxide (549).

In the course of a study of the effectiveness of various single and dual cigarette filters, WILLIAMSON et al. (582) focused on a group of substances they termed "semivolatiles", which under regular smoking conditions "are retained on a Cambridge filter but which can be volatilized from it" by flowing gas, particularly at elevated temperatures. This kind of material was isolated as follows: Particulate matter from four English cigarettes with fluecured Virginia tobaccos, smoked under standard conditions, was collected on a Cambridge filter in an all-glass holder, which was then connected to a U-shaped narrow bore glass tube immersed in liquid oxygen. The Cambridge filter assembly was placed in an air oven, heated to $180-190{ }^{\circ} \mathrm{C}$ and flushed for $45 \mathrm{~min}$ in reverse direction (compared to smoking) with a stream of argon (10 $25 \mathrm{~mL} / \mathrm{min}$ ). The substances, which were volatilized from the filter and subsequently condensed and solidified in the glass tube, the "semi-volatiles", were taken up after melting in a small volume of acetone and analyzed by gas chromatography. More than 40 distinct major peaks were recorded and many more components presumed to be present. Compounds identified by peak trapping and rechromatography on various other columns, using infrared spectrophotometry, were limonene, benzene, toluene, phenol, $o$-cresol, furfural, diacetyl and nicotine. The behavior of these substances is plausible in view of their boiling points lying approximately between 50 and $250{ }^{\circ} \mathrm{C}$. This explanation, though, is not tenable regarding nicotine, which is trapped and solidly retained on the Cambridge filter protonized as a salt; thermal deprotonation has to be assumed as the mechanism of setting it free from the filter. The "semi-volatiles" represent a diversified but typical group of chemicals with important functions in tobacco products, such as various flavoring agents (e.g., menthol), humectants (e.g., 1,2-propylene glycol) and tobacco and filter additives (e.g., triacetin). 
Less formally, the term "semi-volatiles" is used for smoke constituents, which are not divided up clean-cut by Cambridge filters and are both retained on the filter and passing through it.

In a study of the less volatile substances with boiling points between $100{ }^{\circ} \mathrm{C}$ and $200{ }^{\circ} \mathrm{C}$ in the gas phase of plain cigarettes, GROB (583) observed the influence of temperature on the separation efficiency of a Cambridge filter. When the holder assembly was enclosed in a block of aluminum heated to $60{ }^{\circ} \mathrm{C}(584)$ rather than kept at ambient temperature the concentration of high boiling, more polar substances, passing the filter and subsequently recorded in gas chromatograms, approximately doubled while the low boiling, less polar components were not markedly increased.

SAKUMA et al. (585) refined the quantification of low boiling carbonyls in whole mainstream smoke of various plain cigarettes by formation of $p$-nitrophenylhydrazones followed by capillary gas chromatography. Thereby, passage of several compounds through Cambridge filters was determined (no details reported) and retention rates were found to be quite variable: Formaldehyde $42 \%$, acetaldehyde $2 \%$, propionaldehyde $15 \%$, acrolein $7 \%$ and acetone $66 \%$. In our view, the high retention levels for formaldehyde and acetone are related to water retained on the filter. For many years Cambridge filters have been an integral component of the smoking standards mandated by FTC (19) and ISO (105). These represent highly sophisticated and delicately balanced methodology in terms of technical equipment, operating parameters and data evaluation. With the advent of the more intense smoking regimes decreed by HEALTH CANADA (22) and the COMMONWEALTH OF MASSACHUSETTS (20), which nonetheless rely on conventional instrumentation, the known performance of Cambridge filters may be critically influenced by higher concentration and increased water content of the smoke, higher temperature of the smoke, intensified flow through the pad and larger loads on the filter.

The problematic performance of Cambridge filters (and holders) when burdened with a "high-water" aerosol stream had been reported in 1990 by BORGERDING et al. (586) in connection with cigarettes that heat instead of burn tobacco (361). The novel design produces an aerosol with a considerable higher amount and proportion of water (and glycerol) than conventional cigarettes; the possibility of non-quantitative water measurements was a problem that required special attention but could be controlled by adequate procedure. When determining a novel cigarette TPM materials balance using Cambridge filters it was found that TPM, nicotine, glycerol and propylene glycol could be measured appropriately while water - present in relative abundance - was quantified to differing degrees greatly depending on filter/holder handling practices. Consequently, the calculation of "tar" by difference weighing required careful selection of water values. Electrostatic precipitation avoided these difficulties and proved to be the method of choice for novel cigarette "tar" determination. CôTÉ et al. (587) followed up their supposition that loss of water from the Cambridge filter assembly may occur after weighing the pad and while preparing it for extraction. They compared the procedure described in ISO 4387 (219) to a modification when the pad was weighed "directly in the extraction flask". Three Canadian cigarettes (4-14 mg ISO "tar") were smoked in compliance with ISO 3308 (105) or the more intense smoking conditions, mandated in Canada (22) and Massachusetts (20). Under the two intense regimes, NFDPM ("tar") values were found to be $14-26 \%$ higher when the ISO procedure, rather than the modification, was followed in Cambridge filter processing. The difference was less significant when smoking was done according to ISO. Nicotine amounts were not affected under any conditions.

Subsequently, these observations were directionally confirmed in a larger study by CôTÉ and VERREAULT (588).

GHOSH and JEANNET (589) developed a solution to the problem of incomplete quantification of water in cigarette smoke by modifying the Cambridge filter pad holder in combination with a new procedure for weighing the filter assembly and extracting the material trapped inside. Any underestimation of water will inevitably lead to exaggerated levels of NFDPM (nicotine-free dry particulate matter = "tar"). Reasons for the inadequate measurement of water may be loss from the opened filter assembly and during handling of the filter pad, but also adsorption on holder surfaces inside. To overcome these problems, a 44-mm Cambridge filter pad was positioned before smoking in a newly designed metallic holder, which was then closed, sealed at both ends by two caps containing a septum and weighed. After removing the caps the filter assembly was used in machine smoking, eventually sealed again without delay and weighed once more (fully assembled) for determining the weight difference, which represents total particulate matter (TPM). The entire material collected inside the filter assembly (still hermetically sealed) was then extracted in situ using two syringes with needles pierced through the septa at both ends, which flushed $10 \mathrm{~mL}$ isopropanol repeatedly up and down through the assembly $(30 \mathrm{~min}$ at a flow of approximately $20 \mathrm{~mL} / \mathrm{min}$ ). The resulting solvent extract was collected for further analysis.

To evaluate the new method three test pieces were smoked under both the ISO and Canadian Intense regimes with TPM collection according to ISO or using the new holder and extraction procedure: the 3R4F Kentucky reference cigarette (9 mg ISO "tar"/cigarette), a conventional cigarette (6 mg ISO "tar"/cigarette) and a so-called Tobacco Heated Product, from which the aerosol was created by heating a specially designed tobacco stick with a battery operated device ( $9 \mathrm{mg}$ ISO “tar"/cigarette). For all $(3 \times 4)$ test combinations, TPM, water and nicotine were measured (besides glycerol and carbon monoxide) and NFDPM ("tar") levels calculated. The results were remarkable. As expected, for all three test pieces TPM was higher under Canadian Intense than ISO smoking conditions but did not differ when the ISO holder and the new holder were compared. Water in TPM per 3R4F cigarette, however, increased with new holder use under ISO conditions from 0.7 to $1.1 \mathrm{mg}(+70 \%)$ and under Canadian Intense conditions from 13.3 to $20.5 \mathrm{mg}$ ( $+54 \%$ ). Concerning the conventional cigarette, amounts rose under ISO conditions from 0.7 to $0.9 \mathrm{mg}(+35 \%)$ and under Canadian Intense conditions from 12.6 to $15.8 \mathrm{mg}(+26 \%)$; for the Tobacco Heated Product water, which contributed exceedingly to 
aerosol weight, increased under ISO conditions from 18.8 to $23.2 \mathrm{mg}(+24 \%)$ and under Canadian Intense conditions from 37.6 to $44.7 \mathrm{mg}(+19 \%)$. As nicotine levels were not affected by the holder and extraction systems, the increases in TPM water content measured with the new holder immediately diminished "tar" levels per test piece (for the conventional cigarettes used in this study under ISO conditions by $3-4 \%$ and under Canadian Intense conditions by $17-23 \%)$.

The use of the new holder and extraction technique had no effect on total particulate matter (TPM) and nicotine in TPM levels, assured the more accurate quantification of water in TPM and, as a consequence, reduced the calculated quantities of "tar" (NFDPM) per cigarette. Compared to ISO conditions, these effects were more pronounced with the Canadian Intense smoking regime for which the new methodology for TPM collection and analysis seems to be particularly appropriate and needed.

\subsection{Electrostatic precipitation}

As early as 1919, TOLMAN et al. (590) described an apparatus for the electrostatic precipitation of particulate matter from smoke, independent of its origin and chemical nature. The essential parts were a central threaded platinum wire (as cathode) surrounded by a very thin aluminum foil (as anode), housed in a glass tube (Figure 56). The system worked with an electric potential difference of up to 15,000 volts; material was collected on the foil and could be measured gravimetrically.

BAUMBERGER (91) used electrostatic precipitation for the determination of the total amounts of suspended solid and liquid particles (including nicotine) in fresh as well as exhaled tobacco smoke. The study was conducted to investigate the total amount of mainstream smoke produced from a cigarette and the portion retained in the body of a smoker (puffing or inhaling). Retention rates were found to be $66.7 \%$ in smokers when puffing, and $88.2 \%$ when inhaling - data confirmed by more recent studies.

An electrostatic trap of identical design with a tungsten wire (diameter: $0.5 \mathrm{~mm}$ ) and an aluminum tube (inner diameter: $4 \mathrm{~cm}$ ), mounted in a glass cylinder and operated at 12,000 volts, was used in 1958 by ESCHLE (591) for the determination of cigarette mainstream smoke TPM and nicotine in a study focused on cigarette filter efficiency. Electrostatic precipitation of smoke particulates on a cathode made from silver-plated brass was the approach taken by GOLAZ et al. (592) to the assessment of the relationship of volume and nicotine content of individual puffs.

Possible adverse effects of the very strong electrical fields required for electrostatic precipitation were given consideration by two research groups. WALTZ et al. (112) measured the amount of ozone formed - under the influence of 18,000 volts - when mimicking the sequential "smoking" of 10 unlighted cigarettes in $75 \mathrm{~min}$, and detected $0.12 \mathrm{mg}$ ozone in total. However, under conditions of real smoking ozone could not be discovered and was assumed to have reacted with smoke constituents although artifact formation was never clearly demonstrated. KUHN and MAREK (593) observed increased retention of particulates and nicotine by cellulose acetate filters, obviously caused by

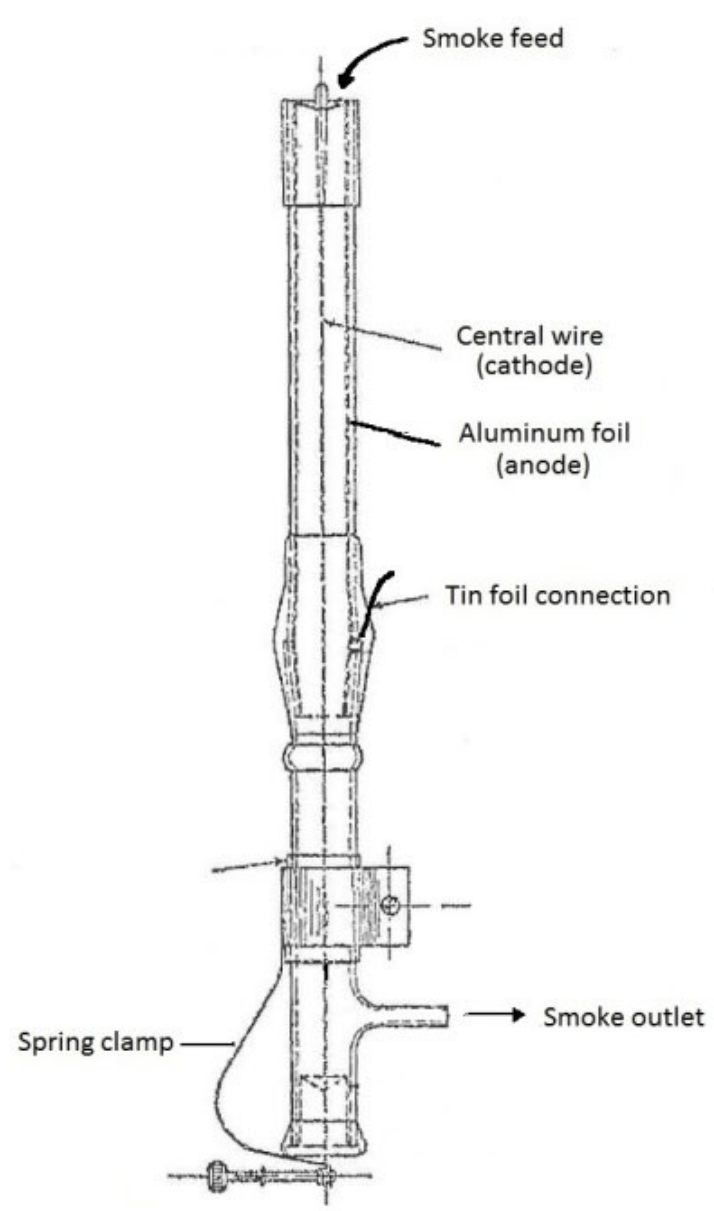

Figure 56. This electrostatic precipitator, developed by the U.S. Chemical Warfare Service and described by TOLMAN et al. (590) in 1919, was used for the analysis of any kind of smoke.

the static charging the filter plug by the neighboring high voltage field of an electrostatic trap and simulating filter efficiency strengthened by $25-30 \%$.

WILLIAMSON and ALLMAN (594) compared the distribution patterns of 17 volatile cigarette mainstream smoke components - range of boiling points from $21^{\circ} \mathrm{C}$ (acetaldehyde) to $111^{\circ} \mathrm{C}$ (toluene) - between the particulate and gaseous phases under three experimental circumstances: after passing through either an electrostatic trap or a Cambridge filter, and following gravitational settling of the whole smoke for 15, 55 and $95 \mathrm{~min}$ in a flexible vapor-impermeable bag. Gas chromatographic peak heights of the vapor phases were remarkably similar under all three conditions - with the exception of three compounds (acrolein, acetonitrile, diacetyl) after gravitational settling, which was assumed to be the (aging) effect of continued contact with particulate matter. After volatilization of the compounds inadvertently collected in the electrostatic precipitator or on the Cambridge filter by heating for $30 \mathrm{~min}$ in boiling water, it was observed that (only) between $0 \%$ and $18 \%$ of individual constituents had been intercepted - with a nearly identical pattern for both trapping techniques. It was concluded that the composition of the vapor after passage through an electrostatic trap or a Cambridge filter was very similar to that in the original smoke aerosol. 
Techniques for the collection of cigarette smoke condensate were evaluated in 1961 by BENTLEY and BURGAN (595) of the UK Tobacco Manufacturers' Standing Committee. Using a specific set of smoking parameters (one 25-mL puff of $2 \mathrm{sec}$ duration every min), based on a survey of 312 representative UK smokers, two different automatic smoking machines - a constant volume machine, i.e., the "Autosmoker" described by ILES and SHARMAN (90) and the constant flow Cigarette Components Ethel Mark VII smoking machine - were shown to give concordant results. Three condensate trapping systems were compared: an electrostatic precipitator, the Cambridge glass fiber filter and a cold trap immersed in dry ice/ acetone. After condensate in each trap had been taken up in methanol the following analytical parameters were determined: Condensate gravimetrically after oven drying, water by the Karl Fischer method and nicotine by UV spectrometry. All three condensate collection methods were found to give reliable and comparable results. In the authors' experience, Cambridge filters distinguished themselves by simplicity of use, and when using an electrostatic precipitator the cigarette holder needed to be efficiently grounded to prevent electrification of the cigarette butt end - a distorting factor also noted by KUHN and MAREK (593). In 1979, the German VERBAND DER CigaretTENINDUSTRIE (VDC, Cigarette Manufacturers Association) (596) conducted a comprehensive and collaborative comparative study of the two smoke trapping systems, the Cambridge glass fiber filter and the electrostatic trap. First, it was experimentally confirmed that both devices met the specifications of ISO 3308:1977 (10), paragraph 6.2, which required the retention of at least $99.9 \%$ of all particles in a dioctyl phthalate aerosol of at least $0.3 \mu \mathrm{m}$ diameter. Smoking was done on a Borgwaldt RM20CS machine (128) under conditions of ISO 3308 (10) with either a central Cambridge filter ( $92 \mathrm{~mm}$ diameter) or a central electrostatic precipitator, both receiving the smoke from 20 cigarettes. In a first round, 5 German brands were examined in 2 laboratories and, subsequently, 26 German brands in 5 laboratories ( 4 company labs and 1 government lab). Data were produced for smoke condensate (TPM), smoke nicotine and water in TPM, and statistically evaluated in considerable detail with focus on within-lab and between-lab variations. It was concluded that the differences systematically observed between the two trapping systems (amounts of smoke condensate and nicotine, on average, smaller with electrostatic precipitation) had no direct bearing on the outcome of testing in practice.

The electrostatic trap for collecting cigarette mainstream smoke condensate was an integral part of the rotary head smoking machines developed and manufactured in Germany after 1960 (114-116, 120). Its use for the quantitative determination of smoke condensate and nicotine levels was specified in the instructions of the German Cigarette Industry Association (50), the German Standard DIN 10240, part 2 (123), and the International Standard ISO 8453 (174). The problematic influence of an electrostatic field on smoke yields and filter efficiency had been practically eliminated by the design of the electrostatic trap and the use of recommended voltages as described in DIN 10240 (123).

The electrostatic precipitator has developed into the preferred - and practically indispensable - tool for the determination of (trace) metals and other elements in smoke. This is due to the fact that Cambridge filters are known to release significant inorganic impurities, which cannot be eliminated by prior cleaning. Two additional precautionary considerations are important. To prevent even minute contamination of smoke during generation KALCHER et al. (597) designed a compact, valve-controlled smoking machine for cigarettes and cigars with as few metallic parts as possible and a preference for quartz glass, acrylic glass and teflon. The system included an electrostatic precipitator. With the objective of stringent control of the analytical blank, RHOADES and WHITE (598) described "extremely clean laboratory conditions during sample preparation" when they determined six trace elements ( $\mathrm{Al}, \mathrm{As}, \mathrm{Cd}, \mathrm{Cr}, \mathrm{Ni}$ and $\mathrm{Si}$ ) in cigarette mainstream smoke condensates. Optimum working conditions required positive room pressure with HEPA-filtered air and constant temperature and humidity control. Smoke was produced using a RM20CS smoking machine, condensate collected in an electrostatic trap and analyzed - following predigestion with concentrated nitric acid under an IR lamp in an ultraclean "environmental evaporation chamber" and sample dissolution by microwave heating with concentrated nitric acid and concentrated hydrofluoric acid inside sealed teflon vessels to remove the organic matrix - by graphite furnace atomic absorption spectrometry and inductively coupled plasma optical emission spectrometry (ICP-OES). Distinct concentrations (ng/cigarette) above the (very low) limits of quantification were measurable only for arsenic, cadmium and silicon.

An early instance of trace metal analysis in cigarette tobacco and smoke was reported by PERINELLI and CARUGNO (599). Condensate was produced using a simple customized smoking device combined with an electrostatic precipitator. Tobacco was digested sequentially with concentrated sulfuric acid and concentrated nitric acid, and TPM taken up in methanol (and treated with sulfuric and nitric acid if necessary) prior to analysis by atomic absorption spectrophotometry (flameless, sporadically flame method) for their content in lead, zinc, cadmium and nickel. Transfer rates were calculated and found to be very low. Three commercial cigarette brands from Yugoslavia were examined by IVIČIC et al. (600) for cadmium and lead content in tobacco, butts and crude smoke condensate - the latter produced with a Borgwaldt BAT-RM 20/68 smoking machine with an electrostatic trap. Following thorough digestion, samples were analyzed by differential pulse anodic stripping voltammetry. Rates were calculated for metal transfer into smoke and retention in the butts. In two very comprehensive investigations (a local and an inter-laboratory study), SCHNEIDER and KRIVAN (601) and KRIVAN et al. (602) examined as many as 57 elements in cigarette tobacco and smoke condensate, using four different analytical methods. Test pieces were the topselling German brand in 1990 and the experimental cigarette C-20 (representative for the German market at this time). Their tobacco was isolated, and smoke condensate was produced under standard conditions by means of a Borgwaldt BAT-RM 20/68 smoking machine and a modified electrostatic precipitator specially equipped with a quartz tube and a high purity aluminum electrode for 

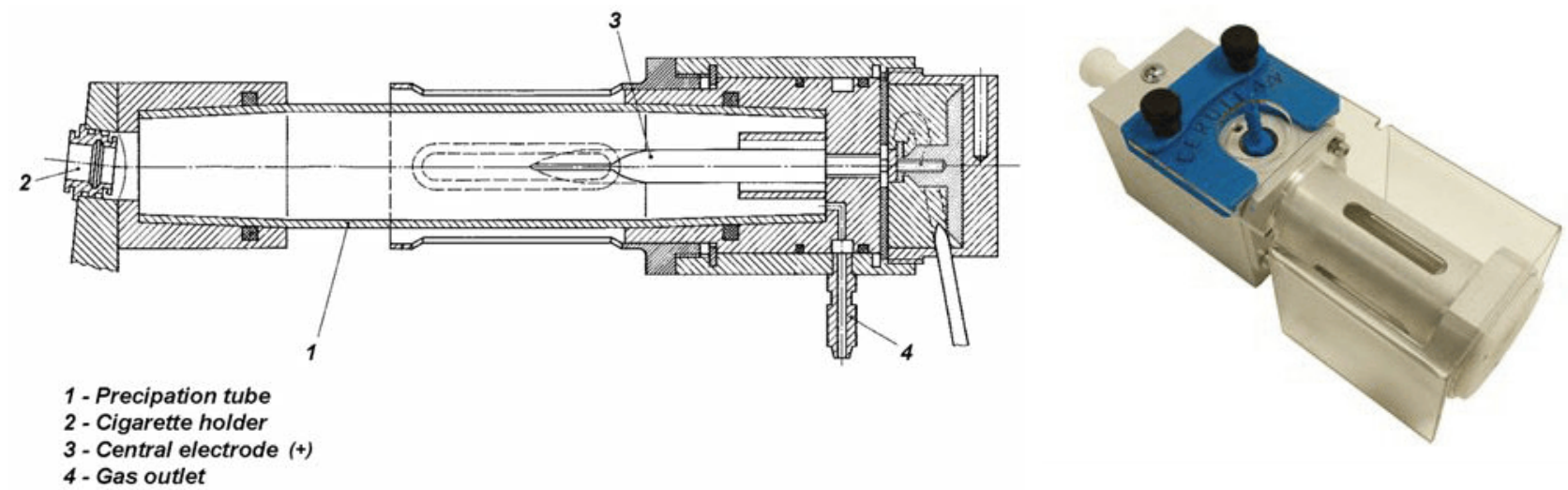

Figure 57. The constructional drawing of an electrostatic trap, shown in DIN 10240, part 2 of 1978 (123), reveals the working principle of the modern device of CERULEAN (643), which operates with an electrode voltage variable up to $20 \mathrm{kV}$ and an output current limited to $1 \mathrm{~mA}$ for safety reasons.

minimizing contaminations (Al was not analyzed in the studies). Trace elements were measured by inductively coupled plasma mass spectrometry (ICP-MS), total reflection X-ray fluorescence analysis and atomic absorption spectrometry (these three methods required prior pressure microwave decomposition of the matrix) as well as instrumental neutron activation analysis, which permits direct irradiation and counting of undigested samples. The availability of four different methods and the fact that several elements could be determined by at least two of them provided useful means for broad analyte coverage and detailed data cross-checking. Levels of trace elements found in the studies were frequently (and occasionally strikingly) lower than those reported previously - one reason obviously being the efficient control of contamination during sample production and processing.

The major components, carbon, hydrogen and nitrogen, were determined by combustion analysis, and sulfur by precipitation microtitrimetry after reduction to hydrogen sulfide. These 4 elements amounted to around $75 \%$ of the condensates with oxygen estimated at approximately $25 \%$. All trace elements determined in these studies added up to less than $0.01 \%$.

In view of their concentrations in TPM and their known toxicological characteristics, the most relevant elements in cigarette smoke condensate were judged (602) to be arsenic, cadmium and lead. Interestingly, two liquid traps with concentrated nitric acid connected to the outlet pipe of the TPM collection system did not retain any nickel (above the limit of detection) that might have been volatilized as tetracarbonyl derivative. This confirmed earlier reasoning of PAILER and KUHN (603) that nickel tetracarbonyl was not expected to exist in cigarette mainstream smoke for thermodynamic reasons.

The determination of mercury in cigarette mainstream smoke requires special operating procedures due to the high volatility of the analyte and the ensuing risk of major losses during sample processing. Using two smoking regimes - mandated by the FTC (19) and the COMMONWEALTH OF MASSACHUSETTS (20) - MCDANIEL et al. (604) produced both the particulate and gas phases from 1R4F Kentucky reference cigarettes and two commercial U.S. brands by means of a Borgwaldt RM20CSR smoking machine, combined in sequence with an electrostatic preci- pitator and two impinger traps filled with acidic potassium permanganate solution for the efficient collection of mercury. Modifications aimed at preventing contamination included the use of polypropylene for smoking machine inner seal surfaces and cigarette holders as well as a quartz tube and a high purity tungsten wire with sharpened end (rather than aluminum) as precipitator parts. The fractions collected in the electrostatic trap and the impingers were isolated, digested by microwave technique and analyzed by cold vapor atomic absorption spectrometry - either without (gas phase) or with amalgamation on gold-platinum gauze (particulate phase, which required pre-concentration before quantification). The distribution of mercury between the two smoke fractions proved quite remarkable: While the mercury content of the particulate phase was negligible $(<0.2 \mathrm{ng}$ per $1 \mathrm{R} 4 \mathrm{~F}$ cigarette), the overwhelming share was found in the gas phase ( $4.9 \mathrm{ng} /$ cigarette).

SNEDDON $(605,606)$ discussed the theory and principles of electrostatic precipitation and the design, construction and evaluation of a device suitable for trapping air-borne metals for analysis by atomic absorption spectrometry. Copper, lead, manganese and mercury were determined in direct and near-real-time mode in laboratory atmospheres. Levels found and detection limits were in the range of lowto-sub $\mathrm{ng} / \mathrm{m}^{3}$.

The electrostatic trap was set down in the German standard DIN 10240, part 2 of 1978 (123) as collection device for the determination of crude condensate and nicotine free dry condensate in cigarette smoke (Figure 57).

The use of an electrostatic precipitator was specified in the 1999 Health Canada Official Method T-109 (130) for the determination of $\mathrm{Ni}, \mathrm{Pb}, \mathrm{Cd}, \mathrm{Cr}$, As and $\mathrm{Se}$ in mainstream tobacco smoke.

Modern, commercially available electrostatic tobacco smoke precipitation systems (Figure 57) consist of a positive needle-sized metallic electrode positioned in the center of a glass tube of about $3 \mathrm{~cm}$ diameter. The tube is enveloped by the cylindrical negative electrode usually made from stainless steel. The electrodes supply a voltage variable from 0 to $20 \mathrm{kV}$ creating an electrical field, which is traveled through by smoke during puffing. Positively charged smoke aerosol particles are collected on the inner surface of the glass tube. Vapor phase constituents pass through the trap and may be collected subsequently. 


\subsection{Jet impaction traps}

The jet impaction trap for the collection of mainstream smoke condensate operates on the principle of smoke aerosol traveling at high speed through a capillary or through an orifice and then being allowed to impact a flat surface at short distance. The following discussion is focused on collection efficiency in comparison to other trapping methods.

Already in the 1930s, WENUSCH (42) forced the coagulation of smoke particulates by passage through a long zshaped capillary of $0.5 \mathrm{~mm}$ inner diameter and collected the viscous droplets in a flask (Figure 58). The method was subsequently modified by CUZIN (66) for use with an advanced analytical smoking machine. NEURATH and KRÖGER (274) combined an apparatus designed for reverse smoking (described on page 178) with a nozzle of $0.4 \mathrm{~mm}$ diameter for the production of coagulated native condensate with a soft, paste-like consistency for direct application in skin painting tests without solvent interference and risk of aging - with yields of 60-70\% compared to electrostatic precipitation.

In the mid-1960s, SEEHOFER and HANßEN (288-290) invented the "capillary press" smoking machine, which combined - upon completion of its development - a rotary smoking head (capable of free and restricted smoking) and an automated piston pump (for puffing and subsequent pressurizing the smoke to 2 atmospheres) with a rather simple unit for capillary coagulation and collection of smoke condensate. The capillary had a length of $40 \mathrm{~mm}$ and an inner diameter of $0.5 \mathrm{~mm}$. Collection efficiency was about $90 \%$ compared to electrostatic precipitation. Using a supplementary appliance, partially remarkable increases of plain cigarette mainstream smoke yields (condensate, nicotine, total steam volatile phenols, benzo $[a]$ pyrene and benzo[e]pyrene) were confirmed when switching from the restricted to the free smoking mode. The use of the "capillary press" allowed the puff-by-puff analysis of the interrelated effects of smoke constituent production by smoldering and their loss in escaping smolder smoke under the conditions of free smoking.

In two publications, MATHEWSON $(291,607)$ of Cigarette Components Ltd. (London, UK) addressed a broad range of theoretical considerations and experimental steps concerning high velocity impaction for collecting and fractionating cigarette mainstream smoke. With sufficient momentum, aerosol particles at a narrowing collide, impinge due to particle inertia and condense either inside the nozzle or on a nearby flat surface. This process is most effective while flow is laminar; turbulent flow, however, brings about "forced coagulation" inside narrow-bore capillary tubes, a process that follows different physical rules. Underlying physical factors include the flow characteristics of an aerosol through a tube, its droplet size distribution, adhesive forces and electrical effects (subsidiary and only with low velocities). Collection efficiency is determined by the dimension of jet and impaction surface as well as aerosol velocity. One of the papers (291) includes an informative discussion of the essential theoretical aspects.

The controlled and effective collection of aerosol by impaction required a smoking machine, which fulfilled the key requirement: an almost exactly rectangular profile of highly constant flow during the puff. An apparatus was developed, which consisted of a vertically positioned 24port rotating disk with the condensate trap mounted behind the fixed outlet, where the cigarettes were positioned while being puffed ( $35 \mathrm{~mL}$ in $2 \mathrm{sec})$. Suction was provided by a vacuum pump and controlled by various valves, meters and gauges so that smoke was drawn through the orifice by permanently reduced pressure of about $190 \mathrm{~mm}$ of mercury. However, the system did not become widely used. It should be pointed out that the way cigarettes were smoked did not prevent uncontrollable temperature and air convection effects and the contamination of mainstream smoke by sidestream smoke.

The impaction devices used were distinguished by simplicity in design and versatility in application. For prepara-

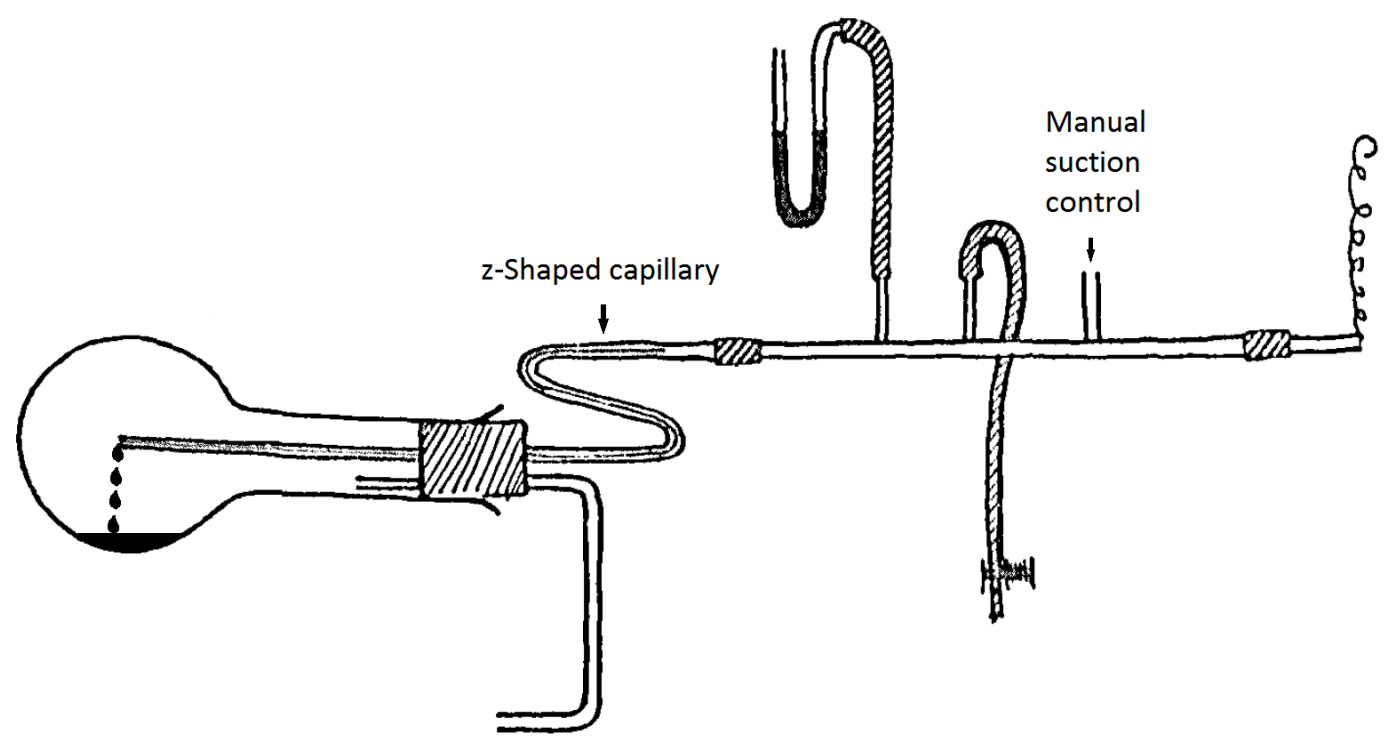

Figure 58. A capillary bent into sharp curves was fundamental to collecting smoke particulates in the device described by WENUSCH (42) in 1939. 
tive purposes, jets of less than $1 \mathrm{~mm}$ length (to prevent clogging) and a diameter of $0.4 \mathrm{~mm}$ were used at a distance of 1-2 $\mathrm{mm}$ from the impaction surface. Downstream pressure was $120-190 \mathrm{~mm}$ of mercury below atmospheric. Mainstream smoke aerosol was routed through a nozzle at high speed and allowed to impact at a short distance onto a flat surface, from which it could be washed away with acetone from a solvent reservoir either batch-wise or continuously, producing condensate from as many as hundreds of cigarettes (Figure 59).

The comparison with Cambridge filters showed 97.5\% collection efficiency of non-volatiles but, at the same time, revealed certain quantitative (not qualitative) differences, which could be explained by the distinctive behavior of the more volatile condensate constituents. Analysis of condensate obtained by impaction produced lower values per cigarettes for wet "tar", water and dry "tar" compared to Cambridge filters while nicotine content was the same - an observation explained by the more efficient retention of low boiling "semi-volatiles" on the filter. Incomplete collection of smaller particles by impaction may have contributed as well. The whole system could also be used for analytical purposes, for studying particle size distribution and the equilibrium between smoke particulate and gaseous phases, and as a source of condensate for in vitro assays.

Maximum retention efficiencies were obtained by allowing the raw smoke to remain in a coagulation chamber $(50 \mathrm{~mL})$ for about $1 \mathrm{~min}$ before the aerosol particles, grown to larger size, were drawn through the impaction orifice. This intermediate step, which did not appear to affect chemical composition, increased collection efficiency from the average $97.5 \%$ to $>99.9 \%$ compared to Cambridge filters. For trapping whole smoke it was suggested to combine an impactor with an empty U-tube immersed in liquid oxygen; after first removing all particulate material, vapor phase constituents could be condensed out much more effectively.

The exceptional influence of orifice diameter $(0.8 \mathrm{~mm}$ decreasing to $0.4 \mathrm{~mm}$ ) and the resulting flow velocity through the jet $(1,990-8,550 \mathrm{~cm} / \mathrm{sec}$ with reduced puff volume of $20 \mathrm{~mL}$ in $2 \mathrm{sec}$ ) was manifested in escalating retention rates of non-volatile material (1.4-92.0\%). As deposition by impaction is strongly determined by particle dimensions (smaller ones requiring higher flow velocities) these observations suggested a working approach to smoke particle fractionation by size.

Generally, jet impaction traps are not widely accepted for preparative and analytical purposes. Their main drawbacks are the tendency to become clogged and the need for controlling flow and pressure conditions during operation with particular care.

\section{6. $\quad$ Cold traps}

Whenever it is desirable to collect tobacco smoke - whole or a fraction - in its most original "native" and un-aged form and with minimal risk of artifact formation cold traps are the method of choice. For their operation no solvents are needed and there is no influence of electrical fields. In addition, the cold temperatures used (possibly as low as $-185{ }^{\circ} \mathrm{C}$ with liquid air) prevent or, at least, minimize

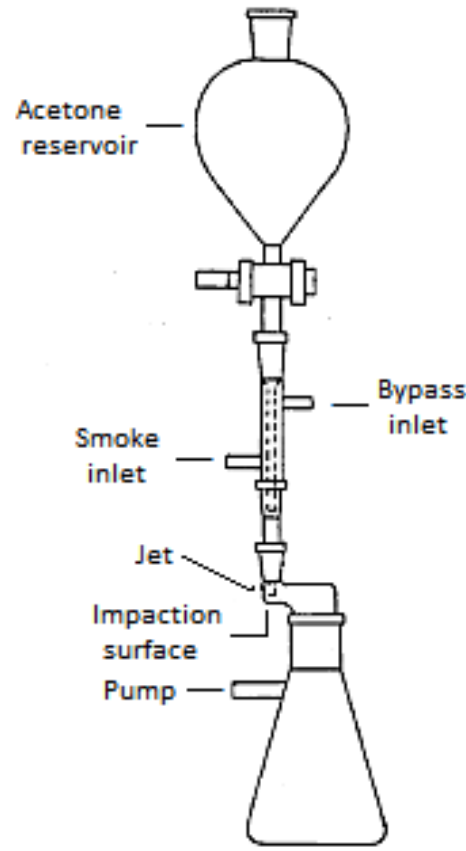

Figure 59. In the mass impaction trap developed by MATHEWSON (291) acetone was used to recover accumulated smoke condensate.

chemical reactions of and between individual smoke constituents. Depending on their design cold traps may be used for collecting condensates from whole smoke or the gaseous fraction after passage through a Cambridge filter. Initially, the identification and quantification of individual volatile constituents of tobacco smoke was the driving force behind the use of cold traps - occasionally arranged in intricate systems. In the mid-1950s at Duke University (Durham, NC, USA), OSBORNE et al. (608) and PHILIPPE and HOBBS (609) produced under controlled conditions mainstream smoke of experimental cigarettes made from the typical tobacco components of an American blend: Burley, cased Burley (10\% dextrose added), bright Virginia and Turkish (Oriental) tobacco as well as 50:50 blends of bright Virginia tobacco with Burley or cased Burley. The condensable fraction of the gaseous phase after removal of particulates by a cellulose filter - was collected puff by puff in a chamber cooled to liquid air temperature (approx. $-185{ }^{\circ} \mathrm{C}$ ) and then fractionated laboriously - by means of low temperature trap-to-trap distillation in a closed system by allowing the trapping temperature to rise in up to 8 controlled steps to $-15^{\circ} \mathrm{C}$. Samples were analyzed with a dual beam compensating infrared spectrophotometer; compensation describes the nullification of an observed characteristic infrared absorption band by a single known compound present in the reference cell. The range of (then) known gas phase constituents, such as air, the carbon oxides, hydrogen sulfide, hydrogen cyanide, ammonia, dicyan, acetylene and unspecified saturated and unsaturated hydrocarbons, was extended considerably by the quantitative characterization of "all of its major components and a considerable number of minor constituents". However, the methodology was time consuming, required the correct manipulation of com- 
plex equipment and clearly did not lend itself to routine application.

In 1964, this investigation was followed up by PHILIPPE et al. (610) with a study focused on the hydrocarbons present in the condensable fraction of the gaseous phase of cigarette mainstream smoke and using improved methodology. The laboratory equipment consisted of an advanced smoking apparatus with a mercury-filled leveling bulb for drawing puffs, a Cambridge filter for removing particulate matter, and a relatively simple train of cold traps using liquid air for the collection and concentration of condensable material. Gas chromatographic, infrared spectophotometric and mass spectrometric techniques were combined for analyte identification and quantification. After removal of carbon dioxide and water from the condensate by treatment with Ascarite ${ }^{\circledR}$ and Drierite, some 37 compounds were detected by gas chromatography on alumina - 34 of them hydrocarbons and 20 not previously reported in cigarette smoke. Finally, the authors speculated freely why some 14 hydrocarbons, which could be expected to exist in cigarette smoke, were not yet identified (that is, in 1964). In fact, the presence of five of them has since been verified while the other nine are still unconfirmed.

The analytical capability of the combination of a "conventional" smoking machine equipped with a Cambridge filter, the relatively simple all-glass apparatus developed by LE RoY (611) for the trapping (at $-196{ }^{\circ} \mathrm{C}$ ) and low temperature evaporative distillation of condensable multicomponent gas mixtures, and a gas chromatograph was examined by IRBY and HARLOW (580) with regard to a number of cigarette mainstream smoke gas phase constituents. Comparative quantitative data were produced with three test pieces containing the same tobacco blend with different filter configurations (plain; cellulose acetate/soft paper; cellulose acetate/soft paper impregnated with activated charcoal).

With special attention to the levels of acetaldehyde, acrolein and acetone in cigarette mainstream smoke, the instrumental set-up was further improved and simplified by LAURENE et al. (612) and LAURENE and HARBIN (613). Puffs were produced by means of a modified vial filler using a $50 \mathrm{~mL}$ syringe fitted with a synchronous motor. A cycle indicator and micro-switches controlled the puffing cycle (one 2-sec puff/min, puff volume not reported). A 6-port precision gas sampling valve was used for routing the smoke through a syringe to the smoke trap, which was connected to the column of the gas chromatograph. Smoke vapor phase was transferred from the trap to the GC column by means of the carrier gas, helium. The cold trap consisted of coiled copper tubing of $60 \mathrm{~cm}$ length, immersed first in liquid nitrogen for collecting condensable components and then in an oil bath of $125{ }^{\circ} \mathrm{C}$ for their volatilization and subsequent transfer to a chromatographic packed column filled with Chromosorb W, coated with $10 \%(w / w)$ Upon oil 50 HB-280-X, which allowed good separation of the compounds of interest. Further analysis was done by thermal conductivity detection of the column eluate and by mass spectrometry of quantitatively collected peak material.

The method developed by HORTON and GUERIN (614) for the determination of acetaldehyde and acrolein in the vapor phase of cigarette smoke after passing through a Cam- bridge filter collected condensable material puff-by-puff directly on the column head (cooled to $-75^{\circ} \mathrm{C}$ ) of a cryothermal gas chromatograph. No information on the smoking apparatus was furnished. Trapped compounds were subsequently eluted by means of a temperature program and detected by flame ionization. Performance characteristics of the method were assessed, employing a broad range of experimental and some commercial cigarettes.

GROB (615) collected the gas phase of mainstream smoke behind a Cambridge filter in a glass spiral of $50 \mathrm{~cm}$ length and $2 \mathrm{~mm}$ inner diameter, cooled in dry ice slurry. Plain cigarettes were smoked drawing every minute a $35-\mathrm{mL}$ puff of $4 \mathrm{sec}$ duration. This way, flow velocity in the glass spiral was reduced to $8.75 \mathrm{~mL} / \mathrm{sec}$ (down from the routine $17.5 \mathrm{~mL} / \mathrm{sec}$ with 2 -sec puffs). When smoking was finished nitrogen was blown through the spiral at $10 \mathrm{~mL} / \mathrm{sec}$ for $10 \mathrm{~min}$ with only the last coil still immersed in the cold bath. This way the collected material was concentrated for subsequent dissolution in ether and analysis. Although the highly volatile smoke constituents were lost intentionally in the process, as many as 167 smoke components were recorded (certain or probable identifications, chemical formulas, molecular weights) by VöLLMIN et al. (616) using the direct combination of capillary gas chromatography with superior separation efficiency and high resolution mass spectrometry - an approach also suitable and used today for routine analysis. This technique had already been explored in 1963 by DORSEY et al. (617) for the fractionation and identification of light hydrocarbon mixtures and C9 paraffins in reformate (a high-octane liquid petroleum product), and in 1965 by RYHAGE et al. (618) for the separation of fatty acid methyl esters prepared from peanut oil.

The collection, handling and storage of whole cigarette mainstream smoke (specifically, its particulate and condensable components) was a major topic in the comprehensive paper of SCHUR and RICKARDS (252) on the design and operation of a multiple cigarette smoking machine. Horizontal helical glass coils of Pyrex tubing with an internal diameter of approximately $10 \mathrm{~mm}$, immersed in a dry ice/acetone bath, served as collection devices. Each coil was fitted at the inlet with the cigarette holder (featuring a rubber diaphragm or viscous cement of dextrose paste for sealing) and connected at the outlet end to 1 of the 20 ports in the horizontal circular selector plate of the suction mechanism (a third opening was available for controlled relief of residual vacuum after a puff). Up to 30 cigarettes were smoked into each of the 20 coils attached to the smoking machine. The low internal volume of a coil allowed the collected material - after warming up to room temperature and in a rather elaborate procedure - to be taken up in as little as $2 \mathrm{~mL}$ solvent (preferably a 3:2 mixture of ethanol and toluene). Collection of particulate material in the coils was $>95 \%$ effective compared to smoke trapping on a filter plus condensation in a large, glass wool packed U-tube at $-80^{\circ} \mathrm{C}$.

DE SOUZA and SCHERBAK (619) collected whole cigarette smoke in glass spiral traps cooled with a dry ice/acetone mixture when they examined the influence of glycerol, added to tobacco, on the production of benzo[ $a]$ pyrene in mainstream smoke (no effect was observed). 
Whole cigarette mainstream cigarette smoke was analyzed by NEWMAN et al. (620) with minimal delay by combining a smoking machine with a cryogenic gas chromatograph. Smoke was collected within less than $1 \mathrm{sec}$ from a single or several standard puffs in a stainless steel tube filled with glass beads and kept at $-190{ }^{\circ} \mathrm{C}$. Gradual increase of the oven temperature to $230^{\circ} \mathrm{C}$ released material directly into the column for gas chromatographic analysis.

Cold traps, containing glass helices and immersed in solid carbon dioxide/acetone, were used to obtain condensate from whole smoke for the determination of total steamvolatile acids, individual steam-volatile phenols and certain metals (cadmium, cobalt, lead and nickel) according to standard methods described by the CHEMICAL Sub-COMMITTEE OF THE UK TOBACCO ADVISORY COUNCIL (621).

In 1965, a rather special cold trap was developed by ELMENHORST (270) for preparatory purposes, which had the capacity of collecting cigarette mainstream smoke condensate of about 6,000 cigarettes (a standard cold trap suggested earlier by CORESTA was unable to accept the smoke from more than 10 cigarettes and was suited for analytical work only). The large amounts of condensate were required for evaluating its tumorigenicity by application on mouse skin. Occasionally, the trap was also used for the analytical determination of specific smoke constituents, such as aliphatic and aromatic primary and secondary amines (622). The trap consisted of a cylindrical closed flask (volume approximately $1,000 \mathrm{~cm}^{3}$ ) with two joints and a small bulge at the bottom (Figure 60). A glass tube, its upper end linked to the suction device, was vertically inserted into the flask, down into the bulge, through the central joint at the top. The tube was fitted on its lower end inside the flask with a sleeve of about $2 \mathrm{~cm}$ length, made from stainless steel, forming a very narrow gap between the lower edge of the sleeve and the bottom of the bulge. Alternatively, when clogging was caused by sticky precipitates, the bulge was filled with a layer of stainless steel spirals. The whole flask was connected by the lateral joint to the smoking machine.

Precipitation of mainstream smoke condensate was accomplished by first cooling the trap in a dry ice/methanol mixture. As the first puffs were drawn through the trap, condensation was not very efficient; but after smoke particles and ice crystals had formed a porous solid framework in the bulge at the bottom of the trap, collection improved significantly because the precipitated smoke condensate acted as a filter. Once this built-up had occurred, an additional large quantity of smoke condensate could be collected, filling up the flask as long as the temperature was maintained at $-80^{\circ} \mathrm{C}$. According to ELMENHORST the quality of the trapped condensate did not differ from that collected electrostatically. This, however, is not fully convincing as it was based on the measurement of only three analytical values per cigarette in dry smoke condensate (amount, nicotine and total steam volatile phenols). A number of gas phase constituents are definitely known to be collected in a cold trap but not by electrostatic precipitation. The trap has become the standard for the collection of large quantities of smoke condensate for biological and - to some extent - chemical analysis.

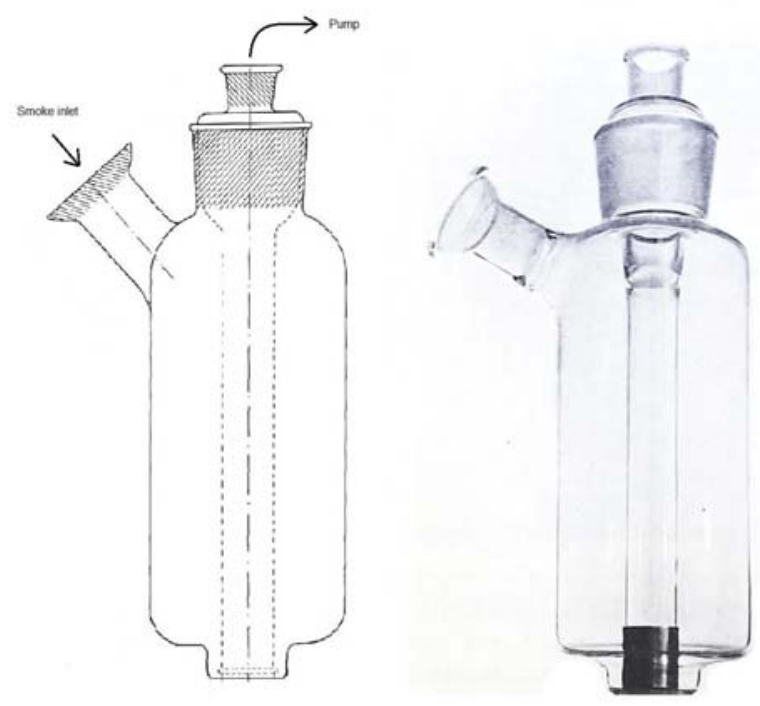

Figure 60. The cold trap developed by ELMENHORST (270) became very popular for the gentle collection of large amounts of smoke condensate.

\subsection{Liquid traps}

The use of impingers (gas wash bottles) - filled with an aqueous solution or a solvent (placed in a cold bath if necessary) - is a rather convenient and very effective way of collecting constituents from both whole smoke and the gaseous phase after passage through a Cambridge filter or an electrostatic trap. In fact, the procedure is an essential step in several analytical standard methods. Impingers of traditional design may be loaded with different liquids depending on the chemical nature and subsequent use of the collected material.

For instance, the following liquids are in use for the determination of these analytes: Diluted sulfuric acid for ammonia in mainstream smoke under Canadian standard T-101 (623); acidified potassium permanganate solution for mercury in mainstream smoke under Canadian standard T-108 (624); acidified 2,4-dinitrophenylhydrazine solution in acetonitrile for eight selected carbonyls in mainstream smoke (formaldehyde, acetaldehyde, acetone, acrolein, propionaldehyde, crotonaldehyde, 2-butanone and n-butyraldehyde) under CORESTA recommended method \# 74 (181) und Canadian standard T-104 (625); cryogenic methanol for five selected volatiles in mainstream smoke (1,3-butadiene, isoprene, acrylonitrile, benzene and toluene) under CORESTA recommended method \# 70 (180) and Canadian standard T-116 (626); and again cryogenic methanol for pyridine, quinoline and styrene in mainstream smoke under Canadian standard T-112 (627). In a study of volatile $N$-nitrosamines in cigarette mainstream and sidestream smoke, BRUNNEMANN et al. (182) used impinger traps with an aqueous buffer solution $(\mathrm{pH}$ 4.5) containing ascorbic acid to avoid the artificial generation of this kind of compounds during sampling, as pointed out by HOFFMANN et al. (557).

Several standard methods compiled and edited (and released in 1986) by the CHEMICAL SUB-COMMITTEE OF THE UK TOBACCO ADVISORY COUNCIL (621) specified the use of cryogenic traps for collecting whole smoke in methanol or vapor phase in ethanol for the determination 


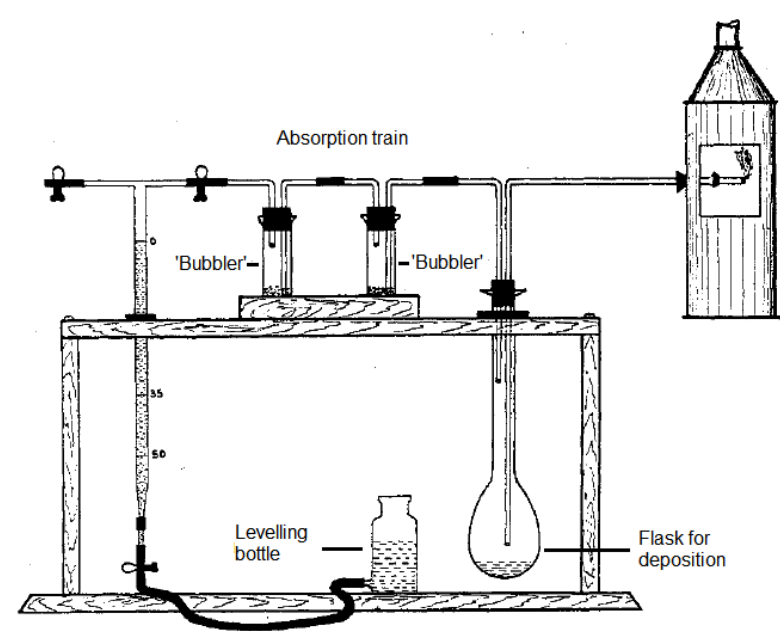

Figure 61. In an earlier smoking system, BRADFORD et al. (4) combined particle deposition by gravity in a flask with trapping gaseous components in "bubblers".

of a number of smoke constituents.

An early example of the use of wash-bottles (impingers) in combination with prior deposition by gravity for collecting smoke aerosol was reported by BRADFORD et al. (4) in 1936. The system (Figure 61) was the integral part of a relatively simple puffing device and consisted of a longnecked flask for the sedimentation of smoke particles and a short train of two bubblers - the first with alcohol and the second with water.

Departing from the traditional form of impingers, more sophisticated solvent traps were developed. In these devices, highly dispersed solvents are used for collecting smoke constituents. In response to his serious concerns (536) over the chemical changes likely to occur in smoke condensate during collection, RochUs (628) of the Medical Research Institute of the German Max-PlanckGesellschaft developed in 1962 a particular liquid trap for the production of "native" tobacco "tar" from cigarettes for biological testing. Machine made smoke was fed without delay into a horizontally positioned glass cylinder of $400 \mathrm{~mm}$ length and $80 \mathrm{~mm}$ diameter, which contained a horizontal driveshaft with several paddles and was surrounded by a jacket for water cooling; the device was protected against light. The rotating shaft $(1,500 \mathrm{rpm})$ distributed some $250-300 \mathrm{~mL}$ solvent (preferably acetone) thoroughly inside the cylinder for continuous smoke extraction. Solvent loaded during operation could be drawn off and fresh solvent added intermittently. A cold trap was attached to the exit outlet of the cylinder for trapping solvent vapors and gaseous smoke constituents. This smoke collection device was directly connected to a rotary smoking machine. Its head was equipped with 60 ports, arranged in two rows of 30 ports each. Puffs were generated by a piston pump or a vacuum pump. While RocHUS considered his system the only available method for collecting native tobacco "tar" and its components, in today's view the formation of artifacts cannot be excluded. The smoke collection system was not adopted by other researchers.

Trapping whole cigarette smoke in a vigorously agitated solvent was also the working principle of a device designed by BARKEMEYER and SEEHOFER (269). A threenecked round bottom flask of $2,000 \mathrm{~mL}$ capacity, placed in a cooling bath, was filled with $100-400 \mathrm{~mL}$ acetone (Figure 62). Smoke entered through a lateral neck (the other lateral neck was for functional use). A glass tube was vertically inserted into the flask through the (third) central neck with its upper end connected to the suction device. At its lower end the tube was closed with porous cotton fabric and slightly immersed in the liquid. In operation, the tube was partly filled by suction with a column of solvent and made to vibrate at a frequency of $50 \mathrm{~Hz}$ for spraying the solvent inside the flask. Trapping efficiency was claimed to exceed $99 \%$ (no details reported). The collection system was used by PAILER et al. (629) for the determination of benzo $[a]$ pyrene in smoke condensate obtained from plain cigarettes.

Details of the collection device (269) were improved by ELMENHORST (268), such as the replacement of the cotton cloth at the lower end of the vibration tube (now closed) by a line-up of 4 slots to prevent clogging. Combined with a modified BAT-RM 30/65 smoking machine (116) throughput was raised to 1,000 cigarettes per day and the amount of smoke material collected at $-78{ }^{\circ} \mathrm{C}$ during several days in the very same flask increased to $80 \mathrm{~g}$ condensate in $100 \mathrm{~mL}$ acetone, produced from 2,000-3,000 cigarettes.

BARKEMEYER (630) described another trap for collecting cigarette mainstream smoke, specifically its gaseous phase, in a solvent. A cylindrical flask was fitted with a vertical rotating tube - with its upper end outside the flask connected to a suction device and its lower end inside the flask laterally equipped with four curved short tubes and immersed in the solvent (for instance, ethanol or acetone). Smoke was introduced into the flask through a lateral joint. A Cambridge filter was installed between smoking

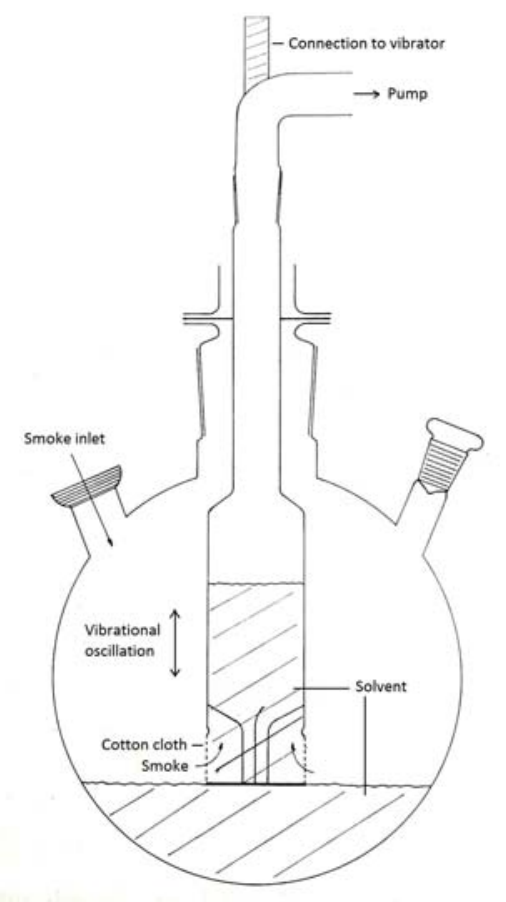

Figure 62. Vibration was used by BARKEMEYER and SEEHOFER (269) to disperse acetone for dissolving smoke particulates. 
machine and collection device for trapping only gas phase constituents in the flask. In operation, the flask was cooled with dry ice/methanol and the rotor set in motion at 2,000 rpm; this ensured efficient mixing inside the flask and prevented upward movement of solvent inside the rotating tube. The trap allowed the collection of representative gas phase samples (except carbon monoxide and the permanent gases) for analytical purposes. To our knowledge, the system was not adopted by other research groups.

\section{8. $\quad$ Solid traps}

The collection of smoke components by adsorption on solid materials, followed by thermal or solvent-mediated desorption, was the approach chosen in certain situations to produce concentrated samples for analysis. Solid adsorbents used include activated carbon and natural or synthetic polymers, optionally coated with reactive chemicals for specific interactions. Solid traps, however, are not without problems; the formation of artifacts represents a major risk, differences in adsorbent quality may come into play, and the quantitative completeness of adsorption and desorption may not always be assured. These complications have limited the application of solid traps.

For the extraction of semi-volatile constituents GROB and VÖLLMIN (631) passed cigarette smoke directly into in a glass tube, which was - to the proximal fourth of its full length $(40 \mathrm{~cm})$ - filled with activated carbon and kept at $120{ }^{\circ} \mathrm{C}$ by a heating jacket. Under these conditions the carbon adsorbed semi-volatiles quite effectively - in con- trast to water. When smoking was completed, the tube was detached, evacuated and completely sealed. The carbon was slowly heated to $150{ }^{\circ} \mathrm{C}$ and trapped material recollected by condensation at the empty end of the tube, which was immersed in ice water; remarkably, it took one day to complete the process. The condensate distinguished itself by remarkably low water content but was lacking in certain semi-volatile constituents, such as higher aromatic compounds, which were irreversibly retained on the carbon.

In several studies, sodium hydroxide coated silica (Ascarite ${ }^{\circledR}$; originally, the carrier material was asbestos) was found to be the preferred reagent for absorbing hydrogen cyanide from tobacco smoke. The Ascarite ${ }^{\mathbb{R}}$ was enclosed in a tube placed immediately behind the cigarette and eventually extracted with water. Hydrogen cyanide was quantified either by colorimetry $(632,633)$ or potentiometrically by titration with silver nitrate (634) or using a cyanide selective electrode (635). REIF (636) compared different methods, observed comparable results and recommended ion-selective potentiometry for its simplicity. The trapping on Ascarite ${ }^{\mathbb{B}}$ was used up to the year 2000 for the routine determination of hydrogen cyanide in cigarette mainstream smoke.

ZELDES and HORTON (637) used Tenax-GC (porous poly$p$-2,6-diphenylphenylene oxide) for trapping the gas phase of cigarette mainstream smoke. Using a single port machine smoke was produced from 1R1 Kentucky reference cigarettes and passed through a Cambridge filter; a sample from the middle of the puff was diverted and transferred to the Tenax trap by means of a nitrogen sweep. Collected

\section{Traditional trapping devices}

Solid traps: Solid adsorbents in use included activated carbon and natural or synthetic polymers (e.g., Tenax, Ascarite ${ }^{\circledR}$ ), optionally coated with reactive chemicals for specific interactions

Use: Collection of mainstream smoke vapor phase and semivolatile constituents

Problems: Batch to batch variation of trapping material quality; possibly incomplete desorption (could not be controlled easily); possible artifact formation (could not be controlled easily)

Impaction traps (TPM collection with capillaries or comparable devices): Smoke aerosol was moved by high pressure through a capillary or a nozzle; under these circumstances smoke particles coagulate and can be collected on a suitable surface

Use: Collection of mainstream smoke condensate for immediate application on mouse skin or bacterial cultures

Problems: Clogging of capillaries or orifices, sealing problems due to the required high pressure, not suitable for collection of large amounts

TPM deposition/collection by gravity: While smoke stays in a suitable container particulates coagulate forming small droplets, which settle at the bottom by gravity

Use: Until the 1940s primarily to obtain tobacco/cigarette smoke condensate for analytical purposes

Problems: TPM collection is not quantitative; generation of artifacts is to be expected (no issue until the 1940s)

TPM deposition/collection with cotton wool traps: Smoke passed through a tube filled with cotton wool; particles were collected on the cotton wool and then eluted with a suitable solvent, such as acetone or chloroform

Use: Until the 1940s primarily to obtain tobacco/cigarette smoke condensate for analytical purposes

Problems: Generation of artifacts is to be expected (no issue until the 1960s) 
low boiling constituents, such as isoprene, acetaldehyde and acrolein, were then thermally desorbed and determined by gas chromatography using a cryothermal packed column. HigGins et al. (638) improved the method and assessed the deliveries of 34 constituents in the gas phase of a range of commercial ultra-low "tar" cigarettes, trapped on Tenax-GC, by thermal desorption and capillary gas chromatography.

\section{Currently used trapping devices}

Cambridge filters: A tool for collecting smoke condensate. It is a glass fiber pad, about $2 \mathrm{~mm}$ thick, with small amounts of polyacrylate or polyvinylacetate as binder; it is specified to retain $99.9 \%$ of a test aerosol, consisting of dioctyl phthalate with a particle diameter equal to or greater than $0.3 \mu \mathrm{m}$ at a linear air velocity of $140 \mathrm{~mm} / \mathrm{s}$

Use: Collection of mainstream smoke particulate phase for routine determination of NFDPM and smoke nicotine (using ISO 3308 or FTC methodology), and for the determination of various particulate phase constituents, e.g., polycyclic aromatic hydrocarbons (PAHs) or tobacco specific N-nitrosamines (TSNAs)

Separation of mainstream smoke into the gaseous and particulate phases, e.g., for the determination of NO or $\mathrm{CO}$ yields; collection of mainstream smoke particulate phase for toxicological evaluation by in vitro assays (Ames test, comet assay, sister chromatid exchange, others)

Electrostatic traps: A device for collecting smoke condensate. It consists of a glass tube, about $3 \mathrm{~cm}$ in diameter and enveloped in an outer negatively charged electrode, and an inner positively charged needle-size electrode. Under the influence of an electrical field of $>15 \mathrm{KV}$, the smoke aerosol disintegrates and the smoke particles are collected on the inside wall of the glass tube

Use: Collection of the particulate phase of smoke aerosol; until 1990: Collection of mainstream smoke particulate phase for routine determination of NFDPM and smoke nicotine (e.g., according to DIN 10240); since 1990: Collection of mainstream smoke particulate phase for the determination of specific heavy metals

Problems: Occasional formation of artifacts due to ozone, formed by high voltage; this is no issue if collected material is used for heavy metal analysis

Cold traps: A device usually made from glass and cooled with dry ice in acetone or methanol or with liquid air

Use: Collection of large amounts of mainstream smoke condensate (TPM, semivolatiles and certain vapor phase components). Highly volatile components may escape from TPM when collected material is warmed to room temperature

Large amounts of smoke condensate in homogenous, reproducible quality are necessary for application on mouse skin to evaluate its tumorigenicity; until the 1970s: Large amounts of condensate (collected in cold traps) were also necessary for the determination of specific smoke components, e.g., benzo[a]pyrene (no longer required due to progress in analytical techniques)

Problems: Formation of artifacts cannot be excluded

Liquid traps: Whole mainstream smoke or specific smoke constituents or groups of components are collected in suitable liquids (e.g., acetone, methanol, aqueous systems)

Use: In the early 1960s, mainstream smoke for mouse skin painting from more than 1000 cigarettes was collected at room temperature in specifically designed liquid traps; today, liquid traps (impingers containing the liquid) are primarily used for collection (also with cooling and by derivatization) and analysis of groups of specific gas phase compounds (volatile carbonyls and $\mathrm{N}$-nitrosamines, benzene, isoprene, butadiene, etc.)

Problems: Depending on the liquid generation of artifacts cannot be excluded 


\section{8.} PERSPECTIVE

A survey of devices available today for the generation and collection of, and exposure to, cigarette mainstream smoke and a retrospect of developments during more than 100 years reveals a remarkable pattern: from simple to sophisticated, from adventitious to well considered, from manual and mechanic to automated and electronic, from optional to standardized - with pertinent publications from copiously narrated to concisely reported (though not in all cases). The course of past events is fascinating, and the present status achieved is very impressive. How is this likely to continue?

Certain fields of action obviously are highlights of the past. Massive production of smoke condensate for the isolation and identification of certain constituents has become unnecessary in view of modern analytical methods. Inhalation studies - some with enormous numbers of experimental animals - are unlikely to be repeated or continued on a large scale, if at all.

At the same time, smoking machines for analytical purposes have reached an impressive level of flexibility, reliability and automation. A continuing task will be the full adaptation of equipment - matched quite perfectly with established regulatory requirements and procedures - to new more "intense" smoking regimes. In particular, the harmonization of linear and rotary smoking machines laboriously but successfully resolved regarding the use of FTC/ISO smoking conditions - concerning their functioning under the presently most notable Canadian Intense regime remains a difficult task for the future. Similarly, the relationship of analytical smoking machine performance and (average and individual) human smoking behavior will remain a technical and philosophical challenge.

In vitro toxicity testing will certainly grow in importance, if only for reducing or avoiding the consumption of experimental animals. The use of air-liquid interface (ALI) exposure technology is particularly promising in light of significant recent progress in instrumentation, dosimetry, the development of ever more complex and realistic cellular and tissue systems (especially of human origin), and inroads into epigenetic toxicology. The workshop conferences organized by the U.S.-based Institute for In Vitro Sciences $(525,639)$ - with impressively compiled proceedings and conclusions - are exemplary opportunities for the interaction and future cooperation of scientists representing regulatory agencies, academia, industry (tobacco and biological) and animal protection. Addressing the research priorities articulated by the FDA'S CENTER FOR TOBACCO PRODUCTS (640) specific conference topics were exposure systems and dosimetry assessment for inhaled tobacco products, and models for diseases like chronic obstructive pulmonary disease (COPD). The standardization and regulatory acceptance of specified tests is now being tackled in collaborative studies. In addition, the toxicological assessment of e-cigarette vapors is coming up conspicuously on the horizon.

\section{ACKNOWLEDGEMENT}

The authors would like to thank everyone who contributed to this review, in particular the reviewers for numerous helpful comments. 
Table A. Early smoke generating devices and modern analytical smoking machines. Page numbers refer to the publication: Klus, $H$., B. Boenke-Nimphius, and L. Müller: Cigarette Mainstream Smoke: The Evolution of Methods and Devices for Generation, Exposure and Collection; Beitr. Tabakforsch. Int. 27 (2016) 137-274.

\begin{tabular}{|c|c|c|c|c|}
\hline Year & Author (Reference) & Country & Specifics & Page \\
\hline 1892 & $\begin{array}{l}\text { CONNECTICUT AGRI- } \\
\text { CULTURAL EXPERIMENT } \\
\text { STATION (15) }\end{array}$ & US & $\begin{array}{l}\text { - single port for cigars } \\
\text { - intermittent puffing using a siphon }\end{array}$ & 141 \\
\hline 1900 & THOMS (26) & $\mathrm{DE}$ & $\begin{array}{l}\text { - single port device for cigars } \\
\text { - continuous suction using an aspirator }\end{array}$ & 141 \\
\hline 1901 & HABERMANN $(16,30)$ & KK & $\begin{array}{l}\text { - single port for cigars, cigarettes and (conditionally) pipes } \\
\text { - flexible conditions of intermittent puffing accomplished by the operating } \\
\text { principle of a siphon }\end{array}$ & 142 \\
\hline 1906 & GARNER (31) & US & $\begin{array}{l}\text { - 4-port device for cigars } \\
\text { - intermittent puffing using a siphon }\end{array}$ & 142 \\
\hline 1927 & PFYL and SCHMITT (38) & $\mathrm{DE}$ & $\begin{array}{l}\text { - operated manually } \\
\text { - single port for cigars and cigarettes } \\
\text { - puffing by intermittent action of a water jet pump }\end{array}$ & 143 \\
\hline 1931 & WENUSCH (43) & AT & $\begin{array}{l}\text { - operated manually } \\
\text { - single port } \\
\text { - intermittent puffing by rubber balloon or falling mercury column } \\
\text { - suction source placed between port and impinger train } \\
\text { - equal amounts of tobacco smoked per puff }\end{array}$ & 144 \\
\hline 1933 & PFYL et al. (17) & $\mathrm{DE}$ & $\begin{array}{l}\text { - operated manually } \\
\text { - single port for cigars and cigarettes } \\
\text { - puffing by intermittent action of a falling mercury column } \\
\text { - fine-tuned smoking parameters } \\
\text { - device commercially available }\end{array}$ & 146 \\
\hline 1935 & JENSEN and HALEY (69) & US & $\begin{array}{l}\text { - semiautomatic operation } \\
\text { - single port for cigarettes } \\
\text { - constant time/constant flow mode } \\
\text { - puffing with vacuum reservoir combined with rotating valve and high- } \\
\text { vacuum pump } \\
\text { - unusual smoking parameters }\end{array}$ & 147 \\
\hline 1936 & BRADFORD et al. (4) & US & $\begin{array}{l}\text { "robot for mass smoking" (4), also called "American Tobacco Company } \\
\text { Machine": } \\
\text { - semi-automatic linear 4-port machine for cigarettes } \\
\text { - constant time/constant flow mode } \\
\text { - falling water column combined with rotating valves operated by a timer- } \\
\text { actuated motor } \\
\text { - smoke collection by gravitational deposition and with impingers } \\
\text { - exemplary smoking parameters }\end{array}$ & 148 \\
\hline 1936 & WENUSCH (47) & AT & $\begin{array}{l}\text { - operated manually } \\
\text { - single port for cigarettes } \\
\text { - puffing by intermittent action of a water jet pump in combinatoon with } \\
\text { manometer and regulator for effective pressure control } \\
\text { - equal amounts of tobacco smoked per puff }\end{array}$ & 145 \\
\hline 1953 & WAHL and HEIL (61) & $\mathrm{DE}$ & $\begin{array}{l}\text { - operated manually } \\
\text { - single port for cigars and cigarettes } \\
\text { - puffing by intermittent pump action } \\
\text { - flow control by manometer, flowmeter and inlet valve }\end{array}$ & 146 \\
\hline 1953 & STAUB and FURRER (63) & $\mathrm{CH}$ & $\begin{array}{l}\text { - operated manually } \\
\text { - single port for cigarettes } \\
\text { - three-way cock control } \\
\text { - puffing by intermittent action of a falling mercury column }\end{array}$ & 146 \\
\hline 1954 & STAUB and FURRER (87) & $\mathrm{CH}$ & $\begin{array}{l}\text { - automated electronic one port machine with } \\
\text { - adjustable piston pump } \\
\text { - smoke collection in impingers }\end{array}$ & 150 \\
\hline 1955 & $\begin{array}{l}\text { CIGARETTE COMPONENTS } \\
(75)\end{array}$ & GB & $\begin{array}{l}\text { - manually operated "simple all-glass smoking machine" comparable to } \\
\text { the American Tobacco Company machine (4) } \\
\text { - constant volume mode }\end{array}$ & 151 \\
\hline 1955 & DECKER et al. (110) & $\mathrm{CH}$ & $\begin{array}{l}\text { - 5-port rotary machine with vertical smoke head } \\
\text { - puffing using aspirator with motor-driven valves } \\
\text { - smoke collection in impingers }\end{array}$ & 154 \\
\hline
\end{tabular}


Table A. Contd.

\begin{tabular}{|c|c|c|c|c|}
\hline Year & Author (Reference) & Country & Specifics & Page \\
\hline 1957 & ILES and SHARMAN (90) & GB & $\begin{array}{l}\text { "Autosmoker": } \\
\text { - single port smoking unit for cigarettes - several units combinable } \\
\text { - puffing using bellows } \\
\text { - direct electrostatic precipitation }\end{array}$ & 151 \\
\hline 1957 & KEITH and NEWSOME (84) & US & $\begin{array}{l}\text { - semi-automatic linear 6-port machine for cigarettes } \\
\text { - constant volume/constant time mode } \\
\text { - puffing using a vacuum reservoir with pressure drop regulator and } \\
\text { several solenoid valves }\end{array}$ & 149 \\
\hline 1958 & CuZIN (66) & FR & $\begin{array}{l}\text { - semi-automatic constant time mode } \\
\text { - 7-port ring-shaped or 10-port bar-shaped manifold } \\
\text { - puffing using a vacuum tank with timer-controlled valve } \\
\text { - smoke collection in a scrubbing tower or by capillary coagulation }\end{array}$ & 146 \\
\hline 1958 & O'KEEFFE and LIESER (85) & US & $\begin{array}{l}\text { - semi-automatic linear 8-port machine for cigarettes } \\
\text { - constant volume/constant time mode } \\
\text { - puffing using individual syringes with electronic timing }\end{array}$ & 150 \\
\hline 1958 & $\begin{array}{l}\text { CIGARETTE COMPONENTS } \\
(76)\end{array}$ & GB & $\begin{array}{l}\text { Ethel Mark VI: } \\
\text { - automated linear 4-channel machine } \\
\text { - constant time/constant pressure mode with rotary vacuum pump } \\
\text { - condensate collection preferentially by electrostatic precipitation }\end{array}$ & 152 \\
\hline 1959 & SEEHOFER (113) & $\mathrm{DE}$ & $\begin{array}{l}\text { BAT-RM 15: } \\
\text { - first rotary 15-port machine } \\
\text { - constant time/constant flow mode } \\
\text { - condensate trapped centrally in cotton wool } \\
\text { - lax smoking parameters acceptable for routine operating control } \\
\text { - manufactured by Borgwaldt }\end{array}$ & 155 \\
\hline 1961 & MUMPOWER et al. (77) & US & $\begin{array}{l}\text { - comparison of two single port machines: falling water column for } \\
\text { constant volume mode vs .vacuum reservoir and adjustable orifice for } \\
\text { constant volume/constant time mode }\end{array}$ & 149 \\
\hline 1961 & WALTZ et al. (111) & $\mathrm{CH}$ & $\begin{array}{l}\text { - 10-port rotary machine with vertical smoke head } \\
\text { - constant volume mode } \\
\text { - puffing using piston pump with magnetic valve } \\
\text { - central electrostic precepitator }\end{array}$ & 154 \\
\hline 1961 & SEEHOFER et al. (114) & $\mathrm{DE}$ & $\begin{array}{l}\text { BAT-RM 15/61: } \\
\text { - rotary 15-port machine } \\
\text { - improved version of BAT-RM } 15 \\
\text { - constant time or constant volume mode } \\
\text { - condensate collected in an electrostatic trap } \\
\text { - stringent smoking parameters } \\
\text { - manufactured by Borgwaldt }\end{array}$ & 155 \\
\hline 1962 & $\begin{array}{l}\text { WILLIAMSON and CLARK } \\
\text { (94) }\end{array}$ & GB & $\begin{array}{l}\text { CSM 10: } \\
\text { - automated linear 4-port machine with vacuum pump and mechanical } \\
\text { timers } \\
\text { - wide choice of smoking parameters } \\
\text { - constant square wave puffs through an automatic volume compensator } \\
\text { - improved model using electronic timers = CSM } 12\end{array}$ & 152 \\
\hline 1965 & SEEHOFER et al. (116) & $\mathrm{DE}$ & $\begin{array}{l}\text { BAT-RM 30/65: } \\
\text { - } \text { rotary 30-port machine } \\
\text { - enlargement of BAT-RM 15/61 } \\
\text { - condensate collected for TPM analysis in an electrostatic trap } \\
\text { - discretionary use of cold or liquid traps } \\
\text { - manufactured by Borgwaldt }\end{array}$ & 155 \\
\hline 1967 & WILEY and FERRI (8) & US & $\begin{array}{l}\text { - automated linear } 20 \text {-port smoking machine } \\
\text { - constant volume/constant time mode } \\
\text { - puffing using } 20 \text { individual syringes controlled with cams and solenoid } \\
\text { valves } \\
\text { - } 20 \text {-port electric lighter for simultaneous ignition } \\
\text { - automatic puff counting } \\
\text { - "string cut-off" technique for butt length control } \\
\text { - widely accepted machine standard }\end{array}$ & 150 \\
\hline 1968 & SEEHOFER (115) & $\mathrm{DE}$ & $\begin{array}{l}\text { BAT-RM 20/68: } \\
\text { - automatic rotary 20-port machine } \\
\text { - constant time/constant flow mode } \\
\text { - various trapping systems } \\
\text { - manufactured by Borgwaldt }\end{array}$ & \\
\hline
\end{tabular}


Table A. Contd.

\begin{tabular}{|c|c|c|c|c|}
\hline Year & Author (Reference) & Country & Specifics & Page \\
\hline 1970 & FILTRONA (95) & GB & $\begin{array}{l}\text { SM 100: } \\
\text { - linear 4-port smoking machine } \\
\text { - } \text { replaced CSM } 10 / 12 \\
\text { - } \text { completely solid state } \\
\text { - } \text { automatic critical flow orifice } \\
\text { - } 12 \text {-port version = SM } 101\end{array}$ & 152 \\
\hline 1970 & FILTRONA & GB & $\begin{array}{l}\text { SM } 300 \text { : } \\
\text { - linear 20-port machine } \\
\text { - commercial adoption of the machine of WILEY and FERRI ( } 8 \text { ) } \\
\text { - corresponds to the Phipps and Bird 20-port machine with updated } \\
\text { electronics } \\
\text { - over } 100 \text { machines produced } \\
\text { - version with automatic collection bag and built-in CO analyzer = SM } 350\end{array}$ & 152 \\
\hline 1972 & LORENZ et al. (120) & $\mathrm{DE}$ & $\begin{array}{l}\text { BAT-RM 20/71: } \\
\text { - automatic rotary 20-port machine } \\
\text { - improved version of BAT-RM 20/68 } \\
\text { - constant time/constant flow mode } \\
\text { - restricted or free smoking } \\
\text { - rectangular or bell-shaped puff profiles } \\
\text { - in compliance with DIN 10240:1969 } \\
\text { - manufactured by Borgwaldt }\end{array}$ & 156 \\
\hline 1974 & FILTRONA (100) & GB & $\begin{array}{l}\text { SM 302: } \\
\text { - linear 8-port machine with reduced dead volume } \\
\text { - suitable for gas phase component }(\mathrm{CO}, \mathrm{NO} / \mathrm{NOx}) \text { measurement puff-by- } \\
\text { puff or per cigarette } \\
\text { - became a lab standard } \\
\text { - } \text { over } 200 \text { machines produced }\end{array}$ & 153 \\
\hline 1985 & BORGWALDT (129) & $\mathrm{DE}$ & $\begin{array}{l}\text { RM20CS: } \\
\text { - rotary 20-port machine } \\
\text { - improved version of BAT-RM 20/71 } \\
\text { - piston pump for bell-shaped puff profile }\end{array}$ & 157 \\
\hline 1990 & BORGWALDT (129) & DE & $\begin{array}{l}\text { RM20CSR: } \\
\text { - rotary 20-port machine } \\
\text { - retrofitted with exhaust hood, } \\
\text { - discretionary anemometer, } \\
\text { - labyrinth seals and } \\
\text { - neoprene washers, and } \\
\text { - a central } 92 \text { mm Cambridge filter - for compliance with ISO 3308:1991 }\end{array}$ & 157 \\
\hline 1992 & FILTRONA (99) & GB & $\begin{array}{l}\text { SM 400: } \\
\text { - linear 20-port machine using computers and microprocessors } \\
\text { - Cambridge filters for condensate collection } \\
\text { - new hood design } \\
\text { - in compliance with ISO } 3308: 1991 \\
\text { - replacing model SM } 300\end{array}$ & 153 \\
\hline 1992 & FILTRONA (103) & GB & $\begin{array}{l}\text { SM 342: } \\
\text { - linear 8-port machine } \\
\text { - Cambridge filters for condensate collection } \\
\text { - in compliance with ISO 3308:1991 } \\
\text { - replacing model SM } 302\end{array}$ & 153 \\
\hline 1994 & BORGWALDT (129) & $\mathrm{DE}$ & $\begin{array}{l}\text { RM200: } \\
\text { - first fully automatic rotary } 20 \text {-port machine } \\
\text { - Cambridge filters for condensate collection } \\
\text { - } 10 \text { consecutive smoke runs without operator intervention in compliance } \\
\text { with ISO 3308:1991 } \\
\text { - optional integrated CO analyzer (available since 2002) }\end{array}$ & 157 \\
\hline 1998 & BORGWALDT (129) & $\mathrm{DE}$ & $\begin{array}{l}\text { RM20: } \\
\text { - semi-automatic rotary 20-port machine based on the concept of } \\
\text { RM20CS and improved for compliance with ISO 3308:1991 } \\
\text { - optional embedded CO analyser }\end{array}$ & 157 \\
\hline 2004 & CERULEAN (106) & GB & $\begin{array}{l}\text { SM 450: } \\
\text { - linear 20-port machine } \\
\text { - different simultaneous smoking regimes in compliance with ISO } \\
\text { 3308:1991 } \\
\text { - "Hoffmann shelf" for collecting special analytes }\end{array}$ & 153 \\
\hline
\end{tabular}


Table A. Contd.

\begin{tabular}{|c|c|c|c|c|}
\hline Year & Author (Reference) & Country & Specifics & Page \\
\hline 2005 & CERULEAN (106) & GB & $\begin{array}{l}\text { SM } 405 \text { and SM 410: } \\
\text { - linear 5-port and 10-port versions of SM } 450\end{array}$ & 153 \\
\hline 2006 & BORGWALDT (108) & $\mathrm{DE}$ & $\begin{array}{l}\text { RM2OH: } \\
\text { - automatic rotary 20-port machine } \\
\text { - revised RM20 } \\
\text { - flexible smoking parameters } \\
\text { - por combination with various trapping systems } \\
\text { - particularly suited for Hoffmann analytes }\end{array}$ & 157 \\
\hline 2006 & BORGWALDT (108) & $\mathrm{DE}$ & $\begin{array}{l}\text { RM200A: } \\
\text { - fully automatic rotary 20-port machine } \\
\text { - improved version of RM200 } \\
\text { - intregrated CO module and NO/NOx analyzer }\end{array}$ & 157 \\
\hline 2008 & CERULEAN (106) & GB & $\begin{array}{l}\text { SM 450RH: } \\
\text { - linear 20-port machine } \\
\text { - modified SM } 450 \text { with increased flexibility }\end{array}$ & 153 \\
\hline 2008 & BORGWALDT (108) & $\mathrm{DE}$ & $\begin{array}{l}\text { RM20D: } \\
\text { - manual rotary 20-port machine } \\
\text { - successor of RM20CSR } \\
\text { - for combination with various trapping systems in compliance with ISO } \\
\text { 3308:1991 }\end{array}$ & 157 \\
\hline 2011 & CERULEAN (106) & GB & $\begin{array}{l}\text { SM 450N: } \\
\text { - linear 20-port machine } \\
\text { - upgraded SM } 450 \text { with integrated chemiluminescence analyzer }\end{array}$ & 153 \\
\hline 2014 & CERULEAN (106) & GB & $\begin{array}{l}\text { SM 450i: } \\
\text { - semi-automated linear 20-port machine with optimized air management } \\
\text { system } \\
\text { up to } 20 \text { different simultaneous smoking regimes }\end{array}$ & 153 \\
\hline 2015 & BORGWALDT (108) & $\mathrm{DE}$ & $\begin{array}{l}\text { RM200A2: } \\
\text { - fully automatic rotary } 20 \text {-port machine } \\
\text { - improved version of RM200A } \\
\text { - capable of smoking } 120 \mathrm{~mm} \text { cigarettes and accommodating alternative } \\
\text { trapping systems }\end{array}$ & 157 \\
\hline
\end{tabular}


Table B. Smoking machines for condensate production and in vivo toxicity testing. Page numbers refer to the publication: Klus, $H$. B. Boenke-Nimphius, and L. Müller: Cigarette Mainstream Smoke: The Evolution of Methods and Devices for Generation, Exposure and Collection; Beitr. Tabakforsch. Int. 27 (2016) 137-274.

\begin{tabular}{|c|c|c|c|c|}
\hline Year & Author & Country & Specifics & Page \\
\hline 1939 & CAMPBELL (5) & GB & $\begin{array}{l}\text { - single cigarette in glass holder } \\
\text { - } 14-15 \text { large puffs produced with aspirator rubber bulb } \\
\text { - condensate collected in a cool glass side bulb }\end{array}$ & 174 \\
\hline 1953 & WYNDER et al. (72) & US & $\begin{array}{l}\text { - manually operated manifold machine with six } 10 \text {-port arms for } 60 \\
\text { vertically positioned cigarettes } \\
\text { - } 350 \text { ml puff volume } \\
\text { - cold trap for TPM collection }\end{array}$ & 175 \\
\hline 1956 & KosAK et al. (250) & US & $\begin{array}{l}\text { - manifold machine with twelfe } 20 \text {-port arms for } 240 \text { horizontally positioned } \\
\text { cigarettes } \\
\text { - large volume puffs generated in constant time mode by a rotary air pump } \\
\text { producing non-uniform puff volumes per cigarette }(35-40 \mathrm{ml}) \\
\text { cold traps for TPM collection }\end{array}$ & 175 \\
\hline 1957 & $\begin{array}{l}\text { WYNDER and WRIGHT } \\
\quad(248)\end{array}$ & US & $\begin{array}{l}\text { - automatic manifold machine with } 10 \text {-port arms for up to } 150 \text { vertically } \\
\text { positioned cigarettes } \\
\text { - } 350 \mathrm{ml} \text { puff volume } \\
\text { - cold traps for TPM collection }\end{array}$ & 175 \\
\hline 1957 & $\begin{array}{l}\text { ENGELBRETH-HOLM et al. } \\
\quad(251)\end{array}$ & DK & $\begin{array}{l}\text { - device for smoking } 50 \text { vertically positioned cigarettes simultaneously } \\
\text { using a pulsator } \\
\text { - smoke collection in a cotton wool trap }\end{array}$ & 176 \\
\hline 1957 & $\begin{array}{l}\text { SCHUR and RICKARDS } \\
(252)\end{array}$ & US & $\begin{array}{l}\text { - 20-port machine with rotating vacuum head } \\
\text { - constant time mode } \\
\text { - individual condensate collection in a cold glass coil }\end{array}$ & 176 \\
\hline 1959 & CLARK and BOCK (7) & US & $\begin{array}{l}\text { - automatic manifold machine with six } 100 \text {-port rings for } 600 \text { horizontally } \\
\text { positioned cigarettes } \\
\text { - } 1,050 \mathrm{ml} \text { puff volume } \\
\text { - common condensate collection in cold traps }\end{array}$ & 176 \\
\hline 1963 & $\begin{array}{l}\text { NEURATH and KRÖGER } \\
(274)\end{array}$ & DE & $\begin{array}{l}\text { - reverse smoking device with single cigarette in a receptacle } \\
\text { - condensate production by nozzle passage for immediate application }\end{array}$ & 178 \\
\hline 1964 & $\begin{array}{l}\text { SEEHOFER and HANßEN } \\
(288)\end{array}$ & DE & $\begin{array}{l}\text { - single cigarette machine with piston pump } \\
\text { - constant volume mode } \\
\text { - smoke forcefully exhausted through capillary for coagulation } \\
\text { - "capillary press" }\end{array}$ & 179 \\
\hline 1965 & HACKNEY et al. (258) & US & $\begin{array}{l}\text { - semi-automatic modification of }(252) \\
\text { - vertically rotating } 15-\text { port machine with fixed vacuum head } \\
\text { - common condensate collection in cold traps }\end{array}$ & 176 \\
\hline 1965 & LANG and MOSHEY (261) & US & $\begin{array}{l}\text { - automatic machine with horizontally rotating } 90 \text {-port ring and stationary } \\
10 \text {-arm inner vacuum manifold } \\
\text { - } 350 \mathrm{ml} \text { puff volume } \\
\text { - common condensate collection }\end{array}$ & 176 \\
\hline 1965 & ELMENHORST (268) & $\mathrm{DE}$ & $\begin{array}{l}\text { - slightly modified rotary } 30 \text {-port machine BAT-RM 30/65 (116) combined } \\
\text { with a modified solvent-containing vibrational trap or a novel solvent-free } \\
\text { cold trap (270) }\end{array}$ & 177 \\
\hline 1965 & $\begin{array}{l}\text { SEEHOFER and HANßEN } \\
(289)\end{array}$ & $\mathrm{DE}$ & $\begin{array}{l}\text { - automatic smoking of } 15 \text { cigarettes using rotary } 30 \text {-port machine (268) } \\
\text { and capillary press technique }\end{array}$ & 179 \\
\hline 1966 & $\begin{array}{l}\text { SEEHOFER and HANßEN } \\
(290)\end{array}$ & DE & $\begin{array}{l}\text { - BAT-RM 15/66: automatic smoking machine (289) modified for free and } \\
\text { restricted smoking, manufactured by Borgwaldt }\end{array}$ & 179 \\
\hline 1966 & MATHEWSON (291) & GB & $\begin{array}{l}\text { - rotary 24-port machine with vertically positioned smoking head } \\
\text { - constant flow and rectangular puff profile for smoke collection by } \\
\text { impaction }\end{array}$ & 234 \\
\hline 1966 & ELMENHORST (271) & $\mathrm{DE}$ & - fully automated rotary 30-port machine improved version of (268) & 177 \\
\hline 1966 & $\begin{array}{l}\text { MORELL and VARSELL } \\
(284)\end{array}$ & US & $\begin{array}{l}\text { - reverse smoking device with single cigarette in a receptacle } \\
\text { - comprehensive collection of mainstream and sidestream smoke, butt } \\
\text { and ash }\end{array}$ & 178 \\
\hline 1969 & $\begin{array}{l}\text { ELMENHORST and HENNIG } \\
(272)\end{array}$ & DE & $\begin{array}{l}\text { - improved rotary } 30 \text {-port machine }(271) \\
\text { - automatic butt length control }\end{array}$ & 178 \\
\hline 1971 & JENKINS et al. (286) & US & $\begin{array}{l}\text { - modified reverse smoking device (284) } \\
\text { - improved trapping techniques }\end{array}$ & 179 \\
\hline
\end{tabular}


Table C. Smoke generation for animal inhalation studies. Page numbers refer to the publication: Klus, H., B. Boenke-Nimphius, and L. Müller: Cigarette Mainstream Smoke: The Evolution of Methods and Devices for Generation, Exposure and Collection; Beitr. Tabakforsch. Int. 27 (2016) 137-274.

\begin{tabular}{|c|c|c|c|c|}
\hline Year & Author & Country & Specifics & Page \\
\hline 1936 & CAMPBELL (296) & GB & $\begin{array}{l}\text { - single cigarette in glass holder } \\
\text { - } 14-15 \text { large puffs produced with aspirator rubber bulb and blown into } 580 \\
\text { Itr chamber for whole body exposure }\end{array}$ & 181 \\
\hline 1952 & ESSENBERG (298) & US & $\begin{array}{l}\text { - automatic 12-port machine with vertical rotary carriage } \\
\text { - single cigarette continuous puffing by evacuation of } 56 \text { Itr chamber for } \\
\text { whole body exposure }\end{array}$ & 181 \\
\hline 1958 & $\begin{array}{l}\text { LEUCHTENBERGER et al. } \\
\quad(299)\end{array}$ & US & - slightly modified smoking/exposure system (298) & 182 \\
\hline 1960 & HAAG et al. (305) & US & $\begin{array}{l}\text { - 12-port machine with circularly arranged cigarettes } \\
\text { - puff volume of } 420 \mathrm{ml} \text { drawn every } 60 \text { seconds by means of a moving } \\
\text { diaphragm } \\
\text { - connected to a } 23 \mathrm{~L} 2 \text {-tier chamber for whole body exposure }\end{array}$ & 182 \\
\hline 1962 & $\begin{array}{l}\text { DONTENWILL and MOHR } \\
(308)\end{array}$ & $\mathrm{DE}$ & $\begin{array}{l}\text { - automatic linear } 10-\text { port machine } \\
\text { - standard parameter intermittent puffing by evacuation of } 20 \text { Itr animal } \\
\text { exposure chamber } \\
\text { - whole body exposure }\end{array}$ & 183 \\
\hline 1963 & Отто (306) & $\mathrm{DE}$ & $\begin{array}{l}\text { - partially evacuated } 400 \text { Itr exposure chamber intermittently connected to } \\
\text { attached manifold with lit cigarettes } \\
\text { - whole body exposure }\end{array}$ & 182 \\
\hline 1964 & DONTENWILL (309) & DE & $\begin{array}{l}\text { - automatic rotary 8-port machine with small smoke collection chamber } \\
\text { surrounded by eight animal holders for head-only exposure }\end{array}$ & 183 \\
\hline 1967 & DONTENWILL et al. (314) & DE & $\begin{array}{l}\text { Type "Hamburg" series } \\
\text { - automatic rotary } 30 \text {-port RM } 30 / 65 \text { machine (116) with a } 525 \mathrm{ml} \text { smoke } \\
\text { collection chamber } \\
\text { - constant flow mode } \\
\text { - suitable for six or ten animal holders for head-only exposure }\end{array}$ & 184 \\
\hline 1968 & WYNDER et al. (307) & US & $\begin{array}{l}\text { - partially evacuated } 17 \text { Itr exposure chamber intermittently connected } \\
\text { through a flow orifice with a lit cigarette } \\
\text { - whole body exposure }\end{array}$ & 182 \\
\hline 1973 & DAVIS et al. (324) & GB & $\begin{array}{l}\text { "Harrogate smoker" } \\
\text { - manually operated single cigarette machine with a } 100 \mathrm{ml} \text { chamber for } \\
\text { intermittent smoke collection, dilution and single animal nose-only } \\
\text { exposure } \\
\text { - puffing using latex diaphragm }\end{array}$ & 185 \\
\hline 1973 & BATISTA et al. (390) & US & $\begin{array}{l}\text { "ADL (Arthur D. Little) machine" } \\
\text { - single cigarette machine with two syringes in tandem, operated by air } \\
\text { pressure and spring tension } \\
\text { - smoke collected in holding tube for animal active inhalation }\end{array}$ & 194 \\
\hline 1975 & $\begin{array}{l}\text { SCHULTZ and WAGNER } \\
(344)\end{array}$ & US & $\begin{array}{l}\text { - automatic reverse smoking machine with two 15-port carrousels } \\
\text { positioned vertically side by side } \\
\text { - puffing using two reciprocating sealing puff chambers } \\
\text { - allowing continuous or intermittent animal exposure }\end{array}$ & 188 \\
\hline 1976 & BEVEN (345) & GB & $\begin{array}{l}\text { - rotary 24-port machine with vertically positioned smoke head } \\
\text { - allowing continuous and intermittent animal exposure }\end{array}$ & 188 \\
\hline 1977 & $\begin{array}{l}\text { REININGHAUS and } \\
\text { HACKENBERG (363) }\end{array}$ & $\mathrm{DE}$ & $\begin{array}{l}\text { - automatic rotary } 30 \text {-port reverse smoking machine in a pressurized } \\
\text { cabinet } \\
\text { continuous head-only exposure of } 120 \text { rats developed by INBIFO and } \\
\text { converted to SM-85 (366) }\end{array}$ & 191 \\
\hline 1978 & MADDOx et al. (336) & US & $\begin{array}{l}\text { - semi-automatic single cigarette machine using a piston pump } \\
\text { - intermittent nose-only exposure developed by ORNL }\end{array}$ & 187 \\
\hline 1979 & STOKELY et al. (333) & US & $\begin{array}{l}\text { "Walton Horizontal Smoking Machine" } \\
\text { - semi-automatic 3-port reverse smoking machine } \\
\text { - puffing using reciprocating air-pressurized dome } \\
\text { - intermittent nose-only exposure }\end{array}$ & 186 \\
\hline 1979 & MONEYHUN et al. (337) & US & $\begin{array}{l}\text { SEM II: } \\
\text { - fully automaticd } 30 \text {-port rotary reverse smoking machine } \\
\text { - puffing mechanism under a pressurized sealed dome } \\
\text { - constant pressure mode } \\
\text { - continuous nose-only exposure of } 120-480 \text { mice }\end{array}$ & 187 \\
\hline
\end{tabular}


Table C. Contd.

\begin{tabular}{|c|c|c|c|c|}
\hline Year & Author & Country & Specifics & Page \\
\hline 1980 & $\begin{array}{l}\text { BAUMGARTNER and } \\
\text { COGGINS (352) }\end{array}$ & $\mathrm{CH}$ & $\begin{array}{l}\text { "Battelle Mk III" - "AMESA Mark III (A)" } \\
\text { - automatic rotary 30-port machine with horizontally positioned cigarettes } \\
\text { using a 4-piston pump } \\
\text { - continuous nose-only exposure of } 72 \text { rats in three superposed trays }\end{array}$ & 189 \\
\hline 1989 & CHEN et al. (355) & US & AMESA Mark III (352) with certain components improved & 190 \\
\hline 1990 & AYERS et al. (360) & US & AMESA Mark III (352) with several components improved & 190 \\
\hline 1992 & CHEN et al. (356) & US & AMESA Mark IIIA (352) further improved to become AMESA Type 1300 & 190 \\
\hline 1998 & CH TECHNOLOGIES (362) & US & $\begin{array}{l}\text { type BJG-CSM and JB Series } 2070 \ldots 2090 \\
\text { - } 30 \text {-port rotary smoking machines redesign of the AMESA original (352) } \\
\text { and AMESA } 1300 \text { (356) machines }\end{array}$ & 190 \\
\hline 2001 & APPLETON ET AL. (369) & US & $\begin{array}{l}\text { "Condor" series } \\
\text { - automatic rotary 30-port machine manufactured by KC Automation }\end{array}$ & 191 \\
\hline
\end{tabular}

Table D. Smoking machines for in vitro smoke toxicity testing. Page numbers refer to the publication: Klus, H., B. Boenke-Nimphius, and L. Müller: Cigarette Mainstream Smoke: The Evolution of Methods and Devices for Generation, Exposure and Collection; Beitr. Tabakforsch. Int. 27 (2016) 137-274.

\begin{tabular}{|c|c|c|c|c|}
\hline Year & Author & Country & Specifics & Page \\
\hline 1960 & BORGWALDT (315) & DE & $\begin{array}{l}\text { RM 1/G: } \\
\text { - } \text { manually operated versatile single cigarette machine with piston pump } \\
\text { - } \quad \text { bell-shaped puff profile } \\
\text { also used for ALI smoke exposure }\end{array}$ & 207 \\
\hline 2000 & SIBATA (526) & JP & $\begin{array}{l}\text { SG-200: } \\
\text { - } \text { automatic rotary 40-port machine with vertically positioned smoking } \\
\text { head } \\
\text { - } \text { uncontrolled air flow around cigarettes } \\
\text { - not in compliance with ISO 3308:1991 }\end{array}$ & 225 \\
\hline 2002 & VITROCELL (450) & DE & $\begin{array}{l}\text { "Smoking Robot" VC 10: } \\
\text { - } \quad \text { automatic rotary 10-port machine with single syringe } \\
\text { - } \quad \text { smoke transfer through up to } 5 \text { ports for dilution and ALI exposure }\end{array}$ & 208 \\
\hline 2002 & BORGWALDT (108) & $\mathrm{DE}$ & $\begin{array}{l}\text { RM20S: } \\
\text { - fully automatic rotary 4-port machine with four dedicated independent } \\
\text { syringes } \\
\text { - } \quad \text { expandable to eight syringes }(474) \\
\text { - in conformity with ISO 3308:1991 } \\
\text { - intended for ALI exposure }\end{array}$ & 214 \\
\hline 2007 & $\begin{array}{l}\text { BURGHART TABAKTECHNIK } \\
(518)\end{array}$ & $\mathrm{DE}$ & $\begin{array}{l}\text { MSB-01: } \\
\text { 5-port machine with five syringe pumps } \\
\text { smoke collected in a mixing bag and then dispersed by a distribution } \\
\text { manifold onto a 96-microwell plate }\end{array}$ & 222 \\
\hline 2010 & $\begin{array}{l}\text { BURGHART MESSTECHNIK } \\
(507)\end{array}$ & $\mathrm{DE}$ & $\begin{array}{l}\text { SM 2000: } \\
\text { - } \quad \text { fully automatic rotary 30-port machine using a programmable dual } \\
\text { - } \quad \text { continuous smoke production } \\
\text { - in conformity with ISO 3308:1991 } \\
\text { - intended for ALI exposure }\end{array}$ & 220 \\
\hline
\end{tabular}




\section{REFERENCES}

All references with an unmarked link were accessed and available on the internet in September 2016. For links no longer in function the date of the last retrieval is indicated in brackets.

1. Zeise, W.C.: Untersuchung der Producte von der trockenen Destillation des Tabaks und über die chemische Beschaffenheit des Tabakrauchs [Investigation of the Products Generated by Dry Distillation of Tobacco and on the Chemical Nature of Tobacco Smoke]; Justus Liebigs Ann. Chem. 47 (1843) 212-225.

2. Melsens, M.: Note sur la Nicotine [Note Concerning Nicotine]; Ann. Chim. Phys. 3 (1843) 465-472.

3. Zebrowski, E.v.: Zur Frage vom Einfluß des Tabakrauches auf Tiere [On the Effects of Tobacco Smoke on Animals]; Centralbl. Allg. Pathol. Pathol. Anat. 19 (1908) 609-617.

4. Bradford, J.A., W.R. Harlan, and H.R. Hanmer: Nature of Cigaret Smoke. Technic of Experimental Smoking; Ind. Eng. Chem. 28 (1936) 836-839.

5. Campbell, A. J.: Carcinogenic Agents Present in the Atmosphere and Incidence of Primary Lung Tumours in Mice; Brit. J. Exp. Path. 20 (1939) 122-132.

6. Wartman Jr, W.B., E.C. Cogbill, and E.S. Harlow: Determination of Particulate Matter in Concentrated Aerosols. Application to Analysis of Cigarette Smoke; Anal. Chem. 31 (1959) 1705-1709.

7. Clark, P.C. and F.G. Bock: Experience with the Large Scale Production of Cigarette Smoke Condensate Using the Process and Instruments Automatic Smoking Machine; presented at the $13^{\text {th }}$ Tobacco Chemists' Research Conference, Lexington, KY, USA, Abstract 7, 1959.

8. Wiley, R.M. and J.A. Ferri: The Design of an Automated Syringe-Type Smoking Machine; Tob. Sci. 11 (1967) 140-143 and Beitr. Tabakforsch. 4 (1967) 59-64. DOI: 10.2478/cttr-2013-0168

9. U.S. Federal Trade Commission (FTC): Cigarettes Testing for Tar and Nicotine Content; Federal Register 32 (1967) 11178.

10. International Organization for Standardization (ISO): International Standard ISO 3308:1977 - Tobacco and Tobacco Products -- Routine Analytical CigaretteSmoking Machine -- Definitions, Standard Conditions and Auxiliary Equipment; ISO, Geneva, Switzerland, 1977.

11. Task Force on the Review of Smoking Methods: CORESTA Report 1991-1. The Determination of Repeatability [r] and Reproducibility [R] for the Measurement of Nicotine-Free Dry Particulate Matter (NFDPM), Nicotine, Water Using CORESTA Recommended Methods 7, 8, 21, 22, 23 and 25; CORESTA, 1991. Available at: https://www.coresta. org/determination-repeatability-and-reproducibilitymeasurement-nicotine-free-dry-particulate-matter

12. International Organization for Standardization (ISO): International Standard ISO 3308:1991 - Routine Analytical Cigarette-Smoking Machine -- Definitions and Standard Conditions; $3^{\text {rd }}$ edition, ISO, Geneva,
Switzerland, 1991

13. Massey, E., M. Aufderheide, W. Koch, H. Lodding, G. Pohlmann, H. Windt, P. Jarck, and J.W. Knebel: Micronucleus Induction in V79 Cells after Direct Exposure to Whole Cigarette Smoke; Mutagenesis 13 (1998) 145-149.

14. UCSF Library: Truth Tobacco Industry Documents. Available at https://www.industrydocumentslibrary. ucsf.edu/tobacco/

15. Johnson, S.W.: Experiments in Growing Tobacco with Different Fertilizers; in: Annual Report of the Connecticut Agricultural Experiment Station for 1892, State of Connecticut, New Haven, CT, USA, 1893, pp. 1-24.

16. Habermann, J.: Beiträge zur Kenntniss des Cigarrenrauches [Contributions to the Knowledge of Cigar Smoke]; Z. Physiol. Chem. 33 (1901) 55-125.

17. Pfyl, B., R. Kölliker, B. Dwilling, and M. Obermiller: Zur Bestimmung des Nicotins im Tabakrauch. II. Normung des künstlichen Verrauchens der Tabakerzeugnisse [Contribution to the Determination of Nicotine in Tobacco Smoke. II. Standardization of the Artificial Smoking of Tobacco Products]; Z. Untersuch. Lebensm. 66 (1933) 501-510.

18. Wissenschaftliche Forschungsstelle im Verband der Cigarettenindustrie: Einheitliche Vorschriften für die Analyse von Tabak und Tabakrauch [Standardized Regulations for the Analysis of Tobacco and Tobacco Smoke]; Beitr. Tabakforsch. 1 (1961) 31-34. DOI: $10.2478 / \mathrm{cttr}-2013-0005$

19. Pillsbury, H.C., C.C. Bright, K.J. O'Connor, and F.W. Irish: Tar and Nicotine in Cigarette Smoke; J. Assoc. Off. Anal. Chem. 52 (1969) 458-462.

20. The Commonwealth of Massachusetts, Tobacco Disclosure Act: Cigarette and Smokeless Tobacco Products: Reports of Added Constituents and Nicotine Ratings; General Laws of Massachusetts, Chapter 94, Section 307B 105 CMR 660.000, 1997.

21. Texas Department of Health: Texas Register 22, No. 79, December 5, 1997, pp. 11939-11941. Available at: http://texashistory.unt.edu/ark:/67531/metapth $176720 / \mathrm{ml} /$

22. Government of Canada, Minister of Justice: Tobacco Reporting Regulations SOR/2000-273 of June 26, 2000, current to June 17, 2015. Available at: http://laws-lois.justice.gc.ca/PDF/SOR-2000-273.pdf

23. Theoi Greek Mythology: Ariadne. Available at: http://www.theoi.com/Georgikos/Ariadne.html

24. Pinner, A.: Ueber Nicotin - II. Mitteilung [On Nicotine - Second Communication]; Arch. Pharm. 233 (1895) 572-612.

25. Kißling, R.: Der Gehalt des Cigarrenrauches an Nikotin unter gleichzeitiger Berücksichtigung der giftig wirkenden Verbrennungsproducte des Tabakes [On the Nicotine Content of Cigar Smoke with Concurrent Regard to the Toxic Combustion Products of Tobacco]; Dingler's Polytechn. J. 244 (1882) 64-71 and 234-246.

26. Thoms, H.: Über die Rauchprodukte des Tabaks [On Smoke Products of Tobacco]; Ber. Dtsch. Pharm. Ges. 10 (1900) 19-31.

27. Pontag, J.J.: Untersuchungen des russischen Rauch- 
tabaks und des Cigarettenrauches [Investigation on the Russian Smoking Tobacco and on Cigarette Smoke]; Z. Untersuch. Nahrung Genussm. 6 (1903) 673-691.

28. Kißling, R.: Kurzgefasstes Handbuch der Tabakkunde [Short Compendium on Tobacco Knowledge]; Parey Verlag, Berlin, 1893.

29. Habermann, J.: Ueber den Blausäuregehalt des Cigarrenrauches [On the Hydrogen Cyanide Content of Cigar Smoke]; Z. Physiol. Chem. 37 (1902-03) 1-17.

30. Habermann, J.: Beiträge zur Kenntniss des Zigaretten- und des Pfeifenrauches [Contributions to the Knowledge of Cigarette Smoke and Pipe Smoke]; Z. Physiol. Chem. 40 (1903-04) 148-161.

31. Garner, W.W.: Methods for Testing the Burning Quality of Cigar Tobacco; U.S. Department of Agriculture, Bureau of Plant Industry, Bulletin 100, Part. IV (1906) 5-14.

32. Garner, W.W.: The Relation of Nicotine to the Quality of Tobacco; U.S. Dept. Agric., Bureau of Plant Industry, Bulletin 141 (1909) 5-16.

33. Preissecker, K., H. Brezina, and A. Wenusch: Tabakstreckung und Tabakersatz [Thinning of Tobacco and Tobacco Substitution]; Fachl. Mitt. Österr. Tabakregie 18 (1918) 1-13.

34. German Empire: Bekanntmachung über tabakähnliche Waren [Announcement Concerning Products Similar to Tobacco]; Berlin, 27. Oktober 1917, Reichsgesetzblatt (1917) 974.

35. Austrian - Hungarian Department of Finances: Kundmachung des Finanzministeriums vom 1. Mai 1918, betreffend die Erklärung der Tabakersatz- und -zusatzmittel als Gegenstände des Tabakmonopols [Announcement of the Department of Finances of May 1, 1918, Concerning Tobacco Substitutes and Tobacco Additives as a Matter of the Tobacco Monopoly]; Reichsgesetzblatt Nr. 167, 8. Mai 1918.

36. German Empire: Gesetz über den Verkehr mit Lebensmitteln und Bedarfsgegenständen (Lebensmittelgesetz) vom 5. Juli 1927 [Law Concerning Food-Stuffs and Consumer Goods (Food-Law), July 5, 1927]; Reichsgesetzblatt Teil 1, Nr. 26 vom 8. Juli 1927.

37. Frank, L.: Nikotinarme Tabakfabrikate und das neue Lebensmittelgesetz [Tobacco Products, Low in Nicotine, and the New Food Law]; Med. Welt 31 (1928) 1-6.

38. Pfyl, B. and O. Schmitt: Zur Bestimmung von Nicotin in Tabak und Tabakrauch [The Determination of Nicotine in Tobacco and Tobacco Smoke]; Z. Untersuch. Lebensm. 54 (1927) 60-78.

39. Winterstein, A. and E. Aronson: Beiträge zur Kenntnis des Tabakgenusses; II. Mitteilung. Über den Verbleib des Nicotins beim Tabakrauchen [Contributions to the Understanding of Tobacco Consumption; Part 2 - On the Fate of Nicotine in Tobacco Smoking]; Z. Hyg. Infektionskr. 108 (1928) 530-553.

40. Fühner, H.: Die quantitative Bestimmung des Nicotins auf biologischem Wege [The Quantification of Nicotine Using a Biological Method]; Biochem. Z. 92 (1918) 355-363.

41. Wenusch, A.: Beitrag zur Bestimmung des Nikotins in Rauchgasen [Contribution to Nicotine Determination in Smoke Gases]; Fachl. Mitt. Österr. Tabakregie 1 (1931) 1-8.

42. Wenusch, A.: Der Tabakrauch [Tobacco Smoke]; Arthur Geist Verlag, Bremen, Germany, 1939, pp. 23-26.

43. Wenusch, A.: Beitrag zur Bestimmung des Nikotins in Rauchgasen. II. Teil [Contribution to Nicotine Determination in Smoke Gases. Part 2]; Fachl. Mitt. Österr. Tabakregie 2 (1931) 1-3.

44. Neurath, G., B. Pirmann, W. Lüttich, and H. Wichern: Zur Frage der N-Nitroso-Verbindungen im Tabakrauch II [On N-Nitroso Compounds in Tobacco Smoke II]; Beitr. Tabakforsch. 3 (1965) 251-262. DOI: $10.2478 /$ cttr-2013-0117

45. Kallianos, A.G., R.E. Means, and J.D. Mold: Effect of Nitrates in Tobacco on the Catechol Yield in Cigarette Smoke; Tob. Sci. 12 (1968) 125-129.

46. Schönherr, H., H.J. Klimisch, and H.P. Harke: Alterung von Cigarettenrauchkondensat: Quantitative Untersuchungen der Artefaktbildung durch Gas- und Partikelphasenreaktionen [Altering of Cigarette Smoke Condensate: Quantitative Determination of the Formation of Artifact Produced by Gas-Phase and Particulate Phase Reaction]; Beitr. Tabakforsch. 7 (1973) 18-23. DOI: 10.2478/cttr-2013-0304

47. Wenusch, A.: Über den Nicotingehalt des Hauptstromrauches von dicken und dünnen Zigaretten [On the Nicotine Content of Mainstream Smoke of Thick and Thin Cigarettes]; Z. Untersuch. Lebensm. 72 (1936) 213-218.

48. German Empire: Erlaß des Reichsministers des Inneren - II A 1780/8.4. - betr. nicotinarme und nicotinfreie Tabakfabrikate, vom 23. April 1929 [Decree of the German Secretary of the Interior Concerning Low-Nicotine and Nicotine-Free Tobacco Products, of April 23, 1929] Z. Unters. Lebensm. Suppl. (1929) 104-105.

49. Petri, J.W.: Zur Beurteilung des Nicotingehaltes von Tabaken [Criteria for Evaluation the Nicotine Content of Tobaccos]; Z. Untersuch. Lebensm. 62 (1931) 126-137.

50. Wissenschaftliche Forschungsstelle im Verband der Cigarettenindustrie: Einheitliche Vorschriften für die Analyse von Tabak und Tabakrauch [Standardized Regulations for the Analysis of Tobacco and Tobacco Smoke]; Beitr. Tabakforsch. 1 (1962) 307-314. DOI: $10.2478 /$ cttr-2013-0038

51. Cooperation Centre for Scientific Research Relative to Tobacco (CORESTA): CORESTA Standard Method No 10: Machine Smoking of Cigarettes, Determination of Crude and Dry Condensate; CORESTA, Paris, France, 1968.

52. German Empire: Gesetz über den Verkehr mit Lebensmitteln und Bedarfsgegenständen (Lebensmittelgesetz) in der Fassung vom 17. Januar 1936 [Law Concerning Food-Stuffs and Consumer Goods (Food-Law) in its Form of January 17, 1936]; Reichsgesetzblatt Teil 1, 1926, pp. 18-22.

53. German Empire: Verordnung über nikotinarmen und nikotinfreien Tabak vom 12. Mai 1939 [Ordinance Concerning Tobacco with Low or No Nicotine]; 
Reichsgesetzblatt Teil I (1939) 912.

54. Bundesrepublik Deutschland: Gesetz zur Neuordnung und Bereinigung des Rechts im Verkehr mit Lebensmitteln, Tabakerzeugnissen, kosmetischen Mitteln und sonstigen Bedarfsgegenständen (Gesetz zur Gesamtreform des Lebensmittelrechts) [Act Concerning the Rearrangement of the Law Concerning Food, Tobacco Products, Cosmetics, and Other Products (Act Concerning the Total Reform of Foodstuff Regulations)]; Bundesgesetzblatt I (1974) 1945-1966. Available at: http://www.bgbl.de/xaver/ bgbl/start.xav?startbk=Bundesanzeiger_BGBl\&jum pTo=bgbl174095.pdf

55. Bundesrepublik Deutschland, Bundesminister der Justiz: Verordnung über Tabakerzeugnisse (Tabakverordnung) vom 20. Dezember 1977 [Federal Republic of Germany: Ordinance on Tobacco Products (Tobacco Ordinance) of Dec. 20, 1977]; Bundesgesetzblatt I (1977) 2831-2837. Available at: http://www.bgbl.de/xaver/bgbl/start.xav?startbk= Bundesanzeiger_BGB1\&jumpTo=bgbl177089.pdf

56. Wenusch, A.: Uéber die Abrauchmethode von B. Pfyl [On the Smoking Method of B. Pfyl]; Pharm. Zentralhalle Dtschl. 78 (1937) 189-191.

57. Preiß, W.: Ueber die Abrauchmethode von B. Pfyl [On the Smoking Method of B. Pfyl]; Pharm. Zentralhalle Dtschl. 78 (1937) 331-333.

58. Wenusch, A.: Ueber die Abrauchmethode von B. Pfyl [On the Smoking Method of B. Pfyl ]; Pharm. Zentralhalle Dtschl. 78 (1937) 578-581.

59. Preiß, W.: Ueber die Abrauchmethode von B. Pfyl [On the Smoking Method of B. Pfyl]; Pharm. Zentralhalle Dtschl. 78 (1937) 581-582.

60. Wenusch, A.: Ueber die Abrauchmethode von B. Pfyl [On the Smoking Method of B. Pfyl]; Pharm. Zentralhalle Dtschl. 78 (1937) 703-706.

61. Wahl, R. and O. Heil: Die Entwicklung einer neuen Rauchapparatur unter besonderer Berücksichtigung der Strömungsverhältnisse [The Development of a New Smoking Device with Special Consideration to Flow Conditions]; Tabak-Forschung, Sonderheft (1953) 16-20.

62. Marek, J.: Vergleichende Untersuchungen mit der Abrauchapparatur von A. Wenusch und der Apparatur von Wahl und O. Hell [Comparative Studies of the Smoking Device of A. Wenusch and the Device of Wahl and O. Hell]; Fachl. Mitt. Österr. Tabakregie 1 (1954) 16-18.

63. Staub, M. and H. Furrer: Die Nikotin- und Teerbestimmung im Tabakrauch [Determination of Nicotine and Tar in Tobacco Smoke]; Mitt. Geb. Lebensmittelunters. Hyg. 44 (1953) 371-377.

64. Staub, M. and H. Furrer: Die Prüfung von Nikotinfiltern [Testing of Nicotine Filters]; Mitt. Geb. Lebensmittelunters. Hyg. 44 (1953) 472-474.

65. Staub, M. and H. Furrer: Kritische Untersuchungen über die Nikotinbestimmungsmethoden in Tabak [Critical Investigation of the Methods for Determination of Nicotine in Tobacco]; Mitt. Geb. Lebensmittelunters. Hyg. 43 (1952) 490-498.

66. Cuzin, I.L.: The Smoking Machines at the Research Laboratories of S.E.I.T.A.; Deuxième Congres Scien- tifique International du Tabac, Brussels, Belgium, June 1958, 513-520.

67. Waltz, P., M. Häusermann, and E. Nyari: Untersuchungen zur Rauchmethodik [Studies on Smoking Methodology]; Mitt. Geb. Lebensmittelunters. Hyg. 50 (1959) 166-185.

68. Waltz, P. and M. Häusermann: Untersuchungen zur Rauchmethodik II - Beschreibung der eigenen Methode und Uebersicht der zur Zeit üblichen Methodik der Zigarettenvorbereitung und des künstlichen Abrauchens [Studies on Smoking Methodology II. Description of Proprietary Method and Review of Currently Used Methodology of Cigarette Preparation and Artificial Smoking]; Mitt. Geb. Lebensmittelunters. Hyg. 51 (1960) 303-320.

69. Jensen, C.O. and D.E. Haley: Studies on the Nicotine Content of Cigarette Smoke; J. Agric. Res. 51 (1935) 267-276.

70. Doll, R. and A.B. Hill: Smoking and Carcinoma of the Lung. Preliminary Report; Brit. Med. J. 2 (1950) 739-748.

71. Wynder, E.L. and E.A. Graham: Tobacco Smoking as a Possible Etiological Factor in Bronchogenic Carcinoma: A Study of Six Hundred and Eighty-Four Proved Cases; J. Am. Med. Assoc. 143 (1950) 329-336.

72. Wynder, E.L., E.A. Graham, and A.B. Croninger: Experimental Production of Carcinoma with Cigarette Tar; Cancer Res. 13 (1953) 855-864.

73. Wynder, E.L. and J.A. Mann: A Study of Tobacco Carcinogenesis III. Filtered Cigarettes; Cancer 10 (1957) 1201-1205.

74. United States. Public Health Service. Office of the Surgeon General: Smoking and Health - Report of the Advisory Committee to the Surgeon General of the Public Health Service; Public Health Service Publication No. 1103, 1964.

75. Cigarette Components Ltd.: Simple Smoking Machine; Sept. 1955. Available at: http://industry documents.library.ucsf.edu/tobacco/docs/nywh0197

76. Cigarette Components Ltd.: Bulletin Ethel Mk. VI Smoking Machine; Cigarette Components, London, UK, 1958

77. Mumpower II, R.C., J.E. Kiefer, and G.P. Touey: Effect of the Duration of a Constant-Volume Puff on the Efficiency of Cigarette Filters; Tob. Sci. 5 (1961) 31-35.

78. Johnson, J.C.: Development of Machine Smoking Parameters for Measurement of Cigarette Tar Yield in the United Kingdom; J. Assoc. Off. Anal. Chem. 69 (1986) 598-600.

79. Baker, R.R.: Temperature Variation Within a Cigarette Combustion Coal During the Smoking Cycle; High Temp. Sci. 7 (1975) 236-247.

80. Baker, R.R.: Variation of the Gas Formation Regions Within a Cigarette Combustion Coal During the Smoking Cycle; Beitr. Tabakforsch. Int. 11 (1981) 1-17. DOI: $10.2478 /$ cttr-2013-0491

81. Seehofer, F. and D. Wennberg: Einfluß verschiedener Zugvolumenprofile auf die Ausbeute und $\mathrm{Zu}-$ sammensetzung des Rauches beim maschinellen Abrauchen von Cigaretten [The Effect of Puff 
Profiles on Yield and Composition of Mainstream Smoke of Cigarettes Smoked on a Smoking Machine]; Beitr. Tabakforsch. 6 (1971) 7-11.

DOI: $10.2478 /$ cttr-2013-0259

82. Klus, H. and H. Kuhn: Verteilung verschiedener Tabakrauchbestandteile auf Haupt- und Nebenstromrauch. Eine Übersicht [Distribution of Various Tobacco Smoke Components Among Mainstream and Sidestream Smoke. A Survey]; Beitr. Tabakforsch. Int. 11 (1982) 229-267.

DOI: $10.2478 /$ cttr-2013-0517

83. Procter, C.J., C. Martin, J.L. Beven, and H.F. Dymond: Evaluation of an Apparatus Designed for Collection of Sidestream Smoke; Analyst, 113 (1988) 1509-1513.

84. Keith, C.H. and J.R. Newsome: Quantitative Studies on Cigarette Smoke. I. An Automatic Smoking Machine; Tob. Sci. 1 (1957) 51-57.

85. O'Keeffe, A.E. and R.C. Lieser: An Improved Smoking Machine; Tob. Sci. 2 (1958) 73-76.

86. DeBardeleben, M.Z., J.E. Wickham, and W.F. Kuhn: The Determination of Tar and Nicotine in Cigarette Smoke from an Historical Perspective; Rec. Adv. Tob. Sci. 17 (1991) 115-148.

87. Staub, M. and H. Furrer: Tabakuntersuchungen IV Eine neue automatische Abrauchapparatur [Studies on Tobacco IV. A New Smoking Apparatus]; Mitt. Geb. Lebensmittelunters. Hyg. 45 (1954) 473-476.

88. Staub, M. and H. Furrer: Tabakuntersuchungen V Die Reproduzierbarkeit der Rauchuntersuchungen [Studies on Tobacco V. The Reproducibility of Smoke Studies]; Mitt. Geb. Lebensmittelunters. Hyg. 48 (1957) 94-116.

89. Cooperation Centre for Scientific Research Relative to Tobacco (CORESTA): About Us - History. Available at: https://www.coresta.org/history29294.html

90. Iles, W.G. and C.F. Sharman: A Versatile Automatic Smoking Machine; J. Appl. Chem. 7 (1957) 384-387.

91. Baumberger, J.P.: The Amount of Smoke Produced from Tobacco and its Absorption in Smoking as Deter mined by Electrical Precipitation; J. Pharmacol. Exp. Ther. 21 (1923) 47-57.

92. Bentley, H.R. and J.G. Burgan: Polynuclear Hydrocarbons in Tobacco and Tobacco Smoke; Analyst 83 (1958) 442-447.

93. Roe, F.J.C., M.H. Salaman, and J. Cohen: Incomplete Carcinogens in Cigarette Smoke Condensate: Tumour-Promotion by a Phenolic Fraction. With an appendix by J.G. Burgan; Brit. J. Cancer, 13 (1959) 623-633.

94. Williamson, J.T. and P.J. Clark: Eine neue automatische Rauchmaschine zur Einstellung auf die verschiedensten Abrauchbedingungen [A New Automated Smoking Machine Allowing to Examine Various Smoking Conditions]; Beitr. Tabakforsch. 1 (1962) 187-191. DOI: 10.2478/cttr-2013-0029

95. Filtrona Instruments: Filtrona Smoking Machine Model 100; Publication ALP. 170, Filtrona Instruments \& Automation Ltd., Milton Keynes, UK.

96. British Standards Institution: UK Report on a Comparison of the Performance of the Phipps \& Bird,
CSM 10, CSM 12 and Borgwaldt RM 20 Smoking Machines (1970); Implementation of Draft Resolution 15 of the Meeting of ISO/TC126, The Hague, Netherlands, September 1969.

97. Rothwell, K. and C.A. Grant (Eds.): Standard Methods for the Analysis of Tobacco Smoke; Tobacco Research Council, London, UK, Research Paper 11, 1972, $2^{\text {nd }}$ edition was published in 1974 and $3^{\text {rd }}$ edition was published in 1986 .

98. Cumming, E.M.: A Smoking Machine Attachment for the Analysis of Carbon Monoxide, Carbon Dioxide and Nitric Oxide in the Vapour Phase of Cigarette Smoke; Beitr. Tabakforsch. 8 (1975) 153-157. DOI: $10.2478 /$ cttr-2013-0373

99. Filtrona Instruments \& Automation Ltd: Filtrona SM400 Twenty Channel Harmonised Smoking Machine; Data Sheet, 1992. Available at: http://industrydocuments.library.ucsf.edu/tobacco/ docs/rxgv0016

100. Cigarette Components, Ltd. - Data Sheet: Filtrona Model 302 Smoking Machine (1974), Bates No. 650333020-650333025. Available at: http://legacy. library.ucsf.edu/tid/bdu08c00/pdf

101. Cigarette Components, Ltd.: Automated Carbon Monoxide Measurements; Filtrona Facets, April 1980, Bates No. 105158818-105158851. Available at: http://legacy.library.ucsf.edu/tid/kqq25a99/pdf

102. Dymond, H.F.D. and A.E. Yallup: Gas Detection; United States Patent, 4,257,777, British American Tobacco Company Ltd., London, UK, March 24, 1981.

103. Filtrona Instruments \& Automation Ltd: Filtrona SM342 Eight Channel Harmonised Smoking Machine; Data Sheet, 1992. Available at: http://industrydocuments.library.ucsf.edu/tobacco/ docs/fnjc0054

104. Molins plc: Our History (2016). Available at: http://www.molins.com/about-us/our-history

105. International Organization for Standardization (ISO): International Standard ISO 3308:2012 - Routine Analytical Cigarette-Smoking Machine-- Definitions and Standard Conditions; $5^{\text {th }}$ edition, ISO, Geneva, Switzerland, 2012.

106. Cerulean: Smoking Machines (2016). Available at: http://www.cerulean.com/product-services/tobacco/ smoking-machines

107. Cerulean: Comparing Air Flow Stability Between the Cerulean SM450 and the New Generation SM450i (2015). Available at: http://www.cerulean.com/ media/412760/Comparison-air-flow-SM450-andSM450i.pdf

108. Borgwaldt KC: Instruments (2016). Available at: http://borgwaldt.hauni.com/en/instruments/smokingmachines.html

109. Lorton, N. and E.M. Cumming: A Multi-Purpose, Programmable Smoking Machine; Beitr. Tabakforsch. Int. 14 (1987) 45-51. DOI: 10.2478/cttr-2013-0584

110. Decker, C., A. Girardet, P. Golaz, and R. Regamy: Appareil automatique multiple pour le dosage de la nicotine et des goudrons dans la fumée des cigarettes [Multiple Automatic Apparatus for the Determination of Nicotine and Tars in the Smoke of Cigarettes]; 
Mitt. Geb. Lebensmittelunters. Hyg. 46 (1955) 178-182.

111. Waltz, P., M. Häusermann, E. Nyári, and G. Montandon: Eine neue Maschine zum gleichzeitigen Abrauchen mehrerer Cigaretten [A New Smoking Machine for Simultaneous Smoking of Several Cigarettes]; Beitr. Tabakforsch. 1 (1961) 1-10.

DOI: $10.2478 /$ cttr-2013-0001

112. Waltz, P., M. Häusermann, and E. Nyári: Erfahrungen mit einer Maschine zum gleichzeitigen Abrauchen mehrerer Cigaretten [Experiences with an Equipment for Simultaneous Smoking of Several Cigarettes]; Beitr. Tabakforsch. 1 (1961) 83-91. DOI: $10.2478 /$ cttr-2013-0013

113. Seehofer, F.: Abrauchmaschine für Serienanalysen [Smoking Machine for Serial Analyses]; TabakForschung 13 (1959) 100.

114. Seehofer, F., H. Barkemeyer, and H. Borowski: Automatische Abrauchmaschine für Serienanalysen [An Automatic Smoking Machine for Serial Analyses]; Beitr. Tabakforsch. 1 (1961) 11-14. DOI: $10.2478 /$ cttr-2013-0002

115. Seehofer, F.: Rauchmaschinen für analytische Bestimmungen sowie für chemische und biologische Untersuchungen des Tabakrauches [Smoking Machines for Analytical Determinations and for Chemical and Biological Investigations of Tobacco Smoke]; Technisch-wissenschaftliche Schriftenreihe der Staatlich Anerkannten Ingenieurschule HamburgBergedorf, Nr. 69 (Oktober 1968) 1-13.

116. Seehofer, F., J.E. Miller, and H. Elmenhorst: Eine automatische Schnellrauchmaschine für Serienanalysen und für die präparative Gewinnung von Rauchkondensaten [An Automatic High-Speed Smoking Machine for Batch Analyses and the Preparative Collection of Smoke Condensates]; Teil 1: Seehofer, F. and J.E. Miller: Automatische Schnellrauchmaschine für Serienanalysen [Automatic High-Speed Smoking Machine for Batch Analyses]; Beitr. Tabakforsch. 3 (1965) 75-78.

DOI: $10.2478 / \mathrm{cttr}-2013-0098$

117. Lipp, G.: Vergleich der Rauchausbeuten von drei verschiedenen Rauchmaschinen I [Comparison of the Smoke Condensate and Nicotine Yields Obtained from Three Different Smoking Machines I]; Beitr. Tabakforsch. 5 (1969) 39-42.

DOI: $10.2478 /$ cttr-2013-0214

118. International Organization for Standardization (ISO): Draft Report of the Second Plenary Meeting of ISO/TC 126 - Tobacco and Tobacco Products: Resolution 15; The Hague, Netherlands, September 1969.

119. Deutsches Institut für Normung (DIN): DIN 10240 Tabak und Tabakerzeugnisse-Untersuchung - Maschinelles Abrauchen von Zigaretten; Bestimmung des feuchten und trockenen Rauchkondensats [Tobacco and Tobacco Products-Analysis - Machine Smoking of Cigarettes and Determination of Moist and Dry Smoke Condensate]; DIN, Berlin 1969.

120. Lorenz, H.-W., H. Kirchner, and F. Seehofer: Die RM 20 - eine Rauchmaschine für analytische Untersuchungen [RM 20 - A Smoking Machine for Analytical Investigations]; Beitr. Tabakforsch. 6
(1972) 199-204. DOI: 10.2478/cttr-2013-0293

121. Seehofer, F. and W. Schulz: Zur Kenntnis des Glimmstromes von Cigaretten [On Smoulder Stream from Cigarettes]; Beitr. Tabakforsch. 3 (1965) 151156. DOI: $10.2478 /$ cttr-2013-0107

122. Deutsches Institut für Normung (DIN): DIN 10240 Teil 1. Untersuchung von Tabak und Tabakerzeugnissen - Maschinelles Abrauchen von Zigaretten und Bestimmung des Rauchkondensats: Anforderungen an eine analytische Rauchmaschine [Analysis of Tobacco and Tobacco Products - Machine Smoking of Cigarettes and Determination of the Smoke Condensate: Specifications of an Analytical Smoking Machine]; DIN, Berlin, Germany, 1978.

123. Deutsches Institut für Normung (DIN): DIN 10240 Untersuchung von Tabak und Tabakerzeugnissen Maschinelles Abrauchen von Zigaretten und Bestimmung des Rauchkondensats: Abrauchverfahren. Teil 2. [Analysis of Tobacco and Tobacco Products - Machine Smoking of Cigarettes and Determination of the Smoke Condensate: Smoking Method. Part 2]; DIN, Berlin, Germany, 1978.

124. Deutsches Institut für Normung (DIN): DIN 10240 Untersuchung von Tabak und Tabakerzeugnissen Maschinelles Abrauchen von Zigaretten und Bestimmung des Rauchkondensats: Bestimmung des Rohkondensats und des nikotinfreien, trockenen Rauchkondensats. Teil 3 [Analysis of Tobacco and Tobacco Products - Machine Smoking of Cigarettes and Determination of the Smoke Condensate: Determination of the Raw Condensate and of the Nicotine Free, Dry Smoke Condensate. Part 3]; DIN, Berlin, Germany, 1978.

125. British Standards Institution (BSI): BS 5668-1:1987. Routine Analytical Cigarette Smoking Machine. Part 1. Specification and Standard Conditions; BSI, London, UK, 1987.

126. Schulz, W. and F. Seehofer: Über den Einfluß von Abrauchparametern auf die Rauchausbeuten [The Influences of Different Smoking Conditions on Smoke Yields]; Beitr. Tabakforsch. 7 (1973) 105-110. DOI: $10.2478 /$ cttr-2013-0317

127. Ayres, C.I. and M.G. Ivinson: "Free" and "Restricted" Smoking; Paper No. 17, presented at the $18^{\text {th }}$ Tobacco Chemists' Research Conference, Raleigh, NC, USA, 1964.

128. Heinrich Borgwaldt GmbH \& Co: Smoking Machine RM 20 CS; Hamburg, Germany, 1977. Bates No. 103508128-103508146. Available at: http://legacy. library.ucsf.edu/tid/yug13a99/pdf

129. Rose, N.: Development of Rotary Smoking Machines in the Mirror of Regulatory Requirements; Paper, presented at the $67^{\text {th }}$ Tobacco Science Research Conference, September 15-18, 2013, Williamsburg, VA, USA.

130. Health Canada: Official Method T-109 - Determination of $\mathrm{Ni}, \mathrm{Pb}, \mathrm{Cd}, \mathrm{Cr}, \mathrm{As}$ and $\mathrm{Se}$ in Mainstream Tobacco Smoke; Health Canada, Ottawa, Canada, 1999.

131. Borgerding, M. and H. Klus: Analysis of Complex Mixtures-Cigarette Smoke; Exp. Toxicol. Pathol. 57 (2005) Suppl. 1, 43-73. 
132. U.S. Federal Trade Commission (FTC): News Release - Letters to Each of the Nation's Major Cigarette Manufacturers; FTC Office of Information, Washington D.C. USA, March 25, 1966.

133. Ogg, C.L.: Determination of Particulate Matter and Alkaloids (As Nicotine) in Cigarette Smoke; J. Assoc. Off. Anal. Chem. 47 (1964) 356-362.

134. U.S. Federal Trade Commission (FTC): Cigarettes and Related Matters. Methods to be Employed in Determining Tar and Nicotine Content; Notice of Public Hearing; Federal Register 31, No. 215 (November 4, 1966) p. 14278.

135. The American Tobacco Company, Brown \& Williamson Tobacco Corporation, Liggett \& Myers Tobacco Company, Philip Morris Incorporated, and RJ. Reynolds Tobacco Company: Cigarettes and Related Matters - Methods to be Employed in Determining Tar and Nicotine Content; written presentation before the Federal Trade Commission, November 28, 1966.

136. The American Tobacco Company, Brown \& Williamson Tobacco Corporation, Liggett \& Myers Tobacco Company, Philip Morris Incorporated, and RJ. Reynolds Tobacco Company: Determination and Reporting of Total Particulate Matter, Water in Total Particulate Matter, and Nicotine in Cigarette Smoke - Technical Data and Recommendations; Joint technical memorandum before the Federal Trade Commission, November 28, 1966.

137. Hetsko, C.F., A. Yeaman, F.P. Haas, P.D. Smith, and H.H. Ramm: Cigarettes and Related Matters: Methods to Be Employed in Determining Tar and Nicotine Content. Supplementary Observations Following November 30, 1966 Hearing Before the Federal Trade Commission, Dec. 20, 1966. Available at: http://industrydocuments.library.ucsf.edu/tobacco/ docs/nmmk0172

138. U.S. Federal Trade Commission (FTC): News Release - FTC to Begin Cigarette Testing; FTC Office of Information, Washington DC, USA; August $1,1967$.

139. U.S. Federal Trade Commission (FTC): Cigarettes and Related Matters - Carbon Monoxide, "Tar" and Nicotine Content of Cigarette Smoke; Description of New Machine and Methods to be Used in Testing; Federal Register 45 (1980) 46483-46487.

140. Merfeld, G.L. and H.C. Pillsbury: A 20-Port Sequential Smoking Machine for the Determination of Carbon Monoxide in Cigarette Smoke; Presentation at the $32^{\text {nd }}$ Tobacco Chemists' Research Conference, November 1978, Montreal, Canada.

141. U.S. Federal Trade Commission (FTC): "Tar", Nicotine, and Carbon Monoxide of the Smoke of 1294 Varieties of Domestic Cigarettes for the Year 1998 (report issued 2000); FTC, 2000.

142. Peeler, C.L.: Cigarette Testing and the Federal Trade Commission: A Historical Overview; in: U.S. Department of Health and Human Services, Public Health Service, National Institutes of Health Publication No. 96-4028: The FTC Cigarette Test Method for Determining Tar, Nicotine, and Carbon Monoxide Yields of U.S. Cigarettes - Report of the NCI Expert
Committee; Smoking and Tobacco Control Monograph 7 (1996) pp. 1-8.

143. Pillsbury, Jr, H.C.: Review of the Federal Trade Commission Method for Determining Cigarette Tar and Nicotine Yield; in: U.S. Department of Health and Human Services, Public Health Service, National Institutes of Health Publication No. 96-4028: The FTC Cigarette Test Method for Determining Tar, Nicotine, and Carbon Monoxide Yields of U.S. Cigarettes - Report of the NCI Expert Committee; Smoking and Tobacco Control Monograph 7 (1996) pp. 9-11.

144. U.S. Federal Trade Commission (FTC): FTC Rescinds Guidance from 1966 on Statements Concerning Tar and Nicotine Yields; Press Release, November 28, 2008. Available at: http://www.ftc. gov/news-events/press-releases/2008/11/ftc-rescindsguidance-1966-statements-concerning-tar-nicotine

145. U.S. Food and Drug Administration: Tobacco Products - Harmful and Potentially Harmful Constituents (HPHCs); last updated September 2015. Available at: http://www.fda.gov/TobaccoProducts/ Labeling/ProductsIngredientsComponents/ucm 2003 5927.htm

146. Kuhn, H.: The CORESTA Smoke Study Group - A Historical Review of its Activities; CORESTA 1956-1981. CORESTA Inform. Bull., Suppl. 1 (1982) 68-86.

147. Cooperation Centre for Scientific Research Relative to Tobacco (CORESTA): CORESTA Standard Method No 1: Machine Smoking of Cigarettes and Determination of Moist and Anhydrous Smoke Condensate; CORESTA, Paris, France, 1966.

148. Cooperation Centre for Scientific Research Relative to Tobacco (CORESTA): CORESTA Standard Method No 2: Determination of Total Alkaloids as Nicotine in Tobacco; CORESTA, Paris, France, 1966.

149. Cooperation Centre for Scientific Research Relative to Tobacco (CORESTA): CORESTA Standard Method No 3: Determination of Total Alkaloids as Nicotine in Tobacco Smoke Condensates; CORESTA, Paris, France, 1966.

150. Cooperation Centre for Scientific Research Relative to Tobacco (CORESTA): CORESTA Standard Method No 4: Determination of Nicotine Alkaloid Retention by Cigarette Filters; CORESTA, Paris, France, 1966.

151. Cooperation Centre for Scientific Research Relative to Tobacco (CORESTA): CORESTA Standard Method No 12: Determination of Alkaloids in Cigarette Smoke Condensates; CORESTA, Paris, France, 1968.

152. Cooperation Centre for Scientific Research Relative to Tobacco (CORESTA): CORESTA Standard Method No 13: Determination of Alkaloid Retention by Cigarette Filters; CORESTA, Paris, France, 1968.

153. Cooperation Centre for Scientific Research Relative to Tobacco (CORESTA): CORESTA Standard Method No 20: Determination of Alkaloids in Manufactured Tobacco; CORESTA, Paris, France, 1968. 
154. International Organization for Standardization (ISO): International Standard ISO 3400:1976. Tobacco and Tobacco Products - Determination of Alkaloids in Cigarette Smoke Condensates - Spectrophotometric Method; $1^{\text {st }}$ edition, ISO, Geneva, Switzerland, 1976.

155. International Organization for Standardization (ISO): International Standard ISO 3401:1977. Tobacco and Tobacco Products - Determination of Alkaloid Retention by Filters of Cigarettes; $1^{\text {st }}$ edition, ISO, Geneva, Switzerland, 1977.

156. International Organization for Standardization ISO: International Standard ISO 2881:1977. Tobacco and Tobacco Products - Determination of Alkaloids in Tobacco - Spectrophotometric Method; $1^{\text {st }}$ edition, ISO, Geneva, Switzerland, 1977.

157. Deutsches Institut für Normung (DIN): DIN $10240-$ Untersuchung von Tabak und Tabakerzeugnissen Maschinelles Abrauchen von Zigaretten und Bestimmung des Rauchkondensats: Zusätzliche Bedingungen für kanalspezifisch ventilierte Zigaretten. Teil 4 [Analysis of Tobacco and Tobacco Products Machine Smoking of Cigarettes and Determination of the Smoke Condensate: Additional Requirements for Channel-Ventilated Cigarettes. Part 4]; DIN, Berlin, Germany, 1985.

158. CORESTA: Minutes of the Meeting of the Scientific Commission in Paris on May 18-19, 1972.

159. N.N.: Reference Products Used in Tobacco and Smoke Analyses; Tob. J. Int. (2013) 150-154. Available at: https:/www.coresta.org/sites/default/ files/pages/tji0213-p150-154-refproducts.pdf

160. Morgareidge, K.: Evaluation of Variables in Cigarette Smoke Collection and Analysis; I. The Use of a Monitor Sample; $13^{\text {th }}$ Tobacco Chemists' Research Conference, October 1959, Lexington KY, USA, Paper No. 21.

161. Diana, J.N. and A. Vaught: The Reference Cigarette; Tobacco and Health Research Institute, The University of Kentucky Printing Services, Lexington, KY, USA, 1990.

162. Benner, J.F.: Tentative Summary of Leaf and Smoke Analysis of the University of Kentucky Reference and Alkaloid Series Cigarettes; Proceedings of the Tobacco and Health Conference, University of Kentucky Tobacco and Health Research Institute, Lexington, KY, USA, February 24-25, 1970. Conference Report 2 (1970) 30-34.

163. Chambers, O., H. Ji, and J. Geary: Information on the Cooperative Agreement Between the Center for Tobacco Products and the University of Kentucky to Develop a "Cigarette Tobacco Reference Products Program"; Presentation at the CORESTA Congress of the Smoke Science/Product Technology Groups, October 2014, Québec City, Canada.

164. University of Kentucky, College of Agriculture, Reference Cigarette Program: The Reference Cigarette. Available at: http://www.ca.uky.edu/refcig/ 1R5F\%20Brochure\%20New.pdf (accessed August 2015).

165. University of Kentucky, Kentucky Tobacco Research \& Development Center: 3R4F Preliminary Analysis. Available at: https://ctrp.uky.edu
166. Shelton, B.J., S.J.P. Cañete, and O.D. Chambers: Certificate of Analysis - 1R6F Certified Reference Cigarette; University of Kentucky Center for Tobacco Reference Products, Lexington, KY, USA, 2016. Available at: https://ctrp.uky.edu

167. University of Kentucky Center for Tobacco Reference Products: Product Category Proficiency Studies. Available at: https://ctrp.uky.edu

168. Cooperation Centre for Scientific Research Relative to Tobacco (CORESTA): CORESTA Board: Minutes of the Meeting in Madrid, Spain, 20 January 1994. Available at: http://industrydocuments.library.ucsf. edu/tobacco/docs/ntyv0079

169. International Organization for Standardization (ISO): International Standard ISO 16055:2003. Tobacco and Tobacco Products - Monitor Test Piece - Requirements and Use; $1^{\text {st }}$ edition, February 1, 2003, ISO, Geneva, Switzerland, 2003.

170. CORESTA Guide No. 8 - CORESTA Monitor Test Piece Production and Evaluation Requirements (April 2009). Available at: https://www.coresta.org/corestamonitor-test-piece-production-and-evaluation-require ments-29477.html

171. CORESTA Routine Analytical Chemistry SubGroup: Technical Report, 2013 Collaborative Study of CORESTA Monitor \#7 (CM7) for the Determination of Test Piece Weight, TPM, Water, Nicotine, NFDPM, Carbon Monoxide and PuffCount Obtained Under Mainstream 'ISO' and 'Intense' Smoking Regimes; February 2015. Available at: https://www. coresta.org/2012-collaborative-study-coresta-monitor7-cm7-determination-test-piece-weight-tpm-waternicotine

172. CORESTA Routine Analytical Chemistry SubGroup: Technical Report - 2014-2015 Collaborative Study of CORESTA Monitor Test Pieces CM 7 and CM 8 for the Determination of Test Piece Weight, TPM, Water, Nicotine, NFDPM, Carbon Monoxide and Puff Count Obtained under Mainstream 'ISO' Smoking Regime (August 2015). Available at: https://www.coresta.org/2014-2015-collaborativestudy-coresta-monitor-test-pieces-cm-7-and-cm-8determination-test-piece

173. International Organization for Standardization (ISO): International Standard ISO 4387:1987 - Cigarettes -Determination of Total and Dry Particulate Matter Using a Routine Analytical Cigarette-Smoking Machine -- Glass Fibre Filter Smoke Trap Method; ISO, Geneva, Switzerland, 1987.

174. International Organization for Standardization (ISO): International Standard ISO 8453:1987 - Cigarettes -Determination of Total and Dry Particulate Matter Using a Routine Analytical Cigarette-Smoking Machine. Electrostatic Smoke Trap Method; ISO, Geneva, Switzerland, 1987.

175. Baker, R.R.: The Development and Significance of Standards for Smoking-Machine Methodology; Beitr. Tabakforsch. Int. 20 (2002) 23-41. DOI: $10.2478 / \mathrm{cttr}-2013-0728$

176. Council of the European Union: Council Directive 90/239 EEC 17 May 1990 on the Approximation of the Laws, Regulations and Administrative Provisions 
of the Member States Concerning the Maximum Tar Yield of Cigarettes; Off. J. L 137 (1990) 36-37.

177. European Parliament, Council of the European Union: Directive 2001/37/EC of the European Parliament and of the Council of 5 June 2001 on the Approximation of the Laws, Regulations and Administrative Provisions of the Member States Concerning the Manufacture, Presentation and Sale of Tobacco Products - Commission Statement; Off. J. L194 (2001) 26-35.

178. Bourlas, M.C. and H.F.D. Dymond: Report of the Chairmen of the Review of Methods Task Force to the Smoke Study Group; CORESTA Symposium, Thessaloniki, Greece, October 7-11, 1990.

179. Thomsen, H.V.: International Reference Method for the Smoking of Cigarettes; Rec. Adv. Tob. Sci. 18 (1992) 69-94.

180. Cooperation Centre for Scientific Research Relative to Tobacco (CORESTA): CORESTA Recommended Method No. 70 - Determination of Selected Volatile Organic Compounds in Mainstream Cigarette Smoke by GC-MS (July 2014); CORESTA, Paris, France, 2014. Available at: https://www.coresta.org/ determination-selected-volatile-organic-compoundsmainstream-smoke-cigarettes-gas-chromatography

181. Cooperation Centre for Scientific Research Relative to Tobacco (CORESTA): CORESTA Recommended Method No. 74 - Determination of Selected Carbonyls in Mainstream Cigarette Smoke by HPLC) (July 2014); CORESTA, Paris, France, 2014. Available at: https://www.coresta.org/determinationselected-carbonyls-mainstream-cigarette-smokehigh-performance-liquid-chromatography

182. Brunnemann, K.D., L. Yu., and D. Hoffmann: Chemical Studies on Tobacco Smoke XVII. Assessment of Carcinogenic Volatile N-Nitrosamines in Tobacco and in Mainstream and Sidestream Smoke from Cigarettes; Cancer Res. 37 (1977) 3218-3222.

183. International Organization for Standardization (ISO): Technology Moves On - What About ISO Standards?. Available at: http://www.iso.org/iso/home/ faqs/faqs_standards.htm

184. International Organization for Standardization (ISO): International Standard ISO 3308:2000 - Routine Analytical Cigarette-Smoking Machine -- Definitions and Standard Conditions; $4^{\text {th }}$ edition, ISO, Geneva, Switzerland, 2000.

185. Cahours, X., T. Verron, and R. Julien: Collaborative Studies - Definitions, Concepts \& Outcomes; presented at the CORESTA Congress in Québec City, Canada, October 12-16, 2014.

186. Verron, T., M. Czechowicz, W.D. Heller, X. Cahours, and S.W. Purkis: Aspects of the Design Protocol and the Statistical Methods for Analysis of Tar, Nicotine and Carbon Monoxide Yields in Cigarette Smoke That Can Affect the Measurement Variability within Collaborative Studies; Regul. Toxicol. Pharmacol. 67 (2013) 252-265.

187. Sakaki, T.: The Twenty Years Activities of Asia Collaborative Study; presented at the CORESTA Congress, Sapporo, Japan, September 2012.

188. Intorp, M., S. Purkis, and W. Wagstaff:
Determination of Selected Volatiles in Cigarette Mainstream Smoke. The CORESTA 2009 Collaborative Study and Recommended Method; Beitr. Tabakforsch. Int. 24 (2011) 243-251.

DOI: $10.2478 /$ cttr-2013-0904

189. Intorp, M., S. Purkis, and W. Wagstaff: Determination of Carbonyl Compounds in Cigarette Mainstream Smoke. The CORESTA 2010 Collaborative Study and Recommended Method; Beitr. Tabakforsch. Int. 25 (2012) 361-374.

DOI: $10.2478 /$ cttr-2013-0915

190. Intorp, M., S. Purkis, and W. Wagstaff: Determination of Tobacco Specific Nitrosamines in Cigarette Mainstream Smoke: The CORESTA 2011 Collaborative Study; Beitr. Tabakforsch. Int. 25 (2012) 507-519. DOI: 10.2478/cttr-2013-0926

191. Cooperation Centre for Scientific Research Relative to Tobacco (CORESTA): CORESTA Recommended Method No. 75: Determination of Tobacco Specific Nitrosamines in Mainstream Cigarette Smoke by LCMS/MS; CORESTA, Paris, France. Available at: https://www.coresta.org/determination-tobaccospecific-nitrosamines-mainstream-cigarette-smokelc-msms-29198.html

192. CORESTA Task Force Alterative Smoking Regimes: Final Report (July 12, 2006). Available at: https://www.coresta.org/task-force-alternative-smokingregimes-29284.html

193. Mariner, D., W.-D. Heller, J. Sarabia, S.W. Purkis, M. Meger, H.J. Eberhardt, and M. Czechowicz: The 2010 ISO TC 126 Working Group 10 Collaborative Study on Intense Machine Smoking; CORESTA Joint Study Group Meeting, Presentation ST 05, Graz, Austria, 2011, CORESTA 2011 Abstracts (2001) 30.

194. Centers for Disease Control and Prevention National Center for Environmental Health: Global Collaborative Study - Method Validation of TNCO in Mainstream Cigarette Smoke, Intense Smoking Regime; Centers for Disease Control and Prevention, Atlanta, GA, USA, 2010.

195. Parrish, M.E. and C.N. Harward: Measurement of Formaldehyde in a Single Puff of Cigarette Smoke Using Tunable Diode Laser Infrared Spectroscopy; Appl. Spectrosc. 54 (2000) 1665-1677.

196. Li, S., J.L. Banyasz, M.E. Parrish, J. Lyons-Hart, and K.H. Shafer: Formaldehyde in the Gas Phase of Mainstream Cigarette Smoke; J. Anal. Appl. Pyrol. 65 (2002) 137-145.

197. Li, S., J.L. Banyasz, R.M. Olegario, C.B. Huang, E.A. Lambert, and K.H. Shafer: Brief Communication: The Flame Effect on Benzo[a]pyrene in Cigarette Smoke; Combust. Flame 128 (2002) 314-319.

198. Li, S., R.M. Olegario, J.L. Banyasz, and K.H. Shafer: Gas Chromatographic-Mass Spectrometry Analysis of Polycyclic Aromatic Hydrocarbons in Single Puff of Cigarette Smoke; J. Anal. Appl. Pyrol. 66 (2003) 155-163.

199. Adam, T., R.R. Baker, and R. Zimmermann: Investigation, by Single Photon Ionization (SPI)Time-of-Flight Mass Spectrometry (TOFMS), of the Effect of Different Cigarette-Lighting Devices on the 
Chemical Composition of the First Cigarette Puff; Anal. Bioanal. Chem. 387 (2007) 575-584.

200. World Health Organization (WHO): WHO TobLabNet Official Method SOP 01: Standard Operating Procedure for Intense Smoking of Cigarettes; WHO, Geneva, Switzerland, 2012.

201. Tindall, I., L. Crumpler, and P. Jordan: Air Flow, Turbulences and Smoke Yields. The Unexpected Consequences of Machine Design; Paper, presented at the CORESTA Smoke Science and Product Technology Study Groups' Meeting, Sept. 29 - Oct. 3, 2013, Seville, Spain.

202. Darrall, K.G.: Smoking Machine Parameters and Cigarette Smoke Yields; Sci Total Environ. 74 (1988) 263-278.

203. Scherer, G., J. Engl, M. Urban, G. Gilch, D. Janket, and K. Riedel: Relationship Between MachineDerived Smoke Yields and Biomarkers in Cigarette Smokers in Germany; Regul. Toxicol. Pharmacol. 47 (2007) 171-183.

204. The State of Texas: Disclosure of Ingredients in Cigarettes and Tobacco Products; Texas $75^{\text {th }}$ Legislature - Regular Session, Chapter 1216, House Bill 119, Section 1, Chapter 161, Health and Safety Code, Subchapter N, 1997, Bates-No. 471323781-3. Available at: https://industrydocuments.library.ucsf. edu/tobacco/docs/\#id=nnxm0054

205. Rickert, W.S.: Smoking Under Realistic Conditions: Develpoment of Minimum and Maximum Values for Toxic Costituents in Tobacco Smoke; Labstat Inc. Tobacco Characterization Program, Project Report DSS File No 35SS.H4078-5-C105, September 30, 1996. Available at: http://industrydocuments.library. ucsf.edu/tobacco/docs/pllx0091

206. Province of British Columbia: Tobacco Testing and Disclosure Regulation; Order of the Lieutenant Governor in Council, B.C. Reg. 282/98, Section 1 to 6, 1998. Available at: http://industrydocuments. library.ucsf.edu/tobacco/docs/stxv0063

207. Province of British Columbia: Tobacco Testing and Disclosure Regulation; Order of the Lieutenant Governor in Council No. 423 of April 2, 2001, amending B.C. Reg. 282/98, 2001. Available at: http://www. bclaws.ca/civix/content/oic/arc_oic/2000_2009/ oic2001/2001_0400_0499/?xsl=/te $\overline{m p l a t e s} /$ browse.xsl

208. Health Canada: Proposed Tobacco (Reporting) Regulations; Health Protection Branch, Information Letter No. 819, 1998 , Bates-No. TA67945-70. Available at: https://industrydocuments.library.ucsf. edu/tobacco/docs/\#id=syxx0149

209. Health Canada: Official Method T-115 - Determination of "Tar", Nicotine and Carbon Monoxide in Mainstream Tobacco Smoke; Health Canada, Ottawa, Canada, 1999.

210. International Organization for Standardization (ISO): International Standard ISO 4387:1991. Cigarettes Determination of Total and Nicotine-Free Dry Particulate Matter Using a Routine Analytical Smoking Machine; Second Edition, ISO, Geneva, Switzerland, 1991.

211. Word Health Organization (WHO): WHO Study
Group on Tobacco Product Regulation (TobReg). Available at: http://www.who.int/tobacco/global_inter action/tobreg/en/

212. WHO Study Group on Tobacco Product Regulation (TobReg): Recommendation 1: Guiding Principles for the Development of Tobacco Product Research and Testing Capacity and Proposed Protocols for the Initiation of Tobacco Product Testing; WHO, Geneva, Switzerland, 2004.

213. Pickworth, W., P. Houlgate, M. Schorp, M. Dixon, M. Borgerding, and G. Zaatari: Report of the ad hoc WG9 Smoking Behaviour Review Team to ISO/TC 126 WG9: A Review of Human Smoking Behaviour Data and Recommendations for a New ISO Standard for the Machine Smoking of Cigarettes (2005). Available at: https://industrydocuments.library.ucsf. edu/tobacco/docs/\#id=kkgm0006 This document was subsequently released as: International Organization for Standardardization (ISO): ISO/TR 17219:2013 - Review of Human Smoking Behaviour and Recommendations for a New ISO Standard for the Machine Smoking of Cigarettes; ISO, Geneva, Switzerland, 2013.

214. Purkis, S.W., V. Troude, G. Duputié, and C. Tessier: Limitations in the Characterisation of Cigarette Products Using Different Machine Smoking Regimes; Regul. Toxicol. Pharmacol. 58 (2010) 501-515.

215. Dixon, M. and M.F. Borgerding: Recent Advances in the Application and Understanding of Alternative Smoking Regimes; Rec. Adv. Tob. Sci. 32 (2006) 3-83.

216. Purkis, S.W.: Issues, Paradigms and Paradoxes That Need to Be Addressed by Both Manufacturers and Regulators in the Measurement and Interpretation of Cigarette Smoke Yields of Harmful and Potentially Harmful Constituents (HPHPCs); Rec. Adv. Tob. Sci. 39 (2013) 3-58.

217. Purkis; S.W., X. Cahours, M. Rey, B. Teillet, V. Troude, and T. Verron: Some Consequences of Using Cigarette Machine Smoking Regimes with Different Intensities on Smoke Yields and Their Variability; Regul. Toxicol. Pharmacol. 59 (2011) 293-309.

218. Counts, M.E., M.J. Morton, S.W. Laffon, R.H. Cox, and P.J. Lipowicz: Smoke Composition and Predicting Relationships for Internal Commercial Cigarettes Smoked with Three Machine-Smoking Conditions; Regul. Toxicol. Pharmacol. 41 (2005) 185-227.

219. International Organization for Standardization (ISO): ISO 4387:2000/Amd.1:2008 - Cigarettes -Determination of Total and Nicotine-Free Dry Particulate Matter Using a Routine Analytical Smoking Machine; $3^{\text {rd }}$ edition, ISO, Geneva, Switzerland, 2008.

220. Blackley, R.L., J.L. Nelson, R.A. Vernon, W.D. Brown, D.W. Bowman, J.M. Gwyn, and D.D. Henry: Development of a Holder Designed to Block $50 \%$ of Cigarette Filter Ventilation; ACD Test Memorandum, R.J. Reynolds Tobacco Company, 1999.

221. Rickert, W.S.: A New More "Intense" ISO Smoking Regime: Observations and Implications; Paper presented at the 13th Asian Collaborative Study 
(ACS) Meeting, Taipei, Taiwan, 2006.

222. Marian, C., R.J. O'Connor, M.V. Djordjevic, V.W. Rees, D.K. Hatsukami, and P.G. Shields: Reconciling Human Smoking Behavior and Machine Smoking Patterns: Implications for Understanding Smoking Behavior and the Impact on Laboratory Studies; Cancer Epidemiol. Biomarkers Prev. 18 (2009) 3305-3320.

223. Cooperation Centre for Scientific Research Relative to Tobacco (CORESTA): CORESTA Recommended Method No. 58: Determination of Benzo $[a]$ pyrene in Mainstream Cigarette Smoke by GC-MS (July 2014); CORESTA, Paris, France. Available at: https://www. coresta.org/determination-benzoapyrene-cigarette-mainstream-smoke-gas-chromatography-massspectrometry-29181

224. Purkis, S.W., M. Intorp, and A. Hauleithner: Updates of CORESTA Recommended Methods after Further Collaborative Studies Carried out Under Both ISO and Health Canada Intense Smoking Regimes; Beitr. Tabakforsch. Int. 25 (2013) 700-707.

DOI: $10.2478 / \mathrm{cttr}-2013-0946$

225. Tindall, I.F., L.P. Crumpler, and T.J.P. Mason: Smoking Machine Design and Yield Errors under Intense Smoke Regimes. Part 1: The Influence of Dead Volume on Yield; presented at the CORESTA Smoke Science and Product Technology Study Groups' Meeting, Sapporo, Japan 2012, Abstract SSPT06.

226. Tindall, I.: The Impact of Smoking Machine Design on Yield During Canadian Intense Smoking; Presentation at ISO/TC 126, WG 10, Ad hoc Group Meeting, London, UK, April, 2012.

227. Tindall, I.F., L.P. Crumpler, and T.J.P. Mason: Smoking Machine Design and Yield Errors under Intense Smoke Regimes. Part 2: The Influence of Puff Volume on Desorption of Volatile Smoke Components; Paper presented at the CORESTA Smoke Science and Product Technology Study Groups Meeting Sapporo, Japan 2012, Abstract SSPT07.

228. Tindall, I.F., A. Cole, and L. Crumpler: Dead Volume and Impinger Capture: Will Machine Design Change Puffing Conditions?; Paper presented at the CORESTA Smoke Science \& Product Technology Meeting, 4-8 October 2015, Jeju, Korea; Abstract ST 39.

229. Purkis, S: A Caution Note: Reference to ISO Standards is Not a Sufficient Means of Providing Validation for Non ISO Methods; Poster STPOST 05 presented at the CORESTA Congress, 12-16 October 2014, Quebec, Canada.

230. Herdt, D.: The Influence of Smoke Path Geometry on TNCO Results Using Smoking Machines Complying with ISO 3308; Poster STPOST 04 presented at the CORESTA Congress, 12-16 October 2014, Quebec, Canada.

231. Deutsches Institut für Normung (DIN): DIN ISO 4387:2011-01 - Zigaretten - Bestimmung des Rohkondensats und des nikotinfreien Trockenkondensats unter Verwendung einer Zigaretten-Abrauchmaschine für Routineanalysen (ISO 4387:2000 + Amd.1:2008) [DIN ISO 4387:2011-01 - Cigarettes - Determination of Total and Nicotine-Free Dry Particulate Matter Using a Routine Analytical Smoking Machine (ISO 4387:2000 + Amd.1:2008)]; DIN, Berlin, Germany, 2011.

232. European Parliament, Council of the European Union: Directive 2014/40/EU of the European Parliament and of the Council of $3^{\text {rd }}$ April 2014 on the Approximation of the Laws, Regulations and Administrative Provisions of the Member States Concerning the Manufacture, Presentation and Sale of Tobacco and Related Products and Repealing Directive 2001/37/EC; Official Journal European Union L127 (2014) 1-38.

233. Brosch, A.: Theoretische und Experimentelle Untersuchungen zur Pathogenesis und Histogenesis der malignen Geschwülste [Theoretical and Experimental Investigations on Pathogenesis and Histogenesis of Malignant Tumors]; Virchows Arch. Pathol. Anat. Physiol. Klin. Med.162 (1900) 32-84.

234. Tillmanns, H.: Ueber Theer-, Russ- und Tabakkrebs [On Cancer Caused by "Tar", Soot and Tobacco]; Dtsch. Z. Chir. 13 (1880) 519-535.

235. Ludwig, E.: Ueber einige Bestandtheile des Tabakrauches [On Several Components of Tobacco Smoke]; Arch. Klin. Chir. 20 (1876).

236. Wacker, L. and A. Schmincke: Experimentelle Untersuchungen zur kausalen Genese atypischer Epithelwucherungen [Experimental Investigations on the Causal Generation of Atypic Epithelial Tumours]; Münch. Med. Wochenschr. 58 (1911) 1607-1613 and 1680-1682.

237. Hoffmann, E., H.T. Schreus, and E. Zurhelle: Beobachtungen zur experimentellen Geschwulsterzeugung durch Teer verschiedener Herkunft und Paraffin [Observations on the Experimental Formation of Tumors by Tars of Different Origins and Paraffin]; Dtsch. Med. Wochenschr. 49 (1923) 633-634.

238. Helwig, F.C.: The Growth-Producing Effects of Extracts of Tobacco on Mice; J.A.M.A. 91 (1928) 150-151.

239. Roffo, A.H.: Desarrollo de un carcinoma en el conejo por el tabaco [Tobacco-Induced Carcinoma in Rabbits]; Boletin del Instituto de Medicina Experimental para el Estudio y Tratamiento del Cancer, Buenos Aires, University Press 7 (1930) 501-538.

240. Roffo, A.H.: Durch Tabak beim Kaninchen entwickeltes Carcinom [Carcinoma Initiated by Tobacco in Rabbits]; Z. Krebsforsch. 33 (1931) 321-332.

241. Roffo, A.H.: Der Tabak als krebserzeugendes Agens [Tobacco as Carcinogenic Agent]; Dtsch. Med. Wochenschr. 63 (1937) 1267-1271.

242. Roffo, A.H.: Carcinome développé chez un lapin après trois ans, par l'effet du tabac [Carcinoma Developed in a Rabbit After Three Years by the Action of Tobacco]; Les Neoplasmes 11 (1932) 77-89.

243. Roffo, A.H.: Krebserzeugendes Benzpyren, gewonnen aus Tabakteer [Cancer Causing Benzpyrene Obtained from Tobacco Tar]; Z. Krebsforsch. 49 (1939) 588-597.

244. Kosak, A.I.: The Composition of Tobacco Smoke; 
Experientia 10 (1954) 69-71.

245. Lü-Fu-hua: Über die Erzeugung von Krebs durch Tabakteerpinselung beim Kaninchen [On the Production of Carcinomas by Skin Painting Rabbits with Tobacco Tar]; Frankf. Z. Pathol. 46 (1934) 513-522.

246. Sugiura, K.: Observations on Animals Painted with Tobacco Tars; Am. J. Cancer 38 (1940) 41-49.

247. Flory, C.M.: The Production of Tumors by Tobacco Tars; Cancer Res. 1 (1941) 252-276.

248. Wynder, E.L. and G. Wright: A Study of Tobacco Carcinogenesis. I. The Primary Fractions; Cancer 10 (1957) 255-271.

249. Wynder, E.L. and D. Hoffmann: Tobacco and Tobacco Smoke. Studies in Experimental Carcinogenesis; Academic Press, New York, USA, 1967.

250. Kosak, A.I., J.S. Swinehart, and D. Taber: The Components of Cigarette Smoke. I. Preliminary Studies; J. Nat. Cancer Inst. 17 (1956) 375-389.

251. Engelbreth-Holm, J. and J. Ahlmann: Production of Carcinoma in ST/Eh Mice with Cigarette Tar; Acta Pathol. Microbiol. Scand. 41 (1957) 267-272.

252. Schur, M.O. and J.C. Rickards: Design and Operation of a Multiple Cigarette Smoking Machine; Tob. Sci. 1 (1957) 13-20.

253. Haag, H.B. and P.S. Larson: Some Basic Facts of Medical Interest Concerning Cigarettes and Their Smoke; Interne 14 (1948) 110-112 and 119.

254. Wolman, W.: A Study of Cigarettes, Cigarette Smoke, and Filters. 4. Regular Cigarettes, King-Size Cigarettes, and Additional Filter-Tip Cigarettes; J. Am. Med. Assoc. 157 (1955) 1309-1312.

255. Consumers Union of U.S. Inc.: Cigarettes. What CU's Tests Showed. The Industry and its Advertising ... and: How Harmful Are They?; Consumer Reports 18 (1953) 58-74.

256. Waser, E. and M. Stähli: Untersuchungen am Tabakrauch. I. [Investigations on Tobacco Smoke. I.]; Z. Untersuch. Lebensm. 64 (1932) 470-485.

257. Pyriki, C.: Untersuchungen über den Rauch von Zigaretten [Investigations on the Smoke from Cigarettes]; Z. Lebensm. Unters. Forsch. 88 (1948) 254-269.

258. Hackney, E.J., W.R. Weidlich, and J.B. Williams: A Large Capacity Smoking Machine; Tob. Sci. 9 (1965) 112-115.

259. Bock, F.G. and G.E. Moore: Carcinogenic Activity of Cigarette-Smoke Condensate. I. Effect of Trauma and X Irradiation; J. Nat.Cancer Inst. 22 (1959) 401-411.

260. Bock, F.G., G.E. Moore, J.E. Dowd, and P.C. Clark: Carcinogenic Activity of Cigarette Smoke Condensate. Biological Activity of Refined Tar from Certain Brands of Cigarettes; JAMA 181 (1962) 668-673.

261. Lang, R.E. and R.J. Moshy: An Improved Automatic Smoking Machine for the Large Scale Collection of Cigarette Smoke Condensate; presented at the $19^{\text {th }}$ Tobacco Chemists' Research Conference, Lexington, KY, USA, 1965.

262. National Cancer Institute Smoking and Health Program: Report No. 1 - Toward Less Hazardous Cigarettes. The First Set of Experimental Cigarettes; edited by G.E. Gori, U.S. Department of Health, Education and Welfare, Publication No. (NIH)
76-905, National Institutes of Health, National Cancer Institute, 1976.

263. National Cancer Institute Smoking and Health Program: Report No. 2 - Toward Less Hazardous Cigarettes. The Second Set of Experimental Cigarettes; edited by G.E. Gori, U.S. Department of Health, Education and Welfare, Publication No. (NIH) 76-1111, National Institutes of Health, National Cancer Institute, 1976.

264. National Cancer Institute Smoking and Health Program: Report No. 3 - Toward Less Hazardous Cigarettes. The Third Set of Experimental Cigarettes; edited by G.E. Gori, U.S. Department of Health, Education and Welfare, Publication No. (NIH) 77-1280, National Institutes of Health, National Cancer Institute, 1977.

265. National Cancer Institute Smoking and Health Program: Report No. 4 - Toward Less Hazardous Cigarettes. The Fourth Set of Experimental Cigarettes; edited by G.E. Gori, U.S. Department of Health, Education and Welfare, National Institutes of Health, National Cancer Institute, 1980.

266. National Cancer Institute Smoking and Health Program: Report No. 5 - Toward Less Hazardous Cigarettes. Summary: Four Skin Painting Bioassays Using Condensate from Experimental Cigarettes; edited by G.E. Gori, U.S. Department of Health, Education and Welfare, 1980.

267. Bombick, D.W., P.H. Ayres, and D.J. Doolittle: Cytotoxicity Assessment of Whole Smoke and Vapor Phase of Mainstream and Sidestream Cigarette Smoke from Three Kentucky Reference Cigarettes; Toxicol. Methods 7 (1997) 177-190.

268. Seehofer, F, J.E. Miller, and H. Elmenhorst.: Eine automatische Schnellrauchmaschine für Serienanalysen und für die präparative Gewinnung von Rauchkondensaten [An Automatic High-Speed Smoking Machine for Batch Analyses and the Preparative Collection of Smoke Condensates]; Teil 2: Elmenhorst, H.: Automatische Schnellrauchmaschine zur präparativen Gewinnung von Tabakrauchkondensaten [Automatic High-Speed Smoking Machine for the Preparative Collection of Smoke Condensates]; Beitr. Tabakforsch. 3 (1965) 78-82. DOI: $10.2478 /$ cttr-2013-0098

269. Barkemeyer, H. and F. Seehofer: Über eine neue Methode zur Niederschlagung von Tabakrauch in Flüssigkeiten [A New Method for the Collection of the Particle Phase of Tobacco Smoke in Liquids]; Beitr. Tabakforsch. 1 (1961) 93-96.

DOI: $10.2478 /$ cttr-2013-0014

270. Elmenhorst, H.: Eine neue Kältefalle zur Gewinnung großer Mengen von Tabakrauchkondensat [A New Cold Trap for the Collection of Large Amounts of Tobacco Smoke Condensate]; Beitr. Tabakforsch. 3 (1965) 101-107. DOI: 10.2478/cttr-2013-0103

271. Elmenhorst, H.: Eine vollautomatische Rauchmaschine für die Herstellung großer Mengen von Cigarettenrauch Kondensat [An Automatic Smoking Machine for the Collection of Large Quantities of Cigarette Smoke Condensate]; Beitr. Tabakforsch. 3 (1966) 545-553. DOI: 10.2478/cttr-2013-0152 
272. Elmenhorst, H. and W. Hennig: Eine vollautomatische Rauchmaschine. II. Mitteilung: Automatische Kontrolle der Stummellänge und ihre Verwendung als Steuerprinzip [An Automatic Smoking Machine. 2nd Report: The Automatic Control of Butt Lengths and its Use as Control Device]; Beitr. Tabakforsch. 5 (1969) 25-27. DOI: 10.2478/cttr-2013-0211

273. Dontenwill, W., H. Elmenhorst, H.-P. Harke, G. Reckzeh, and K.H. Weber: Experimentelle Untersuchungen über die tumorenerzeugende Wirkung von Zigarettenrauch-Kondensaten an der Mäusehaut. I. Mitteilung: Gesamtversuch [Experimental Studies on Tumorigenic Activity of Cigarette Smoke Condensates on the Skin of Mice. $1^{\text {st }}$ Communication: Total Experiment]; Z. Krebsforsch. 73 (1970) 265-284.

274. Neurath, G. and R. Kröger: Druckverrauchen - Eine neue Abrauchmethode und Möglichkeiten ihrer Anwendung [Mechanical Smoking Using a Pressure Procedure - A New Smoking Procedure and its Application Conditions]; Beitr. Tabakforsch. 2 (1963) 81-88. DOI: $10.2478 /$ cttr-2013-0058

275. Food Protection Committee, Food and Nutrition Board: Problems in the Evaluation of Carcinogenic Hazard from Use of Food Additives; U.S. Natl. Acad. Sci., Natl. Res. Council Publ. 749, Washington, DC, USA, 1959.

276. Neurath, G. and H. Ehmke: Apparatur zur Untersuchung des Nebenstromrauches [An Apparatus for Sidestream Studies]; Beitr. Tabakforschung 2 (1964) 117-121. DOI: $10.2478 /$ cttr-2013-0064

277. Brunnemann, K.D. and D. Hoffmann: The $\mathrm{pH}$ of Tobacco Smoke; Food Cosmetics Toxicol. 12 (1974) 115-124.

278. Brunnemann, K.D., L. Yu, and D. Hoffmann: Assessment of Carcinogenic N-Nitrosamines in Mainstream and Sidestream Smoke from Cigarettes; Cancer Res. 37 (1977) 3218-3222.

279. Brunnemann, K.D., J.D. Adams, D.P.S. Ho, and D. Hoffmann: The Influence of Tobacco Smoke on Indoor Atmospheres II. Volatile and Tobacco Specific Nitrosamines in Main- and Sidestream Smoke and Their Contribution to Indoor Pollution; Proceed. $4^{\text {th }}$ Joint Conference on Sensing of Environmental Pollutants, 1977, pp. 876-880, published by the Am. Chem. Soc., Washington D.C., USA, 1978.

280. Adams J.D., K.D. Brunnemann, and D. Hoffmann: Determination of Nitric Oxide in Unaged Smoke by GSC-TEA; Paper presented at the $32^{\text {nd }}$ Tobacco Chemists Research Conference, 1978, Montreal, Canada.

281. Brunnemann, K.D., H. Lee, and D. Hoffmann: Chemical Studies on Tobacco Smoke XLVII. On the Quantitative Analysis of Catechols and Their Reduction; Anal. Letters 9 (1976) 934-955.

282. Brunnemann, K.D., S. Stanke, and D. Hoffmannn: Chemical Studies on Tobacco Smoke LXI. Volatile Pyridines: Quantitative Analysis in Mainstream and Sidestream Smoke of Cigarettes and Cigars; Anal. Letters A11 (1978) 545-560.

283. Brunnemann, K.D. and D. Hoffmann: Chemical
Studies on Tobacco Smoke XXXIV. Gaschromatographic Determination of Ammonia in Cigarette and Cigar Smoke; J Chrom. Sci. 13 (1975) 159-163.

284. Morrell, F.A. and C. Varsel: A Total Combustion Product Cigarette Smoking Machine - Analyses of Radioactive Labeled Cigarette Paper; Tob. Sci. 10 (1966) 45-50.

285. O'Keefe, A.E. and G.T. Smith: A Proximity Cigarette Lighter; Paper presented at the $13^{\text {th }}$ Tobacco Chemists Research Conference, October 1959, Lexington, KY, USA.

286. Jenkins, R.W. Jr, M.K. Chavis, R.H. Newman, and F.A. Morrell: The Quantitative Recovery of Smoke from Radioactively Labeled Cigarettes; Int. J. Appl. Radiat. Isotopes, 22 (1971) 691-697.

287. Jenkins Jr, R.W., C. Goldey, and T.G. Williamson: Neutron Activation Analysis in Tobacco and Cigarette Smoke Studies: 2R1 Cigarette Composition, Smoke Transference and Butt Filtration; Beitr. Tabakforsch. Int. 13 (1985) 59-65.

DOI: $10.2478 /$ cttr-2013-0556

288. Seehofer, F. and D. Hanßen: Die Kapillarpresse, eine Rauchmaschine zur Gewinnung von nativem Rauchkondensat. 1. Mitteilung [The Capillary Press, a Smoking Machine for Collecting Native Smoke Condensate. $1^{\text {st }}$ Report]; Beitr. Tabakforsch. 2 (1964) 303-310. DOI: 10.2478/cttr-2013-0083

289. Seehofer, F. and D. Hanßen: Die Kapillarpresse, eine Rauchmaschine zur Gewinnung von nativem Rauchkondensat. 2. Mitteilung; Die automatische Kapillarpresse [The Capillary Press, a Smoking Machine for Collecting Native Smoke Condensate. $2^{\text {nd }}$ report. The Automatic Capillary Press]; Beitr. Tabakforsch. 3 (1965) 291-300. DOI: 10.2478/cttr-2013-0121

290. Seehofer, F. and D. Hanßen: Die Kapillarpresse, eine Rauchmaschine zur Gewinnung von nativem Rauchkondensat. 3. Mitteilung. Die automatische Kapillarpresse mit einem Rauchkopf für offenes und geschlossenes Abrauchen [The Capillary Press, a Smoking Machine for Collecting Native Smoke Condensate. $3^{\text {rd }}$ Report. An Automatic Smoking Machine Equipped with a Supplementary Appliance for Free and Restricted Smoking]; Beitr. Tabakforsch. 3 (1966) 358-370. DOI: 10.2478/cttr-2013-0128

291. Mathewson, H.D.: The Direct Preparation of Cigarette Smoke Condensate by High Velocity Impaction; Beitr. Tabakforsch. 3 (1966) 430-437.

DOI: $10.2478 / \mathrm{cttr}-2013-0137$

292. Walaszek Z., M. Hanausek, and T.J. Slaga: The Role of Skin Painting in Predicting Lung Cancer; Int. J. Toxicol. 26 (2007) 345-351.

293. Lickint, F.: Tabak und Tabakrauch als ätiologischer Faktor des Carcinoms [Tobacco and Tobacco Smoke as Etiologic Factor of Carcinoma]; Z. Krebsforsch. 30 (1929) 349-365.

294. Mertens, V.E.: Zigarettenrauch eine Ursache des Lungenkrebses? (Eine Anregung.) [Cigarette Smoke a Cause of Lung Cancer? (A Suggestion)]; Z. Krebsforsch. 32 (1930) 82-91.

295. Mertens, V.E.: Noch einmal Zigarettenrauch und Lungenkrebs [Once More: Cigarette Smoke and Lung Cancer]; Z. Krebsforsch. 51 (1941) 183-192. 
296. Campbell, J.A.: The Effect of Exhaust Gases from Internal Combustion Engines and Tobacco Smoke Upon Mice with Special Reference to Incidence of Tumours of the Lung; Br. J. Exp. Pathol. 17 (1936) $146-158$

297. Lorenz, E., H.L. Stewart, J.H. Daniel, and C.V. Nelson: The Effects of Breathing Tobacco Smoke on Strain A Mice; Cancer Res. 3 (1943) 123.

298. Essenberg, J.M.: Cigarette Smoke and the Incidence of Primary Neoplasm of the Lung in the Albino Mouse; Science 116 (1952) 561-562.

299. Leuchtenberger, C., R. Leuchtenberger, P.F. Doolin, and P. Shaffer: A Correlated Histological, Cytological, and Cytochemical Study of the Tracheobronchial Tree and Lungs of Mice Exposed to Cigarette Smoke. I. Bronchitis with Atypical Epithelial Changes in Mice Exposed to Cigarette Smoke; Cancer 11 (1958) 490-506.

300. Leuchtenberger, C., R. Leuchtenberger, F. Ruch, K. Tanaka, and T. Tanaka: Cytological and Cytochemical Alterations in the Respiratory Tract of Mice after Exposure to Cigarette Smoke, Influence Virus, and Both; Cancer Res. 23 (1963) 555-565.

301. Leuchtenberger, C., R. Leuchtenberger, and S. Weiss: Increase of Carbon Monoxide (CO) in Blood of Mice Exposed to Inhalation of Cigarette Smoke; Proc. Am. Assoc. Cancer Res. 6 (1965) 39.

302. Leuchtenberger, C. and R. Leuchtenberger: Einfluss von frischem Zigarettenrauch auf die Entwicklung von Lungentumoren und auf Lungenkulturen bei der Snell-Maus [Influence of Fresh Cigarette Smoke on the Development of Lung Tumors and on Lung Explant Cultures Using the Snell Mouse]; Schweiz. med. Wschr. 101 (1971) 1374-1381.

303. Holland, R.H., R.H. Wilson, D. Morris, M.S. McCall, and H. Lanz: The Effect of Cigarette Smoke on the Respiratory System of the Rabbit - A Preliminary Report; Cancer 11 (1958) 709-712.

304. Holland, R.H., E.J. Kozlowski, and L. Booker: The Effect of Cigarette Smoke on the Respiratory System of the Rabbit - A Final Report; Cancer 16 (1963) 612-615.

305. Haag, H.B., P.S. Larson, and J.H. Weatherby: The Effect on Rats of Chronic Exposure to Cigarette Smoke; Ann. N.Y. Acad. Sci. 90 (1960) 227-238.

306. Otto, H.: Experimentelle Untersuchungen an Mäusen mit passiver Zigarettenrauchbeatmung [Experimental Studies of Passive Inhalation of Cigarette Smoke by Mice]; Frankfurter Z. Patholog. 73 (1963) 10-23.

307. Wynder, E.L., K.T. Taguchi, V. Baden, and D. Hoffmann: Tobacco Carcinogenesis. IX. Effect of Cigarette Smoke on Respiratory Tract of Mice after Passive Inhalation; Cancer 21 (1968) 134-153.

308. Dontenwill, W. and U. Mohr: Experimentelle Untersuchungen zum Problem der Carcinomentstehung im Respirationstrakt. II. Die Wirkung von Tabakrauchkondensaten und Zigarettenrauch auf die Lunge des Goldhamsters [Experimental Investigation to the Problem of the Generation of Carcinomas in the Respiatory Tract. II. The Effect of Tobacco Smoke Condensates and Cigarette Smoke in the Lung of Golden Hamsters]; Z. Krebsforsch. 65 (1962) 62-68.
309. Dontenwill, W.: Inhalationsversuche mit Tabakrauch [Inhalation Experiments With Tobacco Smoke]; in: Alkylierend wirkende Verbindungen, Tagungsbericht zur 1. Konferenz über N-Nitroso-Verbindungen und Lactone [Alkylating Compounds, Report of the $1^{\text {st }}$ Conference on N-Nitroso Compounds and Lactones], Hamburg, Sept. 1963, Wissenschaftliche Forschungsstelle im Verband der Cigarettenindustrie, 1964, pp. 133-137.

310. Dontenwill, W. and B. Wiebecke: Tracheal and Pulmonary Alterations Following the Inhalation of Cigarette Smoke by the Golden Hamster; in: Lung Tumours in Animals - Proceedings of the Third Quadrennial Conference on Cancer, University of Perugia, June 24-29, 1965, edited by L. Severi, Perugia, Division of Cancer Research, 1966, pp. 519-526.

311. Dontenwill, W., G. Reckzeh, and L. Stadler: Inhalationsexperimente mit Cigarettenrauch [Inhalation Experiments with Cigarette Smoke]; Beitr. Tabakforsch. 3 (1966) 438-448. DOI: $10.2478 /$ cttr-2013-0138

312. Harris, R.J.C. and G. Negroni: Cigarette Smoking and the Induction of Lung Cancer in Mice; in: Lung Tumours in Animals - Proceedings of the Third Quadrennial Conference on Cancer, University of Perugia, June 24-29, 1965, edited by L. Severi, Perugia, Division of Cancer Research, 1966, pp. 497-512.

313. Harris, R.J.C. and G. Negroni: Production of Lung Carcinomas in C57BL Mice Exposed to a Cigarette Smoke and Air Mixture; Brit. Med. J. 4 (1967) 637-641.

314. Dontenwill, W., G. Reckzeh, and L. Stadler: Berauchungsapparatur für Laboratoriumstiere [An Inhalation Apparatus Designed for Laboratory Animals]; Beitr. Tabakforsch. 4 (1967) 45-49. DOI: $10.2478 /$ cttr-2013-0166

315. Heinr. Borgwaldt GmbH: Smoking Machines - Inhalation Apparatus Type "Hamburg II"; Bulletin, Borgwaldt, Hamburg, Germany. Available at: https://industrydocuments.library.ucsf.edu/tobacco/ docs/\#id=hmmm0075

316. Rücker, K. and W. Dontenwill: Tierexperimentelle Untersuchungen über die Beeinflussung der Atmung während der Inhalation von Cigarettenrauch [Animal Experiments on the Influence of Cigarette Smoke Inhalation on the Respiratory Frequency]; Beitr. Tabakforsch. 4 (1967) 40-44.

DOI: $10.2478 /$ cttr-2013-0165

317. Reckzeh, G., K. Rücker, H.-P. Harke, and W. Dontenwill: Untersuchungen zur Bestimmung der akuten und chronischen Toxizität von Cigarettenrauch bei passiver Berauchung von Versuchstieren [Studies to Determine the Acute and Chronic Toxicity of Cigarette Smoke in Experimental Animals after Passive Smoking]; Arzneimittelforschung 19 (1969) 237-241.

318. Dontenwill, W.: Experimental Investigations on the Effect of Cigarette Smoke Inhalation on Small Laboratory Animals; Inhalation Carcinogenesis, U.S. AEC Symposium Series 18 (1970) 389-412. 
319. Houseman, T.H. and E. Heneage: Studies of Cigarette Smoke Transfer Using Radioisotopically Labelled Tobacco Constituents; Part I: The Preparation of Radioisotopically Labelled Cigarettes; Beitr. Tabakforsch. 7 (1973) 138-141. DOI: $10.2478 / \mathrm{cttr}-2013-0321$

320. Reznik, G., N. Kmoch, and U. Mohr: Inhalation Experiments with ${ }^{14} \mathrm{C}$-Labelled Cigarette Smoke. I. Determination of the Effectiveness of Two Different Smoking Systems with Labelled Cigarettes; Toxicology 4 (1975) 363-371.

321. Reznik, G. and U. Mohr: A Modified Method for Locating Labeled Smoke Particles in Organs of Syrian Golden and European Hamsters; in: Experimental Lung Cancer; edited by E. Karbe and J.F. Park, Springer, Berlin, Germany (1974) 300-308.

322. Carruthers, W. and R.A.W. Johnston: Composition of a Paraffin Wax Fraction from Tobacco Leaf and Tobacco Smoke; Nature 184 (1959) 1131-1132.

323. Carugno, N.: Determination of Paraffin Waxes of Tobacco and Tobacco Smoke by Gas-Liquid Chormatography; Natl. Cancer Inst. Monograph 9 (1962) 171-178.

324. Davis, B.R., T.H. Houseman, and H.R. Roderick: Studies of Cigarette Smoke Transfer Using Radioisotopically Labelled Tobacco Constituents; Part III: The Use of Dotriacontane-16, $17-{ }^{14} \mathrm{C}$ as a Marker for the Deposition of Cigarette Smoke in the Respiratory System of Experimental Animals; Beitr. Tabakforsch. 7 (1973)148-153. DOI: 10.2478/cttr-2013-0323

325. Kmoch, N., G. Reznik, and U. Mohr: Inhalation Experiments with ${ }^{14} \mathrm{C}$-Labelled Cigarette Smoke; II. The Distribution of Cigarette Smoke Particles in the Hamster Respiratory Tract after Exposure in Two Different Smoking Systems; Toxicology 4 (1975) 373-381.

326. Dontenwill, W.P., H.J. Chevalier, H.P. Harke, U. Lafrenz, G. Reckzeh, and B. Schneider: Investigations on the Effects of Chronic Cigarette-Smoke Inhalation in Syrian Golden Hamsters; J. Natl. Cancer Inst. 51 (1973) 1781-1832.

327. Wehner, A.P., R.H. Busch, R.J. Olson, and D.K. Craig: Chronic Inhalation of Asbestos and Cigarette Smoke by Hamsters; Environ. Res. 10 (1975) 368383.

328. Davis, B.R., E.R. Harris, and E. Heneage: A New Device for Exposing Experimental Animals to Cigarette Smoke; Bates-No. 105439902-9919 Available at: http://beta.industrydocuments.library. ucsf.edu/tobacco/docs/mmdb0208 (accessed February 2015).

329. Bättig, K. and V. Hrubes: The Effect of Filtrate Fractions of Tobacco Smoke on Swimming Endurance in the Rat; Z. Präventivmed. 16 (1971) 205-210

330. Driscoll, P. and K. Bättig: The Effect of Filtrate Fractions of Tobacco Smoke on the Avoidance Behavior of Rats Under Extinction Procedure; Z. Präventivmed. 16 (1971) 197-203.

331. Walton, R.R.: Smoking Machine; U.S. Patent 3,476,119, Nov. 4, 1969.

332. Morrissey, C.A.: Cigarette Smoking Machine; U.S.
Patent 3,528,435, Sept. 15, 1968.

333. Stokely, J.R., J.H. Moneyhun, M.R. Guerin, L. Florant, and J. Greenspan: Description and Characteristics of the Walton Horizontal Inhalation Exposure Smoking Machine; in: Tobacco Smoke Inhalation Bioassay Chemistry, edited by M.R. Guerin, J.R. Stokely, C.E. Higgins, and G.W. Griffith, Oak Ridge National Laboratory, Oak Ridge, TN, USA, 1979, pp. 1-17.

334. Guerin, M.R., J.R. Stokely, C.E. Higgins, J.H. Moneyhun, and R.W. Holmberg: Inhalation Bioassay Chemistry - Walton Horizontal Smoking Machine for Inhalation Exposure of Rodents to Cigarette Smoke; J. Natl. Cancer Inst. 63 (1979) 441-448.

335. Chen, B.T., J. Namenyi, H.C. Yeh, J.L. Mauderly, and R.G. Cuddihy: Physical Characterization of Cigarette Smoke Aerosol Generated from a Walton Smoke Machine; Aerosol Sci. Technol. 12 (1990) 364-375.

336. Maddox, W.L., W.E. Dalbey, M.R. Guerin, J.R. Stokely, D.A. Creasia, and J. Kendrick: A Tobacco Smoke Inhalation Exposure Device for Rodents; Arch. Environm. Health 33 (1978) 64-71.

337. Moneyhun, J.H., J.R. Stokely, and L. Florant: Process and Instruments Corporation Automatic Smoke Exposure Machine - SEM II; in: Tobacco Smoke Inhalation Bioassay Chemistry, edited by M.R. Guerin, J.R. Stokely, C.E. Higgins, and G.W. Griffith, Report ORNL-5424, pp. 19-33, Oak Ridge National Laboratory, Oak Ridge, TN, USA 1979.

338. Henry, C.J., J.E. Caton, J.R. Stokely, M.R. Guerin, A. Lopez, M.D. Avery, D.R. Dansie, G.M. Henderson, T. Gayle, C.E. Whitmire, and R.E. Kouri: Deposition and Distribution of the Total Particulate Matter of Cigarette Smoke in Mice Using a Large-Capacity Smoke Exposure System; Toxicol. Appl. Pharmacol. 58 (1981) 399-409.

339. Henry, C.J., C.E. Whitmire, A. Lopez, D.R. Dansie. M.D. Avery, J.E. Caton, J.R. Stokely, R.W. Holmberg, M.R. Guerin, and R.E. Kouri: The Dosimetry and Distribution of Whole Cigarette Smoke Particulates in Inbred Strains of Mice: Comparison of a Large Smoke Exposure Machine (SEM) with a Small-Capacity Smoke-Exposure Machine (Walton); Pulonary Toxicology of Respirable Particles; edited by G.E. Dage and J.A. Mahafley, U.S. Technical Information Center, Department of Energy, 1980, pp. 177-192.

340. Henry, C.J., D.R. Dansie, K.K. Kanagalingam, R.E. Kouri, T. Gayle, M. Guerin, R. Holmberg, L. Florant, and J. Greenspan: Chronic Inhalation Studies in Mice. I. Facilities and Equipment for "Nose-Only" Exposure to Cigarette Smoke; Beitr. Tabakforsch. Int. 13 (1985) 37-53. DOI: 10.2478/cttr-2013-0554

341. Henry, C.J. and R.E. Kouri: Chronic Inhaltation Studies in Mice. II. Effects of Long-Term Exposure to 2R1 Cigarette Smoke on (C57BL/Cum $x$ C3H/AnfCum)F ${ }_{1}$ Mice; J. Natl. Cancer Inst. 77 (1986) 203-212.

342. Gori, G.B., (ed.): Proceedings of the Tobacco Smoke Inhalation Workshop on Experimental Methods in Smoking and Health Research; DHEW Publ. No. (NIH) 75-906, Washington, D.C., U.S. Gov. Print. 
Office, 1975.

343. Guerin, M., W.L. Maddox, and J.R. Stokely: Tobacco Smoke Inhalation Exposure: Concepts and Devices; in: Proceedings of the Tobacco Smoke Inhalation Workshop on Experimental Methods in Smoking and Health Research, edited by G.B. Gori, Bethesda, ML, USA, June 19-21, 1974, DHEW Publ. No. (NIH) 75906, 1975, pp. 31-48.

344. Schultz, F.J. and J.R. Wagner: A Thirty-Port Smoking Machine for Continuous Smoke Generation; Beitr. Tabakforsch. 8 (1975) 53-59.

DOI: $10.2478 /$ cttr-2013-0355

345. Beven, J.L.: Inhalation Toxicity Studies on Cigarette Smoke. I. A Versatile Exposure System for Inhalation Toxicity Studies on Cigarette Smoke; Toxicology 6 (1976) 189-196.

346. Binns, R., J.L. Beven, L.V. Wilton, and W.G.D. Lugton: Inhalation Toxicity Studies on Cigarette Smoke. II. Tobacco Smoke Inhalation Dosimetry Studies on Small Laboratory Animals; Toxicology 6 (1976) 197-206.

347. Binns, R., J.L. Beven, L.V. Wilton, and W.G.D. Lugton: Inhalation Toxicity Studies on Cigarette Smoke. III. Tobacco Smoke Inhalation Dosimetry Study on Rats; Toxicology 6 (1976) 207-217.

348. Bilimoria, M.H. and D.J. Ecobichon: Responses of Rodent Hepatic, Renal and Pulmonary Aryl Hydrocarbon Hydroxylase Following Exposure to Cigarette Smoke; Toxicology 15 (1980) 83-89.

349. Bilimoria, M.H. and D.J. Ecobichon: The Induction of Rat Lung Aryl Hydrocarbon Hydroxylase by Diluted Smoke from Commercial Cigarettes; Toxicology 22 (1981) 205-210.

350. Bilimoria, M.H. and D.J. Ecobichon: Protective Antioxidant Mechanisms in Rat and Guinea Pig Tissues Challenged by Acute Exposure to Cigarette Smoke; Toxicology 72 (1992) 131-144.

351. Celanese Fibers Marketing Company: Cytrel $^{\mathrm{TM}}$ Smoking Material Manual; Charlotte, NC, USA, 1969.

352. Baumgartner, H. and C.R.E. Coggins: Description of a Continuous-Smoking Inhalation Machine for Exposing Small Animals to Tobacco Smoke; Beitr. Tabakforsch. Int. 10 (1980) 169-174.

DOI: $10.2478 /$ cttr-2013-0487

353. Coggins, C.R.E., R. Lam, and K.T. Morgan: Chronic Inhalation Study in Rats, Using Cigarettes Containing Different Amounts of Cytrel Tobacco Supplement; Toxicology 22 (1982) 287-296.

354. Coggins, C.R.E., X.L.M. Fouillet, R. Lam, and K.T. Morgan: Cigarette Smoke Induced Pathology of the Rat Respiratory Tract: A Comparison of the Effects of the Particulate and Vapour Phases; Toxicology 16 (1980) 83-101.

355. Chen, B.T., W.E. Bechtold, E.B. Barr, Y.-S. Cheng, J.L. Mauderly, and R.G. Cuddihy: Comparison of Cigarette Exposure Atmospheres in Different Exposure and Puffing Modes; Inhal. Toxicol. 1 (1989) 331-347.

356. Chen, B.T., W.E. Bechtold, and J.L. Mauderly: Description and Evaluation of a Cigarette Smoke Generation System for Inhalation Studies; J. Aerosol
Med. 5 (1992) 19-30.

357. Briggs, C.D., J. McCready, and A. Schweitzer: The Chemical Evaluation of a Continuous-smoking Inhalation Machine; Beitr. Tabakforsch. Int. 10 (1980) 175-184. DOI: $10.2478 / \mathrm{cttr}-2013-0488$

358. New Smoking Materials Ltd.: An Introduction to NSM Tobacco Substitute; NSM Ltd., Wilmslow, UK, 1977. Bates-No. 500538417-500538423. Available at: https://www.industrydocumentslibrary.ucsf.edu/ tobacco/docs/\#id=ghlf0099

359. Coggins, C.R.E., R.K. Haroz, R. Lam, and K.T. Morgan: The Tumourigenicity of Smoke Condensates From Cigarettes Containing Different Amounts of Cytrel, as Assessed by Mouse Skin Painting; Toxicology 23 (1982) 177-185.

360. Ayres, P.H., A.T. Mosberg, and C.R.E. Coggins: Modernization of Nose-Only Smoking Machines for Use in Animal Inhalation Studies; J. Am. College Toxicol. 9 (1990) 441-446.

361. R.J. Reynolds Tobacco Co.: Chemical and Biological Studies on New Cigarette Prototypes That Heat Instead of Burn Tobacco; R.J. Reynolds Tobacco Co., Winston-Salem, NC, USA, 1988, Bates No. 533436276-533436657. Available at: http://legacy. library.ucsf.edu/tid/brt81b00

362. CH Technologies (USA): Catalog 2015. Available at: http://chtechusa.com (accessed May 2016)

363. Reininghaus, W. and U. Hackenberg: Anlage zur Langzeit-Inhalation mit Zigarettenrauch für kleine Labortiere [System for Long-Term Inhalation with Cigarette Smoke by Small Laboratory Animals]; Med. Technik 97 (1977) 5-6.

364. Vanscheeuwijck, P.M., A. Teredesai, P.M. Terpstra, J. Verbeeck, P. Kuhl, B. Gerstenberg, S. Gebel, and E.L. Carmines: Evaluation of the Potential Effects of Ingredients Added to Cigarettes. Part 4: Subchronic Inhalation Toxicity; Food Chem. Toxicol. 40 (2002) 113-131.

365. Haussmann, H.J., E. Anskeit, D. Becker, P. Kuhl, W. Stinn, A. Teredesai, P. Voncken, and R.A. Walk: Comparison of Fresh and Room-Aged Cigarette Sidestream Smoke in a Chronic Inhalation Study on Rats; Toxicol. Sci. 41 (1998) 100-116.

366. INBIFO Institut für biologische Forschung $\mathrm{GmbH}$ : Description and Design of the 30-Port INBIFO Smoking Machine (SM-85), August 1999. Available at: http://industrydocuments.library.ucsf.edu/tobacco/ docs/gyfc0062

367. Carmines, E.L., C.L. Gaworski, A.S. Faqi, and N. Rajendran: In Utero Exposure to 1R4F Reference Cigarette Smoke: Evaluation of Developmental Toxicity; Toxicol. Sci. 75 (2003) 134-147.

368. Gavorski, C.L., E.L. Carmines, A.S. Faqi, and N. Rajendran: In Utero and Lactation Exposure of Rats to 1R4F Reference Cigarette Mainstream Smoke: Effect on Prenatal and Postnatal Development; Toxicol. Sci. 79 (2004) 157-169.

369. Appleton, S., G.R. Krautter, J.H. Lauterbach, and N. Rajendran: Validation of a New Smoking Machine for Rodent Inhalation Studies; Paper presented at the $55^{\text {th }}$ Tobacco Science Research Conference, Greensboro, NC, USA, 2001, Abstract 66. 
370. Appleton, S., G.R. Krautter, J.H. Lauterbach, R. Morrissey, and N. Rajendran: Validation of a NoseOnly Rodent Inhalation Smoking Protocol; Paper presented a the $55^{\text {th }}$ Tobacco Science Research Conference, Greensboro, NC, USA, 2001, Abstract 67.

371. Adams, S.M., S. Appleton, J.H. Lauterbach, and N.E. Takenaka.: Design and Performance of a New Reference Cigarette for Biological Studies on Cigarette Smoke; Paper presented at the $55^{\text {th }}$ Tobacco Science Research Conference, Greensboro, NC, USA, 2001, Abstract 65.

372. Obot, C.J., K.M. Lee, A.F. Fuciarelli, R.A. Renne, and W.J. McKinney: Characterization of Mainstream Cigarette Smoke-Induced Biomarker Responses in ICR and C57Bl/6 Mice; Inhal. Toxicol. 16 (2004) 701-719.

373. Coggins, C.R.E.: A Review of Chronic Inhalation Studies with Mainstream Cigarette Smoke in Rats and Mice; Toxicol. Pathol. 26 (1998) 307-314.

374. Coggins, C.R.E.: A Review of Chronic Inhalation Studies with Mainstream Cigarette Smoke, in Hamsters, Dogs, and Nonhuman Primates; Toxicol. Pathol. 29 (2001) 550-557.

375. Coggins, C.R.E.: An Updated Review of Inhalation Studies with Cigarette Smoke in Laboratory Animals; Int. J. Toxicol. 26 (2007) 331-338.

376. Coggins, C.R.E.: Letter to the Editor; Toxicol. Pathol. 28 (2000) 754.

377. Hahn, F.F., A.P. Gigliotti, J.A. Hutt, T.H. March, and J.L. Mauderly: A Review of the Histopathology of Cigarette Smoke-Induced Lung Cancer in Rats and Mice; Int. J. Toxicol. 26 (2007) 307-313.

378. Mauderly, J.L., A.P. Gigliotti, E.B. Barr, W.E. Bechtold, S.A. Belinsky, F.F. Hahn, C.A. Hobbs, T.H. March, S.K. Seilkop, and G.L. Finch: Chronic Inhalation Exposure to Mainstream Cigarette Smoke Increases Lung and Nasal Tumor Incidence in Rats; Toxicol. Sci. 81 (2004) 280-292.

379. Hutt, J.A., B.R. Vuillemenot, E.B. Barr, M.J. Grimes, F.F. Hahn, C.H. Hobbs, T.H. March, A.P. Gigliotti, S.K. Seilkop, G.L. Finch, J.L. Mauderly, and S.A. Belinsky: Life-Span Inhalation Exposure to Mainstream Cigarette Smoke Induces Lung Cancer in B6C3F1 Mice Through Genetic and Epigenetic Pathways; Carcinogenesis 26 (2005) 1999-2009

380. Teague, S.V., K.E. Pinkerton, M. Goldsmith, A. Gebremichael, and S. Chang: Sidestream Cigarette Smoke Generation and Exposure System for Environmental Tobacco Smoke Studies; Inhal. Toxicol. 6 (1994) 79-93.

381. Sun, W., R. Wu, and J.A. Last: Effects of Exposure to Environmental Tobacco Smoke on a Human Tracheobronchial Epithelial Cell Line; Toxicology 100 (1995) 163-174.

382. Aufderheide, M., D. Ritter, J.W. Knebel, and G. Scherer: A Method for In Vitro Analysis of the Biological Activity of Complex Mixtures Such as Sidestream Cigarette Smoke; Exp. Toxic. Pathol. 53 (2001) 141-152.

383. Wolz, L., G. Krause, G. Scherer, M. Aufderheide, and U. Mohr: In Vitro Genotoxic Assay of Side- stream Smoke Using a Human Bronchial Epithelial Cell Line; Food Chem. Toxicol. 40 (2002) 845-850.

384. Bair, W.J., N.S. Porter, D.P. Brown, and A.P. Wehner: Apparatus for Direct Inhalation of Cigarette Smoke by Dogs; J. Appl. Physiol. 26 (1969) 847-850.

385. Cross, F.T., R.F. Palmer, R.E. Filipy, G.E. Dagle, and B.O. Stuart: Carcinogenic Effects of Radon Daughters, Uranium Ore Dust, and Cigarette Smoke in Beagle Dogs; Health Phys. 42 (1982) 33-52.

386. Park, S.S., Y. Kikkawa, Y. Goldring, M.M. Daly, M. Zeiefsky, C. Shim, M. Spierer, and T. Morita: An Animal Model of Cigarette Smoking in Beagle Dogs. Correlative Evaluation of the Effects on Pulmonary Function, Defense and Morphology; Am. Rev. Respir. Dis. 115 (1977) 971-979.

387. Cahan, W.G. and D. Kirman: An Effective System and Procedure for Cigarette Smoking by Dogs; J. Surg. Res. 8 (1968) 567-575.

388. Hammond, E.C., O. Auerbach, D. Kirman, and L. Garfinkel: Effects of Cigarette Smoking on Dogs. I. Design of Experiment, Mortality, and Findings in Lung Parenchyma; Arch. Environm. Health 21 (1970) 740-753.

389. Auerbach, O., E.C. Hammond, D. Kirman, and L. Garfinkel: Effects of Cigarette Smoking on Dogs. II. Pulmonary Neoplasms; Arch. Environm. Health 21 (1970) 754-768.

390. Battista, S.P., M.R. Guerin, G.B. Gori, and C.J. Kensler: A New System for Quantitatively Exposing Laboratory Animals by Direct Inhalation; Arch. Environm. Health 27 (1973) 376-382.

391. Hazleton Laboratories America Inc.: Inhalation Bioassay of Cigarette Smoke in Dogs. Effects of Nicotine and Carbon Monoxide on Atherogenesis; Report submitted to the National Cancer Institute, Division of Cancer Cause and Prevention, Smoking and Health Program, 1981, Vienna, VA, USA, pp. 1-291.

392. Piccirillo, V.J.: Inhalation Bioassay of Cigarette Smoke in Dogs: Nicotine and PMO effects; Report submitted to the National Cancer Institute Smoking and Health Program, Borriston Laboratories, Temple Hills, MD, USA, 1981.

393. Brazell, R.S., A.C. Stiff, G.M. Henderson, R.A. Jenkins, P.L. Röming, and O. Auerbach: Plasma Nicotine and Cotinine in Tobacco Smoke Exposed Beagle Dogs; Toxicol. Appl. Pharamcol. 73 (1984) 152-158.

394. Darwin, C.: The Descent of Man; John Murray, London, 1871, Vol. 1, Chapter 1, p. 12.

395. Jarvik, M.E.: Tobacco Smoking in Monkeys; Ann. N.Y. Acad. Sci. 142 (1967) Art. 1, 280-294.

396. Ando, K. and T. Yanagita: Cigarette Smoking in Rhesus Monkeys; Psychopharmacology 72 (1981) 117-127.

397. McGill, H.C. Jr, W.R. Rogers, R.L. Wilbur, and D.E. Johnson: Cigarette Smoking Baboon Model: Demonstration of Feasibility; Proc. Soc. Exp. Biol. Med. 157 (1978) 672-676.

398. Rogers, W.R., R.L. Bass $3^{\text {rd }}$, D.E. Johnson, A.W. Kruski, C.A. McMahan, M.M. Montiel, G.E. Mott, 
R.L. Wilbur, and H.C. McGill, Jr: AtherosclerosisRelated Responses to Cigarette Smoking in the Baboon; Circulation 61 (1980) 1188-1193.

399. Rogers, W.R., K.D. Carey, C.A. McMahan, M.M. Montiel, G.E. Mott, H.S. Wigodsky, and H.C. McGill: Cigarette Smoking, Dietary Hyperlipidemia, and Experimental Atherosclerosis in the Baboon; Exp. Mol. Pathol. 48 (1988) 135-141.

400. Rogers, W.R., B. McCullough, and J.E. Caton: Cigarette Smoking by Baboons: In Vivo Assessment of Particulate Inhalation Using Bronchoalveolar Lavage to Recover $\left[{ }^{14} \mathrm{C}\right]$ Dotriacontane; Toxicology 20 (1981) 309-321.

401. Raymond, T.L., A.J. Delucia, and L.R. Bryant: Failure of Chronic Cigarette Smoke Exposure to Alter Plasma Lipoproteins of Stumptailed Macaques (Macaca arcoides); Atherosclerosis 41 (1982) 27-33.

402. Sopori, M.L., C.C. Gairola, A.J. Delucia, L.R. Bryant, and S. Cherian: Immune Responsiveness of Monkeys Exposed Chronically to Cigarette Smoke; Clin. Immunol. Immunopathol. 36 (1985) 338-344.

403. Bundesrepublik Deutschland: Tierschutzgesetz in der Neufassung vom 25. Mai 1998 [Animal Protection Law, Revised Version of 25 May 1998]; Bundesgesetzblatt I (1998) 1105-1120. Available at: http://www.bgbl.de/xaver/bgbl/start.xav?startbk= Bundesanzeiger_BGBl\&jumpTo=bgbl198030.pdf

404. Peta Deutschland: Tierversuche für die Tabakindustrie [Animal Experiments in the Tobacco Industry]; Stuttgart, Germany. Available at: http://www.peta.de/tierversuche-fuer-die-tabak industrie\#.V8mDG6UkprR

405. European Parliament, Council of the European Union: Regulation (EC) 1907/2006 of the European Parliament and of the Council of 18 December 2006 Concerning the Registration, Evaluation, Authorisation and Restriction of Chemicals (REACH), Establishing a European Chemicals Agency; Off. J. L 396 (2006) 1-849.

406. FRAME - Fund for the Replacement of Animals in Medical Experiments. Available at: http://www. frame.org.uk/

407. BéruBé, K., M. Aufderheide, D. Breheny, R. Clothier, R. Combes, R. Duffin, B. Forbes, M. Gaça, A. Gray, I. Hall, M. Kelly, M. Lethem, M. Liebsch, L. Merolla, J.-P. Morin, J.C. Seagrave, M.A. Swartz, T.D. Tetley, and M. Umachandran: In Vitro Models of Inhalation Toxicity and Disease - The Report of a FRAME Workshop; Altern. Lab. Anim. 37 (2009) 89-141.

408. Organisation for Economic Co-operation and Development (OECD): Guidelines for the Testing of Chemicals, Section 4: Health Effects. Available at: http://www.oecd-ilibrary.org/environment/oecdguidelines-for-the-testing-of-chemicals-section-4health-effects 20745788

409. Putnam, K.P., D.W. Bombick, and D.J. Doolittle: Evaluation of Eight In Vitro Assays for Assessing the Cytotoxicity of Cigarette Smoke Condensate; Toxicol. In Vitro 16 (2002) 599-607.

410. Borenfreund, E. and J.A. Puerner: A Simple Quantitative Procedure Using Monolayer Cultures for
Cytotoxicity Assays (HTD/NR-90); J. Tissue Cult. Methods 9 (1984) 7-9.

411. Babich, H. and E. Borenfreund: Applications of the Neutral Red Cytotoxicity Assay to In Vitro Toxicology; ATLA 18 (1990) 129-144.

412. Repetto, G., A. del Peso, and J. L. Zurita: Neutral Red Uptake Assay for the Estimation of Cell Viability/Cytotoxicity; Nat. Protoc. 3 (2008) 1125-1131.

413. Ames, B.N., F.D. Lee, and W.E. Durston: An Improved Bacterial Test System for the Detection and Classification of Mutagens and Carcinogens; Proc. Nat. Acad. Sci. 70 (1973) 782-786.

414. Ames, B.N., W.E. Durston, E. Yamasaki, and F.D. Lee: Carcinogens are Mutagens: A Simple Test System Combining Liver Homogenates for Activation and Bacteria for Detection; Proc. Nat. Acad. Sci. 70 (1973) 2281-2285.

415. Mortelmans, K. and E. Zeiger: The Ames Salmonella/Microsome Mutagenicity Assay; Mutat. Res. 455 (2000) 29-60.

416. Crooks, I., D.M. Dillon, J.K. Scott, M. Ballantyne, and C. Meredith: The Effect of Long Term Storage on Tobacco Smoke Particulate Matter in In Vitro Genotoxicity and Cytotoxicity Assays; Regul. Toxicol. Pharmacol. 65 (2013) 196-200.

417. Rickert, W.S., A.H. Trivedi, R.A. Momin, W.G. Wright, and J.H. Lauterbach: Effect of Smoking Conditions and Methods of Collection on the Mutagenicity and Cytotoxicity of Cigarette Mainstream Smoke; Toxicol. Sci. 96 (2007) 285-293.

418. Mumpower, R.C. and G.P. Touey: Selective Removal of Phenol from Cigarette Smoke with Filters; Technical Report, January1967, Tennessee Eastman Company (now Tennessee Eastman Division of Eastman Chemical Company). Available at: https://industrydocuments.library.ucsf.edu/tobacco/ docs/qhx10207

419. Hopkin, J.M., V.S. Tomlinson, and R.M. Jenkins: Variation in Response to Cytotoxicity of Cigarette Smoke; Brit. Med. J. 283 (1981) 1209-1211.

420. Kaltenbach J.P., M.H. Kaltenbach, and W.B. Lyons: Nigrosin as a Dye for Differentiating Live and Dead Ascites Cells; Exp. Cell. Res. 15 (1958) 112-7.

421. Bombick, D.W. and D.J. Doolittle: The Role of Chemical Structure and Cell Type in the Cytotoxicity of Low-Molecular-Weight Aldehydes and Pyridines; In Vitro Toxicol. 8 (1995) 349-356.

422. Kier, L., E. Yamasaki, and B.N. Ames: Detection of Mutagenic Activity in Cigarette Smoke Condensates; Proc. Nat. Acad. Sci. USA 71 (1974) 4159-4163.

423. Wynder, E.L. and D. Hoffmann: Experimental Tobacco Carcinogenesis; Science 162 (1968) 862-871.

424. Hutton, J. and C. Hackney: Metabolism of Cigarette Smoke Condensates by Human and Rat Homogenates to Form Mutagens Detectable by Salmonella Typhimurium TA1538; Cancer Res. 35 (1975) 2461-2468.

425. De Raat, W.K.: Comparison of the Induction by Cigarette Smoke Condensates of Sister-Chromatid Exchanges in Chinese Hamster Cells and of Mutations in Salmonella Typhimurium; Mutat. Res. 66 (1979) 253-259. 
426. Hopkin, J.M. and H.J. Evans: Cigarette Smoke Condensates Damage DNA in Human Lymphocytes; Nature 279 (1979) 241-242.

427. Hopkin, J.M. and H.J. Evans: Cigarette SmokeInduced DNA Damage and Lung Cancer Risks; Nature 283 (1980) 388-390.

428. Andreoli, C., D. Gigante, and A. Nunziata: A Review of In Vitro Methods to Assess the Biological Activity of Tobacco Smoke with the Aim of Reducing the Toxicity of Smoke; Toxicol. in Vitro 17 (2003) 587-594.

429. ICH - International Council for Harmonization. Available at: http://www.ich.org/home.html

430. Johnson, M.D., J. Schilz, M.V. Djordjevic, J.R. Rice, and P.G. Shields: Evaluation of In Vitro Assays for Assessing the Toxicity of Cigarette Smoke and Smokeless Tobacco; Cancer Epidemiol. Biomarkers Prev. 12 (2009) 3263-3304.

431. CORESTA In Vitro Toxicology Task Force: The Rationale and Strategy for Conducting In Vitro Toxicology Testing of Tobacco Smoke (May 2004). Available at: https://www.coresta.org/rationale-andstrategy-conducting-vitro-toxicology-testing-tobaccosmoke-29237.html

432. Deutsches Institut für Normung (DIN): Toxikologische Bewertung von Zusatzstoffen für Tabakprodukte - Ein Leitfaden [Toxicological Assessment of Additives for Tobacco Products - A Guidance]; DIN SPEC 10133:2014-09; DIN Deutsches Institut für Normung e.V., Berlin, Germany, 2014.

433. European Parliament, Council of the European Union: Directive 2014/40/EU of the European Parliament and of the Council of $3^{\text {rd }}$ April 2014 on the Approximation of the Laws, Regulations and Administrative Provisions of the Member States Concerning the Manufacture, Presentation and Sale of Tobacco and Related Products and Repealing Directive 2001/37/EC; Official Journal European Union L127 (2014) 1-38. AUCH 232

434. Voisin, C., C. Aerts, A.B. Tonnel, J.-L. Houdret, and P. Ramon: Mise en survie en phase gazeuse et reconstitution "in vitro" du microenvironnement naturel des macrophages alvéolaires [Survival in Gaseous Phase and Reconstitution "In Vitro" of the Natural Micro-Enviroment of Alveolar Macrophages]; Path. Biol. 23 (1975) 453-459.

435. Voisin, C., C. Aerts, E. Jakubczak, and A.B. Tonnel: La culture cellulaire en phase gazeuse - Un nouveau modèle expérimental d'étude in vitro des activités des macrophages alvéolaires [Cell Culture in the Gas Phase - A New Experimental Model for the In Vitro Study of Alveolar Macrophages]; Bull. Europ. Physiopath. Resp. 13 (1977) 69-82.

436. Voisin, C., C. Aerts, E. Jakubczak, J.L. Houdret, and A.B. Tonnel: Effects du bioxyde d'azote sur les macrophages alvéolaires en survie en phase gazeuse - Un nouveau modèle expérimental pour l'étude in vitro de la cytotoxicité des gaz nocifs [Effects of Nitrogen Dioxide on Alveolar Macrophages Surviving in the Gas Phase - A New Experimental Model for the Study of In Vitro Cytotoxicity of Toxic Gases]; Bull. Europ. Physiopath. Resp. 13 (1977)
137-144.

437. Tarkington B.K., R. Wu, W.-M. Sun, K.J. Nikula, D.W. Wilson, and J.A. Last: In Vitro Exposure of Tracheobronchial Epithelial Cells and of Tracheal Explants to Ozone; Toxicology 88 (1994) 51-68.

438. Aufderheide, M. and U. Mohr: CULTEX - A New System and Technique for the Cultivation and Exposure of Cells at the Air/Liquid Interface; Exp. Toxicol. Pathol. 51 (1999) 489-490.

439. Mohr, U. and M. Aufderheide: Kulturvorrichtung und Verfahren zur Kultivierung von Zellen oder Gewebekomponenten; German Patent DE 19801763 C2, publication date: July 22, 1999.

440. Aufderheide, M. and U. Mohr: CULTEX -An Alternative Technique or Cultivation and Exposure of Cells of the Respiratory Tract to Airborne Pollutants at the Air/Liquid Interface; Exp. Toxicol. Pathol. 52 (2000) 265-270.

441. Ritter, D., J.W. Knebel, and M. Aufderheide: In Vitro Exposure of Isolated Cells to Native Gaseous Compounds - Development and Validation of an Optimized System for Human Lung Cells; Exp. Toxicol. Pathol. 53 (2001) 373-386.

442. Aufderheide, M., J.W. Knebel, and D. Ritter: A Method for the In Vitro Exposure of Human Cells to Environmental and Complex Gaseous Mixtures: Application to Various Types of Atmosphere; ATLA 30 (2002) 433-441.

443. Aufderheide, M., J.W. Knebel, and D. Ritter: Novel Approaches for Studying Pulmonary Toxicity In Vitro; Toxicol. Lett. 140-141 (2003) 205-211.

444. Ritter, D., J.W. Knebel, and M. Aufderheide: Exposure of Human Lung Cells to Inhalable Substances: A Novel Test Strategy Involving Clean Air Exposure Periods Using Whole Diluted Cigarette Mainstream Smoke; Inhal. Toxicol. 15 (2003) 67-84.

445. Aufderheide, M., J.W. Knebel, and D. Ritter: An Improved In Vitro model for Testing the Pulmonary Toxicity of Complex Mixtures Such as Cigarette Smoke; Exp. Toxicol. Pathol. 55 (2003) 51-57.

446. Ritter, D., J.W. Knebel, and M. Aufderheide: Comparative Assessment of Toxicities of Mainstream Smoke from Commercial Cigarettes; Inhal. Toxicol. 16 (2004) 691-700.

447. CORESTA In vitro Toxicology Task Force: In Vitro Exposure of Cells to Smoke at the Air Liquid Interface (2007). Available at: https:/www.coresta. org/vitro-exposure-cells-smoke-air-liquid-interface29235.html

448. Aufderheide, M.: Direct Exposure Methods for Testing Native Atmospheres; Exp. Toxicol. Pathol. 57 (2005) 213-226.

449. Aufderheide M.: An Efficient Approach to Study the Toxicological Effects of Complex Mixtures; Exp. Toxicol. Pathol. 60 (2008) 163-180.

450. VITROCELL ${ }^{\circledR}$ Systems GmbH: Advanced In Vitro Exposure Systems - VITROCELL ${ }^{\circledR}$ VC $10^{\circledR}$ SMOKING ROBOT; Catalogue, edition 03/2016, pp. $70-77$.

451. Okuwa, K., M. Tanaka, Y. Fukano, H. Nara, Y. Nishijima, and T. Nishino: In Vitro Micronucleus Assay for Cigarette Smoke Using a Whole Smoke 
Exposure System: A Comparison of Smoking Regimens; Exp. Toxicol. Pathol. 62 (2010) 433-440.

452. Nara, H., Y. Fukano, T. Nishino, and M. Aufderheide: Detection of the Cytotoxicity of WaterInsoluble Fraction of Cigarette Smoke by Direct Exposure to Cultured Cells at an Air-Liquid Interface; Exp. Toxicol. Pathol. 65 (2013) 683-688.

453. Aufderheide, M. and U. Mohr: A Modified CULTEX $^{\circledR}$ System for the Direct Exposure of Bacteria to Inhalable Substances; Exp. Toxicol. Pathol. 55 (2004) 451-454.

454. Aufderheide, M. and H. Gressmann: A Modified Ames Assay Reveals the Mutagenicity of Native Cigarette Mainstream Smoke and its Gas Vapour Phase; Exp. Toxicol. Pathol. 58 (2007) 383-392.

455. Aufderheide, M. and H. Gressmann: Mutagenicity of Native Cigarette Mainstream Smoke and its Gas/Vapour Phase by Use of Different Tester Strains and Cigarettes in a Modified Ames Assay; Mut. Res. 656 (2008) 82-87.

456. Paur, H.R., F.R. Cassee, J. Teeguarden, H. Fissan, S. Diabate, M. Aufderheide, W.G. Kreyling, O. Hänninen, G. Kasper, M. Riediker, B. RothenRutishauser, and O. Schmid: In-Vitro Cell Exposure Studies for the Assessment of Nanoparticle Toxicity in the Lung - A Dialog Between Aerosol Science and Biology; J. Aerosol Sci. 42 (2011) 668-692.

457. Bakand, S. and A. Hayes: Troubleshooting Methods for Toxicity Testing of Airborne Chemicals In Vitro; J. Pharmacol. Toxicol. Methods 61 (2010) 76-85.

458. Aufderheide, M., S. Scheffler, N. Möhle, B. Halter, and D. Hochrainer: Analytical In Vitro Approach for Studying Cyto- and Genotoxic Effects of Particular Airborne Material; Anal. Bioanal. Chem. 401 (2011) 3213-3220.

459. Aufderheide, M., B. Halter, N. Möhle, and D. Hochrainer: The CULTEX RFS: A Comprehensive Technical Approach for the In Vitro Exposure of Airway Cells to Particulate Matter at the Air-Liquid Interface; BioMed Res. Int. (2013) 734137. DOI: $10.1155 / 2013 / 734137$

460. Rach, J., J. Budde, N. Möhle, and M. Aufderheide: Direct Exposure at the Air-Liquid Interface: Evaluation of an In Vitro Approach for Simulating Inhalation of Airborne Substances; J. Appl. Toxicol. 34 (2014) 506-515.

461. Ishikawa, S., T. Suzuki, and Y. Nagata: Analysis of Cigarette Smoke Deposition Within an In Vitro Exposure System for Simulating Exposure in the Human Respiratory Tract; Beitr. Tabakforsch. Int. 27 (2016) 20-29. DOI: 10.1515/cttr-2016-0004

462. Steinritz, D., N. Möhle, C. Pohl, M. Papritz, B. Stenger, A. Schmidt, C.J. Kirkpatrick, H. Thiermann, R. Vogel, S. Hoffmann, and M. Aufderheide: Use of the CULTEX ${ }^{\circledR}$ Radial Flow System as an In Vitro Exposure Method to Assess Acute Pulmonary Toxicity of Fine Dusts and Nanoparticles with Special Focus on the Intra- and Inter-Laboratory Reproducibility; Chem. Biol. Interact. 206 (2013) 479-490.

463. Hartung T., S. Bremer, S. Casati, S. Coecke, R. Corvi, S. Fortaner, L. Gribaldo, M. Halder, S. Hoffmann, A. Janusch Roi, P. Prieto, E. Sabbioni,
L. Scott, A. Worth, and V. Zuang: A Modular Approach to the ECVAM Principles on Test Validity; ATLA 32 (2004) 467-472.

464. European Commisson - Joint Research Centre: European Union Reference Laboratory for Alternatives to Animal Testing (EURL ECVAM). Available at: https://eurl-ecvam.jrc.ec.europa.eu/

465. Aufderheide, M., S. Scheffler, S. Ito, S. Ishikawa, and M. Emura: Ciliatoxicity in Human Primary Bronchiolar Epithelial Cells after Repeated Exposure at the Air-Liquid Interface with Native Mainstream Smoke of K3R4F Cigarettes With and Without Charcoal Filter; Exp. Toxicol. Pathol. 67 (2015) 407-411.

466. Walker, T.R. and J.E. Kiefer: Ciliastatic Components in the Gas Phase of Cigarette Smoke; Science 15 (1966) 1248-1250.

467. Scheffler, S., H. Dieken, O. Krischenowsk, C. Förster, D. Branscheid, and M. Aufderheide: Evaluation of E-Cigarette Liquid Vapor and Mainstream Cigarette Smoke After Direct Exposure of Primary Human Bronchial Epithelial Cells; Int. J. Environ. Res. Public Health 12 (2015) 3915-3925. DOI: $10.3390 /$ ijerph120403915

468. Scheffler, S., H. Dieken, N. Moehle, M. Aufderheide, and T. Schmidt: A New In Vitro Testing Module for the Cytotoxic Evaluation of E-Cigarette Vapor; Poster presented at the CORESTA Smoke Science \& Product Technology Meeting, 4-8 October 2015, Jeju, Korea, Poster STPOST 10.

469. Aufderheide, M., C. Förster, M. Beschay, D. Branscheid, and M. Emura: A New Computer-Controlled Air-Liquid Interface Cultivation System for the Generation of Differentiated Cell Cultures of the Airway Epithelium; Exp. Toxicol. Pathol. (2015). DOI: 10.1016/j.etp.2015.10.001.

470. Phillips, J., J. Williamson, and E.D. Massey: A Method to Expose Mammalian Cells In Vitro to Whole Smoke at the Air-Liquid Interface; Mutagenesis 17 (2002) 566.

471. Massey, E.D., J. Williamson, and J.F.N. Phillips: An Exposure Device; International Patent, WO 03/100417 A1 (publication date: December 4, 2003).

472. Phillips, J., B. Kluss, A. Richter, and E.D. Massey: Exposure of Bronchial Epithelial Cells to Whole Cigarette Smoke: Assessment of Cellular Responses; Altern. Lab. Anim. 33 (2005) 239-248.

473. Maunders, H., S. Patwardhan, J. Phillips, A. Clack, and A. Richter: Human Bronchial Epithelial Cell Transcriptome: Gene Expression Changes Following Acute Exposure to Whole Cigarette Smoke In Vitro; Am. J. Physiol. Lung Cell. Mol. Physiol. 292 (2007) L1248-L1256.

474. Adamson, J., D. Azzopardi, G. Errington, C. Dickens, J. McAughey, and M.D. Gaça: Assessment of an In Vitro Whole Cigarette Smoke Exposure System: The Borgwaldt RM20S 8-Syringe Smoking Machine; Chem. Cent. J. 5 (2011) 50.

475. Adamson, J., S. Hughes, D. Azzopardi, J. McAughey, and M.D. Gaça: Real-Time Assessment of Cigarette Smoke Particle Deposition In Vitro; Chem. Cent. J. 6:98 (2012). Available at: https://ccj.springeropen. 
com/articles/10.1186/1752-153X-6-98

476. Adamson J., D. Thorne, J. McAughey, D. Dillon, and C. Meredith: Quantification of Cigarette Smoke Particle Deposition In Vitro Using a Triplicate Quartz Crystal Microbalance Exposure Chamber; BioMed Res. Int. (2013) 685074. DOI:10.1155/2013/685074

477. Garcia-Canton, C., G. Errington, A. Anadon, and C. Meredith: Characterisation of an Aerosol Exposure System to Evaluate the Genotoxicity of Whole Mainstream Cigarette Smoke Using the In Vitro $\gamma \mathrm{H} 2 \mathrm{AX}$ Assay by High Content Screening; BMC Pharmacol. Toxicol. 15 (2014) 41.

DOI: $10.1186 / 2050-6511-15-41$

478. Kaur, N., M. Lacasse, J.-P. Roy, J.-L. Cabral, J. Adamson, G. Errington, K.C. Waldron, M. Gaça, and A. Morin: Evaluation of Precision and Accuracy of the Borgwaldt RM20S ${ }^{\circledR}$ Smoking Machine Designed for In Vitro Exposure; J. Inhal. Toxicol. 22 (2010) 1174-1183.

479. Thorne, D., J. Wilson, T.S. Kumaravel, E.D. Massey, and M. McEwan: Measurement of Oxidative DNA Damage Induced by Mainstream Cigarette Smoke in Cultured NCI-H292 Human Pulmonary Carcinoma Cells; Mutat. Res. 673 (2009) 3-8.

480. McAughey, J., T. Adam, C. McGrath, C. Mocker, and R. Zimmermann: Simultaneous On-Line Size and Chemical Analysis of Gas Phase and Particulate Phase of Mainstream Tobacco Smoke; J. Physics: Conference Series 151 (2009) 012017. DOI: $10.1088 / 1742-6596 / 151 / 1 / 012017$

481. Sauerbrey, G.: Verwendung von Schwingquarzen zur Wägung dünner Schichten und zur Mikrowägung [The Use of Oscillator Quartz Crystals for Weight Determination of Thin Layers and for Microweighing]; Z. Physik 155 (1959) 206-22.

482. Thorne, D. and J. Adamson: A Review of In Vitro Cigarette Smoking Systems; Exp. Toxicol. Pathol. 65 (2013) 1183-1193.

483. St-Laurent, J., L.-I. Proulx, L.-P. Boulet, and E. Bissonnette: Comparison of Two In Vitro Models of Cigarette Smoke Exposure; Inhal. Toxicol. 21 (2009) 1148-1153.

484. Gualerzi, A., M. Sciarabba, G. Tartaglia, C. Sforza, and E. Donetti: Acute Effects of Cigarette Smoke on Three-Dimensional Cultures of Normal Human Oral Mucosa; Inhal. Toxicol. 24 (2012) 382-389.

485. Garcia-Canton, C., A. Anadon, and C. Meredith: $\gamma \mathrm{H} 2 \mathrm{AX}$ as a Novel Endpoint to Detect DNA Damage: Application of the Assessment of the In Vitro Genotoxicity of Cigarette Smoke; Toxicol. In Vitro 26 (2012) 1075-1086.

486. Garcia-Canton C., A. Anadon, and C. Meredith: Assessment of the In Vitro $\gamma \mathrm{H} 2 \mathrm{AX}$ Assay by High Content Screening as a Novel Genotoxicity Test; Mutat. Res. 757 (2013) 158-166.

487. World Health Organization - International Agency for Research on Cancer (IARC): Agents Classified by the IARC Monographs, Volumes 1-113; last update: June 26, 2015. Available at: http://monographs. iarc.fr/ENG/Classification/

488. Li, X., C. Nie, P. Shang, F. Xie, H. Liu, and J. Xie: Evaluation Method for the Cytotoxicity of Cigarette
Smoke by In Vitro Whole Smoke Exposure; Exp. Toxicol. Pathol. 66 (2014) 27-33.

489. Fields, W.R., K.W. Fowler, K.H. Hargreaves, L.D. Walker, and B.D. Bombick: Validation of the Vitro Cell VT 10 Smoke Exposure System for in Vitro Assays; Poster, presented at the CORESTA Smoke Science \& Product Technology Study Groups Meeting, Sept. 29 - Oct. 3, 2013, Seville, Spain.

490. Thorne, D., J. Kilford, R. Payne, J. Adamson, K. Scott, A. Dalrymple, C. Meredith, and D. Dillon: Characterisation of a Vitrocell ${ }^{\circledR}$ VC 10 In Vitro Smoke Exposure System Using Dose Tools and Biological Analysis; Chem. Cent. J. 7 (2013) 146155.

491. Thorne, D., J. Kilford, R. Payne, L. Haswell, A. Dalrymple, C. Meredith, and D. Dillon: Development of a BALB/c 3T3 Neutral Red Uptake Cytotoxicity Test Using a Mainstream Cigarette Smoke Exposure System; BMC Research Notes 7 (2014) 367.

492. Adamson, J., D. Thorne, A. Dalrymple, D. Dillon, and C. Meredith: Assessment of Cigarette Smoke Particle Deposition Within the Vitrocell ${ }^{\circledR}$ Exposure Module Using Quartz Crystal Microbalances; Chem. Cent. J. 7 (2013) 50.

493. Adamson, J., D. Thorne, G. Errington, W. Fields, X. Li, R. Payne, T. Krebs, A. Dalrymple, K. Fowler, D. Dillon, F. Xie, and C. Meredith: An Inter-Machine Comparison of Tobacco Smoke Particle Deposition In Vitro from Six Independent Smoke Exposure Systems; Toxicol. In Vitro 28 (2014) 1320-1328

494. Kilford J., D. Thorne, R. Payne, A. Dalrymple, J. Clements, C. Meredith, and D. Dillon: A Method for Assessment of the Genotoxicity of Mainstream Cigarette-Smoke by Use of the Bacterial ReverseMutation Assay and an Aerosol-Based Exposure System; Mutat. Res. 769 (2014) 20-28.

495. Thorne, D., J. Kilford, M. Hollings, A. Dalrymple, M. Ballantyne, C. Meredith, and D. Dillon: The Mutagenic Assessment of Mainstream Cigarette Smoke Using the Ames Assay: A Multi-Strain Approach; Mutat. Res. Genet. Toxicol. Environ. Mutagen, 2015. DOI: 10.1016/j.mrgentox.2015.03.006

496. Breheny, D., F. Cunningham, J. Kilford, R. Payne, D. Dillon, and C. Meredith: Application of a Modified Gaseous Exposure System to the In Vitro Toxicological Assessment of Tobacco Smoke Toxicants; Environ. Mol. Mutagen. 55 (2014) 662-672.

497. Binder H. and W. Lindner: Bestimmung von Äthylenoxid im Rauch garantiert unbegaster Zigaretten [The Determination of Ethylene Oxide in the Smoke of Definitely Unfumigated Cigarettes]; Fachl. Mitt. Österr. Tabakregie 13 (1972) 215-220.

498. Bach, B., H. Kuhn, and F. Kuffner: Über die Verminderung des Nicotingehalts von Tabaken mittels Äthylenoxides [On the Reduction of the Nicotine Content of Tobaccos with Ethylene Oxide]; Fachl. Mitt. Österr. Tabakregie 4 (1963) 53-60.

499. Pailer, M. and W. Hübsch: Über die Umsetzung von Äthylenoxid mit Tabakinhaltsstoffen, ausgenommen Nikotin, bei extremen Begasungsverhältnissen [On the Reaction of Ethylene Oxide with Tobacco 
Components - Except Nicotine - by Intensive Fumigation]; Fachl. Mitt. Österr. Tabakregie 8 (1967) 140-142.

500. Gross, P.M. and L.F. Dixon: Method of Reducing the Nicotine Content of Tobacco; United States Patent 1,962,145, June 12, 1934.

501. Samfield, M.M., E.E. Locklair, and B.A. Brock: Reducing the Nicotine Content of Tobacco Smoke; United States Patent 2,760,495, August 28, 1956.

502. Weber S., M. Hebestreit, T. Wilms, L.L. Conroy, and G. Rodrigo: Comet Assay and Air-Liquid Interface Exposure System: A New Combination to Evaluate Genotoxic Effects of Cigarette Whole Smoke in Human Lung Cell Lines; Toxicol. in Vitro 27 (2013) 1987-1991.

503. Vitrocell ${ }^{\circledR}$ Systems GmbH: Advanced in Vitro Exposure Systems. VITROCELL ${ }^{\circledR}$ Dilution Systems; Edition 03/2014, p. 68.

504. Vitrocell ${ }^{\circledR}$ Systems GmbH: Advanced in Vitro Exposure Systems. VITROCELL ${ }^{\circledR} 24 / 24$ Cultivation and Exposure Module; Edition 03/2014, pp. 30-31.

505. Vitrocell ${ }^{\circledR}$ Systems GmbH: Advanced in Vitro Exposure Systems. VITROCELL ${ }^{\circledR}$ 24/48 Cultivation and Exposure Module; Edition 03/2014, pp. 32-34.

506. Majeed S., S. Frentzel, S. Wagner, D. Kuehn, P. Leroy, P.A. Guy, A. Knorr, J. Hoeng, and M.C. Peitsch: Characterization of the Vitrocell ${ }^{\circledR} 24 / 48$ In Vitro Aerosol Exposure System Using Mainstream Cigarette Smoke; Chem. Cent. J. 8 (2014) 62-72.

507. Burghart Messtechnik GmbH: Instructions - Automated Smoking Machine SM2000 - 8589, 2010.

508. Karp, P.M., T.O. Moninger, S.P. Weber, T.S. Nesselhauf, J.L. Launspach, J. Zabner, and M.J. Welsch: An In Vitro Model of Differentiated Human Airway Epithelia. Methods for Establishing Primary Cultures; Methods Mol. Biol. 188 (2002) 115-137.

509. Baxter, A. and E. Minet: Primary Human Airway Epithelial Cells (MucilAirTM) Express Key Tobacco Toxicant Metabolizing Enzymes in Short Term Air Liquid Interface Cultures; Poster presented at the International Meeting of the Society for Research on Nicotine and Tobacco, POS4-47, March 13-16, 2013, Boston, MA, USA.

510. Mathis, C., C. Poussin, D. Weisensee, S. Gebel, A. Hengstermann, A. Sewer, V. Belcastro, Y. Xiang, S. Ansari, S. Wagner, J. Hoeng, and M.C. Peitsch: Human Bronchial Epithelial Cells Exposed In Vitro to Cigarette Smoke at the Air-Liquid Interface Resemble Bronchial Epithelium from Human Smokers; Am. J. Physiol. Lung Cell Mol. Physiol. 304 (2013) L489-L503.

511. Pezzulo, A.A., T.D. Starner, T.E. Scheetz, G.L. Traver, A.E. Tilley, B.G. Harvey, R.G. Crystal, P.B. McCray Jr, and J. Zaber: The Air Liquid Interface and Use of Primary Cell Cultures Are Important to Recapitulate the Transcriptional Profile of In Vivo Airway Epithelia; Am. J. Physiol. Lung Cell Mol. Physiol. 300 (2011) L25-L31.

512. Subramanian, A., P. Tamayo, V.K. Mootha, S. Mukherjee, B.L. Ebert, M.A. Gillette, A. Paulovich, S.L. Pomeroy, T.R. Golub, E.S. Lander, and J.P. Mesirov: Gene Set Enrichment Analysis: A Know-
ledge-Based Approach for Interpreting Genome-Wide Expression Profiles; PNAS 102 (2005) 15545-15550.

513. Iskandar A.R., F. Martin, M. Talikka, W.K. Schlage, R. Kostadinova, C. Mathis, J. Hoeng, and M.C. Peitsch: Systems Approaches Evaluating the Perturbation of Xenobiotic Metabolism in Response to Cigarette Smoke Exposure in Nasal and Bronchial Tissues; Biomed Res. Int. (2013) 512086. DOI: $10.1155 / 2013 / 512086$

514. Schlage, W.K., A.R. Iskandar, R. Kostadinova, Y. Xiang, A. Sewer, S. Majeed, D. Kuehn, S. Frentzel, M. Talikka, M. Geertz, C. Mathis, N. Ivanov, J. Hoeng, and M.C. Peitsch: In Vitro Systems Toxicology Approach to Investigate the Effects of Repeated Cigarette Smoke Exposure on Human Buccal and Gingival Organotypic Epithelial Tissue Cultures; Toxicol. Mech. Meth. 24 (2014) 470-487.

515. Röper, W. and R. Wieczoreck: In Vitro Cytotoxicity of Cigarette Mainstream Smoke. Evaluation of Different Cell Exposure Methods, Including "Native" Smoke Aerosol Exposure; Paper presented at CORESTA Congress, 22-27 September 2002, New Orleans, LA, USA.

516. Riss, T.L. and R.A. Moravec: Comparison of MTT, XTT, and a Novel Tetrazolium Compound MTS for In Vitro Proliferation and Chemosensitivity Assays; Mol. Biol. Cell. 3 (1992) Suppl. 184a.

517. Wieczorek, R., W. Röper, and H. Burghart: Validation of a New Smoking Machine for the Exposure of 96 Well Cell Culture Plates to "Native" Cigarette Mainstream Smoke Aerosol. Course of Action and Some In Vitro Toxicological Data; Paper presented at CORESTA Congress, 4-8 September 2005, Stratford, U.K.

518. Heinrich Burghart GmbH: Bedienungsanleitung und Technische Dokumentation für die MSB-01 Mimic Smoker Burghart [Operating Instructions and Technical Documentation for the MSB-01 Mimic Smoker Burghart]; Burghart, Hamburg, Germany, 2007.

519. Scian, M.J., M.J. Oldham, D.B. Kane, J.S. Edmiston, and W.J. McKinney: Characterization of a Whole Smoke In Vitro Exposure System (Burghart Mimic Smoker - 01); Inhal. Toxicol. 21 (2009) 234-243.

520. Berner, A. and J. Marek: Untersuchungen über die Verteilung einzelner Rauchinhaltsstoffe in Rauchpartikeln verschiedener Größe [Investigation on the Distribution of Individual Smoke Constituents in Smoke Particles of Different Size]; Fachl. Mitt. Österr. Tabakregie 7 (1967) 118-127.

521. Jones, R.T., W.G.D. Lugton, S.R. Massey, and R.B. Richardson: The Distribution with Respect to Smoke Particle Size of Dotriacontane, Hexadecane, and Decachlorobiphenyl Added to Cigarettes; Beitr. Tabakforsch. 8 (1975) 89-82. DOI: 10.2478/cttr-2013-0361

522. Morie, G.P. and M.S. Baggett: Observations on the Distribution of Certain Tobacco Smoke Components with Respect to Particle Size; Beitr. Tabakforsch. 9 (1977) 72-78. DOI: 10.2478/cttr-2013-0430

523. Jenkins, R.W., R.W. Francis, H. Flachsbart, and W. Stöber: Chemical Variability of Mainstream Cigarette Smoke as a Function of Aerodynamic Particle Size; 


\section{J. Aerosol Sci. 10 (1979) 355-362.}

524. Scian, M.J., M.J. Oldham, J.H. Miller, D.B. Kane, J.S. Edmiston, and W.J. McKinney: Chemical Analysis of Cigarette Smoke Particulate Generated in the MSB-01 In Vitro Whole Smoke Exposure System; Inhal. Toxicol. 21 (2009) 1040-1052.

DOI: $10.1080 / 08958370802712705$

525. The Institute for In Vitro Sciences, Inc.: In Vitro Exposure Systems and Dosimetry Assessment Tools for Inhaled Tobacco Products; Conference on April 4-6, 2016, in Bethesda, MD, USA, Report 2016. Available at: http://www.w2ww.iivs.org/home/resources/ documents

526. Sibata Scientific Technology Ltd. Available at: https://www.sibata.co.jp/english/company-profile

527. Fukano, Y., M. Ogura, K. Eguchi, M. Shibagaki, and M. Suzuki: Modified Procedure of a Direct In Vitro Exposure System for Mammalian Cells to Whole Cigarette Smoke; Exp. Toxicol. Pathol. 55 (2004) 317-323.

528. Fukano, Y., H. Yoshimura, and T. Yoshida: Heme Oxigenase-1 Gene Expression in Human Alveolar Epithelial Cells (A549) Following to Whole Cigarette Smoke in a Direct In Vitro Exposure System; Exp. Toxicol. Pathol. 57 (2006) 411-418.

529. Kasagi, S., K. Seyama, H. Mori, S. Souma, T. Sato, T. Akiyoshi, H. Suganuma, and Y. Fukuchi: Tomato Juice Prevents Senescence-Accelerated Mouse P1 Strain from Developing Emphysema Induced by Chronic Exposure to Tobacco Smoke; Am. J. Physiol. Lung Cell Mol. Physiol. 290 (2006) L396-L404. DOI: 10.1152/ajplung.00483.2004

530. Hoshino, Y., T. Nakamura, M. Mishima, J. Yodoi, and H. Nakamura: Neurotropin Demonstrates Cytoprotective Effects in Lung Cells Through the Induction of Thioredoxin-1; Am. J. Respir. Cell Mol. Biol. 37 (2007) 438-446.

531. Mori, H., N. Takashi, K. Ishitani, S. Kasagi, S. Souma, T. Akiyoshi, Y. Kodama, T. Mori, M. Kondo, S. Sasaki, A. Iwase, K. Takahashi, Y. Fukuchi, and K. Seyama: Phoshodiesterase 4 Inhibitior GPD-1116 Markedly Attenuates the Development of Cigarette Smoke-Induced Emphysema in SenescenceAccelerated Mice P1 Strain; Am. J. Physiol. Lung Cell Mol. Physiol. 294 (2008) L196-L204.

532. Sato, A., Y. Hoshino, T. Hara, S. Muro, H. Nakamura, M. Mishima, and J. Yodoi: Thioredoxin-1 Ameliorates Cigarette Smoke-Induced Lung Inflammation and Emphysema in Mice; J. Pharmacol. Exp. Ther. 325 (2008) 380-388.

533. Nakajima, Y., Y. Nakamura, W. Shigeeda, M. Tomoyasu, H. Deguchi, T. Tanita, and K. Yamauchi: The Role of Tumor Necrosis Factor- $\alpha$ and Interferon$\gamma$ in Regulating Angiomotin-Like Protein 1 Expression in Lung Microvascular Endothelial Cells; Allergol. Int. 62 (2013) 309-322.

534. Benner, J.F. and C.K. Keene: Condensate Preparation and Fractionation; Proc. Univ. Kentucky Tobacco and Health Res. Inst. - Tobacco and Health Workshop Conference, Report 3 (1972) pp. 185-190.

535. Dube, M.F. and C.R. Green: Methods of Collection of Smoke for Analytical Purposes; Rec. Adv. Tob.
Sci. 8 (1982) 42-102.

536. Rochus, W.: Grundsätzliche Überlegungen zur chemischen Zusammensetzung des Tabakrauches und seiner Abscheidungsprodukte [Fundamental Reflections on the Chemical Composition of Tobacco Smoke and its Deposition Products]; Beitr. Tabakforsch. 1 (1962) 155-164.

DOI: $10.2478 /$ cttr-2013-0024

537. Day, T.D.: Carcinogenic Action of Cigarette Smoke Condensate on Mouse Skin. An Attempt of a Quantitative Study; Brit. J. Cancer 21 (1967) 56-81.

538. Schüller, D., C.J. Drews, and H.P. Harke: Analytische Untersuchungen am Gasphasenkondensat des Cigarettenrauches [Analytical Examination of Gas Phase Condensate of Cigarette Smoke]; Beitr. Tabakforsch. 6 (1971) 84-88. DOI: $10.2478 /$ cttr-2013-0272

539. Elmenhorst, H. and H.P. Harke: Die präparative Gewinnung eines Gasphasen-Kondensates [The Preparative Production of a Gas Phase Condensate]; Z. Naturforsch. 23b (1968) 1271-1272.

540. Diels, O. and K. Alder: Synthesen in der hydroaromatischen Reihe. III Mitteilung. Synthese von Terpenen, Camphern, hydroaromatischen und heterocyclischen Systemen [Synthesis of Hydroaromatics. III Report. Synthesis of Terpenes, Camphor, Hydroaromatic and Heterocyclic Systems]; Ann. Chem. 470 (1929) 62.

541. Harke, H.P., C.J. Drews, and D. Schüller: Über das Vorkommen von Norbornenderivaten in Tabakrauchkondensaten [About the Presence of Norbornene Derivatives in Tobacco Smoke Condensates]; Tetrahedron Lett. 43 (1970) 3789-3790.

542. Einolf, W.N., R.N. Ferguson, J.F. Whidby, and J.F. DeBardeleben: Isolation and Indentification of 2,6Dimethyl- and 2,2,6-Trimethyl-4-piperidone, Artifacts Produced by the Reaction of Cigarette Smoke Condensate and Acetone; Beitr. Tabakforsch. Int. 9 (1978) 208-213. DOI: 10.2478/cttr-2013-0449

543. Nall, J.F.: Complexed Cyanide in Collected Cigarette Smoke; $20^{\text {th }}$ Tobacco Chemists' Research Conference, November 1-3, 1966, Winston-Salem, NC, USA.

544. Norman, V. and C.H. Keith: Nitrogen Oxides in Tobacco Smoke; Nature 205 (1965) 915-916.

545. Barkemeyer, H. and F. Seehofer: Zur Untersuchung der Gas-Dampfphase des Cigarettenrauches: 2. Mitteilung. Zur Bestimmung des Stickstoffmonoxides (NO) aus der Gasphase des Cigarettenrauches [On the Gas-Vapour Phase of Cigarette Smoke: 2. Report. On the Determination of the Nitric Oxide Content in the Gaseous Phase of Cigarette Smoke]; Beitr. Tabakforsch. 4 (1968) 278-282.

DOI: $10.2478 /$ cttr-2013-0197

546. Greig, J.O. and P.G. Hall: Thermal Oxidations of Nitric Oxide at Low Concentrations; Trans. Faraday Soc. 63 (1967) 655.

547. Sloan, C.H. and J.E. Kiefer: Determination of NO and $\mathrm{NO}_{2}$ in Cigarette Smoke from Kinetic Data; Tob. Sci. 13 (1969) 180-182.

548. Cooper, P.H. and R.B. Hege, Jr: The Oxidation of $\mathrm{NO}$ to $\mathrm{NO}_{2}$ in Cigarette Smoke; $32^{\text {nd }}$ Tobacco 
Chemists Research Conference, Montreal, Canada, $30^{\text {th }}$ October $-1^{\text {st }}$ November 1978.

549. British American Tobacco Group Research \& Development: Method - Determination of Nitric Oxide in Mainstream Smoke; edited March 31, 2008. Available at: http://www.bat-science.com/groupms/ sites/bat_9gvjxs.nsf/vwPagesWebLive/DO7AXGC L/\$FILE/medMD7DAEA9.pdf?openelement

550. Philippe, R.J. and E.J. Hackney: The Presence of Nitrous Oxide and Methyl Nitrite in Cigarette Smoke and Tobacco Pyrolysis Gases; Tob. Sci. 3 (1959) 139-143.

551. Vilcins, G. and J.P. Lephardt: Ageing Processes of Cigarette Smoke: Formation of Methyl Nitrite; Chem. Ind. (London) 22 (1975) 974-975.

552. Klus, H. and H. Kuhn: Die Bestimmung von Nitrophenolen im Tabakrauchkondensat (Vorläufige Ergebnisse) [The Determination of Nitrophenols in Tobacco Smoke Condensate (Preliminary Results)]; Fachl. Mitt. Österr. Tabakregie 15 (1974) 276-288.

553. Neurath, G., B. Pirmann, and H. Wichern: Zur Frage der N-Nitrosoverbindungen im Tabakrauch [On the N-Nitroso Compounds in Tobacco Smoke]; Beitr. Tabakforsch. 2 (1964) 311-319.

DOI: $10.2478 /$ cttr-2013-0084

554. Neurath, G., B. Pirmann, and M. Dünger: Identifizierung von N-Nitroso-Verbindungen and asymmetrischen Hydrazinen als 5-Nitro-2-HydroxyBenzal-Derivate and Anwendung im Mikromaßstab [Identification of N-Nitroso Compounds and Asymmetric Hydrazines as 5-Nitro-2-Hydroxy-Benzal Derivatives and Use on a Micro Scale]; Chem. Ber. 97 (1964) 1631-1638.

555. Neurath G., M. Dünger, and F.G. Pein: Interaction of Nitrogen Oxides, Oxygen and Amines in Gaseous Mixtures; in: Environmental N-Nitroso Compounds Analysis and Formation, edited by E.A. Walker, P. Bogovskii, and L. Griciute, IARC Scientific Publ. No. 14, International Agency for Research on Cancer, Lyon, France, 1976, pp. 215-225.

556. Mirvish, S.S., L. Wellcave, M. Eagen, and P. Shubik: Ascorbate-Nitrite Reaction: Possible Means of Blocking the Formation of Carcinogenic N-Nitroso Compounds; Science 177 (1972) 65-68.

557. Hoffmann, D., S.S. Hecht, I. Schmeltz, K.D. Brunnemann, and E.L. Wynder: New Separation Techniques for Classes of Smoke Compounds; Rec. Adv. Tob. Sci. 1 (1976) 97-122.

558. Grob, K.: Zur Gewinnung und Behandlung frischer Gasphase aus Cigarettenrauch [Isolation and Treatment of Fresh Gas Phase from Cigarette Smoke]; Beitr. Tabakforsch. 3 (1965) 243-250. DOI: $10.2478 /$ cttr-2013-0116

559. Mori, G. and C.H. Sloan: The Use of Cryogenic Temperature Gas Chromatography for the Determination of Carbon Monoxide and Carbon Dioxide in Cigarette Smoke; Beitr. Tabakforsch. 6 (1972) 178-181. DOI: $10.2478 /$ cttr-2013-0289

560. Newsome, J.R., V. Norman, and C.H. Keith: Vapor Phase Analysis of Tobacco Smoke; Tob. Sci. 9 (1965) 102-110.

561. Norman, V., J.R. Newsome, and C.H. Keith:
Smoking Machines for the Analysis of the Vapor Phase of Cigarette Smoke; Tob. Sci. 10 (1966) 216-221.

562. Koller, K.B., C.E. Thomas, M.E. Parrish, and D.E. Leyden: Puff by Puff Determination of Gas Phase Acetaldehyde, HCN, NO and CO Using FT-IR Spectroscopy; Philip Morris (1990). Available at: http://industrydocuments.library.ucsf.edu/tobacco/d ocs/yymf0131

563. Thomas, C.E. and K.B. Koller: Puff-by-Puff Mainstream Smoke Analysis by Multiplex Gas Chromatography-Mass Spectrometry; Beitr. Tabakforsch. Int. 19 (2001) 345-351. DOI: 10.2478/cttr-2013-0722

564. Vilcins, G.: Determination of Ethylene and Isoprene in the Gas Phase of Cigarette Smoke by Infrared Spectroscopy; Beitr. Tabakforsch. 8 (1975) 181-185. DOI: $10.2478 /$ cttr-2013-0377

565. Collins, P.F. and J.F. Williams: An Automated Procedure for CO in Cigarette Smoke, Using a 20Port Syringe Smoking Machine; Beitr. Tabakforsch. 7 (1973) 67-72 594). September 20, 2016. DOI: $10.2478 /$ cttr-2013-0311

566. Williams, T.B.: The Determination of Nitric Oxide in Gas Phase Cigarette Smoke by Non-Dispersive Infrared Analysis; Beitr. Tabakforsch. Int. 10 (1980) 91-99. DOI: 10.2478/cttr-2013-0474

567. Sloan, C.H., J.S. Lewis, and G.P. Morie: Computerization of the Gas Phase Analysis of Cigarette Smoke; Tob. Sci. 21 (1977) 57.

568. Cooperation Centre for Scientific Research Relative to Tobacco (CORESTA): CORESTA Recommended Method No. 5: Determination of Carbon Monoxide in the Mainstream Smoke of Cigarettes by NonDispersive Infrared Analysis; CORESTA, Paris, France, 1993. Available at: https:/www.coresta.org/ determination-carbon-monoxide-mainstream-smokecigarettes-non-dispersive-infrared-analysis-29139

569. Bates, W.W., R.B. Griffith, E.S. Harlow, M. Senkus, and H. Wakeham: Determination and Reporting of Total Particulate Matter, Water in Total Particulate Matter, and Nicotine in Cigarette Smoke; Tob. Sci. 12 (1968) 192-196.

570. International Organization for Standardization (ISO): International Standard ISO $10362-1: 2011$. Cigarettes - Determination of Water in Smoke Condensates. Part 1. Gas Chromatographic Method; ISO, Geneva, Switzerland, 2011. Available at: http:www.iso.org

571. International Organization for Standardization (ISO): International Standard ISO 10362-2:2011. Cigarettes - Determination of Water in Smoke Condensates. Part 2. Karl Fischer Method; ISO, Geneva, Switzerland, 2011. Available at: http:www.iso.org

572. International Organization for Standardization (ISO): International Standard ISO 10315:2008. Cigarettes Determination of Nicotine in Smoke Condensates Gas-Chromtographic Method; ISO, Geneva, Switzerland, 2008. Available at: http:www.iso.org

573. Cooperation Centre for Scientific Research Relative to Tobacco (CORESTA): CORESTA Recommended Method No. 63: Determination of Tobacco Specific Nitrosamines in Cigarette Mainstream Smoke - GC- 
TEA Method; CORESTA, Paris, France, 2005. Available at: https://www.coresta.org/determinationtobacco-specific-nitrosamines-cigarette-mainstreamsmoke-gc-tea-method-29186.html

574. Health Canada: Official Method T-103 - Determination of Benzo[a]pyrene in Mainstream Tobacco Smoke; Health Canada, Ottawa, Canada, 1999.

575. McConnell, W.V., R.C. Mumpower II, and G.P. Touey: Evaluation of Cigarette Filter Efficiency by Photofluorometry; Tob. Sci. 4 (1960) 56-62.

576. Burton, F.G., J.T. Veal, and D.W. Phelps: Quantitative Analysis of Cigarette Tars Using Fluorescent Spectroscopy; Paper presented at the American Chemical Society (ACS) Spring National Meeting, New Orleans, LA, USA, March 20-25, 1977.

577. Sloan, C.H. and J.G. Curran: Spectrophotometric Determination of Filtration Efficiency of Cigarette Filters; $33^{\text {rd }}$ Tobacco Chemists' Research Conference 1979, Lexington, KY, USA.

578. Thomas, C.E.: Automated Method for FTC Tar for Low Delivery Cigarettes Based Upon Fluorescence of TPM Extracts; Tob. Sci. 24 (1980) 64-68.

579. Borgerding, M.F., R.D. Hicks, J.E. Bodnar, D.M. Riggs, E.J. Nanni, G.W. Fulp, Jr, W.C. Hamling, Jr, and J.A. Giles: Cigarette Smoke Composition. Part 1. Limitations of FTC Method When Applied to Cigarettes That Heat Instead of Burn Tobacco; J. Assoc. Off. Anal. Chem. 73 (1990) 605-609.

580. Irby, R.M. Jr and E.S. Harlow: Cigarette Smoke I. Determination of Certain Vapor Phase Constituents; Tob. Sci. 3 (1959) 52-56.

581. International Organization for Standardization (ISO): International Standard ISO 8454:2009. Cigarettes Determination of Carbon Monoxide in the Vapour Phase of Cigarette Smoke - NDIR Method; ISO, Geneva, Switzerland, 2009. Available at: http:www.iso.org

582. Williamson, J.T., J.F. Graham, and D.R. Allman: The Modification of Cigarette Smoke by Filter Tips; Beitr. Tabakforsch. 3 (1965) 233-242. DOI: $10.2478 / \mathrm{cttr}-2013-0115$

583. Grob, K.: Gas Chromatography of Cigarette Smoke. Part III. Separation of the Overlap Region of Gas and Particulate Phase by Capillary Columns; J. Gas Chrom. 3 (1965) 52-65.

584. Grob, K.: Zur Gaschromatographie des Cigarettenrauches, 2. Teil: Verfeinerte Trennung mit Hilfe von Kapillarkolonnen [Gas Chromatography of Cigarette Smoke, Part II: Refined Separation Using Golay Columns]; Beitr. Tabakforsch. 1 (1962) 315-323. DOI: $10.2478 /$ cttr-2013-0039

585. Sakuma, H., M. Kusama, N. Shimojima, and S. Sugawara: Gas Chromatographic Analysis of the pNitrophenylhydrazones of Low Boiling Carbonyl Compounds in Cigarette Smoke; Tob. Sci. 22 (1976) 156-158

586. Borgerding, M.F., L.A. Milhous, Jr, R.D. Hicks, and J.A. Giles: Cigarette Smoke Composition. Part 2. Method for Determining Major Components in Smoke of Cigarettes that Heat Instead of Burn Tobacco; J. Assoc. Off. Anal. Chem. 73 (1990) 610 615.
587. Côté, F., J. Verreault, and F.K. St.Charles: Accuracy of Tar Yield Determination and Intense Smoking Regimes; $63^{\text {rd }}$ Tobacco Science Research Conference, 27-30 September 2009, Amelia Island, FL, USA.

588. Côté, F. and J. Verreault: Overestimation of Tar Yields at Canadian Intense Smoking Regime: Factors Affecting the Accuracy; Paper presented at CORESTA Congress, 12-16 September 2010, Edinburgh, Scotland, Abstr. SSPT38.

589. Ghosh, D. and C. Jeannet: An Improved Cambridge Filter Pad Extraction Methodology to Obtain More Accurate Water and “Tar" Values: In Situ Cambridge Filter Pad Extraction Methodology; Beitr. Tabakforsch. Int. 26 (2014) 38-49.

DOI: $10.2478 /$ cttr-2014-0008

590. Tolman, R.C., L.H. Reyerson, A.P. Brooks, and H.D. Smyth: An Electrical Precipitator for Analyzing Smokes; J. Am. Chem. Soc. 41 (1919) 587-589.

591. Eschle, K.: Beitrag zur vergleichenden Analyse der Wirkung von Zigarettenfiltern [Analytical Contribution to the Comparison of Effects of Cigarette Filters]; Mitt. Geb. Lebensmittelunters. Hyg. 49 (1958) 275-289.

592. Golaz, P., A. Girardet, and R. Regamey: Considérations sur la récupération des constituants de la fumée de cigarettes [Considerations on the Recovery of the Constituents of Cigarette Smoke]; Mitt. Geb. Lebensmittelunters. Hyg. 50 (1959) 18-39.

593. Kuhn, H. and J. Marek: Beitrag zur Bestimmung des Rauchkondensates von Filterzigaretten [Contribution to the Determination of Smoke Condensate of Filter Cigarettes]; Fachl. Mitt. Österr. Tabakregie 1 (1961), $1-6$.

594. Williamson, J.T. and D.R. Allman: The Distribution of Tobacco Smoke Constituents Between the Vapour and Particulate Phases; Beitr. Tabakforsch. 3 (1966) 590-596. DOI: $10.2478 /$ cttr-2013-0158

595. Bentley, H.R. and J.G. Burgan: Cigarette Condensate: Preparation and Routine Laboratory Estimation; Tobacco Manufacturers' Standing Comm., Research Paper No 4, $2^{\text {nd }}$ ed., 1961.

596. Verband der Cigarettenindustrie (VdC): Vergleichende Untersuchung der Rauchfallensysteme Cambridge Filter $(\mathrm{CF})=$ Glasfaserfilter und Elektrostatische Rauchfalle (EL) - für die analytische Erfassung der Tabakrauchdaten Kondensat und Rauchnikotin [Comparison of Two Smoke-Trapping Systems - Cambridge Filter $(\mathrm{CF})=$ Glass-Fiber Filter and Electrostatic Smoke Trap (EL) - for the Analytical Determination of Two Tobacco Smoke Constituents, Condensate and Smoke Nicotine]; Internal Document, VdC, Hamburg, Germany, 1979. BatesNo. 104577784-104577845. Available at: http://legacy. library.ucsf.edu/tid/bar25a99

597. Kalcher, K., A. Nitsch, R. Pietsch, and C. Jorde: Schwermetalle in Tabaken und Tabakrauch. I. Eine kompakte Laborrauchmaschine zum Abrauchen von Zigaretten und Zigarren [Heavy Metals in Tobacco and Tobacco Smoke. I. A Compact Laboratory Smoking Machine for Cigarettes and Cigars]; Fresenius Z. Anal. Chem. 332 (1988) 17-21.

598. Rhoades, C.B., Jr and R.T. White Jr: Mainstream 
Smoke Collection by Electrostatic Precipitation for Acid Dissolution in a Microwave Digestion System Prior to Trace Metal Determination; J. AOAC Int. 80 (1997) 1320-1331.

599. Perinelli, M.A. and N. Carugno: Determination of Trace Metals in Cigarette Smoke by Flameless Atomic Absorption Spectrometry; Beitr. Tabakforsch. Int. 9 (1978) 214-217. DOI: 10.2478/cttr-2013-0450

600. Ivičić, N., L. Tomić, and V.I Simeon: Cadmium and Lead in Cigarettes and Smoke Condensate; Arh. Hig. Rada. Toksikol. 36 (1985) 157-164.

601. Schneider, G. and V. Krivan: Multi-Element Analysis of Tobacco Smoke Condensate by Instrumental Neutron Activation Analysis and Atomic Absorption Spectrometry; Int. J. Environ. Anal. Chem. 53 (1993) 87-100.

602. Krivan, V., G. Schneider, H. Baumann, and U. Reus: Multi-Element Characterization of Tobacco Smoke Condensate; Fres. J. Anal. Chem. 348 (1994) 218-225.

603. Pailer, M. and H. Kuhn: Kurzer Bericht über das Vorkommen von Nickel im Zigarettenrauch [Short Report on the Presence of Nickel in Cigarette Smoke]; Fachl. Mitt. Österr. Tabakregie 4 (1963) 61-63.

604. McDaniel, R.L., K.M. Torrence, D.A. Self, and M.J. Chang: Determination of Mercury in Mainstream Cigarette Smoke by Conventional and Amalgamation Cold Vapor Atomic Absorption Spectrometry; Beitr. Tabakforsch. Int. 19 (2001) 267-276.

DOI: $10.2478 /$ cttr-2013-0713

605. Sneddon, J.: Electrostatic Precipitation Atomic Absorption Spectrometry; Appl. Spectrosc. 44 (1990) 1562-1565.

606. Sneddon, J.: Direct and Near-Real-Time Determination of Lead, Manganese and Mercury in Laboratory Air by Electrostatic Precipitation-Atomic Absorption Spectrometry; Anal. Chim. Acta 245 (1991) 203-206.

607. Mathewson, H.D.: The Jet Impaction Trap. Some Applications in the Collection and Fractionation of Tobacco Smoke Aerosols; Tob. Sci. 12 (1968) 41-49.

608. Osborne, J., S.S. Adamek, and M.E. Hobbs: Some Components of Gas Phase of Cigarette Smoke; Anal. Chem. 28 (1956) 211-215.

609. Philippe, R.J. and M.E. Hobbs: Some Components of Gas Phase of Cigarette Smoke; Anal. Chem. 28 (1956) 2002-2006.

610. Philippe, R.J., H. Moore, G. Honeycutt, and J.M. Ruth: Some Hydrocarbons of the Gas Phase of Cigarette Smoke; Anal. Chem. 36 (1964) 859-865.

611. Le Roy, D.J.: An Apparatus for the Low Temperature Fractionation of Small Gas Samples; Can. J. Research 28R (1950) 492-499.

612. Laurene, A.H., L.A. Lyerly, and G.W. Young: Direct Vapor Chromatographic Determination of Acetaldehyde, Acrolein, and Acetone in Cigarette Smoke; Tob. Sci. 8 (1964) 150-153.

613. Laurene, A.H. and B.A. Harbin: Improved Gas Chromatographic Determination of Acetaldehyde, Acrolein, and Acetone in Cigarette Smoke; Tob. Sci. 11 (1967) 37.
614. Horton, A.D. and M.R. Guerin: Determination of Acetaldehyde and Acrolein in the Gas Phase of Cigarette Smoke Using Cryothermal Gas Chromatography; Tob. Sci. 18 (1974) 19-22.

615. Grob, K.: Zur Gaschromatographie des Cigarettenrauches. 4. Teil Identifikation mit Hilfe des Massenspektrometers [Gas Chromatographic Analysis of Cigarette Smoke. Part 4 Mass Spectrometric Analysis]; Beitr. Tabakforsch. 3 (1966) 403-408. DOI: $10.2478 /$ cttr-2013-0133

616. Völlmin, J.A., I. Omura, J. Seibl, K. Grob, and W. Simon: Einsatz gas-chromatographischer Kolonnen hoher Trennleistung in direkter Kombination mit Massenspektrometer [Use of Gaschromatographic Columns with High Separation Efficiency in Direct Combination with Mass Spectrometer]; Helv. Chim. Acta 49 (1966) 1768-1778.

617. Dorsey, J.A., R.H. Hunt, and M.J. O’Neal: RapidScanning Mass Spectrometry - Continuous Analysis of Fractions From Capillary Gas Chromatography; Anal. Chem. 35 (1963) 511-515.

618. Ryhage, R., S. Wikstrom, and G.R. Waller: Mass Spectrometer Used as Detector and Analyzer for Effluent Emerging from Capillary Gas Liquid Chromatography Column; Anal. Chem. 37 (1965) 435-436.

619. De Souza, J.F. and M. Scherbak: The Effect of Glycerol Added to Tobacco on the Constituents of Cigarette Smoke; Analyst 89 (1964) 735-739.

620. Newman, R.H., W.L. Jones, and R.W. Jenkins: Automated Device for the Evaluation of Total Mainstream Cigarette Smoke; Anal. Chem. 41 (1969) 543-545.

621. Tobacco Advisory Council (U.K.): Standard Methods for the Analysis of Cigarette Smoke; Research Paper $11,3^{\text {rd }}$ edition, 1986. Available at: http://industry documents.library.ucsf.edu/tobacco/docs/hnjx0113

622. Pailer, M., W.J. Hübsch, and H. Kuhn: Untersuchungen der aliphatischen und aromatischen primären und sekundären Amine des Zigarettenrauches mit Hilfe der Gaschromatographie und Massenspektrometrie [Investigation of the Aliphatic and Aromatic Primary and Secondary Amines of Cigarette Smoke by Means of Gas Chromatography and Mass Spectrometry]; Fachl. Mitt. Österr. Tabakregie, 7 (1967) 109-118.

623. Health Canada: Official Method T-101 Determination of Ammonia in Mainstream Tobacco Smoke; Health Canada, Ottawa, Canada, 1999.

624. Health Canada: Official Method T-108 Determination of Mercury in Mainstream Tobacco Smoke; Health Canada, Ottawa, Canada, 1999.

625. Health Canada: Official Method T-104 Determination of Selected Carbonyls in Mainstream Tobacco Smoke; Health Canada, Ottawa, Canada, 1999.

626. Health Canada: Official Method T-116 - Determination of 1,3-Butadiene, Isoprene, Acrylonitrile, Benzene und Toluene in Mainstream Tobacco Smoke; Health Canada, Ottawa, Canada, 1999.

627. Health Canada: Official Method T-112 Determination of Pyridine, Quinoline and Styrene in 
Mainstream Tobacco Smoke; Health Canada, Ottawa, Canada, 1999.

628. Rochus, W.: Eine neue maschinelle Abrauch- und Rauchabscheidungsapparatur zur Gewinnung von "nativem" Tabakteer [A New Smoking Machine and Smoke Collection Device for Production of "Native" Tobacco-Tar]; Beitr. Tabakforsch. 1 (1962) 165-169. DOI: $10.2478 /$ cttr-2013-0025

629. Pailer, M., W. Hübsch, and H. Kuhn: Beitrag zur Bestimmung des Benzo[a]pyrens in Tabakrauchkondensaten [Contribution to the Determination of Benzo[a]pyrene in Tobacco Smoke Condensates]; Fachl. Mitt. Österr. Tabakregie, Sonderheft, 1965, $1-10$.

630. Barkemeyer, H.: Zur Untersuchung der Gas-DampfPhase des Cigarettenrauches. 1. Mitteilung: Der Rotationsabscheider, ein Gerät zur Absorption von Aerosolen, Dämpfen und Gasen in Flüssigkeiten [Investigation of the Gas/Vapor-Phase of Cigarette Smoke. $1^{\text {st }}$ Report: The Rotation Separator, an Apparatus for the Collection of Aerosols, Vapors and Gases in Liquids]; Beitr. Tabakforsch. 3 (1965) 91-93. DOI: 10.2478/cttr-2013-0101

631. Grob, K. and J.A. Völlmin: Analyse der „SemiVolatiles" aus Cigarettenrauch mit Hilfe einer Kombination von hochauflösender Gaschromatographie mit Massenspektrometrie [Analysis of the Semi-Volatiles with the Aid of Combination High Resolution Gaschromatography and Mass Spectrometry]; Beitr. Tabakforsch. 5 (1969) 52-57.

DOI: $10.2478 /$ cttr-2013-0216

632. Collins, P.F., N.M. Sarji, and J.F. Williams: An Automated Method for Determination of Hydrogen Cyanide in Cigarette Smoke; Tob. Sci. 14 (1970) $12-15$.

633. Kuhn, H. and C. Braun: Beitrag zur Blausäurebestimmung im Zigarettenrauch [Contribution to the Analysis of Hydrogen Cyanide in Cigarette Smoke]; Fachl. Mitt. Österr. Tabakregie 11 (1970) 175-180.

634. Mattina, C.F.: A Potentiometric Method for the Determination of Hydrogen Cyanide and Hydrogen Sulfide in Cigarette Smoke; Tob. Sci. 16 (1972) 113-114.

635. Vickroy, D.G., and G.L. Gaunt, Jr: Determination of Cyanide in Cigarette Smoke by a Cyanide IonSelective Electrode; Tob. Sci. 16 (1972) 22-25.

636. Reif, H.: Die Verwendung der ionenselektiven Elektrode zur Cyanidbestimmung im Zigarettenrauch [The Use of the Ion-Selective Electrode for Cyanide Determination in Cigarette Smoke]; Fachl. Mitt. Österr. Tabakregie 13 (1972) 220-224.
637. Zeldes, S.G. and A.D. Horton: Trapping and Determination of Labile Compounds in the Gas Phase of Cigarette Smoke; Anal. Chem. 50 (1978) 779-782.

638. Higgins, C.E., W.H. Griest, and G. Olerich: Application of Tenax Trapping to Analysis of Gas Phase Organic Compounds in Ultra-Low Tar Cigarette Smoke; J. Assoc. Off. Anal. Chem. 66 (1983) 1074-1083.

639. Behrsing, H., H. Raabe, J. Manuppello, B. Bombick, R. Curren, K. Sullivan, S. Sethi, R. Phipps, Y. Tesfaigzi, S. Yan, C. D’Ruiz, R. Tarran, S. Constant, G. Phillips, M. Gaça, P. Hayden, X. Cao, C. Mathis, J. Hoeng, A. Braun, and E. Hill: Assessment of In Vitro COPD Models for Tobacco Regulatory Science: Workshop Proceedings, Conclusions and Paths Forward for In Vitro Model Use; ATLA 44 (2016) 129-166.

640. Food and Drug Administration - Center for Tobacco Products: Research Priorities (January 2012). Available at: http://www.fda.gov/downloads/tobacco products/newsevents/ucm293998.pdf

641. Kamber, M.: Comparison of 4 Cytotoxicity Assays Performed Sequentially on the Same Cell Sample; Poster presented at the 37th Annual meeting of the European Environmental Mutagen Society, September 9-13, 2007, Basel, Switzerland.

642. Adamson J., L.E Haswell, G. Phillips, and M.D. Gaça: In Vitro Models of Chronic Obstructive Pulmonary Disease (COPD); in: Bronchitis, edited by Ignacio Martin-Loeches, InTech (2011) pp. 41-66. Available at: http://www.intechopen.com/books/ bronchitis

643. Cerulean Data Sheet: Electrostatic Precipitation Traps ETS 100/101/102 (2014). Available at: http://www. cerulean.com/product-services/tobacco/smokingmachines/smoking-accessories/ets 100101102

Corresponding author:

Lutz Müller

Stralsunder Str. 1

01109 Dresden, Germany

E-mail: lutz.mueller.dd@t-online.de 